\title{
$\pi-\pi$ Stacked Polymeric Micelles: From Optimization to Early Preclinical Evaluation
}

Mahsa Bagheri

2021 


\section{$\pi-\pi$ Stacked Polymeric Micelles: From Optimization to Early Preclinical Evaluation}

Mahsa Bagheri

Department of Pharmaceutics, Utrecht Institute for Pharmaceutical Sciences (UIPS), Faculty of Science, Utrecht University, Utrecht, the Netherlands
Author: Mahsa Bagheri
Cover design: Sandra Tukker
Printed by: Ridderprint | www.ridderprint.nl
ISBN: 978-90-393-7373-6

The research described in this thesis has been done within the Nanomed network. The funding was provided from the European Union's Horizon 2020 research and innovation programme Marie Sklodowska-Curie Innovative Training Networks (ITN) under grant No. 676137.

Printing of this thesis was partially financially supported by Utrecht Institute for Pharmaceutical Sciences (UIPS), Utrecht University, Utrecht, the Netherlands

(C) Mahsa Bagheri, 2021

All rights reserved. No parts of this thesis may be reproduced, stored in a retrieval system or transmitted in any form or by any means without permission of the author. 


\section{$\pi-\pi$ Stacked Polymeric Micelles: From Optimization to Early Preclinical Evaluation}

$\pi-\pi$ Gestapelde Polymere Micellen: Van Optimalisatie tot vroeg-preklinische evaluatie

(met een samenvatting in het Nederlands)

\section{Proefschrift}

ter verkrijging van de graad van doctor aan de Universiteit Utrecht

op gezag van de rector magnificus, prof.dr. H.R.B.M. Kummeling, ingevolge het besluit van het college voor promoties in het openbaar te verdedigen op

woensdag 26 mei 2021 des middags te 12.15 uur

door

\section{Mahsa Bagheri}

geboren op 6 januari 1988

te Mashhad, Iran 


\section{Promotor:}

Prof. dr. ir. W.E. Hennink

Copromotoren:

Dr. M. Heger

Dr. C.F. van Nostrum 
To my dear family 



\section{Contents}

Chapter 1

General Introduction

Chapter 2

Utility of Intravenous Curcumin Delivery Systems in the Context of In Vivo Pharmacokinetics and Anti-Cancer Pharmacodynamics

\section{Chapter 3}

The Effect of Formulation and Processing Parameters on the Size of mPEG-b-p(HPMA-Bz) Polymeric Micelles

\section{Chapter 4}

Tuning Size and Morphology of mPEG-b-p(HPMA-Bz) Copolymer Self-Assemblies Using Microfluidics

\section{Chapter 5}

In Vitro and In Vivo Studies on HPMA-based Polymeric Micelles Loaded with Curcumin

\section{Chapter 6}

In vitro Evaluation of Curcumin-loaded $\pi$ - $\pi$-Stacked Polymeric Micelles in a $2 \mathrm{D}$ monoculture and a 3D stroma-containing Pancreatic Tumor Model: an introductory study

\section{Chapter 7}

Summary and Discussion

\section{Appendices}

Nederlandse Samenvatting

Curriculum Vitae

List of Publications

Acknowledgement 



\section{Chapter 1}

\section{General Introduction}




\section{Nano-sized Drug Delivery Systems for Cancer Therapy}

Cancer, the unregulated growth of malignant cells, is one of the leading causes of death wordwide $^{1-2}$. The standard treatment modalities for cancer therapy are surgery, radiation, chemotherapy and, more recently, immunotherapy. Chemotherapy is still the primary strategy to treat cancer, especially for unresectable and metastatic tumors, both in the early and late stages of the disease and in combination with other treatments ${ }^{3-5}$. However, many chemotherapeutic agents suffer from poor aqueous solubility, limited therapeutic efficacy and off-target side-effects as a result of non-specific cytotoxicity ${ }^{6-8}$. Therefore, a variety of nano-sized drug delivery systems have been developed to improve the therapeutic efficacy of anticancer drugs ${ }^{9-16}$. Among them, polymeric based nanomedicine have gained considerable attention ${ }^{17-19}$. In general, they are based on synthetic or natural polymers which are degraded by hydrolytic processes (chemical or enzymatic action) and/or excreted by the kidneys and can improve the aqueous solubility and stability of drugs by physical entrapment or chemical conjugation. Also, drug delivery systems can prolong the circulation kinetics, which is essential for disposition of the loaded drugs in tumors exploiting the enhanced permeation and retention effect (EPR) and thereby improving the therapeutic efficacy. The EPR effect is based on pathophysiological properties of many solid tumors such as high angiogenesis, fenestrated blood capillaries and impaired lymphatic system, which results in the accumulation of nanoparticles and macromolecules in tumors ${ }^{20-22}$. In recent years, however, the importance and the existence of the EPR effect in human patients are under debate regarding its high heterogeneity, both inter- and intra-individually ${ }^{23-26}$. Therefore, more personalized therapies are demanded by applying strategies to monitor and predict nanomedicine accumulation and efficacy ${ }^{23,27-28}$.

It has been shown that the size of nanomedicines significantly impacts the therapeutic efficacy of loaded drugs. In recent years, many studies have been focused on understanding the effect of nanomedicine size on the efficacy of cancer treatment ${ }^{29-32}$. The circulation kinetics, tumor accumulation and penetration are size-dependent. Achieving prolonged circulation kinetics is essential for tumor targeting exploiting the EPR effect. Large-sized nanoparticles (>200 $\mathrm{nm}$ ) activate the complement system and have a higher clearance tendency by the mononuclear phagocytic system (MPS) and thereby show high liver and spleen accumulation ${ }^{29,33}$. Nanoparticles smaller than $\sim 250 \mathrm{~nm}$ are able to extravasate through the leaky tumor vasculature and accumulate in solid tumors ${ }^{32}$. Similarly, the vasculature permeability decreases with increasing the size of nanoparticles ${ }^{34}$. In preclinical research, nanomedicines $<100 \mathrm{~nm}$ have been shown to extravasate to a greater extent than larger particles and accumulate in tumors. Once extravasated, smaller nanoparticles penetrate more deeply into tumors as compared to the larger nanoparticles to increase the therapeutic effect of the loaded anticancer drugs ${ }^{10,30-31}$.

\section{Polymeric Micelles as Drug Delivery Systems}

Polymeric micelles are one of the most extensively investigated nanocarriers in the field of cancer therapy ${ }^{35-37}$. Polymeric micelles are based on amphiphilic block copolymers composed of a hydrophilic and hydrophobic block. Therefore, in an aqueous environment, they self-assemble into nano-size structures consisting of a hydrophobic core and a hydrophilic shell, mostly based on poly(ethylene glycol) (PEG). The size is usually less than $100 \mathrm{~nm}$, which is favorable for tumor accumulation via the EPR effect ${ }^{38-39}$. These structures 
form spontaneously above the critical micelle concentration (CMC), and they have lower CMC values than surfactant-based micelles ${ }^{40}$. Generally, poorly water-soluble drug molecules can be accommodated in the hydrophobic core of polymeric micelles, while the hydrophilic shell provides colloidal stability and stealth properties ${ }^{37,41-44}$. However, recently, stabilizing interactions between the hydrophilic block and loaded drugs have been reported to contribute to the solubilizing capacity polymeric micelles which highlights the complexity of the core-shell structure and the need for rational drug-carrier optimization ${ }^{45-46}$. Importantly, the physicochemical properties of polymeric micelles can be tailored by changing the chemical composition of the polymers, polymer molecular weight and the hydrophilic-hydrophobic ratio of the blocks ${ }^{35,37}$. As discussed above, size plays an important role in many biological phenomena such as circulation kinetics, clearance, extravasation through leaky vasculature, tumor penetration and mononuclear phagocytic system uptake. Therefore, in recent years, several studies have been conducted to tailor the size of the micelles and improve its effect on the efficacy of cancer therapy. Tuning the size of polymeric micelles can be achieved by modulating polymer properties and processing conditions ${ }^{47-48}$. For instance, Cabral et al. ${ }^{30}$ prepared 1,2-diaminocyclohexane-platinum(II) (DACHPt)loaded PEG- $b$-p(glutamic acid) (PEG- $b$-P(Glu)) polymeric micelles of different sizes based on spontaneous self-assembly in water resulting from the interaction of the platinum of DACHPt and the carboxylic moieties of PEG- $b-\mathrm{P}(\mathrm{Glu})$. The size of the micelles was controlled by addition of poly(glutamic acid) ( $\mathrm{p}(\mathrm{Glu})$ ) homopolymer to PEG- $b$ - $\mathrm{p}(\mathrm{Glu})$ copolymer. The size of micelles varied between 30 and $100 \mathrm{~nm}$, without homopolymer and at 0.3 homopolymer/copolymer molar ratio, respectively. The study showed that both micelles could penetrate into tumors that are highly permeable. In contrast, only polymeric micelles $<50 \mathrm{~nm}$ penetrated into tumors with poor permeability to result in antitumor effects.

The stability of micelles and their drug retention capability are important factors for prolonged circulation of the payloads, which can be provided by physical interactions ${ }^{49-51}$, chemical crosslinking of the core ${ }^{52}$ and by covalent coupling of the drug to the core of the micelles ${ }^{53}$. Physical crosslinking using $\pi$ - $\pi$ stacking is a promising method to improve the stability of drug-loaded micelles. This interaction is due to the electron configuration of aromatic conjugated systems and takes place between two aromatic moieties. $\pi-\pi$ Stacking between polymer chains containing monomers with aromatic rings and drug molecules with aromatic rings in their structures can improve the stability of the micelles and the retention of the drug, respectively ${ }^{50,54}$. For instance, recently, Shi et al. ${ }^{55-56}$ developed mPEG- $b$ p(HPMA-Bz) and showed considerable improvement in the stability, drug loading capacity and retention of paclitaxel in micelles based on these block copolymers. Similarly, docetaxel was efficiently loaded in these $\Pi$ electron-stabilized polymeric micelles up to a loading capacity of 23 weight $\%$. The micellar docetaxel formulation induced chemotherapeutic responses in multiple advanced-stage GI cancer mouse models and outperformed free docetaxel $^{57}$. Also, Hahn et al. ${ }^{58}$ studied the influence of $\pi-\pi$ stacking between small molecules and polymers to increase the drug loading and stability of poly(2-oxazoline)-based micelles by changing the aromatic content of the polymers and drug models. They observed that the drug loading decreased with increasing the relative aromatic amount in the polymer when paclitaxel with the lowest relative aromatic content was used. In contrast, the loading for curcumin having a higher relative aromatic content increased with increasing relative aromatic amount in the polymer. Also, the drug loading of schizandrin A with intermediate relative aromatic content was not influenced significantly by the aromatic content of the polymers. Furthermore, poly(2-phenyl-2-oxazoline) with the highest aromatic content showed a lower solubilization efficiency for curcumin and schizandrin A compared to poly(2- 
benzyl-2-oxazoline). The authors did not provide a conclusive explanation but corroborated that the latter observation could be due to the reduced molecular flexibility and close proximity of phenyl rings to the polymer backbone. Therefore, it was concluded that the interaction between the polymers and drugs is rather complicated and the effect should be examined for each carrier and drug combination.

\section{Nanoprecipitation Method to Prepare Drug-Loaded Polymeric Micelles}

There are various technologies for the preparation of polymeric based nanoparticles such as emulsion-based nanoprecipitation or direct dissolution methods ${ }^{59-60}$. Nanoprecipitation is a fast and straightforward method to prepare different nanostructures, such as polymeric micelles. By performing this approach in an appropriate way, tailorable polymeric micelle characteristics (e.g size and shape) can be achieved. Also, it is a simple strategy and can be easily applied in the upscale production of large batches ${ }^{61}$. Nanoprecipitation methods require that the solvent applied to dissolve the polymer and drug is miscible with the selected nonsolvent. Briefly, the amphiphilic block copolymer, in combination with the drug, is dissolved in a suitable organic solvent. Subsequently, the solution is added to a large amount of nonsolvent, almost exclusively water leading to the formation of nanoparticles. Next, the organic solvent is removed by either evaporation or dialysis ${ }^{62}$. The presence of the non-solvent water for the hydrophobic block induces supersaturation, resulting in spontaneous nuclei formation. It has been shown that the size of nanoparticles is dependent on the nucleation rate $^{63}$. To briefly explain, a low supersaturation state yields few stable nuclei while higher supersaturation increases the number of formed nuclei which in turn will result in smaller micelles. Obtaining a homogeneous supersaturation state demands a rapid mixing of the solvent and non-solvent. Therefore, the quality of the mixing process should be controlled efficiently ${ }^{62,64}$. In general, tailoring the size of micelles can be achieved by controlling the formulation and processing parameters (e.g. polymer molecular weight, concentration, addition rate, etc.) during the nanoprecipitation procedure ${ }^{47-48}$.

\section{Microfluidics to Prepare Drug-Loaded Polymeric Micelles}

Microfluidics is referred to as the technology that deals with and manipulates small volumes of fluids in microscale channels in a precise and controlled way. This platform has gained considerable attention in chemical engineering and pharmaceutical sciences for the production of drug-loaded nanoparticles ${ }^{65-69}$. Generally, it offers many advantages over bulk processing methods, such as providing a homogenous reaction environment, controlling highly robust and continuous processes, and maintaining controllable mixing rates. Microfluidics has been recognized as a highly reliable technology to control the selfassembly process of amphiphilic polymers in terms of size and morphology ${ }^{70}$. The mixing rate of the solvents in the microfluidic channels is drastically reduced compared to the macroscopic vessels used in batch mode as a result of small dimensions and thus higher surface to volume ratio ${ }^{71-72}$. The tunable mixing conditions critically impact kinetically controlled processes such as nanoprecipitation and thereby efficiently control the size and size distribution of the obtained nanoparticles ${ }^{73}$. Besides, microfluidics is based on a continuous flow process that can maintain the same mixing quality condition over time, avoiding batch to batch variation throughout the production. Therefore it offers great potential in achieving a reliable and upscalable production method for nanomedicines ${ }^{68,74-75}$. 


\section{Pharmacokinetics and Pharmacodynamics}

Pharmacokinetics (PK) encompasses the absorption, distribution, metabolism and excretion (ADME) of drugs ${ }^{76}$. In other words, it describes how the body affects drugs upon administration. Pharmacodynamics (PD) is the study of the physiological effects of drugs, through interaction with e.g, receptors, resulting in therapeutic and/or side effects ${ }^{76-78}$. The outcome of PK and PD studies determines the relationship between dose and effect. It is essential to study both PK and PD as neither of them alone can provide a complete understanding of the drug action ${ }^{79}$. Generally speaking, anticancer agents have poor pharmacokinetics showing rapid metabolism/clearance and non-specific tissue distribution that substantially impacts the therapeutic efficacy. Nanomedicines can improve the pharmacokinetics of drugs by prolonging the retention time in the body and changing tissue distribution among which tumor disposition, thus enhancing the therapeutic efficacy and reducing adverse side effects ${ }^{80-81}$. In general, the encapsulated drug is protected from degradation and metabolism as compared to the drug in its free form. The disposition of drugloaded nanoformulation depends on the nanocarrier and not on the drug provided that the drug-loaded nanoformulation is stable in the circulation. The released fraction of the drug is pharmacologically active and has the same metabolism and clearance route as the unformulated drug ${ }^{82}$. Also, the larger size of nanomedicines compared to the cut-off of renal filtration (approximately $5 \mathrm{~nm}$ ) decreases their clearance ${ }^{83}$. The extended circulation time of nanosized drug delivery systems allows higher accumulation in tumor tissue due to the EPR effect $^{84,80,85}$. Polymeric micelles, provided that they have sufficient stability and drug retention in the circulation, could increase the systemic exposure of many anticancer drugs. However, polymeric micelles have a dynamic nature and could be destabilized due to dilution in the circulation below $\mathrm{CMC}$ and/or due to binding of the micelle forming amphiphilic block copolymers to blood proteins upon administration ${ }^{86-88}$. Therefore the colloidal stability of the drug-loaded micelles should be enhanced as discussed earlier by either physical or chemical interactions to prevent dissociation of the micelles and/or premature drug release and finally benefit from the prolonged circulation kinetics and tissue distribution ${ }^{35}$. Currently, several polymeric micelle formulations are under clinical evaluation for the treatment of different types of cancer, showing promising results ${ }^{35,43}$. One very recent successful example is CPC634 (CriPec ${ }^{\circledR}$ Docetaxel), docetaxel covalently entrapped in a stabilized $65 \mathrm{~nm}$ sized core-crosslinked polymeric micelles, which is now under phase I and II clinical trials. The first-in-human trial demonstrated longer systemic circulation kinetics than the clinically used Taxotere formulation. The safety evaluation showed less neutropenia with skin toxicity as the main dose-limiting effect and early indications of efficacy have also been observed ${ }^{89-91}$.

\section{D Tumor Spheroids in Cancer Research}

The rate of success in translation of promising nanomedicines for cancer therapy into the clinic is relatively low despite the number of drug delivery systems developed and evaluated in preclinical models ${ }^{92-93}$. This is partly due to the fact that the cellular models used in the early-stage research, i.e. 2D-cell cultures, are not closely aligned to the patient tumor in clinical situation ${ }^{94}$. In $2 \mathrm{D}$ cell models, cells are in monolayer forms, attached to a solid surface without proper cell-cell and cell-matrix interactions that are present in tumors ${ }^{95}$. Threedimensional cell culture models such as spheroids more accurately represent the in vivo situation. They resemble tumor microenvironments in a better way, which potentially can lead to better predictive in vitro results. Also, 3D-spheroids are excellent substitutes for costly 
animal models in line with the strict regulation of reducing the number of animal testing in many countries ${ }^{96}$. Importantly, 3D spheroids more effectively facilitate nanomedicine screening as they can better display the penetration and distribution of therapeutic agents in the context of a complex 3D network ${ }^{96-98}$. In recent years, heterotype multicellular spheroids composed of cancer cells and stroma have been developed as better platforms since they resemble cellular heterogeneity and cancer tumor microenviroment ${ }^{97}$, 99 . For instance, Priwitaningrum et al. ${ }^{97}$ described tumor-stroma containing heterospheroids and demonstrated that this $3 \mathrm{D}$ co-cultured platform is very suitable for a thorough evaluation of the factors influencing the penetration of nanoparticles.

\section{Curcumin, a Natural Compound with Anticancer Activity}

Curcumin,[(1E,6E)-1,7-bis-(4-hydroxy-3-methoxyphenyl)-1,6-heptadiene-3,5-

dione/diferuloyl-methane], is a natural substance with a feature of being yellow to orange, extracted from the rhizome of the Curcuma longa. Traditionally the crude turmeric product has been used as a spice, dietary supplement and eastern traditional medicinal herb. The commercial turmeric product also contains two structurally related compounds, namely demethoxycurcumin and bisdemethoxycurcumin ${ }^{100-102}$. In recent years, several pharmacological activities of curcumin have been discovered against many chronic diseases through interference via different cellular pathways ${ }^{103-105}$. In particular, its pharmacological effects against cancer, inflammation, and oxidative stress have been the subject of extensive research ${ }^{106}$. The distinct characteristics of curcumin have both contributed to and challenged its application in cancer prevention and therapy. In contrast to most anticancer drugs, curcumin exhibits pleiotropic properties by inhibiting several vital pathways in cancer cells ${ }^{103}$. Also, its safety margin is relatively high, allowing administration of very high doses to patients ${ }^{107-108}$. However, its low aqueous solubility, low bioavailability after oral administration, chemical instability and rapid metabolism and clearance substantially compromise its use as a drug product against cancer as well as other life-threatening and chronic diseases ${ }^{102,109}$. The solubility of curcumin in acidic aqueous buffer of pH 5.0 is very low (around $11 \mathrm{ng} / \mathrm{mL})^{110}$. Also, the main degradation pathway of curcumin is by autoxidation and results in the formation of bicyclopentadione ${ }^{111-112}$. Due to these limitations, efforts have been made to improve the pharmacokinetics and stability of curcumin and thus prolonging curcumin exposure in the blood circulation. One such approach has been the design and development of nano-sized delivery systems loaded with curcumin. Several curcumin nanoformulations, including liposomes, polymeric nanoparticles and micelles are under investigation in both preclinical and clinical stages ${ }^{113-116}$.

\section{Aim and Outline of the Thesis}

The research described in this thesis has been done within the Nanomed network. This consortium aimed to train a new generation of multi-disciplinary nanotechnology experts with the vision of managing the successful translation of pharmaceutical innovations into clinically relevant therapeutic solutions. The funding was received from the European Union's Horizon 2020 research and innovation programme Marie Sklodowska-Curie Innovative Training Networks.

The aim of this thesis was to further develop and optimize the previously investigated formulation of polymeric micelles based on poly(ethylene glycol)- $b$-poly(N-2- 
benzoyloxypropyl methacrylamide) (mPEG- $b$-p(HPMA-Bz)) as nanocarriers for hydrophobic drugs in the framework of the thesis of Dr. Yang Shi ${ }^{117}$ so that it is potentially suitable for up-scale manufacturing processes and clinical translation. Also, this project was conducted in close collaboration with Dr. Jaleesa Bresseleers, an early-stage researcher in Nanomed consortium focusing on developing scalable processes for the manufacturing nanocarriers. Her thesis entitled "Development of scalable processes for the manufacture of nanocarriers" was published recently ${ }^{118}$.

Chapter 2 gives an overview of the literature regarding the pharmacokinetics of different curcumin nanoformulations upon intravenous administration in preclinical studies. In this chapter the pharmacokinetic parameters of free curcumin and curcumin nanoformulations are systematically compared. The extent to which nanocarriers improve the pharmacokinetics of curcumin is evaluated and discussed. Also, the relationship between the PK and PD is evaluated by correlating the therapeutic efficacy outcomes with curcumin exposures in both mouse and rat models.

Chapter 3 describes the influence of formulation and processing parameters on the size of mPEG- $b$-p(HPMA-Bz) polymeric micelles. The aim of the study was to tailor the size of the micelles in the range that is suitable for therapeutic application $(25-100 \mathrm{~nm})$. The effects of several parameters such as block copolymer molecular weight, hydrophilic to hydrophobic ratio, presence of homopolymer, rate of addition, the type of organic solvents used for the preparation of micelles and so on were comprehensively studied. The obtained polymeric micelles were thoroughly characterized using different advanced techniques, in particular, Dynamic Light Scattering (DLS), Multi-Angle Laser Light Scattering (MALLS), and CryoTransmission Electron Microscopy (Cryo-TEM) to investigate the parameters that influence micelle size.

In Chapter 4, the self-assembly of mPEG- $b$-p(HPMA-Bz) block copolymers was carried out in a microfluidic device. As explained in this Introduction, the nanostructures obtained in batch mode are sensitive and variable depending on the processing parameters. Therefore, the aim of the study was to investigate the effect of formulation and processing parameters on the size and morphology of mPEG- $b$-p(HPMA-Bz) nanoparticles using microfluidics.

In Chapter 5 mPEG- $b$-p(HPMA-Bz) polymeric micelles were used to improve the poor pharmacokinetics of curcumin. The stability of different curcumin micellar dispersions was comprehensively studied in buffer and plasma using asymmetrical flow field-flow fractionation (AF4). The most stable formulation was selected for in vivo studies and the pharmacokinetics of both curcumin and micelles was assessed in mice. Finally, an introductory therapeutic efficacy study was performed in Neuro2A-bearing mice.

In Chapter 6, the penetration and efficacy of curcumin-loaded micelles were studied in stroma-rich 3D heterospheroids. The micelles were formulated in two distinct size ranges, and the cytotoxicity of micelles loaded with curcumin and the penetration depth of fluorescently-labeled micelles were evaluated in 2D mono-culture and 3D heterospheroids, respectively.

Chapter 7 gives a summary of the main findings of this thesis and provides a discussion on future perspectives for further development and application of mPEG- $b$-p(HPMA-Bz) polymeric micelles for cancer therapy. 


\section{References}

1. Bray, F.; Ferlay, J.; Soerjomataram, I.; Siegel, R. L.; Torre, L. A.; Jemal, A., Global cancer statistics 2018: GLOBOCAN estimates of incidence and mortality worldwide for 36 cancers in 185 countries. Ca-Cancer J. Clin. 2018, 68 (6), 394-424.

2. Siegel, R. L.; Miller, K. D.; Jemal, A., Cancer statistics, 2019. Ca-Cancer J. Clin. 2019, 69 (1), 7-34.

3. Liu, H.; Lv, L.; Yang, K., Chemotherapy targeting cancer stem cells. Am. J. Cancer Res. 2015, 5 (3), 880-893.

4. Gustavsson, B.; Carlsson, G.; Machover, D.; Petrelli, N.; Roth, A.; Schmoll, H.-J.; Tveit, K.M.; Gibson, F., A Review of the evolution of systemic chemotherapy in the management of colorectal cancer. Clin. Colorectal Cancer 2015, 14 (1), 1-10.

5. Pérez-Herrero, E.; Fernández-Medarde, A., Advanced targeted therapies in cancer: Drug nanocarriers, the future of chemotherapy. Eur. J. Pharm. Biopharm. 2015, 93, 52-79.

6. Crawford, J.; Dale, D. C.; Lyman, G. H., Chemotherapy-induced neutropenia. Cancer 2004, 100 (2), 228-237.

7. Oun, R.; Moussa, Y. E.; Wheate, N. J., The side effects of platinum-based chemotherapy drugs: a review for chemists. Dalton Trans. 2018, 47 (19), 6645-6653.

8. Staff, N. P.; Grisold, A.; Grisold, W.; Windebank, A. J., Chemotherapy-induced peripheral neuropathy: A current review. Ann. Neurol. 2017, 81 (6), 772-781.

9. Bobo, D.; Robinson, K. J.; Islam, J.; Thurecht, K. J.; Corrie, S. R., Nanoparticle-based medicines: a review of FDA-approved materials and clinical trials to date. Pharm. Res. 2016, 33 (10), 2373-2387.

10. Hare, J. I.; Lammers, T.; Ashford, M. B.; Puri, S.; Storm, G.; Barry, S. T., Challenges and strategies in anti-cancer nanomedicine development: An industry perspective. Adv. Drug Delivery Rev. 2017, 108, 25-38.

11. Shi, J.; Kantoff, P. W.; Wooster, R.; Farokhzad, O. C., Cancer nanomedicine: progress, challenges and opportunities. Nat. Rev. Cancer 2017, 17 (1), 20-37.

12. Edgar, J. Y. C.; Wang, H., Introduction for design of nanoparticle based drug delivery systems. Curr. Pharm. Des. 2017, 23 (14), 2108-2112.

13. Wicki, A.; Witzigmann, D.; Balasubramanian, V.; Huwyler, J., Nanomedicine in cancer therapy: challenges, opportunities, and clinical applications. Journal of controlled release : official journal of the Controlled Release Society 2015, 200, 138-157.

14. van Elk, M.; Murphy, B. P.; Eufrásio-da-Silva, T.; O’Reilly, D. P.; Vermonden, T.; Hennink, W. E.; Duffy, G. P.; Ruiz-Hernández, E., Nanomedicines for advanced cancer treatments: Transitioning towards responsive systems. Int. J. Pharm. 2016, 515 (1), 132-164.

15. Wakaskar, R. R., Cancer therapy with drug delivery systems. J. Pharmacogenomics Pharmacoproteomics 2017, 8, 1-2.

16. Rodallec, A.; Fanciullino, R.; Lacarelle, B.; Ciccolini, J., Seek and destroy: improving PK/PD profiles of anticancer agents with nanoparticles. Expert Rev. Clin. Pharmacol. 2018, 11 (6), 599-610.

17. Masood, F., Polymeric nanoparticles for targeted drug delivery system for cancer therapy. Mater. Sci. Eng., C 2016, 60, 569-578.

18. Prabhu, R. H.; Patravale, V. B.; Joshi, M. D., Polymeric nanoparticles for targeted treatment in oncology: current insights. Int J Nanomedicine 2015, 10, 1001-1018. 
19. Begines, B.; Ortiz, T.; Pérez-Aranda, M.; Martínez, G.; Merinero, M.; Argüelles-Arias, F.; Alcudia, A., Polymeric nanoparticles for drug delivery: Recent developments and future prospects. Nanomaterials 2020, 10 (7).

20. Fang, J.; Nakamura, H.; Maeda, H., The EPR effect: Unique features of tumor blood vessels for drug delivery, factors involved, and limitations and augmentation of the effect. Adv. Drug Delivery Rev. 2011, 63 (3), 136-151.

21. Maeda, H.; Wu, J.; Sawa, T.; Matsumura, Y.; Hori, K., Tumor vascular permeability and the EPR effect in macromolecular therapeutics: a review. Journal of controlled release : official journal of the Controlled Release Society 2000, 65 (1), 271-284.

22. Torchilin, V., Tumor delivery of macromolecular drugs based on the EPR effect. Adv. Drug Delivery Rev. 2011, 63 (3), 131-135.

23. van der Meel, R.; Sulheim, E.; Shi, Y.; Kiessling, F.; Mulder, W. J. M.; Lammers, T., Smart cancer nanomedicine. Nat. Nanotechnol. 2019, 14 (11), 1007-1017.

24. Danhier, F., To exploit the tumor microenvironment: Since the EPR effect fails in the clinic, what is the future of nanomedicine? J Control Release 2016, 244 (Pt A), 108-121.

25. Shi, Y.; van der Meel, R.; Chen, X.; Lammers, T., The EPR effect and beyond: Strategies to improve tumor targeting and cancer nanomedicine treatment efficacy. Theranostics 2020, 10 (17), 7921-7924.

26. Lammers, T., Macro-nanomedicine: Targeting the big picture. Journal of controlled release : official journal of the Controlled Release Society 2019, 294, 372-375.

27. Lammers, T.; Rizzo, L. Y.; Storm, G.; Kiessling, F., Personalized nanomedicine. Clin. Cancer Res. 2012, 18 (18), 4889-94.

28. Golombek, S. K.; May, J.-N.; Theek, B.; Appold, L.; Drude, N.; Kiessling, F.; Lammers, T., Tumor targeting via EPR: Strategies to enhance patient responses. Adv. Drug Delivery Rev. 2018, 130, $17-38$.

29. Yu, W.; Liu, R.; Zhou, Y.; Gao, H., Size-tunable strategies for a tumor targeted drug delivery system. ACS Cent. Sci. 2020, 6 (2), 100-116.

30. $\quad$ Cabral, H.; Matsumoto, Y.; Mizuno, K.; Chen, Q.; Murakami, M.; Kimura, M.; Terada, Y.; Kano, M. R.; Miyazono, K.; Uesaka, M.; Nishiyama, N.; Kataoka, K., Accumulation of sub-100 nm polymeric micelles in poorly permeable tumours depends on size. Nat. Nanotechnol. 2011, 6 (12), 815823.

31. Wang, J.; Mao, W.; Lock, L. L.; Tang, J.; Sui, M.; Sun, W.; Cui, H.; Xu, D.; Shen, Y., The role of micelle size in tumor accumulation, penetration, and treatment. ACS Nano 2015, 9 (7), 71957206.

32. Sun, Q.; Ojha, T.; Kiessling, F.; Lammers, T.; Shi, Y., Enhancing tumor penetration of nanomedicines. Biomacromolecules 2017, 18 (5), 1449-1459.

33. Hoshyar, N.; Gray, S.; Han, H.; Bao, G., The effect of nanoparticle size on in vivo pharmacokinetics and cellular interaction. Nanomedicine 2016, 11 (6), 673-692.

34. Jain, R. K.; Stylianopoulos, T., Delivering nanomedicine to solid tumors. Nat. Rev. Clin. Oncol. 2010, 7 (11), 653-664.

35. Varela-Moreira, A.; Shi, Y.; Fens, M. H. A. M.; Lammers, T.; Hennink, W. E.; Schiffelers, R. M., Clinical application of polymeric micelles for the treatment of cancer. Mater. Chem. Front. 2017, $l(8), 1485-1501$.

36. Houdaihed, L.; Evans, J. C.; Allen, C., Overcoming the road blocks: advancement of block copolymer micelles for cancer therapy in the clinic. Mol. Pharmaceutics 2017, 14 (8), 2503-2517. 


\section{Chapter 1}

37. Cabral, H.; Miyata, K.; Osada, K.; Kataoka, K., Block copolymer micelles in nanomedicine applications. Chem. Rev. 2018, 118 (14), 6844-6892.

38. Rana, V.; Sharma, R., Chapter 5 - Recent advances in development of nano drug delivery. In Applications of targeted nano drugs and delivery systems, Mohapatra, S. S.; Ranjan, S.; Dasgupta, N.; Mishra, R. K.; Thomas, S., Eds. Elsevier: 2019; pp 93-131.

39. Fang, J.; Islam, W.; Maeda, H., Exploiting the dynamics of the EPR effect and strategies to improve the therapeutic effects of nanomedicines by using EPR effect enhancers. Adv. Drug Delivery Rev. 2020.

40. Kataoka, K.; Harada, A.; Nagasaki, Y., Block copolymer micelles for drug delivery: Design, characterization and biological significance. Adv. Drug Delivery Rev. 2012, 64, 37-48.

41. Deng, C.; Jiang, Y.; Cheng, R.; Meng, F.; Zhong, Z., Biodegradable polymeric micelles for targeted and controlled anticancer drug delivery: Promises, progress and prospects. Nano Today 2012, 7 (5), 467-480.

42. Mikhail, A. S.; Allen, C., Block copolymer micelles for delivery of cancer therapy: transport at the whole body, tissue and cellular levels. Journal of controlled release : official journal of the Controlled Release Society 2009, 138 (3), 214-223.

43. Cabral, H.; Kataoka, K., Progress of drug-loaded polymeric micelles into clinical studies. Journal of controlled release : official journal of the Controlled Release Society 2014, 190, 465-476.

44. $\quad$ Cagel, M.; Tesan, F. C.; Bernabeu, E.; Salgueiro, M. J.; Zubillaga, M. B.; Moretton, M. A.; Chiappetta, D. A., Polymeric mixed micelles as nanomedicines: Achievements and perspectives. Eur. J. Pharm. Biopharm. 2017, 113, 211-228.

45. Cao, C.; Zhao, J.; Chen, F.; Lu, M.; Khine, Y. Y.; Macmillan, A.; Garvey, C. J.; Stenzel, M. H., Drug-Induced Morphology Transition of Self-Assembled Glycopolymers: Insight into the DrugPolymer Interaction. Chemistry of Materials 2018, 30 (15), 5227-5236.

46. Haider, M. S.; Lübtow, M. M.; Endres, S.; Forster, S.; Flegler, V. J.; Böttcher, B.; Aseyev, V.; Pöppler, A.-C.; Luxenhofer, R., Think Beyond the Core: Impact of the Hydrophilic Corona on Drug Solubilization Using Polymer Micelles. ACS Applied Materials \& Interfaces 2020, 12 (22), 2453124543.

47. Li, X.; Iocozzia, J.; Chen, Y.; Zhao, S.; Cui, X.; Wang, W.; Yu, H.; Lin, S.; Lin, Z., From precision synthesis of block copolymers to properties and applications of nanoparticles. Angew. Chem., Int. Ed. 2018, 57 (8), 2046-2070.

48. Makino, A.; Hara, E.; Hara, I.; Ozeki, E.; Kimura, S., Size control of core-shell-type polymeric micelle with a nanometer precision. Langmuir 2014, 30 (2), 669-74.

49. Yuan, X.; Jiang, M.; Zhao, H.; Wang, M.; Zhao, Y.; Wu, C., Noncovalently connected polymeric micelles in aqueous medium. Langmuir 2001, 17 (20), 6122-6126.

50. Shi, Y.; Lammers, T.; Storm, G.; Hennink, W. E., Physico-chemical strategies to enhance stability and drug retention of polymeric micelles for tumor-targeted drug delivery. Macromol. Biosci. 2017, 17 (1), 1600160.

51. Kang, N.; Perron, M.-È.; Prud'homme, R. E.; Zhang, Y.; Gaucher, G.; Leroux, J.-C., Stereocomplex block copolymer micelles: Core-shell nanostructures with enhanced stability. Nano Lett. 2005, 5 (2), 315-319.

52. van Nostrum, C. F., Covalently cross-linked amphiphilic block copolymer micelles. Soft Matter 2011, 7 (7), 3246-3259.

53. Hu, Q.; Rijcken, C. J.; Bansal, R.; Hennink, W. E.; Storm, G.; Prakash, J., Complete regression of breast tumour with a single dose of docetaxel-entrapped core-cross-linked polymeric micelles. Biomaterials 2015, 53, 370-8. 
54. Zhuang, W.-R.; Wang, Y.; Cui, P.-F.; Xing, L.; Lee, J.; Kim, D.; Jiang, H.-L.; Oh, Y.-K., Applications of $\pi-\pi$ stacking interactions in the design of drug-delivery systems. Journal of controlled release : official journal of the Controlled Release Society 2019, 294, 311-326.

55. Shi, Y.; van der Meel, R.; Theek, B.; Oude Blenke, E.; Pieters, E. H. E.; Fens, M. H. A. M.; Ehling, J.; Schiffelers, R. M.; Storm, G.; van Nostrum, C. F.; Lammers, T.; Hennink, W. E., Complete regression of xenograft tumors upon targeted delivery of paclitaxel via $\Pi-\Pi$ stacking stabilized polymeric micelles. ACS Nano 2015, 9 (4), 3740-3752.

56. Shi, Y.; van Steenbergen, M. J.; Teunissen, E. A.; Novo, L.; Gradmann, S.; Baldus, M.; van Nostrum, C. F.; Hennink, W. E., Pi-pi stacking increases the stability and loading capacity of thermosensitive polymeric micelles for chemotherapeutic drugs. Biomacromolecules 2013, 14 (6), 1826-1837.

57. $\quad$ Liang, C.; Bai, X.; Qi, C.; Sun, Q.; Han, X.; Lan, T.; Zhang, H.; Zheng, X.; Liang, R.; Jiao, J.; Zheng, Z.; Fang, J.; Lei, P.; Wang, Y.; Möckel, D.; Metselaar, J. M.; Storm, G.; Hennink, W. E.; Kiessling, F.; Wei, H.; Lammers, T.; Shi, Y.; Wei, B., П electron-stabilized polymeric micelles potentiate docetaxel therapy in advanced-stage gastrointestinal cancer. Biomaterials 2021, 266, 120432 .

58. Hahn, L.; Lübtow, M. M.; Lorson, T.; Schmitt, F.; Appelt-Menzel, A.; Schobert, R.; Luxenhofer, R., Investigating the influence of aromatic moieties on the formulation of hydrophobic natural products and drugs in poly(2-oxazoline)-based amphiphiles. Biomacromolecules 2018, 19 (7), 3119-3128.

59. Pagels, R. F.; Edelstein, J.; Tang, C.; Prud'homme, R. K., Controlling and predicting nanoparticle formation by block copolymer directed rapid precipitations. Nano Lett. 2018, 18 (2), 11391144.

60. Reddy, B. P.; Yadav, H. K.; Nagesha, D. K.; Raizaday, A.; Karim, A., Polymeric micelles as novel carriers for poorly soluble drugs--A review. J. Nanosci. Nanotechnol. 2015, 15 (6), 4009-18.

61. Martínez Rivas, C. J.; Tarhini, M.; Badri, W.; Miladi, K.; Greige-Gerges, H.; Nazari, Q. A.; Galindo Rodríguez, S. A.; Román, R.; Fessi, H.; Elaissari, A., Nanoprecipitation process: From encapsulation to drug delivery. Int. J. Pharm. 2017, 532 (1), 66-81.

62. Lepeltier, E.; Bourgaux, C.; Couvreur, P., Nanoprecipitation and the "Ouzo effect": Application to drug delivery devices. Adv. Drug Delivery Rev. 2014, 71, 86-97.

63. D'Addio, S. M.; Prud'homme, R. K., Controlling drug nanoparticle formation by rapid precipitation. Adv. Drug Delivery Rev. 2011, 63 (6), 417-426.

64. Zhang, C.; Pansare, V. J.; Prud'homme, R. K.; Priestley, R. D., Flash nanoprecipitation of polystyrene nanoparticles. Soft Matter 2012, 8 (1), 86-93.

65. Zhang, J.; Yan, S.; Yuan, D.; Alici, G.; Nguyen, N.-T.; Ebrahimi Warkiani, M.; Li, W., Fundamentals and applications of inertial microfluidics: a review. Lab Chip 2016, 16 (1), 10-34.

66. Valencia, P. M.; Pridgen, E. M.; Rhee, M.; Langer, R.; Farokhzad, O. C.; Karnik, R., Microfluidic platform for combinatorial synthesis and optimization of targeted nanoparticles for cancer therapy. ACS Nano 2013, 7 (12), 10671-10680.

67. Chen, R.; Wulff, J. E.; Moffitt, M. G., Microfluidic processing approach to controlling drug delivery properties of curcumin-loaded block copolymer nanoparticles. Mol. Pharmaceutics 2018, 15 (10), 4517-4528.

68. Liu, D.; Zhang, H.; Fontana, F.; Hirvonen, J. T.; Santos, H. A., Current developments and applications of microfluidic technology toward clinical translation of nanomedicines. Adv. Drug Delivery Rev. 2018, 128, 54-83. 


\section{Chapter 1}

69. Xu, J.; Zhang, S.; Machado, A.; Lecommandoux, S.; Sandre, O.; Gu, F.; Colin, A., Controllable microfluidic production of drug-loaded PLGA nanoparticles using partially watermiscible mixed solvent microdroplets as a precursor. Sci. Rep. 2017, 7 (1), 4794.

70. Wang, L.; Sanchez, S., Self-assembly via microfluidics. Lab Chip 2015, 15 (23), 4383-4386.

71. Liu, D.; Zhang, H.; Fontana, F.; Hirvonen, J. T.; Santos, H. A., Microfluidic-assisted fabrication of carriers for controlled drug delivery. Lab Chip 2017, 17 (11), 1856-1883.

72. Liu, D.; Cito, S.; Zhang, Y.; Wang, C.-F.; Sikanen, T. M.; Santos, H. A., A versatile and robust microfluidic platform toward high throughput synthesis of homogeneous nanoparticles with tunable properties. Adv. Mater. 2015, 27 (14), 2298-2304.

73. Chiesa, E.; Dorati, R.; Modena, T.; Conti, B.; Genta, I., Multivariate analysis for the optimization of microfluidics-assisted nanoprecipitation method intended for the loading of small hydrophilic drugs into PLGA nanoparticles. Int. J. Pharm. 2018, 536 (1), 165-177.

74. Ding, S.; Anton, N.; Vandamme, T. F.; Serra, C. A., Microfluidic nanoprecipitation systems for preparing pure drug or polymeric drug loaded nanoparticles: an overview. Expert Opin. Drug Delivery 2016, 13 (10), 1447-60.

75. Martins, J. P.; Torrieri, G.; Santos, H. A., The importance of microfluidics for the preparation of nanoparticles as advanced drug delivery systems. Expert Opin. Drug Delivery 2018, 15 (5), 469-479.

76. Currie, G. M., Pharmacology, part 2: Introduction to pharmacokinetics. J. Nucl. Med. Technol. 2018, 46 (3), 221-230.

77. Currie, G. M., Pharmacology, part 1: Introduction to pharmacology and pharmacodynamics. J. Nucl. Med. Technol. 2018, 46 (2), 81-86.

78. Gabrielsson, J.; Green, A. R., Quantitative pharmacology or pharmacokinetic pharmacodynamic integration should be a vital component in integrative pharmacology. J. Pharmacol. Exp. Ther. 2009, 331 (3), 767.

79. Dingemanse, J.; Appel-Dingemanse, S., Integrated pharmacokinetics and pharmacodynamics in drug development. Clin. Pharmacokinet. 2007, 46 (9), 713-737.

80. Choi, Y. H.; Han, H.-K., Nanomedicines: current status and future perspectives in aspect of drug delivery and pharmacokinetics. J. Pharm. Invest. 2018, 48 (1), 43-60.

81. Cuong, N. V.; Hsieh, M. F., Recent advances in pharmacokinetics of polymeric excipients used in nanosized anti-cancer drugs. Curr. Drug Metab. 2009, 10 (8), 842-50.

82. Lucas, A. T.; Madden, A. J.; Zamboni, W. C., Formulation and physiologic factors affecting the pharmacology of carrier-mediated anticancer agents. Expert Opin. Drug Metab. Toxicol. 2015, 11 (9), 1419-1433.

83. Li, S.-D.; Huang, L., Pharmacokinetics and biodistribution of nanoparticles. Mol. Pharmaceutics 2008, 5 (4), 496-504.

84. Kobayashi, H.; Watanabe, R.; Choyke, P. L., Improving conventional enhanced permeability and retention (EPR) effects; what is the appropriate target? Theranostics 2013, 4 (1), 81-89.

85. Petschauer, J. S.; Madden, A. J.; Kirschbrown, W. P.; Song, G.; Zamboni, W. C., The effects of nanoparticle drug loading on the pharmacokinetics of anticancer agents. Nanomedicine 2015, 10 (3), 447-463.

86. Miller, T.; Rachel, R.; Besheer, A.; Uezguen, S.; Weigandt, M.; Goepferich, A., Comparative investigations on in vitro serum stability of polymeric micelle formulations. Pharm. Res. 2012, 29 (2), 448-59.

87. Kim, S.; Shi, Y.; Kim, J. Y.; Park, K.; Cheng, J. X., Overcoming the barriers in micellar drug delivery: loading efficiency, in vivo stability, and micelle-cell interaction. Expert Opin. Drug Delivery 2010, 7 (1), 49-62. 
88. Talelli, M.; Barz, M.; Rijcken, C. J.; Kiessling, F.; Hennink, W. E.; Lammers, T., Corecrosslinked polymeric micelles: Principles, Preparation, biomedical applications and clinical translation. Nano Today 2015, 10 (1), 93-117.

89. Atrafi, F.; Dumez, H.; Mathijssen, R. H. J.; Menke van der Houven van Oordt, C. W.; Rijcken, C. J. F.; Hanssen, R.; Eskens, F. A. L. M.; Schöffski, P., A phase I dose-escalation and pharmacokinetic study of a micellar nanoparticle with entrapped docetaxel (CPC634) in patients with advanced solid tumours. Journal of controlled release : official journal of the Controlled Release Society 2020, 325, 191-197.

90. Braal, C. L.; de Bruijn, P.; Atrafi, F.; van Geijn, M.; Rijcken, C. J. F.; Mathijssen, R. H. J.; Koolen, S. L. W., A new method for the determination of total and released docetaxel from docetaxelentrapped core-crosslinked polymeric micelles (CriPec $\left.{ }^{\circledR}\right)$ by LC-MS/MS and its clinical application in plasma and tissues in patients with various tumours. J. Pharm. Biomed. Anal. 2018, 161, 168-174.

91. Atrafi, F.; van Eerden, R. A. G.; van Hylckama Vlieg, M. A. M.; Oomen-de Hoop, E.; de Bruijn, P.; Lolkema, M. P.; Moelker, A.; Rijcken, C. J.; Hanssen, R.; Sparreboom, A.; Eskens, F. A. L. M.; Mathijssen, R. H. J.; Koolen, S. L. W., Intratumoral comparison of nanoparticle entrapped docetaxel (CPC634) with conventional docetaxel in patients with solid tumors. Clin. Cancer Res. 2020, 26 (14), 3537.

92. Venditto, V. J.; Szoka, F. C., Jr., Cancer nanomedicines: so many papers and so few drugs! Adv Drug Deliv Rev 2013, 65 (1), 80-8.

93. Ioannidis, J. P. A.; Kim, B. Y. S.; Trounson, A., How to design preclinical studies in nanomedicine and cell therapy to maximize the prospects of clinical translation. Nat. Biomed. Eng. 2018, 2 (11), 797-809.

94. Sant, S.; Johnston, P. A., The production of 3D tumor spheroids for cancer drug discovery. Drug Discovery Today: Technol. 2017, 23, 27-36.

95. Nath, S.; Devi, G. R., Three-dimensional culture systems in cancer research: Focus on tumor spheroid model. Pharmacol. Ther. 2016, 163, 94-108.

96. Lu, H.; Stenzel, M. H., Multicellular tumor spheroids (MCTS) as a 3D in vitro evaluation tool of nanoparticles. Small 2018, 14 (13), 1702858.

97. Priwitaningrum, D. L.; Blondé, J.-B. G.; Sridhar, A.; van Baarlen, J.; Hennink, W. E.; Storm, G.; Le Gac, S.; Prakash, J., Tumor stroma-containing 3D spheroid arrays: A tool to study nanoparticle penetration. Journal of controlled release : official journal of the Controlled Release Society 2016, 244 (Part B), 257-268.

98. Astashkina, A.; Grainger, D. W., Critical analysis of 3-D organoid in vitro cell culture models for high-throughput drug candidate toxicity assessments. Adv. Drug Delivery Rev. 2014, 69-70, 1-18.

99. Lazzari, G.; Couvreur, P.; Mura, S., Multicellular tumor spheroids: a relevant 3D model for the in vitro preclinical investigation of polymer nanomedicines. Polym. Chem. 2017, 8 (34), 4947-4969.

100. Sharma, R. A.; Gescher, A. J.; Steward, W. P., Curcumin: The story so far. European Journal of Cancer 2005, 41 (13), 1955-1968.

101. Kotha, R. R.; Luthria, D. L., Curcumin: Biological, pharmaceutical, nutraceutical, and analytical aspects. Molecules 2019, 24 (16), 2930.

102. Anand, P.; Kunnumakkara, A. B.; Newman, R. A.; Aggarwal, B. B., Bioavailability of curcumin: problems and promises. Mol. Pharmaceutics 2007, 4 (6), 807-818.

103. Heger, M.; van Golen, R. F.; Broekgaarden, M.; Michel, M. C., The molecular basis for the pharmacokinetics and pharmacodynamics of curcumin and its metabolites in relation to cancer. Pharmacol. Rev. 2014, 66 (1), 222-307. 


\section{Chapter 1}

104. Shanmugam, M. K.; Rane, G.; Kanchi, M. M.; Arfuso, F.; Chinnathambi, A.; Zayed, M. E.; Alharbi, S. A.; Tan, B. K.; Kumar, A. P.; Sethi, G., The multifaceted role of curcumin in cancer prevention and treatment. Molecules 2015, 20 (2), 2728-69.

105. Allegra, A.; Innao, V.; Russo, S.; Gerace, D.; Alonci, A.; Musolino, C., Anticancer activity of curcumin and its analogues: Preclinical and clinical studies. Cancer Invest. 2017, 35 (1), 1-22.

106. Yeung, A. W. K.; Horbańczuk, M.; Tzvetkov, N. T.; Mocan, A.; Carradori, S.; Maggi, F.; Marchewka, J.; Sut, S.; Dall'Acqua, S.; Gan, R.-Y.; Tancheva, L. P.; Polgar, T.; Berindan-Neagoe, I.; Pirgozliev, V.; Šmejkal, K.; Atanasov, A. G., Curcumin: Total-scale analysis of the scientific literature. Molecules 2019, 24 (7), 1393.

107. Vareed, S. K.; Kakarala, M.; Ruffin, M. T.; Crowell, J. A.; Normolle, D. P.; Djuric, Z.; Brenner, D. E., Pharmacokinetics of curcumin conjugate metabolites in healthy human subjects. Cancer Epidemiol., Biomarkers Prev. 2008, 17 (6), 1411-1417.

108. Lao, C. D.; Ruffin, M. T.; Normolle, D.; Heath, D. D.; Murray, S. I.; Bailey, J. M.; Boggs, M. E.; Crowell, J.; Rock, C. L.; Brenner, D. E., Dose escalation of a curcuminoid formulation. BMC Complementary Altern. Med. 2006, 6 (1), 10.

109. Dei Cas, M.; Ghidoni, R., Dietary curcumin: correlation between bioavailability and health potential. Nutrients 2019, 11 (9), 2147.

110. Tønnesen, H. H.; Másson, M.; Loftsson, T., Studies of curcumin and curcuminoids. XXVII. Cyclodextrin complexation: solubility, chemical and photochemical stability. Int. J. Pharm. 2002, 244 (1), 127-135.

111. Naksuriya, O.; van Steenbergen, M. J.; Torano, J. S.; Okonogi, S.; Hennink, W. E., A kinetic degradation study of curcumin in its free form and loaded in polymeric micelles. AAPS J. 2016, 18 (3), 777-787.

112. Schneider, C.; Gordon, O. N.; Edwards, R. L.; Luis, P. B., Degradation of curcumin: from mechanism to biological implications. J. Agric. Food Chem. 2015, 63 (35), 7606-7614.

113. Keitaro, S., Curcumin towards nanomedicine. Recent Pat. Nanomed. 2012, 2 (2), 133-145.

114. Peng, J.-R.; Qian, Z.-Y., Drug delivery systems for overcoming the bioavailability of curcumin: not only the nanoparticle matters. Nanomedicine 2014, 9 (6), 747-750.

115. Naksuriya, O.; Okonogi, S.; Schiffelers, R. M.; Hennink, W. E., Curcumin nanoformulations: A review of pharmaceutical properties and preclinical studies and clinical data related to cancer treatment. Biomaterials 2014, 35 (10), 3365-3383.

116. Yallapu, M. M.; Nagesh, P. K. B.; Jaggi, M.; Chauhan, S. C., Therapeutic applications of curcumin nanoformulations. AAPS J. 2015, 17 (6), 1341-1356.

117. Shi, Y. Polymeric micelles for drug delivery from synthesis to in vivo studies. Ph.D. thesis, Utrecht University, The Netherlands, 2014, ISBN: 978-90-393-6219-8.

118. Bresseleers, J. Development of scalable processes for the manufacture of nanocarriers. Ph.D. thesis, TU Eindhoven, The Netherlands, 2020, ISBN: 978-90-356-5150-7. 




\section{Chapter 2}

\section{Utility of Intravenous Curcumin Delivery Systems in the Context of In Vivo Pharmacokinetics and Anti-Cancer Pharmacodynamics}

Mahsa Bagheri ${ }^{1}$, Cornelus F. van Nostrum ${ }^{1}$, Robbert Jan Kok ${ }^{1}$, Gert Storm ${ }^{1}$, Wim E. Hennink $^{1}$, Michal Heger ${ }^{1,2}$

${ }^{I}$ Department of Pharmaceutics, Utrecht Institute for Pharmaceutical Sciences (UIPS), Utrecht University, 3508 TB Utrecht, the Netherlands

${ }^{2}$ Department of Pharmaceutics, Jiaxing Key Laboratory for Photonanomedicine and Experimental Therapeutics, College of Medicine, Jiaxing University, Jiaxing 314001, Zhejiang, P. R. China

Manuscript in preparation 


\begin{abstract}
Curcumin is a natural compound that is under investigation as a potential anti-cancer agent. However, its low aqueous solubility and extensive biotransformation by the intestine and liver as well as rapid renal excretion account for poor bioavailability and pharmacokinetics, which hampers its pharmaceutical application. Considerable efforts have been made to improve curcumin pharmacokinetics and prolong its exposure to diseased tissues by formulating it in nano-sized delivery systems. In this review, the pharmacokinetic profiles of intravenously administrated curcumin in free form and as nanoformulation in two preclinical animal models (mice and rats) are comprehensively analyzed. Thus, the impact of the variable absorption step on bioavailability is excluded providing a more precise overview of curcumin circulation kinetics and therapeutic efficacy. Interestingly, the results indicate that the majority of the nanoformulations improves the area under the curve (AUC) of curcumin only slightly between 1.3-5 folds higher than the free form. This means that curcumin is not well retained in the particles and thus they essentially act as solubilizers for this compound. Also, in studies which report both pharmacokinetics (PK) and pharmacodynamic (PD) data, curcumin nanoformulations result in stronger tumor growth inhibition than the free form in line with the slightly improved pharmacokinetics. A negative trend is observed between the total injected dose and relative tumor growth inhibition (\% TGI) in treatment groups receiving curcumin nanoformulations and free curcumin, possibly due to tumor inhibition at higher dose regimen even for free curcumin with unfavorable PK. Furthermore, notable PD effects are not exclusively observed for the more stable nanoformulations and thus solubilization is sufficient for tumor growth inhibition. This review sheds light onto the current status of nanosized delivery systems for improving curcumin PK, highlights common weaknesses in study designs and brings the reader's attention to important factors (yet often neglected) affecting curcumin pharmacokinetics.
\end{abstract}

Keywords: drug delivery; nanomedicine; nanocarrier; micelles; nanoparticles, ADME, cancer therapy 


\section{Introduction}

Curcumin is a polyphenolic phytochemical derived from the rhizome of Curcuma longa. The crude root (turmeric) traditionally serves as a spice and dietary supplement ${ }^{1-2}$. Curcumin, the principal bioactive constituent in turmeric, is used for the prevention and treatment of numerous diseases and conditions, owing to its advantageous pharmacological properties ${ }^{3-7}$ and clinical safety profile ${ }^{8-9}$. The complete curcumin research spectrum is presented in Figure 1. Curcumin-related research has drastically intensified over the last decade, attesting to its potential utility as an active pharmaceutical ingredient.

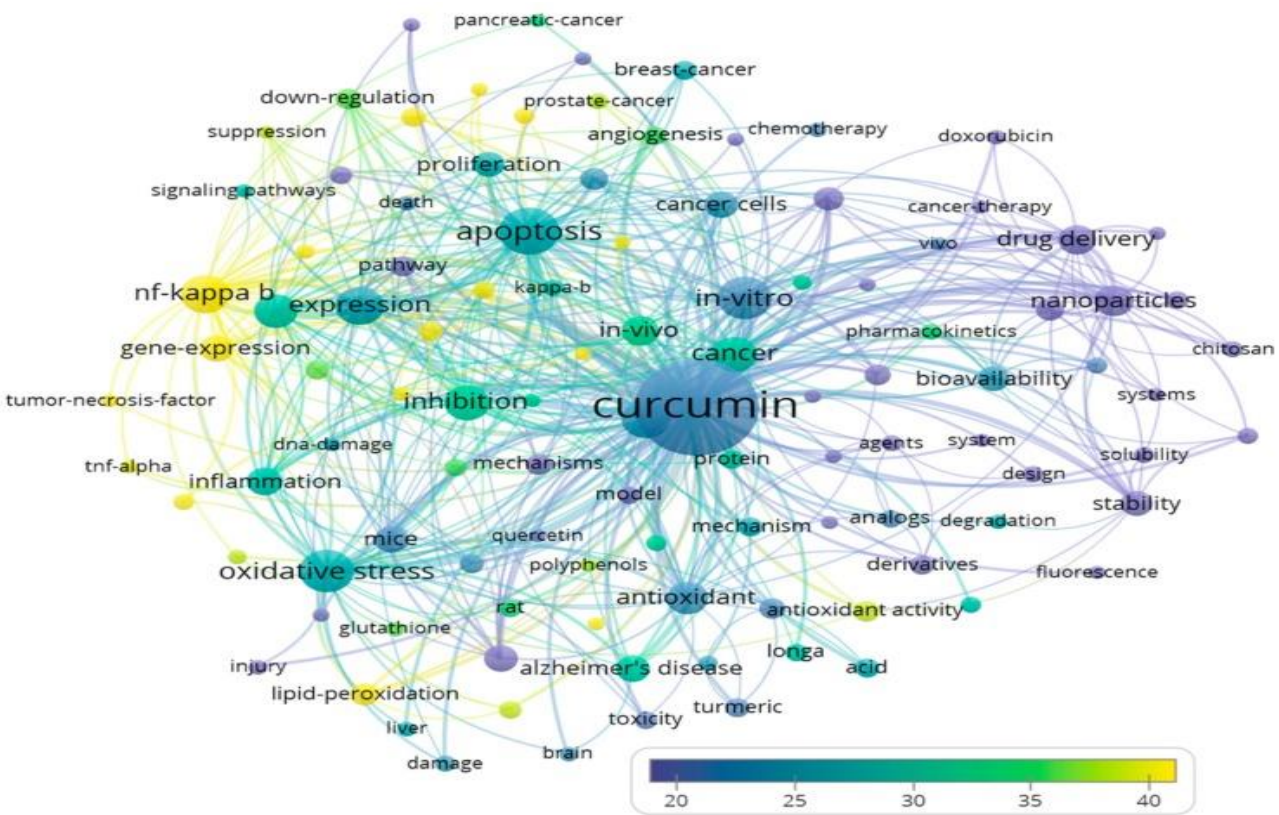

Figure 1. Bubble map of thematic keywords assembled from 18,036 publications on curcumin, showing most profound research interest in chronic disease fields that encompass oxidative stress, inflammation, and cancer as well as drug delivery systems and nanotechnology. Artwork reproduced with permission from ${ }^{10}$.

Curcumin is widely investigated as a cancer therapeutic in light of its apoptosis-inducing effects in hyperproliferative cells $\mathrm{s}^{11-14}$. However, the compound is pharmacodynamically (PD) fierce but pharmacokinetically (PK) feeble when it comes to treatment of cancer ${ }^{6}$. Curcumin inhibits more than 40 vital metabolic pathways in malignant cells as a result of pleiotropic interactions with biomolecules, ultimately causing apoptotic cell death ${ }^{15}$. Non-to-low proliferative healthy cells remain largely unafflicted ${ }^{16-18}$. Although proof-of-concept regarding curcumin's anti-cancer effects has been abundantly provided in mouse models of various types of human cancer ${ }^{19-20}$, the results have suffered translationally insofar as no notable therapeutic benefits have materialized in the clinical oncological setting ${ }^{6,21}$. Two main reasons lie at the basis of this disconnect between preclinical and clinical outcomes. First, many of the animal studies have been focused on systemically injected curcumin, while most clinical studies hitherto have been conducted with orally dosed curcumin. Oral dosing 
gives rise to the second reason, which is that oral curcumin is associated with dismal bioavailability ${ }^{22-23}$ due to poor intestinal absorption, extensive first-pass metabolism (phases I-III in enterocytes), degradation in pH-neutral aqueous medium, and second pass metabolism (phases I-III in hepatocytes) ${ }^{6}$. Consequently, the plasma levels achieved with intravenous infusion - those that account for the favorable PD in vivo - can never be reached outside the enterohepatic circulation with orally administered curcumin. Orally dosed curcumin is therefore less propitious against cancer in humans as it is for non-oncological indications ${ }^{7}$.

Considerable efforts have been invested in improving the PK profile of curcumin to improve PD in humans, including chemical modifications ${ }^{24-25}$, co-administration with P-glycoprotein inhibitors such as piperine ${ }^{26-28}$, and first pass circumventive approaches that require encapsulation into nanoparticulate delivery systems for intravenous administration ${ }^{29-31}$. The latter methods have been instrumental in improving the PK of different hydrophobic drugs ${ }^{32-}$ ${ }^{35}$, of which several have been approved for clinical use by regulatory agencies ${ }^{36}$. Nanoencapsulation of curcumin stabilizes curcumin in aqueous solution ${ }^{37}$ and render the compound water-soluble properties. Nanoformulations for curcumin hence warrant close scrutiny, especially given the previous successes with other drugs.

This study therefore addressed the following questions: (1) does intravenously administered nanoformulated curcumin improve PK compared to intravenously administered free curcumin; (2) which nanoformulations are associated with the greatest improvement in PK; and (3) do improved PK translate to improved PD in murine models of human cancer?

\section{Data Curation and Analysis}

Readers should note that references to supplemental material are indicated with prefix ' $\mathrm{S}$ ' (for example Figure S1 or section S3).

PK data were collected from mouse and rat studies in which intravenously administered curcumin nanoformulations had been compared with free curcumin. It is remarked that plasma extraction and curcumin quantification methods differed between the studies. Extraction was chiefly performed by protein precipitation with organic solvent (e.g., acetonitrile and methanol) ${ }^{38-52}$ or liquid phase extraction with ethyl acetate ${ }^{53-61}$. Curcumin was quantified by absorbance, fluorescence-measurements, or mass spectrometry. Almost all studies employed a chromatography system coupled to a spectroscopic detector, while a handful of studies used a cuvette-based or plate reader spectrometer $^{39,44,47,50-51}$.

For the reproduction of curcumin plasma concentration-time curves, mouse and rat studies were selected where the PK of free curcumin could be relatively accurately extrapolated from the respective figure $(n=15)$. The figures were imported into Adobe Photoshop from the PDF version of the publication at 600 dpi resolution and lines were protracted from the data point to the $y$-axis and $x$-axis to guestimate the curcumin concentration at a given time point, respectively. Time points were verified by cross-referencing with the methods section where available. Data were normalized to the plasma concentration at the earliest time point and plotted in GraphPad Prism (GraphPad Software, San Diego, CA, USA). In a separate analysis, normalized plasma concentrations were fitted with a two-phase decay fit function to reflect distribution (KFast segment of the curve) and clearance (KSlow segment of the curve). Fitting was performed on the entire measurement interval, which in some studies 
extended to $24 \mathrm{~h}$ post-injection. Given the rapid decay in curcumin plasma concentration, only the first $4 \mathrm{~h}$ post-injection are presented. Eleven of the 15 studies conformed to this model and yielded a goodness of fit $\left(\mathrm{R}^{2}\right)$ value of $\geq 0.9970$.

The plasma curcumin concentration over time is typically analyzed by non-compartmental and compartmental models. Among the common PK parameters, the maximum concentration $\left(\mathrm{C}_{\max }\right)$, area under the curve (AUC), and circulation half-life $\left(\mathrm{t}_{1 / 2}\right)$ are frequently reported. Conversely, only a few studies report clearance $(\mathrm{CL})$ and distribution volume $\left(\mathrm{V}_{\mathrm{d}}\right)$, even though $C L$ and $V_{d}$ can be calculated from the plasma concentration-time curve ${ }^{62}$. In combination with AUC and $t_{1 / 2}$, these parameters indicate how quickly a compound is eliminated and reflect the propensity of the drug to stay in the circulation or distribute to other tissue compartments ${ }^{63}$. The CL and $\mathrm{V}_{\mathrm{d}}$ were calculated using the data available for AUC and $\mathrm{t}_{1 / 2}$ in instances where CL and $\mathrm{V}_{\mathrm{d}}$ were not reported (see section S2 and Figure $\mathrm{S} 1$, for more detailed information). To enable interstudy comparative analysis of PK parameters, values were converted to harmonize the units and the AUCs were subsequently normalized to the administered curcumin dose.

\section{Nanoformulations Improve Multiple Curcumin Pharmacokinetics Parameters Compared to Non-Formulated, Free Curcumin}

\subsection{Pharmacokinetics of Intravenously Administered Free Curcumin}

Representative PK profiles of free curcumin in mice and rats are depicted in Figure 2. Plasma concentration kinetics follow a biphasic pattern that is characterized by a rapid concentration decay due to biodistribution and a slower decay due to clearance (Figure S1). In fact, more than $50 \%$ of the injected dose is no longer retrievable from plasma $10 \mathrm{~min}$ after administration. Curcumin undergoes renal and hepatobiliary clearance ${ }^{40,64-66}$ and biodistributes to multiple organs, including the liver, kidneys, lungs, spleen, and brain ${ }^{40,47,53}$ The switch from distribution to clearance typically occurs between 20-30 min post-injection, at which point only $13 \pm 10 \%$ (mean $\pm \mathrm{SD}, \mathrm{n}=14$ ) of the injected dose remains in the circulation that gradually dissipates during the course of the subsequent $3.5 \mathrm{~h}$. 
A

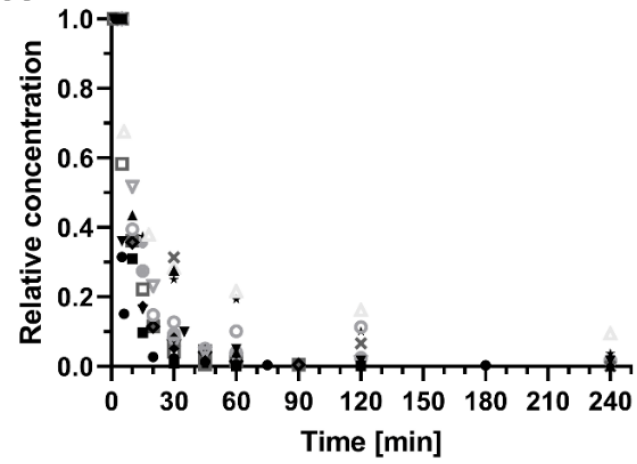

B

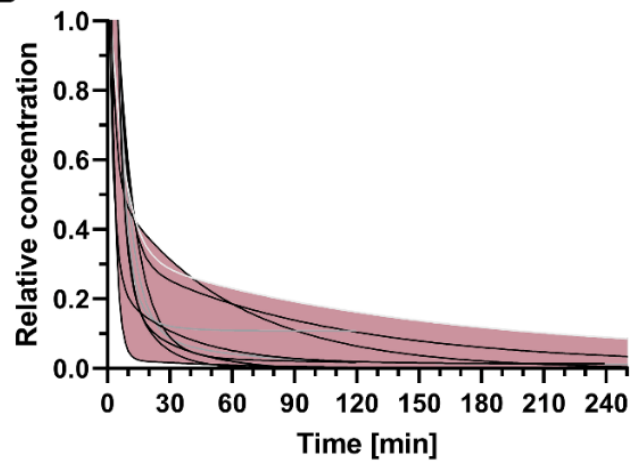

Figure 2. Pharmacokinetics of free curcumin following intravenous administration in mice and rats as reported in literature. The $x$-axis and $y$-axis data were extracted from published figures and verified from the text. Plasma concentrations were normalized to the concentration measured at the earliest time point. Normalized concentrations are provided in $(\mathbf{A})$ as a function of circulation time. Data compiled from 15 studies ${ }^{41,46-47,49,51-53,57-58,60-61,67-70}$. The points were fitted with a two-phase decay fit function to reflect distribution (fast phase) and clearance (slow phase) (B). Eleven studies conformed to this PK model $\left(\mathrm{R}^{2} \geq 0.9970\right)^{41,47,49,51-53,57-58,61,69-70}$. The maximum and minimum concentration comprise the outer bounds of the pink region. Compartmental deflection generally occurred between 10 and 30 min following intravenous administration.

Table 1. Descriptive statistics of normalized AUC $(\mu \mathrm{g} \bullet \mathrm{h} / \mathrm{L}) /(\mathrm{mg} / \mathrm{kg})$ of free curcumin and curcumin nanoformulations in mice and rats.

\begin{tabular}{ccccc}
\hline & \multicolumn{2}{c}{ Free curcumin } & \multicolumn{2}{c}{ Curcumin nanoformulations } \\
\cline { 2 - 5 } & Mice & Rats & Mice & Rats \\
\hline Number of studies & 7 & 23 & 8 & 24 \\
Minimum & 36 & 1 & 62 & 8 \\
Maximum & 4,075 & 167,000 & 149,705 & 632,000 \\
Median & 73 & 170 & 3,944 & 714 \\
Mean & 1,260 & 9526 & 22,107 & 36,395 \\
SEM & 658 & 7412 & 18,291 & 26,996 \\
\hline
\end{tabular}

Abbreviations: SEM, standard error of the mean

Note: The circulating blood volume is $78-80 \mathrm{~mL} / \mathrm{kg}$ in mice and $50-70 \mathrm{~mL} / \mathrm{kg}$ in rats ${ }^{71}$. Data assembled from $^{38,40-47,49-61,67-70,72-77}$. Statistical analysis was not significant with $\mathrm{P}$ value $>0.05$ (Kruskal-Wallis test with Dunn's post-hoc correction).

It is important to point out two considerations. First, inter-species differences notwithstanding, the administered dose, type of solvent/vehicle, experimental design, and analytical method may differentially affect PK parameters ${ }^{78}$. This is illustrated by the rather wide relative concentration range per time point as presented in Figure 2. Some of the solvents/solubilizers that were used are micelle-forming surfactants such as Kolliphore and Tween. The consequences of these factors on PK are further addressed in section S3.1. Second, with the therapeutic efficacy of intravenously administered curcumin being so low, it is not difficult to fathom how therapeutically impotent orally dosed curcumin is in oncological patients. Bioavailability and therefore systemic concentrations are significantly hampered by the aforementioned absorption and metabolism issues (section 1) and ultimately yield systemic concentrations that are utterly futile in an oncotherapeutic setting. However, for certain non-oncological indications (such as systematic inflammation, oxidative stress, etc.), the achieved plasma levels of oral curcumin are clinically adequate ${ }^{7}$. 


\subsection{Nano-Encapsulation of Curcumin Improves the Measured $\mathbf{C}_{\max }$}

For the analysis of $\mathrm{C}_{\max }, 27$ studies were included in which free and nano-encapsulated curcumin were administered intravenously into mice and rats at equimolar curcumin doses. For intravenously administered compounds, the measured $\mathrm{C}_{\max }$ is the highest concentration of compound in plasma detected immediately after injection and in theory approximates the injected dose per $\mathrm{mL}$ blood when the interval between injection and blood sampling narrows. The steep distribution phase of free curcumin lasts at least $20 \mathrm{~min}$ (Figure 2). The range of the injection-measurement intervals was 1-15 min for the included studies ${ }^{38,40-47,49-53,55-61,67-}$ 70, 72-77. The $\mathrm{C}_{\max }$ values, stratified by injected dose, are presented in Figure 3 . The selected timeframe allowed the detection of PK differences in the distribution phase only.

A

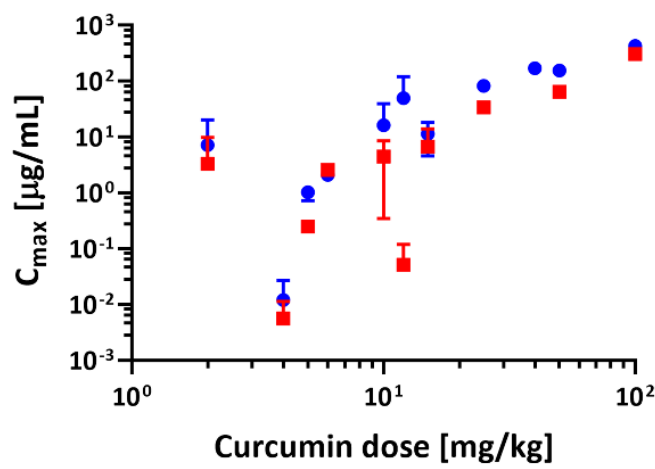

B

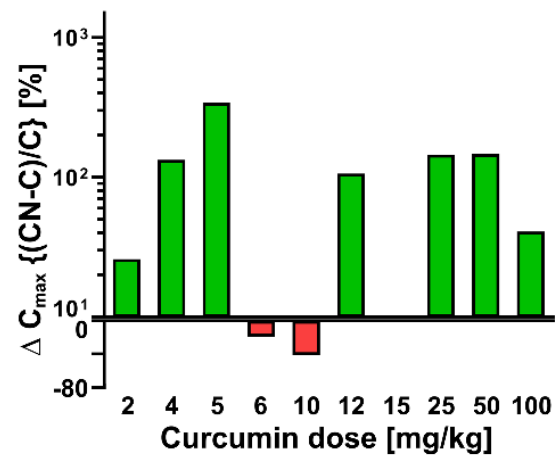

Figure 3. (A) $\mathrm{C}_{\max }$ of free curcumin ( $\square$ ) and curcumin nanoformulations $(\bullet)$ plotted as a function of injected dose in mice and rats. (B) Fold-increase (green bars, log scale) and fold-decrease (red bars, linear scale) in the $\mathrm{C}_{\max }$ of nano-encapsulated curcumin relative to the $\mathrm{C}_{\max }$ of free curcumin, plotted as a percentage and as a function of injected curcumin dose. Abbreviations: $\mathrm{CN}$ : curcumin nanoformulation, $\mathrm{C}$ : free curcumin.

In line with expectations, the $\mathrm{C}_{\max }$ of curcumin increased with injected dose for both free and nanoformulated forms. The injected dose- $\mathrm{C}_{\max }$ relationship for nano-encapsulated curcumin (Spearman's rho of $0.909 ; \mathrm{p} \leq 0.001$ ), shows stronger correlation compared to the injected dose and $C_{\max }$ for the free form (Spearman's rho of $0.793 ; \mathrm{p} \leq 0.01$ ) (Figure $3 \mathrm{~A}$ ). Of the 27 studies, the majority of studies $(n=19)$ yielded a higher $\mathrm{C}_{\max }$ for the nanoformulations compared to the free form (Figure 3B). The fold increase in $\mathrm{C}_{\max }$ was around $100 \%$ for most dose comparisons. The data indicate that formulating curcumin into nanocarriers therefore generally improves the $\mathrm{C}_{\max }$ and systemic exposure to the phytochemical during the distribution phase, although this is not a ubiquitous rule for every type of nanoparticulate carrier.

Currently it is not entirely clear why injected dose- $\mathrm{C}_{\max }$ correlation is stronger for nanoformulations than for the free form and why nano-encapsulation improves the $\mathrm{C}_{\max }$ so considerably. One plausible reason is that free curcumin rapidly settles into the membranes of blood cells upon entry into the systemic circulation ${ }^{79}$. This fraction of blood is not included in the plasma analysis, which does not apply to the non-cell associated nanoparticulate curcumin that remains in the plasma fraction during sample processing. Another (co-) possibility is that hepatic and renal clearance already contribute to the concentration decline 
in the distribution phase, and that the clearance favors free curcumin due to steric factors in terms of particle size relative to the size of the endothelial fenestrations in the kidneys, liver, and the cutaneous microcirculation. Fast biliary clearance of curcumin after intravenous injection, evidenced by the detection of curcumin in bile as early as 5 min post i.v. administration, was shown by Wahlström et al. in rats ${ }^{80}$. It should be noted that, as was recently also demonstrated by our group ${ }^{81}$, most nanoparticulate curcumin carriers act as solubilizers and do not firmly retain the curcumin in the nanoparticle following dispersion in blood. Clearance of nanoparticulate curcumin is also quite steep during the distribution phase $^{41,46-47,49,51-53,57-58,60-61,67-70,73}$, albeit more delayed compared to free curcumin as a result of the more gradual release of curcumin from the nanoparticles.

\subsection{Curcumin AUC Is Improved by Nano-Encapsulation}

The AUC represents the total drug exposure during which the drug can exert its aimed pharmacological effect. Curcumin is heavily biotransformed and therefore rapidly cleared from the circulation via renal and hepatic clearance ${ }^{82-83}$. The normalized AUC (AUC per dose of injection) values of intravenously administered free curcumin or curcumin nanoformulations in mice and rats are summarized in supplementary information Table S14. In different studies, the AUC values were reported as either $\mathrm{AUC}_{0-\mathrm{t}}{ }^{38,41-42,49,51,55,58,68}$ or $\mathrm{AUC}_{0 \text {-infinity }}{ }^{43-48,50,53-54,56-57,59-61,67,69-70,74-77}$. There are also studies in which it is not clearly stated which AUC parameter is reported ${ }^{39-40,52,73}$. The normalized AUC for free curcumin and loaded in nanoformulations are plotted in Figure 4A. Table 1 reports the range of normalized AUC values of free curcumin and nanoformulations and shows the median and mean of the data in both mice and rats. It is clear that the range of normalized AUC values is rather broad, and therefore there is a great variation (as indicated by a large SEM value) in the mean values. Also, the median and mean of the data for free curcumin and curcumin nanoformulations in rats are substantially different from each other. The mean is highly affected by two very high values ${ }^{61,76}$, but this is not the case for the median. The reason for having these high AUC values was not discussed by the authors. It is likely that the difference is due to data miscalculation (see Table S5). Further statistical analysis of AUC results (Table 1) shows no significant difference between free and nanoformulated curcumin in mice and rats (Kruskal-Wallis test with Dunn's post-hoc correction). Also, the $\mathrm{C}_{\max }$ and AUC values reported in rats and mice are plotted against the injected dose in Figure 3A and 4B since the range of values in these two animals are overlapping. The corresponding AUC values do not show similar dose-dependency as those of $\mathrm{C}_{\max }$. The variation in some of the doses is quite high, and it needs more systematic studies to really understand the pharmacokinetics of curcumin. Possible reasons might be the dissimilarities in the pharmacokinetics of curcumin in different animals or the experimental setups (e.g. animal characteristics, monitoring time, the sensitivity of the analytical method). The point that has been so far neglected but has a great impact on PK of curcumin, is how curcumin distributes over blood cells. Essentially, curcumin with a relatively high $\log P$ of $2.5^{84}$ distributes extensively over the blood cells, especially erythrocytes ${ }^{79}$. Therefore, during sample preparation, free curcumin partitions in the membranes of red blood cells (RBC) which are discarded after centrifugation, while curcumin that is stably loaded in the nanocarriers remains in the plasma fraction as the centrifugal force is not sufficient to spin down the nanoparticles. Therefore, the curcumin fraction that ends up in the red blood cells is discounted from the pharmacokinetic analysis. 


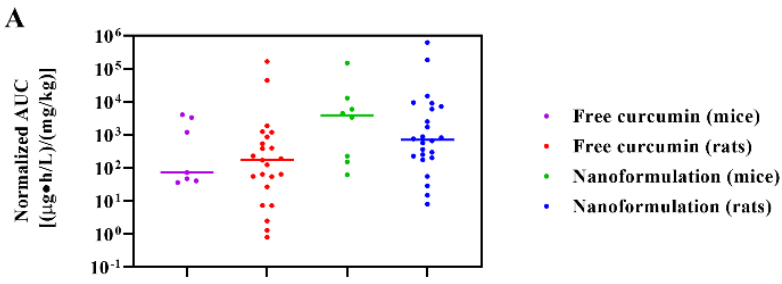

B

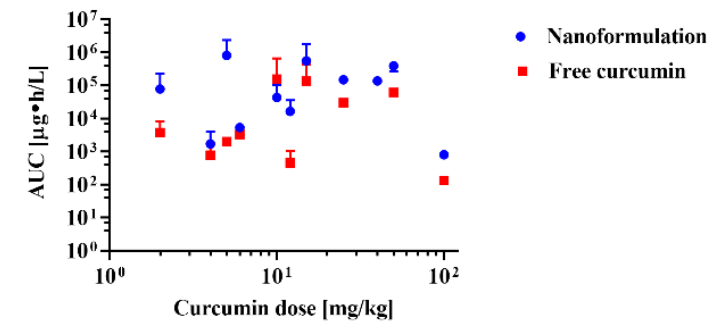

C

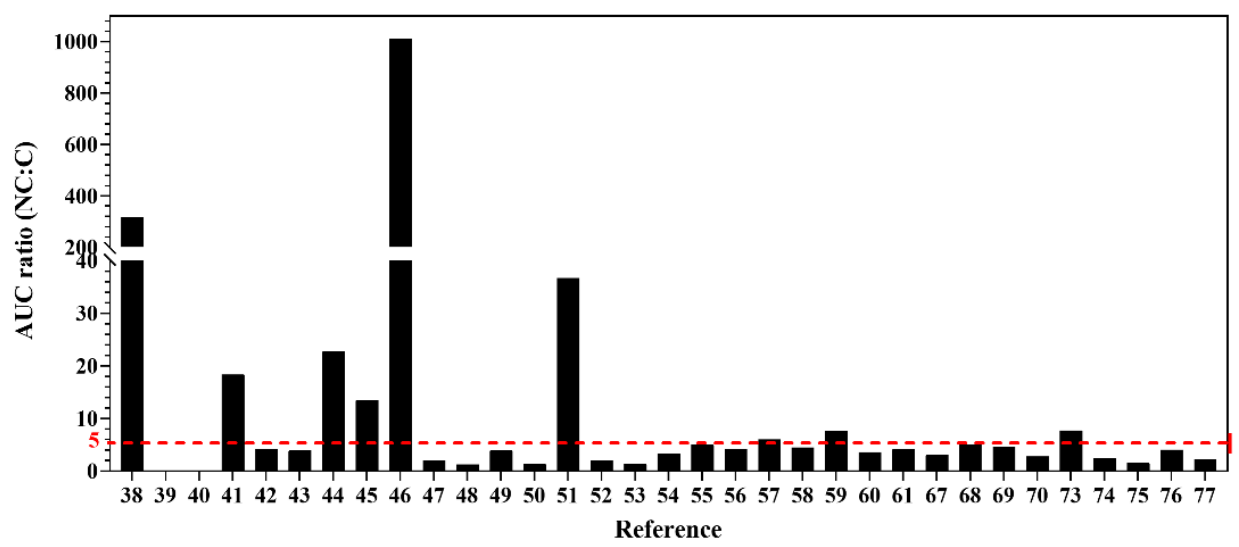

Figure 4. (A) Normalized AUC of free curcumin and curcumin nanoformulations. The horizontal line indicates the median of the data set. (B) AUC of free curcumin ( $\bullet$ ) and curcumin nanoformulations $(\bullet)$ as a function of injected dose. (C) The AUC ratio of curcumin nanoformulations (NC) versus free curcumin $(\mathrm{C})$ in mice and rats.

Surprisingly, the ratio of nano-free curcumin is quite comparable, and the majority of the nanoformulations (22 out of 31 studies) improves the AUC of the curcumin by factor 1.3-5 (Figure 4C). This clearly demonstrates that most of the nanoformulations do not stably retain curcumin in the circulation and thus only acts as a solubilizer. Among the studies, a few reported AUC ratios of 13.5, 19, 23, and 3741,44-45, 51 (Figure 4C). Sun et al. ${ }^{38}$ and Yoon et al. $^{46}$ even reported AUC ratios of curcumin nanoformulations to free curcumin of 300 and 1000 , respectively. In these studies a variety of delivery systems (micelles, nanoparticles, liposomes and nanocrystals) were used to formulate curcumin and the physicochemical properties are summarized in Table2. The particle sizes were $>100 \mathrm{~nm}$ and even as large as $200 \mathrm{~nm}$ for the two studies with the very high AUC ratios ${ }^{38,46}$. Moreover, the zeta potentials 
of the particles were almost neutral $(-0.8 \text { to }-5.3)^{46,51}$ or negative $(<-10 \mathrm{mV})^{38,44-45}$, which favors prolonged circulation ${ }^{85-87}$.

The delivery systems (reported in Table 2) showed different release profiles depending on the nature of the platform, such as the presence of $\mathrm{pH}$-sensitive bonds or degradable polymers. Also, since the release medium and the setup of the release experiments were different between the studies, it is not possible to directly compare the results. Therefore, a standardized procedure using a suitable releasing medium which ensures sink condition is mandatory to perform and compare release studies of hydrophobic molecules like curcumin. A large volume of releasing medium or addition of surfactants or cosolvents are recommended strategies to maintain sink conditions ${ }^{88-89}$. Although these recommendations are not always followed, the general release profiles of curcumin nanoformulations with both marginal and high AUC ratios are comparable. There are several stable nanoformulations with low curcumin release of approximately $20 \%$ in $24 \mathrm{~h}$ with AUC ratio range of $4.1-1011^{38}$, $42,45-46,55,57,59,68,73$.

The discussions provided by the authors to explain the AUC ratios are diverse without pinpointing the very high values observed in their studies with similar nanoformulations in the literature. For instance, Liu et al. ${ }^{44}$ explained that higher micellar stability and better drug retention of the curcumin-loaded crosslinked micelles resulted in lower clearance of curcumin and thus higher AUC. Ji et al. ${ }^{45}$ argued that hyaluronic acid (HA) grafting of curcumin nanocrystals using EDC chemistry improved stability and slowed down curcumin release $(100 \%$ curcumin release within $1 \mathrm{~h}$ and $15 \%$ within $24 \mathrm{~h}$ in PBS $+0.5 \%$ Tween, $\mathrm{pH}$ 5.0 for uncoated and HA-coated curcumin nanocrystals, respectively). Similarly, the longcirculating property of zein-poly(sulfobetaine methacrylate) micelles (zein-PSBMA) (halflife not reported) composed of zein (a protein extracted from corn) as the core and poly(sulfobetaine methacrylate) as the shell in addition to the high retention of the loaded curcumin resulted in 37 times higher AUC ratio of the curcumin nanoformulation compared to free curcumin ${ }^{51}$. Also, sustained curcumin release from poly(d,l-lactic acid)-glycerol (PDLLA-G)-based nanoparticles was suggested by Yoon et al. ${ }^{46}$ to explain the high AUC ratio of curcumin nanoformulation compared to the free form. Generally, the improved pharmacokinetics of intravenously administered nanoformulations is dependent on the stability of the nanoparticles, ensuring a prolonged circulation time, and high drug retention rather than a sustained-release property in vitro. Specifically, in this study ${ }^{46}$ with the highest reported AUC ratio of 1000 , the $\mathrm{C}_{\max }$ of free curcumin was $\sim 0.1$ compared to $100 \mu \mathrm{g} / \mathrm{mL}$ for the nanoformulation at an equal dose of injection $(12 \mathrm{mg} / \mathrm{kg})$. Thus, it is very likely that this high AUC ratio mainly resulted from the very low AUC of free curcumin than from exceptionally high stability of the nanoformulation.

Among the studies with high AUC ratios, only three performed in vivo imaging using a fluorescent dye either encapsulated in or conjugated to the formulation. DIR-loaded crosslinked micelles showed prolonged circulation time (half-life was not reported) and higher tumor accumulation compared to the non-crosslinked micelles and free dye as controls ${ }^{44}$. However, the in vivo imaging of DIR-loaded crosslinked micelles was performed in tumor-bearing mice while PK was conducted in rats. Cy5.5-labeled zein-PSBMA micelles showed more intense fluorescence and the fluorescence signal was detectable $72 \mathrm{~h}$ after injection in mice compared to a significant decrease in the fluorescence signal of free Cy5.5 in $6 \mathrm{~h}$ followed by disappearance after $48 \mathrm{~h}$, indicating prolonged circulation of zein-PSBMA micelles ${ }^{51}$. In another study, healthy mice received intravenous injection of HA-coated 
liposomes loaded with DID, a lipophilic near-infrared fluorescent membrane dye, and were terminated after $12 \mathrm{~h}$ to select the optimized formulation in terms of HA molecular weight and grafting density by comparing the fluorescence signal in the organs (especially the liver and spleen $)^{38}$. The mice that were used for the in vivo imaging were tumor-free and, therefore, could not demonstrate possible tumor accumulation. Further, it is recommended to covalently attach the fluorescent dye to the nanocarrier because the loaded dye can be released from the system and interfere with imaging of the nanocarrier. This was not the case for some studies ${ }^{38}$, ${ }^{44}$ and only Cy5.5 was covalently attached to the micelles by the reaction between NHS ester and amine ${ }^{51}$. Also, none of the studies quantitively reported the circulation kinetics of the nanoparticles and thus no firm conclusions can be drawn because of experimental design weaknesses. Further research is therefore needed to understand the particle stability in the blood circulation and its effect on the improved pharmacokinetics of curcumin. 


\section{Chapter 2}

Table 2. Summary of the physicochemical properties of intravenously administered nanoformulations with an AUC ratio of $>5$ compared to free curcumin.

\begin{tabular}{|c|c|c|c|c|c|c|c|c|c|}
\hline $\begin{array}{l}\text { Curcumin } \\
\text { nanoformulation }\end{array}$ & $\begin{array}{l}\text { Size } \\
{[\mathbf{n m}]}\end{array}$ & $\begin{array}{l}\mathbf{Z P} \\
{[\mathbf{m V}]}\end{array}$ & $\begin{array}{c}\text { Loading } \\
\text { efficiency } \\
{[\%]}\end{array}$ & Release profile & $\begin{array}{l}\text { Free curcumin } \\
\text { vehicle }\end{array}$ & Species & $\begin{array}{c}\text { ID } \\
{[\mathrm{mg} / \mathrm{kg}]}\end{array}$ & $\begin{array}{l}\text { AUC ratio } \\
\text { NC:C }\end{array}$ & Ref. \\
\hline $\mathrm{HA}-$ curc-NC ${ }^{\mathrm{a}}$ & 161 & -25 & $3.3 \%$ & $\begin{array}{c}15 \% \text { release in } \mathrm{PBS}+0.5 \% \text { Tween at } \mathrm{pH}=5.0 \text {. The } \\
\text { release increased in the presence of HAase over } 24 \mathrm{~h} \text {. } \\
40 \%, 60 \% \text {, and } 80 \% \text { release within } 24 \mathrm{~h} \text { in } \mathrm{PBS}+ \\
0.5 \% \text { Tween containing } 0.3 \mu \mathrm{M} \text { HAase at } \mathrm{pH}=7.4 \\
\text { (blood), } 6.5 \text { (cancer site), and } 5.0 \text { (lysosome), } \\
\text { respectively. }\end{array}$ & NA & SD rats & 2 & 13.5 & 45 \\
\hline $\begin{array}{l}\text { mPEG-PLGA } \\
\text { nanoparticles }^{\mathrm{b}}\end{array}$ & 120 & NA & NA & $\begin{array}{c}70 \% \text { release within } 27 \mathrm{~h} \text { in } \mathrm{PBS} \text { at } \mathrm{pH}=5.8 . \text { Release } \\
\text { reached } 90 \% \text { in } 144 \mathrm{~h} .\end{array}$ & NA & SD rats & 4 & 19 & 41 \\
\hline $\begin{array}{l}\text { mPEG- } b \text {-PHEMA- } \\
\text { 5HA micelles }{ }^{c}\end{array}$ & 104 & -19 & $17.8 \%$ & $\begin{array}{l}90 \% \text { and } 80 \% \text { release after } 30 \mathrm{~h} \text { in } \mathrm{PBS} \text { at } \mathrm{pH}=7.4 \text { for } \\
\text { non-crosslinked and crosslinked micelles, respectively. } \\
\text { Higher release }(35 \%) \text { for } \mathrm{pH} \text {-sensitive crosslinked } \\
\text { micelles in acidic environment }(\mathrm{pH}=5.0) \text { was } \\
\text { observed compared to non-crosslinked micelles }(25 \%) \text {. }\end{array}$ & NA & SD rats & 5 & 23 & 44 \\
\hline $\begin{array}{l}\text { Zein-PSBMA } \\
\text { micelles }^{\mathrm{d}}\end{array}$ & 155 & -5.3 & $3.6 \%$ & $77 \%$ curcumin release in PBS $\mathrm{pH}=7.4$ after $168 \mathrm{~h}$. & $\begin{array}{l}\text { Saline with } 1 \% \\
\text { Tween } 20\end{array}$ & $\begin{array}{l}\mathrm{BALB} / \mathrm{c} \\
\text { mice }\end{array}$ & 2 & 37 & 51 \\
\hline HA-Cur-LPs ${ }^{\mathrm{e}}$ & 210 & -37 & $13.2 \%$ & $\begin{array}{l}100 \% \text { release after } 100 \mathrm{~h} \text { in } \mathrm{PBS}+0.2 \% \text { Tween } 80 \text { at } \\
\qquad \mathrm{pH}=7.4 .\end{array}$ & DMSO & $\begin{array}{l}\text { BALB/c } \\
\text { mice }\end{array}$ & 10 & 317 & 38 \\
\hline $\begin{array}{l}\text { (PDLLA-G)-based } \\
\text { nanoparticles }^{\mathrm{f}}\end{array}$ & 200 & -0.8 & NA & $\begin{array}{c}10 \% \text { and } 45 \% \text { release after } 168 \mathrm{~h} \text { in } \mathrm{PBS} \text { at } \mathrm{pH}=7.4 \\
\text { and } \mathrm{pH}=5.5 \text {, respectively, as a result of the polymer } \\
\text { degradation and higher stability of curcumin at acidic } \\
\mathrm{pH}\end{array}$ & $\begin{array}{c}37.5 \% \text { PEG } 400 \\
v / v\end{array}$ & SD rats & 12 & 1011 & 46 \\
\hline
\end{tabular}

Abbreviations: ZP, Zeta-potential; ID, injected dose; DMSO, dimethyl sulfoxide; PEG, polyethylene glycol; NA, not available; NC:C, curcumin nanoformulation:free curcumin; SD, Sprague Dawley.

Notes nanoformulations: ${ }^{a}$ hyaluronic acid-coated curcumin nanocrystals; ${ }^{\mathrm{b}}$ (polyethylene glycol)-poly (lactic-co-glycolic acid); ${ }^{\mathrm{c}} \mathrm{pH}-\mathrm{responsive}$ reversibly crosslinked micelles poly(ethylene glycol)-b-poly(2-methacrylate ethyl 5-hexynoicate); ${ }^{\mathrm{d}}$ zein-poly(sulfobetaine methacrylate); ${ }^{\mathrm{e}}$ hyaluronic acid modified liposomes; ${ }^{\mathrm{f}}$ poly(D,L-lactic acid)-glycerol-based nanoparticles. 


\subsection{Clearance and Distribution Volume Are Affected by Nano-Encapsulation}

In line with higher AUC ratios, the clearance of curcumin as nanoformulation was lower compared to that of free curcumin. However, the clearance and volume of distribution should be evaluated together since the reduction in the blood concentration is not only due to the metabolism or removal from the body (excretion) but also due to disposition from the circulation into different tissues ${ }^{90-91}$. The distribution volume was calculated as explained in the supplement when the values were not reported by the authors. The calculated values, marked with an asterisk (Table S1-4), show the distribution volumes at the terminal phase of elimination (schematically explained in Figure $\mathrm{S} 1$ ). Generally, the range of $\mathrm{V}_{\mathrm{d}}$ in different studies is broad $(0.002$ to $1376 \mathrm{~L} / \mathrm{kg}$ and 0.06 to $882 \mathrm{~L} / \mathrm{kg}$ for free curcumin and as nanoformulation, respectively) that can be due to both variation in PK results and different distribution volumes used to describe $\mathrm{V}_{\mathrm{d}}{ }^{62}$. Surprisingly two studies ${ }^{54,61}$ reported very low and unrealistic $\mathrm{V}_{\mathrm{d}}$ values ( 2 and $4 \mathrm{~mL} / \mathrm{kg}$ for free curcumin) even lower than the average blood volume in mice and rats $(50-80 \mathrm{~mL} / \mathrm{kg} \text { or } 7-8 \% \text { body weight })^{92-93}$ assuming curcumin distribution is limited to the blood circulation.

Additionally, the $\mathrm{V}_{\mathrm{d}}$ of curcumin nanoformulations was either lower ${ }^{41-46,51,57,59,68,73}$, unchanged $^{48,69,74-75}$ or unexpectedly higher ${ }^{50,52-54,56,58,60-61,67,70,76-77}$ than free curcumin. The reduction in $\mathrm{V}_{\mathrm{d}}$ of curcumin nanoformulations indicates that curcumin is stably retained in the nanoparticles, while nanoformulations that do not change or marginally affect $\mathrm{V}_{\mathrm{d}}$ most likely act as solubilizers (Figure 5). However, in many studies, the change in volume of distribution was not reported or the authors only reported $V_{d}$ without proper discussion. Duan et al. ${ }^{54}$ and $\mathrm{Ma}$ et al. ${ }^{76}$ observed a substantial increase in $\mathrm{V}_{\mathrm{d}}$ for curcumin loaded in chitosan/poly(butyl cyanoacrylate) nanoparticles and methoxy poly(ethylene oxide)-blockpoly( $\varepsilon$-caprolactone) micelles, respectively. The authors explained that this might be due to the sequestering of larger curcumin-loaded nanoparticles by reticuloendothelial (RES) or other tissues which released the encapsulated curcumin as a reservoir. 


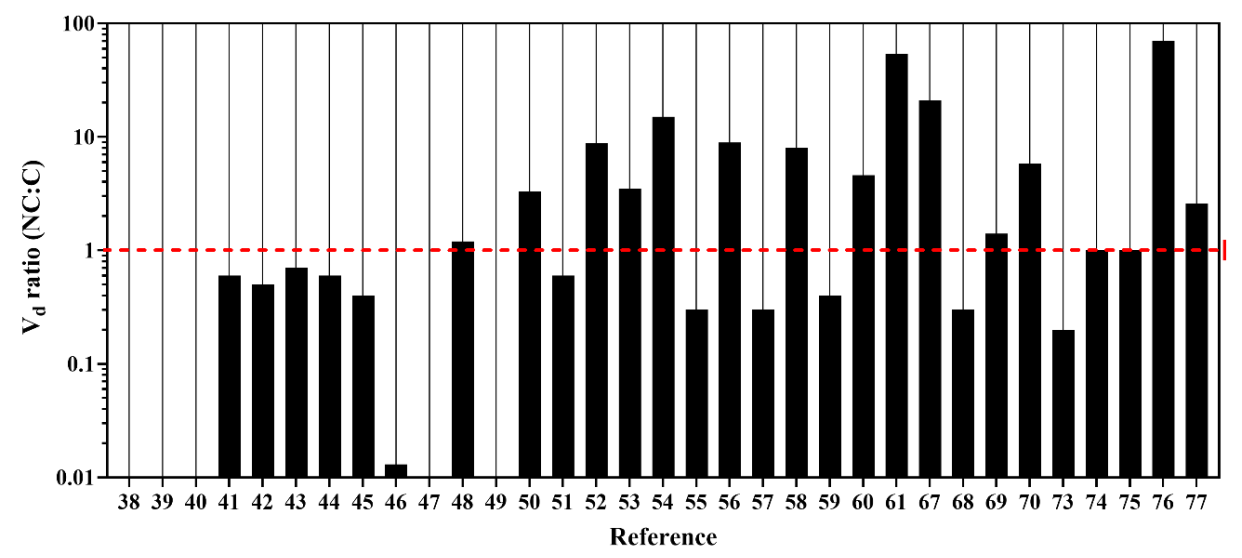

Figure 5. The distribution volume ratio of curcumin nanoformulation (NC) to free curcumin (C) in mice and rats. The $\mathrm{V}_{\mathrm{d}}$ ratio could not be calculated for references ${ }^{38-40,47,49}$ due to insufficient data.

\section{Correlation of Pharmacokinetics and Therapeutic Efficacy of Curcumin Nanoformulations}

The studies that reported both PK and PD aspects of the nanoformulations are summarized in Table 3. Generally, the treatment groups that received curcumin nanoformulations showed stronger tumor reduction compared to free curcumin, in line with the increase in AUC of curcumin upon loading in nano-sized delivery systems. However, one important weakness in some studies was the difference in the animal model used in PK and in vivo antitumor experiments (Table 3). To explain, the PK parameters were reported in rats, whereas the therapeutic efficacy was always evaluated in tumor bearing mice. The preference of using rats for PK studies than mice is due to the possibility of withdrawing higher blood volumes and more frequent samples from rats at the cost of discrepancy in animal models. In addition to the in vivo antitumor effects, some studies demonstrated anti-angiogenic effects of curcumin (reduction in microvessel density) using alginate-encapsulated tumor cell assay (in vitro), zebra fish or simply performing CD31 (platelet endothelial cell adhesion molecule-1), COX-1 (cyclooxygenase-1) or VEGF (vascular endothelial growth factor) staining in tumor slices $^{54-55,57,68,73}$. The results of tumor staining for the presence of microvessels in treatment groups vs control showed higher angiogenesis inhibition following treatment with curcumin nanoformulations. Also, the presence of high vascular density in the stained tumor sections of the control group receiving physiologic buffer indirectly confirmed that the tumor xenograft models were adequately vascularized to benefit from the enhanced permeability and retention (EPR) effect. 
Table 3. Summary of the therapeutic efficacy studies of intravenously administered curcumin nanoformulations.

\begin{tabular}{|c|c|c|c|c|c|c|c|c|c|c|}
\hline $\begin{array}{c}\text { Curcumin } \\
\text { nanoformulation }\end{array}$ & $\begin{array}{l}\text { Size } \\
{[\mathrm{nm}]}\end{array}$ & $\begin{array}{c}\mathbf{Z P} \\
{[\mathbf{m V}]}\end{array}$ & $\begin{array}{c}\text { Animal } \\
\text { model } \\
\text { PK }\end{array}$ & $\begin{array}{l}\text { Animal model, } \\
\text { xenograft } \\
\text { PD }\end{array}$ & Curcumin dosing schedule & $\begin{array}{l}\text { Monitoring } \\
\text { time } \\
{[d]}\end{array}$ & $\begin{array}{l}\text { AUC } \\
\text { ratio } \\
\text { NC:C }\end{array}$ & $\begin{array}{l}\% \text { TGI } \\
\text { (NC) }\end{array}$ & $\begin{array}{l}\% \text { TGI } \\
\text { (C) }\end{array}$ & Ref. \\
\hline $\begin{array}{l}\text { Curcumin-PBCA } \\
\text { nanoparticles }^{\mathrm{a}}\end{array}$ & 200 & 29 & $\mathrm{SD}$ rats & $\begin{array}{l}\text { athymic } \\
\text { BALB/c nude } \\
\text { mice, HepG2, } \\
\text { s.c. flank }\end{array}$ & $\begin{array}{l}3 \text { times per week for } 28 \text { days } \\
\text { (curcumin dose unknown) }\end{array}$ & 28 & 3.4 & 55 & NA & 54 \\
\hline mPEG-PLA micelles ${ }^{b}$ & 30 & -0.3 & $\mathrm{SD}$ rats & $\begin{array}{l}\text { BALB/c mice, } \\
\text { CT26 s.c. flank }\end{array}$ & $\begin{array}{c}50 \mathrm{mg} / \mathrm{kg} \text { every } 3 \text { days for } \\
15 \text { days (total dose: } 300 \\
\mathrm{mg} / \mathrm{kg} \text { ) }\end{array}$ & 25 & 5.0 & 80 & 60 & 55 \\
\hline $\begin{array}{c}\text { mPEG-PCL-Phe }(\text { Boc }) \\
\text { micelles }^{\mathrm{c}}\end{array}$ & 23 & NA & ICR mice & $\begin{array}{l}\text { BALB/c nude } \\
\text { mice, } \\
\text { K562/ADR s.c. } \\
\text { armpit }\end{array}$ & $\begin{array}{l}40 \mathrm{mg} / \mathrm{kg} \text { daily for } 21 \text { days } \\
\text { (total dose: } 840 \mathrm{mg} / \mathrm{kg} \text { ) }\end{array}$ & 21 & 3.1 & 65 & NA & 67 \\
\hline mPEG-PCL micelles ${ }^{\mathrm{d}}$ & 37 & -0.8 & $\begin{array}{l}\mathrm{C} 57 \mathrm{BL} / 6 \\
\text { mice }\end{array}$ & $\begin{array}{l}\text { C57BL/6 mice, } \\
\text { LL/2 s.c. flank } \\
\text { and pulmonary } \\
\text { metastases i.v. }\end{array}$ & $\begin{array}{c}25 \mathrm{mg} / \mathrm{kg} \text { every } 2 \text { days for } \\
14 \text { days (total dose: } 200 \\
\mathrm{mg} / \mathrm{kg} \text { ) }\end{array}$ & 28 & 5.0 & 52 & 30 & 68 \\
\hline mPEG-PLA-PAE micelles ${ }^{\mathrm{e}}$ & $\begin{array}{c}\mathrm{pH}=7.4 \\
171 \\
\mathrm{pH}=5.5 \\
23\end{array}$ & $\begin{array}{c}\mathrm{pH}=7.4,4 ; \\
\mathrm{pH}=5.5,25\end{array}$ & $\begin{array}{l}\mathrm{BALB} / \mathrm{c} \\
\text { nude mice }\end{array}$ & $\begin{array}{l}\text { BALB/c nude } \\
\text { mice, } \mathrm{MCF}-7 \\
\text { s.c. flank }\end{array}$ & $\begin{array}{c}40 \mathrm{mg} / \mathrm{kg} \text { on days } 0,2,4,6 \\
\text { and } 8 \text { (total dose: } 200 \\
\mathrm{mg} / \mathrm{kg} \text { ) }\end{array}$ & 25 & NA & 65 & NA & 39 \\
\hline mPEG-PCL micelles ${ }^{\mathrm{d}}$ & 27 & NA & $\mathrm{SD}$ rats & $\begin{array}{l}\text { BALB/c mice, } \\
\text { C26 s.c. }\end{array}$ & $\begin{array}{l}25 \mathrm{mg} / \mathrm{kg} \text { daily for } 10 \text { days } \\
\text { (total dose: } 250 \mathrm{mg} / \mathrm{kg} \text { ) }\end{array}$ & 18 & 6.0 & 50 & 30 & 57 \\
\hline $\begin{array}{l}\text { mPEG-b-PHEMA-5HA } \\
\text { micelles }^{\mathrm{f}}\end{array}$ & 109 & -19 & $\mathrm{SD}$ rats & $\begin{array}{l}\mathrm{BALB} / \mathrm{c} \text { mice, } \\
4 \mathrm{~T} 1 \text { s.c. }\end{array}$ & $\begin{array}{l}20 \mathrm{mg} / \mathrm{kg} \text { every } 3 \text { days for } \\
21 \text { days (total of } 5 \text { injection) } \\
\text { (total dose: } 100 \mathrm{mg} / \mathrm{kg} \text { ) }\end{array}$ & 21 & 23 & 40 & 25 & 44 \\
\hline HA-curc-NC ${ }^{g}$ & 161 & -25 & $\mathrm{SD}$ rats & $\begin{array}{l}\mathrm{BALB} / \mathrm{c} \text { mice, } \\
4 \mathrm{~T} 1 \text { s.c. flank } \\
\text { breast }\end{array}$ & $\begin{array}{l}5 \mathrm{mg} / \mathrm{kg} \text { every } 2 \text { days for } 10 \\
\text { days (total dose: } 30 \mathrm{mg} / \mathrm{kg} \text { ) }\end{array}$ & 10 & 13.5 & 75 & 20 & 45 \\
\hline $\begin{array}{c}\text { Curcumin nanosuspension } \\
\text { stabilized by mPEG-DSPE } \\
\text { and } \mathrm{SPC}^{\mathrm{h}}\end{array}$ & 186 & -19 & $\mathrm{SD}$ rats & $\begin{array}{l}\text { ICR mice, } \mathrm{H} 22 \\
\text { s.c. armpit }\end{array}$ & $\begin{array}{l}10 \mathrm{mg} / \mathrm{kg} \text { every other day } \\
\text { (total dose: } 40 \mathrm{mg} / \mathrm{kg} \text { ) }\end{array}$ & 6 & 4.5 & 60 & 15 & 58 \\
\hline
\end{tabular}




\begin{tabular}{|c|c|c|c|c|c|c|c|c|c|c|}
\hline $\begin{array}{c}\text { Curcumin } \\
\text { nanoformulation }\end{array}$ & $\begin{array}{l}\text { Size } \\
{[\mathrm{nm}]}\end{array}$ & $\begin{array}{c}\mathbf{Z P} \\
{[\mathbf{m V}]}\end{array}$ & $\begin{array}{l}\text { Animal } \\
\text { model } \\
\text { PK }\end{array}$ & $\begin{array}{l}\text { Animal model, } \\
\text { xenograft } \\
\text { PD }\end{array}$ & Curcumin dosing schedule & $\begin{array}{l}\text { Monitoring } \\
\text { time } \\
{[d]}\end{array}$ & $\begin{array}{l}\text { AUC } \\
\text { ratio } \\
\text { NC:C }\end{array}$ & $\begin{array}{c}\text { \% TGI } \\
\text { (NC) }\end{array}$ & $\begin{array}{c}\text { \% TGI } \\
\text { (C) }\end{array}$ & Ref. \\
\hline mPEG-PCL micelles ${ }^{\mathrm{d}}$ & 30 & -4 & Rats & $\begin{array}{l}\text { BALB/c mice, } \\
\text { CT26 s.c. flank }\end{array}$ & $\begin{array}{c}50 \mathrm{mg} / \mathrm{kg} \text { every } 2 \text { days (total } \\
\text { dose: } 500 \mathrm{mg} / \mathrm{kg} \text { ) }\end{array}$ & 18 & 7.7 & 20 & 16 & 73 \\
\hline HSA nanoparticles ${ }^{\mathrm{i}}$ & 166 & -27 & Rats & $\begin{array}{l}\text { BALB/ c mice, } \\
\text { HT-29 s.c. } \\
\text { dorsal flank }\end{array}$ & $\begin{array}{c}10 \mathrm{mg} / \mathrm{kg} \text { every other day } \\
\text { for } 10 \text { days (total dose: } 60 \\
\mathrm{mg} / \mathrm{kg} \text { ) }\end{array}$ & 20 & 2.5 & 45 & 18 & 74 \\
\hline
\end{tabular}

The percentage of tumor growth inhibition (\% TGI) is calculated as the difference in the ratio of tumor volume in the treatment group receiving curcumin nanoformulation compared to the untreated control group (\% TGI (NC)) or the difference in the ratio of tumor volume of the treatment group receiving free curcumin vs the untreated control group (\% TGI (C)) on the last day of tumor monitoring.

Abbreviations: ZP, zeta potential; PK, pharmacokinetics; PD, pharmacodynamics; d, days; NC, curcumin nanoformulation; C, free curcumin; TGI, tumor growth inhibition; Ref., reference; NA, not available; s.c., subcutaneous; i.v., intravenous.

Notes nanoformulations: ${ }^{\mathrm{a}}$ (cationic poly(butyl) cyanoacrylate (PBCA) nanoparticles coated with chitosan); ${ }^{\mathrm{b}}$ monomethoxy poly(ethylene glycol)poly(lactide); c methoxy-poly(ethyleneglycol)-block-poly(e-caprolactone) and N-(tert-butoxycarbonyl)-L-phenylalanine end-capped; d methoxy poly(ethylene glycol)block-poly( $\varepsilon$-caprolactone); ${ }^{\mathrm{e}} \mathrm{pH}$-sensitive methoxy poly(ethylene glycol)-poly(lactide)-poly( $\beta$-amino ester); ${ }^{\mathrm{f}} \mathrm{pH}$-responsive reversibly crosslinked micelles poly(ethylene glycol)-b-poly(2-methacrylate ethyl 5-hexynoicate); ${ }^{\mathrm{g}}$ hyaluronic acid-modified curcumin nanocrystals; ${ }^{\mathrm{h}}$ curcumin nanosuspension stabilized by mPEG2000-DSPE and soybean lecithin; ${ }^{\mathrm{i}}$ human serum albumin nanoparticles

Cell lines: HepG2, human hepatocellular carcinoma; CT26, mouse fibroblasts from colon carcinoma; K562/ADR, human chronic myelogenous leukemia with selected resistance to doxorubicin; LL/2, mouse Lewis lung carcinoma; MCF-7, human mammary gland carcinoma; C26, mouse colon carcinoma; 4T1, mouse mammary gland carcinoma; H22, mouse hepatocellular carcinoma; HT-29, human colorectal adenocarcinoma. 
To evaluate possible relationships between the therapeutic efficacy and pharmacokinetics, the \% TGI of curcumin nanoformulations at the end of in vivo experiment relative to the control group and free curcumin is plotted against AUC, total injected dose and the AUC ratio of curcumin nanoformulation to free curcumin (figure 6). Here again, the PK data in mice and rats are plotted together regardless of the animal species since the corresponding AUC range of these animals overlaps with each other, and even in many studies, the PK and PD animal models were not the same (Table 3). Correlation analysis performed on different datasets indicates no relationship between the parameters except a negative relationship (correlation coefficient, -0.826; P-value $<0.05$ ) between total injected dose and \%TGI of nanoformulation relative to free curcumin (Figure 6B, green symbols). Thus, the results show that an increase in AUC does not result in improved therapeutic outcome in terms of tumor volume reduction. This might be explained due to several possible reasons such as variety in the nanoformulations, tumor models, the discrepancy between PK/PD animal models or different time frames that animals were monitored. However, the correlation between the TGI\% of the treatment group receiving curcumin nanoformulation compared to free curcumin is stronger than that of the untreated control group (green vs red symbols). In this way, the influence of animal models could be offset to some extent, as we expect to observe lower tumor reduction in the free curcumin group of mice with more resistance tumor and vice versa. Observing a negative correlation between the total injected dose and tumor growth inhibition of nanoformulation relative to free curcumin is unexpected. One possible explanation could be that at higher cumulative dose regimen, there is sufficient curcumin deposited in the tumor to exert tumor inhibition even in the case of free curcumin despite its unfavorable PK. As a consequence, the difference between the treatment group receiving curcumin nanoformulation and free curcumin becomes smaller. It is further noted that the effect of cofounders such as the difference in the performance of nano-sized delivery systems (different AUC ratios), the number of injections, tumor models, number of available studies and the monitoring time frame on the result of the correlation study should not be underrated. Therefore, more controlled studies are required to elucidate and confirm such findings. This is similar for the relationship between the stability of curcumin nanoformulations, i.e. higher AUC ratios, and pharmacodynamic effects. The results so far do not show a clear indication of achieving better therapeutic outcome with stable nanoformulations ${ }^{54-55,58,67-68,74}$. Even nanoformulations that act as only solubilizers for curcumin exert considerable anti-tumor effects (Figure 6). 
Legends: • (Nano-CTRL) • (Free-CTRL) • (Nano-free)
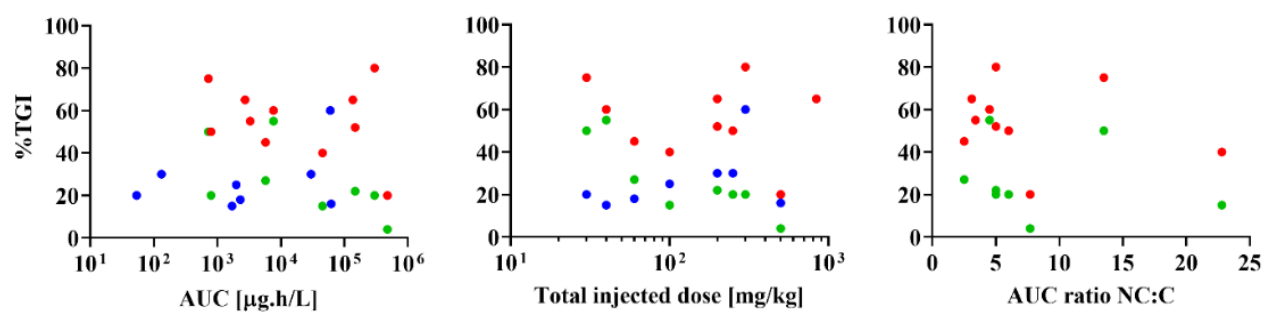

Figure 6. Correlation between therapeutic efficacy and pharmacokinetics of curcumin nanoformulations and free curcumin. The percentage of tumor growth inhibition (TGI\%) is calculated as the difference in the ratio of tumor volume in the treatment group compared to the control group on the last day of tumor monitoring. Red: The tumor growth inhibition of nanoformulation relative to the untreated control (CTRL) group. Blue: The tumor growth inhibition of free curcumin relative to the untreated control group. Green: The tumor growth inhibition of curcumin nanoformulation relative to free curcumin group. Correlation analysis Spearman test was performed using Graphpad Prism. Abbreviation: NC, curcumin nanoformulation; $\mathrm{C}$, free curcumin.

Figure 7 exclusively depicts the correlation between therapeutic efficacy and PK of only one type of copolymer that is widely used to formulate curcumin. Copolymers of methoxy poly(ethylene glycol)-block-poly( $\varepsilon$-caprolactone) (mPEG-PCL) are frequently used for drug delivery of hydrophobic drugs ${ }^{94}$ and it is also used as a platform to deliver curcumin (Table 3). In these studies, mPEG-PCL copolymers with similar hydrophilic and hydrophobic molecular weights of $2000 \mathrm{~g} / \mathrm{mol}$ were used. Despite the similarity of the polymer platform, the AUC ratio of mPEG-PCL micelles to the free curcumin was different among the studies. Gong et al. ${ }^{68}$, Gou et al. ${ }^{57}$ and $\mathrm{Hu}$ et al. ${ }^{73}$ reported AUC ratios of 5.0, 6 and 7, respectively, whereas Gong et al. found a AUC value of $1.1^{67}$. Interestingly, the therapeutic efficacy of curcumin-loaded mPEG-PCL micelles was not comparable in different studies, and no correlation was observed between TGI\% and AUC or total dose of injection (Figure 7). Gong et al. ${ }^{68}$ and Gou et al. ${ }^{57}$ reported around $50 \%$ tumor reduction compared to the untreated group after receiving $25 \mathrm{mg} / \mathrm{kg}$ curcumin-loaded mPEG-PCL micelles every 2 days for two weeks in mice bearing Lewis lung carcinoma (LL/2) and $25 \mathrm{mg} / \mathrm{kg}$ daily for 10 days in mice bearing C26, respectively. On the contrary, curcumin-loaded mPEG-PCL micelles did not exhibit tumor reduction compared to the control group in mice-bearing K567/ADR after higher curcumin dosing of $40 \mathrm{mg} / \mathrm{kg}$ every day for three weeks. This could be due to the similar PK of curcumin-loaded mPEG-PCL with free curcumin reported in this study (no difference in AUC), in combination to the multidrug-resistant characteristic of the tumor model. In this particular study only $\mathrm{N}$-(tert-butoxycarbonyl)-L-phenylalanine end-capped $\mathrm{mPEG}-\mathrm{PCL}$ with higher stability, as a result of $\pi-\pi$ stacking interaction, demonstrated $65 \%$ tumor reduction compared to the untreated group (Figure 7 , reference $67^{*}$ ). 
Legends: • (Nano-CTRL) • (Free-CTRL) • (Nano-free)
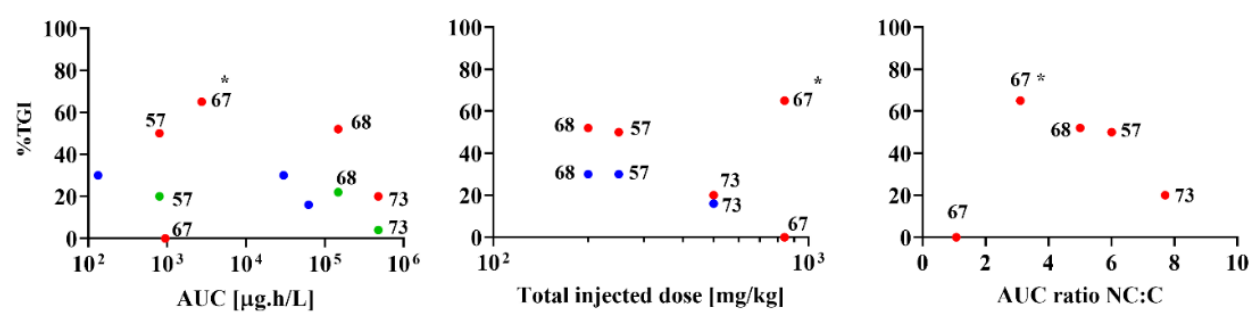

Figure 7. Correlation between therapeutic efficacy and pharmacokinetics of curcumin-loaded mPEGPCL micelles and free curcumin. The percentage of tumor growth inhibition (TGI\%) is calculated as the difference in the ratio of tumor volume in the treatment group compared to the control group on the last day of tumor monitoring. Red: The tumor growth inhibition of nanoformulation relative to the untreated control (CTRL) group. Blue: The tumor growth inhibition of free curcumin relative to the untreated control group. Green: The tumor growth inhibition of curcumin nanoformulation relative to free curcumin group. The number next to each point shows the reference from which the data is collected. Reference 67 marked with an asterisk, presents curcumin-loaded mPEG-PCL-Phe (Boc) micelles and the study did not have free curcumin treatment group in antitumor efficacy experiment. Correlation analysis Spearman test was performed using Graphpad Prism. Abbreviation: NC, curcumin nanoformulation; $\mathrm{C}$, free curcumin.

\section{Preliminary Clinical Outcomes of the Intravenous Curcumin Nanoformulations}

At present, there are only two intravenously injectable curcumin nanoformulations (Lipocurc $^{\mathrm{TM}}$ and CUC-01) listed in the clinicaltrials.gov website. So far, the efficacy and safety of CUC-01, a curcuminoid formulation in polyoxyl castor oil (Kolliphor® ELP) as a nonionic solubilizer, was evaluated in patients with metastatic breast cancer in combination with paclitaxel. The authors reported that the combination therapy was superior to paclitaxel plus placebo without major safety concerns ${ }^{95}$.

The PK and biodistribution profile of Lipocurc ${ }^{\mathrm{TM}}$, a liposomal curcumin formulation, was extensively studied in dogs and humans in several studies. The impact of the duration of intravenous infusions of Lipocurc ${ }^{\mathrm{TM}}$ on curcumin metabolism and tissue distribution was assessed in dogs. The tissue levels of curcumin and its metabolite tetrahydrocurcumin in the lung, spleen and liver were substantially higher after longer infusion time of the eight-hour compared to the two-hour. Also, longer infusion time resulted in a higher tissue partition coefficient for curcumin and tetrahydrocurcumin. The ratio of the metabolite to curcumin was lower during longer infusion regimens and different in a tissue-specific manner. The authors argued that the extended infusion might facilitate the distribution of curcumin into tissues by a transporter-dependent mechanism and higher tissue concentrations of curcumin might inhibit or saturate a putative reductase enzyme converting curcumin to its metabolite ${ }^{96}$. However, later the authors suggested another mechanism to substantiate these observations by performing complementary in vitro experiments that are discussed in the next paragraph. The pharmacokinetic profile, safety and tolerability of Lipocurc ${ }^{\mathrm{TM}}$ were studied in phase 1 clinical trial upon a single-dose intravenous injection in the range of $10-400 \mathrm{mg} / \mathrm{m}^{2}$. The plasma concentration of curcumin and tetrahydrocurcumin increased in a dose-dependent 
manner, and shortly after discontinuation of the infusion (6-60 min), the concentrations were below the detection limit. The intravenous dosing was safe, and above $120 \mathrm{mg} / \mathrm{m}^{2}$ a transient change in the morphology of red blood cells (RBC) was noticed. Therefore, short-term infusion of Lipocurc ${ }^{\mathrm{TM}}$ was safe up to $120 \mathrm{mg} / \mathrm{m}^{2}$ and higher doses represented dose-limiting toxicity in $\mathrm{RBCs}^{97}$. They also showed in vitro that curcumin and liposomal curcumin caused morphological changes in RBCs in a dose-dependent manner ${ }^{98}$.

In the light of these observations, the cellular distribution and metabolism of curcumin, formulated as Lipocure ${ }^{\mathrm{TM}}$ were investigated in vitro in RBCs and peripheral blood mononuclear cells (PBMCs). It was shown that curcumin rapidly distributed into RBCs and PBMCs. The authors assumed that Lipocurc ${ }^{\mathrm{TM}}$ is absorbed by the cell membrane, and curcumin subsequently diffuses from the cell membrane into the intracellular compartment. A blood-based metabolism, in particular by RBCs, was observed as $92 \%$ and $68 \%$ of curcumin disappeared from the medium in $15 \mathrm{~min}$ after the addition of dog and human RBCs, respectively. Thus, incorporation of curcumin and further metabolization into tetrahydrocurcumin and likely other metabolites is apparent in human and dog RBCs. The formation of tetrahydrocurcumin indicates that a reductase activity must be present in RBCs. Although the type of enzyme responsible for the metabolism is not yet clear, the authors suggested a similar enzyme as dihydrocurcumin reductase in gut microorganisms or Cytochrome b5 reductase that simulations showed preferential binding to curcumin. It was as well argued that the finding is highly relevant for the PK profile of curcumin. Together with organ-based metabolism, it can explain why after infusion, steady-state levels of curcumin were not achieved, or extremely short plasma half-lives were observed. Also, the authors hypothesized that RBCs might serve as a vehicle to distribute curcumin to tissues due to their significant presence in the blood. So, it may explain why a longer infusion time of eight-hour resulted in higher curcumin concentrations in tissues than two-hour infusion. Furthermore, when reported in terms of per cell basis, curcumin has a higher concentration in PBMCs compared to RBCs that can be of potential therapeutic value in the treatment of tumors with lymphocytic origin ${ }^{79}$. Follow up to this novel observation, the authors showed higher curcumin distribution into PBMCs in CLL patient-derived PBMC cells compared to healthy donors ${ }^{99}$ and higher uptake in multiple myeloma cell lines ${ }^{100}$, suggesting therapeutic benefit in the treatment of hematological cancers.

\section{Conclusions and Future Perspectives}

Considerable research has been performed to improve the poor pharmacokinetic profile of curcumin. Generally, curcumin loading in nanocarriers prolongs the circulation kinetics of curcumin compared to the free form upon intravenous administration in preclinical studies. Most of the nanoformulations to some extent increases the area under the curve (between 1.3-5 folds) compared to free curcumin. Also, curcumin nanoformulations demonstrated higher tumor growth inhibition compared to free curcumin in different tested tumor models, which is in line with improved PK. Unfortunately, the poor experimental design in some studies, such as inadequate sampling time points or using different animal models for PK and therapeutic experiments, caused concerns about the reliability of the information in some cases. Interestingly, the results indicate that even nanoformulations with solubilizing effect exert considerable tumor inhibition. Despite the improved PK of curcumin, there is no conclusive correlation between AUC and antitumor efficacy of the nanoformulations. The relatively low number of available PK-PD data so far plus the wide variation in 
nanoformulations (e.g. stability profile) and some experimental design weaknesses makes it hard to correlate PK and PD outcomes effectively. Therefore, there is still a lot to be learnt about the pharmacokinetics and the oncotherapeutic effects of curcumin nanoformulations. In light of the recent observation regarding the rapid distribution of curcumin to blood cells and the proposed blood-based metabolism, more research is required to understand the effect of this large compartment on curcumin. It is highly recommended to take full blood as the biological matrix to quantify curcumin instead of plasma, since discarding blood cells during sample preparation could result in underestimating curcumin concentration in the blood circulation. It is also possible that curcumin redistributes from the cellular compartment to the plasma prolonging the circulation time. Relevant questions are the whole blood-plasma partition of curcumin, the fraction of protein-bound and curcumin retained in the nanocarrier during its circulation time in the blood. Such complementary information could help us better comprehend the curcumin PK and its relationship with therapeutic efficacy when formulated as nano-sized delivery systems.

\section{Acknowledgements}

This project was supported by the European Union's Horizon 2020 research and innovation program Marie Sklodowska-Curie Innovative Training Networks (ITN) under grant No. 676137. Michal Heger was supported by grants from the Dutch Cancer Foundation (KWF project \# 10666), a Zhejiang Provincial Foreign Expert Program Grant, Zhejiang Provincial Key Natural Science Foundation of China (\#Z20H160031), and a grant for the establishment of the Jiaxing Key Laboratory for Photonanomedicine and Experimental Therapeutics. Michal Heger is chief formulation officer at Nurish.Me LLC and Camelina Sun LTD and has equity in those companies. There are no other conflicts of interest. 


\section{References}

1. Sharma, R. A.; Gescher, A. J.; Steward, W. P., Curcumin: The story so far. Eur. J. Cancer 2005, 41 (13), 1955-1968.

2. Kotha, R. R.; Luthria, D. L., Curcumin: Biological, pharmaceutical, nutraceutical, and analytical aspects. Molecules 2019, 24 (16), 2930.

3. Doello, K.; Ortiz, R.; Alvarez, P. J.; Melguizo, C.; Cabeza, L.; Prados, J., Latest in Vitro and in Vivo Assay, Clinical Trials and Patents in Cancer Treatment using Curcumin: A Literature Review. Nutr. Cancer 2018, 70 (4), 569-578.

4. $\quad$ Patel, S. S.; Acharya, A.; Ray, R. S.; Agrawal, R.; Raghuwanshi, R.; Jain, P., Cellular and molecular mechanisms of curcumin in prevention and treatment of disease. Crit. Rev. Food Sci. Nutr. 2020, 60 (6), 887-939.

5. Shehzad, A.; Wahid, F.; Lee, Y. S., Curcumin in Cancer Chemoprevention: Molecular Targets, Pharmacokinetics, Bioavailability, and Clinical Trials. Arch. Pharm. 2010, 343 (9), 489-499.

6. Heger, M.; van Golen, R. F.; Broekgaarden, M.; Michel, M. C., The molecular basis for the pharmacokinetics and pharmacodynamics of curcumin and its metabolites in relation to cancer. Pharmacol. Rev. 2014, 66 (1), 222-307.

7. Heger, M., Drug screening: Don't discount all curcumin trial data. Nature 2017, 543 (7643), 40.

8. Balaji, S.; Chempakam, B., Toxicity prediction of compounds from turmeric (Curcuma longa L). Food Chem. Toxicol. 2010, 48 (10), 2951-2959.

9. $\quad$ Cheng, A. L.; Hsu, C. H.; Lin, J. K.; Hsu, M. M.; Ho, Y. F.; Shen, T. S.; Ko, J. Y.; Lin, J. T.; Lin, B. R.; Ming-Shiang, W.; Yu, H. S.; Jee, S. H.; Chen, G. S.; Chen, T. M.; Chen, C. A.; Lai, M. K.; Pu, Y. S.; Pan, M. H.; Wang, Y. J.; Tsai, C. C.; Hsieh, C. Y., Phase I clinical trial of curcumin, a chemopreventive agent, in patients with high-risk or pre-malignant lesions. Anticancer Res 2001, 21 (4b), 2895-900.

10. Yeung, A. W. K.; Horbańczuk, M.; Tzvetkov, N. T.; Mocan, A.; Carradori, S.; Maggi, F.; Marchewka, J.; Sut, S.; Dall'Acqua, S.; Gan, R.-Y.; Tancheva, L. P.; Polgar, T.; Berindan-Neagoe, I.; Pirgozliev, V.; Šmejkal, K.; Atanasov, A. G., Curcumin: Total-scale analysis of the scientific literature. Molecules 2019, 24 (7), 1393.

11. Willenbacher, E.; Khan, S. Z.; Mujica, S. C. A.; Trapani, D.; Hussain, S.; Wolf, D.; Willenbacher, W.; Spizzo, G.; Seeber, A., Curcumin: New Insights into an Ancient Ingredient against Cancer. Int. J. Mol. Sci. 2019, 20 (8), 1808.

12. Duvoix, A.; Blasius, R.; Delhalle, S.; Schnekenburger, M.; Morceau, F.; Henry, E.; Dicato, M.; Diederich, M., Chemopreventive and therapeutic effects of curcumin. Cancer Lett. 2005, 223 (2), 181-190.

13. Shanmugam, M. K.; Rane, G.; Kanchi, M. M.; Arfuso, F.; Chinnathambi, A.; Zayed, M. E.; Alharbi, S. A.; Tan, B. K.; Kumar, A. P.; Sethi, G., The multifaceted role of curcumin in cancer prevention and treatment. Molecules 2015, 20 (2), 2728-69.

14. Ismail, N. I.; Othman, I.; Abas, F.; N, H. L.; Naidu, R., Mechanism of apoptosis induced by curcumin in colorectal cancer. Int J Mol Sci 2019, 20 (10).

15. Giordano, A.; Tommonaro, G., Curcumin and Cancer. Nutrients 2019, 11 (10).

16. Ravindran, J.; Prasad, S.; Aggarwal, B. B., Curcumin and Cancer Cells: How Many Ways Can Curry Kill Tumor Cells Selectively? AAPS J. 2009, 11 (3), 495-510.

17. Syng-Ai, C.; Kumari, A. L.; Khar, A., Effect of curcumin on normal and tumor cells: role of glutathione and bcl-2. Mol. Cancer Ther. 2004, 3 (9), 1101-8. 
18. Chang, R.; Sun, L.; Webster, T. J., Short communication: selective cytotoxicity of curcumin on osteosarcoma cells compared to healthy osteoblasts. Int. J. Nanomed. 2014, 9, 461-465.

19. Mehta, H. J.; Patel, V.; Sadikot, R. T., Curcumin and lung cancer-a review. Target Oncol. 2014, 9 (4), 295-310.

20. Bimonte, S.; Barbieri, A.; Leongito, M.; Piccirillo, M.; Giudice, A.; Pivonello, C.; de Angelis, C.; Granata, V.; Palaia, R.; Izzo, F., Curcumin AntiCancer Studies in Pancreatic Cancer. Nutrients 2016, $8(7), 433$.

21. Shureiqi, I.; Baron, J. A., Curcumin chemoprevention: The long road to clinical translation. Cancer Prev. Res. 2011, 4 (3), 296.

22. Dhillon, N.; Aggarwal, B. B.; Newman, R. A.; Wolff, R. A.; Kunnumakkara, A. B.; Abbruzzese, J. L.; Ng, C. S.; Badmaev, V.; Kurzrock, R., Phase II Trial of curcumin in patients with advanced pancreatic cancer. Clin. Cancer Res. 2008, 14 (14), 4491.

23. Cheng, A. L.; Hsu, C. H.; Lin, J. K.; Hsu, M. M.; Ho, Y. F.; Shen, T. S.; Ko, J. Y.; Lin, J. T.; Lin, B. R.; Ming-Shiang, W.; Yu, H. S.; Jee, S. H.; Chen, G. S.; Chen, T. M.; Chen, C. A.; Lai, M. K.; Pu, Y. S.; Pan, M. H.; Wang, Y. J.; Tsai, C. C.; Hsieh, C. Y., Phase I clinical trial of curcumin, a chemopreventive agent, in patients with high-risk or pre-malignant lesions. Anticancer Res. 2001, 21 (4b), 2895-900.

24. Mohamed, S. A.; El-Shishtawy, R. M.; Al-Bar, O. A. M.; Al-Najada, A. R., Chemical modification of curcumin: Solubility and antioxidant capacity. Int. J. Food Prop. 2017, 20 (3), 718724.

25. Subramaniam, D.; May, R.; Sureban, S. M.; Lee, K. B.; George, R.; Kuppusamy, P.; Ramanujam, R. P.; Hideg, K.; Dieckgraefe, B. K.; Houchen, C. W.; Anant, S., Diphenyl difluoroketone: A curcumin derivative with potent in vivo anticancer activity. Cancer Res. 2008, 68 (6), 1962.

26. Li, Q.; Zhai, W.; Jiang, Q.; Huang, R.; Liu, L.; Dai, J.; Gong, W.; Du, S.; Wu, Q., Curcuminpiperine mixtures in self-microemulsifying drug delivery system for ulcerative colitis therapy. Int. J. Pharm. 2015, 490 (1), 22-31.

27. Shoba, G.; Joy, D.; Joseph, T.; Majeed, M.; Rajendran, R.; Srinivas, P. S. S. R., Influence of piperine on the pharmacokinetics of curcumin in animals and human volunteers. Planta Med 1998, 64 (04), 353-356.

28. Mirzaei, H.; Shakeri, A.; Rashidi, B.; Jalili, A.; Banikazemi, Z.; Sahebkar, A., Phytosomal curcumin: A review of pharmacokinetic, experimental and clinical studies. Biomed. Pharmacother. 2017, 85, 102-112.

29. Naksuriya, O.; Okonogi, S.; Schiffelers, R. M.; Hennink, W. E., Curcumin nanoformulations: A review of pharmaceutical properties and preclinical studies and clinical data related to cancer treatment. Biomaterials 2014, 35 (10), 3365-3383.

30. Yallapu, M. M.; Jaggi, M.; Chauhan, S. C., Curcumin nanoformulations: a future nanomedicine for cancer. Drug discovery today 2012, 17 (1-2), 71-80.

31. Mohanty, C.; Das, M.; Sahoo, S. K., Emerging role of nanocarriers to increase the solubility and bioavailability of curcumin. Expert Opin. Drug Delivery 2012, 9 (11), 1347-64.

32. Patra, J. K.; Das, G.; Fraceto, L. F.; Campos, E. V. R.; Rodriguez-Torres, M. d. P.; AcostaTorres, L. S.; Diaz-Torres, L. A.; Grillo, R.; Swamy, M. K.; Sharma, S.; Habtemariam, S.; Shin, H.-S., Nano based drug delivery systems: recent developments and future prospects. J. Nanobiotechnol. 2018, $16(1), 71$.

33. Mirza, A. Z.; Siddiqui, F. A., Nanomedicine and drug delivery: a mini review. Int. Nano Lett. 2014, 4 (1), 94. 
34. Varela-Moreira, A.; Shi, Y.; Fens, M. H. A. M.; Lammers, T.; Hennink, W. E.; Schiffelers, R. M., Clinical application of polymeric micelles for the treatment of cancer. Mater. Chem. Front. 2017, 1 (8), 1485-1501.

35. Deng, C.; Jiang, Y.; Cheng, R.; Meng, F.; Zhong, Z., Biodegradable polymeric micelles for targeted and controlled anticancer drug delivery: Promises, progress and prospects. Nano Today 2012, 7 (5), 467-480.

36. Bobo, D.; Robinson, K. J.; Islam, J.; Thurecht, K. J.; Corrie, S. R., Nanoparticle-based medicines: a review of FDA-approved materials and clinical trials to date. Pharm. Res. 2016, 33 (10), 2373-2387.

37. Naksuriya, O.; van Steenbergen, M. J.; Torano, J. S.; Okonogi, S.; Hennink, W. E., A kinetic degradation study of curcumin in its free form and loaded in polymeric micelles. AAPS J. 2016, 18 (3), 777-787.

38. Sun, D.; Zhou, J.-K.; Zhao, L.; Zheng, Z.-Y.; Li, J.; Pu, W.; Liu, S.; Liu, X.-S.; Liu, S.-J.; Zheng, Y.; Zhao, Y.; Peng, Y., Novel curcumin liposome modified with hyaluronan targeting CD44 plays an anti-leukemic role in acute myeloid leukemia in vitro and in vivo. ACS Appl. Mater. Interfaces 2017, 9 (20), 16857-16868.

39. Yu, Y.; Zhang, X.; Qiu, L., The anti-tumor efficacy of curcumin when delivered by size/charge-changing multistage polymeric micelles based on amphiphilic poly(beta-amino ester) derivates. Biomaterials 2014, 35 (10), 3467-79.

40. Z Zou, P.; Helson, L.; Maitra, A.; Stern, S. T.; McNeil, S. E., Polymeric Curcumin Nanoparticle Pharmacokinetics and Metabolism in Bile Duct Cannulated Rats. Mol. Pharmaceutics 2013, 10 (5), 1977-1987.

41. Duan, D.; Wang, A.; Ni, L.; Zhang, L.; Yan, X.; Jiang, Y.; Mu, H.; Wu, Z.; Sun, K.; Li, Y., Trastuzumab- and Fab' fragment-modified curcumin PEG-PLGA nanoparticles: preparation and evaluation in vitro and in vivo. Int. J. Nanomed. 2018, 13, 1831-1840.

42. Wu, W.; Wu, J.; Fu, Q.; Jin, C.; Guo, F.; Yan, Q.; Yang, Q.; Wu, D.; Yang, Y.; Yang, G., Elaboration and characterization of curcumin-loaded Tri-CL-mPEG three-arm copolymeric nanoparticles by a microchannel technology. Int. J. Nanomed. 2019, 14, 4683-4695.

43. Lollo, G.; Ullio-Gamboa, G.; Fuentes, E.; Matha, K.; Lautram, N.; Benoit, J.-P., In vitro anticancer activity and pharmacokinetic evaluation of curcumin-loaded lipid nanocapsules. Mater Sci Eng C Mater Biol Appl 2018, 91, 859-867.

44. Liu, Y.; Chen, F.; Zhang, K.; Wang, Q.; Chen, Y.; Luo, X., pH-Responsive reversibly crosslinked micelles by phenol-yne click via curcumin as a drug delivery system in cancer chemotherapy. J. Mater. Chem. B 2019, 7 (24), 3884-3893.

45. Ji, P.; Wang, L.; Chen, Y. W.; Wang, S. Q.; Wu, Z. H.; Qi, X. L., Hyaluronic acid hydrophilic surface rehabilitating curcumin nanocrystals for targeted breast cancer treatment with prolonged biodistribution. Biomater. Sci. 2020, 8 (1), 462-472.

46. Yoon, I.-S.; Park, J.-H.; Kang, H. J.; Choe, J. H.; Goh, M. S.; Kim, D.-D.; Cho, H.-J., Poly(d,1lactic acid)-glycerol-based nanoparticles for curcumin delivery. Int. J. Pharm. 2015, 488 (1), 70-77.

47. Wu, Z.; Cai, M.; Xie, X.; He, L.; Huang, L.; Chen, Y.; Luo, X., The effect of architecture/composition on the $\mathrm{pH}$ sensitive micelle properties and in vivo study of curcuminin-loaded micelles containing sulfobetaines. RSC Adv. 2015, 5 (129), 106989-107000.

48. Sun, J.; Bi, C.; Chan, H. M.; Sun, S.; Zhang, Q.; Zheng, Y., Curcumin-loaded solid lipid nanoparticles have prolonged in vitro antitumour activity, cellular uptake and improved in vivo bioavailability. Colloids Surf., B 2013, 111, 367-375. 
49. Xu, Y.; Asghar, S.; Yang, L.; Chen, Z.; Li, H.; Shi, W.; Li, Y.; Shi, Q.; Ping, Q.; Xiao, Y., Nanoparticles based on chitosan hydrochloride/hyaluronic acid/PEG containing curcumin: In vitro evaluation and pharmacokinetics in rats. Int. J. Biol. Macromol. 2017, 102, 1083-1091.

50. Zhai, S.; Ma, Y.; Chen, Y.; Li, D.; Cao, J.; Liu, Y.; Cai, M.; Xie, X.; Chen, Y.; Luo, X., Synthesis of an amphiphilic block copolymer containing zwitterionic sulfobetaine as a novel $\mathrm{pH}-$ sensitive drug carrier. Polym. Chem. 2014, 5 (4), 1285-1297.

51. Chen, S.; Li, Q.; Li, H.; Yang, L.; Yi, J.-Z.; Xie, M.; Zhang, L.-M., Long-circulating zeinpolysulfobetaine conjugate-based nanocarriers for enhancing the stability and pharmacokinetics of curcumin. Mater. Sci. Eng., C 2020, 109, 110636.

52. Chen, D.; Sun, J., In vitro and in vivo evaluation of PEG-conjugated ketal-based chitosan micelles as pH-sensitive carriers. Polym. Chem. 2015, 6 (6), 998-1004.

53. Song, Z.; Feng, R.; Sun, M.; Guo, C.; Gao, Y.; Li, L.; Zhai, G., Curcumin-loaded PLGAPEG-PLGA triblock copolymeric micelles: Preparation, pharmacokinetics and distribution in vivo. $J$. Colloid Interface Sci. 2011, 354 (1), 116-123.

54. Duan, J.; Zhang, Y.; Han, S.; Chen, Y.; Li, B.; Liao, M.; Chen, W.; Deng, X.; Zhao, J.; Huang, B., Synthesis and in vitro/in vivo anti-cancer evaluation of curcumin-loaded chitosan/poly(butyl cyanoacrylate) nanoparticles. Int. J. Pharm. 2010, 400 (1), 211-220.

55. Gao, X.; Zheng, F.; Guo, G.; Liu, X.; Fan, R.; Qian, Z.-y.; Huang, N.; Wei, Y.-q., Improving the anti-colon cancer activity of curcumin with biodegradable nano-micelles. J. Mater. Chem. B 2013, 1 (42), 5778-5790.

56. Sun, M.; Gao, Y.; Guo, C.; Cao, F.; Song, Z.; Xi, Y.; Yu, A.; Li, A.; Zhai, G., Enhancement of transport of curcumin to brain in mice by poly(n-butylcyanoacrylate) nanoparticle. J. Nanopart. Res. 2010, 12 (8), 3111-3122.

57. Gou, M.; Men, K.; Shi, H.; Xiang, M.; Zhang, J.; Song, J.; Long, J.; Wan, Y.; Luo, F.; Zhao, X.; Qian, Z., Curcumin-loaded biodegradable polymeric micelles for colon cancer therapy in vitro and in vivo. Nanoscale 2011, 3 (4), 1558-1567.

58. Hong, J. Y.; Liu, Y. Y.; Xiao, Y.; Yang, X. F.; Su, W. J.; Zhang, M. Z.; Liao, Y. H.; Kuang, H. X.; Wang, X. T., High drug payload curcumin nanosuspensions stabilized by mPEG-DSPE and SPC: in vitro and in vivo evaluation. Drug Deliv. 2017, 24 (1), 109-120.

59. Yang, C.; Chen, H.; Zhao, J.; Pang, X.; Xi, Y.; Zhai, G., Development of a folate-modified curcumin loaded micelle delivery system for cancer targeting. Colloids Surf., B 2014, 121, 206-213.

60. Song, Z.; Zhu, W.; Yang, F.; Liu, N.; Feng, R., Preparation, characterization, in vitro release, and pharmacokinetic studies of curcumin-loaded mPEG-PVL nanoparticles. Polym. Bull. 2015, 72 (1), 75-91.

61. Feng, R.; Song, Z.; Zhai, G., Preparation and in vivo pharmacokinetics of curcumin-loaded PCL-PEG-PCL triblock copolymeric nanoparticles. Int. J. Nanomed. 2012, 7, 4089-4098.

62. Smith, D. A.; Beaumont, K.; Maurer, T. S.; Di, L., Volume of distribution in drug design. J. Med. Chem 2015, 58 (15), 5691-5698.

63. Mansoor, A.; Mahabadi, N., Volume of distribution. In StatPearls, StatPearls Publishing LLC.: Treasure Island (FL), 2020.

64. Ravindranath, V.; Chandrasekhara, N., Absorption and tissue distribution of curcumin in rats. Toxicology 1980, 16 (3), 259-265.

65. Ravindranath, V.; Chandrasekhara, N., Metabolism of curcumin-studies with $[3 \mathrm{H}]$ curcumin. Toxicology 1981, 22 (4), 337-344. 


\section{Chapter 2}

66. Holder, G. M.; Plummer, J. L.; Ryan, A. J., The metabolism and excretion of curcumin (1,7bis-(4-hydroxy-3-methoxyphenyl)-1,6-heptadiene-3,5-dione) in the rat. Xenobiotica 1978, 8 (12), 7618 .

67. Gong, F.; Chen, D.; Teng, X.; Ge, J.; Ning, X.; Shen, Y. L.; Li, J.; Wang, S., Curcuminloaded blood-stable polymeric micelles for enhancing therapeutic effect on erythroleukemia. Mol. Pharmaceutics 2017, 14 (8), 2585-2594.

68. Gong, C.; Deng, S.; Wu, Q.; Xiang, M.; Wei, X.; Li, L.; Gao, X.; Wang, B.; Sun, L.; Chen, Y.; Li, Y.; Liu, L.; Qian, Z.; Wei, Y., Improving antiangiogenesis and anti-tumor activity of curcumin by biodegradable polymeric micelles. Biomaterials 2013, 34 (4), 1413-1432.

69. Song, Z.; Zhu, W.; Song, J.; Wei, P.; Yang, F.; Liu, N.; Feng, R., Linear-dendrimer type methoxy-poly (ethylene glycol)-b-poly ( $\varepsilon$-caprolactone) copolymer micelles for the delivery of curcumin. Drug Deliv. 2015, 22 (1), 58-68.

70. $\quad$ Song, Z.; Zhu, W.; Liu, N.; Yang, F.; Feng, R., Linolenic acid-modified PEG-PCL micelles for curcumin delivery. Int. J. Pharm. 2014, 471 (1), 312-321.

71. Removal of blood from laboratory mammals and birds. First report of the BVA/FRAME/RSPCA/UFAW Joint Working Group on Refinement. Lab. Anim. 1993, 27 (1), 1-22.

72. Yu, Y.; Zhang, X.; Qiu, L., The anti-tumor efficacy of curcumin when delivered by size/charge-changing multistage polymeric micelles based on amphiphilic poly( $\beta$-amino ester) derivates. Biomaterials 2014, 35 (10), 3467-3479.

73. Hu, Y. Z.; He, Y. H.; Ji, J. R.; Zheng, S. P.; Cheng, Y. Z., Tumor Targeted Curcumin Delivery by Folate-Modified MPEG-PCL Self-Assembly Micelles for Colorectal Cancer Therapy. Int. J. Nanomed. 2020, 15, 1239-1252.

74. Song, Z.; Lu, Y.; Zhang, X.; Wang, H.; Han, J.; Dong, C., Novel curcumin-loaded human serum albumin nanoparticles surface functionalized with folate: characterization and in vitro/vivo evaluation. Drug Des Devel Ther 2016, 10, 2643-2649.

75. Jiang, K.; Shen, M.; Xu, W., Arginine, glycine, aspartic acid peptide-modified paclitaxel and curcumin co-loaded liposome for the treatment of lung cancer: in vitro/vivo evaluation. Int. J. Nanomed. 2018, 13, 2561-2569.

76. Ma, Z.; Shayeganpour, A.; Brocks, D. R.; Lavasanifar, A.; Samuel, J., High-performance liquid chromatography analysis of curcumin in rat plasma: application to pharmacokinetics of polymeric micellar formulation of curcumin. Biomed. Chromatogr. 2007, 21 (5), 546-552.

77. Li, L.; Xiang, D.; Shigdar, S.; Yang, W.; Li, Q.; Lin, J.; Liu, K.; Duan, W., Epithelial cell adhesion molecule aptamer functionalized PLGA-lecithin-curcumin-PEG nanoparticles for targeted drug delivery to human colorectal adenocarcinoma cells. Int. J. Nanomed. 2014, 9, 1083-1096.

78. Jamwal, R., Bioavailable curcumin formulations: A review of pharmacokinetic studies in healthy volunteers. J. Integr. Med. 2018, 16 (6), 367-374.

79. Bolger, G. T.; Licollari, A.; Tan, A.; Greil, R.; Vcelar, B.; Majeed, M.; Helson, L., Distribution and metabolism of Lipocurc ${ }^{\mathrm{TM}}$ (liposomal curcumin) in dog and human blood cells: species selectivity and pharmacokinetic relevance. Anticancer Res 2017, 37 (7), 3483-3492.

80. Wahlström, B.; Blennow, G., A study on the fate of curcumin in the rat. Acta Pharmacol. Toxicol. 1978, 43 (2), 86-92.

81. Bagheri, M.; Fens, M. H.; Kleijn, T. G.; Capomaccio, R. B.; Mehn, D.; Krawczyk, P. M.; Scutigliani, E. M.; Gurinov, A.; Baldus, M.; van Kronenburg, N. C. H.; Kok, R. J.; Heger, M.; van Nostrum, C. F.; Hennink, W. E., In vitro and In vivo studies on HPMA-based polymeric micelles loaded with curcumin. Mol Pharm 2021. In press.

82. Anand, P.; Kunnumakkara, A. B.; Newman, R. A.; Aggarwal, B. B., Bioavailability of curcumin: problems and promises. Mol. Pharmaceutics 2007, 4 (6), 807-818. 
83. Metzler, M.; Pfeiffer, E.; Schulz, S. I.; Dempe, J. S., Curcumin uptake and metabolism. BioFactors 2013, 39 (1), 14-20.

84. Fujisawa, S.; Atsumi, T.; Ishihara, M.; Kadoma, Y., Cytotoxicity, ROS-generation activity and radical-scavenging activity of curcumin and related compounds. Anticancer Res. 2004, 24 (2B), 563-570.

85. Yu, X.; Trase, I.; Ren, M.; Duval, K.; Guo, X.; Chen, Z., Design of nanoparticle-based carriers for targeted drug delivery. J. Nanomater. 2016, 2016, 1087250.

86. Wang, B.; He, X.; Zhang, Z.; Zhao, Y.; Feng, W., Metabolism of nanomaterials in vivo: Blood circulation and organ clearance. Acc. Chem. Res. 2013, 46 (3), 761-769.

87. Dawidczyk, C. M.; Kim, C.; Park, J. H.; Russell, L. M.; Lee, K. H.; Pomper, M. G.; Searson, P. C., State-of-the-art in design rules for drug delivery platforms: Lessons learned from FDA-approved nanomedicines. J. Controlled Release 2014, 187, 133-144.

88. Abouelmagd, S. A.; Sun, B.; Chang, A. C.; Ku, Y. J.; Yeo, Y., Release kinetics study of poorly water-soluble drugs from nanoparticles: Are we doing it right? Mol. Pharmaceutics 2015, 12 (3), 997-1003.

89. Sheybanifard, M.; Beztsinna, N.; Bagheri, M.; Miriam Buhl, E.; Bresseleers, J.; VarelaMoreira, A.; Shi, Y.; van Nostrum, C. F.; van der Pluijm, G.; Storm, G.; Hennink, W. E.; Lammers, T.; Metselaar, J. M., Systematic evaluation of design features enables efficient selection of $\Pi$ electronstabilized polymeric micelles. Int. J. Pharm. 2020, 119409.

90. Felder-Flesch, D., Dendrimers in Nanomedicine. Jenny Stanford Publishing: 2016.

91. Boussery, K.; Belpaire, F. M.; Van de Voorde, J., Chapter 23 - Physiological Aspects Determining the pharmacokinetic properties of drugs. In The Practice of Medicinal Chemistry (Fourth Edition), Wermuth, C. G.; Aldous, D.; Raboisson, P.; Rognan, D., Eds. Academic Press: San Diego, 2008; pp 539-559.

92. The Johns Hopkins University. Animal care and use committee. http://web.jhu.edu/animalcare/procedures/mouse.html (accessed accessed July 24, 2020).

93. Riches, A. C.; Sharp, J. G.; Thomas, D. B.; Smith, S. V., Blood volume determination in the mouse. J. Physiol. 1973, 228 (2), 279-284.

94. Grossen, P.; Witzigmann, D.; Sieber, S.; Huwyler, J., PEG-PCL-based nanomedicines: A biodegradable drug delivery system and its application. J. Controlled Release 2017, 260, 46-60.

95. Saghatelyan, T.; Tananyan, A.; Janoyan, N.; Tadevosyan, A.; Petrosyan, H.; Hovhannisyan, A.; Hayrapetyan, L.; Arustamyan, M.; Arnhold, J.; Rotmann, A.-R.; Hovhannisyan, A.; Panossian, A., Efficacy and safety of curcumin in combination with paclitaxel in patients with advanced, metastatic breast cancer: A comparative, randomized, double-blind, placebo-controlled clinical trial. Phytomedicine 2020, 70, 153218.

96. Matabudul, D.; Pucaj, K.; Bolger, G.; Vcelar, B.; Majeed, M.; Helson, L., Tissue distribution of (Lipocurc ${ }^{\mathrm{TM}}$ ) liposomal curcumin and tetrahydrocurcumin following two- and eight-hour infusions in Beagle dogs. Anticancer Res 2012, 32 (10), 4359-64.

97. Storka, A.; Vcelar, B.; Klickovic, U.; Gouya, G.; Weisshaar, S.; Aschauer, S.; Bolger, G.; Helson, L.; Wolzt, M., Safety, tolerability and pharmacokinetics of liposomal curcumin in healthy humans. Int. J. Clin. Pharmacol. Ther. 2015, 53 (1), 54-65.

98. Storka, A.; Vcelar, B.; Klickovic, U.; Gouya, G.; Weisshaar, S.; Aschauer, S.; Helson, L.; Wolzt, M., Effect of liposomal curcumin on red blood cells in vitro. Anticancer Res 2013, 33 (9), 362934. 
99. Bolger, G. T.; Licollari, A.; Tan, A.; Greil, R.; Pleyer, L.; Vcelar, B.; Majeed, M.; Sordillo, P., Distribution of curcumin and THC in peripheral blood mononuclear cells isolated from healthy individuals and patients with chronic lymphocytic leukemia. Anticancer Res 2018, 38 (1), 121-130.

100. Bolger, G. T.; Licollari, A.; Bagshaw, R.; Tan, A.; Greil, R.; Vcelar, B.; Majeed, M.; Sordillo, P., Intense Uptake of Liposomal Curcumin by Multiple Myeloma Cell Lines: Comparison to normal lymphocytes, red blood Cells and chronic lymphocytic leukemia cells. Anticancer Res 2019, 39 (3), $1161-1168$. 


\section{Supporting Information}

\section{S2. Data Curation and Analysis}

The not-reported clearance and volume of distribution are calculated as follows:

The reported $\mathrm{t}_{1 / 2}$ or $\mathrm{t}_{1 / 2 \beta}$ was used in the equations

Clearance $(\mathrm{CL})=$ dose $/ \mathrm{AUC}, \mathrm{CL}=\mathrm{k} \cdot \mathrm{V}_{\mathrm{d}}$, whereby $\mathrm{k}=0.693 / \mathrm{t}_{1 / 2}$

CL unit: volume/time/kg

Dose (D) unit: amount

Elimination half-life $\left(\mathrm{t}_{1 / 2}\right)$ unit: time

Area under the plasma concentration-time curve (AUC) unit: amount•time/volume

Elimination rate constant $(\mathrm{k})$ unit: time ${ }^{-1}$

Apparent volume of distribution $\left(\mathrm{V}_{\mathrm{d}}\right)$ unit: volume $/ \mathrm{kg}$

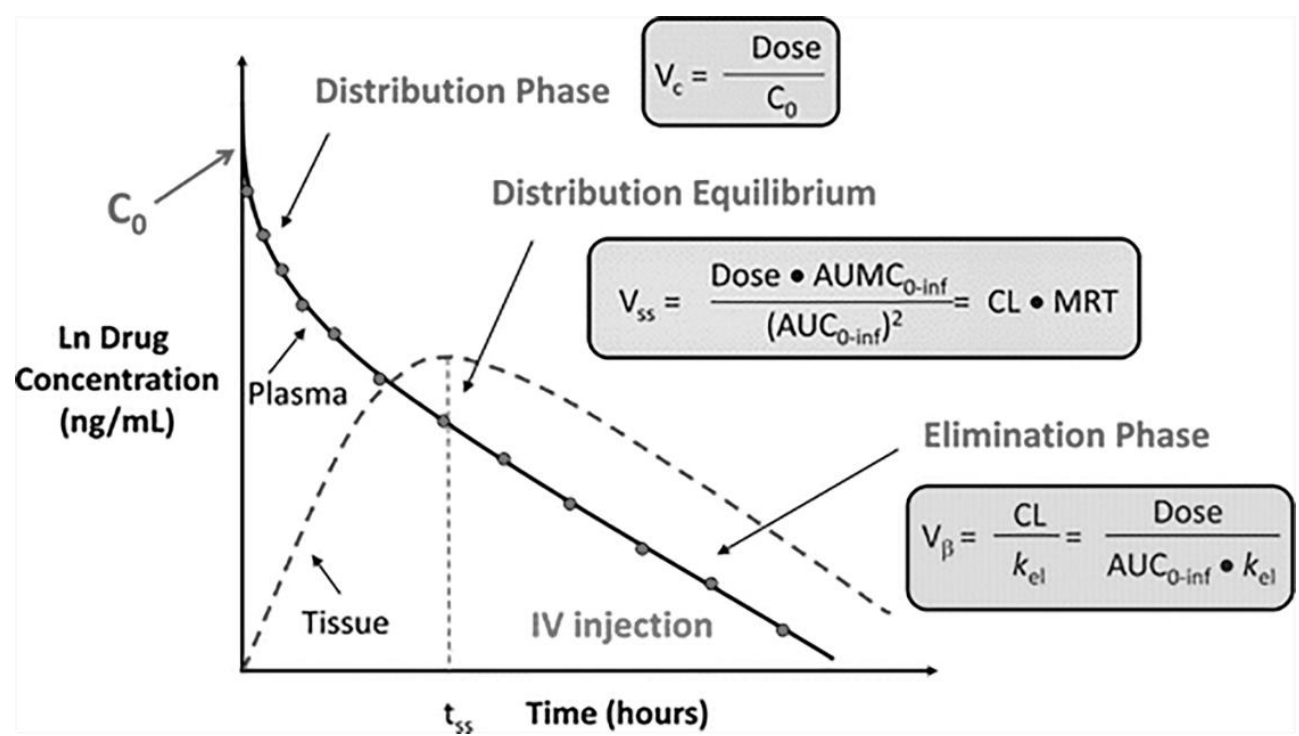

Figure S1. Definition of the different volumes of distribution of drugs after IV administration. Reprinted with permission from reference ${ }^{62}$, copyright 2015, American Chemical Society. Abbreviations: AUC, area under the plasma concentration versus time curve; AUMC, area under the first-moment curve; $\mathrm{CL}$, clearance; $\mathrm{k}_{\mathrm{el}}$, elimination rate constant; MRT, mean residence time; $\mathrm{V}_{\beta}$, volume of distribution at pseudodistribution equilibrium; $\mathrm{V}_{\mathrm{ss}}$, volume of distribution at steady state; $\mathrm{V}_{\mathrm{c}}$, volume of the central compartment. 


\section{S3. Nanoformulations Improve Multiple Curcumin Pharmacokinetics Parameters Compared to Non-Formulated, Free Curcumin}

\section{S3.1. Pharmacokinetics of Intravenously Administered Free Curcumin}

The free curcumin controls used in the in vivo studies were prepared using a solvent or solvent mixture comprising mainly $\mathrm{DMSO}^{38,40,42,52,67}$, DMA + PEG400 + isotonic dextrose or glucose $\mathrm{e}^{47-48,50,53,56,59,61,69-70,76}$, or solubilizers such as Kolliphor EL (the new name for Cremophor EL) $)^{55,57,74}$, Kolliphor HS15 ${ }^{43}$, Tween $20^{51}$ or Tween $80^{58}$. The type of vehicle can impact the PK profile which has already been demonstrated for Kolliphor as a vehicle of hydrophobic drugs. The PK of paclitaxel formulated in Kolliphor EL was unpredictable and non-linear in plasma, probably due to the entrapment of paclitaxel in the Kolliphor EL micelles, leading to lower clearance and prolonged exposure ${ }^{101-104}$.

Also, PK calculations were based on different sampling time periods. Most of the studies perform sampling during $12 \mathrm{~h}^{44,46,49,53,59-61,67,69-70}, 24 \mathrm{~h}^{39,42-43,45,47,50-52,55-58,68,73-77}, 48 \mathrm{~h}^{40-}$ ${ }^{41}$, or $72 \mathrm{~h}^{38}$. One study reported PK parameters $\left(\mathrm{AUC}_{0 \text {-inf }}\right.$ and $\left.\mathrm{t}_{1 / 2}\right)$ based on data acquired during the first hour after administration ${ }^{48}$; This study was therefore excluded from the analysis. Differences in the sampling time could influence the reliability of the PK values due to the poor characterization of the initial or terminal elimination rate ${ }^{105}$. To explain, PK analysis should include a sufficient number of sample acquisition points during the first hour after intravenous administration to properly cover the distribution phase. Similarly, sampling should continue until curcumin is no longer detected in the plasma to fully account for the elimination phase. The same issues were underscored in a recent review of clinical studies in which different sampling times after oral curcumin administration had been used ${ }^{78}$. 
Table S1. Summary of PK parameters for intravenously administered free curcumin in mice.

\begin{tabular}{|c|c|c|c|c|c|c|c|c|c|}
\hline Solvent system & Dose $[\mathrm{mg} / \mathrm{kg}]$ & $\begin{array}{l}\mathbf{t}_{1 / 2} \\
{[\mathbf{h}]}\end{array}$ & $\begin{array}{c}\mathbf{C}_{\max } \\
{[\mu \mathrm{g} / \mathrm{mL}]}\end{array}$ & $\begin{array}{c}\mathrm{AUC} \\
{[\mu \mathrm{g} \cdot \mathbf{h} / \mathbf{L}]}\end{array}$ & $\begin{array}{l}\text { Normalized AUC } \\
{[\mu \mathrm{g} \cdot \mathrm{h} / \mathrm{L}] /[\mathrm{mg} / \mathrm{kg}]}\end{array}$ & $\begin{array}{c}\mathbf{k} \\
{\left[\mathbf{h}^{-1}\right]}\end{array}$ & $\mathrm{CL}[\mathrm{L} / \mathrm{h} / \mathrm{kg}]$ & $\begin{array}{c}\mathbf{V}_{\mathrm{d}} \\
{[\mathbf{L} / \mathrm{kg}]}\end{array}$ & Ref. \\
\hline DMSO & 12 & 0.2 & 0.003 & 878 & 73 & 3.7 & 13.7 & 3.8 & 67 \\
\hline NA & 25 & 1.5 & 33.9 & 29,894 & 1,196 & 0.45 & 0.84 & $1.8^{*}$ & 68 \\
\hline Mixture & 10 & $\begin{array}{c}\mathrm{t}_{1 / 2 \alpha}=0.02 \\
\mathrm{t}_{1 / 2 \beta}=0.3\end{array}$ & 3.7 & 474 & 47 & 2.7 & 21.1 & $7.7^{*}$ & 53 \\
\hline Mixture & 10 & $\begin{array}{c}\mathrm{t}_{1 / 2 \alpha}=0.02 \\
\mathrm{t}_{1 / 2 \beta}=0.9\end{array}$ & 3.7 & 361 & 36 & 0.8 & 29 & $35.3^{*}$ & 56 \\
\hline DMSO & 10 & NA & $\sim 0.01$ & 407 & 41 & NA & 5.8 & NA & 38 \\
\hline Mixture & 2 & $\begin{array}{c}\mathrm{t}_{1 / 2 \alpha}=0.02 \\
\mathrm{t}_{1 / 2 \beta}=1.5\end{array}$ & 0.15 & 6,708 & 3,354 & 0.5 & 0.3 & $0.6^{*}$ & 50 \\
\hline $\begin{array}{c}\text { Saline }+1 \% \\
\text { Tween } 20\end{array}$ & 2 & 0.3 & 13 & 8,150 & 4,075 & 2.4 & $0.3^{*}$ & $0.1^{*}$ & 51 \\
\hline
\end{tabular}

The calculated clearance (CL) and volume of distribution $(\mathrm{Vd})$ values using the above formula are indicated by an asterisk. The solvent mixture that used to solubilize free curcumin contained $15 \% \mathrm{DMA}+45 \%$ PEG + 40\% dextrose or glucose.

Abbreviations: t1/2, half-life; Cmax, maximum concentration; $k$, elimination rate constant; AUC, area under the plasma concentration versus time curve; CL, clearance; volume of distribution (Vd); Ref., reference; NA, not available; DMA, dimethylformamide; DMSO, dimethyl sulfoxide; PEG, polyethylene glycol. 


\section{Chapter 2}

Table S2. Summary of PK parameters of intravenously administered curcumin nanoformulations in mice.

\begin{tabular}{|c|c|c|c|c|c|c|c|c|c|c|c|}
\hline Formulation & $\begin{array}{l}\text { Size } \\
{[\mathrm{nm}]}\end{array}$ & $\begin{array}{c}\mathbf{Z P} \\
{[\mathbf{m V}]}\end{array}$ & $\begin{array}{c}\text { Dose } \\
{[\mathrm{mg} / \mathrm{kg}]}\end{array}$ & $\begin{array}{l}\mathbf{t}_{1 / 2} \\
{[\mathrm{~h}]}\end{array}$ & $\underset{[\mu \mathrm{g} / \mathrm{mL}]}{\operatorname{Cmax}}$ & $\begin{array}{c}\mathbf{A U C} \\
{[\mu \mathrm{g} \bullet \mathbf{h} / \mathbf{L}]}\end{array}$ & $\begin{array}{l}\text { Normalized AUC } \\
{[\mu \mathrm{g} \bullet \mathrm{h} / \mathrm{L}] /[\mathrm{mg} / \mathrm{kg}]}\end{array}$ & $\begin{array}{c}\mathbf{k} \\
{[\mathbf{h}-1]}\end{array}$ & $\begin{array}{c}\text { CL } \\
{[\mathbf{L} / \mathbf{h} / \mathbf{k g}]}\end{array}$ & $\begin{array}{c}\mathbf{V}_{\mathrm{d}} \\
{[\mathrm{L} / \mathrm{kg}]}\end{array}$ & Ref. \\
\hline $\begin{array}{c}\text { mPEG-PCL- } \\
\text { Phe(Boc) } \\
\text { micelles }^{\text {a }}\end{array}$ & 23 & NA & 12 & 1.2 & 0.01 & 2,730 & 228 & 0.6 & 4.4 & 7.8 & 67 \\
\hline $\begin{array}{l}\text { mPEG-PCL } \\
\text { micelles }^{b}\end{array}$ & 37 & -0.8 & 25 & 2.2 & 83.0 & 148,079 & 5,923 & 0.3 & 0.2 & $0.5^{*}$ & 68 \\
\hline $\begin{array}{l}\text { mPEG-PLA-PAE } \\
\text { micelles }^{\mathrm{c}}\end{array}$ & 171 & 4 & 40 & $\begin{array}{r}\mathrm{t}_{1 / 2 \mathrm{a}}=0.14 \\
\mathrm{t}_{1 / 2 \beta}=1.03\end{array}$ & $\sim 170$ & 136,228 & 3,405 & 0.7 & 5.9 & 7.2 & 72 \\
\hline $\begin{array}{c}\text { PLGA-PEG- } \\
\text { PLGA micelles }^{\mathrm{d}}\end{array}$ & 26 & -0.7 & 10 & $\begin{array}{c}\mathrm{t}_{1 / 2 \alpha}=0.057 \\
\mathrm{t}_{1 / 2 \beta}=1.15\end{array}$ & 2.2 & 622 & 62.2 & 0.6 & 16.1 & $26.8^{*}$ & 53 \\
\hline $\begin{array}{c}\text { PBCN } \\
\text { nanoparticles }^{\mathrm{e}}\end{array}$ & 152 & 0 & 5 & $\begin{aligned} \mathrm{t}_{1 / 2 \alpha} & =10.8 \\
\mathrm{t}_{1 / 2 \beta} & =33.1\end{aligned}$ & 1.3 & 758 & 152 & 0.02 & 7.5 & $315^{*}$ & 56 \\
\hline HA-Curc-LPs ${ }^{f}$ & 210 & -36.8 & 10 & NA & $\sim 31.6$ & 128,998 & 12,900 & NA & 0.1 & NA & 38 \\
\hline $\begin{array}{c}\text { PCL-PDEA- } \\
\text { PSBMA micelles }\end{array}$ & 143 & 40 & 2 & $\begin{array}{l}\mathrm{t}_{1 / 2 \mathrm{a}}=0.2 \\
\mathrm{t}_{1 / 2 \beta}=6.5\end{array}$ & 0.19 & 8,964 & 4,482 & 0.11 & 0.2 & $2.1^{*}$ & 50 \\
\hline $\begin{array}{l}\text { Zein-PSBMA- } \\
\text { based micelles }\end{array}$ & 155 & -5.3 & 2 & 6.4 & 27 & 299,410 & 149,705 & 0.11 & $0.006^{*}$ & $0.06^{*}$ & 51 \\
\hline
\end{tabular}

The calculated clearance $(\mathrm{CL})$ and volume of distribution $\left(\mathrm{V}_{\mathrm{d}}\right)$ values using the above formula are indicated by asterisk.

Abbreviations: $\mathrm{t}_{1 / 2}$, half-life; $\mathrm{C}_{\max }$, maximum concentration; $\mathrm{k}$, elimination rate constant; AUC, area under the plasma concentration versus time curve; CL, clearance; volume of distribution $\left(\mathrm{V}_{\mathrm{d}}\right)$; $\mathrm{ZP}$, zeta potential; Ref., reference; NA, not available.

Notes nanoformulations: ${ }^{a} \mathrm{~N}$-(tert-butoxycarbonyl)-L-phenylalanine end-capped methoxy-poly(ethylene glycol)-b-poly( $\varepsilon$-caprolactone); ${ }^{b}$ monomethyl poly(ethylene glycol)-poly( $\varepsilon$-caprolactone); ${ }^{\mathrm{c}} \mathrm{pH}$-sensitive methoxy poly(ethylene glycol)-poly(lactide)-poly( $\beta$-amino ester); ${ }^{\mathrm{d}}$ poly $(\mathrm{D}$,L-lactide-co-glycolide)$b$-poly(ethylene glycol)- $b$-poly(D,L-lactide-co-glycolide); ${ }^{\text {e }}$ polybutylcyanoacrylate nanoparticles coated with polysorbate $80 ;{ }^{\mathrm{f}}$ curcumin liposome modified 
with hyaluronic acid; ${ }^{\mathrm{g}} \mathrm{pH}$-sensitive poly( $\varepsilon$-caprolactone)- $b$-poly(diethylaminoethyl methacrylate)- $b$-poly(sulfobetaine methacrylate); ${ }^{\mathrm{h}}$ zein-poly(sulfobetaine methacrylate).

Table S3. Summary of PK parameters of intravenously administered free curcumin in rats.

\begin{tabular}{|c|c|c|c|c|c|c|c|c|c|}
\hline Solvent system & $\begin{array}{c}\text { Dose } \\
{[\mathrm{mg} / \mathrm{kg}]}\end{array}$ & $\begin{array}{l}\mathbf{t}_{1 / 2} \\
{[\mathbf{h}]}\end{array}$ & $\begin{array}{c}\mathrm{C}_{\max } \\
{[\mu \mathrm{g} / \mathbf{m L}]}\end{array}$ & $\begin{array}{c}\mathbf{A U C} \\
{[\mu \mathrm{g} \bullet \mathbf{h} / \mathbf{L}]}\end{array}$ & $\begin{array}{l}\text { Normalized AUC } \\
{[\mu g \bullet h / L] /[\mathrm{mg} / \mathrm{kg}]}\end{array}$ & $\begin{array}{c}\mathbf{K} \\
{\left[\mathbf{h}^{-1}\right]}\end{array}$ & $\begin{array}{c}\text { CL } \\
{[\mathrm{L} / \mathrm{h} / \mathbf{k g}]}\end{array}$ & $\begin{array}{c}\mathbf{V}_{\mathrm{d}} \\
{[\mathrm{L} / \mathrm{kg}]}\end{array}$ & Ref. \\
\hline NA & 10 & $\mathrm{t}_{1 / 2 \beta}=0.4$ & NA & 1,922 & 192 & 1.9 & 0.005 & 0.002 & 54 \\
\hline Cremophor & 50 & 1.5 & 67.7 & 60,325 & 1,206 & 0.5 & $0.8 *$ & $1.8^{*}$ & 55 \\
\hline Cremophor & 100 & 0.33 & 306 & 132 & 1 & 2.1 & $755^{*}$ & $355^{*}$ & 57 \\
\hline NA & 5 & $\mathrm{t}_{1 / 2 \beta}=0.44$ & $\sim 0.25$ & 1,990 & 398 & 1.6 & 2.5 & 15.8 & 44 \\
\hline NA & 2 & 11.13 & 0.02 & 54 & 27 & 0.06 & 77 & 1.2 & 45 \\
\hline $\begin{array}{l}\text { DMSO:Tween } 80 \\
(1: 1 v / v)\end{array}$ & 10 & 1.81 & 3.7 & 1,705 & 171 & 0.4 & 3.8 & $15.3^{*}$ & 58 \\
\hline NA & 50 & 0.81 & 61.7 & 62,280 & 1,246 & 0.9 & $0.8^{*}$ & $0.9^{*}$ & 73 \\
\hline Cremophor & 10 & 0.08 & 1.8 & 2,298 & 230 & 8.7 & 16.7 & 0.5 & 74 \\
\hline NA & 4 & 0.77 & 0.01 & 3 & 1 & 0.9 & $1238^{*}$ & $1376^{*}$ & 41 \\
\hline
\end{tabular}




\section{Chapter 2}

\begin{tabular}{|c|c|c|c|c|c|c|c|c|c|}
\hline Solvent system & $\begin{array}{c}\text { Dose } \\
{[\mathrm{mg} / \mathrm{kg}]}\end{array}$ & $\begin{array}{l}\mathbf{t}_{1 / 2} \\
{[\mathbf{h}]}\end{array}$ & $\underset{[\mu \mathrm{g} / \mathrm{mL}]}{\mathrm{C}_{\max }}$ & $\begin{array}{c}\mathrm{AUC} \\
{[\mu \mathrm{g} \bullet \mathbf{h} / \mathbf{L}]}\end{array}$ & $\begin{array}{l}\text { Normalized AUC } \\
{[\mu g \bullet h / L] /[\mathrm{mg} / \mathrm{kg}]}\end{array}$ & $\begin{array}{c}\mathbf{K} \\
{\left[\mathbf{h}^{-1}\right]}\end{array}$ & $\begin{array}{c}\text { CL } \\
{[\mathbf{L} / \mathbf{h} / \mathbf{k g}]}\end{array}$ & $\begin{array}{c}\mathbf{V}_{d} \\
{[\mathbf{L} / \mathbf{k g}]}\end{array}$ & Ref. \\
\hline NA & 6 & 3.6 & $\sim 2.6$ & 3,287 & 548 & 0.2 & 11.2 & $9.5^{*}$ & 75 \\
\hline DMSO & 2 & 2.41 & 0.12 & 110 & 55 & 0.3 & 28.5 & $62.8^{*}$ & 42 \\
\hline Kolliphor HS15 & 10 & 1.15 & 13.1 & 18,770 & 1,877 & 0.6 & 0.13 & $0.9^{*}$ & 43 \\
\hline Mixture & 10 & 0.57 & $\sim 10$ & $1,670,000$ & 167,000 & 1.2 & 5.5 & 2.1 & 76 \\
\hline PEG400 & 10 & NA & $\sim 0.1$ & 72 & 7 & NA & 17.0 & NA & 49 \\
\hline Mixture & 15 & 0.09 & 19.8 & 109 & 7 & 7.7 & $137 *$ & $17.8^{*}$ & 59 \\
\hline $37.5 \%(v / v)$ PEG400 & 12 & 0.11 & $\sim 0.1$ & 30 & 3 & 6.3 & 409 & 62 & 46 \\
\hline Mixture & 10 & $\mathrm{t}_{1 / 2 \alpha}=0.133$ & 3.7 & 8,688 & 869 & NA & 1.2 & NA & 47 \\
\hline NA & 15 & 0.31 & $\sim 3$ & 835 & 56 & 2.2 & 18.0 & 8.1 & 60 \\
\hline Mixture & 2 & 0.22 & NA & 112 & 56 & 3.21 & 18.1 & 5.7 & 48 \\
\hline Mixture & 15 & 0.2 & $\sim 4$ & 967 & 65 & 3.5 & 15.5 & 4.4 & 69 \\
\hline
\end{tabular}




\begin{tabular}{|c|c|c|c|c|c|c|c|c|c|}
\hline Solvent system & $\begin{array}{c}\text { Dose } \\
{[\mathrm{mg} / \mathrm{kg}]}\end{array}$ & $\begin{array}{l}\mathbf{t}_{1 / 2} \\
{[\mathrm{~h}]}\end{array}$ & $\underset{[\mu \mathrm{g} / \mathrm{mL}]}{\mathrm{C}_{\max }}$ & $\begin{array}{c}\text { AUC } \\
{[\mu \mathrm{g} \cdot \mathbf{h} / \mathbf{L}]}\end{array}$ & $\begin{array}{c}\text { Normalized AUC } \\
{[\mu \mathrm{g} \cdot \mathrm{h} / \mathrm{L}] /[\mathrm{mg} / \mathrm{kg}]}\end{array}$ & $\begin{array}{c}\mathbf{K} \\
{\left[\mathbf{h}^{-1}\right]}\end{array}$ & $\begin{array}{c}\text { CL } \\
{[\mathrm{L} / \mathrm{h} / \mathbf{k g}]}\end{array}$ & $\begin{array}{c}\mathbf{V}_{\mathrm{d}} \\
{[\mathrm{L} / \mathrm{kg}]}\end{array}$ & Ref. \\
\hline Mixture & 15 & 0.14 & $\sim 2.5$ & 668415 & 44561 & 5.1 & 0.022 & 0.004 & 61 \\
\hline DMSO & 10 & $\begin{array}{c}\mathrm{t}_{1 / 2 \alpha}=0.068 \\
\mathrm{t}_{1 / 2 \beta}=0.56\end{array}$ & 5.1 & 1250 & 125 & 1.24 & 4.1 & 0.8 & 52 \\
\hline NA & 4 & $\mathrm{t}_{1 / 2 \beta}=1.1$ & 0.002 & 1529 & 382 & 0.7 & 2.4 & $4.0 *$ & 77 \\
\hline Mixture & 15 & 0.2 & $\sim 4.5$ & 967 & 64.5 & 3.5 & 15.5 & 4.4 & 70 \\
\hline
\end{tabular}

The calculated clearance $(\mathrm{CL})$ and volume of distribution $\left(\mathrm{V}_{\mathrm{d}}\right)$ values using the above formula are indicated by asterisk. The solvent mixture that was used to solubilized free curcumin contained $15 \%$ DMA $+45 \%$ PEG $+40 \%$ dextrose or glucose.

Abbreviations: $\mathrm{t}_{1 / 2}$, half-life; $\mathrm{C}_{\max }$, maximum concentration; $\mathrm{k}$, elimination rate constant; $\mathrm{AUC}$, area under the plasma concentration versus time curve; $\mathrm{CL}$, clearance; volume of distribution $\left(\mathrm{V}_{\mathrm{d}}\right)$; Ref., reference; NA, not available; DMA, dimethylformamide; DMSO, dimethyl sulfoxide; PEG, polyethylene glycol. 


\section{Chapter 2}

Table S4. Summary of PK parameters of intravenously administered curcumin nanoformulations in rats.

\begin{tabular}{|c|c|c|c|c|c|c|c|c|c|c|c|}
\hline Formulation & $\begin{array}{l}\text { Size } \\
{[\mathrm{nm}]}\end{array}$ & $\begin{array}{c}\mathbf{Z P} \\
{[\mathbf{m V}]}\end{array}$ & $\begin{array}{c}\text { Dose } \\
{[\mathrm{mg} / \mathrm{kg}]}\end{array}$ & $\begin{array}{l}\mathbf{t}_{1 / 2} \\
{[\mathrm{~h}]}\end{array}$ & $\underset{[\mu \mathrm{g} / \mathrm{mL}]}{\mathrm{C}_{\max }}$ & $\begin{array}{c}\mathrm{AUC} \\
{[\mu \mathrm{g} \bullet \mathbf{h} / \mathrm{L}]}\end{array}$ & $\begin{array}{l}\text { Normalized AUC } \\
{[\mu \mathrm{r} \cdot \mathrm{h} / \mathrm{L}] /[\mathrm{mg} / \mathrm{kg}]}\end{array}$ & $\begin{array}{c}\mathbf{k} \\
{\left[\mathbf{h}^{-1}\right]}\end{array}$ & $\begin{array}{c}\text { CL } \\
{[\mathbf{L} / \mathbf{h} / \mathbf{k g}]}\end{array}$ & $\begin{array}{c}\mathbf{V}_{\mathbf{d}} \\
{[\mathrm{L} / \mathrm{kg}]}\end{array}$ & Ref. \\
\hline $\begin{array}{c}\text { Curcumin-PBCA } \\
\text { nanoparticles }^{\mathrm{a}}\end{array}$ & 200 & 29.1 & 5 & $\mathrm{t}_{1 / 2 \beta}=18.7$ & NA & 3,302 & 660 & 0.04 & 0.002 & 0.103 & 54 \\
\hline $\begin{array}{l}\text { mPEG-PLA } \\
\text { micelles }^{\text {b }}\end{array}$ & 30 & -0.3 & 50 & 2.4 & 166 & 300,125 & 6,003 & 0.3 & $0.2^{*}$ & $0.6^{*}$ & 55 \\
\hline $\begin{array}{l}\text { mPEG-PCL } \\
\text { micelles }^{c}\end{array}$ & 27 & NA & 100 & 0.6 & 431 & 798 & 8 & 1.2 & $125^{*}$ & $103^{*}$ & 57 \\
\hline $\begin{array}{c}\text { mPEG- } b \text {-PHEMA- } \\
5 \mathrm{HA}^{\mathrm{d}}\end{array}$ & 104 & -19 & 5 & $\mathrm{t}_{1 / 2 \beta}=6.2$ & $\sim 1.1$ & 45,340 & 9,068 & 0.11 & 0.11 & 0.3 & 44 \\
\hline HA-Curc-NC ${ }^{e}$ & 161 & -25 & 2 & 53.1 & 0.08 & 724 & 362 & 0.01 & 6.0 & 0.4 & 45 \\
\hline $\begin{array}{c}\text { Curcumin } \\
\text { nanosuspension } \\
\text { stabilized by } \\
\text { mPEG-DSPE and } \\
\text { SPC }^{\mathrm{f}}\end{array}$ & 186 & -19 & 10 & 65 & 1.5 & 7,672 & 767 & 0.01 & 0.21 & $122 *$ & 58 \\
\hline mPEG-PCL ${ }^{\mathrm{c}}$ & 30 & -3.6 & 50 & 1.4 & 147 & 478,600 & 9,572 & 0.49 & $0.1^{*}$ & $0.2^{*}$ & 73 \\
\hline HSA nanoparticles ${ }^{g}$ & 165 & -27.3 & 10 & 0.4 & 1 & 5,761 & 576 & 1.9 & 3.2 & $1.0^{*}$ & 74 \\
\hline $\begin{array}{c}\text { Polymeric } \\
\text { nanoparticles } \\
\text { composed of } \\
\text { NIPAAM, VP, and } \\
\text { AA }^{\mathrm{h}}\end{array}$ & 92 & -20.1 & 10 & 3.2 & 25.5 & 151,135 & 15,113 & 0.22 & 0.07 & 0.3 & 40 \\
\hline
\end{tabular}




\begin{tabular}{|c|c|c|c|c|c|c|c|c|c|c|c|}
\hline Formulation & $\begin{array}{l}\text { Size } \\
{[\mathrm{nm}]}\end{array}$ & $\begin{array}{c}\mathbf{Z P} \\
{[\mathbf{m V}]}\end{array}$ & $\begin{array}{c}\text { Dose } \\
{[\mathrm{mg} / \mathrm{kg}]}\end{array}$ & $\begin{array}{l}\mathbf{t}_{1 / 2} \\
{[\mathrm{~h}]}\end{array}$ & $\begin{array}{c}\mathrm{C}_{\max } \\
{[\mu \mathrm{g} / \mathrm{mL}]}\end{array}$ & $\begin{array}{c}\text { AUC } \\
{[\mu \mathrm{g} \cdot \mathbf{h} / \mathrm{L}]}\end{array}$ & $\begin{array}{l}\text { Normalized AUC } \\
{[\mu \mathrm{g} \bullet \mathrm{h} / \mathrm{L}] /[\mathrm{mg} / \mathrm{kg}]}\end{array}$ & $\begin{array}{c}\mathbf{k} \\
{\left[\mathbf{h}^{-1}\right]}\end{array}$ & $\begin{array}{c}\text { CL } \\
{[\mathrm{L} / \mathrm{h} / \mathrm{kg}]}\end{array}$ & $\begin{array}{c}\mathbf{V}_{\mathbf{d}} \\
{[\mathrm{L} / \mathrm{kg}]}\end{array}$ & Ref. \\
\hline $\begin{array}{l}\text { mPEG-PLGA } \\
\text { nanoparticles }^{i}\end{array}$ & 120 & NA & 4 & 9.1 & 0.02 & 59 & 15 & 0.08 & $67 *$ & $882 *$ & 41 \\
\hline Liposomes & 130 & -6.3 & 6 & 6.1 & $\sim 2.1$ & 5,322 & 887 & 0.11 & 6.2 & $9.9^{*}$ & 75 \\
\hline $\begin{array}{l}\text { Tri-CL-mPEG } \\
\text { nanoparticles }^{\mathrm{j}}\end{array}$ & 116 & -12.2 & 2 & 5.0 & 1.45 & 456 & 228 & 0.1 & 4.1 & $31.6^{*}$ & 42 \\
\hline Lipid nanocapsules & 55 & -11 & 10 & 3.3 & 70.1 & 72,980 & 7,298 & 0.2 & 0.04 & $0.6^{*}$ & 43 \\
\hline $\begin{array}{l}\text { MePEO- } b \text {-PCL } \\
\text { micelles }^{\mathrm{k}}\end{array}$ & 78 & NA & 5 & 61 & $\sim 0.7$ & $3,160,000$ & 632,000 & 0.01 & 1.8 & 147 & 76 \\
\hline $\begin{array}{l}\text { Nanoparticles based } \\
\text { on CSH/HA/PEG }\end{array}$ & 246 & -27 & 10 & NA & $\sim 0.3$ & 287 & 29 & NA & 4.5 & NA & 49 \\
\hline $\begin{array}{l}\text { mPEG-PLA } \\
\text { micelles }^{\mathrm{b}}\end{array}$ & 70 & 3 & 15 & 0.3 & 20.7 & 835 & 56 & 2.4 & $18.0^{*}$ & $7.5^{*}$ & 59 \\
\hline $\begin{array}{c}\text { (PDLLA-G)-based } \\
\text { nanoparticles }^{\mathrm{m}}\end{array}$ & 200 & -0.8 & 12 & 1.43 & $\sim 100$ & 30,168 & 2,514 & 0.48 & 0.4 & 0.1 & 46 \\
\hline $\begin{array}{l}\text { PCL-PDEASB } \\
\text { micelles }^{\mathrm{n}}\end{array}$ & 103 & 56.6 & 10 & $\mathrm{t}_{1 / 2 \alpha}=0.2$ & 11.6 & 17,376 & 1,737 & NA & 0.6 & NA & 47 \\
\hline $\begin{array}{c}\text { mPEG-PVL } \\
\text { nanoparticles }^{\circ}\end{array}$ & 132 & -2.1 & 15 & 5.1 & $\sim 10$ & 3,006 & 200 & 0.1 & 5.0 & 36.8 & 60 \\
\hline SLN $^{p}$ & 153 & -21.4 & 2 & 0.3 & NA & 139 & 70 & 2.10 & 14.5 & 6.9 & 48 \\
\hline
\end{tabular}




\begin{tabular}{|c|c|c|c|c|c|c|c|c|c|c|c|}
\hline Formulation & $\begin{array}{l}\text { Size } \\
{[\mathrm{nm}]}\end{array}$ & $\begin{array}{c}\mathbf{Z P} \\
{[\mathbf{m V}]}\end{array}$ & $\begin{array}{c}\text { Dose } \\
{[\mathrm{mg} / \mathrm{kg}]}\end{array}$ & $\begin{array}{l}\mathbf{t}_{1 / 2} \\
{[\mathrm{~h}]}\end{array}$ & $\begin{array}{c}\mathrm{C}_{\max } \\
{[\mu \mathrm{g} / \mathrm{mL}]}\end{array}$ & $\begin{array}{c}\mathrm{AUC} \\
{[\mu \mathrm{g} \cdot \mathbf{h} / \mathbf{L}]}\end{array}$ & $\begin{array}{l}\text { Normalized AUC } \\
{[\mu \mathrm{h} \bullet / \mathrm{L}] /[\mathrm{mg} / \mathrm{kg}]}\end{array}$ & $\begin{array}{c}\mathbf{k} \\
{\left[\mathbf{h}^{-1}\right]}\end{array}$ & $\begin{array}{c}\text { CL } \\
{[\mathbf{L} / \mathbf{h} / \mathbf{k g}]}\end{array}$ & $\begin{array}{c}\mathbf{V}_{\mathbf{d}} \\
{[\mathbf{L} / \mathbf{k g}]}\end{array}$ & Ref. \\
\hline $\begin{array}{c}\text { Linear-dendrimer } \\
\text { mPEG-PCL } \\
\text { micelles }^{\mathrm{q}}\end{array}$ & 108 & -9.3 & 15 & 1.3 & $\sim 15$ & 4,464 & 298 & 0.54 & 3.4 & 6.3 & 69 \\
\hline $\begin{array}{l}\text { PCL-PEG-PCL } \\
\text { triblock } \\
\text { nanoparticles }^{\mathrm{r}}\end{array}$ & 62 & -4.3 & 15 & 27.6 & $\sim 2.5$ & $2,772,523$ & 184,835 & 0.03 & 0.005 & 0.21 & 61 \\
\hline $\begin{array}{c}\text { mPEG-chitosan- } \\
\text { Ketal micelles }\end{array}$ & 50 & NA & 10 & $\begin{array}{c}\mathrm{t}_{1 / 2 \mathrm{a}}=0.21 \\
\mathrm{t}_{1 / 2 \beta}=3.7\end{array}$ & 2.3 & 2,530 & 253 & 0.2 & 2.0 & 2.0 & 52 \\
\hline $\begin{array}{c}\text { Lipid-polymer- } \\
\text { lecithin hybrid } \\
\text { nanoparticles }\end{array}$ & 86 & -26.9 & 4 & 5.9 & 0.002 & 3,321 & 830 & 0.12 & 1.2 & $10.3^{*}$ & 77 \\
\hline $\begin{array}{l}\text { Linolenic acid- } \\
\text { modified PEG-PCL } \\
\text { micelles }\end{array}$ & 21 & -2.6 & 15 & 3.2 & $\sim 9$ & 2,662 & 177 & 0.22 & 5.6 & 25.6 & 70 \\
\hline
\end{tabular}

The calculated clearance $(\mathrm{CL})$ and volume of distribution $\left(\mathrm{V}_{\mathrm{d}}\right)$ values using the above formula are indicated by asterisk.

Abbreviations: $\mathrm{t}_{1 / 2}$, half-life; $\mathrm{C}_{\max }$, maximum concentration; $\mathrm{k}$, elimination rate constant; AUC, area under the plasma concentration versus time curve; CL, clearance; volume of distribution $\left(\mathrm{V}_{\mathrm{d}}\right)$; ZP, zeta potential; Ref., reference; NA, not available

Notes nanoformulations: ${ }^{a}$ cationic poly(butyl) cyanoacrylate (PBCA) nanoparticles coated with chitosan; ${ }^{\text {b }}$ monomethoxy poly(ethylene glycol)poly(lactide) copolymer; ${ }^{\mathrm{c}}$ monomethoxy poly(ethylene glycol)- poly( $\varepsilon$-caprolactone); ${ }^{\mathrm{d}}$ crosslinked poly(ethylene glycol)-b-poly(2-methacrylate ethyl 5-hexynoicate); hyaluronic acid surface modified curcumin nanocrystals; ${ }^{\mathrm{f}}$ curcumin nanosuspension stabilized by mPEG2000-DSPE and soybean lecithin; ${ }^{\mathrm{g}}$ human serum albumin; ${ }^{\mathrm{h}}$ polymeric nanoparticles composed of $\mathrm{N}$-isopropylacrylamide, vinylpyrrolidone, acrylic acid; ${ }^{\mathrm{i}}$ (methoxypolyethylene glycol)-poly (lactic-coglycolic acid); ${ }^{\mathrm{j}}$ three-arm amphiphilic copolymer tricarballylic acid-poly ( $\epsilon$-caprolactone)-methoxypolyethylene glycol; ${ }^{\mathrm{k}}$ methoxy poly(ethylene oxide)- $b$ poly( $\varepsilon$-caprolactone); ${ }^{l}$ nanoparticles based on chitosan hydrochloride/hyaluronic acid/polyethylene glycol; ${ }^{\mathrm{m}}$ poly(d,l-lactic acid)-glycerol; ${ }^{\mathrm{n}}$ poly( $\varepsilon$ caprolactone)- $b$-poly(N,N-diethylaminoethylmethacrylate)-r-poly(N-(3-sulfopropyl)-N-methacryloxyethy-N,N-diethylammoniumbetaine); o methoxy poly(ethylene glycol)-b-poly( $\delta$-valerolactone); ${ }^{\text {}}$ solid lipid nanoparticles; ${ }^{\mathrm{a}}$ linear dendrimer-type methoxy-poly(ethylene glycol)-b-poly $(\varepsilon$-caprolactone); poly( $\varepsilon$-caprolactone)-poly(ethylene glycol)-poly ( $\varepsilon$-caprolactone). 


\section{Analysis of Studies with Very High AUC Range (References ${ }^{61,76}$ )}

Plasma concentration-time curves of curcumin nanoformulations and free curcumin were reproduced by extrapolating the data from the respective PK figures. The following studies ${ }^{50,53,68-70,74}$ were used to verify the analysis method. The AUC is calculated using PkSolver 2.0 add-in template in Microsoft Excel. Calculations are based on noncompartmental analysis.

Table S5. Comparison of reported AUCs and reanalyzed AUC value of free curcumin and curcumin nanoformulations using PkSolver.

\begin{tabular}{|c|c|c|c|c|c|c|c|}
\hline \multirow[b]{2}{*}{$\begin{array}{c}\text { Dose } \\
{[\mathrm{mg} / \mathrm{kg}]}\end{array}$} & \multicolumn{3}{|c|}{ Free curcumin } & \multicolumn{3}{|c|}{ Curcumin nanoformulation } & \multirow[b]{2}{*}{ Ref } \\
\hline & $\begin{array}{c}\text { Reported AUC } \\
{[\mu \mathrm{g} . \mathrm{h} / \mathrm{L}]}\end{array}$ & $\begin{array}{c}\text { Calculated AUC } \\
{[\mu \mathrm{g} \cdot \mathrm{h} / \mathrm{L}]}\end{array}$ & $\begin{array}{c}\text { AUC ratio } \\
\text { calculated:reported [\%] }\end{array}$ & $\begin{array}{c}\text { Reported AUC } \\
{[\mu \mathrm{g} . \mathrm{h} / \mathrm{L}]}\end{array}$ & $\begin{array}{c}\text { Calculated AUC } \\
{[\mu \mathrm{g} . \mathrm{h} / \mathrm{L}]}\end{array}$ & $\begin{array}{c}\text { AUC ratio } \\
\text { calculated:reported [\%] }\end{array}$ & \\
\hline 10 & 474 & 425 & 90 & 622 & 662 & 106 & 53 \\
\hline 25 & 29894 & 33041 & 111 & 148079 & 159542 & 108 & 68 \\
\hline 15 & 967 & 1130 & 117 & 4464 & 3860 & 86 & 69 \\
\hline 2 & 6708 & 6370 & 95 & 8964 & 10705 & 119 & 50 \\
\hline 10 & 1705 & 1717 & 101 & 7672 & 8288 & 108 & 70 \\
\hline 10 & 2298 & 2745 & 119 & 5761 & 7742 & 134 & 74 \\
\hline 15 & 668415 & 577 & 0.09 & 2772523 & 2187 & 0.08 & 61 \\
\hline $10 / 5^{*}$ & 1670000 & 2718 & 0.16 & 3160000 & 1504 & 0.05 & 76 \\
\hline
\end{tabular}

(*) the dose of free curcumin and curcumin nanoformulation was 10 and $5 \mathrm{mg} / \mathrm{kg}$, respectively.

Abbreviations: AUC, area under the curve; Ref, reference. 


\section{References}

1. Sharma, R. A.; Gescher, A. J.; Steward, W. P., Curcumin: The story so far. European Journal of Cancer 2005, 41 (13), 1955-1968.

2. Kotha, R. R.; Luthria, D. L., Curcumin: Biological, pharmaceutical, nutraceutical, and analytical aspects. Molecules 2019, 24 (16), 2930.

3. Doello, K.; Ortiz, R.; Alvarez, P. J.; Melguizo, C.; Cabeza, L.; Prados, J., Latest in Vitro and in Vivo Assay, Clinical Trials and Patents in Cancer Treatment using Curcumin: A Literature Review. Nutrition and Cancer 2018, 70 (4), 569-578.

4. $\quad$ Patel, S. S.; Acharya, A.; Ray, R. S.; Agrawal, R.; Raghuwanshi, R.; Jain, P., Cellular and molecular mechanisms of curcumin in prevention and treatment of disease. Critical Reviews in Food Science and Nutrition 2020, 60 (6), 887-939.

5. Shehzad, A.; Wahid, F.; Lee, Y. S., Curcumin in Cancer Chemoprevention: Molecular Targets, Pharmacokinetics, Bioavailability, and Clinical Trials. Arch. Pharm. 2010, 343 (9), 489-499.

6. Heger, M.; van Golen, R. F.; Broekgaarden, M.; Michel, M. C., The molecular basis for the pharmacokinetics and pharmacodynamics of curcumin and its metabolites in relation to cancer. Pharmacol. Rev. 2014, 66 (1), 222-307.

7. Heger, M., Drug screening: Don't discount all curcumin trial data. Nature 2017, 543 (7643), 40.

8. Balaji, S.; Chempakam, B., Toxicity prediction of compounds from turmeric (Curcuma longa L). Food Chem. Toxicol. 2010, 48 (10), 2951-2959.

9. $\quad$ Cheng, A. L.; Hsu, C. H.; Lin, J. K.; Hsu, M. M.; Ho, Y. F.; Shen, T. S.; Ko, J. Y.; Lin, J. T.; Lin, B. R.; Ming-Shiang, W.; Yu, H. S.; Jee, S. H.; Chen, G. S.; Chen, T. M.; Chen, C. A.; Lai, M. K.; Pu, Y. S.; Pan, M. H.; Wang, Y. J.; Tsai, C. C.; Hsieh, C. Y., Phase I clinical trial of curcumin, a chemopreventive agent, in patients with high-risk or pre-malignant lesions. Anticancer Res 2001, 21 (4b), 2895-900.

10. Yeung, A. W. K.; Horbańczuk, M.; Tzvetkov, N. T.; Mocan, A.; Carradori, S.; Maggi, F.; Marchewka, J.; Sut, S.; Dall'Acqua, S.; Gan, R.-Y.; Tancheva, L. P.; Polgar, T.; Berindan-Neagoe, I.; Pirgozliev, V.; Šmejkal, K.; Atanasov, A. G., Curcumin: Total-scale analysis of the scientific literature. Molecules 2019, 24 (7), 1393.

11. Willenbacher, E.; Khan, S. Z.; Mujica, S. C. A.; Trapani, D.; Hussain, S.; Wolf, D.; Willenbacher, W.; Spizzo, G.; Seeber, A., Curcumin: New Insights into an Ancient Ingredient against Cancer. International journal of molecular sciences 2019, 20 (8), 1808.

12. Duvoix, A.; Blasius, R.; Delhalle, S.; Schnekenburger, M.; Morceau, F.; Henry, E.; Dicato, M.; Diederich, M., Chemopreventive and therapeutic effects of curcumin. Cancer Lett. 2005, 223 (2), 181-190.

13. Shanmugam, M. K.; Rane, G.; Kanchi, M. M.; Arfuso, F.; Chinnathambi, A.; Zayed, M. E.; Alharbi, S. A.; Tan, B. K.; Kumar, A. P.; Sethi, G., The multifaceted role of curcumin in cancer prevention and treatment. Molecules 2015, 20 (2), 2728-69.

14. Ismail, N. I.; Othman, I.; Abas, F.; N, H. L.; Naidu, R., Mechanism of Apoptosis Induced by Curcumin in Colorectal Cancer. Int J Mol Sci 2019, 20 (10).

15. Giordano, A.; Tommonaro, G., Curcumin and Cancer. Nutrients 2019, 11 (10).

16. Ravindran, J.; Prasad, S.; Aggarwal, B. B., Curcumin and Cancer Cells: How Many Ways Can Curry Kill Tumor Cells Selectively? The AAPS Journal 2009, 11 (3), 495-510.

17. Syng-Ai, C.; Kumari, A. L.; Khar, A., Effect of curcumin on normal and tumor cells: role of glutathione and bcl-2. Mol. Cancer Ther. 2004, 3 (9), 1101-8. 
18. Chang, R.; Sun, L.; Webster, T. J., Short communication: selective cytotoxicity of curcumin on osteosarcoma cells compared to healthy osteoblasts. International journal of nanomedicine 2014, 9 , 461-465.

19. Mehta, H. J.; Patel, V.; Sadikot, R. T., Curcumin and lung cancer-a review. Target Oncol. 2014, 9 (4), 295-310.

20. Bimonte, S.; Barbieri, A.; Leongito, M.; Piccirillo, M.; Giudice, A.; Pivonello, C.; de Angelis, C.; Granata, V.; Palaia, R.; Izzo, F., Curcumin AntiCancer Studies in Pancreatic Cancer. Nutrients 2016, $8(7), 433$.

21. Shureiqi, I.; Baron, J. A., Curcumin Chemoprevention: The Long Road to Clinical Translation. Cancer Prev. Res. 2011, 4 (3), 296.

22. Dhillon, N.; Aggarwal, B. B.; Newman, R. A.; Wolff, R. A.; Kunnumakkara, A. B.; Abbruzzese, J. L.; Ng, C. S.; Badmaev, V.; Kurzrock, R., Phase II Trial of Curcumin in Patients with Advanced Pancreatic Cancer. Clinical Cancer Research 2008, 14 (14), 4491.

23. Cheng, A. L.; Hsu, C. H.; Lin, J. K.; Hsu, M. M.; Ho, Y. F.; Shen, T. S.; Ko, J. Y.; Lin, J. T.; Lin, B. R.; Ming-Shiang, W.; Yu, H. S.; Jee, S. H.; Chen, G. S.; Chen, T. M.; Chen, C. A.; Lai, M. K.; Pu, Y. S.; Pan, M. H.; Wang, Y. J.; Tsai, C. C.; Hsieh, C. Y., Phase I clinical trial of curcumin, a chemopreventive agent, in patients with high-risk or pre-malignant lesions. Anticancer Res. 2001, 21 (4b), 2895-900.

24. Mohamed, S. A.; El-Shishtawy, R. M.; Al-Bar, O. A. M.; Al-Najada, A. R., Chemical modification of curcumin: Solubility and antioxidant capacity. International Journal of Food Properties 2017, 20 (3), 718-724.

25. Subramaniam, D.; May, R.; Sureban, S. M.; Lee, K. B.; George, R.; Kuppusamy, P.; Ramanujam, R. P.; Hideg, K.; Dieckgraefe, B. K.; Houchen, C. W.; Anant, S., Diphenyl Difluoroketone: A Curcumin Derivative with Potent \&lt;em\&gt;In vivo\&lt;/em\&gt; Anticancer Activity. Cancer Research 2008, 68 (6), 1962.

26. Li, Q.; Zhai, W.; Jiang, Q.; Huang, R.; Liu, L.; Dai, J.; Gong, W.; Du, S.; Wu, Q., Curcuminpiperine mixtures in self-microemulsifying drug delivery system for ulcerative colitis therapy. International Journal of Pharmaceutics 2015, 490 (1), 22-31.

27. Shoba, G.; Joy, D.; Joseph, T.; Majeed, M.; Rajendran, R.; Srinivas, P. S. S. R., Influence of Piperine on the Pharmacokinetics of Curcumin in Animals and Human Volunteers. Planta Med 1998, 64 (04), 353-356.

28. Mirzaei, H.; Shakeri, A.; Rashidi, B.; Jalili, A.; Banikazemi, Z.; Sahebkar, A., Phytosomal curcumin: A review of pharmacokinetic, experimental and clinical studies. Biomedicine \& Pharmacotherapy 2017, 85, 102-112.

29. Naksuriya, O.; Okonogi, S.; Schiffelers, R. M.; Hennink, W. E., Curcumin nanoformulations: A review of pharmaceutical properties and preclinical studies and clinical data related to cancer treatment. Biomaterials 2014, 35 (10), 3365-3383.

30. Yallapu, M. M.; Jaggi, M.; Chauhan, S. C., Curcumin nanoformulations: a future nanomedicine for cancer. Drug discovery today 2012, 17 (1-2), 71-80.

31. Mohanty, C.; Das, M.; Sahoo, S. K., Emerging role of nanocarriers to increase the solubility and bioavailability of curcumin. Expert opinion on drug delivery 2012, 9 (11), 1347-64.

32. Patra, J. K.; Das, G.; Fraceto, L. F.; Campos, E. V. R.; Rodriguez-Torres, M. d. P.; AcostaTorres, L. S.; Diaz-Torres, L. A.; Grillo, R.; Swamy, M. K.; Sharma, S.; Habtemariam, S.; Shin, H.-S., Nano based drug delivery systems: recent developments and future prospects. Journal of Nanobiotechnology 2018, 16 (1), 71.

33. Mirza, A. Z.; Siddiqui, F. A., Nanomedicine and drug delivery: a mini review. International Nano Letters 2014, 4 (1), 94. 
34. Varela-Moreira, A.; Shi, Y.; Fens, M. H. A. M.; Lammers, T.; Hennink, W. E.; Schiffelers, R. M., Clinical application of polymeric micelles for the treatment of cancer. Mater. Chem. Front. 2017, $l(8), 1485-1501$.

35. Deng, C.; Jiang, Y.; Cheng, R.; Meng, F.; Zhong, Z., Biodegradable polymeric micelles for targeted and controlled anticancer drug delivery: Promises, progress and prospects. Nano Today 2012, 7 (5), 467-480.

36. Bobo, D.; Robinson, K. J.; Islam, J.; Thurecht, K. J.; Corrie, S. R., Nanoparticle-based medicines: a review of FDA-approved materials and clinical trials to date. Pharm. Res. 2016, 33 (10), 2373-2387.

37. Naksuriya, O.; van Steenbergen, M. J.; Torano, J. S.; Okonogi, S.; Hennink, W. E., A kinetic degradation study of curcumin in its free form and loaded in polymeric micelles. AAPS J. 2016, 18 (3), 777-787.

38. Sun, D.; Zhou, J.-K.; Zhao, L.; Zheng, Z.-Y.; Li, J.; Pu, W.; Liu, S.; Liu, X.-S.; Liu, S.-J.; Zheng, Y.; Zhao, Y.; Peng, Y., Novel curcumin liposome modified with hyaluronan targeting CD44 plays an anti-leukemic role in acute myeloid leukemia in vitro and in vivo. ACS Appl. Mater. Interfaces 2017, 9 (20), 16857-16868.

39. Yu, Y.; Zhang, X.; Qiu, L., The anti-tumor efficacy of curcumin when delivered by size/charge-changing multistage polymeric micelles based on amphiphilic poly(beta-amino ester) derivates. Biomaterials 2014, 35 (10), 3467-79.

40. Zou, P.; Helson, L.; Maitra, A.; Stern, S. T.; McNeil, S. E., Polymeric Curcumin Nanoparticle Pharmacokinetics and Metabolism in Bile Duct Cannulated Rats. Molecular Pharmaceutics 2013, 10 (5), 1977-1987.

41. Duan, D.; Wang, A.; Ni, L.; Zhang, L.; Yan, X.; Jiang, Y.; Mu, H.; Wu, Z.; Sun, K.; Li, Y., Trastuzumab- and Fab' fragment-modified curcumin PEG-PLGA nanoparticles: preparation and evaluation in vitro and in vivo. International journal of nanomedicine 2018, 13, 1831-1840.

42. Wu, W.; Wu, J.; Fu, Q.; Jin, C.; Guo, F.; Yan, Q.; Yang, Q.; Wu, D.; Yang, Y.; Yang, G., Elaboration and characterization of curcumin-loaded Tri-CL-mPEG three-arm copolymeric nanoparticles by a microchannel technology. International journal of nanomedicine 2019, 14, 46834695.

43. Lollo, G.; Ullio-Gamboa, G.; Fuentes, E.; Matha, K.; Lautram, N.; Benoit, J.-P., In vitro anticancer activity and pharmacokinetic evaluation of curcumin-loaded lipid nanocapsules. Mater Sci Eng C Mater Biol Appl 2018, 91, 859-867.

44. Liu, Y.; Chen, F.; Zhang, K.; Wang, Q.; Chen, Y.; Luo, X., pH-Responsive reversibly crosslinked micelles by phenol-yne click via curcumin as a drug delivery system in cancer chemotherapy. Journal of Materials Chemistry B 2019, 7 (24), 3884-3893.

45. Ji, P.; Wang, L.; Chen, Y. W.; Wang, S. Q.; Wu, Z. H.; Qi, X. L., Hyaluronic acid hydrophilic surface rehabilitating curcumin nanocrystals for targeted breast cancer treatment with prolonged biodistribution. Biomater. Sci. 2020, 8 (1), 462-472.

46. Yoon, I.-S.; Park, J.-H.; Kang, H. J.; Choe, J. H.; Goh, M. S.; Kim, D.-D.; Cho, H.-J., Poly(d,1lactic acid)-glycerol-based nanoparticles for curcumin delivery. International Journal of Pharmaceutics 2015, 488 (1), 70-77.

47. Wu, Z.; Cai, M.; Xie, X.; He, L.; Huang, L.; Chen, Y.; Luo, X., The effect of architecture/composition on the $\mathrm{pH}$ sensitive micelle properties and in vivo study of curcuminin-loaded micelles containing sulfobetaines. RSC Advances 2015, 5 (129), 106989-107000.

48. Sun, J.; Bi, C.; Chan, H. M.; Sun, S.; Zhang, Q.; Zheng, Y., Curcumin-loaded solid lipid nanoparticles have prolonged in vitro antitumour activity, cellular uptake and improved in vivo bioavailability. Colloids Surf., B 2013, 111, 367-375. 
49. Xu, Y.; Asghar, S.; Yang, L.; Chen, Z.; Li, H.; Shi, W.; Li, Y.; Shi, Q.; Ping, Q.; Xiao, Y., Nanoparticles based on chitosan hydrochloride/hyaluronic acid/PEG containing curcumin: In vitro evaluation and pharmacokinetics in rats. International Journal of Biological Macromolecules 2017, 102, 1083-1091.

50. Zhai, S.; Ma, Y.; Chen, Y.; Li, D.; Cao, J.; Liu, Y.; Cai, M.; Xie, X.; Chen, Y.; Luo, X., Synthesis of an amphiphilic block copolymer containing zwitterionic sulfobetaine as a novel $\mathrm{pH}-$ sensitive drug carrier. Polym. Chem. 2014, 5 (4), 1285-1297.

51. Chen, S.; Li, Q.; Li, H.; Yang, L.; Yi, J.-Z.; Xie, M.; Zhang, L.-M., Long-circulating zeinpolysulfobetaine conjugate-based nanocarriers for enhancing the stability and pharmacokinetics of curcumin. Mater. Sci. Eng., C 2020, 109, 110636.

52. Chen, D.; Sun, J., In vitro and in vivo evaluation of PEG-conjugated ketal-based chitosan micelles as pH-sensitive carriers. Polymer Chemistry 2015, 6 (6), 998-1004.

53. Song, Z.; Feng, R.; Sun, M.; Guo, C.; Gao, Y.; Li, L.; Zhai, G., Curcumin-loaded PLGAPEG-PLGA triblock copolymeric micelles: Preparation, pharmacokinetics and distribution in vivo. $J$. Colloid Interface Sci. 2011, 354 (1), 116-123.

54. Duan, J.; Zhang, Y.; Han, S.; Chen, Y.; Li, B.; Liao, M.; Chen, W.; Deng, X.; Zhao, J.; Huang, B., Synthesis and in vitro/in vivo anti-cancer evaluation of curcumin-loaded chitosan/poly(butyl cyanoacrylate) nanoparticles. Int. J. Pharm. 2010, 400 (1), 211-220.

55. Gao, X.; Zheng, F.; Guo, G.; Liu, X.; Fan, R.; Qian, Z.-y.; Huang, N.; Wei, Y.-q., Improving the anti-colon cancer activity of curcumin with biodegradable nano-micelles. J. Mater. Chem. B 2013, 1 (42), 5778-5790.

56. Sun, M.; Gao, Y.; Guo, C.; Cao, F.; Song, Z.; Xi, Y.; Yu, A.; Li, A.; Zhai, G., Enhancement of transport of curcumin to brain in mice by poly(n-butylcyanoacrylate) nanoparticle. J. Nanopart. Res. 2010, 12 (8), 3111-3122.

57. Gou, M.; Men, K.; Shi, H.; Xiang, M.; Zhang, J.; Song, J.; Long, J.; Wan, Y.; Luo, F.; Zhao, X.; Qian, Z., Curcumin-loaded biodegradable polymeric micelles for colon cancer therapy in vitro and in vivo. Nanoscale 2011, 3 (4), 1558-1567.

58. Hong, J. Y.; Liu, Y. Y.; Xiao, Y.; Yang, X. F.; Su, W. J.; Zhang, M. Z.; Liao, Y. H.; Kuang, H. X.; Wang, X. T., High drug payload curcumin nanosuspensions stabilized by mPEG-DSPE and SPC: in vitro and in vivo evaluation. Drug Deliv. 2017, 24 (1), 109-120.

59. Yang, C.; Chen, H.; Zhao, J.; Pang, X.; Xi, Y.; Zhai, G., Development of a folate-modified curcumin loaded micelle delivery system for cancer targeting. Colloids and Surfaces B: Biointerfaces 2014, 121, 206-213.

60. Song, Z.; Zhu, W.; Yang, F.; Liu, N.; Feng, R., Preparation, characterization, in vitro release, and pharmacokinetic studies of curcumin-loaded mPEG-PVL nanoparticles. Polymer Bulletin 2015, $72(1), 75-91$.

61. Feng, R.; Song, Z.; Zhai, G., Preparation and in vivo pharmacokinetics of curcumin-loaded PCL-PEG-PCL triblock copolymeric nanoparticles. International Journal of Nanomedicine 2012, 7, 4089-4098.

62. Smith, D. A.; Beaumont, K.; Maurer, T. S.; Di, L., Volume of Distribution in Drug Design. Journal of Medicinal Chemistry 2015, 58 (15), 5691-5698.

63. Mansoor, A.; Mahabadi, N., Volume of Distribution. In StatPearls, StatPearls Publishing Copyright (C) 2020, StatPearls Publishing LLC.: Treasure Island (FL), 2020.

64. Ravindranath, V.; Chandrasekhara, N., Absorption and tissue distribution of curcumin in rats. Toxicology 1980, 16 (3), 259-265. 


\section{Chapter 2}

65. Ravindranath, V.; Chandrasekhara, N., Metabolism of curcumin-studies with $[3 \mathrm{H}]$ curcumin. Toxicology 1981, 22 (4), 337-344.

66. Holder, G. M.; Plummer, J. L.; Ryan, A. J., The metabolism and excretion of curcumin (1,7bis-(4-hydroxy-3-methoxyphenyl)-1,6-heptadiene-3,5-dione) in the rat. Xenobiotica; the fate of foreign compounds in biological systems 1978, 8 (12), 761-8.

67. Gong, F.; Chen, D.; Teng, X.; Ge, J.; Ning, X.; Shen, Y. L.; Li, J.; Wang, S., Curcuminloaded blood-stable polymeric micelles for enhancing therapeutic effect on erythroleukemia. Mol. Pharmaceutics 2017, 14 (8), 2585-2594.

68. Gong, C.; Deng, S.; Wu, Q.; Xiang, M.; Wei, X.; Li, L.; Gao, X.; Wang, B.; Sun, L.; Chen, Y.; Li, Y.; Liu, L.; Qian, Z.; Wei, Y., Improving antiangiogenesis and anti-tumor activity of curcumin by biodegradable polymeric micelles. Biomaterials 2013, 34 (4), 1413-1432.

69. Song, Z.; Zhu, W.; Song, J.; Wei, P.; Yang, F.; Liu, N.; Feng, R., Linear-dendrimer type methoxy-poly (ethylene glycol)-b-poly ( $\varepsilon$-caprolactone) copolymer micelles for the delivery of curcumin. Drug Delivery 2015, 22 (1), 58-68.

70. Song, Z.; Zhu, W.; Liu, N.; Yang, F.; Feng, R., Linolenic acid-modified PEG-PCL micelles for curcumin delivery. International Journal of Pharmaceutics 2014, 471 (1), 312-321.

71. Removal of blood from laboratory mammals and birds. First report of the BVA/FRAME/RSPCA/UFAW Joint Working Group on Refinement. Laboratory animals 1993, 27 (1), $1-22$.

72. Yu, Y.; Zhang, X.; Qiu, L., The anti-tumor efficacy of curcumin when delivered by size/charge-changing multistage polymeric micelles based on amphiphilic poly( $\beta$-amino ester) derivates. Biomaterials 2014, 35 (10), 3467-3479.

73. Hu, Y. Z.; He, Y. H.; Ji, J. R.; Zheng, S. P.; Cheng, Y. Z., Tumor Targeted Curcumin Delivery by Folate-Modified MPEG-PCL Self-Assembly Micelles for Colorectal Cancer Therapy. International Journal of Nanomedicine 2020, 15, 1239-1252.

74. Song, Z.; Lu, Y.; Zhang, X.; Wang, H.; Han, J.; Dong, C., Novel curcumin-loaded human serum albumin nanoparticles surface functionalized with folate: characterization and in vitro/vivo evaluation. Drug Des Devel Ther 2016, 10, 2643-2649.

75. Jiang, K.; Shen, M.; Xu, W., Arginine, glycine, aspartic acid peptide-modified paclitaxel and curcumin co-loaded liposome for the treatment of lung cancer: in vitro/vivo evaluation. International journal of nanomedicine 2018, 13, 2561-2569.

76. Ma, Z.; Shayeganpour, A.; Brocks, D. R.; Lavasanifar, A.; Samuel, J., High-performance liquid chromatography analysis of curcumin in rat plasma: application to pharmacokinetics of polymeric micellar formulation of curcumin. Biomedical Chromatography 2007, 21 (5), 546-552.

77. Li, L.; Xiang, D.; Shigdar, S.; Yang, W.; Li, Q.; Lin, J.; Liu, K.; Duan, W., Epithelial cell adhesion molecule aptamer functionalized PLGA-lecithin-curcumin-PEG nanoparticles for targeted drug delivery to human colorectal adenocarcinoma cells. International journal of nanomedicine 2014, 9, 1083-1096.

78. Jamwal, R., Bioavailable curcumin formulations: A review of pharmacokinetic studies in healthy volunteers. Journal of Integrative Medicine 2018, 16 (6), 367-374.

79. Bolger, G. T.; Licollari, A.; Tan, A.; Greil, R.; Vcelar, B.; Majeed, M.; Helson, L., Distribution and metabolism of Lipocurc ${ }^{\mathrm{TM}}$ (liposomal curcumin) in dog and human blood cells: species selectivity and pharmacokinetic relevance. Anticancer Res 2017, 37 (7), 3483-3492.

80. Wahlström, B.; Blennow, G., A study on the fate of curcumin in the rat. Acta pharmacologica et toxicologica 1978, 43 (2), 86-92.

81. Bagheri, M.; Fens, M. H.; Kleijn, T. G.; Capomaccio, R. B.; Mehn, D.; Krawczyk, P. M.; Scutigliani, E. M.; Gurinov, A.; Baldus, M.; van Kronenburg, N. C. H.; Kok, R. J.; Heger, M.; van 

and Anti-Cancer Pharmacodynamics

Nostrum, C. F.; Hennink, W. E., In Vitro and In Vivo Studies on HPMA-Based Polymeric Micelles Loaded with Curcumin. Mol Pharm 2021.

82. Anand, P.; Kunnumakkara, A. B.; Newman, R. A.; Aggarwal, B. B., Bioavailability of curcumin: problems and promises. Mol. Pharmaceutics 2007, 4 (6), 807-818.

83. Metzler, M.; Pfeiffer, E.; Schulz, S. I.; Dempe, J. S., Curcumin uptake and metabolism. BioFactors 2013, 39 (1), 14-20.

84. Fujisawa, S.; Atsumi, T.; Ishihara, M.; Kadoma, Y., Cytotoxicity, ROS-generation activity and radical-scavenging activity of curcumin and related compounds. Anticancer Res. 2004, 24 (2B), 563-570.

85. Yu, X.; Trase, I.; Ren, M.; Duval, K.; Guo, X.; Chen, Z., Design of Nanoparticle-Based Carriers for Targeted Drug Delivery. Journal of Nanomaterials 2016, 2016, 1087250.

86. Wang, B.; He, X.; Zhang, Z.; Zhao, Y.; Feng, W., Metabolism of Nanomaterials in Vivo: Blood Circulation and Organ Clearance. Accounts of Chemical Research 2013, 46 (3), 761-769.

87. Dawidczyk, C. M.; Kim, C.; Park, J. H.; Russell, L. M.; Lee, K. H.; Pomper, M. G.; Searson, P. C., State-of-the-art in design rules for drug delivery platforms: Lessons learned from FDA-approved nanomedicines. Journal of Controlled Release 2014, 187, 133-144.

88. Abouelmagd, S. A.; Sun, B.; Chang, A. C.; Ku, Y. J.; Yeo, Y., Release kinetics study of poorly water-soluble drugs from nanoparticles: Are we doing it right? Mol. Pharmaceutics 2015, 12 (3), 997-1003.

89. Sheybanifard, M.; Beztsinna, N.; Bagheri, M.; Miriam Buhl, E.; Bresseleers, J.; VarelaMoreira, A.; Shi, Y.; van Nostrum, C. F.; van der Pluijm, G.; Storm, G.; Hennink, W. E.; Lammers, T.; Metselaar, J. M., Systematic evaluation of design features enables efficient selection of $\Pi$ electronstabilized polymeric micelles. Int. J. Pharm. 2020, 119409.

90. Felder-Flesch, D., Dendrimers in Nanomedicine. Jenny Stanford Publishing: 2016.

91. Boussery, K.; Belpaire, F. M.; Van de Voorde, J., Chapter 23 - Physiological Aspects Determining the Pharmacokinetic Properties of Drugs. In The Practice of Medicinal Chemistry (Fourth Edition), Wermuth, C. G.; Aldous, D.; Raboisson, P.; Rognan, D., Eds. Academic Press: San Diego, 2008; pp 539-559.

92. The Johns Hopkins University. Animal care and use committee. http://web.jhu.edu/animalcare/procedures/mouse.html (accessed accessed July 24, 2020).

93. Riches, A. C.; Sharp, J. G.; Thomas, D. B.; Smith, S. V., Blood volume determination in the mouse. J. Physiol. 1973, 228 (2), 279-284.

94. Grossen, P.; Witzigmann, D.; Sieber, S.; Huwyler, J., PEG-PCL-based nanomedicines: A biodegradable drug delivery system and its application. Journal of Controlled Release 2017, 260, 4660 .

95. Saghatelyan, T.; Tananyan, A.; Janoyan, N.; Tadevosyan, A.; Petrosyan, H.; Hovhannisyan, A.; Hayrapetyan, L.; Arustamyan, M.; Arnhold, J.; Rotmann, A.-R.; Hovhannisyan, A.; Panossian, A., Efficacy and safety of curcumin in combination with paclitaxel in patients with advanced, metastatic breast cancer: A comparative, randomized, double-blind, placebo-controlled clinical trial. Phytomedicine 2020, 70, 153218.

96. Matabudul, D.; Pucaj, K.; Bolger, G.; Vcelar, B.; Majeed, M.; Helson, L., Tissue distribution of (Lipocurc ${ }^{\mathrm{TM}}$ ) liposomal curcumin and tetrahydrocurcumin following two- and eight-hour infusions in Beagle dogs. Anticancer Res 2012, 32 (10), 4359-64.

97. Storka, A.; Vcelar, B.; Klickovic, U.; Gouya, G.; Weisshaar, S.; Aschauer, S.; Bolger, G.; Helson, L.; Wolzt, M., Safety, tolerability and pharmacokinetics of liposomal curcumin in healthy humans. International journal of clinical pharmacology and therapeutics 2015, 53 (1), 54-65. 
98. Storka, A.; Vcelar, B.; Klickovic, U.; Gouya, G.; Weisshaar, S.; Aschauer, S.; Helson, L.; Wolzt, M., Effect of liposomal curcumin on red blood cells in vitro. Anticancer Res 2013, 33 (9), 362934.

99. Bolger, G. T.; Licollari, A.; Tan, A.; Greil, R.; Pleyer, L.; Vcelar, B.; Majeed, M.; Sordillo, P., Distribution of Curcumin and THC in Peripheral Blood Mononuclear Cells Isolated from Healthy Individuals and Patients with Chronic Lymphocytic Leukemia. Anticancer Res 2018, 38 (1), 121-130.

100. Bolger, G. T.; Licollari, A.; Bagshaw, R.; Tan, A.; Greil, R.; Vcelar, B.; Majeed, M.; Sordillo, P., Intense Uptake of Liposomal Curcumin by Multiple Myeloma Cell Lines: Comparison to Normal Lymphocytes, Red Blood Cells and Chronic Lymphocytic Leukemia Cells. Anticancer Res 2019, 39 (3), 1161-1168.

101. Ma, P.; Mumper, R. J., Paclitaxel Nano-Delivery Systems: A Comprehensive Review. J Nanomed Nanotechnol 2013, 4 (2), 1000164.

102. Wang, F.; Porter, M.; Konstantopoulos, A.; Zhang, P.; Cui, H., Preclinical development of drug delivery systems for paclitaxel-based cancer chemotherapy. Journal of Controlled Release 2017, 267, 100-118.

103. Gelderblom, H.; Verweij, J.; Nooter, K.; Sparreboom, A., Cremophor EL: the drawbacks and advantages of vehicle selection for drug formulation. European journal of cancer (Oxford, England : 1990) 2001, 37 (13), 1590-1598.

104. Stern, S. T.; Martinez, M. N.; Stevens, D. M., When Is It Important to Measure Unbound Drug in Evaluating Nanomedicine Pharmacokinetics? Drug Metabolism and Disposition 2016, 44 (12), 1934.

105. Colucci, P.; Turgeon, J.; Ducharme, M. P., How critical is the duration of the sampling scheme for the determination of half-life, characterization of exposure and assessment of bioequivalence? Journal of pharmacy \& pharmaceutical sciences : a publication of the Canadian Society for Pharmaceutical Sciences, Societe canadienne des sciences pharmaceutiques 2011, 14 (2), 217-26. 




\title{
$\underline{\text { Chapter } 3}$
}

\section{The Effect of Formulation and Processing Parameters on the Size of mPEG- $b$ - p(HPMA-Bz) Polymeric Micelles}

\begin{abstract}
Mahsa Bagheri ${ }^{1}$, Jaleesa Bresseleers ${ }^{2,3}$, Aida Varela-Moreira ${ }^{1,4}$, Olivier Sandre ${ }^{5}$, Silvie A. Meeuwissen $^{2}$, Raymond M. Schiffelers ${ }^{4}$, Josbert M Metselaar ${ }^{6}$, Cornelus F. van Nostrum ${ }^{1}$, Jan C.M. van $\mathrm{Hest}^{3}$, Wim E. Hennink ${ }^{1}$
\end{abstract}

${ }^{1}$ Department of Pharmaceutics, Utrecht Institute for Pharmaceutical Sciences (UIPS), Faculty of Science, Utrecht University, 3508 TB Utrecht, The Netherlands

${ }^{2}$ ChemConnection BV, 5349 AB Oss, The Netherlands

${ }^{3}$ Department of Bio-Organic chemistry, Eindhoven University of Technology, 5600 MB Eindhoven, The Netherlands

${ }^{4}$ Department of Clinical Chemistry and Haematology, University Medical Centre Utrecht, 3584 CX Utrecht, The Netherlands

${ }^{5}$ Laboratoire de Chimie de Polymères Organiques, Université de Bordeaux, UMR 5629 CNRS

Pessac, France

${ }^{6}$ Department of Nanomedicine and Theranostics Institute for Experimental Molecular Imaging RWTH University Clinic, 52074 Aachen, Germany 


\begin{abstract}
Micelles composed of block copolymers of poly(ethylene glycol)- $b$-poly $(N-2-$ benzoyloxypropyl methacrylamide) (mPEG- $b$-p(HPMA-Bz)) have shown great promise as drug delivery carriers due to their excellent stability and high loading capacity. In the present study, parameters influencing micelle size were investigated to tailor sizes in the range of 25 to $100 \mathrm{~nm}$. Micelles were prepared by a nanoprecipitation method and their size was modulated by the block copolymer properties such as molecular weight, their hydrophilic to hydrophobic ratio, homopolymer content, as well as formulation and processing parameters. It was shown that the micelles have a core-shell structure using a combination of dynamic light scattering and transmission electron microscopy analysis. By varying the degree of polymerization of the hydrophobic block $\left(\mathrm{N}_{\mathrm{B}}\right)$ between 68 and 10 , at a fixed hydrophilic block $\mathrm{mPEG}_{5 \mathrm{~K}}\left(\mathrm{~N}_{\mathrm{A}}=114\right)$, it was shown that the hydrophobic core of the micelle was collapsed following the power law of $\left(\mathrm{N}_{\mathrm{B}} \times \mathrm{N}_{\mathrm{agg}}\right)^{1 / 3}$. Further, the calculated brush height was similar for all the micelles examined $(10 \mathrm{~nm})$, indicating that crew-cut micelles were made. Both addition of homopolymer and preparation of micelles at lower concentrations or lower rates of addition of the organic solvent to the aqueous phase increased the size of micelles due to partitioning of the hydrophobic homopolymer chains to the core of the micelles and lower nucleation rates, respectively. Furthermore, it was shown that by using different solvents, the size of the micelles substantially changed. The use of acetone, acetonitrile, ethanol, THF and dioxane, resulted in micelles in the size range from 45 to $60 \mathrm{~nm}$ after removal of the organic solvents. The use of DMF and DMSO led to markedly larger sizes of 75 and $180 \mathrm{~nm}$ respectively. In conclusion, the results show that by modulating polymer properties and processing conditions, micelles with tailorable sizes can be obtained.
\end{abstract}

Keywords: polymeric micelles; nanomedicines; HPMA; nanoprecipitation; core-shell; crew-cut micelles; size control; formulation and processing parameters 


\section{Introduction}

Over the last decades, a large variety of nanomedicines has been developed to improve drug disposition at the target site ${ }^{1-5}$. Particularly polymeric micelles, core-shell structures composed of amphiphilic polymers, with a diameter in the size range of 10-100 nm, have attracted much attention. The shell mainly consists of a hydrophilic block, usually poly(ethylene glycol) (PEG), which offers good colloidal stability as well as stealth properties by protecting the micelles from serum/protein interactions and fast uptake by the reticuloendothelial system after injection. The hydrophobic core can accommodate poorly water-soluble drugs like chemotherapeutics for cancer treatment ${ }^{6-12}$.

In order to have a clinically interesting tumor targeted nanomedicine, the formulation should provide sufficient stability and drug retention in the blood circulation. This stability can either be provided by physical interactions ${ }^{13-15}$, or through chemical crosslinking ${ }^{16}$. Once circulating in the bloodstream, nanomedicines can penetrate the fenestrated blood capillaries of tumors. Due to the lack of lymphatic drainage, the nanoparticles tend to retain in the tumor region. This phenomenon is the so-called enhanced permeation and retention effect (EPR) ${ }^{17-}$ 19. Nanomedicines that exploit the EPR effect have shown to both significantly improve therapeutic effects and reduce systemic side effects ${ }^{20-21}$.

It has been shown that the size of nanomedicines, like drug-loaded polymeric micelles, is an important factor for an improved therapeutic efficacy ${ }^{22-24}$. Therefore, in recent years, many studies have been devoted to understanding the effect of size of nanomedicines on their efficacy of cancer treatment. To highlight some of them, Huang et al. demonstrated that tiopronin coated gold nanoparticles of 2 and $6 \mathrm{~nm}$ have longer blood circulation times and better tumor penetration than $15 \mathrm{~nm}$ nanoparticles ${ }^{25}$. Kataoka et al. prepared micelles of different sizes by adding poly(glutamic acid) (p(Glu)) homopolymer to PEG- $b$-p(Glu) copolymer achieving micelle sizes ranging from $30 \mathrm{~nm}$ without homopolymer to around 100 $\mathrm{nm}$ at a 0.3 homopolymer/copolymer molar ratio. They reported that polymeric micelles in the range of 30 to $100 \mathrm{~nm}$ could penetrate highly permeable tumors while only the micelle formulations that were smaller than $50 \mathrm{~nm}$ penetrated into poorly permeable tumors and showed antitumor effect ${ }^{23}$. Chilkoti et al. showed that dextrans with a molecular weight of 40 to $70 \mathrm{kDa}$ did accumulate in tumors after intravenous administration, whereas dextrans of 3.3 and $10 \mathrm{kDa}$ provided deeper and more homogeneous tumor penetration ${ }^{26}$. Shen et al. prepared micelles, based on PEG and a 10-OH methacrylate ester of 7-ethyl-10hydroxylcamptothecin (PEG-p(HEMASN38)), of 20 to $300 \mathrm{~nm}$ by varying the process parameters. Although the $100 \mathrm{~nm}$ micelles reached a higher concentration at the peripheral side of the tumor compared to the $30 \mathrm{~nm}$ size micelles, due to higher liver accumulation of the $30 \mathrm{~nm}$ size micelles, this did not translate in an improved therapeutic effect since the latter micelles had better tumor penetration ${ }^{22}$. Smaller sized nanoparticles also showed better penetration in tumor stroma-containing 3D spheroids which are a suitable model to study penetration of nanoparticles. The results indicated deeper penetration of $30 \mathrm{~nm}$ silica nanoparticles compared to particles of $100 \mathrm{~nm}^{27}$. In conclusion, various studies have convincingly demonstrated that smaller drug-loaded particles resulted in better tumor penetration and thus better efficacy of the treatment ${ }^{24}$.

Recently we reported on a polymeric micelle formulation based on poly(ethylene glycol)block-poly(N-2-benzoyloxypropyl methacrylamide) (mPEG- $b$-p(HPMA-Bz)). Micelles 
based on this polymer combine excellent particle stability, also in circulation, with improved drug retention as a result of $\pi-\pi$ stacking interactions in the core of the micelles. When loaded with paclitaxel, these micelles have shown very promising results regarding pharmaceutical formulation characteristics (loading and stability) and therapeutic efficacy in animal studies demonstrating complete tumor regression ${ }^{28}$. In the present study, a systematic evaluation was made to understand which parameters affect the size and stability of micelles prepared from mPEG- $b$-p(HPMA-Bz) block copolymers. The goal was to find a robust method to obtain micelles with tailorable sizes in the range of 25 to $100 \mathrm{~nm}$. This was achieved by synthesizing block copolymers with a hydrophilic $5 \mathrm{kDa}$ poly(ethylene glycol) methyl ether mPEG block, but also some studies were done with a $2 \mathrm{kDa}$ mPEG block copolymer, and a varying molecular weight of poly( $N$-2-benzoyloxypropyl methacrylamide) (p(HPMA-Bz)). Furthermore, the effect of formulation variables among which the homopolymer $\mathrm{p}$ (HPMA$\mathrm{Bz}$ ) content, polymer concentration, type of solvent and the effect processing variables, particularly the addition rate of the solution of the block copolymer to the aqueous phase, on the size of polymeric micelles were investigated.

\section{Materials and Methods}

\subsection{Materials}

4-(Dimethylamino)pyridine (DMAP), p-toluenesulfonic acid, 4,4-azobis(4-cyanopentanoic acid) (ABCPA), DL-1-amino-2-propanol, methacryloyl chloride, benzoyl chloride, poly(ethylene glycol) methyl ether (mPEG) $2 \mathrm{kDa}, \mathrm{N}, \mathrm{N}$ '-dicyclohexylcarbodiimide (DCC), trichloroacetyl isocyanate (TAIC), bovine serum albumin and pyrene were obtained from Sigma-Aldrich (Darmstadt, Germany) and used without further purification. mPEG $5 \mathrm{kDa}$ was obtained from Polysciences (Warrington, USA) and dried in a vacuum oven overnight at $70{ }^{\circ} \mathrm{C}$. Easivial PEG standards for GPC analysis were obtained from Agilent (Santa Clara, USA). All solvents were purchased from commercial suppliers and used as received.

\subsection{Optimized Macro-Initiator (MI) Synthesis}

mPEG-ABCPA-mPEG macro-initiators were synthesized through an esterification of mPEG (molecular weight 2.0 or $5.0 \mathrm{kDa}$ ) and ABCPA, using DCC as a coupling reagent and 4(dimethylamino) pyridinium 4-toluenesulfonate (DPTS; which was made by separately dissolving DMAP and p-toluenesulfonic acid in THF and mixing the two solutions using a 1:1 molar equivalence) as a catalyst (Scheme 1) ${ }^{29}$. One equivalent ABCPA, 2 equivalents mPEG and 0.3 equivalents of DPTS (or $0.280 \mathrm{~g} \mathrm{ABCPA,} 10 \mathrm{~g}$ mPEG, $0.094 \mathrm{~g}$ DPTS, respectively) were dissolved in $50 \mathrm{~mL}$ dry DCM and put on ice. Next, 3 equivalents of DCC (0.619 $\mathrm{g}$ DCC) were dissolved in $50 \mathrm{~mL}$ DCM and dropwise added to the mPEG solution under nitrogen atmosphere. After addition of DCC, the ice bath was removed allowing the reaction mixture to reach room temperature. After $16 \mathrm{~h}$ at room temperature, the reaction mixture was filtered to remove the precipitated 1,3-dicyclohexyl urea and the solvent was removed in vacuo. The product was dissolved in water, stirred for $2 \mathrm{~h}$ and dialyzed against water for $72 \mathrm{~h}$ at $4^{\circ} \mathrm{C}$. The sample was freeze-dried to obtain a fluffy white product. 


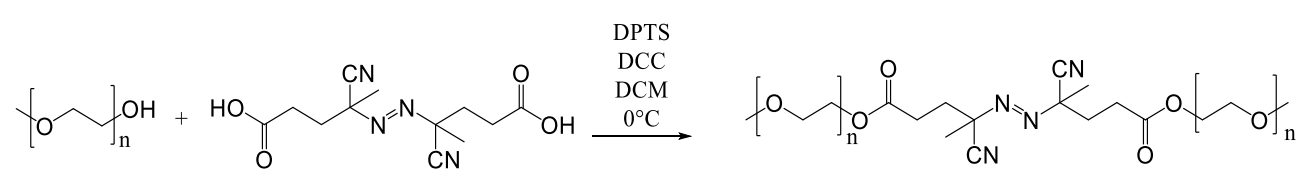

Scheme 1. Synthesis of mPEG-ABCPA-mPEG macro-initiator

The product was analyzed by GPC using a PSS PFG analytical linear S column and PEGs of narrow molecular weights as calibration standards as described previously. Samples were prepared by dissolving approximately $5 \mathrm{mg}$ of the MI in $1 \mathrm{~mL} \mathrm{DMF}$ containing $10 \mathrm{mM} \mathrm{LiCl}$. Samples of $20 \mu \mathrm{L}$ were injected, the eluent was DMF containing $10 \mathrm{mM} \mathrm{LiCl}$, the elution rate was $0.7 \mathrm{~mL} / \mathrm{min}$, the temperature was $40^{\circ} \mathrm{C}$ and detection was done using a refractive index detector ${ }^{30}$.

The product was further analyzed by ${ }^{1} \mathrm{H}-\mathrm{NMR}$ spectroscopy $(20 \mathrm{mg}$ product was dissolved in $700 \mu \mathrm{L} \mathrm{CDCl}_{3}$ ). To determine the unreacted mPEG-OH content, TAIC was added to the sample and analyzed again after 20 minutes using ${ }^{1} \mathrm{H}-\mathrm{NMR}$ spectroscopy. After reaction with TAIC, the signal of the methylene group neighboring the terminal hydroxyl group shifts from 4.2 to $4.4 \mathrm{ppm}$ and the amount of unreacted mPEG-OH can subsequently be determined based on the peak areas ${ }^{31-32}$.

\subsection{Dynamic Light Scattering (DLS)}

The size of the formed micelles was determined by DLS using a Malvern Zetasizer nano series ZS90 with a measurement angle of $90^{\circ}$ and a temperature of $25^{\circ} \mathrm{C}$. Unless stated differently, the concentration of the micellar dispersions was $20 \mathrm{mg} / \mathrm{mL}$.

\subsection{Gas Chromatography Headspace Analysis (GC-headspace)}

GC-headspace was conducted to determine the residual solvent contents in the different micellar dispersions using a Shimadzu GC-2010 equipped with a Flame Ionization Detector and Shimadzu HS-20 headspace auto-sampler. A $30 \mathrm{~m} \times 0.32 \mathrm{~mm}$ capillary column with a film thickness of $0.25 \mu \mathrm{m}$ was used. An internal standard stock solution was prepared by dissolving $150 \mu \mathrm{L}$ 2-propanol (analytical standard) in water in a volumetric $100 \mathrm{~mL}$ flask. 1 $\mathrm{mL}$ of this solution was transferred into a $100 \mathrm{~mL}$ volumetric flask and diluted to the $100 \mathrm{~mL}$ volume with DMF. Samples were prepared by taking $50 \mu \mathrm{L}$ of micellar dispersion and dissolving it in $1 \mathrm{~mL} \mathrm{DMF}$, and subsequently $4 \mathrm{~mL}$ internal standard stock solution was added. The flow rate of nitrogen was $1.8 \mathrm{~mL} / \mathrm{min}$. All measurements were done in triplicate.

\subsection{Cryogenic Transmission Electron Microscopy (cryo-TEM) Analysis}

Cryo-TEM measurements were performed on selected micelles. Samples were prepared on Quantifoil R 2/2 grids. In short, $3 \mu \mathrm{L}$ micellar dispersion was pipetted onto the grid and blotted for 3 seconds using a fully automated vitrification robot (MARK III) at 100\% relative humidity. The grid was subsequently plunged and frozen in liquid ethane. Micrographs were taken using a FEI Tecnai G2 Sphere ( $200 \mathrm{kV}$ electron source) equipped with LaB6 filament utilizing a cryoholder or a FEI Titan $(300 \mathrm{kV}$ electron source) equipped with an autoloader station. 


\subsection{Analysis of the Micelles by Asymmetric Flow Field-Flow Fractionation Connected to Multi-Angle Light Scattering Detector (AF4-MALS)}

The radius of gyration $\left(\mathrm{R}_{\mathrm{g}}\right)$ and weight-average molecular weight of some selected micelles $\left(\mathrm{M}_{\mathrm{w}}\right)$ was determined using a Wyatt Dualtec $\mathrm{AF}_{4}$ instrument connected to a Shimadzu LC2030 Prominence-I system with a Shimadzu LC-2030 auto-sampler. Fractionation was performed on an $\mathrm{AF}_{4}$ short channel with a $10 \mathrm{kDa}$ membrane of regenerated cellulose and a spacer of $350 \mu \mathrm{m}$. The $\mathrm{AF}_{4}$ was connected to a light scattering detector (Wyatt DAWN HELEOS II) installed at 16 different angles ranging from 12.9 to $157.8^{\circ}$ using a laser operating at $664.5 \mathrm{~nm}$ and a refractive index detector (Wyatt Optilab). Bovine serum albumin dissolved in phosphate buffer saline (PBS) pH 7.4 with a concentration of $5 \mathrm{mg} / \mathrm{mL}$ was used for calibration. The data were analyzed using ASTRA software. Also, to be able to calculate the $\mathrm{M}_{\mathrm{w}}$ of micelles using the Zimm plot method ${ }^{33}$, the refraction index increment $(\mathrm{dn} / \mathrm{dc})$ of the polymers was measured in water by injection of $600 \mu \mathrm{L}$ of precisely weighted samples in the range of 6 to $15 \mathrm{mg} / \mathrm{mL}$ and using a flow rate of $0.6 \mathrm{~mL} / \mathrm{min}$ in an Optilab Rex detector (Wyatt technology).

\subsection{Critical Micelle Concentration (CMC) determination}

The CMC of the different block copolymers in water was determined using pyrene as a fluorescent probe ${ }^{34-35}$. Samples were prepared by dissolving the polymers in THF at different concentrations of which $500 \mu \mathrm{L}$ was added to $4.5 \mathrm{~mL} 120 \mathrm{mM}$ ammonium acetate buffer $\mathrm{pH}$ 5.0. This was followed by solvent evaporation. The final polymer concentrations ranged from $1.9 \times 10^{-11}$ to $1.0 \mathrm{mg} / \mathrm{mL}$. A $15 \mu \mathrm{L}$ solution of pyrene in acetone $(0.18 \mathrm{mM})$ was added to the polymer solution in buffer and the solvent was allowed to evaporate overnight. Fluorescence excitation spectra of pyrene between 300 and $360 \mathrm{~nm}$ were recorded with an emission wavelength at $390 \mathrm{~nm}$ at $37{ }^{\circ} \mathrm{C}$ using a UV spectrometer (Jasco FP-8300 Fluorescence Spectrometer). The excitation and emission band slits were 4 and $2 \mathrm{~nm}$, respectively. The intensity ratio of $\mathrm{I}_{338} / \mathrm{I}_{333}$ was plotted against the polymer concentration to calculate the CMC.

\subsection{Polymer Synthesis}

mPEG-block-poly( $N$-2-benzoyloxypropyl methacrylamide) (mPEG- $b$-p(HPMA-Bz)) block copolymers were synthesized via free-radical polymerization as described earlier using mPEG-ABCPA-mPEG as a macro-initiator and N-(2-benzoyloxypropyl methacrylamide) (HPMA-Bz) as the monomer ${ }^{28,34,36}$. mPEG- $b$-p (HPMA-Bz) block copolymers with different molecular weights of the hydrophobic and hydrophilic block were synthesized either by using $\mathrm{mPEG}_{2 \mathrm{~K}}-\mathrm{ABCPA} \mathrm{mPEG}_{2 \mathrm{~K}}$ or $\mathrm{mPEG}_{5 \mathrm{~K}}-\mathrm{ABCPA}_{-\mathrm{mPEG}_{5 \mathrm{~K}}}$ as a macro-initiator and by varying the molar feed ratios of macro-initiator:monomer $(1: 200,1: 100,1: 75,1: 50,1: 25$, $1: 12.5 \mathrm{~mol} / \mathrm{mol}$ ) (Scheme 2). In short, the selected macro-initiator and monomer amounts were dissolved at a total concentration of $0.3 \mathrm{~g} / \mathrm{mL}$ in $20 \mathrm{~mL}$ of acetonitrile. The polymerization was conducted at $70^{\circ} \mathrm{C}$ in a nitrogen atmosphere for $24 \mathrm{~h}$. The resulting polymers were precipitated in cold diethyl ether and collected after centrifugation. Homopolymers of $\mathrm{p}(\mathrm{HPMA}-\mathrm{Bz})$ were synthesized and collected in the same way using ABCPA as initiator and HPMA-Bz as monomer. The feed ratios of initiator:monomer were $1: 200(\mathrm{~mol} / \mathrm{mol}), 1: 100(\mathrm{~mol} / \mathrm{mol})$ and $1: 50(\mathrm{~mol} / \mathrm{mol})$ with a total concentration of $0.3 \mathrm{~g} / \mathrm{mL}$ in $10 \mathrm{~mL}$ acetonitrile to obtain a total of $2 \mathrm{~g}$ homopolymer after precipitation in cold diethyl ether and centrifugation. 

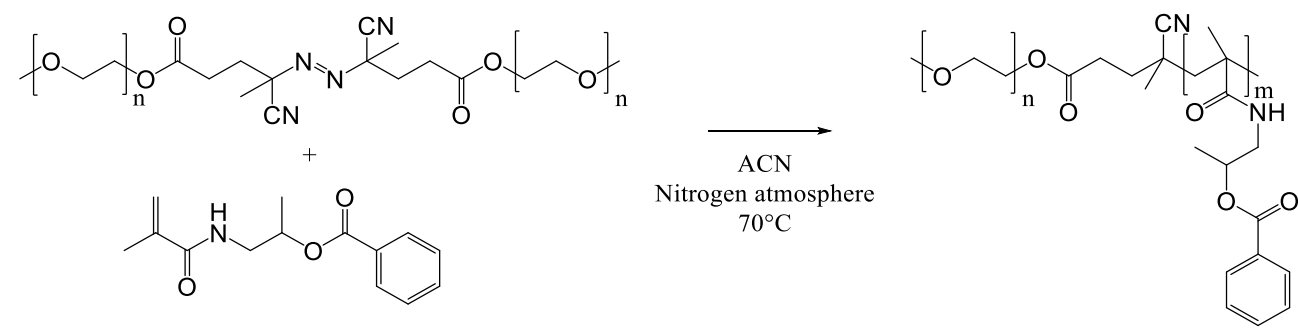

Scheme 2. Synthesis of mPEG- $b$-p(HPMA-Bz)

The synthesized polymers were analyzed by ${ }^{1} \mathrm{H}-\mathrm{NMR}$. To this end, $20 \mathrm{mg}$ of polymer was dissolved in $700 \mu \mathrm{L}$ DMSO- $\mathrm{d}_{6}$ and the obtained polymer solution was analyzed using a 400 $\mathrm{MHz}$ NMR with $5 \mathrm{~mm}$ PABBO BB probe from Bruker. GPC analysis was conducted to determine the number average molecular weight $\left(\mathrm{M}_{\mathrm{n}}\right)$, weight average molecular weight $\left(\mathrm{M}_{\mathrm{w}}\right)$ and polydispersity of the synthesized polymers using a PSS PFG analytical linear S column and PEGs of narrow molecular weights as calibration standards. Samples were prepared by dissolving approximately $5 \mathrm{mg}$ of polymer in $1 \mathrm{~mL}$ DMF containing $10 \mathrm{mM}$ LiCl. Samples of $20 \mu \mathrm{L}$ were injected, the eluent was DMF containing $10 \mathrm{mM} \mathrm{LiCl}$, the elution rate was $0.7 \mathrm{~mL} / \mathrm{min}$, the temperature was $40^{\circ} \mathrm{C}$ and detection was done using a refractive index detector.

\subsection{Micelle Preparation}

mPEG- $b$-p(HPMA-Bz) micelles were prepared in triplo by a nanoprecipitation of the polymer dissolved in THF, using water as non-solvent. First, the polymers were dissolved in THF and the obtained solutions were pipetted into MilliQ at a 1:1 volume ratio while stirring. Subsequently, THF was evaporated overnight at room temperature, resulting in the formation of micelles. To investigate the effect of polymer concentration on micellar sizes, samples were prepared using $5,10,20,30,40 \mathrm{mg} / \mathrm{mL}$ of polymer solution. Additionally, an experiment was carried out where the volume ratio of solvent-to-water was decreased from 1:1 to $0.6: 1$ and $0.3: 1$. Addition of the polymer/solvent solution to water was performed rapidly using a pipette while stirring. The final polymer concentrations in water were 10,20 and $30 \mathrm{mg} / \mathrm{mL}$. Thus, in total nine different conditions were tested. In the remainder of the experiments the polymer concentration was fixed at $20 \mathrm{mg} / \mathrm{mL}$, unless mentioned otherwise. Besides THF, the following solvents were also used: acetonitrile, acetone, 1,4-dioxane, dimethylformamide (DMF), dimethylsulfoxide (DMSO) and ethanol. For the less/nonvolatile solvents (dioxane, DMSO, DMF and ethanol), the residual solvent was removed by dialysis using a Spectra/Por dialysis membrane with a molecular weight cut off of 6-8 kDa. Moreover, the aqueous phase was varied: water, $0.9 \% \mathrm{NaCl}$ solution, $\mathrm{PBS}$ (containing $3.1 \mathrm{~g}$ $\mathrm{Na}_{2} \mathrm{HPO}_{4}, 0.3 \mathrm{~g} \mathrm{NaH}_{2} \mathrm{PO}_{4}, 8.2 \mathrm{~g} \mathrm{NaCl}$ in $1 \mathrm{~L} \mathrm{pH} \mathrm{7.4)} \mathrm{and} 120 \mathrm{mM}$ ammonium acetate buffer $\mathrm{pH} 5.0$ were used. The addition rates were varied by introducing the polymer solution in THF into the aqueous phase using a peristaltic pump (Pharmacia LKB pump P-1, made in Sweden) at 0.15 and $1.5 \mathrm{~mL} / \mathrm{min}$ while stirring using a magnetic stirrer. Also, the effect of adding MilliQ to the polymer solution in THF in a 1:1 volume ratio was investigated. 


\subsection{The Effect of the Presence of p(HPMA-Bz) Homopolymer and the Presence of Free mPEG on the Size of Polymeric Micelles}

Samples of $20 \mathrm{mg}$ mPEG- $b$-p(HPMA-Bz) and 0, 1, 2, 5 and $10 \mathrm{mg}$ p(HPMA-Bz) were dissolved in $1 \mathrm{~mL}$ THF corresponding with weight fractions of $0,5,9,20$ and $33 \mathrm{w} \%$ of the homopolymer. Other samples of $20 \mathrm{mg}$ mPEG- $b$-p(HPMA-Bz) and $0,1,2,5$ and $10 \mathrm{mg}$ mPEG $_{5 \mathrm{~K}}$ were dissolved in $1 \mathrm{~mL}$ THF corresponding with weight fractions of $0,5,9,20$ and $33 \mathrm{w} \%$ of the $\mathrm{mPEG}_{5 \mathrm{~K}}$. Addition of the polymer solution to water was performed rapidly using a pipette while stirring. THF was evaporated overnight at room temperature, resulting in the formation of micelles.

\section{Results and Discussions}

\section{1 mPEG-ABCPA-mPEG Macro-Initiator Synthesis}

The macro-initiator (MI) used for polymerization, mPEG-ABCPA-mPEG, was previously synthesized by the reaction of 2 equivalents $\mathrm{mPEG}$ with 1 equivalent 4,4-azobis(4cyanopentanoic acid) $(\mathrm{ABCPA})^{31}, 37$. According to this previous procedure, all the components except mPEG were dissolved simultaneously in a 1:1 mixture of DCM and dry DMF and put on ice. In this way, the $\mathrm{COOH}$ groups of ABCPA were first activated with DCC and subsequently mPEG was added. After addition of $\mathrm{mPEG}$, the ice bath was removed and the mixture was stirred at room temperature overnight. This resulted in a yield of $\sim 80 \%$ of macroinitiator after precipitation ${ }^{37}$. This strategy however led to a large amount of $\sim 40 \%$ byproduct with a molecular weight of $5 \mathrm{kDa}$ according to GPC analysis (see SI). The shoulder peak in GPC can be due to the presence of either mPEG-ABCPA or unreacted mPEG, or a combination of both. It is known that a DCC-activated ester can undergo a rearrangement reaction to yield an $\mathrm{N}$-acyl iso-urea product, which is not reactive with the primary hydroxyl group of $\mathrm{mPEG}^{38}$. Therefore, there is a possibility that the ABCPA reacts with only one mPEG chain giving mPEG-ABCPA, with or without an acyl urea (see SI), as a byproduct. Consequently, unreacted $\mathrm{mPEG}-\mathrm{OH}$ (free $\mathrm{mPEG)}$ will also be present in the reaction solution. TAIC is a reagent that is used for the quantitative determination of hydroxy endgroups of polymers using ${ }^{1} \mathrm{H}-\mathrm{NMR}^{32,}{ }^{39}$. Therefore, this reagent was used to quantify the amount of free mPEG in the obtained product. Analysis showed the presence of $\sim 30 \%$ unreacted $\mathrm{mPEG}$, leaving the remaining $10 \%$ of the $5 \mathrm{kDa}$ byproduct to be mPEG-ABCPA. Further purification steps such as dialysis could not separate the byproducts from mPEGABCPA-mPEG.

Upon the use of MI contaminated with mPEG-ABCPA for the polymerization of HMPA-Bz, both the p(HPMA-Bz) homopolymer and the aimed mPEG- $b$-p(HPMA-Bz) block copolymer are formed (Figure 1). The presence of the p(HPMA-Bz) is unwanted because it will be solubilized in the core of the micelles, which in turn will result in an increase in micellar size. Therefore, the MI synthesis was optimized to obtain a high yield of mPEG-ABCPA-mPEG and to minimize the amounts of the mPEG-ABCPA/mPEG byproducts.

In the new procedure, all reagents, including mPEG but except DCC were dissolved in DCM. Subsequently DCC dissolved in DCM was added dropwise. ${ }^{29}$ This resulted in activation of the $\mathrm{COOH}$ groups in the presence of $\mathrm{mPEG}$ to allow reaction of its $\mathrm{OH}$ group with the active ester thereby reducing the possibility for the formation of the inactive $\mathrm{N}$-acyl iso-urea product. Furthermore, contrary to the other procedure, no DMF was used and the reaction was therefore conducted in the less polar solvent DCM. 
The new procedure resulted in the successful synthesis of two different mPEG-ABCPAmPEG macro-initiators $\left(\mathrm{mPEG}_{5 \mathrm{~K}}\right.$ and $\mathrm{mPEG}_{2 \mathrm{~K}}$ ) which were obtained in a yield of $\sim 90 \%$ and only contained $\sim 5 \%$ of the mixture of $5 \mathrm{kDa}$ mPEG-ABCPA/free mPEG (GPC analysis, Figure S1). The amount of free mPEG was determined by ${ }^{1} \mathrm{H}-\mathrm{NMR}$ using TAIC to be $4.2 \%$ (Figure S2). This shows that the MI only contained a trace amount of $0.8 \%$ mPEG-ABCPA. Therefore, the MI synthesized according to this new procedure was used for the synthesis of the different mPEG- $b$-p(HPMA-Bz) block copolymers.

\subsection{Synthesis of mPEG- $b$-p(HPMA-Bz) Block Copolymers}

Amphiphilic mPEG- $b$-p(HPMA-Bz) block copolymers with varying molecular weights of the hydrophobic and hydrophilic blocks were synthesized by free-radical polymerization of HPMA-Bz using mPEG-ABCPA-mPEG macro-initiators $\left(\mathrm{mPEG}_{5 \mathrm{~K}}\right.$ or $\mathrm{mPEG}_{2 \mathrm{~K}}$ ) (Figure 1 ) at different macro-initiator/monomer ratios (MI:M; Table 1). The molecular weights $\left(\mathrm{M}_{n}\right.$, $\mathrm{M}_{\mathrm{w}}$ ) of the obtained polymers were determined by ${ }^{1} \mathrm{H}-\mathrm{NMR}$ and GPC analysis. As reported earlier, an increasing trend of molecular weight was observed upon increasing the monomer to initiator ratio ${ }^{34}$.

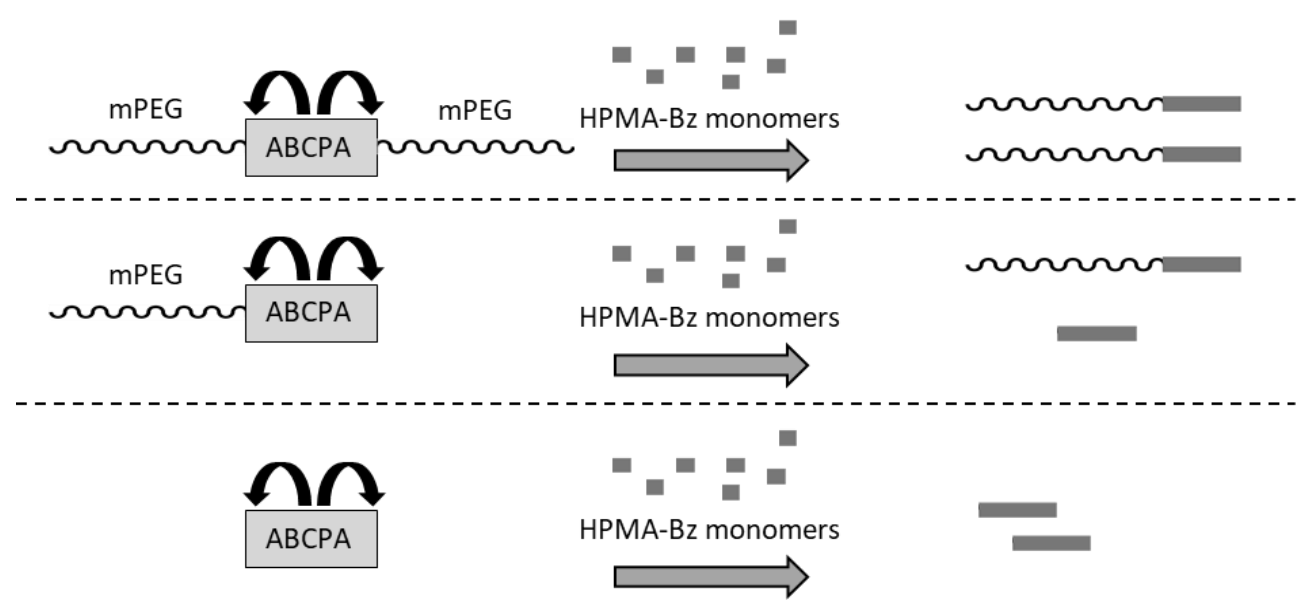

Figure 1. Schematic representation of the polymerization of mPEG- $b-\mathrm{p}(\mathrm{HPMA}-\mathrm{Bz})$ initiated by mPEG-ABCPA-mPEG results in the synthesis of mPEG- $b$-p(HPMA-Bz) diblock copolymers only. Initiation by mPEG-ABCPA will result in a mixture of block copolymer and homopolymer p(HPMA$\mathrm{Bz}$ ). Initiation by ABCPA will result in the formation of homopolymer only. It is hereby assumed that no chain transfer occurs. 
Table 1. Characteristics of the synthesized mPEG- $b$-p(HPMA-Bz) block copolymers as determined by ${ }^{1} \mathrm{H}-\mathrm{NMR}$ and GPC.

\begin{tabular}{ccccccc}
\hline Polymer & MI:M & $\begin{array}{c}\mathbf{M}_{\mathbf{n}} \mathbf{b y}{ }^{1} \mathbf{H}- \\
\mathbf{N M R} \\
(\mathbf{k D a})\end{array}$ & $\begin{array}{c}\mathbf{M}_{\mathbf{n}} \mathbf{b y} \\
\mathbf{G P C} \\
(\mathbf{k D a})\end{array}$ & $\begin{array}{c}\mathbf{M}_{\mathbf{w}} \mathbf{b y} \\
\mathbf{G P C} \\
(\mathbf{k D a})\end{array}$ & $\begin{array}{c}\text { Polydispersity } \\
\left(\mathbf{M}_{\mathrm{w}} / \mathbf{M}_{\mathbf{n}}\right)(\mathbf{G P C})\end{array}$ & $\begin{array}{c}\text { Yield } \\
(\%)\end{array}$ \\
\hline $\mathrm{mPEG}_{5 \mathrm{~K}}-b-\mathrm{p}(\mathrm{HPMA}-\mathrm{Bz})_{18.5 \mathrm{~K}}$ & $1: 200$ & 23.5 & 18.9 & 21.1 & 1.12 & 72 \\
$\mathrm{mPEG}_{5 \mathrm{~K}}-b-\mathrm{p}(\mathrm{HPMA}-\mathrm{Bz})_{9.6 \mathrm{~K}}$ & $1: 100$ & 14.6 & 17.3 & 19.6 & 1.13 & 79 \\
$\mathrm{mPEG}_{5 \mathrm{~K}}-b-\mathrm{p}(\mathrm{HPMA}-\mathrm{Bz})_{7.7 \mathrm{~K}}$ & $1: 75$ & 12.7 & 16.4 & 18.7 & 1.14 & 81 \\
$\mathrm{mPEG}_{5 \mathrm{~K}}-b-\mathrm{p}(\mathrm{HPMA}-\mathrm{Bz})_{4.7 \mathrm{~K}}$ & $1: 50$ & 9.7 & 15.1 & 17.4 & 1.15 & 83 \\
$\mathrm{mPEG}_{5 \mathrm{~K}}-b-\mathrm{p}(\mathrm{HPMA}-\mathrm{Bz})_{2.2 \mathrm{~K}}$ & $1: 25$ & 7.2 & 12.8 & 14.8 & 1.16 & 83 \\
$\mathrm{mPEG}_{5 \mathrm{~K}}-b-\mathrm{p}(\mathrm{HPMA}-\mathrm{Bz})_{1.0 \mathrm{~K}}$ & $1: 12.5$ & 6.0 & 9.8 & 12.2 & 1.23 & 84 \\
\hline $\mathrm{mPEG}_{2 \mathrm{~K}}-b-\mathrm{p}(\mathrm{HPMA}-\mathrm{Bz})_{20.6 \mathrm{~K}}$ & $1: 200$ & 22.6 & 13.5 & 19 & 1.42 & 59 \\
$\mathrm{mPEG}_{2 \mathrm{~K}}-b-\mathrm{p}(\mathrm{HPMA-Bz})_{10.9 \mathrm{~K}}$ & $1: 100$ & 12.9 & 10.7 & 16 & 1.51 & 74 \\
$\mathrm{mPEG}_{2 \mathrm{~K}}-b-\mathrm{p}(\mathrm{HPMA}-\mathrm{Bz})_{7.7 \mathrm{~K}}$ & $1: 75$ & 9.7 & 8.5 & 17.1 & 1.57 & 53 \\
$\mathrm{mPEG}_{2 \mathrm{~K}}-b-\mathrm{p}(\mathrm{HPMA}-\mathrm{Bz})_{5.3 \mathrm{~K}}$ & $1: 50$ & 7.3 & 8.1 & 12.6 & 1.55 & 87 \\
$\mathrm{mPEG}_{2 \mathrm{~K}}-b-\mathrm{p}(\mathrm{HPMA}-\mathrm{Bz})_{2.6 \mathrm{~K}}$ & $1: 25$ & 4.6 & 5.7 & 8.2 & 1.45 & 82 \\
$\mathrm{mPEG}_{2 \mathrm{~K}}-b-\mathrm{p}(\mathrm{HPMA}-\mathrm{Bz})_{1.2 \mathrm{~K}}$ & $1: 12.5$ & 3.2 & 4.4 & 5.7 & 1.31 & 87 \\
\hline
\end{tabular}

The average kinetic chain length for free-radical chain polymerization is defined as the average number of monomers polymerized per initiated chain and is proportional to the monomer concentration $\left[\mathrm{M}_{0}\right]$ divided by the square root of the initiator concentration $\left[\mathrm{I}_{0}\right]^{-1 / 2}$ ${ }^{40-41}$. Plotting the number average molecular weight $\left(\mathrm{M}_{\mathrm{n}}\right)$ as measured by ${ }^{1} \mathrm{H}-\mathrm{NMR}$ spectroscopic analysis against the average kinetic chain length indeed resulted in a linear correlation for both the $\mathrm{mPEG}_{5 \mathrm{~K}}$ and $\mathrm{mPEG}_{2 \mathrm{~K}}$ block copolymers (Figure 2 ) as also observed

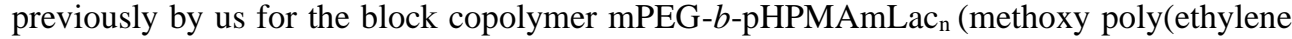
glycol)- $b$-poly[N-(2-hydroxypropyl) methacrylamide-lactate $])^{30}$.

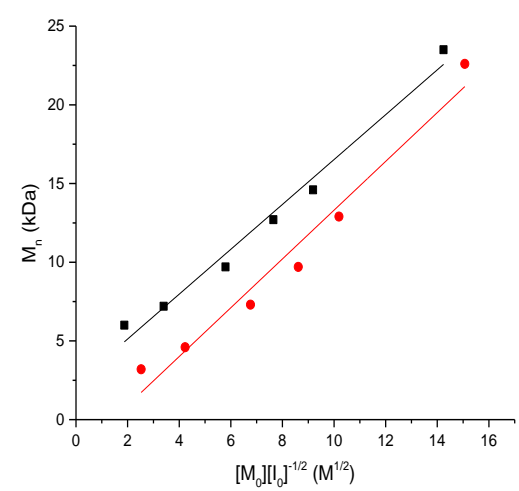

Figure 2. Linear correlation between the number average molecular weight $\left(\mathrm{M}_{\mathrm{n}}\right)$ as measured by ${ }^{1} \mathrm{H}$ NMR of mPEG- $b$-p(HPMA-Bz) as a function of the feed molar concentration of monomer divided by the square root of the feed molar concentration of initiator $\left(\left[\mathrm{M}_{0}\right]\left[\mathrm{I}_{0}\right]^{-1 / 2}\right)$ (black: $\mathrm{mPEG}_{5 \mathrm{k}}-b-\mathrm{p}(\mathrm{HPMA}-$ $\mathrm{Bz})_{\mathrm{n}}$ with $\mathrm{r}^{2}=0.98$; red: $\mathrm{mPEG}_{2 \mathrm{~K}}-b-\mathrm{p}(\mathrm{HPMA}-\mathrm{Bz})_{\mathrm{n}}$ with $\left.\mathrm{r}^{2}=0.97\right)$. 


\subsection{Residual Solvent and Kinetics of Micelle Formation}

To get insight into the kinetics of micelle formation and the rate of removal of THF, in which the $\mathrm{mPEG}_{5 \mathrm{~K}}-b-\mathrm{p}(\mathrm{HPMA}-\mathrm{Bz})_{18.5 \mathrm{~K}}$ polymer was dissolved at $20 \mathrm{mg} / \mathrm{mL}$, the size of micelles was followed in time after direct addition of the THF/polymer solution to water (THF/water was $1: 1 \mathrm{v} / \mathrm{v}$ ). The hydrodynamic diameters of the micelles were measured using DLS, and THF content was measured using GC-headspace analysis directly after addition $(0 \mathrm{~h})$ and at regular time intervals up to $96 \mathrm{~h}$ (Figure 3). Directly after addition of the polymer solution to water, particles with a hydrodynamic diameter of approximately $80 \mathrm{~nm}$ and a polydispersity index of less than 0.1 were formed. After $24 \mathrm{~h}$, the micelles showed a decrease in size to 50 $\mathrm{nm}$, and a residual THF content of $\sim 3000 \mathrm{ppm}$ was detected. At $25 \mathrm{~h}$ the micellar dispersion was spiked with an additional 50 volume percentage of THF, which resulted in an immediate increase in micelle size from 50 to $70 \mathrm{~nm}$. It can therefore be concluded that there is a direct correlation between the remaining amount of THF and the hydrodynamic diameter of the micelles. It should be noted that the final micelle size of $50 \mathrm{~nm}$ was already reached at THF concentrations of less than $10^{5} \mathrm{ppm}$. Addition of THF to the micellar dispersion showed that the core of the micelles can become swollen by accommodating part of the added THF. After $48 \mathrm{~h}$ of evaporation the residual THF content was $\sim 3000 \mathrm{ppm}$, which is not sufficient to obtain a product within the acceptable range below $720 \mathrm{ppm}$ according to the International Council of Harmonization of Technical Requirements for Registration of Pharmaceuticals for Human $\mathrm{Use}^{42}$. Therefore, the evaporation time was extended to $96 \mathrm{~h}$ and the micellar dispersion was also dialyzed against water to remove any residual THF. The final THF content after dialysis and measured by GC headspace was below the detection limit (10 ppm).

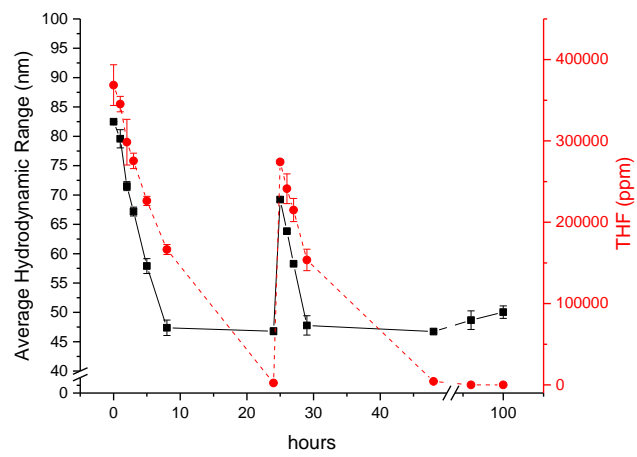

Figure 3. Average hydrodynamic diameters (black) of $\mathrm{mPEG}_{5 \mathrm{~K}}-b-\mathrm{p}(\mathrm{HPMA}-\mathrm{Bz})_{18.5 \mathrm{~K}}$ micelles and THF concentration (red) of the micellar dispersion as a function of time. At $25 \mathrm{~h}$ the dispersion was spiked with THF. After a second overnight evaporation $(48 \mathrm{~h}$ ) and subsequent over weekend evaporation (96 h) the samples were dialyzed overnight, which is represented at time point $100 \mathrm{~h}$ in the graph.

\subsection{Effect of the Hydrophobic/Hydrophilic Block Molecular Weight of mPEG- $b$ - p(HPMA-Bz) on the Micelle Size}

To investigate the effect of the hydrophobic/hydrophilic block molecular weight of the polymers on the micelle size, micelles were prepared from the synthesized polymers of Table 1. The polymers were dissolved in THF at $20 \mathrm{mg} / \mathrm{mL}$ and used to prepare micelles through the nanoprecipitation method in water as described in the Materials and Methods section. All mPEG $_{5 \mathrm{~K}}$ block copolymers formed micelles as was demonstrated by cryo-TEM and DLS analysis. 


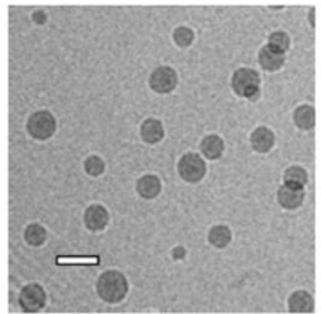

mPEG $_{5 \mathrm{~K}}-\boldsymbol{b}$ p(HPMA-Bz $)_{18.5 K}$

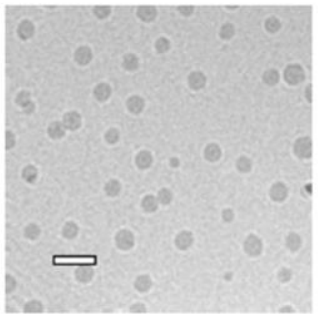

mPEG $_{5 \mathrm{~K}}-\boldsymbol{b}-$ p(HPMA-Bz $)_{9.6 \mathrm{~K}}$

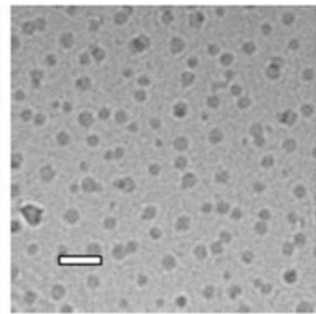

mPEG $_{5 \mathrm{~K}-} \boldsymbol{b}$ p(HPMA-Bz $)_{4.7 \mathrm{~K}}$

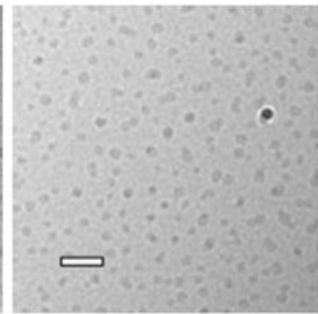

mPEG $_{5 \mathrm{~K}-\boldsymbol{b}}-$ p(HPMA-Bz $)_{2.2 \mathrm{~K}}$

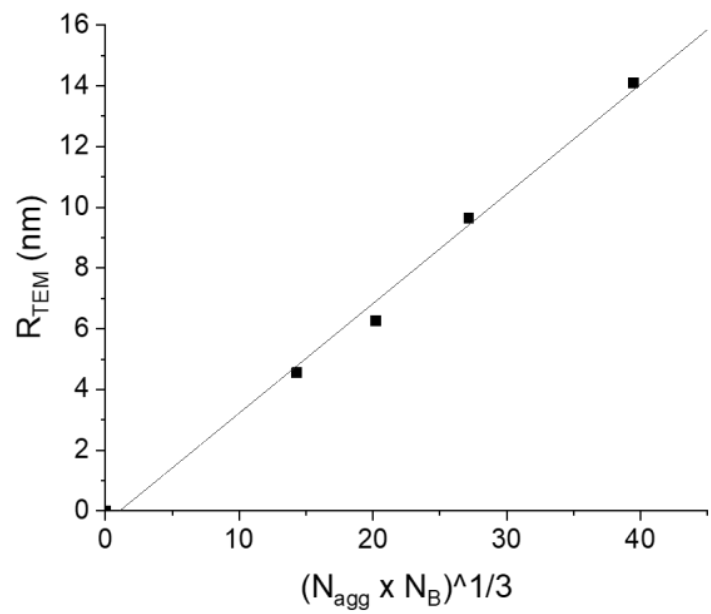

Figure 4. (Top) cryo-TEM images showing particle size variation upon molecular weight changes of the $\mathrm{mPEG}_{5 \mathrm{~K}}$ block copolymers used; scale bars correspond to $50 \mathrm{~nm}$. (Bottom) the average micelle core diameter measured by cryo-TEM as a function of the cubic root of the product of the degree of polymerization $\left(\mathrm{N}_{\mathrm{B}}\right)$ of the hydrophobic blocks of the copolymers as determined by ${ }^{1} \mathrm{H}-\mathrm{NMR}$, and the aggregation number $\left(\mathrm{N}_{\mathrm{agg}}\right)$ of the corresponding micelles revealed by $\mathrm{AF}_{4}-\mathrm{MALS}, \mathrm{r}^{2}=0.99$.

Both the DLS and the cryo-TEM results showed that the micelle size proportionally increased from the smallest to largest molecular weights of the hydrophobic block from 30 to $48 \mathrm{~nm}$ for the hydrodynamic diameter and from 9 to $28 \mathrm{~nm}$ for the cryo-TEM diameter (Figure 4 and Figure S4). The DLS polydispersities were lower than 0.1 pointing to a narrow size distribution, which is in agreement with the results of the TEM pictures. More precisely, the histograms of the TEM diameters based on $\sim 100$ to $\sim 400$ micelles (for $\mathrm{mPEG}_{5 \mathrm{~K}}-b$-p $(\mathrm{HPMA}$ $\mathrm{Bz})_{18.5 \mathrm{~K}}$ and $\mathrm{mPEG}_{5 \mathrm{~K}}-b-\mathrm{p}(\mathrm{HPMA}-\mathrm{Bz})_{2.2 \mathrm{~K}}$ copolymer respectively) exhibited normalized standard deviations of $25 \%$ at most. The hydrated layer of the micelles was estimated by the difference of radii between the radius of hydration $\left(\mathrm{R}_{\mathrm{h}}\right)$ and the radius as determined by TEM $\left(\mathrm{R}_{\text {TEM }}\right)$ and appeared to be constant for the four samples (approximately $10 \mathrm{~nm}$; Table 2).

The radius of gyration $\left(R_{g}\right)$, radius of hydration $\left(R_{h}\right)$ and $M_{w}$ of the micelles based on a selection of $\mathrm{mPEG}_{5 \mathrm{~K}}$ polymers of Table 1 were determined by $\mathrm{AF}_{4}-\mathrm{MALS}$ (Table 2). The ratio of $R_{g} / R_{h}$ is structure sensitive and provides information about the morphology of a system. The ratio for rigid spherical structures with a uniform density is $\sqrt{3 / 5} \approx 0.775^{43-45}$. Structures with a dense core and a partly coiled less dense shell (core-shell structures) show 
a smaller $\mathrm{R}_{\mathrm{g}}$ and therefore have $\mathrm{R}_{\mathrm{g}} / \mathrm{R}_{\mathrm{h}}$ values lower than $0.775^{43-48}$. Based on the MALS data, the produced $\mathrm{mPEG}_{5 \mathrm{~K}}-b$ - $\mathrm{p}(\mathrm{HPMA}-\mathrm{Bz})$ micelles had a core-shell structure because the $\mathrm{R}_{\mathrm{g}} / \mathrm{R}_{\mathrm{h}}$ ratios were between 0.59 and 0.64 and thus lower than that of typical rigid spheres. This corecorona structure was also confirmed using ${ }^{1} \mathrm{H}-\mathrm{NMR}$ analysis of the micelles dispersed in deuterium oxide $\left(\mathrm{D}_{2} \mathrm{O}\right)$ (shown in Figure S6). The ${ }^{1} \mathrm{H}-\mathrm{NMR}$ spectrum of mPEG- $b$-p (HPMA$\mathrm{Bz}$ ) block copolymer dissolved in DMSO- $\mathrm{d}_{6}$ (Figure S6) showed resonances that can be assigned to the protons of both mPEG as the hydrophilic part (3.40-3.60 ppm) and p(HPMA$\mathrm{Bz})$ as the hydrophobic block (5.25 ppm and 7.25-8.25 ppm). The self-assembled structure of the mPEG- $b$-p(HPMA-Bz) block copolymer in $\mathrm{D}_{2} \mathrm{O}$ only showed resonances belonging to the mPEG block of the copolymer while the peaks of the p(HPMA-Bz) block completely disappeared due to suppression of molecular motion of the hydrophobic part inside the core of the micelles ${ }^{49-50}$.

Table 2. Characteristics of mPEG $5 \mathrm{~K}-b$ - $\mathrm{p}(\mathrm{HPMA}-\mathrm{Bz})$ micelles prepared from a $20 \mathrm{mg} / \mathrm{mL}$ THF solution and determined by $\mathrm{AF}_{4}$-MALS.

\begin{tabular}{cccccccccc}
\hline Polymer & $\mathbf{R}_{\mathbf{g}}$ & $\mathbf{R}_{\mathbf{h}}$ & $\mathbf{R}_{\text {TEM }}$ & $\mathbf{R}_{\mathbf{g}} / \mathbf{R}_{\mathbf{h}}$ & $\mathbf{M}_{\mathbf{w}(\text { mic })}$ & $\mathbf{N}_{\mathrm{agg}}$ & $\boldsymbol{\sigma}^{-1}=\mathbf{d}^{2}$ & $\mathbf{H}$ & $\mathbf{R}_{\mathbf{h}}-\mathbf{R}_{\mathrm{TEM}}$ \\
\hline mPEG $_{5 \mathrm{~K}}-b-\mathrm{p}(\mathrm{HPMA}-\mathrm{Bz})_{18.5 \mathrm{~K}}$ & 15 & 24 & 14.1 & 0.63 & 21.2 & 905 & 8.2 & 8.9 & 9.9 \\
mPEG $_{5 \mathrm{~K}}-b-\mathrm{p}(\mathrm{HPMA}-\mathrm{Bz})_{9.6 \mathrm{~K}}$ & 12 & 20 & 9.6 & 0.59 & 7.5 & 513 & 9.3 & 8.4 & 10.4 \\
$\mathrm{mPEG}_{5 \mathrm{~K}}-b-\mathrm{p}(\mathrm{HPMA}-\mathrm{Bz})_{4.7 \mathrm{~K}}$ & 12 & 18 & 6.3 & 0.63 & 4.0 & 416 & 10.0 & 8.3 & 11.7 \\
$\mathrm{mPEG}_{5 \mathrm{~K}}-b-\mathrm{p}(\mathrm{HPMA}-\mathrm{Bz})_{2.2 \mathrm{~K}}$ & 10 & 15 & 4.6 & 0.64 & 2.1 & 291 & 10.0 & 8.4 & 10.4 \\
\hline
\end{tabular}

$\mathrm{R}_{\mathrm{g}}=$ radius of gyration $(\mathrm{nm}) ; \mathrm{R}_{\mathrm{h}}=$ hydrodynamic radius $(\mathrm{nm}) ; \mathrm{R}$ TEM = radius as measured by TEM $(\mathrm{nm}) ; \mathrm{M}_{\mathrm{w}(\mathrm{mic})}=$ weight average molecular weight of the micelles $\left(10^{6} \mathrm{Da}\right) ; \mathrm{N}_{\mathrm{agg}}=$ the micelle aggregation number; = mean surface area per molecule calculated by where is the inter-chain distance $\left(\mathrm{nm}^{2}\right) ; \mathrm{H}=$ shell brush height calculated by the de Gennes-Alexander model $(\mathrm{nm})$.

$\mathrm{AF}_{4}$-MALS also revealed that the micelle aggregation number $\left(\mathrm{N}_{\mathrm{agg}}\right)$, calculated by dividing the weight average molecular weight of the micelles $\left(\mathrm{M}_{\mathrm{w}(\mathrm{mic})}\right)$ by the $\mathrm{M}_{\mathrm{n}}$ of the polymer as determined by ${ }^{1} \mathrm{H}-\mathrm{NMR}$ analysis, decreased with decreasing molecular weight of the hydrophobic block of the block copolymer. This was also observed in dissipative particle dynamic simulations of A-B diblock copolymers by Li et al. and Sheng et al. where the $\mathrm{N}_{\mathrm{agg}}$ increases by either increasing the hydrophobic interaction energy through varying the repulsive parameter within the hydrophobic block B or decreasing the molecular weight of the hydrophilic block $A^{51-52}$. In our system, the molecular weight of the hydrophobic block B was varied between 2.2 and $18.5 \mathrm{kDa}$, which corresponds to degrees of polymerization $\mathrm{N}_{\mathrm{B}}$ between 10 and 68, while the molecular weight of the hydrophilic block A was kept at $5 \mathrm{kDa}$ $\left(\mathrm{N}_{\mathrm{A}}=114\right)$. However, even the lowest $\mathrm{M}_{\mathrm{n}}$ of the hydrophobic block $\mathrm{p}(\mathrm{HPMA}-\mathrm{Bz})$ of $2.2 \mathrm{kDa}$ still provided sufficient hydrophobicity for micelle formation by creating a packed core structure. Already indicated by the disappearance of the B block peaks in ${ }^{1} \mathrm{H} N M R$ spectroscopy, this statement can also be proved by a polymer physics consideration. As shown in Figure 4, the TEM radii that reflect the hydrophobic cores of the micelles follow a power law with the product of $N_{B}$ and $N_{\text {agg }}$ of exponent $1 / 3$, characteristic for a collapsed state of the B block chains ${ }^{53}$. On the opposite, the mPEG chains of the corona are highly swollen by water, making them invisible on the TEM images. The surface area per mPEG chain was calculated by dividing the surface area of the micelles $\left(4 \pi \mathrm{R}_{\mathrm{h}}{ }^{2}\right)$ by the number of molecules $\left(\mathrm{N}_{\mathrm{agg}}\right)$ and assimilated with the square of the inter-chain distance (d), neglecting a geometrical pre-factor. This spacing between mPEG molecules remained approximately the same for all samples $(\mathrm{d} \sim 3.0 \pm 0.1 \mathrm{~nm})$ since both $\mathrm{N}_{\mathrm{agg}}$ and the hydrated radius $\left(\mathrm{R}_{\mathrm{h}}\right)$ decreased 
simultaneously when $\mathrm{N}_{\mathrm{B}}$ was decreased. When using de Gennes-Alexander theory of polymer brushes, the mPEG height was estimated by $\mathrm{H}=\mathrm{N}_{\mathrm{A}} \mathrm{a}_{\mathrm{A}}\left(\mathrm{a}_{\mathrm{A}} / \mathrm{d}\right)^{2 / 3}{ }^{54-56}$. The values obtained by this model are shown in Table 2 and were found approximately constant $\mathrm{H} \sim 8.5$ $\pm 0.3 \mathrm{~nm}$, using $\mathrm{N}_{\mathrm{A}}=114$ and a Kuhn length per mPEG segment $\mathrm{a}_{\mathrm{A}}=0.33 \mathrm{~nm}$ obtained from the bond lengths and coarse grain simulations ${ }^{57}$. In agreement with the constant difference of $\sim 10 \mathrm{~nm}$ that was observed between $\mathrm{R}_{\mathrm{h}}$ and $\mathrm{R}_{\mathrm{TEM}}$, one can deduce that the mPEG chains forming the corona of the micelles are densely packed and in a stretched conformation (brush regime). However, there was no curvature effect on the brush height as there was no variation observed with the micelle core size, which corresponds to the "crew cut" regime of micelles rather than the "star-like" regime that would require longer hydrophilic blocks ${ }^{58}$. Of the $\mathrm{mPEG}_{2 \mathrm{~K}}$ copolymers only $\mathrm{mPEG}_{2 \mathrm{~K}}-b$-p(HPMA-Bz $)_{2.6 \mathrm{~K}}$ and $\mathrm{mPEG}_{2 \mathrm{~K}}-b-\mathrm{p}(\mathrm{HPMA}-\mathrm{Bz})_{1.2 \mathrm{~K}}$ formed clear micellar dispersions with a size of the micelles of $25 \mathrm{~nm}$ and polydispersity lower than 0.2. On the other hand, the block copolymers with higher molecular weight of the hydrophobic block (between 5.3 and $20.6 \mathrm{kDa}$; see Table 1) aggregated after THF evaporation. Cryo-TEM analysis of $\mathrm{mPEG}_{2 \mathrm{~K}}-b$ - $\mathrm{p}(\mathrm{HPMA}-\mathrm{Bz})_{20.6 \mathrm{~K}}$ after nanoprecipitation confirmed that mainly aggregates were formed, yet showing an interesting internal structure appearing as densely packed spherical globules (Figure S9). Typically, the spherical micelle shape is stable if the core diameter does not exceed too much the dimensions of the corona, which is estimated at $3.3 \mathrm{~nm}$ using de Gennes-Alexander formula with $N_{\mathrm{A}}=45$ for $\mathrm{mPEG}_{2 \mathrm{~K}}$. This is the case when the right balance of hydrophilic to hydrophobic ratio is used. When increasing the hydrophobic content, this will eventually cause phase separation, as mentioned by Sheng et al. ${ }^{52}$. In other words, the ratio between the hydrophilic and hydrophobic block influences the critical packing parameter, which in turn can predict whether either micelles or aggregates are formed. These scaling laws state that once the effective hydrophilic surface area at the aggregate solution interface is reached, the volume occupied by the hydrophobic chains in the aggregate core becomes too large to be able to form spherical vesicles ${ }^{59-60}$. Cylindrical and lamellar aggregates, but also aggregated precipitated structures will in these cases be observed as is the case for the $\mathrm{mPEG}_{2 \mathrm{~K}}$ block copolymers with higher molecular weight of the hydrophobic block.

\subsection{Critical Micelle Concentration Determination}

The critical micelle concentrations (CMCs) of the polymers of Table 1 were determined using the commonly used pyrene method. Pyrene is a hydrophobic fluorescent molecule that shows a shift of the excitation wavelength (from 300 to $360 \mathrm{~nm}$ ) as a result of its partitioning in the hydrophobic core of polymeric micelles ${ }^{35}$. Figure 5 shows the CMC values for the block copolymers with a fixed hydrophilic mPEG block of $5 \mathrm{kDa}$ and a varying molecular weight of the hydrophobic $\mathrm{p}(\mathrm{HPMA}-\mathrm{Bz})$ block. For the polymer mPEG $_{5 \mathrm{~K}}-b-\mathrm{p}(\mathrm{HPMA}-\mathrm{Bz})_{18.5 \mathrm{~K}}$ (total $\mathrm{M}_{\mathrm{w}}$ of $23.5 \mathrm{kDa}$ ), the $\mathrm{CMC}$ is $2.3 \mu \mathrm{g} / \mathrm{mL}$. On the other hand, the block copolymer with the smallest hydrophobic block $\mathrm{mPEG}_{5 \mathrm{~K}}-b$-p(HPMA-Bz) 1.0K $_{\text {(total }} \mathrm{M}_{\mathrm{w}}$ of $6 \mathrm{kDa}$ ) had a CMC of $16.4 \mu \mathrm{g} / \mathrm{mL}$. This demonstrates that a block copolymer with only $\sim 5$ monomeric units in the hydrophobic block is already able to form micelles pointing to strong $\pi$ - $\pi$ stacking interactions. It was also shown that the CMCs of the polymers decreased with increasing molecular weight of the hydrophobic block. This trend has previously been reported in literature ${ }^{50,61-62}$. The $\mathrm{CMCs}$ of $\mathrm{mPEG}_{2 \mathrm{~K}}-b-\mathrm{p}(\mathrm{HPMA}-\mathrm{Bz})_{2.6 \mathrm{~K}}$ and $\mathrm{mPEG}_{2 \mathrm{~K}}-b-\mathrm{p}(\mathrm{HPMA}-\mathrm{Bz})_{1.2 \mathrm{~K}}$ are 5.1 and $7.4 \mu \mathrm{g} / \mathrm{mL}$ respectively (Figure 5). These CMCs are still lower compared to the mPEG $_{5 \mathrm{~K}}-b-\mathrm{p}(\mathrm{HPMA}-\mathrm{Bz})_{2.2 \mathrm{~K}}$ and mPEG $_{5 \mathrm{~K}}-b-\mathrm{p}(\mathrm{HPMA}-\mathrm{Bz})_{1.0 \mathrm{~K}}$, with a CMC of 10.2 and 16.4 $\mu \mathrm{g} / \mathrm{mL}$ respectively, where the only difference between those polymers is the molecular weight of the hydrophilic mPEG block being 2 or $5 \mathrm{kDa}$. Therefore, as expected and 
previously shown for other systems, it can be concluded that both the size of the hydrophobic block and the hydrophilic/hydrophobic ratio determine the CMC value ${ }^{63-64}$.

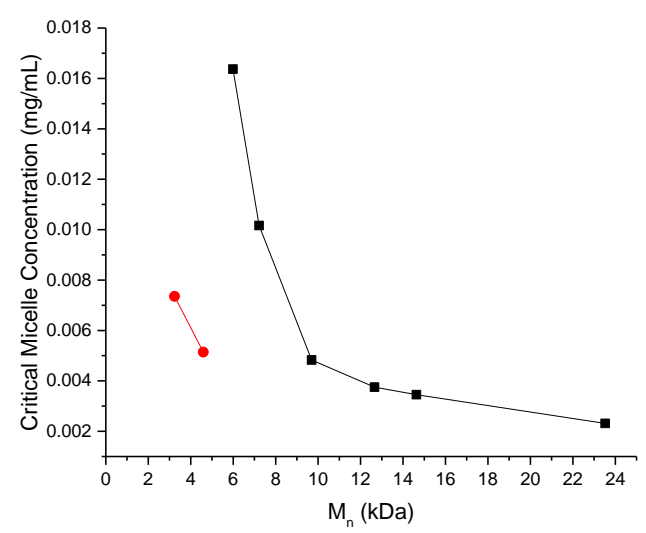

Figure 5. Critical micelle concentration as a function of polymer molecular weight. The black line shows the CMC of the polymers with a fixed $\mathrm{mPEG}$ of $5 \mathrm{kDa}$, whereas the red line shows the CMC of the polymers with a fixed $\mathrm{mPEG}$ of $2 \mathrm{kDa}$.

\subsection{The Effect of Free Homopolymer and Free PEG on the Size of Micelles}

To investigate the effect of homopolymer in the polymer mixture on micelle size, homopolymers $\mathrm{p}(\mathrm{HPMA}-\mathrm{Bz})$ were synthesized with an $\mathrm{M}_{\mathrm{n}}$ of $14.5,11.2$ and $5.5 \mathrm{kDa}$. Subsequently, known amounts of the p(HPMA-Bz $)_{14.5 \mathrm{~K}}$ homopolymer together with mPEG $_{5 \mathrm{~K}}-b$-p(HPMA-Bz $)_{18.5 \mathrm{~K}}$ were dissolved in THF and added to water to obtain micelles with polydispersities lower than 0.2. Figure 6 shows that with increasing amounts of homopolymer in the THF solution, the size of the obtained micelles increased proportionally. Since the p(HPMA-Bz $)_{14.5 \mathrm{~K}}$ homopolymer is very hydrophobic it will very likely partition inside the hydrophobic core of the micelles resulting in an increase in micellar size. Similar results were observed for $\mathrm{mPEG}_{5 \mathrm{~K}}-b$ - $\mathrm{p}(\mathrm{HPMA}-\mathrm{Bz})_{9.6 \mathrm{~K}}$ and $\mathrm{mPEG}_{5 \mathrm{~K}}-b$ - $\mathrm{p}(\mathrm{HPMA}-\mathrm{Bz})_{4.7 \mathrm{~K}}$, upon addition of homopolymers with an $\mathrm{M}_{\mathrm{n}}$ of $11.3 \mathrm{kDa}$ and $5.5 \mathrm{kDa}$ to the feed (Figure S10). Figure S10 shows that the effect of the added homopolymer on the size of the micelles is larger for micelles made of polymers with a smaller hydrophobic domain. A possible explanation is that block copolymers with a smaller hydrophobic block are relatively more soluble in water, as also shown by a higher CMC, making them more susceptible to the conditions at which the mixing and solvent shifting occur. Additionally, the $\mathrm{N}_{\text {agg }}$ of the micelles is lower for the smaller polymers. Therefore, incorporation of homopolymer in the core of the micelles based on lower polymer molecular weight increases the size of micelle more drastically. 


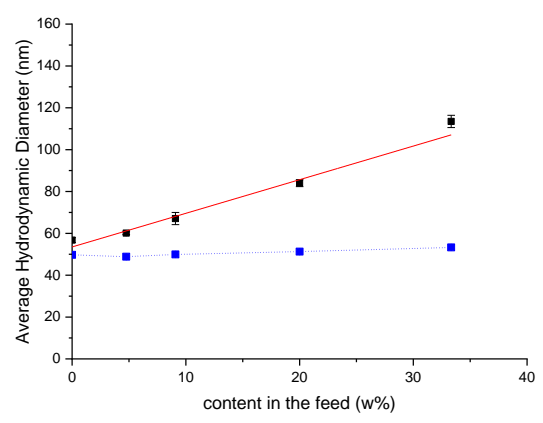

Figure 6. Average hydrodynamic diameter of $\mathrm{mPEG}_{5 \mathrm{~K}}-b-\mathrm{p}(\mathrm{HPMA}-\mathrm{Bz})_{18.5 \mathrm{~K}}$ in black/red as a function of homopolymer content in the feed, and in blue as a function of free $\mathrm{mPEG}_{5 \mathrm{~K}}$ content in the feed $(\mathrm{n}=3)$.

Figure 6 and previous studies of Kataoka et al. ${ }^{23}$ and Kimura et al. ${ }^{65}$ show that the presence of homopolymer in the feed can be exploited to tailor the size of polymer micelles. Kataoka et al. ${ }^{23}$ mixed poly(glutamic acid) homopolymer with PEG- $b$-poly(glutamic acid) to control the size of micelles and Kimura et al. ${ }^{65}$ used poly(L-lactic acid) homopolymer to control the size of (sarcosine)- $b$-(L-lactic acid) nano-particles.

Besides p(HPMA-Bz), the block copolymer mixture might also contain less than $5 \%$ free mPEG (see SI). We therefore also examined the effect of free mPEG on the size of micelles by adding excess amounts of mPEG to the polymer mixture (Figure 6). The presence of up to $40 \%$ of free mPEG did not result in changes in micelle size, which is probably due to the high solubility of $\mathrm{mPEG}_{5 \mathrm{~K}}$ in water.

\subsection{The Effect of Polymer Concentration and Rate of Addition on Micelle Size}

Micelles were prepared by addition of THF with varying concentrations of $\mathrm{mPEG}_{5 \mathrm{~K}}-b$ p(HPMA-Bz) ${ }_{18.5 \mathrm{~K}}$ block copolymers. Figure 7 shows that the hydrodynamic diameter of the formed micelles decreased from approximately 80 to $50 \mathrm{~nm}$ with increasing polymer concentration in THF. Concomitantly, the micelles had a smaller size distribution at higher initial polymer concentrations, as indicated by the decreasing indices from 0.3 to less than 0.1 . Similar results were observed for $\mathrm{mPEG}_{5 \mathrm{~K}}-b$ - $\mathrm{p}(\mathrm{HPMA}-\mathrm{Bz})_{9.6 \mathrm{~K}}$ and $\mathrm{mPEG}_{5 \mathrm{~K}}-b-\mathrm{p}(\mathrm{HPMA}-$ $\mathrm{Bz})_{4.7 \mathrm{~K}}$ from approximately 50 to $42 \mathrm{~nm}$ and from approximately 42 to $36 \mathrm{~nm}$, respectively, upon increasing the polymer concentration in THF (Figure S11). These results suggest that the self-assembly is based on a nucleation-controlled process where the size of micelles is dependent on the nucleation rate. A larger number of nuclei will thereby result in smaller micelles $^{66}$. This trend was also observed in the study of Caron et al. where higher initial concentration of squalenoyl prodrug in the organic phase yielded smaller sized selfassemblies ${ }^{67}$. The concentration dependency on the resulting micelle size was also confirmed in another experiment where the final concentration of the polymer in the water phase after THF evaporation was fixed at 10,20 and $30 \mathrm{mg} / \mathrm{mL}$, but the ratio of organic solvent to water was reduced from 1:1 to 0.3:1 using less THF $(0.3,0.6$ and $1 \mathrm{~mL})$ to dissolve the same amount of polymer (Figure 7). Higher initial polymer concentrations in the organic phase upon mixing with the water phase led to higher supersaturation and consequently to more nuclei and smaller micelles. Also, at higher THF content the supersaturation state is lower, decreasing nucleus formation. Therefore, as expected, smaller micelles were obtained at $0.3: 1$ compared to $1: 1$ volume ratio ${ }^{68}$. Furthermore, the size of the micelles reached a minimum at 
approximately 50-55 nm suggesting a critical particle size was obtained and increasing the concentration did not affect the size anymore ${ }^{69}$.
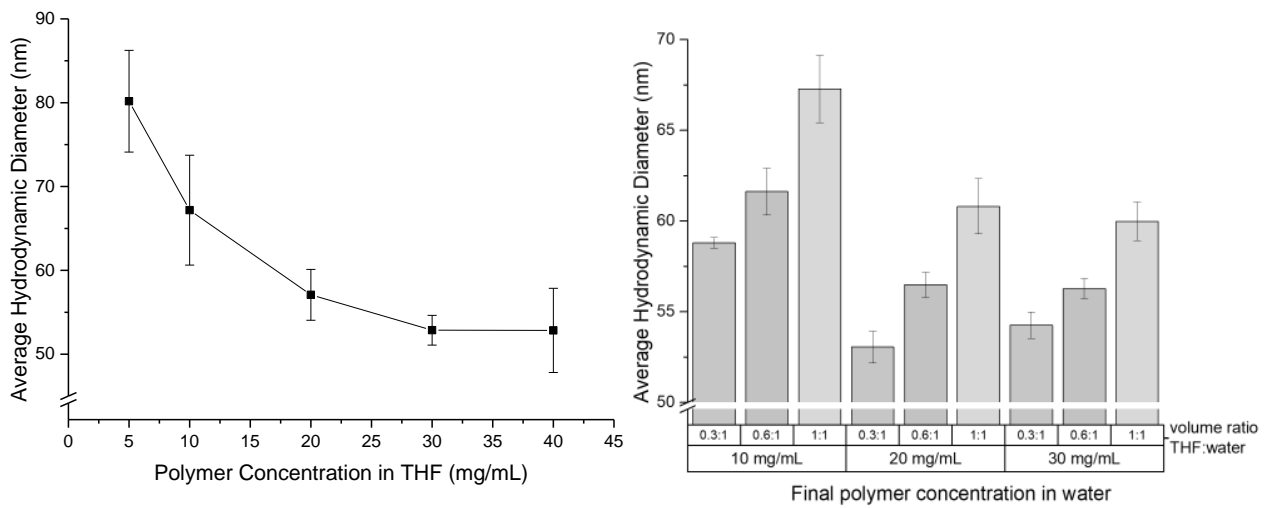

Figure 7. (Left) Hydrodynamic diameters of micelles as a function of the polymer concentration in THF added to water in a 1:1 ratio; (Right) The effect of changing the concentration of polymer in THF using different THF to water volume ratios. The final polymer concentrations were 10,20 and 30 $\mathrm{mg} / \mathrm{mL}(\mathrm{n}=3)$.

As the nucleation rate is dependent on supersaturation and is also affected by the quality of mixing, different rates of addition of organic polymer solution to aqueous phase were used to manipulate the supersaturation state. Thus, micelles were prepared at different addition rates of the polymer solution to water and by fast addition of water to the organic phase (Figure 8). The hydrodynamic diameters of $\mathrm{mPEG}_{5 \mathrm{~K}}-b-\mathrm{p}(\mathrm{HPMA}-\mathrm{Bz})_{18.5 \mathrm{~K}}$ micelles after THF evaporation were 82 and $62 \mathrm{~nm}$ with polydispersities of less than 0.1 and 0.2 at addition rates of 0.15 and $1.5 \mathrm{~mL} / \mathrm{min}$, respectively. Also, the sizes of the micelles upon rapid addition of the polymer solution to the water phase, or the water phase to the polymer solution were 58 and $56 \mathrm{~nm}$, respectively, with polydispersities of less than 0.1 , reaching the minimal micelle size. This is similar to the finding reported by Aliabadi et al. in which no significant difference in size of MePEO- $b$-PCL micelles prepared by addition of water to acetone, or acetone to water was observed ${ }^{70}$. As expected, similar results were observed using $\mathrm{mPEG}_{5 \mathrm{~K}^{-}}$ $b$-p(HPMA-Bz) ${ }_{9.6 \mathrm{~K}}$ and $\mathrm{mPEG}_{5 \mathrm{~K}}-b$ - $\mathrm{p}(\mathrm{HPMA}-\mathrm{Bz})_{4.7 \mathrm{~K}}$ polymers (Figure S12). However, the polydispersities of the micellar dispersions were higher $(0.35$ and 0.45$)$ at $0.15 \mathrm{ml} / \mathrm{min}$ rate of addition (Figure S12). Generally, during nanoprecipitation, both nucleation and particle growth occur in the water/THF mixture even before complete mixing. So, when the polymer solution is added slowly to water, there is a continuous change in the composition of the mixture which results into less homogeneous supersaturation. Fast addition of THF to water, on the other hand, results in a fast mixing which in turn is associated with rapid supersaturation causing the formation of smaller nuclei and thus smaller and more monodisperse micelles ${ }^{71}$. 


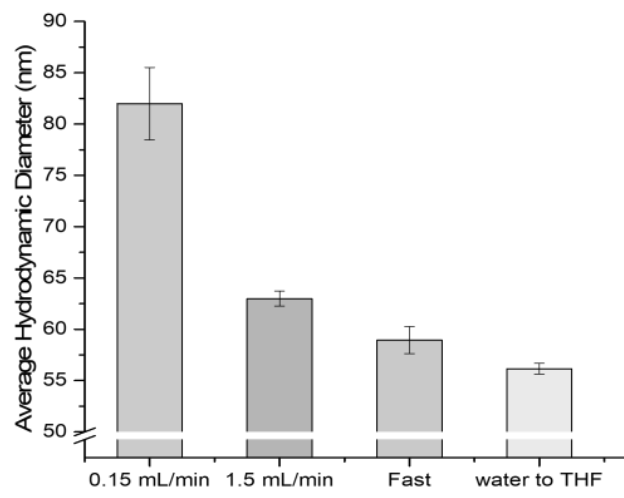

Figure 8. The effect of addition rate of the polymer solution in THF to the aqueous phase on micelle size; the samples were stirred during and until 1 minute after addition of the polymer solution to aqueous buffer $(n=3)$.

\subsection{The Effect of Different Solvents and Buffers on Micelle Size}

The effect of the type of organic solvent on the size of HPMA-Bz micelles was also investigated. THF, acetonitrile, acetone, ethanol, 1,4-dioxane, DMSO and DMF were used because of their miscibility with water and ability to dissolve the mPEG- $b$-p(HPMA-Bz) block copolymers. The polymer concentrations in organic solvent were fixed at $20 \mathrm{mg} / \mathrm{mL}$ to avoid the effect of polymer concentration on the nanoprecipitation process. In the case of ethanol, the polymer was only soluble at temperatures above $\sim 60^{\circ} \mathrm{C}$ and thus the micelle preparation was conducted using polymer solutions and water at $70^{\circ} \mathrm{C}$. Subsequently the solvents were removed by either evaporation (for THF, acetonitrile and acetone) or, in case of the less volatile solvents dioxane, DMSO, DMF and ethanol, by dialysis. As depicted in Figure 9, the use of DMSO and DMF resulted into large micelles, 175 and $75 \mathrm{~nm}$, respectively, compared to the micelles formed using THF, which were $50 \mathrm{~nm}$. Acetone, acetonitrile and dioxane resulted in smaller micelles of approximately $45 \mathrm{~nm}$ with polydispersities lower than 0.2. Generally, solvents with a lower viscosity such as THF, acetone and acetonitrile mix faster with water which causes more uniform supersaturation, leading to smaller micelles ${ }^{71}$. On the other hand, solvents with relatively higher viscosity and surface tension (DMF and DMSO) have slower mixing rates with water, resulting in the growth of micelles and larger self-assemblies. In line with our observations, Kissel et al. reported that nanoparticles prepared using acetone were smaller than particles prepared in THF (140 and $180 \mathrm{~nm}$ respectively) due to its lower viscosity and higher diffusion rate in water $^{72}$. 

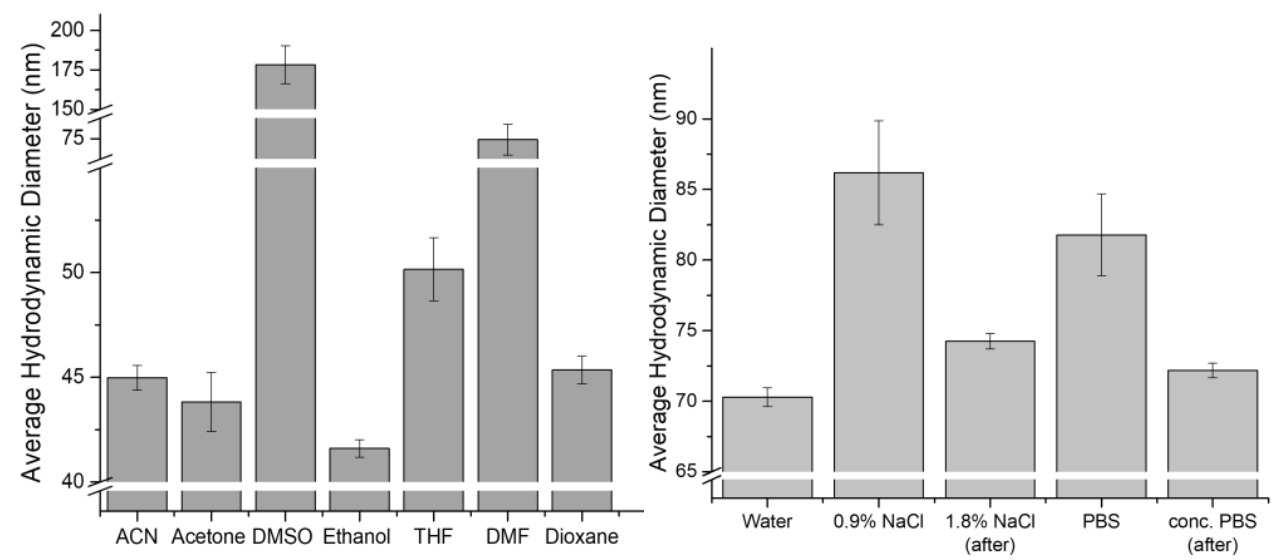

Figure 9. (Left) The effect of different solvents on the hydrodynamic diameter of $\mathrm{mPEG}_{5 \mathrm{~K}}-b-\mathrm{p}(\mathrm{HPMA}-$

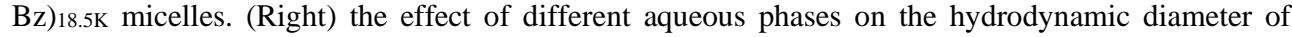
$\mathrm{mPEG}_{5 \mathrm{~K}}-b$-p(HPMA-Bz) ${ }_{18.5 \mathrm{~K}}$ micelles; in the graph, bars depicted with (after), were samples prepared in water and later concentrated solutions of salts were added $(n=3)$.

The effect of the composition of the aqueous phase on the size of the obtained micelles was evaluated by addition of a $20 \mathrm{mg} / \mathrm{mL}$ polymer solution in THF to different aqueous phases. The micelles were slightly larger when they were made in either PBS or $0.9 \% \mathrm{NaCl}$ solution ( 85 and $80 \mathrm{~nm}$ respectively). Addition of salt to water increases the viscosity of the aqueous phase which in turn affects the mixing of solvent and non-solvent and thus nanoprecipitation of micelles in line with previous studies ${ }^{73}$. However, once the micelles were formed in water and the aqueous phase was subsequently adjusted by adding concentrated $1.8 \% \mathrm{NaCl}$ solution or twice concentrated PBS, the size of micelles did not change (Figure 9) showing that the micellar structures are thermodynamically stable after formation.

\section{Conclusion}

The results of this study demonstrate that the self-assembly of mPEG- $b$-p(HPMA-Bz) polymers into micelles can be easily tailored in size. This size-control relies on both the molecular weight of the polymers and the processing methods which change the saturation conditions. In short it can be said that reducing the micelle size can be accomplished by controlling the polymerization step and optimizing the polymer molecular weight by using higher hydrophilic to hydrophobic ratios. The size dependency coming from those ratios, fits excellently into the de Gennes-Alexander theory and scaling law. Reducing the homopolymer content as a potential byproduct of block copolymer synthesis will also optimize the production of smaller micelles. Moreover, in terms of processing conditions, the use of organic solvents with faster mixing quality with water and applying higher rates of addition yield smaller and more homogenous micelles. This systematic study is of great importance as it indicates which parameters during the micelle formation process are critical to allow reproducible formation of micelles with a desired size. We are currently exploring these findings to develop a scalable route toward well-defined micelles for nanomedicine applications. 


\section{Acknowledgement}

The authors are grateful for financial support received from the European Union's Horizon 2020 research and innovation program Marie Sklodowska-Curie Innovative Training Networks (ITN) under grant No. 676137 and HighTech Systems \& Materials (HTSM) grant number 13312 from NWO. 


\section{References}

1. Duncan, R.; Vicent, M. J. Polymer Therapeutics-Prospects for 21st Century: The End of the Beginning. Adv. Drug Delivery Rev. 2013, 65 (1), 60-70.

2. Hare, J. I.; Lammers, T.; Ashford, M. B.; Puri, S.; Storm, G.; Barry, S. T. Challenges and Strategies in Anti-Cancer Nanomedicine Development: An Industry Perspective. Adv. Drug Delivery Rev. 2017, $108,25-38$.

3. Kunjachan, S.; Ehling, J.; Storm, G.; Kiessling, F.; Lammers, T. Noninvasive Imaging of Nanomedicines and Nanotheranostics: Principles, Progress, and Prospects. Chem. Rev. 2015, 115 (19), 10907-10937.

4. Björnmalm, M.; Thurecht, K. J.; Michael, M.; Scott, A. M.; Caruso, F. Bridging Bio-Nano Science and Cancer Nanomedicine. ACS Nano 2017, 11 (10), 9594-9613.

5. van Elk, M.; Murphy, B. P.; Eufrásio-da-Silva, T.; O’Reilly, D. P.; Vermonden, T.; Hennink, W. E.; Duffy, G. P.; Ruiz-Hernández, E. Nanomedicines for Advanced Cancer Treatments: Transitioning Towards Responsive Systems. Int. J. Pharm. 2016, 515 (1), 132-164.

6. Deng, C.; Jiang, Y.; Cheng, R.; Meng, F.; Zhong, Z. Biodegradable Polymeric Micelles for Targeted and Controlled Anticancer Drug Delivery: Promises, Progress and Prospects. Nano Today 2012, 7 (5), 467-480.

7. Mikhail, A. S.; Allen, C. Block Copolymer Micelles for Delivery of Cancer Therapy: Transport at the Whole Body, Tissue and Cellular Levels. J. Controlled Release 2009, 138 (3), 214-223.

8. Cabral, H.; Kataoka, K. Progress of Drug-Loaded Polymeric Micelles into Clinical Studies. J. Controlled Release 2014, 190, 465-476.

9. Cagel, M.; Tesan, F. C.; Bernabeu, E.; Salgueiro, M. J.; Zubillaga, M. B.; Moretton, M. A.; Chiappetta, D. A. Polymeric Mixed Micelles as Nanomedicines: Achievements and Perspectives. Eur. J. Pharm. Biopharm. 2017, 113, 211-228.

10. Houdaihed, L.; Evans, J. C.; Allen, C. Overcoming the Road Blocks: Advancement of Block Copolymer Micelles for Cancer Therapy in the Clinic. Mol. Pharmaceutics 2017, 14 (8), 2503-2517.

11. Varela-Moreira, A.; Shi, Y.; Fens, M. H. A. M.; Lammers, T.; Hennink, W. E.; Schiffelers, R. M. Clinical Application of Polymeric Micelles for the Treatment of Cancer. Mater. Chem. Front. 2017, 1 (8), 1485-1501.

12. Cabral, H.; Miyata, K.; Osada, K.; Kataoka, K. Block Copolymer Micelles in Nanomedicine Applications. Chem Rev 2018, 118 (14), 6844-6892.

13. Yuan, X.; Jiang, M.; Zhao, H.; Wang, M.; Zhao, Y.; Wu, C. Noncovalently Connected Polymeric Micelles in Aqueous Medium. Langmuir 2001, 17 (20), 6122-6126.

14. Shi, Y.; Lammers, T.; Storm, G.; Hennink, W. E. Physico-Chemical Strategies to Enhance Stability and Drug Retention of Polymeric Micelles for Tumor-Targeted Drug Delivery. Macromol. Biosci. 2017, 17 (1), 1600160.

15. Kang, N.; Perron, M.-È.; Prud'homme, R. E.; Zhang, Y.; Gaucher, G.; Leroux, J.-C. Stereocomplex Block Copolymer Micelles: Core-Shell Nanostructures with Enhanced Stability. Nano Letters 2005, 5 (2), 315-319.

16. van Nostrum, C. F. Covalently Cross-Linked Amphiphilic Block Copolymer Micelles. Soft Matter 2011, 7 (7), 3246-3259.

17. Fang, J.; Nakamura, H.; Maeda, H. The EPR Effect: Unique Features of Tumor Blood Vessels for Drug Delivery, Factors Involved, and Limitations and Augmentation of the Effect. Adv. Drug Delivery Rev. 2011, 63 (3), 136-151. 
18. Maeda, H.; Wu, J.; Sawa, T.; Matsumura, Y.;Hori, K. Tumor Vascular Permeability and the EPR Effect in Macromolecular Therapeutics: A Review. J. Controlled Release 2000, 65 (1-2), 271-284.

19. Torchilin, V. Tumor Delivery of Macromolecular Drugs Based on the EPR Effect. Adv. Drug Delivery Rev. 2011, 63 (3), 131-135.

20. Rizzo, L. Y.; Theek, B.; Storm, G.; Kiessling, F.; Lammers, T. Recent Progress in Nanomedicine: Therapeutic, Diagnostic and Theranostic Applications. Curr. Opin. Biotechnol. 2013, 24 (6), 11591166.

21. Bharali, D. J.; Mousa, S. A. Emerging Nanomedicines for Early Cancer Detection and Improved Treatment: Current Perspective and Future Promise. Pharmacol. Ther. 2010, 128 (2), 324-335.

22. Wang, J.; Mao, W.; Lock, L. L.; Tang, J.; Sui, M.; Sun, W.; Cui, H.; Xu, D.; Shen, Y. The Role of Micelle Size in Tumor Accumulation, Penetration, and Treatment. ACS Nano 2015, 9 (7), 7195-7206.

23. Cabral, H.; Matsumoto, Y.; Mizuno, K.; Chen, Q.; Murakami, M.; Kimura, M.; Terada, Y.; Kano, M. R.; Miyazono, K.; Uesaka, M.; Nishiyama, N.; Kataoka, K. Accumulation of Sub-100 Nm Polymeric Micelles in Poorly Permeable Tumours Depends on Size. Nat. Nanotechnol. 2011, 6 (12), 815-823.

24. Sun, Q.; Ojha, T.; Kiessling, F.; Lammers, T.; Shi, Y. Enhancing Tumor Penetration of Nanomedicines. Biomacromolecules 2017, 18 (5), 1449-1459.

25. Huang, K.; Ma, H.; Liu, J.; Huo, S.; Kumar, A.; Wei, T.; Zhang, X.; Jin, S.; Gan, Y.; Wang, P. C.; He, S.; Zhang, X.; Liang, X.-J. Size-Dependent Localization and Penetration of Ultrasmall Gold Nanoparticles in Cancer Cells, Multicellular Spheroids, and Tumors in Vivo. ACS Nano 2012, 6 (5), 4483-4493.

26. Dreher, M. R.; Liu, W.; Michelich, C. R.; Dewhirst, M. W.; Yuan, F.; Chilkoti, A. Tumor Vascular Permeability, Accumulation, and Penetration of Macromolecular Drug Carriers. JNCI, J. Natl. Cancer Inst. 2006, 98 (5), 335-344.

27. Priwitaningrum, D. L.; Blondé, J.-B. G.; Sridhar, A.; van Baarlen, J.; Hennink, W. E.; Storm, G.; Le Gac, S.; Prakash, J. Tumor Stroma-Containing 3d Spheroid Arrays: A Tool to Study Nanoparticle Penetration. J. Controlled Release 2016, 244 (Part B), 257-268.

28. Shi, Y.; van der Meel, R.; Theek, B.; Oude Blenke, E.; Pieters, E. H. E.; Fens, M. H. A. M.; Ehling, J.; Schiffelers, R. M.; Storm, G.; van Nostrum, C. F.; Lammers, T.; Hennink, W. E. Complete Regression of Xenograft Tumors Upon Targeted Delivery of Paclitaxel Via П-П Stacking Stabilized Polymeric Micelles. ACS Nano 2015, 9 (4), 3740-3752.

29. Lo, C. L.; Lin, S. J.; Tsai, H. C.; Chan, W. H.; Tsai, C. H.; Cheng, C. H.; Hsiue, G. H. Mixed Micelle Systems Formed from Critical Micelle Concentration and Temperature-Sensitive Diblock Copolymers for Doxorubicin Delivery. Biomaterials 2009, 30 (23-24), 3961-70.

30. Hu, Q.; Rijcken, C. J. F.; van Gaal, E.; Brundel, P.; Kostkova, H.; Etrych, T.; Weber, B.; Barz, M.; Kiessling, F.; Prakash, J.; Storm, G.; Hennink, W. E.; Lammers, T. Tailoring the Physicochemical Properties of Core-Crosslinked Polymeric Micelles for Pharmaceutical Applications. J. Controlled Release 2016, 244 (Part B), 314-325.

31. Talelli, M.; Rijcken, C. J. F.; Oliveira, S.; van der Meel, R.; van Bergen en Henegouwen, P. M. P.; Lammers, T.; van Nostrum, C. F.; Storm, G.; Hennink, W. E. Nanobody - Shell Functionalized Thermosensitive Core-Crosslinked Polymeric Micelles for Active Drug Targeting. J. Controlled Release 2011, 151 (2), 183-192.

32. De Vos, R.; Goethals, E. J. End Group Analysis of Commercial Poly(Ethylene Glycol) Monomethyl Ether's. Polym. Bull. 1986, 15 (6), 547-549.

33. Zimm, B. H. Apparatus and Methods for Measurement and Interpretation of the Angular Variation of Light Scattering; Preliminary Results on Polystyrene Solutions. J. Chem. Phys. 1948, 16 (12), 10991116. 
34. Naksuriya, O.; Shi, Y.; van Nostrum, C. F.; Anuchapreeda, S.; Hennink, W. E.; Okonogi, S. HpmaBased Polymeric Micelles for Curcumin Solubilization and Inhibition of Cancer Cell Growth. Eur. J. Pharm. Biopharm. 2015, 94, 501-512.

35. Zhao, C. L.; Winnik, M. A.; Riess, G.; Croucher, M. D. Fluorescence Probe Techniques Used to Study Micelle Formation in Water-Soluble Block Copolymers. Langmuir 1990, 6 (2), 514-516.

36. Shi, Y.; van Steenbergen, M. J.; Teunissen, E. A.; Novo, L. s.; Gradmann, S.; Baldus, M.; van Nostrum, C. F.; Hennink, W. E. П-П Stacking Increases the Stability and Loading Capacity of Thermosensitive Polymeric Micelles for Chemotherapeutic Drugs. Biomacromolecules 2013, 14 (6), 1826-1837.

37. Neradovic, D.; van Nostrum, C. F.; Hennink, W. E. Thermoresponsive Polymeric Micelles with Controlled Instability Based on Hydrolytically Sensitive N-Isopropylacrylamide Copolymers. Macromolecules 2001, 34 (22), 7589-7591.

38. Hermanson, G. T. Zero-Length Crosslinkers. Bioconjugate Techniques, 3rd ed.; Academic Press: Boston, 2013, 259-273.

39. Postma, A.; Davis, T. P.; Donovan, A. R.; Li, G.; Moad, G.; Mulder, R.; O'Shea, M. S. A Simple Method for Determining Protic End-Groups of Synthetic Polymers by $1 \mathrm{~h}$ Nmr Spectroscopy. Polymer 2006, 47 (6), 1899-1911.

40. Su, W.-F. Radical Chain Polymerization. Principles of Polymer Design and Synthesis, SpringerVerlag Berlin Heidelberg: Berlin, 2013, 137-183.

41. Odian, G. Radical Chain Polymerization. Principles of Polymerization, John Wiley \& Sons, Inc.: New Jersey, 2004, 198-349.

42. anonymous Ich Harmonised Guidelines, Impurities: Guideline for Residual Solvents Q3c(R6) Step 4.

43. Ma, C.; Pan, P.; Shan, G.; Bao, Y.; Fujita, M.; Maeda, M. Core-Shell Structure, Biodegradation, and Drug Release Behavior of Poly(Lactic Acid)/Poly(Ethylene Glycol) Block Copolymer Micelles Tuned by Macromolecular Stereostructure. Langmuir 2015, 31 (4), 1527-1536.

44. Nie, T.; Zhao, Y.; Xie, Z.; Wu, C. Micellar Formation of Poly(Caprolactone-Block-Ethylene OxideBlock- Caprolactone) and Its Enzymatic Biodegradation in Aqueous Dispersion. Macromolecules 2003, 36 (23), 8825-8829.

45. Nguyen, V. T. A.; De Pauw-Gillet, M.-C.; Sandre, O.; Gauthier, M. Biocompatible Polyion Complex Micelles Synthesized from Arborescent Polymers. Langmuir 2016, 32 (50), 13482-13492.

46. Giacomelli, C.; Schmidt, V.; Aissou, K.; Borsali, R. Block Copolymer Systems: From Single Chain to Self-Assembled Nanostructures. Langmuir 2010, 26 (20), 15734-15744.

47. Kale, T. S.; Klaikherd, A.; Popere, B.; Thayumanavan, S. Supramolecular Assemblies of Amphiphilic Homopolymers. Langmuir 2009, 25 (17), 9660-9670.

48. de Graaf, A. J.; Boere, K. W. M.; Kemmink, J.; Fokkink, R. G.; van Nostrum, C. F.; Rijkers, D. T. S.; van der Gucht, J.; Wienk, H.; Baldus, M.; Mastrobattista, E.; Vermonden, T.; Hennink, W. E. Looped Structure of Flowerlike Micelles Revealed by $1 \mathrm{~h}$ Nmr Relaxometry and Light Scattering. Langmuir 2011, 27 (16), 9843-9848.

49. Li, W.; Nakayama, M.; Akimoto, J.; Okano, T. Effect of Block Compositions of Amphiphilic Block Copolymers on the Physicochemical Properties of Polymeric Micelles. Polymer 2011, 52 (17), 37833790.

50. Soga, O.; van Nostrum, C. F.; Ramzi, A.; Visser, T.; Soulimani, F.; Frederik, P. M.; Bomans, P. H. H.; Hennink, W. E. Physicochemical Characterization of Degradable Thermosensitive Polymeric Micelles. Langmuir 2004, 20 (21), 9388-9395. 
51. Li, Z.; Dormidontova, E. E. Kinetics of Diblock Copolymer Micellization by Dissipative Particle Dynamics. Macromolecules 2010, 43 (7), 3521-3531.

52. Sheng, Y.-J.; Wang, T.-Y.; Chen, W. M.;Tsao, H.-K. A-B Diblock Copolymer Micelles: Effects of Soluble-Block Length and Component Compatibility. J Phys Chem B 2007, 111 (37), 10938-10945.

53. Williams, C.; Brochard, F.; Frisch, H. L. Polymer Collapse. Annu. Rev. Phys. Chem. 1981, 32 (1), 433-451.

54. Alexander, S. Adsorption of Chain Molecules with a Polar Head a Scaling Description. J. Phys. 1977, 38 (8), 983-987.

55. de Gennes, P. G. Polymers at an Interface; a Simplified View. Adv. Colloid Interface Sci. 1987, 27 (3), 189-209.

56. de Gennes, P. G. Conformations of Polymers Attached to an Interface. Macromolecules 1980, 13 (5), 1069-1075.

57. Lee, H.; de Vries, A. H.; Marrink, S.-J.; Pastor, R. W. A Coarse-Grained Model for Polyethylene Oxide and Polyethylene Glycol: Conformation and Hydrodynamics. J. Phys. Chem. B 2009, 113 (40), 13186-13194.

58. Daoud, M.;Cotton, J. P. Star Shaped Polymers: A Model for the Conformation and Its Concentration Dependence. J. Phys. 1982, 43 (3), 531-538.

59. Mai, Y.;Eisenberg, A. Self-Assembly of Block Copolymers. Chem. Soc. Rev. 2012, 41 (18), 59695985.

60. Zhulina, E. B.; Adam, M.; LaRue, I.; Sheiko, S. S.; Rubinstein, M. Diblock Copolymer Micelles in a Dilute Solution. Macromolecules 2005, 38 (12), 5330-5351.

61. Astafieva, I.; Zhong, X. F.; Eisenberg, A. Critical Micellization Phenomena in Block Polyelectrolyte Solutions. Macromolecules 1993, 26 (26), 7339-7352.

62. Zhang, Z. G.; Yin, H. Effect of Polyoxypropylene Chain Length on the Critical Micelle Concentration of Propylene Oxide-Ethylene Oxide Block Copolymers. J. Zhejiang Univ., Sci., B 2005, 6 (3), 219-221.

63. Torchilin, V. P. Structure and Design of Polymeric Surfactant-Based Drug Delivery Systems. J. Controlled Release 2001, 73 (2), 137-172.

64. Ashok, B.; Arleth, L.; Hjelm, R. P.; Rubinstein, I.; Onyuksel, H. In Vitro Characterization of Pegylated Phospholipid Micelles for Improved Drug Solubilization: Effects of Peg Chain Length and Pc Incorporation. J. Pharm. Sci 2004, 93 (10), 2476-2487.

65. Makino, A.; Hara, E.; Hara, I.; Ozeki, E.; Kimura, S. Size Control of Core-Shell-Type Polymeric Micelle with a Nanometer Precision. Langmuir 2014, 30 (2), 669-674.

66. Zhang, C.; Pansare, V. J.; Prud'homme, R. K.; Priestley, R. D. Flash Nanoprecipitation of Polystyrene Nanoparticles. Soft Matter 2012, 8 (1), 86-93.

67. Caron, J.; Maksimenko, A.; Wack, S.; Lepeltier, E.; Bourgaux, C.; Morvan, E.; Leblanc, K.; Couvreur, P.; Desmaële, D. Improving the Antitumor Activity of Squalenoyl-Paclitaxel Conjugate Nanoassemblies by Manipulating the Linker between Paclitaxel and Squalene. Adv. Healthcare Mater. 2013, 2 (1), 172-185.

68. Aubry, J.; Ganachaud, F.; Cohen Addad, J. P.; Cabane, B. Nanoprecipitation of Polymethylmethacrylate by Solvent Shifting: 1. Boundaries. Langmuir 2009, 25 (4), 1970-9.

69. Johnson, B. K.;Prud'homme, R. K. Mechanism for Rapid Self-Assembly of Block Copolymer Nanoparticles. Phys. Rev. Lett. 2003, 91 (11), 118302.

70. Aliabadi, H. M.; Elhasi, S.; Mahmud, A.; Gulamhusein, R.; Mahdipoor, P.; Lavasanifar, A. Encapsulation of Hydrophobic Drugs in Polymeric Micelles through Co-Solvent Evaporation: The 
Effect of Solvent Composition on Micellar Properties and Drug Loading. Int. J. Pharm. 2007, 329 (1), 158-65.

71. Lepeltier, E.; Bourgaux, C.; Couvreur, P. Nanoprecipitation and the "Ouzo Effect": Application to Drug Delivery Devices. Adv. Drug Delivery Rev. 2014, 71, 86-97.

72. Beck-Broichsitter, M.; Rytting, E.; Lebhardt, T.; Wang, X.; Kissel, T. Preparation of Nanoparticles by Solvent Displacement for Drug Delivery: A Shift in the "Ouzo Region" Upon Drug Loading. Eur. J. Pharm. Sci. 2010, 41 (2), 244-253.

73. Huang, W.; Zhang, C. Tuning the Size of Poly(Lactic-Co-Glycolic Acid) (Plga) Nanoparticles Fabricated by Nanoprecipitation. Biotechnol. J. 2017, 13 (1), 1700203. 


\section{Supporting information}
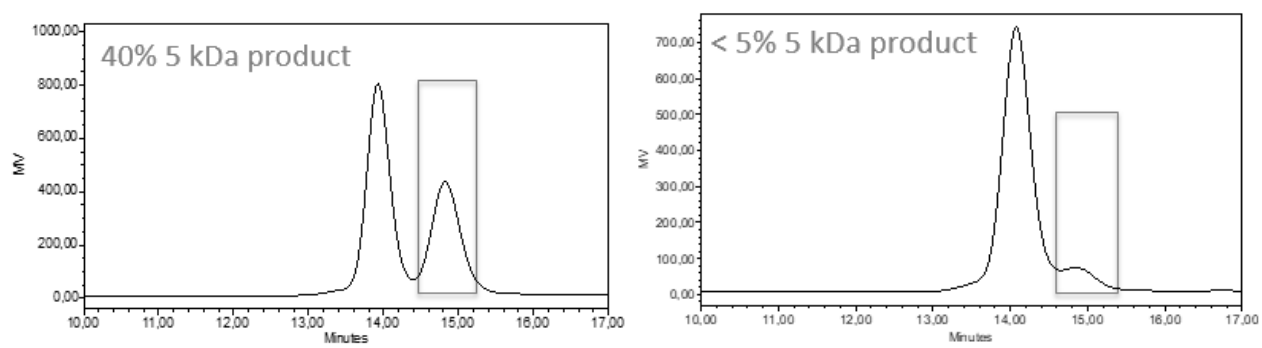

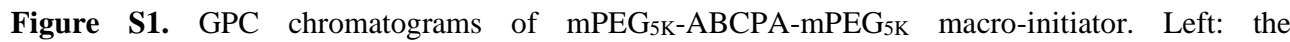
chromatogram of the macroinitiator containing $\sim 40 \%$ of mPEG/mPEG-ABCPA. Right: the chromatogram of the macroinitiator with less than 5\% free mPEG.

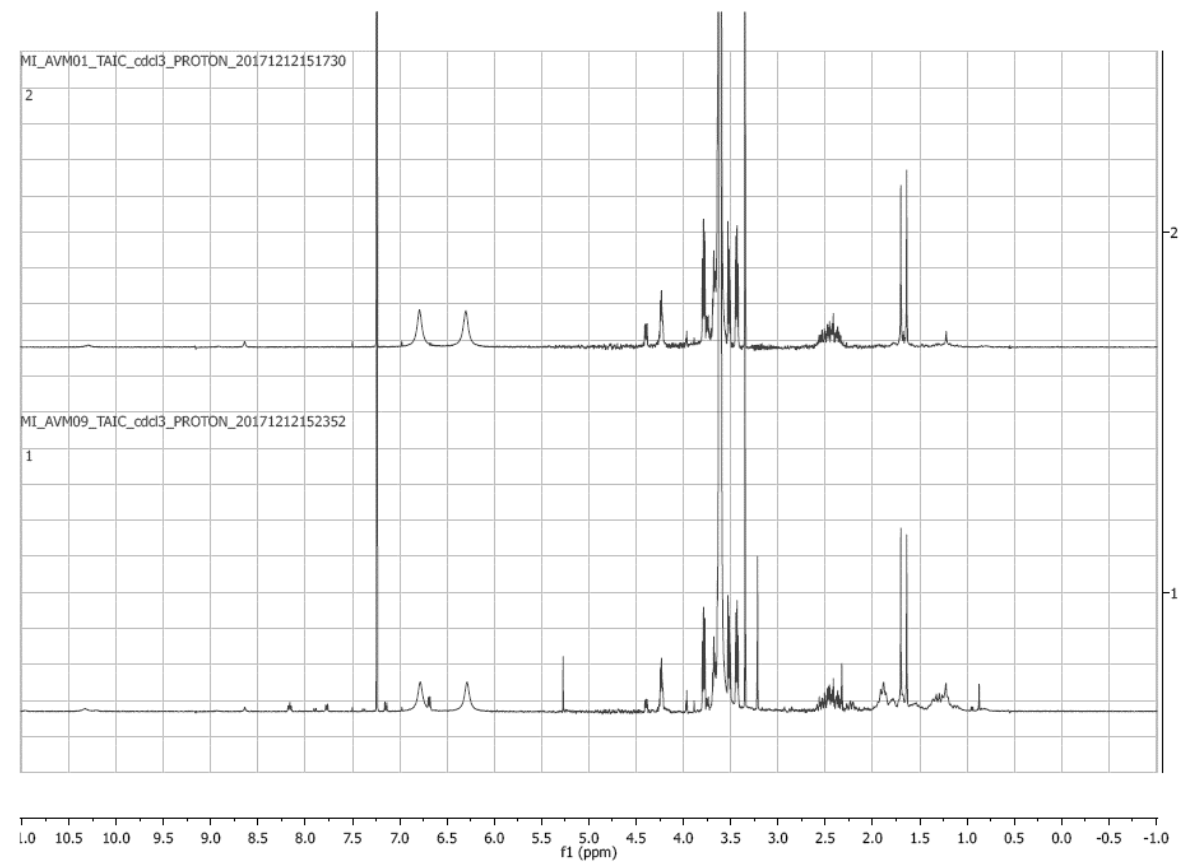

Figure S2. ${ }^{1} \mathrm{H}-\mathrm{NMR}$ results of the $\mathrm{mPEG}_{5 \mathrm{~K}-\mathrm{ABCPA}-\mathrm{mPEG}}$ K macro-initiators using TAIC. Top the spectrum of the macroinitiator containing $~ 40 \%$ of free mPEG/ mPEG-ABCPA (Figure S1). The product contains $30 \%$ mPEG according to ${ }^{1} \mathrm{H}-\mathrm{NMR}$ with TAIC reagent. Bottom: the spectrum of the macroinitiator containing less than $5 \%$ of free $\mathrm{mPEG} / \mathrm{mPEG}-\mathrm{ABCPA}$ (Figure $\mathrm{S} 1$ ). The product contains $4.2 \% \mathrm{mPEG}$ according to ${ }^{1} \mathrm{H}-\mathrm{NMR}$ with TAIC reagent. 


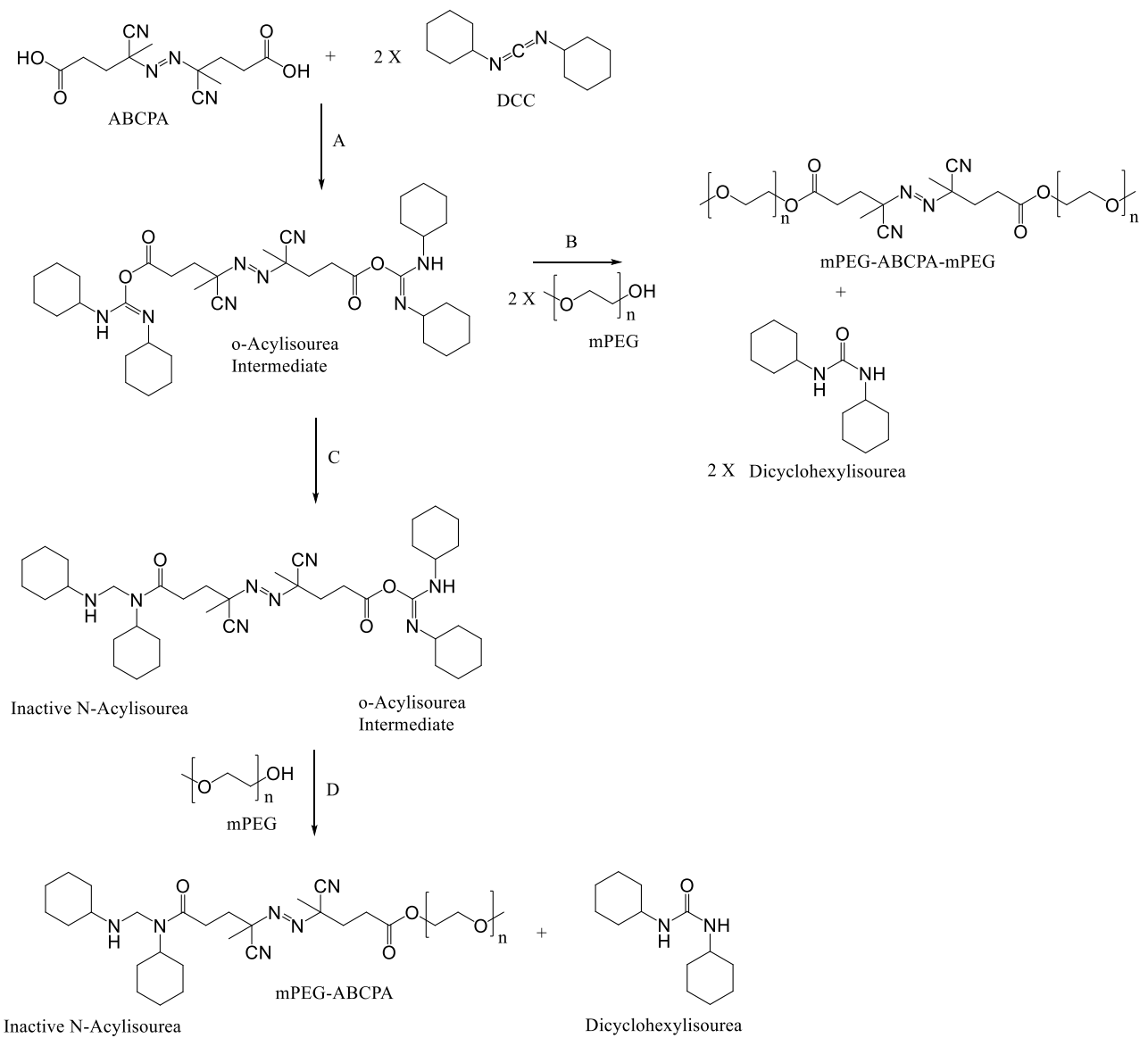

Scheme S1. Reaction A shows the activation of the ABCPA initiator. Reaction B shows the subsequent coupling of $\mathrm{mPEG}$ with the activated ABCPA resulting in the formation of mPEG-ABCPA-mPEG and dicyclohexylisourea. Note that activation of ABCPA and coupling of mPEG to ABCPA can occur at both carboxylic acids subsequently but also at the same time. Reaction $\mathrm{C}$ shows the rearrangement of the activated ABCPA resulting in inactive $\mathrm{N}$-acylisourea. Reaction $\mathrm{D}$ shows the subsequent coupling of mPEG with the partly inactivated ABCPA resulting in the formation of mPEG-ABCPA and dicyclohexylisourea. 

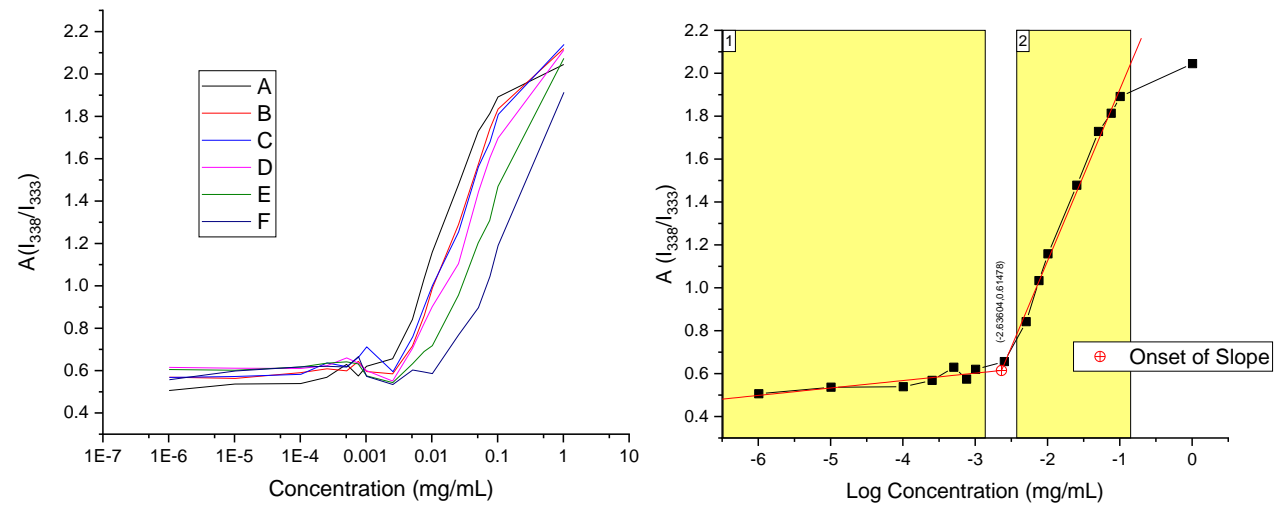

Figure S3. On the left, CMC results depicting the fluorescence of the pyrene against the concentration of the used polymer. A: mPEG5K-b-p(HPMA-Bz)18.5K; B: mPEG5K-b-p(HPMA-Bz)9.6K; C: mPEG5K-b-p(HPMA-Bz)7.7K; D: mPEG5K-b-p(HPMA-Bz)4.7K; E: mPEG5K-b-p(HPMA$\mathrm{Bz}) 2.2 \mathrm{~K}$; F: mPEG5K-b-p(HPMA-Bz)1.0K. On the right, an example of how the actual CMC is determined by the onset of slope and using this value for further calculations.
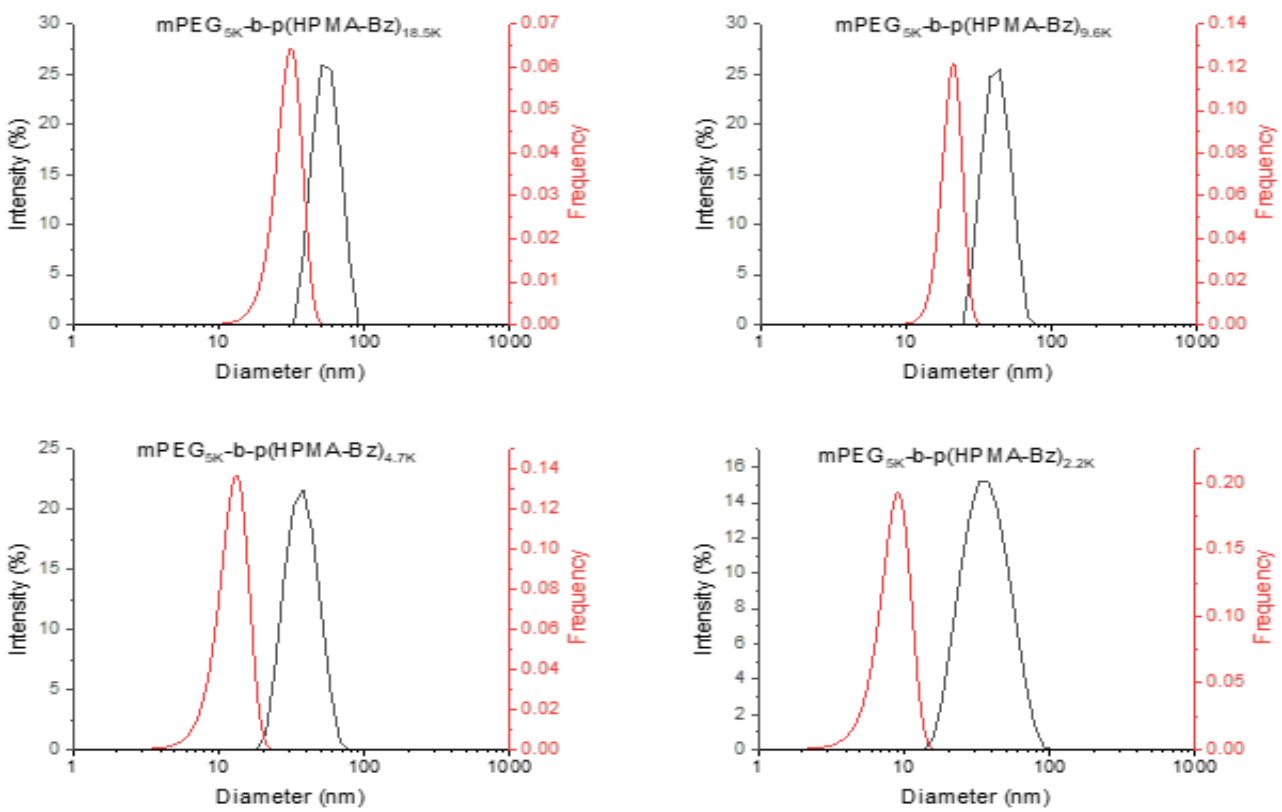

Figure S4. Histograms of diameters measured respectively by DLS (black and depicted by intensity) and by TEM (red and depicted by frequency) for the four studied micelle samples of $\mathrm{mPEG}_{5 \mathrm{~K}}-b$ $\mathrm{p}(\mathrm{HPMA}-\mathrm{Bz})_{18.5 \mathrm{~K}}, \quad \mathrm{mPEG}_{5 \mathrm{~K}}-b-\mathrm{p}(\mathrm{HPMA}-\mathrm{Bz})_{9.6 \mathrm{~K}}, \quad \mathrm{mPEG}_{5 \mathrm{~K}}-b-\mathrm{p}(\mathrm{HPMA}-\mathrm{Bz})_{4.7 \mathrm{~K}}$ and $\mathrm{mPEG}_{5 \mathrm{~K}}-b-$ p(HPMA-Bz) $2.2 \mathrm{~K}$. 


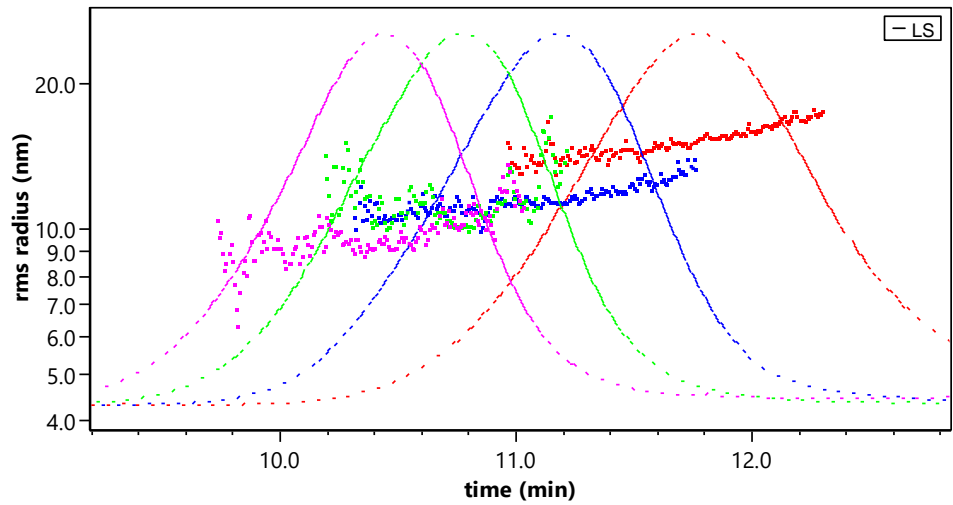

Figure S5. AF4-MALLS results showing the rms radius against time. Pink: $\mathrm{mPEG}_{5 \mathrm{~K}}-b-\mathrm{p}(\mathrm{HPMA}-$ $\mathrm{Bz})_{2.2 \mathrm{~K}}$; green: $\mathrm{mPEG}_{5 \mathrm{~K}}-b-\mathrm{p}(\mathrm{HPMA}-\mathrm{Bz})_{4.7 \mathrm{~K}}$; blue: $\mathrm{mPEG}_{5 \mathrm{~K}}-b-\mathrm{p}(\mathrm{HPMA}-\mathrm{Bz})_{9.6 \mathrm{~K}}$ and red: $\mathrm{mPEG}_{5 \mathrm{~K}}-b-$ p(HPMA-Bz) $18.5 \mathrm{~K}$.

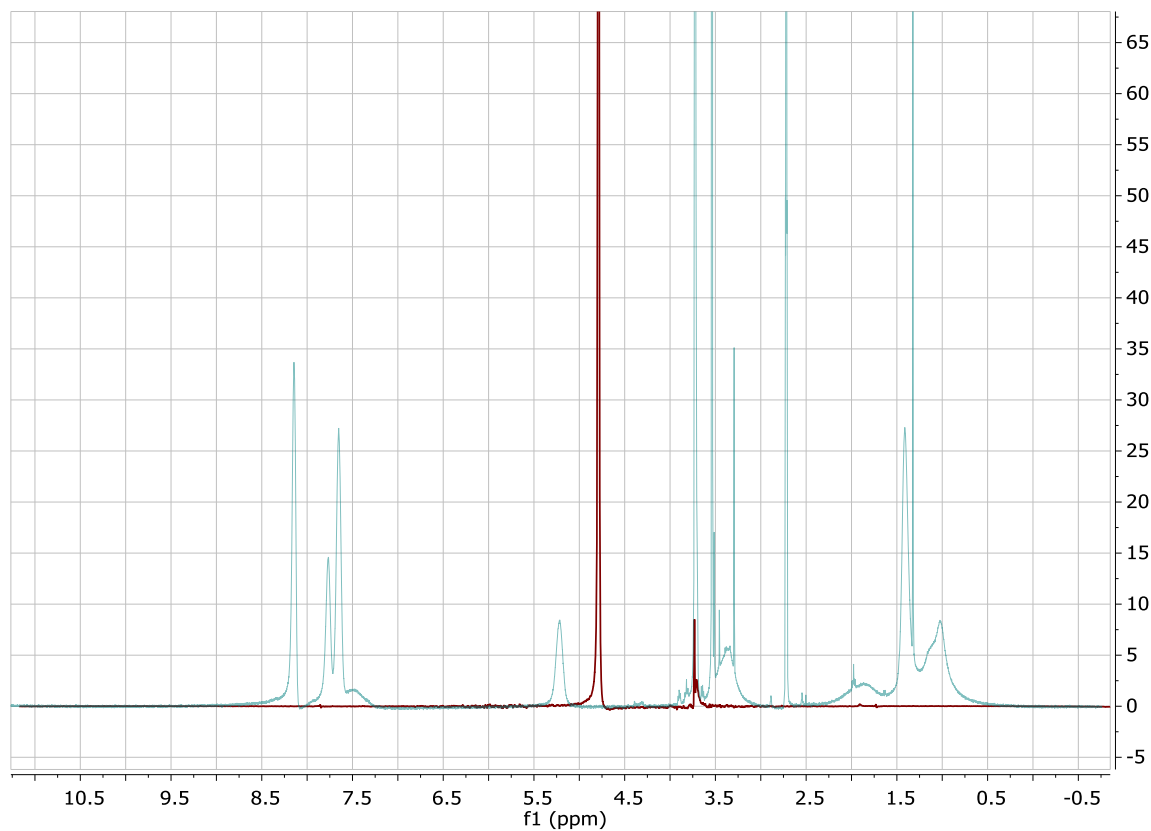

Figure S6. ${ }^{1} \mathrm{H}-\mathrm{NMR}$ spectra of mPEG- $b$-p(HPMA-Bz) copolymer (blue) in DMSO-d 6 and (red) micelle dispersion in $\mathrm{D}_{2} \mathrm{O}$ at $25^{\circ} \mathrm{C}$. 


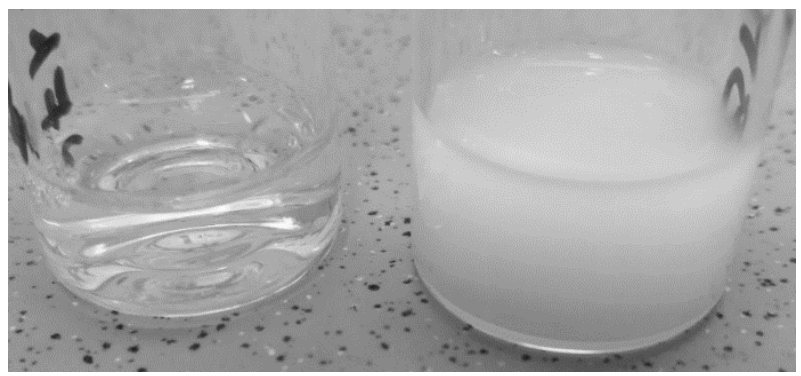

Figure S7. Visual appearance of micelles made of $\mathrm{mPEG}_{5 \mathrm{~K}}$ (left) $\mathrm{mPEG}_{5 \mathrm{~K}}-b-\mathrm{p}(\mathrm{HPMA}-\mathrm{Bz})_{18.5 \mathrm{~K}}$ and (right) $\mathrm{mPEG}_{2 \mathrm{~K}}-b-\mathrm{p}(\mathrm{HPMA}-\mathrm{Bz})_{20.6 \mathrm{~K}}$
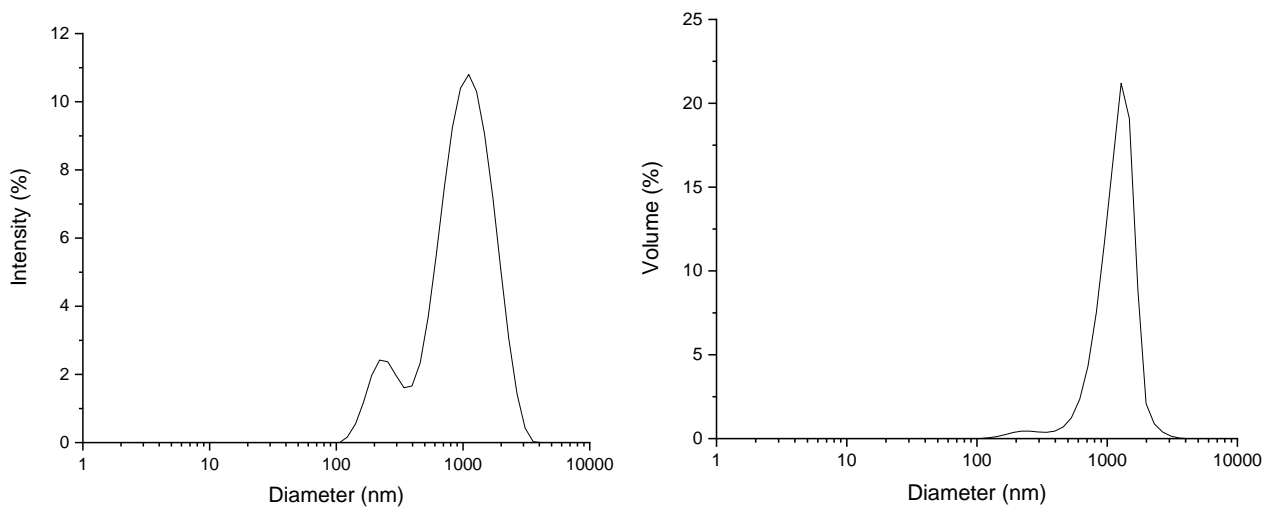

Figure S8. (Left) DLS intensity profile and (right) DLS volume profile of the $\mathrm{mPEG}_{2 \mathrm{~K}}-b$-p $(\mathrm{HPMA}-$ Bz)20.6K aggregates.

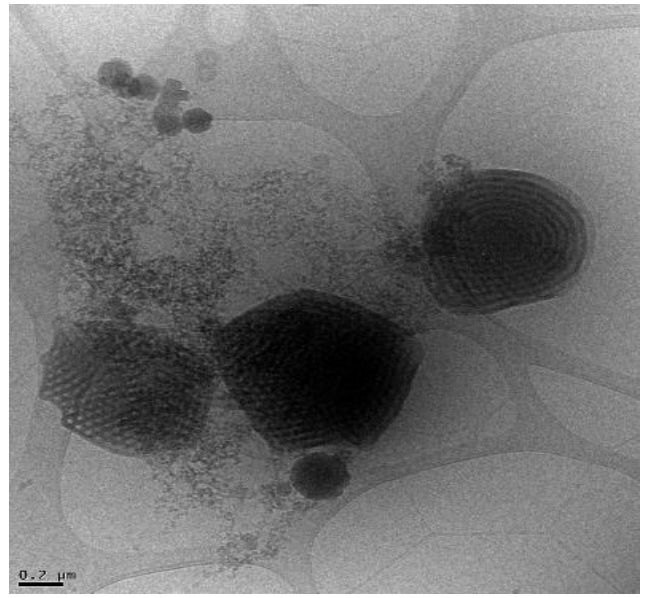

Figure S9. Cryo-TEM image of the $\mathrm{mPEG}_{2 \mathrm{~K}}-b$-p(HPMA-Bz) $20.6 \mathrm{~K}$ aggregates; scale bar corresponds to $0.2 \mu \mathrm{m}$. 


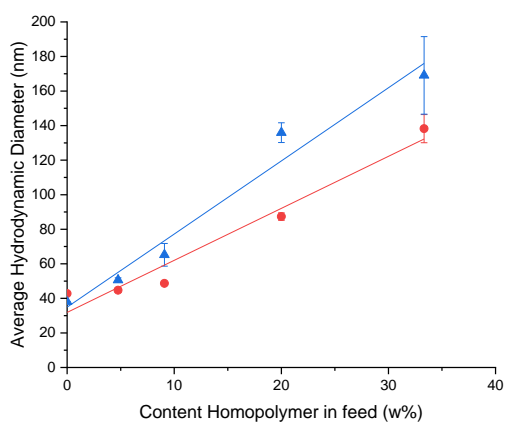

Figure S10. Average hydrodynamic diameter as a function of homopolymer in the feed $(n=3)$. Red: $\mathrm{mPEG}_{5 \mathrm{~K}}-b-\mathrm{p}(\mathrm{HPMA}-\mathrm{Bz})_{9.6 \mathrm{~K}}$ with $\mathrm{p}(\mathrm{HPMA}-\mathrm{Bz})_{11.3}$ and Blue: $\mathrm{mPEG}_{5 \mathrm{~K}}-b-\mathrm{p}(\mathrm{HPMA}-\mathrm{Bz})_{4.7 \mathrm{~K}}$ with $\mathrm{p}(\mathrm{HPMA}-\mathrm{Bz})_{5.5}$.

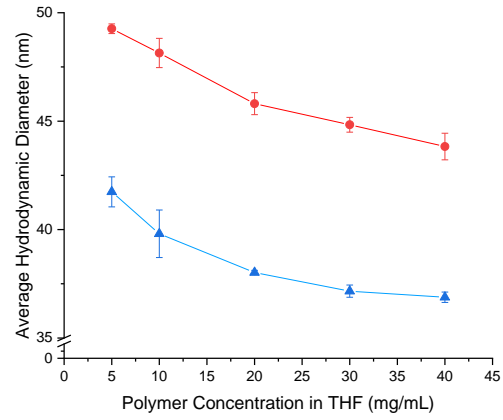

Figure S11. Hydrodynamic diameters of micelles as a function of the polymer concentration in THF added to water in a 1:1 ratio; Red: $\mathrm{mPEG}_{5 \mathrm{~K}}-b$-p(HPMA-Bz) $)_{96}$ and Blue: $\mathrm{mPEG}_{5 \mathrm{~K}}-b-\mathrm{p}(\mathrm{HPMA}-\mathrm{Bz})_{4.7 \mathrm{~K}}$ 

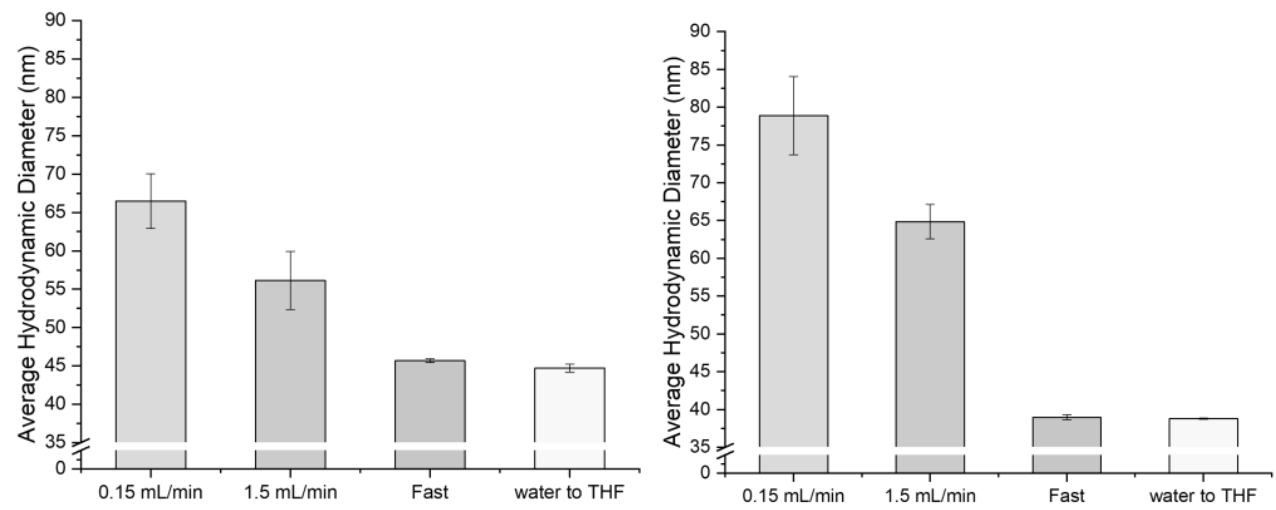

Figure S12. The effect of addition rate of the polymer solution in THF to the aqueous phase on micelle size. Left: $\mathrm{mPEG}_{5 \mathrm{~K}}-b-\mathrm{p}(\mathrm{HPMA}-\mathrm{Bz})_{9.6 \mathrm{~K}}$ and Right: $\mathrm{mPEG}_{5 \mathrm{~K}}-b-\mathrm{p}(\mathrm{HPMA}-\mathrm{Bz})_{4.7 \mathrm{~K}}$; the samples were stirred until 1 minute after addition of the polymer solution to aqueous buffer $(n=3)$.

Table S1: Viscosity of solvents, water and organic solvent mixture (1:1) and surface tension of solvents used in micelle preparation at $25^{\circ} \mathrm{C}$.

\begin{tabular}{cccc}
\hline Solvent & $\begin{array}{c}\text { Viscosity } \\
\text { Water:solvent } \\
(\mathbf{m P a} . \mathbf{1})^{1}\end{array}$ & $\begin{array}{c}\text { Viscosity of pure } \\
\text { solvents }\end{array}$ & $\begin{array}{c}\text { Surface } \\
\text { tension } \\
(\mathbf{m N} / \mathbf{m}) \mathbf{2}\end{array}$ \\
\hline ACN & 0.842 & 0.361 & 28.7 \\
Acetone & 0.806 & 0.3311 & 23.0 \\
THF & 1.651 & 0.481 & 26.7 \\
dioxane & 1.857 & 1.172 & 32.9 \\
DMF & 2.295 & 0.796 & 34.4 \\
DMSO & 2.858 & 1.948 & 42.9 \\
\hline
\end{tabular}




\section{References}

1. Aminabhavi, T. M.; Gopalakrishna, B. Density, Viscosity, Refractive Index, and Speed of Sound in Aqueous Mixtures of N,N-Dimethylformamide, Dimethyl Sulfoxide, N,N-Dimethylacetamide, Acetonitrile, Ethylene Glycol, Diethylene Glycol, 1,4-Dioxane, Tetrahydrofuran, 2-Methoxyethanol, and 2-Ethoxyethanol at 298.15 K. J. Chem. Eng. Data 1995, 40 (4), 856-861.

2. Yaws, C. L.; Richmond, P. C. Surface Tension-Inorganic Compounds. Thermophysical Properties of Chemicals and Hydrocarbons, William Andrew Publishing: Norwich, NY, 2009, pp 782-789. 



\section{Chapter 4}

\section{Tuning Size and Morphology of mPEG- $b$ - p(HPMA-Bz) Copolymer Self-Assemblies Using Microfluidics}

Jaleesa Bresseleers ${ }^{1,2}$, Mahsa Bagheri ${ }^{3}$, Coralie Lebleu ${ }^{4}$, Sébastien Lecommandoux ${ }^{4}$, Olivier Sandre ${ }^{4}$, Imke A. B. Pijpers ${ }^{1}$, Alexander F. Mason ${ }^{1}$, Silvie Meeuwissen ${ }^{2}$, Cornelus F. van Nostrum ${ }^{3}$, Wim E. Hennink ${ }^{3}$ and Jan C.M. van Hest ${ }^{1}$

${ }^{1}$ Department of Bio-Organic chemistry, Eindhoven University of Technology, 5600 MB Eindhoven, the Netherlands

${ }^{2}$ Ardena Oss, 5349 AB Oss, the Netherlands

${ }^{3}$ Department of Pharmaceutics, Utrecht Institute for Pharmaceutical Sciences (UIPS), Faculty of Science, Utrecht University, 3508 TB Utrecht, the Netherlands

${ }^{4}$ Laboratoire de Chimie de Polymères Organiques, Université de Bordeaux, UMR 5629 CNRS, Bordeaux-INP, 33600 Pessac, France

Polymers 2020, 12(11), 2572 


\begin{abstract}
The careful design of nanoparticles, in terms of size and morphology, is of great importance to developing effective drug delivery systems. The ability to precisely tailor nanoparticles in size and morphology during polymer self-assembly was therefore investigated. Four poly(ethylene glycol)- $b$-poly( $N$-2-benzoyloxypropyl methacrylamide) mPEG- $b$-p(HPMA$\mathrm{Bz}$ ) block copolymers with a fixed hydrophilic block of $\mathrm{mPEG} 5 \mathrm{kDa}$ and a varying molecular weight of the hydrophobic p(HPMA-Bz) block (A: 17.1, B: 10.0, C: 5.2 and D: $2.7 \mathrm{kDa}$ ) were self-assembled into nanoparticles by nanoprecipitation under well-defined flow conditions, using microfluidics, at different concentrations. The nanoparticles from polymer A, increased in size from 55 to $90 \mathrm{~nm}$ using lower polymer concentrations and slower flow rates and even polymer vesicles were formed along with micelles. Similarly, nanoparticles from polymer D increased in size from 35 to $70 \mathrm{~nm}$ at slower flow rates and also formed vesicles along with micelles, regardless of the used concentration. Differently, polymers B and $\mathrm{C}$ mainly self-assembled into micelles at the different applied flow rates with negligible size difference. In conclusion, this study demonstrates that the self-assembly of mPEG- $b$ p(HPMA-Bz) block copolymers can be easily tailored in size and morphology using microfluidics and is therefore an attractive option for further scaled-up production activities.
\end{abstract}

Keywords: block copolymers; nanoparticles; micelles; polymersomes; HPMA; size control; nanoprecipitation; microfluidics; micromixer 


\section{Introduction}

During the past few decades, polymeric-based drug delivery nanoparticles, in particular polymeric micelles, have received growing interest for tumor targeting and other therapeutic purpose ${ }^{1-3}$. In general, polymeric micelles are core-shell structures composed of amphiphilic block copolymers. The shell consists of a brush of the hydrophilic block chains, usually poly(ethylene glycol) (PEG), which provides stealth-like properties against non-specific protein adsorption and offers good colloidal stability in physiological conditions. The hydrophobic core, in turn, can be used to accommodate poorly water-soluble drugs ${ }^{4-6}$. A careful design of the topological features of the polymeric micelles is of importance to achieve efficacy of treatment e.g., regarding pharmacokinetics and tumor penetration ${ }^{7-9}$.

The morphological characteristics of nanoparticles such as size and shape have a determinant effect on their in vivo and in vitro performance ${ }^{10}$. In general, the morphology of nanoparticles can impact drug loading and release, pharmacokinetics and biodistribution, cell uptake and biosafety features ${ }^{11,12}$. It was shown in preclinical studies that nanoparticles with sizes $<100$ $\mathrm{nm}$ could extravasate better to target tumors and those with sizes $<50 \mathrm{~nm}$ could even penetrate the tumor deeper, exerting better tumor efficacy ${ }^{7,9,13}$. In terms of shape, many biological processes such as pharmacokinetics and cell uptake could be influenced ${ }^{10,14}$. For instance, it was shown that cellular uptake was more inhibited by nanoparticles with higher aspect ratios compared to spherical particles. So far, polymersomes and micelles are the most frequently studied and advanced polymer-based nanomedicines for cancer therapy. However, achieving a full control over the self-assembly of block copolymer chains into particles still remains a challenge ${ }^{11}$.

Various methods are available to prepare polymeric micelles such as emulsion-based and solvent displacement procedures. The latter, first introduced in the late nineties by Devissaguet and Fessi ${ }^{15}$, is also referred to as nanoprecipitation, which renders tailorable characteristics such as size and size distribution ${ }^{16}$. The nanoprecipitation method is a simple, fast and straightforward technique to produce polymer-based nanoparticles. In short, an amphiphilic block copolymer (possibly in combination with a drug) is dissolved in a watermiscible organic solvent. The obtained solution is then added to an aqueous phase, which acts as a non-solvent for the hydrophobic block and leads to the formation of (drug-loaded) nanoparticles. In the final step, the organic solvent is removed by evaporation or dialysis ${ }^{17-}$ 20 .

The conventional nanoprecipitation method is performed in batch mode i.e., in traditional glassware, which is simple and efficient. Nevertheless, it has its limitations regarding uniformity and reproducibility of mixing. For instance, temperature or concentration inhomogeneity during mixing can have a substantial effect on the final size and structure of the particles ${ }^{16}$. Such issues might be particularly relevant with a block copolymer such as poly(ethylene glycol)- $b$-poly( $N$-2-benzoyloxypropyl methacrylamide) (mPEG- $b$-p(HPMA$\mathrm{Bz})$ ), since the benzyl groups have shown to provide strong п-п stacking interactions and its self-assembly most likely leads to kinetically trapped nanoparticles rather than a dynamic micelle state ${ }^{21}$. Even for a block copolymer without aromatic groups such as poly(ethylene glycol)-block-poly(butyl acrylate) (mPEG- $b$-PBMA), previously reported simulations demonstrated that its self-assembly is controlled by kinetics and the applied process conditions rather than thermodynamics ${ }^{22}$. In this case, with a moderately hydrophobic block, 
the introduction of charges in the hydrophilic block can drive the self-assembly towards dynamic micelles ${ }^{23}$.

Microfluidics is a technology that handles minute volumes of solutions in microscale fluidic devices in a precise and controlled way ${ }^{24,25}$, usually in a laminar flow regime. As a comparison, flash nanoprecipitation in high pressure reactors rather uses turbulent flows that enable them to reach the shortest mixing times of the solvent and non-solvent ${ }^{16,19}$, which is a way to separate nucleation from particle growth ${ }^{26}$, and ultimately better controlling the size distribution of the self-assemblies. It is usually hypothesized that small dimensions of channels lead to a much higher surface to volume ratio of the solutions to be mixed than what is achieved in macroscopic vessels, which in turn reduces the diffusional times. Thus, controlled and tunable mixing is expected to give access to a kinetically controlled nanoprecipitation process, facilitating control over size and size distribution of the formed self-assemblies ${ }^{27,28}$. Therefore, microfluidics has been evaluated in the literature to see if it can be considered as a reliable and up-scalable technology to control the self-assembly of polymeric nanoparticles ${ }^{29-31}$. A self-assembly process is highly dependent upon both external (e.g., temperature) and internal parameters such as interfacial and viscous forces. Parameters such as fluid viscosity and mass density are homogeneous at the scale of microchannel dimensions, therefore one expects that the self-assembly process can be efficiently controlled by flow rates of, respectively, the solvent and anti-solvent ${ }^{29}$. Previous works reported only moderate reduction of size dispersities, but showed at least real optimization in terms of drug encapsulation rates $^{32}$. For instance, Xu et al. described a lab-made coaxial flow chip enabling encapsulation of hydrophobic drugs with high efficiency in poly(lactic-co-glycolic acid) (PLGA) nanoparticles ${ }^{33}$. Furthermore, it is important to remark that microfluidics has a great potential in scaling up production of polymer-based nanomedicines thanks to its continuous flow operational process, which is a major advantage for the production of formulations when moving to clinical (trial) applications ${ }^{34-36}$.

Although there is still some variability in outcome, most of the previously published studies showed that, when using microfluidics, fine-tuning of the flow rates and the ratio of organic solvent to the aqueous buffer enables control over both final particle size and polydispersity index $(\mathrm{PDI})^{36-41}$. As an example, for the preparation of chitosan nanoparticles using microfluidics, varying the flow rates of the polymeric to alkaline water solutions resulted in the formation of smaller nanoparticles of 63 and $102 \mathrm{~nm}$ at, respectively, the shortest and longest applied mixing time in the microfluidic device, as compared to $161 \mathrm{~nm}$ nanosized particles using bulk production ${ }^{37}$. In the same study, it was also observed that the nanoparticles obtained from microfluidics had a narrower size distribution over all applied mixing times compared to the particles prepared using a bulk procedure. Similarly, Bally et al. reported that increasing the flow rates of non-solvent to the polymer solution and thus a faster and more efficient mixing resulted in smaller poly(methyl methacrylate)-based nanoparticles compared to particles prepared in a batch process at similar solvent to nonsolvent ratios (100 and $245 \mathrm{~nm}$, respectively) ${ }^{38}$. In general, microfluidic devices offer control over flow rates, and therefore mixing times, which is of utmost importance to control the self-assembly and to tailor particle size $\mathrm{e}^{29,36,39-41}$.

Previously, we reported on the preparation of size-tunable micelles based on poly(ethylene glycol)-block-poly(N-2-benzoyloxypropyl methacrylamide) (mPEG- $b$-p(HPMA-Bz)) in batch mode ${ }^{42}$. The study showed that the obtained micelles exhibited crew-cut structures and that their sizes were sensitive to the mixing rate of solvents and non-solvents, emphasizing 
the need for a system with robust mixing features. Therefore, in the present study, a microfluidic mixing device was used to investigate the effects of process and formulation parameters on the size of mPEG- $b$-p(HPMA-Bz) micelles. It was shown that the selfassembly of mPEG- $b$-p(HPMA-Bz) block copolymers could be easily tailored in size and morphology. This is of great importance with our aim of achieving a robust method for the production of small $(<100 \mathrm{~nm})$ and well-defined polymeric nanoparticles eventually suitable for drug delivery purposes. More precisely, a commercial glass chip from Dolomite Inc. was used, which belongs to the herringbone-type micromixers employing chaotic laminar flow ${ }^{43}$. This set-up had previously shown its suitability for achieving morphological control via the assembly of block copolymers with respectively poly(trimethylene carbonate) and poly $\left(\gamma^{-}\right.$ benzyl- $L$-glutamate) as the hydrophobic block, and respectively poly(ethylene oxide) and elastin-like polypeptide (ELP) as the hydrophilic block, on the very same chip ${ }^{41,44}$.

\section{Materials and Methods}

\subsection{Materials}

$\mathrm{N}$-(2-benzoyloxypropyl) methacrylamide (HPMA-Bz) monomer and methoxy-poly(ethylene glycol)-(4,4-azobis(4-cyanopentanoic acid)-methoxy-poly(ethylene glycol) (mPEGABCPA-mPEG) macroinitiator (each mPEG block with a molecular weight of $5.0 \mathrm{kDa}$ ) were synthesized and characterized using previously published protocols ${ }^{42,45,46}$. Poly(tetrafluoroethylene) (PTFE) and cellulose acetate syringe disc filters (both $0.22 \mu \mathrm{m}$ ) and bovine serum albumin (BSA) were obtained from Merck (Darmstadt, Germany). PEG standards for gel permeation chromatography (GPC) analysis were obtained from Agilent (Santa Clara, CA, USA). All solvents were purchased from commercial suppliers and used as received.

\subsection{Instrumentation}

\subsubsection{Laminar Chaotic Mixing Microfluidic System}

The core of the microfluidic system consists of a commercial herringbone micromixer glass chip (Part No. 3200401 purchased from Dolomite Center Ltd., Royston, UK). According to the manufacturer, the chip consists of two independent channels with 12 mixing steps with a depth and width alternating between $125 \times 350$ and $50 \times 125 \mu \mathrm{m}^{2}$, creating lamination of the entering flows and even swirling of the flow streams. The whole microfluidic system is constituted of two pressure pumps and two flowmeters (range 30-1000 $\mu \mathrm{L} / \mathrm{min}$ ) connected to a computer to control the pumps with the provided software (Mitos Flow Control Center 2.5.17 software), PTFE tubing, an ethylene tetrafluoroethylene (ETFE) T-connector, a micromixer chip and a fast camera from Dolomite Microfluidics ${ }^{\circledR}$ (Figure 1). Pump A was linked to the chip through inlets $1+3$ using the $\mathrm{T}$-connector, whereas the pump $\mathrm{B}$ was connected directly to inlet 2 . Flow rate calibration as a function of applied pressure and mixing time calculation was done as described in the manual provided by the supplier (Supplementary Information Figure S5 and Table S1). 


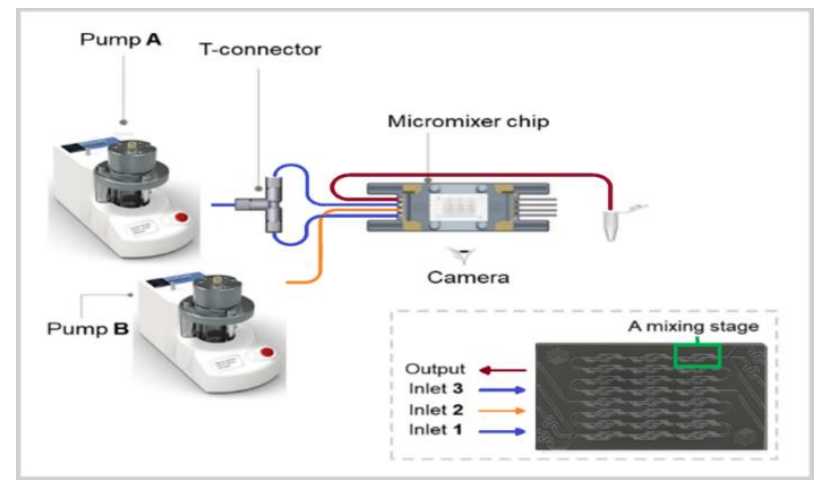

Figure 1. Scheme of the microfluidic system used in the present study (from Dolomite Inc., UK) ${ }^{47}$.

\subsection{Methods}

\subsubsection{Dynamic Light Scattering (DLS) Analysis}

The hydrodynamic diameter of the self-assemblies was determined by DLS analysis using a Malvern Zetasizer nano series ZS90 (Malvern, UK) with a measurement angle of $173^{\circ}$ and a temperature of $25^{\circ} \mathrm{C}$. Prior to measuring, the samples were filtered using a $0.22 \mu \mathrm{m}$ cellulose acetate disk filter to remove any dust and large particles.

\subsubsection{Asymmetric Flow Field-Flow Fractionation Connected to Multi-Angle Laser Light Scattering Detector $\left(A F_{4}-M A L L S\right)$}

The radius of gyration $\left(\mathrm{R}_{\mathrm{g}}\right)$ was determined using a Wyatt Dualtec $\mathrm{AF}_{4}$ instrument connected to a Shimadzu LC-2030 Prominence-I system with a Shimadzu LC-2030 auto-sampler. The fractionation was accomplished on an $\mathrm{AF}_{4}$ short channel with a spacer of $350 \mu \mathrm{m}$ and a 10 $\mathrm{kDa}$ membrane of regenerated cellulose. The $\mathrm{AF}_{4}$ was attached to a light scattering detector (Wyatt DAWN HELEOS II, Santa Barbara, CA, USA) that was installed at 16 different angles ranging from $12.9^{\circ}$ to $157.8^{\circ}$ using a laser operating at $664.5 \mathrm{~nm}$ and a refractive index detector (Wyatt Optilab, Santa Barbara, CA, USA). BSA $(5 \mathrm{mg} / \mathrm{mL})$ dissolved in phosphate buffer saline (PBS) (0.01 M phosphate buffer, $0.0027 \mathrm{M}$ potassium chloride and $0.137 \mathrm{M}$ sodium chloride, $\mathrm{pH} 7.4$, at $25{ }^{\circ} \mathrm{C}$ ) was used for calibration. The data were analyzed using the provided ASTRA software. The refractive index increment $(d n / d c)$ of the polymers was measured by injection of $600 \mu \mathrm{L}$ of precisely weighted samples in the range of 6 to $15 \mathrm{mg} / \mathrm{mL}$ and using a flow rate of $0.6 \mathrm{~mL} / \mathrm{min}$ in an Optilab Rex detector (Wyatt technology). The results of the $d n / d c$ measurements were used to calculate the molecular weight $M_{\mathrm{w}(\mathrm{np})}$ of the scattering nanoparticles using a Zimm plot and to deduce the aggregation number $N_{\text {agg }}$ by dividing the $M_{\mathrm{w}(\mathrm{np})}$ by the weight-averaged molar mass of the polymer chains ${ }^{48}$. Data was analyzed using Astra software.

\subsubsection{Cryo-Transmission Electron Microscopy (Cryo-TEM) Analysis}

Cryo-TEM analysis on selected samples was performed using a FEI CryoTitan (Thermo Fisher Scientific, Hillsboro, OR, USA) equipped with a field emission gun and autoloader and operated at $300 \mathrm{kV}$ acceleration voltage in low-dose bright-field TEM mode. Samples 
for cryo-TEM were prepared by glow-discharging the grids (Lacey carbon coated, $\mathrm{R} 2 / 2, \mathrm{Cu}$, 200 mesh, EM sciences) in a Cressington 208 carbon coater for $40 \mathrm{~s}$. Then, $4 \mu \mathrm{L}$ of the nanoparticle dispersion was pipetted onto the grid and blotted in a Vitrobot MARK III at room temperature and 100\% humidity. The grid was blotted for 3 s (offset -3 ) and subsequently frozen in liquid ethane. Cryo-TEM images were acquired with zero loss energy filtering mode (Gatan GIF 2002, 20eV energy slit) on a charge-coupled device (CCD) camera (Gatan model 794).

\subsubsection{Polymer Synthesis}

mPEG- $b$-p(HPMA-Bz) block copolymers were synthesized by free radical polymerization as described previously (Supplementary information, Scheme S1) ${ }^{42,45,49}$. In short, a 4,4azobis(4-cyanopentanoic acid) (ABCPA) containing macro-initiator, mPEG-ABCPAmPEG, and HPMA-Bz were dissolved in acetonitrile at varying feed ratios (1:25, 1:50, 1:100, 1:200 $\mathrm{mol} / \mathrm{mol}$, respectively). Under a nitrogen atmosphere, the polymerization was conducted at $70{ }^{\circ} \mathrm{C}$ for $24 \mathrm{~h}$. The formed polymer was collected by precipitation in excess of ice-cold diethyl ether, followed by filtration and drying under vacuum. The synthesized block copolymers were analyzed by GPC and ${ }^{1} \mathrm{H}-\mathrm{NMR}$ spectroscopy.

\subsubsection{Preparation of Nanoparticles Based on mPEG-b-p(HPMA-Bz) Using Microfluidics}

The different mPEG- $b$-p(HPMA-Bz) block copolymers were dissolved in THF (concentrations were 5, 10 and $20 \mathrm{mg} / \mathrm{mL}$ ) and ultrapure water was used as a non-solvent. Both solutions were filtered prior to use with cellulose acetate $0.22 \mu \mathrm{m}$ and PTFE $0.22 \mu \mathrm{m}$ syringe filters, respectively. Pump A was filled with ultrapure water and pump $\mathbf{B}$ with the block copolymer solution in THF. The polymer solution and water were mixed at a 1:1 volume ratio at different total flow rates $Q_{\text {tot }}(100,200,350,500$ and $1600 \mu \mathrm{L} / \mathrm{min})$ and the obtained dispersions were collected at the output into a glass vial until a total volume of 2 $\mathrm{mL}$ was obtained. THF was removed by evaporation for $16 \mathrm{~h}$ by leaving the vial uncapped in a fume hood, which leads to less than $1 \mathrm{vol} \%$ of THF remaining according to our previous study ${ }^{42}$. The formed nanoparticles, prepared in triplicate, were characterized using DLS, $\mathrm{AF}_{4}{ }^{-}$ MALLS and cryo-TEM.

\section{Results and Discussion}

\subsection{Synthesis of mPEG- $b$-p(HPMA-Bz) Block Copolymers}

Amphiphilic mPEG- $b$-p(HPMA-Bz) block copolymers were synthesized through free radical polymerization with varying feed ratios of monomer HPMA-Bz to macro-initiator mPEGABCPA-mPEG (M:MI). For all synthesized polymers the yield was approximately $75 \%$. The number- and weight- average molar masses ( $M_{\mathrm{n}}$ and $M_{\mathrm{w}}$, respectively), the degree of polymerization $\left(N_{\text {HPMA-Bz }}\right)$ and the molar mass dispersities $(Đ)$ of the obtained polymers were determined by ${ }^{1} \mathrm{H}-\mathrm{NMR}$ and GPC analysis (Table 1 ). 
Table 1. Characteristics of the synthesized poly(ethylene glycol)- $b$-poly( $N$-2-benzoyloxypropyl methacrylamide) $\mathrm{mPEG}_{5 \mathrm{~K}-} b-\mathrm{p}(\mathrm{HPMA}-\mathrm{Bz}) \mathrm{x}$ block copolymers as determined by ${ }^{1} \mathrm{H}-\mathrm{NMR}$ and gel permeation chromatography (GPC).

\begin{tabular}{|c|c|c|c|c|c|c|c|c|}
\hline \multirow{2}{*}{ Polymer } & \multirow{2}{*}{ M:MI } & \multirow{2}{*}{$M_{\mathrm{n}}$} & \multicolumn{3}{|c|}{ GPC } & \multirow{2}{*}{$N_{\text {HPMA-Bz }}$} & \multirow{2}{*}{$f_{\mathrm{PEG}}$} & \multirow{2}{*}{$\emptyset_{\mathrm{PEG}}$} \\
\hline & & & $M_{\mathrm{n}}$ & $M_{\mathrm{w}}$ & $Đ$ & & & \\
\hline A: $\mathrm{mPEG}_{5 \mathrm{~K}}-b-\mathrm{p}(\mathrm{HPMA}-\mathrm{Bz})_{17.1 \mathrm{~K}}$ & 200 & 22.1 & 15.8 & 20.7 & 1.31 & 69 & 23 & 24 \\
\hline B: $\mathrm{mPEG}_{5 \mathrm{~K}}-b-\mathrm{p}(\mathrm{HPMA}-\mathrm{Bz})_{10.0 \mathrm{~K}}$ & 100 & 15.0 & 13.2 & 17.5 & 1.32 & 40 & 33 & 35 \\
\hline C: $\mathrm{mPEG}_{5 \mathrm{~K}}-b-\mathrm{p}(\mathrm{HPMA}-\mathrm{Bz})_{5.2 \mathrm{~K}}$ & 50 & 10.2 & 10.8 & 14.0 & 1.30 & 21 & 49 & 50 \\
\hline D: mPEG $_{5 \mathrm{~K}}-b-\mathrm{p}(\mathrm{HPMA}-\mathrm{Bz})_{2.7 \mathrm{~K}}$ & 25 & 7.7 & 8.9 & 11.0 & 1.24 & 11 & 65 & 66 \\
\hline
\end{tabular}

M:MI, monomer to macro-initiator ratio ( $\mathrm{mol} / \mathrm{mol}) ; \mathrm{M}_{\mathrm{n}}$, number average molar mass $(\mathrm{kDa}) ; \mathrm{M}_{\mathrm{w}}$, weight average molar mass (kDa); Đ, molar mass dispersity; NHPMA-Bz, degree of polymerization of HPMA$\mathrm{Bz} ; f_{\mathrm{PEG}}$, hydrophilic weight fraction $(\mathrm{wt} \%)$ and $\emptyset_{\mathrm{PEG}}$, hydrophilic volume fraction $(\mathrm{vol} \%)$.

Furthermore, powder mass densities were measured by helium pycnometry. The values for the HPMA-Bz monomer and p(HPMA-Bz) polymer were $1.1796 \pm 0.002$ and $1.1944 \pm$ $0.0012 \mathrm{~g} \cdot \mathrm{cm}^{-3}$, respectively. On the other hand, according to the literature, PEG has a mass density of $1.13 \mathrm{~g} \cdot \mathrm{cm}^{-3}{ }^{50}$. With this information the hydrophilic volume fraction $\left(\emptyset_{\mathrm{PEG}}\right)$ could be estimated (Table 1) by applying the following equation where $f_{\mathrm{PEG}}$ is the calculated hydrophilic weight fraction, $d_{\mathrm{PEG}}$ is the mass density of PEG and $d_{\mathrm{p}(\mathrm{HPMA}-\mathrm{Bz})}$ is the mass density of the $\mathrm{p}(\mathrm{HPMA}-\mathrm{Bz})$ polymer:

$$
\emptyset_{\mathrm{PEG}}=\frac{f_{\mathrm{PEG}} / d_{\mathrm{PEG}}}{\left[f_{\mathrm{PEG}} / d_{\mathrm{PEG}}+\left(1-f_{\mathrm{PEG}}\right) / d_{\mathrm{p}(\mathrm{HPMA}-\mathrm{Bz})}\right]}
$$

Interestingly, the volume fractions $\emptyset_{\mathrm{PEG}}$ were not very different from the weight fractions $f_{\text {PEG }}$. Based on the phase diagram reported by Jain and Bates for the low $T_{\mathrm{g}}$ poly(butadiene)$b$-poly(ethylene glycol) as a function of the degree of polymerization of the hydrophobic block and the hydrophilic fraction $f_{\mathrm{PEG}}{ }^{51}$, the expected equilibrium morphologies of the selfassemblies were vesicles for block copolymer A (mPEG $\left.{ }_{5 \mathrm{~K}}-b-\mathrm{p}(\mathrm{HPMA}-\mathrm{Bz})_{17.1 \mathrm{~K}}\right)$, a blend of vesicles and cylinders for block copolymer $\mathrm{B}\left(\mathrm{mPEG}_{5 \mathrm{~K}}-b-\mathrm{p}(\mathrm{HPMA}-\mathrm{Bz})_{10.0 \mathrm{~K}}\right)$, only cylinders for block copolymer $\mathrm{C}\left(\mathrm{mPEG}_{5 \mathrm{~K}}-b-\mathrm{p}(\mathrm{HPMA}-\mathrm{Bz})_{5.2 \mathrm{~K}}\right)$, and spherical micelles for block copolymer D (mPEG $\left.{ }_{5 \mathrm{~K}}-b-\mathrm{p}(\mathrm{HPMA}-\mathrm{Bz})_{2.7 \mathrm{~K}}\right)$.

\subsection{The Effect of Mixing Time on the Size and Morphology of mPEG- $b$-p(HPMA- Bz) Nanoparticles}

The effect on the size and morphology of mPEG- $b$-p(HPMA-Bz) block copolymer nanoparticles formed by the solvent shift method (nanoprecipitation) was studied using microfluidics. By applying total flow rates $\left(Q_{\text {tot }}\right)$ ranging from 100 to $1600 \mu \mathrm{L} / \mathrm{min}$ the mixing time $\left(\tau_{\mathrm{M}}\right)$ in the micromixer was varied from 1570 to $42 \mathrm{~ms}$ according to the data provided by the manufacturer (Supplementary Information, Table S1).

Figure $2 \mathrm{a}$ demonstrates that block copolymer $\mathrm{A}\left(\mathrm{mPEG}_{5 \mathrm{~K}}-b-\mathrm{p}(\mathrm{HPMA}-\mathrm{Bz})_{17.1 \mathrm{~K}}\right)$, with the largest hydrophobic block and lowest $f_{\mathrm{PEG}}(23 \%)$, at a polymer concentration of $5 \mathrm{mg} / \mathrm{mL}$ assembled into particles increased in size from 55 to $90 \mathrm{~nm}$ when the flow rate decreased from 1600 to $100 \mu \mathrm{L} / \mathrm{min}$. The PDI values for the different nanoparticles were all below 0.2 , 
thereby demonstrating homogeneity in the self-assembly process. Figure $2 \mathrm{a}$ also shows that the observed effect was less pronounced upon increasing the concentration of block copolymer A (mPEG ${ }_{5 \mathrm{~K}}-b$-p $\left.(\mathrm{HPMA}-\mathrm{Bz})_{17.1 \mathrm{~K}}\right)$ to $20 \mathrm{mg} / \mathrm{mL}$. These results can be explained by the nucleation-controlled self-assembly process as the size of the nanoparticle is dependent on the nucleation rate. This is in line with the results from our previous study regarding nanoprecipitation in bulk $^{42}$. In short, the addition of anti-solvent reduces the solubility of block copolymers and induces supersaturation ${ }^{52}$. The nucleation rate is dependent on the supersaturation degree of the block copolymers, which is in turn affected by the used concentration and mixing rate of the polymer-containing solvent and anti-solvent. Slower flow rates result in longer mixing times, which provide a more gradual change in the composition of all the components (solvent, unimers and chain aggregates). This eventually results in less homogeneous supersaturation and slower nucleation and therefore provides a longer growth time of the nanoparticles. Faster flow rates, on the other hand, ensure shorter mixing times. This is associated with rapid and homogenous supersaturation and the formation of more numerous nuclei, which eventually results in smaller and more monodisperse nanoparticles according to the classical nucleation and growth model also called the Lamer model ${ }^{17}$. 
(a)

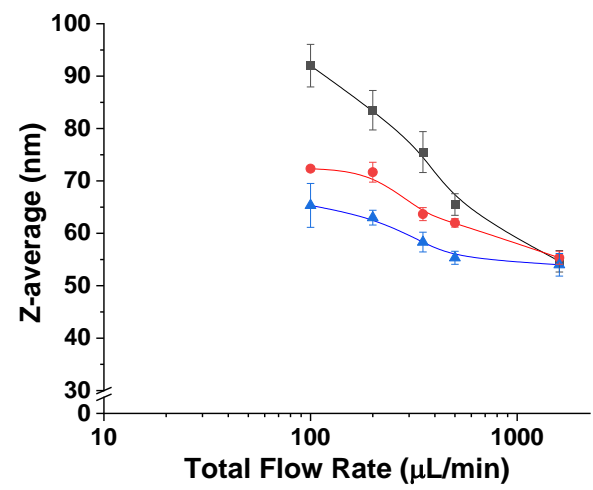

(c)

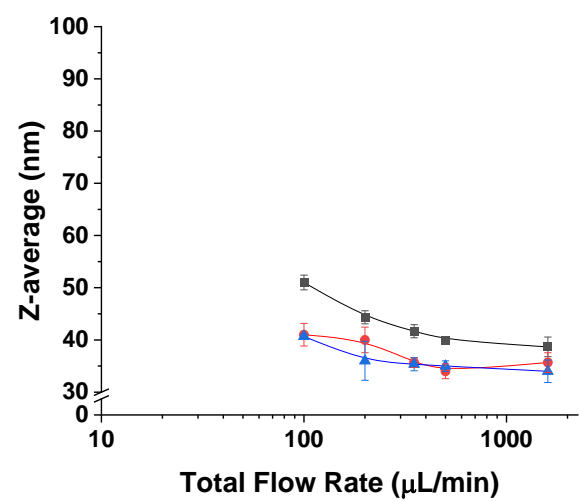

(b)

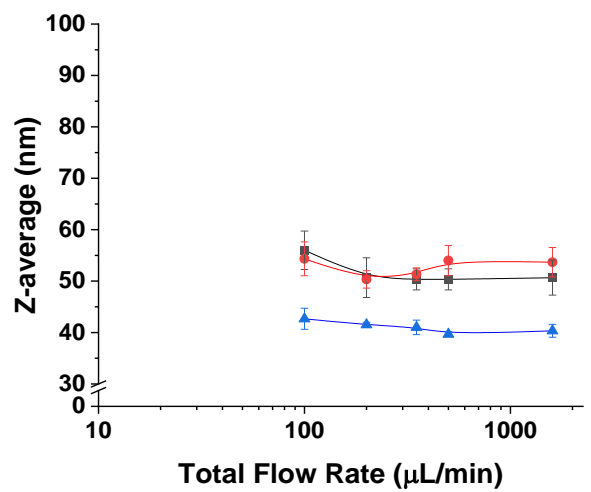

(d)

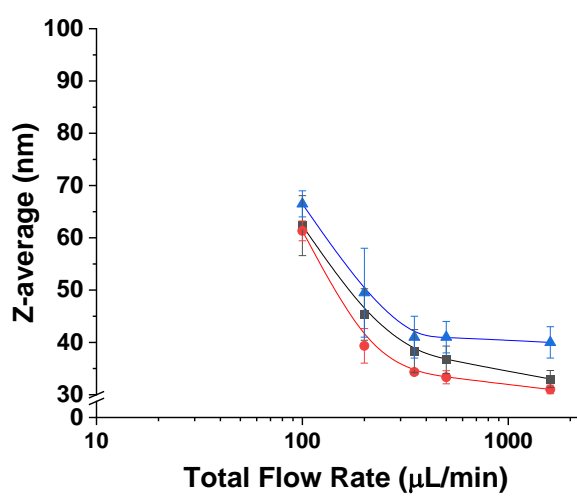

Figure 2. Average hydrodynamic diameter of $\mathrm{mPEG}_{5 \mathrm{~K}}-b-\mathrm{p}(\mathrm{HPMA}-\mathrm{Bz})_{\mathrm{X}}$ nanoparticles as a function of flow rate. (a) mPEG5K-b-p(HPMA-Bz) ${ }_{17.1 \mathrm{~K}}$, (b) mPEG5K- $b$-p(HPMA-Bz) ${ }_{10.0 \mathrm{~K}}$, (c) mPEG $5 \mathrm{~K}-b-$ p(HPMA-Bz) $5.2 \mathrm{~K}$ and (d) mPEG5K- $b$-p(HPMA-Bz) $2.7 \mathrm{~K}$. Black square: $5 \mathrm{mg} / \mathrm{mL}$, red circle: $10 \mathrm{mg} / \mathrm{mL}$ and blue triangle: $20 \mathrm{mg} / \mathrm{mL}$ block copolymer in THF.

The Z-average hydrodynamic diameters of self-assemblies based on the block copolymers with larger hydrophilic weight fraction $f_{\mathrm{PEG}},\left(\mathrm{mPEG}_{5 \mathrm{~K}}-b-\mathrm{p}(\mathrm{HPMA}-\mathrm{Bz})_{10.0 \mathrm{~K}}(\mathrm{~B})\right.$ and $\mathrm{mPEG}_{5 \mathrm{~K}^{-}}$ $b$-p(HPMA-Bz $)_{5.2 \mathrm{~K}}(\mathrm{C})$, did not change significantly when different polymer concentrations or flow rates were used (Figure 2b,c). However, self-assembly of block copolymer D with the smallest hydrophobic block and thus the highest $f_{\mathrm{PEG}}(65 \%), \mathrm{mPEG}_{5 \mathrm{~K}}-b-\mathrm{p}(\mathrm{HPMA}-\mathrm{Bz})_{2.7 \mathrm{~K}}$, resulted in an increase in nanoparticle size from 30 to $65 \mathrm{~nm}$ upon decreasing the flow rate regardless of the polymer concentration (Figure 2d). Along with an increase in particle size, the PDI values also increased moderately upon decreasing the flow rates (Supplementary information, Figure S6).

\subsection{Morphology of mPEG- $b$-p(HPMA-Bz) nanoparticles}

To gain insight into the morphology of the formed nanoparticles based on the largest block copolymer $\mathrm{A} \mathrm{mPEG}_{5 \mathrm{~K}}-b-\mathrm{p}(\mathrm{HPMA}-\mathrm{Bz})_{17.1 \mathrm{~K}}$, the radius of gyration $\left(R_{\mathrm{g}}\right)$, hydrodynamic radius $\left(R_{\mathrm{h}}\right)$ and size distribution (fractograms) were determined using $\mathrm{AF}_{4}-\mathrm{MALLS}$ (Table 2). This 
analytical technique combines the advantages of field-flow fractionation chromatography to separate fractions of nearly monodisperse self-assemblies with the power of multi-angle laser light scattering (MALLS) to get an insight on their morphologies. Interestingly, the $R_{\mathrm{g}} / R_{\mathrm{h}}$ ratio and the weight average molecular weight of the nanoparticles $\left(M_{\mathrm{w}(\mathrm{np})}\right)$ deduced from a Zimm plot gradually increased upon decreasing the flow rate. At the two lower concentrations, 5 and $10 \mathrm{mg} / \mathrm{mL}, R_{\mathrm{g}} / R_{\mathrm{h}}$ ratios of $\sim 1$ were observed for the slowest flow rate $(100 \mu \mathrm{L} / \mathrm{min})$ i.e., longest mixing time $(1570 \mathrm{~ms})$. However, this was not observed for the highest polymer concentration studied $(20 \mathrm{mg} / \mathrm{mL})$ at which $R_{\mathrm{g}} / R_{\mathrm{h}}$ ratios close to 0.8 were measured at all flow rates.

Table 2. Characteristics of block copolymer A (mPEG $\left.5 \mathrm{~K}-b-\mathrm{p}(\mathrm{HPMA}-\mathrm{Bz})_{17.1 \mathrm{~K}}\right)$ nanoparticles as determined by asymmetric flow field-flow fractionation connected to multi-angle laser light scattering detector ( $\mathrm{AF}_{4}$-MALLS).

\begin{tabular}{ccccccc}
\hline $\mathbf{C}$ & $\mathbf{Q}$ & $\boldsymbol{R}_{\mathrm{g}}$ & $\boldsymbol{R}_{\mathbf{h}}$ & $\boldsymbol{R}_{\mathrm{g}} / \boldsymbol{R}_{\mathbf{h}}$ & $\boldsymbol{M}_{\mathbf{w ( n p )}}$ & $\boldsymbol{N}_{\text {agg }}$ \\
\hline 5 & 100 & 46 & 45 & 1.03 & 187 & 8500 \\
5 & 200 & 35 & 39 & 0.90 & 142 & 6400 \\
5 & 350 & 32 & 36 & 0.89 & 131 & 5900 \\
5 & 500 & 30 & 37 & 0.82 & 150 & 6800 \\
5 & 1600 & 21 & 26 & 0.81 & 36 & 1600 \\
\hline 10 & 100 & 34 & 33 & 1.03 & 79 & 3600 \\
10 & 200 & 24 & 30 & 0.82 & 64 & 2900 \\
10 & 350 & 24 & 28 & 0.86 & 42 & 1900 \\
10 & 500 & 22 & 28 & 0.78 & 39 & 1800 \\
10 & 1600 & 17 & 25 & 0.69 & 26 & 1200 \\
\hline 20 & 100 & 24 & 28 & 0.85 & 42 & 1900 \\
20 & 200 & 22 & 26 & 0.82 & 34 & 1600 \\
20 & 350 & 20 & 27 & 0.73 & 69 & 3100 \\
20 & 500 & 21 & 28 & 0.76 & 36 & 1600 \\
20 & 1600 & 20 & 25 & 0.78 & 34 & 1500 \\
\hline
\end{tabular}

C, concentration (mg/mL); Q, flow rate $(\mu \mathrm{L} / \mathrm{min}) ; \mathrm{R}_{\mathrm{g}}$, radius of gyration $(\mathrm{nm}) ; \mathrm{R}_{\mathrm{h}}$, hydrodynamic radius $(\mathrm{nm}) ; \mathrm{M}_{\mathrm{w}}(\mathrm{np})$, weight average molecular weight of the nanoparticles $\left(10^{3} \mathrm{kDa}\right)$ and $\mathrm{N}_{\mathrm{agg}}$, nanoparticle aggregation number.

The $R_{\mathrm{g}} / R_{\mathrm{h}}$ ratio (or shape factor $\rho$ ) is structure sensitive and therefore provides information about the morphology of nanoparticles ${ }^{53}$. In particular, it has been shown that the $R_{\mathrm{g}} / R_{\mathrm{h}}$ ratios for structures with a dense core and less dense shell (core-shell structures) are lower than $0.775^{54-58}$. On the other hand, particles with a rigid spherical structure have in theory $R_{\mathrm{g}} / R_{\mathrm{h}}$ ratios of $\sim \sqrt{3 / 5}$ or $\sim 0.775^{54,55}$. For spherical vesicles like polymersomes, the scattering mass is concentrated on the surface of the sphere yielding a $R_{\mathrm{g}} / R_{\mathrm{h}}$ ratio near one ${ }^{59,60}$. Therefore, the $\mathrm{AF}_{4}-\mathrm{MALLS}$ results for block copolymer $\mathrm{A}\left(\mathrm{mPEG}_{5 \mathrm{~K}}-b-\mathrm{p}(\mathrm{HPMA}-\mathrm{Bz})_{17.1 \mathrm{~K}}\right)$ nanoparticles indicate that polymer vesicles (polymersomes) were formed at slower flow rates, instead of the filled micelles that were formed at higher concentrations and faster flow rates. 
Cryo-TEM analysis of some selected samples was used to corroborate the $\mathrm{AF}_{4}$-MALLS results regarding the nanoparticle morphology of block copolymer A (mPEG ${ }_{5 \mathrm{~K}}-b$-p (HPMA$\mathrm{Bz})_{17.1 \mathrm{~K}}$ ) nanoparticles. Figure 3 provides an overview of all the observed morphologies. It was shown that for the two lowest concentrations $(5$ and $10 \mathrm{mg} / \mathrm{mL})$ using slower flow rates, larger micelles and also polymersomes were formed. Interestingly, at the fastest flow rate of $1600 \mu \mathrm{L} / \mathrm{min}$, regardless of the used concentration, only solid micelles were formed with a diameter of around $35 \mathrm{~nm}$ as measured by cryo-TEM (Figure 4). The hydrodynamic diameters for these samples were around $55 \mathrm{~nm}$ as measured by DLS (Figure 2a). This apparent discrepancy in diameters can be easily explained. Indeed, cryo-TEM only allows visualization the core of the micelles where the aromatic benzyl groups are localized which provide a high scattering density for electrons, whereas DLS includes the hydrated mPEG corona, which is much transparent to the electron beam. Figure 4 also demonstrates that only micelles were formed at $20 \mathrm{mg} / \mathrm{mL}$, independent of the used flow rates.

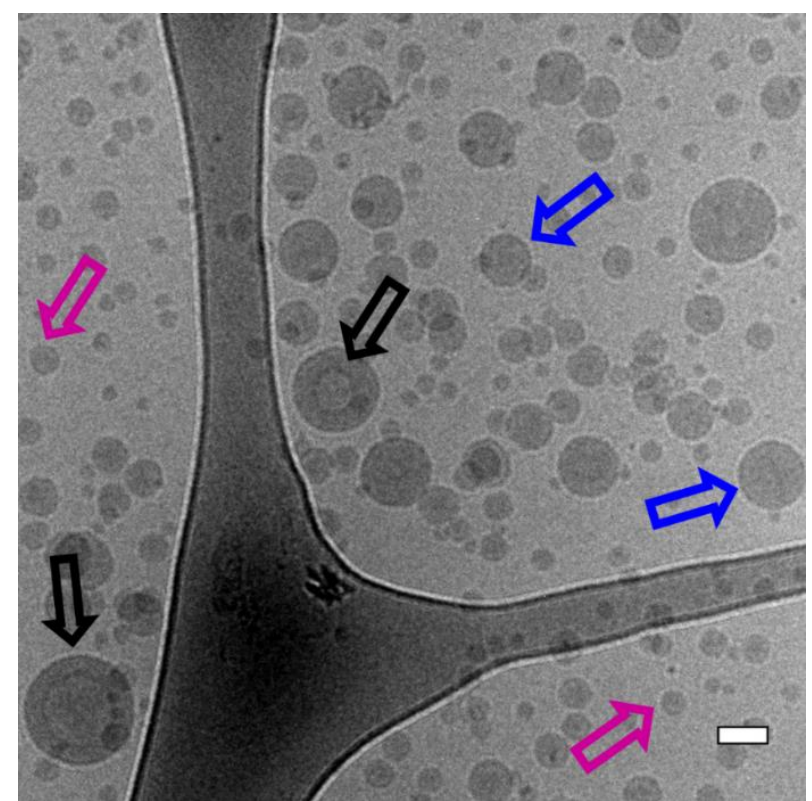

Figure 3. Cryo-TEM overview picture of block copolymer A (mPEG $\left.{ }_{5 \mathrm{~K}}-b-\mathrm{p}(\mathrm{HPMA}-\mathrm{Bz})_{17.1 \mathrm{~K}}\right)$ nanoparticles prepared at a concentration of $5 \mathrm{mg} / \mathrm{mL}$ and a flow rate of $100 \mu \mathrm{L} / \mathrm{min}$. Black arrows point to vesicles such as polymersomes, blue arrows point to bigger micelles and the purple arrows point to smaller filled micelles. Scalebar indicates $50 \mathrm{~nm}$. 
$100 \mathrm{Q}(\mu \mathrm{L} / \mathrm{min})$

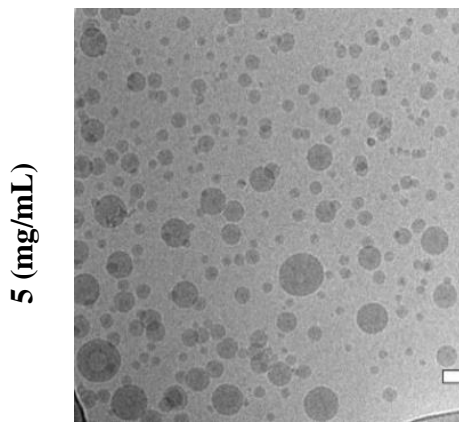

(a)

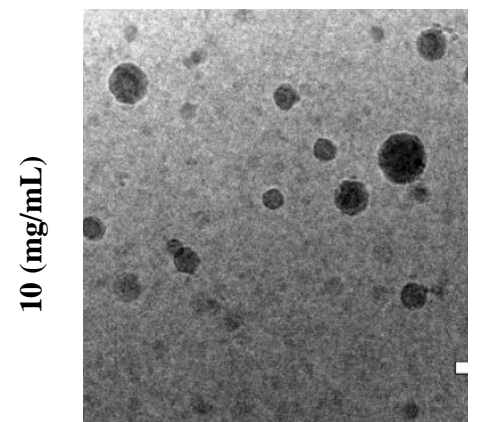

(d)

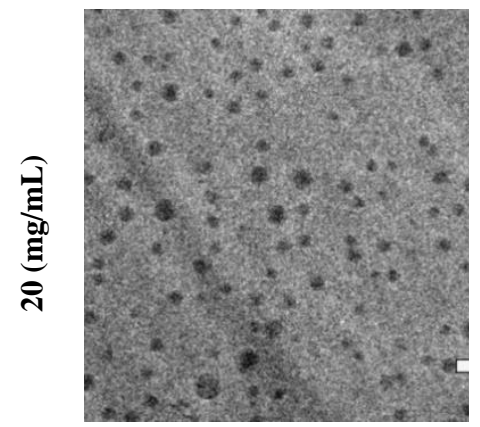

(g)
$350 \mathrm{Q}(\mu \mathrm{L} / \mathrm{min})$

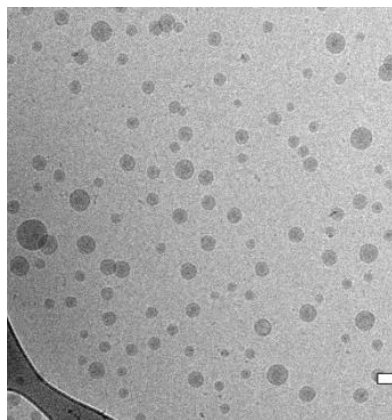

(b)

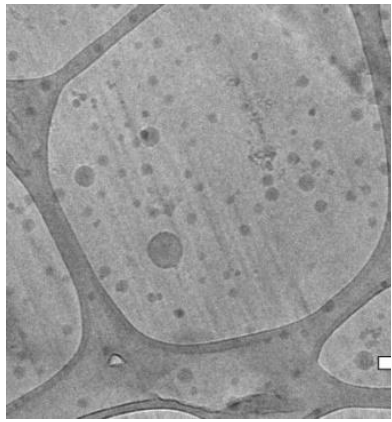

(e)

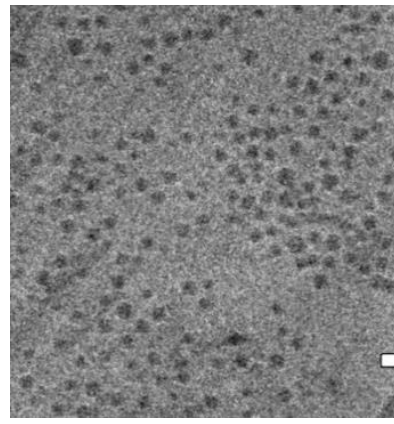

(h)
$1600 \mathrm{Q}(\mu \mathrm{L} / \mathrm{min})$

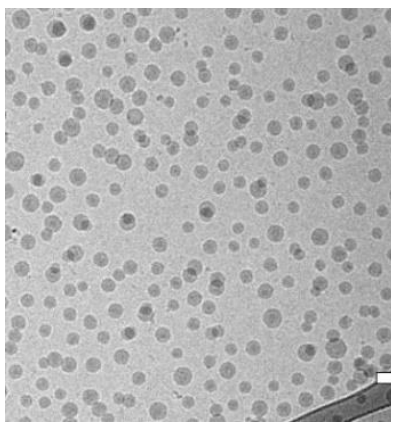

(c)

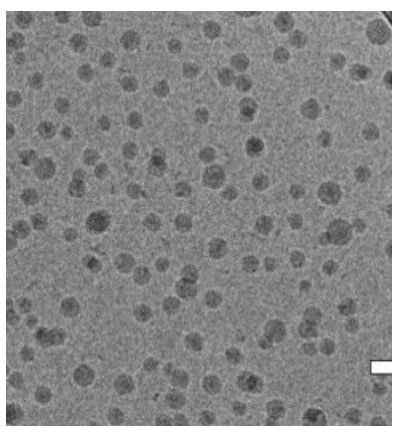

(f)

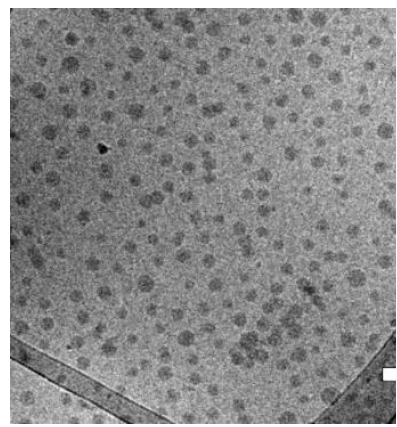

(i)

Figure 4. Cryo-TEM pictures of polymer A (mPEG $5 \mathrm{~K}-b-\mathrm{p}(\mathrm{HPMA}-\mathrm{Bz})_{17.1 \mathrm{~K})}$ nanoparticles prepared using different polymer concentrations and flow rates. (a) $5 \mathrm{mg} / \mathrm{mL}$ and $100 \mu \mathrm{L} / \mathrm{min}$, (b) $5 \mathrm{mg} / \mathrm{mL}$ and $350 \mu \mathrm{L} / \mathrm{min}$, (c) $5 \mathrm{mg} / \mathrm{mL}$ and $1600 \mu \mathrm{L} / \mathrm{min}$, (d) $10 \mathrm{mg} / \mathrm{mL}$ and $100 \mu \mathrm{L} / \mathrm{min}$, (e) $10 \mathrm{mg} / \mathrm{mL}$ and 350 $\mu \mathrm{L} / \mathrm{min}$, (f) $10 \mathrm{mg} / \mathrm{mL}$ and $1600 \mu \mathrm{L} / \mathrm{min}$, (g) $20 \mathrm{mg} / \mathrm{mL}$ and $100 \mu \mathrm{L} / \mathrm{min}$, (h) $20 \mathrm{mg} / \mathrm{mL}$ and 350 $\mu \mathrm{L} / \mathrm{min}$, (i) $20 \mathrm{mg} / \mathrm{mL}$ and $1600 \mu \mathrm{L} / \mathrm{min}$. Scale bars indicate $50 \mathrm{~nm}$.

The fractograms of the $\mathrm{AF}_{4}$-MALLS of the $5 \mathrm{mg} / \mathrm{mL}$ samples for block copolymer A revealed only one peak for the particles prepared at the fastest flow rates (500 and $1600 \mu \mathrm{L} / \mathrm{min})$ and one peak with a tail at higher retention times for particles prepared at microfluidic flow rates 
below $350 \mu \mathrm{L} / \mathrm{min}$, which could not be separated even by adjusting the fractionation method (Figure 5a and supplementary information Figure S7). This observation is in agreement with the cryo-TEM results, which showed that at slower microfluidic flow rates mostly micelles with a size around $30-35 \mathrm{~nm}$ were formed together with some bigger objects of $50-100 \mathrm{~nm}$, presumably micelles and even polymersomes (Figure 4). This transition from homogenous small micelles of 30-35 $\mathrm{nm}$ diameter at high microfluidic flow rates to more polydisperse particles where small micelles coexist with larger micelles and vesicles is rather gradual. This explains the tail in the chromatographic fractogram by $\mathrm{AF}_{4}-\mathrm{MALLS}$.

(a)

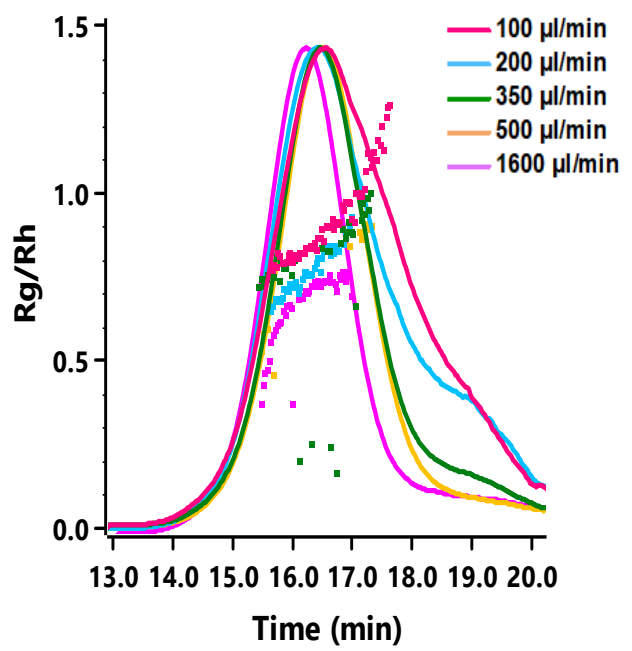

(b)

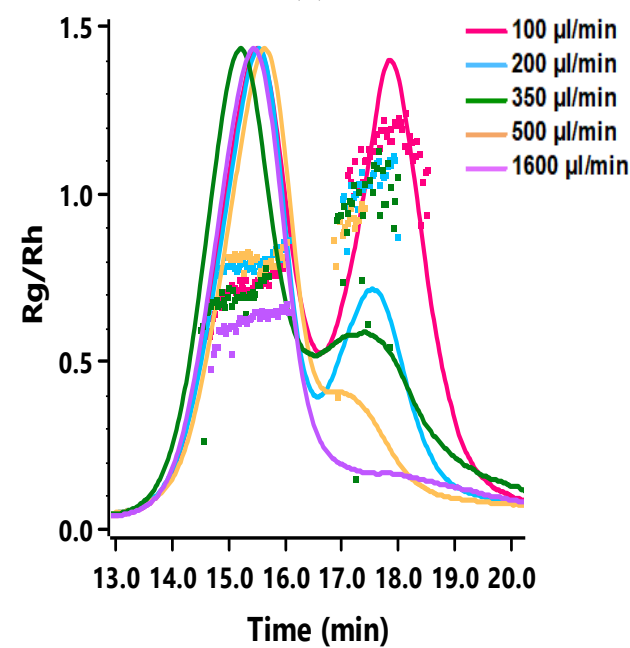

Figure 5. Fractograms of nanoparticles obtained at varying microfluidic flow rates measured with $\mathrm{AF}_{4}-$ MALLS. (a) Block copolymer A (mPEG $\left.{ }_{5 K}-b-\mathrm{p}(\mathrm{HPMA}-\mathrm{Bz})_{17.1 \mathrm{~K}}\right)$ at concentration of $5 \mathrm{mg} / \mathrm{mL}$. (b) Block copolymer D (mPEG $\left.{ }_{5 \mathrm{~K}}-b-\mathrm{p}(\mathrm{HPMA}-\mathrm{Bz})_{2.7 \mathrm{~K}}\right)$ with a concentration of $5 \mathrm{mg} / \mathrm{mL}$.

$\mathrm{AF}_{4}-\mathrm{MALLS}$ results of the samples prepared from the smallest block copolymer D (mPEG${ }_{5 \mathrm{~K}^{-}}$ $b$-p(HPMA-Bz $)_{2.7 \mathrm{~K}}$ ) showed strikingly different fractograms compared to the largest block copolymer A (Figure 5b and supplementary information Figure S12). At slower microfluidic flow rates, two distinct peaks corresponding to two populations of nanoparticles were observed, whereas for the shortest mixing time, only one peak and therefore one population was detected.

The $R_{\mathrm{g}}$ and $R_{\mathrm{h}}$ of the $\mathrm{mPEG}_{5 \mathrm{~K}}-b$-p(HPMA-Bz) ${ }_{2.7 \mathrm{~K}}$ nanoparticles were determined for the separate populations by $\mathrm{AF}_{4}-\mathrm{MALLS}$ (Table 3). Interestingly, the average $R_{\mathrm{g}} / R_{\mathrm{h}}$ ratios of the nanoparticles of the first peaks were all around 0.7 , which points to solid spherical structures $(\sim 0.775)$. On the other hand, the nanoparticles of the second peaks showed higher $R_{\mathrm{g}} / R_{\mathrm{h}}$ values with some even approaching $\sim 1$, suggesting the formation of polymersomes. Moreover, the $M_{\mathrm{w}(\mathrm{np})}$ of the nanoparticles corresponding to the second peak were considerably higher compared to the first peak, between 10-150 MDa and around $3 \mathrm{MDa}$, respectively. The results for the first peak are comparable with the values previously reported for micelles from the same polymer prepared in batch mode ${ }^{42}$. These results demonstrate that, independent of polymer concentration, two separate particle populations of very distinct morphologies were formed when flow rates were decreased and thus mixing times increased. 

Microfluidics

The formation of other morphologies was also substantiated by the increasing PDI values as measured by DLS.

Table 3. Characteristics of polymer D (mPEG $5 \mathrm{~K}-b-\mathrm{p}(\mathrm{HPMA}-\mathrm{Bz})_{2.7 \mathrm{~K})}$ nanoparticles as determined by AF4-MALLS.

\begin{tabular}{|c|c|c|c|c|c|c|c|c|c|c|c|}
\hline \multirow{2}{*}{ C } & \multirow{2}{*}{$\mathbf{Q}$} & \multicolumn{5}{|c|}{ Peak 1} & \multicolumn{5}{|c|}{ Peak 2} \\
\hline & & $R_{\mathrm{g}}$ & $R_{\mathrm{h}}$ & $R_{\mathrm{g}} / \boldsymbol{R}_{\mathrm{h}}$ & $M_{\mathrm{w}(\mathbf{n p})}$ & $N_{\text {agg }}$ & $R_{\mathrm{g}}$ & $R_{\mathrm{h}}$ & $R_{\mathrm{g}} / R_{\mathrm{h}}$ & $M_{\mathrm{w}(\mathrm{np})}$ & $N_{\text {agg }}$ \\
\hline 5 & 100 & 13 & 17 & 0.76 & 3.1 & 400 & 54 & 54 & 0.99 & 98 & 12700 \\
\hline 5 & 200 & 13 & 17 & 0.80 & 3.2 & 420 & 46 & 51 & 0.91 & 68 & 8800 \\
\hline 5 & 350 & 13 & 17 & 0.77 & 3.3 & 430 & 34 & 40 & 0.85 & 91 & 11800 \\
\hline 5 & 500 & 12 & 17 & 0.68 & 3.5 & 450 & 32 & 39 & 0.82 & 171 & 22200 \\
\hline 5 & 1600 & 11 & 17 & 0.63 & 3.4 & 450 & - & - & - & - & - \\
\hline 10 & 100 & 10 & 16 & 0.65 & 2.5 & 320 & 53 & 56 & 0.93 & 70 & 9000 \\
\hline 10 & 200 & 11 & 16 & 0.67 & 2.9 & 380 & 47 & 76 & 0.62 & 11 & 1500 \\
\hline 10 & 350 & 11 & 17 & 0.63 & 3.2 & 420 & 26 & 39 & 0.65 & 625 & 81200 \\
\hline 10 & 500 & 12 & 16 & 0.71 & 2.6 & 340 & - & 39 & - & - & - \\
\hline 10 & 1600 & 13 & 17 & 0.77 & 2.8 & 360 & - & - & - & - & - \\
\hline 20 & 100 & 12 & 16 & 0.75 & 2.3 & 300 & 59 & 54 & 1.09 & 147 & 19100 \\
\hline 20 & 200 & 13 & 16 & 0.81 & 2.3 & 300 & 47 & 45 & 1.04 & 145 & 18780 \\
\hline 20 & 350 & 11 & 16 & 0.71 & 2.5 & 320 & 48 & 45 & 1.06 & 66 & 8500 \\
\hline 20 & 500 & 13 & 16 & 0.81 & 2.3 & 300 & 31 & 36 & 0.87 & 104 & 13600 \\
\hline 20 & 1600 & 11 & 16 & 0.75 & 2.5 & 320 & - & - & - & - & - \\
\hline
\end{tabular}

$\mathrm{C}$, concentration $(\mathrm{mg} / \mathrm{mL})$; $\mathrm{Q}$, flow rate $(\mu \mathrm{L} / \mathrm{min}) ; \mathrm{R}_{\mathrm{g}}$, radius of gyration $(\mathrm{nm}) ; \mathrm{R}_{\mathrm{h}}$, hydrodynamic radius $(\mathrm{nm}) ; \mathrm{M}_{\mathrm{w}}(\mathrm{np})$, weight average molecular weight of the nanoparticles $\left(10^{3} \mathrm{kDa}\right)$ and $\mathrm{N}_{\mathrm{agg}}$, nanoparticle aggregation number.

These results are in accordance with the cryo-TEM results of a selection of $\mathrm{mPEG}_{5 \mathrm{~K}}-b$ p(HPMA-Bz) $)_{2.7 \mathrm{~K}}$ nanoparticles (Figure 6). It was shown that mostly small filled micelles with a size around 15-20 $\mathrm{nm}$ and a few bigger polymersome structures were formed (Supplementary information Figure S13). 
$100 Q(\mu \mathrm{L} / \mathrm{min})$

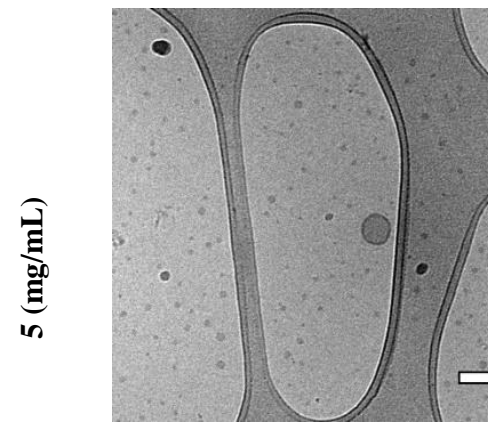

(a)

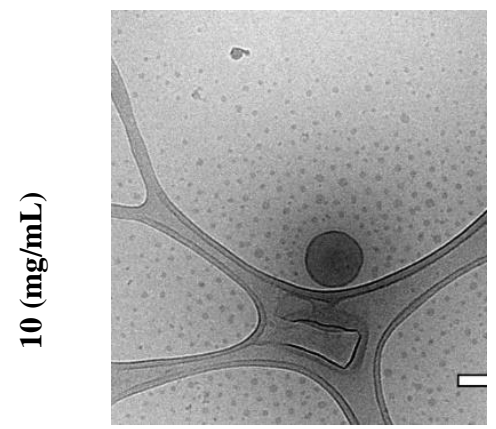

(d)

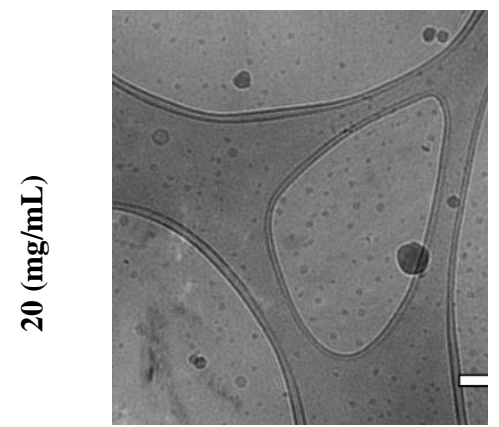

(g)
$350 Q(\mu \mathrm{L} / \mathrm{min})$

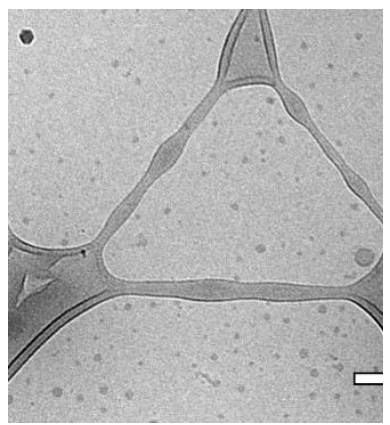

(b)

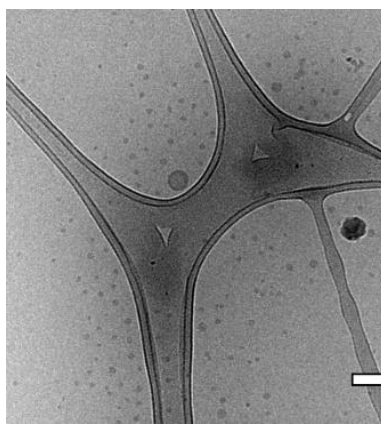

(e)

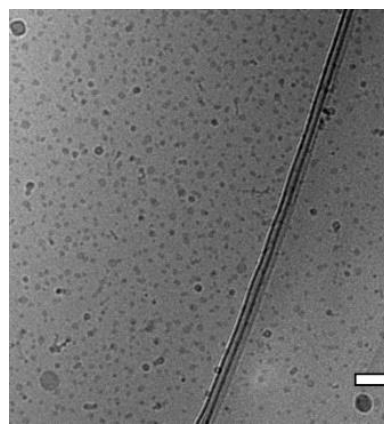

(h)
$1600 Q(\mu \mathrm{L} / \mathrm{min})$

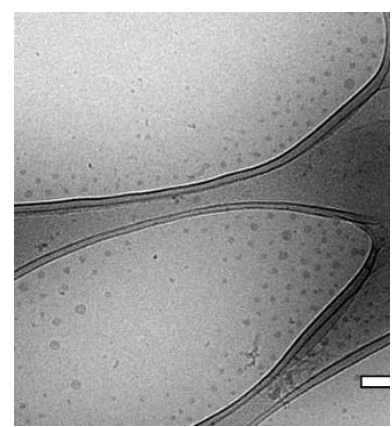

(c)

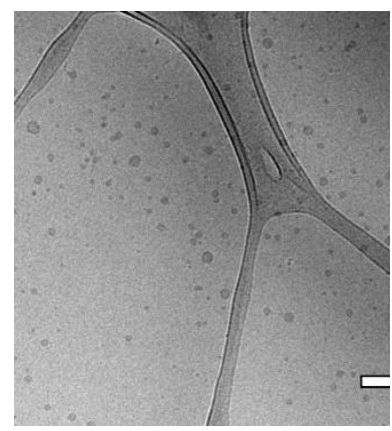

(f)

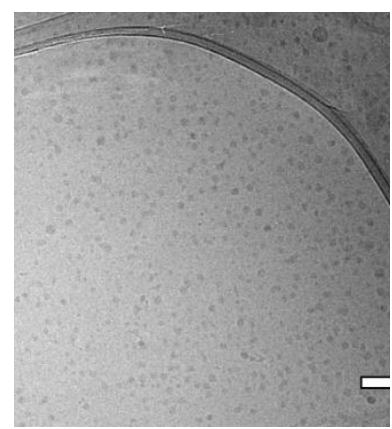

(i)

Figure 6. Cryo-TEM pictures of polymer D (mPEG $5 \mathrm{~K}-b-\mathrm{p}(\mathrm{HPMA}-\mathrm{Bz})_{2.7 \mathrm{~K})}$ nanoparticles prepared using different polymer concentrations and flow rates. (a) $5 \mathrm{mg} / \mathrm{mL}$ and $100 \mu \mathrm{L} / \mathrm{min}$, (b) $5 \mathrm{mg} / \mathrm{mL}$ and $350 \mu \mathrm{L} / \mathrm{min}$, (c) $5 \mathrm{mg} / \mathrm{mL}$ and $1600 \mu \mathrm{L} / \mathrm{min}$, (d) $10 \mathrm{mg} / \mathrm{mL}$ and $100 \mu \mathrm{L} / \mathrm{min}$, (e) $10 \mathrm{mg} / \mathrm{mL}$ and 350 $\mu \mathrm{L} / \mathrm{min}$, (f) $10 \mathrm{mg} / \mathrm{mL}$ and $1600 \mu \mathrm{L} / \mathrm{min}$, (g) $20 \mathrm{mg} / \mathrm{mL}$ and $100 \mu \mathrm{L} / \mathrm{min}$, (h) $20 \mathrm{mg} / \mathrm{mL}$ and 350 $\mu \mathrm{L} / \mathrm{min}$, (i) $20 \mathrm{mg} / \mathrm{mL}$ and $1600 \mu \mathrm{L} / \mathrm{min}$. Scale bars indicate $50 \mathrm{~nm}$. 
The $R_{\mathrm{g}} / R_{\mathrm{h}}$ ratios of block copolymer B $\left(\mathrm{mPEG}_{5 \mathrm{~K}}-b-\mathrm{p}(\mathrm{HPMA}-\mathrm{Bz})_{10.0 \mathrm{~K}}\right)$ and $\mathrm{C}\left(\mathrm{mPEG}_{5 \mathrm{~K}}-b-\right.$ $\mathrm{p}(\mathrm{HPMA}-\mathrm{Bz})_{5.2 \mathrm{~K}}$ ) nanoparticles showed a main value near 0.775 and a second peak with values between 1.13 and 1.73 for block copolymer B and between 0.92 and 1.38 for block copolymer C (Supplementary information Tables S2 and S3, Figures S8 and S10), demonstrating that not only solid micelles were formed but also other structures like vesicles depending on the used concentration and flow rate. Cryo-TEM measurements were in accordance with these results and showed that mostly small filled micelles were formed with a size around 30 and $21 \mathrm{~nm}$, respectively, and a few bigger polymersome structures (Supplementary information Figures S9, S11 and S14).

In the case of $\mathrm{mPEG}_{5 \mathrm{~K}}-b-\mathrm{p}(\mathrm{HPMA}-\mathrm{Bz})$, with a hydrophobic block of high $T_{\mathrm{g}}$ (Supplementary information Figure S15) and aromatic side-groups providing strong $\Pi-\Pi$ interactions, nanoprecipitation at fast mixing rates leads to frozen self-assemblies as soon as water and THF are mixed. The occurrence of different morphologies can be explained by the competition between the kinetic process and the thermodynamically favorable structure. Therefore, by using a microfluidic mixing device and performing nanoprecipitation at mixing times $\tau_{\mathrm{M}}$ that could be tuned between 42 and $1570 \mathrm{~ms}$, snapshots of the kinetic process of block copolymer self-assembly were captured.

The mechanism that is best applicable to vesicle formation from mPEG- $b$-p(HPMA-Bz) block copolymers depends on the size of the hydrophobic block. For the largest block copolymer A $\left(\mathrm{mPEG}_{5 \mathrm{~K}}-b-\mathrm{p}(\mathrm{HPMA}-\mathrm{Bz})_{17.1 \mathrm{~K}}\right)$ vesicles are expected to be formed at the thermodynamic state, from the packing parameter model with a hydrophilic fraction $f_{\mathrm{PEG}} \sim 23 \%$ and a hydrophobic block length $N_{\mathrm{HPMA}-\mathrm{Bz}} \sim 69^{61,62}$. It is envisioned that the vesicles are formed through a mechanism as described in detail by He and Schmid ${ }^{63}$. They stated that vesicles form via self-assembly of micelles that subsequently undergo an internal reorganization to yield vesicular membranes. It was shown that, under dilute conditions, first spherical micelles were formed that continue to grow through a path reminiscent of Ostwald ripening of emulsions into larger micelles. These subsequently transform into semi-vesicles through a flip-flop motion of chains that brings the hydrophilic PEG chains inward and drives solvent diffusion inside and eventually reach full vesicle morphologies. The fact that the different sizes and shapes of the particles could not be separated on $\mathrm{AF}_{4}$-MALLS as described above emphasizes a gradual growth of micelles and eventually a rearrangement into lamellar structures. Therefore, this explains why the fractogram of samples prepared from block copolymer A (mPEG $5_{5 \mathrm{~K}}-b$-p $\left.(\mathrm{HPMA}-\mathrm{Bz})_{17.1 \mathrm{~K}}\right)$ at slower microfluidic flow rates was broader and becomes narrower at faster microfluidic flow rates. The cryo-TEM pictures confirmed the proposed mechanism, and all three structures (micelles, larger micelles and vesicles) were observed for particles prepared at the slowest flow rates and the lowest concentration (Figure $3)$.

From these results, it is apparent that in order to prepare dispersions with only spherical micelles, three factors are important. The first factor is the hydrophobic to hydrophilic ratio, here determined by $f_{\text {PEG. }}$. In this research, it was shown that nanoparticles resulting from all block copolymers resulted mainly into spherical micelles at high concentrations and/or at fast flow rates. This observation is contrary to their equilibrium morphology, which corresponds in theory to vesicles for block copolymer A, a blend of vesicles and of cylindrical (wormlike) micelles for block copolymer B, only cylinders for block copolymer $\mathrm{C}$ and spherical micelles for block copolymer $\mathrm{D}^{51}$. In this work, vesicles were only detected as small 
secondary populations at low concentrations and/or slow flow rates, indicating kinetic control of the self-assembly process rather than thermodynamic. The second important factor is the used polymer concentration which determines the supersaturation condition. It was for example observed for block copolymer A $\left(\mathrm{mPEG}_{5 \mathrm{~K}}-b-\mathrm{p}(\mathrm{HPMA}-\mathrm{Bz})_{17.1 \mathrm{~K}}\right)$ that nanoprecipitation at high supersaturation condition resulting from using high polymer concentrations is needed in order to obtain spherical micelles only. The third important factor is the flow rate of solvents, or equivalently the mixing time during the nanoprecipitation process, which also has an influence on supersaturation conditions. For both block copolymer $\mathrm{A}$ and $\mathrm{D}\left(\mathrm{mPEG}_{5 \mathrm{~K}}-b-\mathrm{p}(\mathrm{HPMA}-\mathrm{Bz})_{17.1 \mathrm{~K}}\right.$ and $\mathrm{mPEG}_{5 \mathrm{~K}}-b-\mathrm{p}(\mathrm{HPMA}-\mathrm{Bz})_{2.7 \mathrm{~K}}$, respectively) it was found that higher flow rates led to faster and better mixing and therefore resulted in the formation of micelles only. On the contrary, the lower flow rates led to slower mixing conditions (with mixing time up to $1.6 \mathrm{sec}$ ), which favors the apparition of self-assemblies with a $R_{\mathrm{g}} / R_{\mathrm{h}}$-value around one. This is a characteristic of vesicles and was even observed for block copolymer $\mathrm{D}$ whose hydrophilic fraction $f_{\mathrm{PEG}} \sim 65 \%$ and hydrophobic block length $N_{\text {HPMA-Bz }} \sim 11$. This indicates a preference for the formation of spherical micelles at thermal equilibrium, according to the classical phase diagram of amphiphilic diblock copolymers ${ }^{51}$. It is hypothesized that vesicle formation proceeds in the case of block copolymer D through a different mechanism. It was proposed that upon mixing a block copolymer solution with a non-solvent for one block, spherical micelles appear first. Then they aggregate through coalescence and grow into larger cylindrical micelles which later fuse into flat membranes that eventually close up on themselves, thereby entrapping solvent to yield vesicles ${ }^{61,62,64}$. Such a scenario of block copolymer self-assembly from micelles to vesicles through cylinders was confirmed with numerical simulation as described by Campos-Villalobos et al. ${ }^{22}$. This is ascribed to a plasticizing effect of THF, enabling chain mobility even at a temperature below the $T_{\mathrm{g}}$.

In general, for reliable nanoprecipitation of mPEG- $b-\mathrm{p}(\mathrm{HPMA}-\mathrm{Bz})$ block copolymers into spherical micelles, of diameters as small as possible to fit the biological applications, a high nucleation rate should be created. This could be achieved by providing high supersaturation conditions by applying fast mixing rates and using high polymer concentrations. The intrinsic propensity of the block copolymers to form other morphologies, based on their hydrophobic to hydrophilic ratio, was hereby bypassed through the kinetic control. Only at lower mixing rates and lower concentrations these thermodynamically more favorable morphologies became apparent. Finally, after one year, all the samples showed no visible precipitation and evolution when measured again using DLS, indicating that the formed nanoparticle suspensions are stable.

\section{Conclusions}

This study demonstrates that the self-assembly of mPEG- $b$-p(HPMA-Bz) block copolymers into nanoparticles can be easily tailored in size and morphology using microfluidics. This control relies partly on the hydrophobic to hydrophilic ratio of the block copolymers and mostly on the processing methods which change the supersaturation conditions. In general, mPEG- $b$-p(HPMA-Bz) block copolymers formed micelles when both concentration and total flow rate were high. Lowering both concentration and flow rate resulted in a considerable effect on the resulting size and morphology of mPEG- $b$-p(HPMA-Bz) self-assembled nanoparticles. Even polymersomes were formed for block copolymers which supposedly self-assemble into spherical micelles at the thermodynamic state. However, other time- 
resolved experiments such X-ray or neutron scattering techniques would be necessary to definitively describe the pathway from unimers to self-assemblies. Importantly, microfluidics is a very suitable method to prepare micelles in a scalable and reproducible manner. For future scaled-up work, using microfluidics is preferred over batch-wise production as it offers more control over the size and morphology of the nanoparticles that are produced.

\section{Funding}

This research was funded by “the European Union's Horizon 2020 research and innovation program Marie Sklodowska-Curie Innovative Training Networks (ITN), grant number 676137 "NANOMED”.

\section{Acknowledgments}

The CPER CAMPUSB project funded by the French state and the Region Nouvelle Aquitaine are gratefully acknowledged for acquisition of the Dolomite micromixer system. Eric Laurichesse from Centre de Recherche Paul Pascal (CNRS, Univ. Bordeaux, France) and Esra Aydinlioglu (LCPO) are kindly acknowledged for the helium pycnometry experiments to measure polymer mass density.

\section{Conflicts of Interest}

The authors declare no conflict of interest. 


\section{References}

1. Bobo, D.; Robinson, K.J.; Islam, J.; Thurecht, K.J.; Corrie, S.R. Nanoparticle-based medicines: A review of FDA-approved materials and clinical trials to date. Pharm. Res. 2016, 33, 2373-2387, doi:10.1007/s11095-016-1958-5.

2. Van der Meel, R.; Lammers, T.; Hennink, W.E. Cancer nanomedicines: Oversold or underappreciated? Expert Opin. Drug Deliv. 2017, 14, 1-5, doi:10.1080/17425247.2017.1262346.

3. Mi, P.; Cabral, H.; Kataoka, K. Ligand-installed nanocarriers toward precision therapy. Adv. Mater. 2020, 32, 1-29, doi:10.1002/adma.201902604.

4. Varela-Moreira, A.; Shi, Y.; Fens, M.H.A.M.; Lammers, T.; Hennink, W.E.; Schiffelers, R.M. Clinical application of polymeric micelles for the treatment of cancer. Mater. Chem. Front. 2017, 1, 1485-1501, doi:10.1039/C6QM00289G.

5. Deng, C.; Jiang, Y.; Cheng, R.; Meng, F.; Zhong, Z. Biodegradable polymeric micelles for targeted and controlled anticancer drug delivery: Promises, progress and prospects. Nano Today 2012, 7, 467480, doi:10.1016/j.nantod.2012.08.005.

6. Cabral, H.; Miyata, K.; Osada, K.; Kataoka, K. Block copolymer micelles in nanomedicine applications. Chem. Rev. 2018, 118, 6844-6892, doi:10.1021/acs.chemrev.8b00199.

7. Wang, J.; Mao, W.; Lock, L.L.; Tang, J.; Sui, M.; Sun, W.; Cui, H.; Xu, D.; Shen, Y. The role of micelle size in tumor accumulation, penetration, and treatment. ACS Nano 2015, 9, 7195-7206, doi:10.1021/acsnano.5b02017.

8. Sun, Q.; Ojha, T.; Kiessling, F.; Lammers, T.; Shi, Y. Enhancing tumor penetration of nanomedicines. Biomacromolecules 2017, 18, 1449-1459, doi:10.1021/acs.biomac.7b00068.

9. Cabral, H.; Matsumoto, Y.; Mizuno, K.; Chen, Q.; Murakami, M.; Kimura, M.; Terada, Y.; Kano, M.R.; Miyazono, K.; Uesaka, M.; et al. Accumulation of sub-100 nm polymeric micelles in poorly permeable tumours depends on size. Nat. Nanotechnol. 2011, 6, 815-823, doi:10.1038/nnano.2011.166.

10. Wang, Z.; Wu, Z.; Liu, J.; Zhang, W. Particle morphology: An important factor affecting drug delivery by nanocarriers into solid tumors. Expert Opin. Drug Deliv. 2018, 15, 379-395, doi:10.1080/17425247.2018.1420051.

11. Pijpers, I.A.B.; Abdelmohsen, L.K.E.A.; Xia, Y.; Cao, S.; Williams, D.S.; Meng, F.; Hest, J.C.M.; Zhong, Z. Adaptive polymersome and micelle morphologies in anticancer nanomedicine: From design rationale to fabrication and proof-of-concept studies. Adv. Ther. 2018, 1, 1800068, doi:10.1002/adtp.201800068.

12. Qin, S.Y.; Cheng, Y.J.; Jiang, Z.W.; Ma, Y.H.; Zhang, A.Q. Morphology control of self-deliverable nanodrug with enhanced anticancer efficiency. Colloids Surf. B Biointerfaces 2018, 165, 345-354, doi:10.1016/j.colsurfb.2018.02.054.

13. Hare, J.I.; Lammers, T.; Ashford, M.B.; Puri, S.; Storm, G.; Barry, S.T. Challenges and strategies in anti-cancer nanomedicine development: An industry perspective. Adv. Drug Deliv. Rev. 2017, 108, 25-38, doi:10.1016/j.addr.2016.04.025.

14. Truong, N.P.; Whittaker, M.R.; Mak, C.W.; Davis, T.P. The importance of nanoparticle shape in cancer drug delivery. Expert Opin. Drug Deliv. 2015, 12, 129-142, doi:10.1517/17425247.2014.950564.

15. Thioune, O.; Fessi, H.; Devissaguet, J.P.; Puisieux, F. Preparation of pseudolatex by nanoprecipitation: Influence of the solvent nature on intrinsic viscosity and interaction constant. Int. J. Pharm. 1997, 146, 233-238, doi:10.1016/S0378-5173(96)04830-2. 

Microfluidics

16. Pagels, R.F.; Edelstein, J.; Tang, C.; Prud'homme, R.K. Controlling and predicting nanoparticle formation by block copolymer directed rapid precipitations. Nano Lett. 2018, 18, 1139-1144, doi:10.1021/acs.nanolett.7b04674.

17. Lepeltier, E.; Bourgaux, C.; Couvreur, P. Nanoprecipitation and the "Ouzo effect": Application to drug delivery devices. Adv. Drug Deliv. Rev. 2014, 71, 86-97, doi:10.1016/j.addr.2013.12.009.

18. Beck-Broichsitter, M.; Rytting, E.; Lebhardt, T.; Wang, X.; Kissel, T. Preparation of nanoparticles by solvent displacement for drug delivery: A shift in the "ouzo region" upon drug loading. Eur. J. Pharm. Sci. 2010, 41, 244-253, doi:10.1016/j.ejps.2010.06.007.

19. Zhang, C.; Pansare, V.J.; Prud'Homme, R.K.; Priestley, R.D. Flash nanoprecipitation of polystyrene nanoparticles. Soft Matter 2012, 8, 86-93, doi:10.1039/c1sm06182h.

20. Aubry, J.; Ganachaud, F.; Addad, J.P.C.; Cabane, B. Nanoprecipitation of polymethylmethacrylate by solvent shifting: 1. Boundaries. Langmuir 2009, 25, 1970-1979, doi:10.1021/la803000e.

21. Nicolai, T.; Colombani, O.; Chassenieux, C. Dynamic polymeric micelles versus frozen nanoparticles formed by block copolymers. Soft Matter 2010, 6, 3111-3118, doi:10.1039/b925666k.

22. Campos-Villalobos, G.; Siperstein, F.R.; Charles, A.; Patti, A. Solvent-induced morphological transitions in methacrylate-based block-copolymer aggregates. J. Colloid Interface Sci. 2020, 572, 133 140, doi:10.1016/j.jcis.2020.03.067.

23. Lejeune, E.; Drechsler, M.; Jestin, J.; Müller, A.H.E.; Chassenieux, C.; Colombani, O. Amphiphilic diblock copolymers with a moderately hydrophobic block: Toward dynamic micelles. Macromolecules 2010, 43, 2667-2671, doi:10.1021/ma902822g.

24. Valencia, P.M.; Pridgen, E.M.; Rhee, M.; Langer, R.; Farokhzad, O.C.; Karnik, R. Microfluidic platform for combinatorial synthesis and optimization of targeted nanoparticles for cancer therapy. ACS Nano 2013, 7, 10671-10680, doi:10.1021/nn403370e.

25. Chen, R.; Wulff, J.E.; Moffitt, M.G. Microfluidic processing approach to controlling drug delivery properties of curcumin-loaded block copolymer nanoparticles. Mol. Pharm. 2018, 15, 4517-4528, doi:10.1021/acs.molpharmaceut.8b00529.

26. LaMer, V.K.; Dinegar, R.H. Theory, production and mechanism of formation of monodispersed hydrosols. J. Am. Chem. Soc. 1950, 72, 4847-4854, doi:10.1021/ja01167a001.

27. Liu, D.; Zhang, H.; Fontana, F.; Hirvonen, J.T.; Santos, H.A. Microfluidic-assisted fabrication of carriers for controlled drug delivery. Lab Chip 2017, 17, 1856-1883, doi:10.1039/c7lc00242d.

28. Liu, D.; Cito, S.; Zhang, Y.; Wang, C.F.; Sikanen, T.M.; Santos, H.A. A versatile and robust microfluidic platform toward high throughput synthesis of homogeneous nanoparticles with tunable properties. Adv. Mater. 2015, 27, 2298-2304, doi:10.1002/adma.201405408.

29. Dou, Y.; Wang, B.; Jin, M.; Yu, Y.; Zhou, G.; Shui, L. A review on self-assembly in microfluidic devices. J. Micromech. Microeng. 2017, 27, 113002, doi:10.1088/1361-6439/aa84db.

30. Wang, L.; Wang, J. Self-assembly of colloids based on microfluidics. Nanoscale 2019, 11, 1670816722, doi:10.1039/c9nr06817a.

31. Wang, L.; Sánchez, S. Self-assembly via microfluidics. Lab Chip 2015, 15, 4383-4386, doi:10.1039/c5lc90116b.

32. Chiesa, E.; Dorati, R.; Modena, T.; Conti, B.; Genta, I. Multivariate analysis for the optimization of microfluidics-assisted nanoprecipitation method intended for the loading of small hydrophilic drugs into PLGA nanoparticles. Int. J. Pharm. 2018, 536, 165-177, doi:10.1016/j.ijpharm.2017.11.044.

33. Xu, J.; Zhang, S.; MacHado, A.; Lecommandoux, S.; Sandre, O.; Gu, F.; Colin, A. Controllable microfluidic production of drug-loaded PLGA nanoparticles using partially water-miscible mixed solvent microdroplets as a precursor. Sci. Rep. 2017, 7, 1-12, doi:10.1038/s41598-017-05184-5. 


\section{Chapter 4}

34. Ding, S.; Anton, N.; Vandamme, T.F.; Serra, C.A. Microfluidic nanoprecipitation systems for preparing pure drug or polymeric drug loaded nanoparticles: An overview. Expert Opin. Drug Deliv. 2016, 13, 1447-1460, doi:10.1080/17425247.2016.1193151.

35. Martins, J.P.; Torrieri, G.; Santos, H.A. The importance of microfluidics for the preparation of nanoparticles as advanced drug delivery systems. Expert Opin. Drug Deliv. 2018, 15, 469-479, doi:10.1080/17425247.2018.1446936.

36. Liu, D.; Zhang, H.; Fontana, F.; Hirvonen, J.T.; Santos, H.A. Current developments and applications of microfluidic technology toward clinical translation of nanomedicines. Adv. Drug Deliv. Rev. 2018, 128, 54-83, doi:10.1016/j.addr.2017.08.003.

37. Soleimani, S.; Hasani-Sadrabadi, M.M.; Majedi, F.S.; Dashtimoghadam, E.; Tondar, M.; Jacob, K.I. Understanding biophysical behaviours of microfluidic-synthesized nanoparticles at nanobiointerface. Colloids Surf. B Biointerfaces 2016, 145, 802-811, doi:10.1016/j.colsurfb.2016.06.002.

38. Bally, F.; Garg, D.K.; Serra, C.A.; Hoarau, Y.; Anton, N.; Brochon, C.; Parida, D.; Vandamme, T.; Hadziioannou, G. Improved size-tunable preparation of polymeric nanoparticles by microfluidic nanoprecipitation. Polymer 2012, 53, 5045-5051, doi:10.1016/j.polymer.2012.08.039.

39. Keßler, S.; Drese, K.; Schmid, F. Simulating copolymeric nanoparticle assembly in the co-solvent method: How mixing rates control final particle sizes and morphologies. Polymer 2017, 126, 9-18, doi:10.1016/j.polymer.2017.07.057.

40. Hamdallah, S.I.; Zoqlam, R.; Erfle, P.; Blyth, M.; Alkilany, A.M.; Dietzel, A.; Qi, S. Microfluidics for pharmaceutical nanoparticle fabrication: The truth and the myth. Int. J. Pharm. 2020, 584, 119408, doi:10.1016/j.ijpharm.2020.119408.

41. Lebleu, C.; Rodrigues, L.; Guigner, J.M.; Brûlet, A.; Garanger, E.; Lecommandoux, S. Selfassembly of PEG-b-PTMC copolymers: Micelles and polymersomes size control. Langmuir 2019, 35, 13364-13374, doi:10.1021/acs.langmuir.9b02264.

42. Bagheri, M.; Bresseleers, J.; Varela-Moreira, A.; Sandre, O.; Meeuwissen, S.A.; Schiffelers, R.M.; Metselaar, J.M.; Van Nostrum, C.F.; Van Hest, J.C.M.; Hennink, W.E. Effect of formulation and processing parameters on the size of mPEG- b-p(HPMA-Bz) polymeric micelles. Langmuir 2018, 34, 15495-15506, doi:10.1021/acs.langmuir.8b03576.

43. Sundararajan, P.; Stroock, A.D. Transport phenomena in chaotic laminar flows. Annu. Rev. Chem. Biomol. Eng. 2012, 3, 473-496, doi:10.1146/annurev-chembioeng-062011-081000.

44. Le Fer, G.; Portes, D.; Goudounet, G.; Guigner, J.M.; Garanger, E.; Lecommandoux, S. Design and self-assembly of PBLG-: B -ELP hybrid diblock copolymers based on synthetic and elastin-like polypeptides. Org. Biomol. Chem. 2017, 15, 10095-10104, doi:10.1039/c7ob01945a.

45. Bresseleers, J.; Bagheri, M.; Storm, G.; Metselaar, J.M.; Hennink, W.E.; Meeuwissen, S.A.; Van Hest, J.C.M. Scale-up of the manufacturing process to produce docetaxel-loaded mPEG-b-p(HPMABz) block copolymer micelles for pharmaceutical applications. Org. Process Res. Dev. 2019, 23, $2707-$ 2715, doi:10.1021/acs.oprd.9b00387.

46. Shi, Y.; Van Steenbergen, M.J.; Teunissen, E.A.; Novo, L.; Gradmann, S.; Baldus, M.; Van Nostrum, C.F.; Hennink, W.E. П-П Stacking increases the stability and loading capacity of thermosensitive polymeric micelles for chemotherapeutic drugs. Biomacromolecules 2013, 14, 18261837, doi:10.1021/bm400234c.

47. Micromixer Chip. Available online: https://www.dolomite-microfluidics.com/product/micromixerchip/ (accessed on 26 May 2020).

48. Zimm, B.H. Apparatus and methods for measurement and interpretation of the angular variation of light scattering; Preliminary results on polystyrene solutions. J. Chem. Phys. 1948, 16, 1099-1116, doi:10.1063/1.1746740. 

Microfluidics

49. Shi, Y.; Van Der Meel, R.; Theek, B.; Oude Blenke, E.; Pieters, E.H.E.; Fens, M.H.A.M.; Ehling, J.; Schiffelers, R.M.; Storm, G.; Van Nostrum, C.F.; et al. Complete regression of xenograft tumors upon targeted delivery of paclitaxel via Pi-Pi stacking stabilized polymeric micelles. ACS Nano 2015, 9, 3740-3752, doi:10.1021/acsnano.5b00929.

50. Nelson, A.; Cosgrove, T. A Small-angle neutron scattering study of adsorbed poly(ethylene oxide) on laponite. Langmuir 2004, 20, 2298-2304, doi:10.1021/la035268t.

51. Jain, S.; Bates, F.S. On the origins of morphological complexity in block copolymer surfactants. Science 2003, 300, 460-464.

52. D'Addio, S.M.; Prud'homme, R.K. Controlling drug nanoparticle formation by rapid precipitation. Adv. Drug Deliv. Rev. 2011, 63, 417-426, doi:10.1016/j.addr.2011.04.005.

53. Kunz, D.; Thurn, A.; Burchard, W. Dynamic light scattering from spherical particles. Colloid Polym. Sci. 1983, 261, 635-644, doi:10.1007/BF01415033.

54. Ma, C.; Pan, P.; Shan, G.; Bao, Y.; Fujita, M.; Maeda, M. Core-shell structure, biodegradation, and drug release behavior of poly(lactic acid)/poly(ethylene glycol) block copolymer micelles tuned by macromolecular stereostructure. Langmuir 2015, 31, 1527-1536, doi:10.1021/1a503869d.

55. Nie, T.; Zhao, Y.; Xie, Z.; Wu, C. Micellar formation of poly(caprolactone-block-ethylene oxideblock-caprolactone) and its enzymatic biodegradation in aqueous dispersion. Macromolecules 2003, 36, 8825-8829, doi:10.1021/ma035131+.

56. Nguyen, V.T.A.; De Pauw-Gillet, M.C.; Sandre, O.; Gauthier, M. Biocompatible polyion complex micelles synthesized from arborescent polymers. Langmuir 2016, 32, 13482-13492, doi:10.1021/acs.langmuir.6b03683.

57. Giacomelli, C.; Schmidt, V.; Aissou, K.; Borsali, R. Block copolymer systems: From single chain to self-assembled nanostructures. Langmuir 2010, 26, 15734-15744, doi:10.1021/la100641j.

58. Kale, T.S.; Klaikherd, A.; Popere, B.; Thayumanavan, S. Supramolecular assemblies of amphiphilic homopolymers. Langmuir 2009, 25, 9660-9670, doi:10.1021/la900734d.

59. Abdelmohsen, L.K.E.A.; Rikken, R.S.M.; Christianen, P.C.M.; van Hest, J.C.M.; Wilson, D.A. Shape characterization of polymersome morphologies via light scattering techniques. Polymer 2016, 107, 445-449, doi:10.1016/j.polymer.2016.06.067.

60. Chécot, F.; Brûlet, A.; Oberdisse, J.; Gnanou, Y.; Mondain-Monval, O.; Lecommandoux, S. Structure of polypeptide-based diblock copolymers in solution: Stimuli-responsive vesicles and micelles. Langmuir 2005, 21, 4308-4315, doi:10.1021/la0468500.

61. Antonietti, M.; Förster, S. Vesicles and liposomes: A self-assembly principle beyond lipids. $A d v$. Mater. 2003, 15, 1323-1333, doi:10.1002/adma.200300010.

62. Nagarajan, R. Molecular packing parameter and surfactant self-assembly: The neglected role of the surfactant tail. Langmuir 2002, 18, 31-38, doi:10.1021/la010831y.

63. He, X.; Schmid, F. Dynamics of spontaneous vesicle formation in dilute solutions of amphiphilic diblock copolymers. Macromolecules 2006, 39, 2654-2662, doi:10.1021/ma052536g.

64. Mai, Y.; Eisenberg, A. Self-assembly of block copolymers. Chem. Soc. Rev. 2012, 41, 5969-5985, doi:10.1039/c2cs35115c. 


\section{Supporting Information}

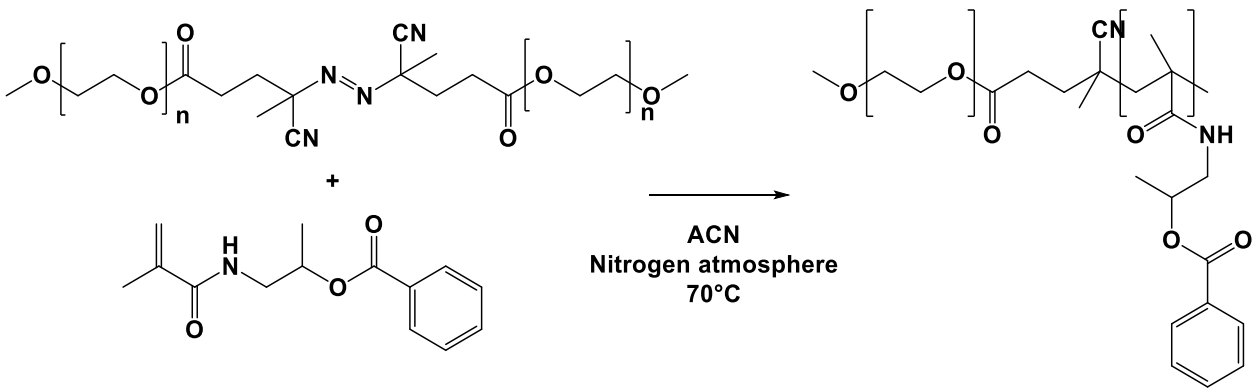

Scheme S1. Synthesis of mPEG- $b-\mathrm{p}($ HPMA-Bz).

${ }^{1} \mathrm{H}-\mathrm{NMR}$ of mPEG- $b$-p(HPMA-Bz): $8.0(\mathrm{~b}, 2 \mathrm{H}$, aromatic $\mathrm{CH}), 7.55(\mathrm{~b}, 1 \mathrm{H}$, aromatic $\mathrm{CH})$, 7.65 (b, 2H, aromatic $\mathrm{CH}), 7.35$ (b, CO-NH- $\left.\mathrm{CH}_{2}\right), 5.0$ (b, $\mathrm{NH}-\mathrm{CH}_{2}-\mathrm{CH}\left(\mathrm{CH}_{3}\right)-\mathrm{O}-(\mathrm{Bz})$ ), 3.40-3.60 (b, mPEG5000 methylene protons, $\mathrm{O}-\mathrm{CH}_{2}-\mathrm{CH}_{2}$ ), 3.1 (b, $\left.\mathrm{NH}-\overline{\mathrm{CH}}_{2}-\mathrm{CH}\right), 0.6-2.2$ (b, the rest of the protons are from the methyl and backbone $\mathrm{CH}_{2}$ protons).

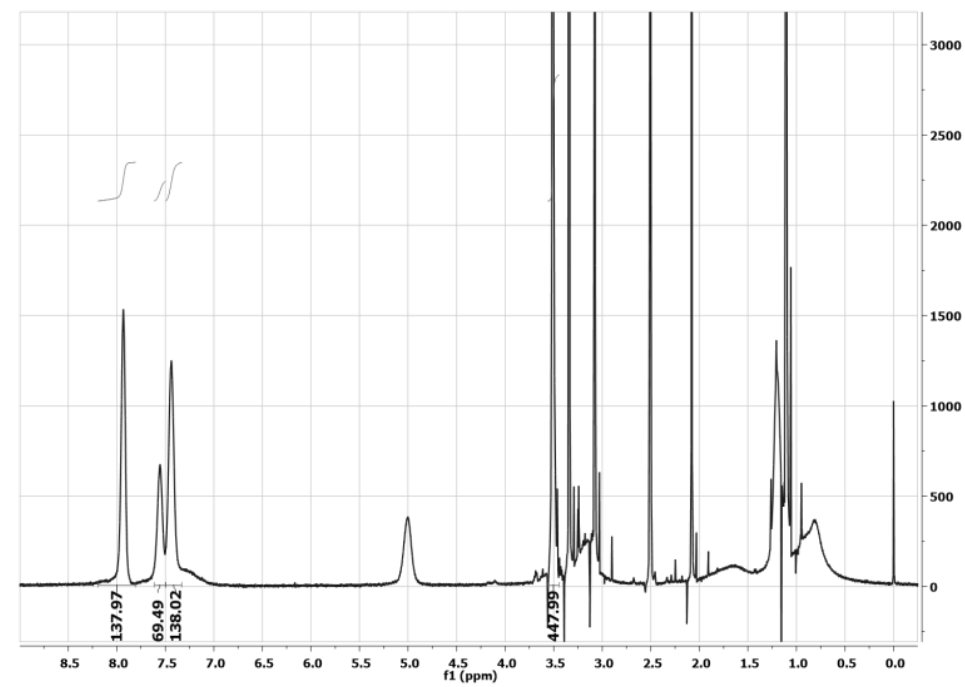

Figure S1. ${ }^{1} \mathrm{H}-\mathrm{NMR}$ of block copolymer A mPEG $5 \mathrm{~K}-b-\mathrm{p}(\mathrm{HPMA}-\mathrm{Bz})_{17.1 \mathrm{~K}}$. 
Tuning Size and Morphology of mPEG-b-p(HPMA-Bz) Copolymer Self-Assemblies Using Microfluidics

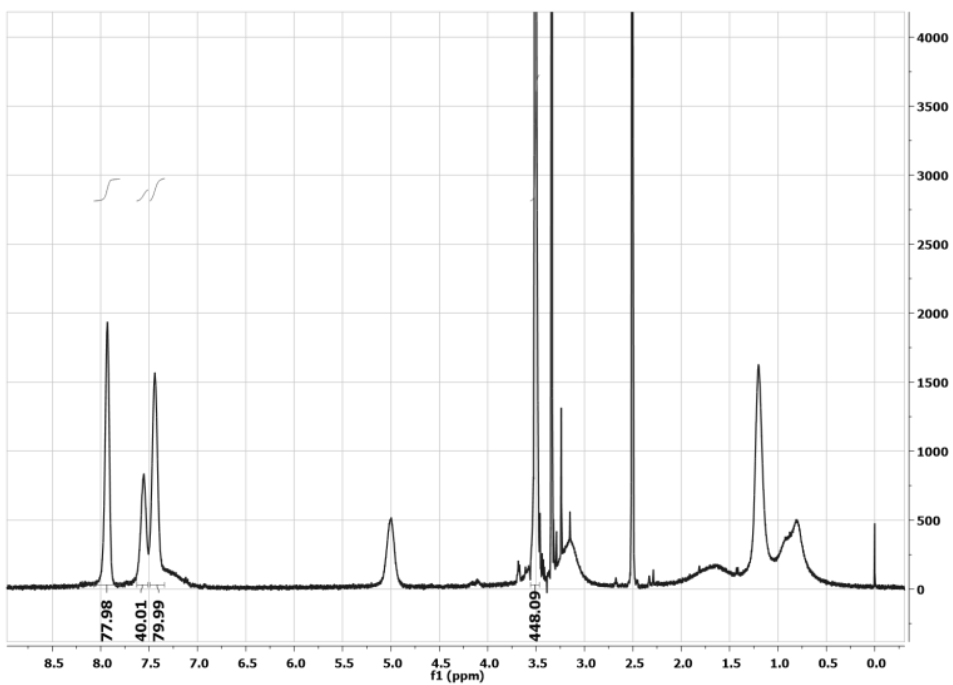

Figure S2. ${ }^{1} \mathrm{H}-\mathrm{NMR}$ of block copolymer B mPEG $5 \mathrm{~K}-b$-p(HPMA-Bz) ${ }_{10.0 \mathrm{~K}}$.

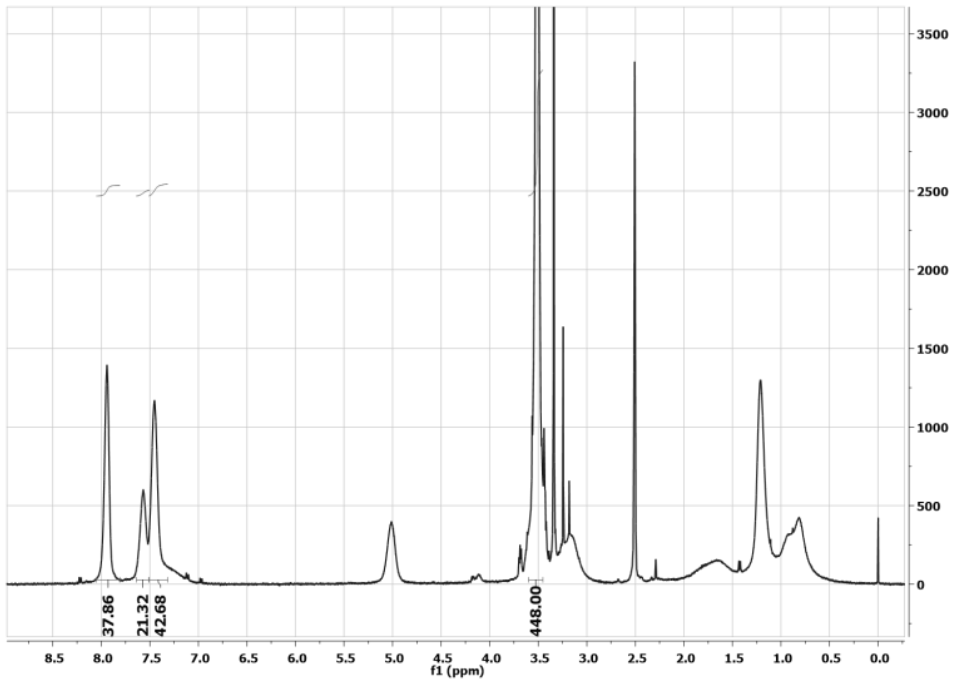

Figure S3. ${ }^{1} \mathrm{H}-\mathrm{NMR}$ of block copolymer $\mathrm{C}$ mPEG $5 \mathrm{~K}-b-\mathrm{p}(\mathrm{HPMA}-\mathrm{Bz}) 5.2 \mathrm{~K}$. 


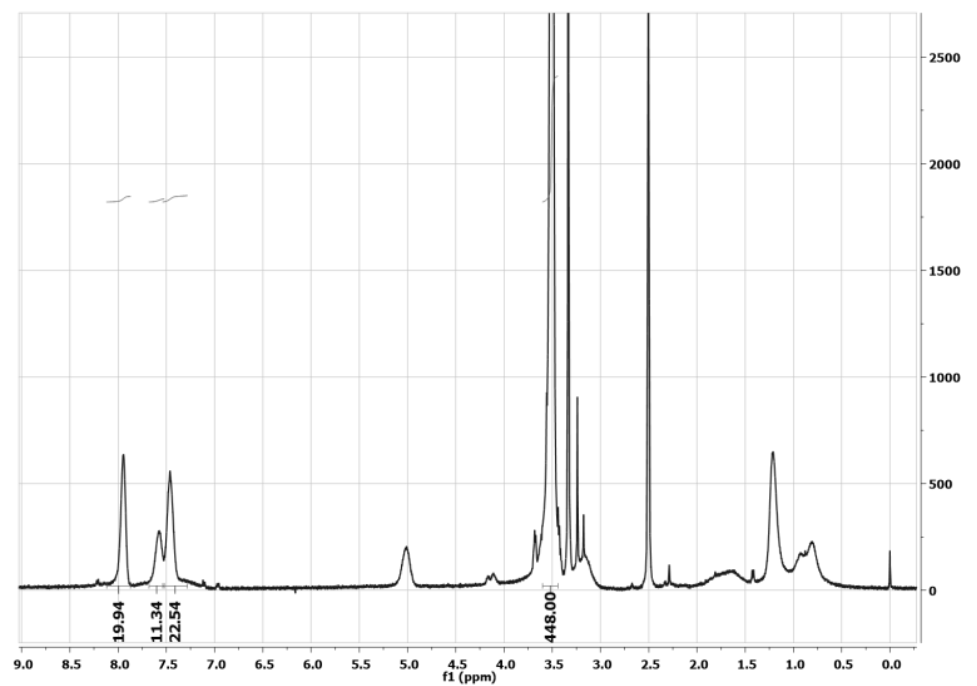

Figure S4. ${ }^{1} \mathrm{H}-\mathrm{NMR}$ of block copolymer D mPEG $5 \mathrm{~K}-b-\mathrm{p}(\mathrm{HPMA}-\mathrm{Bz}) 2.7 \mathrm{~K}$.

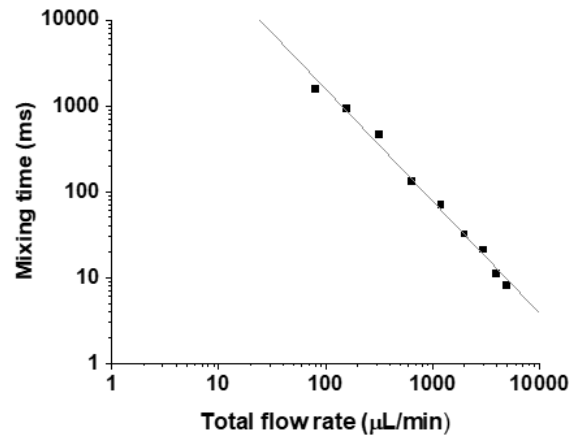

(a)

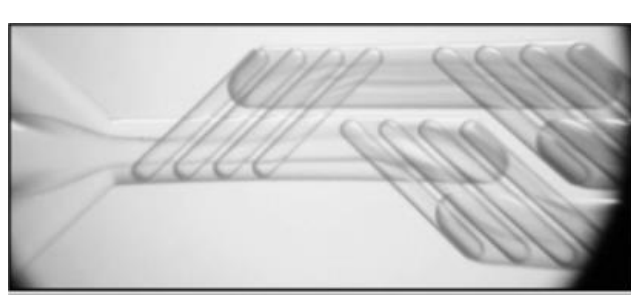

(b)

Figure S5. (a) Mixing time $\tau_{\mathrm{M}}$ (ms) of $\mathrm{NaOH}$ and phenolphthalein solutions plotted against total flowrates $Q_{\text {tot }}(\mu \mathrm{L} / \mathrm{min})$ for 1:1 ratio at each pump and extrapolated to the following equation $\left(\tau_{\mathrm{M}}\right)=$ $6.4133 \cdot 10^{4} Q_{\text {tot }}^{1.306}$. (b) The photograph shows the calibration experiment of the mixing time using two identical flowrates of respectively phenolphthalein and $\mathrm{NaOH}$ solutions. Data and photograph were taken from the specifications on the manufacturer's website ${ }^{1}$. 
Tuning Size and Morphology of mPEG-b-p(HPMA-Bz) Copolymer Self-Assemblies Using Microfluidics

Table S1. Flow rates and their approximated mixing times as calculated using the information from Figure S5.

\begin{tabular}{cc}
\hline $\boldsymbol{Q}_{\text {tot }}(\boldsymbol{\mu} \mathbf{L} / \mathbf{m i n})$ & $\boldsymbol{\tau}_{\mathbf{M}}(\mathbf{m s})$ \\
\hline 100 & 1570 \\
200 & 634 \\
350 & 305 \\
500 & 192 \\
1600 & 42 \\
\hline
\end{tabular}

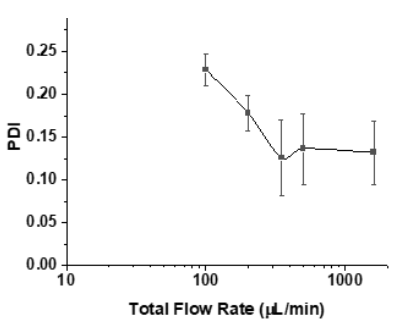

(a)

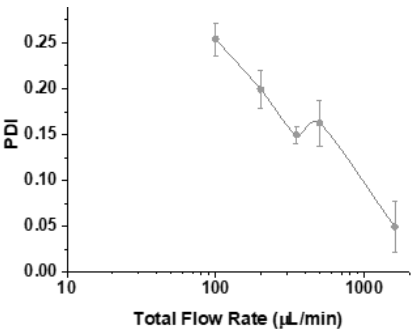

(b)

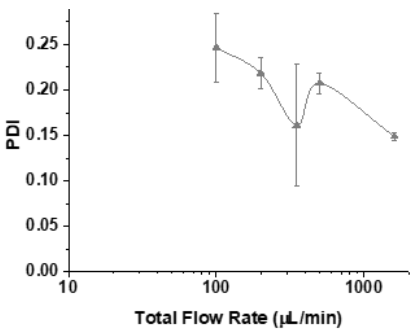

(c)

Figure S6. PDI values of block copolymer D mPEG $5 \mathrm{~K}-b-\mathrm{p}(\mathrm{HPMA}-\mathrm{Bz})_{2.7 \mathrm{~K}}$ nanostructures as a function of mixing time. (a) $5 \mathrm{mg} / \mathrm{mL}$, (b) $10 \mathrm{mg} / \mathrm{mL}$ and (c) $20 \mathrm{mg} / \mathrm{mL}$. 
(a)

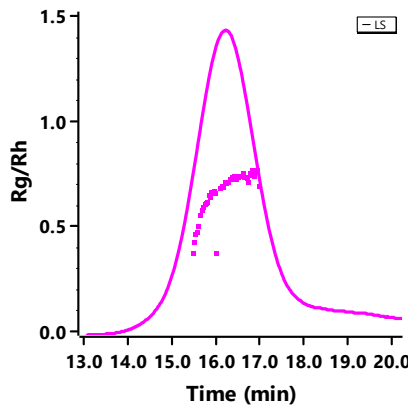

(d)

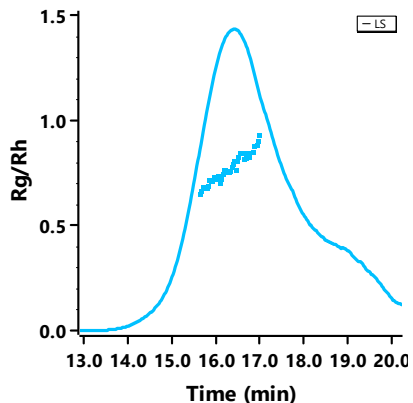

(b)

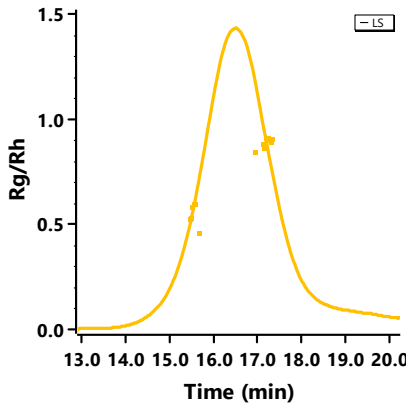

(e)

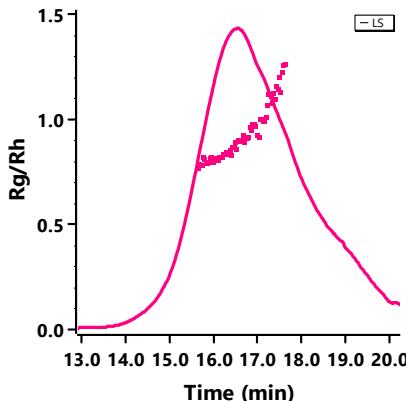

(c)

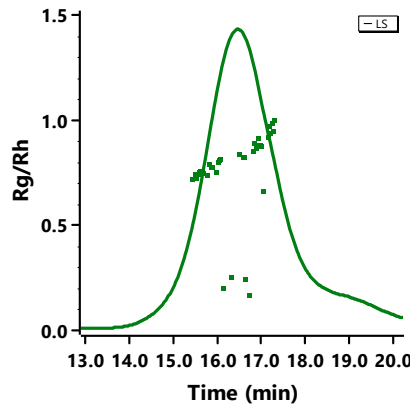

(f)

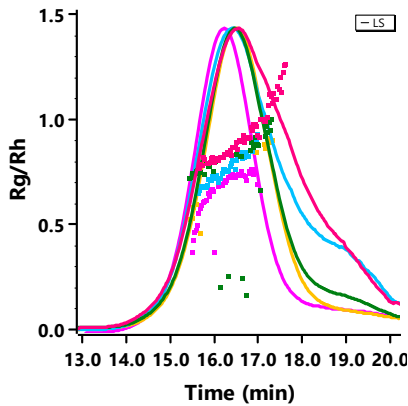

Figure S7. $R_{\mathrm{g}} / R_{\mathrm{h}}$ traces of the $\mathrm{AF}_{4}-\mathrm{MALS}$ fractograms of nanoparticles made with block copolymer A $\mathrm{mPEG}_{5 \mathrm{~K}}-b$ - $\mathrm{p}(\mathrm{HPMA}-\mathrm{Bz})_{17.1 \mathrm{~K}}$ with a concentration of $5 \mathrm{mg} / \mathrm{mL}$ and microfluidic flow rates (a) 1600 $\mu \mathrm{L} / \mathrm{min}$, (b) $500 \mu \mathrm{L} / \mathrm{min}$, (c) $350 \mu \mathrm{L} / \mathrm{min}$, (d) $200 \mu \mathrm{L} / \mathrm{min}$, (e) $100 \mu \mathrm{L} / \mathrm{min}$ and (f) all microfluidic flow rates together in one graph. 
Tuning Size and Morphology of mPEG-b-p(HPMA-Bz) Copolymer Self-Assemblies Using Microfluidics

Table S2. Characteristics of block copolymer B $\mathrm{mPEG}_{5 \mathrm{~K}}-b-\mathrm{p}(\mathrm{HPMA}-\mathrm{Bz})_{10.0 \mathrm{~K}}$ nanoparticles as determined by $\mathrm{AF}_{4}$-MALLS.

\begin{tabular}{cccccccccccc}
\hline \multirow{2}{*}{$\mathbf{C}$} & $\mathbf{Q}$ & \multicolumn{1}{c}{ Peak 1 } & \multicolumn{6}{c}{ Peak 2 } \\
\cline { 3 - 11 } & & $\boldsymbol{R}_{\mathrm{g}}$ & $\boldsymbol{R}_{\mathrm{h}}$ & $\boldsymbol{R}_{\mathrm{g}} / \boldsymbol{R}_{\mathrm{h}}$ & $\boldsymbol{M}_{\text {w(n) }}$ & $\boldsymbol{N}_{\text {agg }}$ & $\boldsymbol{R}_{\mathrm{g}}$ & $\boldsymbol{R}_{\mathrm{h}}$ & $\boldsymbol{R}_{\mathrm{g}} / \boldsymbol{R}_{\mathrm{h}}$ & $\boldsymbol{M}_{\text {w(np) }}$ & $\boldsymbol{N}_{\text {agg }}$ \\
\hline 5 & 100 & 19 & 25 & 0.77 & 16 & 1060 & - & - & - & - & - \\
5 & 200 & 18 & 25 & 0.72 & 16 & 1060 & - & - & - & - & - \\
5 & 350 & 17 & 23 & 0.72 & 15 & 1000 & - & - & - & - & - \\
5 & 500 & 14 & 22 & 0.63 & 13 & 880 & 73 & 49 & 1.49 & 232 & 15500 \\
5 & 1600 & 13 & 22 & 0.59 & 13 & 860 & 84 & 52 & 1.62 & 263 & 17500 \\
\hline 10 & 100 & 15 & 21 & 0.72 & 12 & 770 & 52 & 46 & 1.13 & 1263 & 84200 \\
10 & 200 & 13 & 20 & 0.65 & 11 & 720 & 70 & 46 & 1.52 & 585 & 39000 \\
10 & 350 & 14 & 20 & 0.69 & 11 & 730 & 80 & 48 & 1.67 & 1717 & 114500 \\
10 & 500 & 14 & 21 & 0.69 & 12 & 780 & - & 51 & - & - & - \\
10 & 1600 & 14 & 20 & 0.67 & 11 & 730 & 85 & 49 & 1.73 & 1441 & 96100 \\
\hline 20 & 100 & 14 & 20 & 0.69 & 9.6 & 640 & - & - & - & - & - \\
20 & 200 & 16 & 21 & 0.75 & 9.9 & 660 & - & - & - & - & - \\
20 & 350 & 14 & 20 & 0.70 & 9.6 & 640 & - & - & - & - & - \\
20 & 500 & 11 & 20 & 0.56 & 9.9 & 660 & - & - & - & - & - \\
20 & 1600 & 13 & 20 & 0.65 & 9.9 & 660 & - & - & - & - & - \\
\hline
\end{tabular}

C, concentration $(\mathrm{mg} / \mathrm{mL}) ; \mathrm{Q}$, flow rate $(\mu \mathrm{L} / \mathrm{min}) ; \mathrm{R}_{\mathrm{g}}$, radius of gyration $(\mathrm{nm}) ; \mathrm{R}_{\mathrm{h}}$, hydrodynamic radius $(\mathrm{nm}) ; \mathrm{M}_{\mathrm{w}}(\mathrm{np})$, weight average molecular weight of the nanoparticles $\left(10^{3} \mathrm{kDa}\right)$ and $\mathrm{N}_{\mathrm{agg}}$, nanoparticle aggregation number. 
(a)

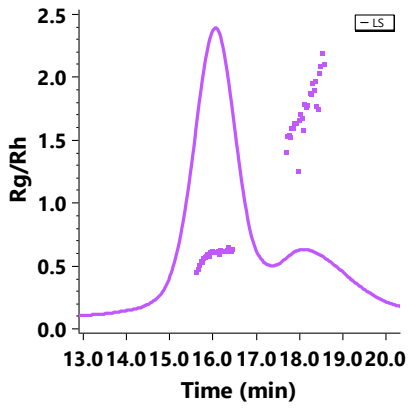

(d)

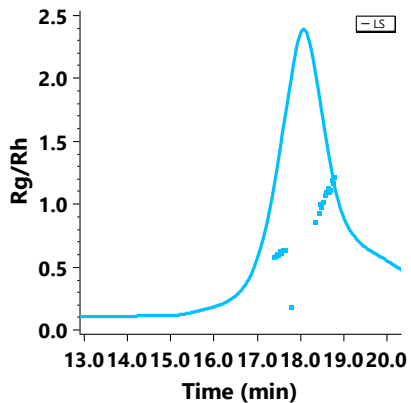

(b)

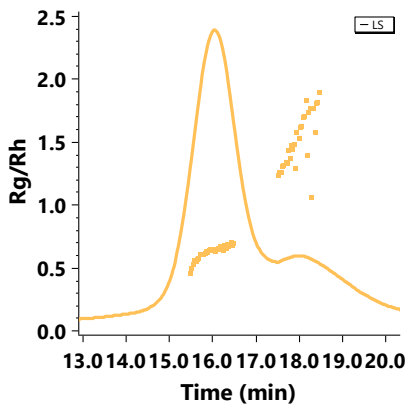

(e)

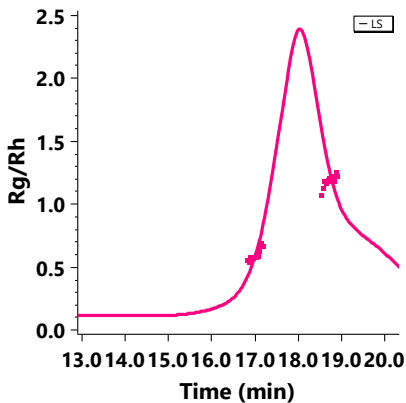

(c)

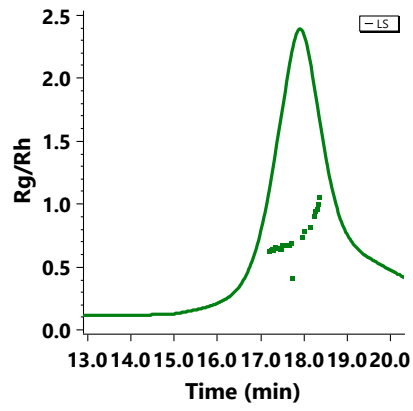

(f)

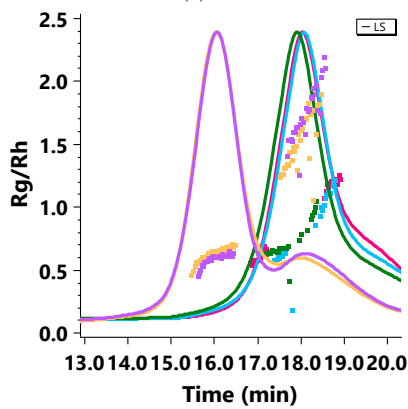

Figure S8. $R_{\mathrm{g}} / R_{\mathrm{h}}$ traces of the $\mathrm{AF}_{4}-\mathrm{MALS}$ fractograms of nanoparticles made with block copolymer $\mathrm{B}$ $\mathrm{mPEG}_{5 \mathrm{~K}}-b-\mathrm{p}(\mathrm{HPMA}-\mathrm{Bz})_{10.0 \mathrm{~K}}$ with a concentration of $5 \mathrm{mg} / \mathrm{mL}$ and microfluidic flow rates (a) 1600 $\mu \mathrm{L} / \mathrm{min}$, (b) $500 \mu \mathrm{L} / \mathrm{min}$, (c) $350 \mu \mathrm{L} / \mathrm{min}$, (d) $200 \mu \mathrm{L} / \mathrm{min}$, (e) $100 \mu \mathrm{L} / \mathrm{min}$ and (f) all microfluidic flow rates together in one graph.

(a)

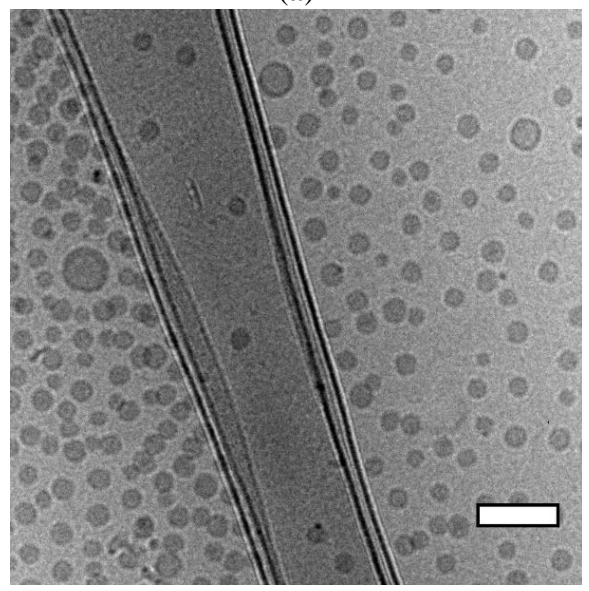

(b)

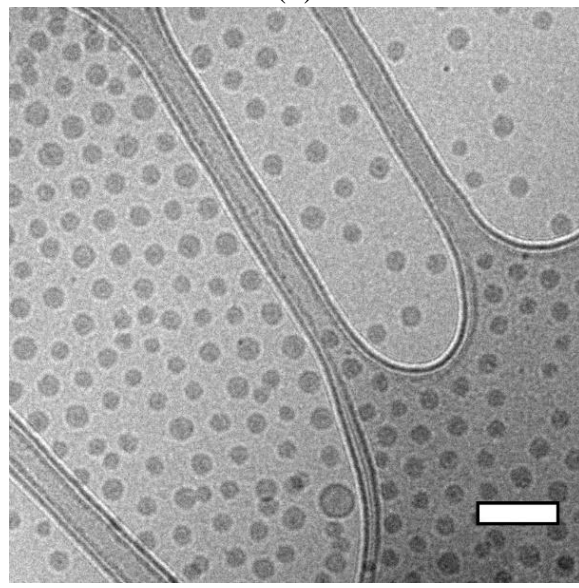

Figure S9. Cryo-TEM pictures of block copolymer B mPEG $_{5 \mathrm{~K}}-b-\mathrm{p}(\mathrm{HPMA}-\mathrm{Bz})_{10.0 \mathrm{~K}}$ nanoparticles prepared at a concentration of $10 \mathrm{mg} / \mathrm{mL}$ and flow rates Scale bars indicate $100 \mathrm{~nm}$. (a) $100 \mu \mathrm{L} / \mathrm{min}$ and (b) $350 \mu \mathrm{L} / \mathrm{min}$. 
Tuning Size and Morphology of mPEG-b-p(HPMA-Bz) Copolymer Self-Assemblies Using Microfluidics

Table S3. Characteristics of polymer C mPEG $5 \mathrm{~K}-b-\mathrm{p}(\mathrm{HPMA}-\mathrm{Bz})_{5.2 \mathrm{~K}}$ nanoparticles as determined by $\mathrm{AF}_{4}$-MALLS.

\begin{tabular}{cccccccccccc}
\hline \multirow{3}{*}{$\mathbf{C}$} & $\mathbf{Q}$ & \multicolumn{1}{c}{ Peak 1 } & \multicolumn{7}{c}{ Peak 2 } \\
\cline { 3 - 12 } & & $\boldsymbol{R}_{\mathrm{g}}$ & $\boldsymbol{R}_{\mathrm{h}}$ & $\boldsymbol{R}_{\mathrm{g}} / \boldsymbol{R}_{\mathrm{h}}$ & $\boldsymbol{M}_{\mathrm{w}(\mathrm{np})}$ & $\boldsymbol{N}_{\text {agg }}$ & $\boldsymbol{R}_{\mathrm{g}}$ & $\boldsymbol{R}_{\mathrm{h}}$ & $\boldsymbol{R}_{\mathrm{g}} / \boldsymbol{R}_{\mathrm{h}}$ & $\boldsymbol{M}_{\mathbf{w}(\mathrm{np})}$ & $\boldsymbol{N}_{\text {agg }}$ \\
\hline 5 & 100 & 12 & 20 & 0.61 & 6.5 & 640 & - & - & - & - & - \\
5 & 200 & 11 & 18 & 0.63 & 5.2 & 510 & 41 & 44 & 0.93 & 96 & 9400 \\
5 & 350 & 11 & 18 & 0.64 & 5.3 & 520 & 35 & 38 & 0.92 & 48 & 4700 \\
5 & 500 & 11 & 19 & 0.56 & 5.9 & 580 & - & - & - & - & - \\
5 & 1600 & 11 & 18 & 0.65 & 5.3 & 520 & - & - & - & - & - \\
\hline 10 & 100 & 14 & 17 & 0.80 & 4.5 & 440 & 58 & 42 & 1.38 & 210 & 20200 \\
10 & 200 & 13 & 17 & 0.77 & 4.7 & 460 & - & - & - & - & - \\
10 & 350 & 11 & 17 & 0.66 & 4.3 & 420 & - & - & - & - & - \\
10 & 500 & 13 & 17 & 0.76 & 4.3 & 420 & - & - & - & - & - \\
10 & 1600 & 12 & 17 & 0.72 & 4.6 & 450 & - & - & - & - & - \\
\hline 20 & 100 & 12 & 17 & 0.73 & 4.1 & 400 & 87 & 116 & 0.75 & 25 & 2500 \\
20 & 200 & 10 & 16 & 0.60 & 3.6 & 360 & - & - & - & - & - \\
20 & 350 & 11 & 16 & 0.68 & 3.9 & 380 & - & - & - & - & - \\
20 & 500 & 11 & 17 & 0.67 & 3.9 & 390 & - & - & - & - & - \\
20 & 1600 & 10 & 16 & 0.63 & 3.6 & 360 & - & - & - & - & - \\
\hline
\end{tabular}

C, concentration $(\mathrm{mg} / \mathrm{mL}) ; \mathrm{Q}$, flow rate $(\mu \mathrm{L} / \mathrm{min}) ; \mathrm{R}_{\mathrm{g}}$, radius of gyration $(\mathrm{nm}) ; \mathrm{R}_{\mathrm{h}}$, hydrodynamic radius $(\mathrm{nm}) ; \mathrm{M}_{\mathrm{w}}(\mathrm{np})$, weight average molecular weight of the nanoparticles $\left(10^{3} \mathrm{kDa}\right)$ and $\mathrm{N}_{\mathrm{agg}}$, nanoparticle aggregation number. 
(a)

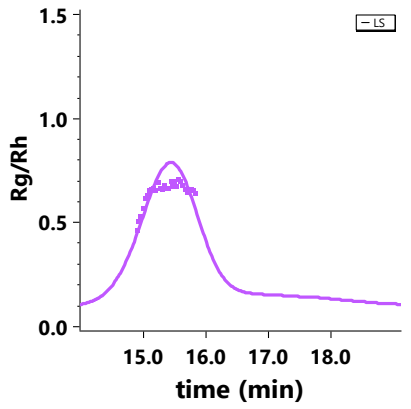

(d)

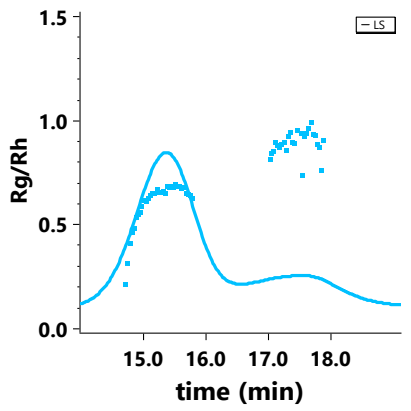

(b)

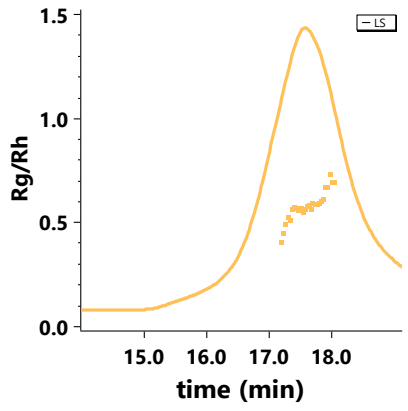

(e)

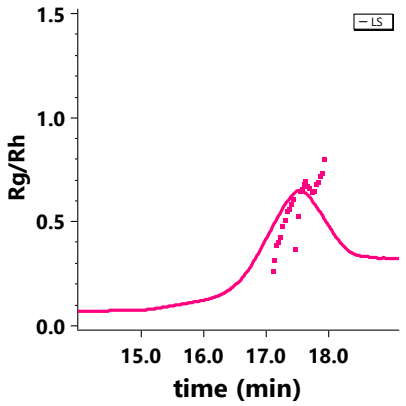

(c)

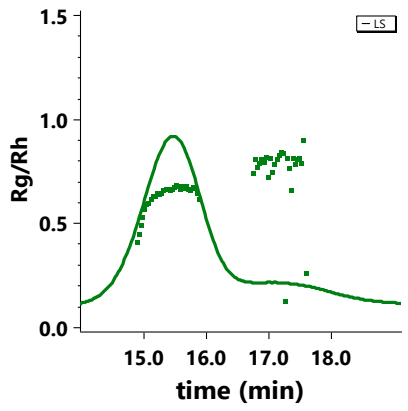

(f)

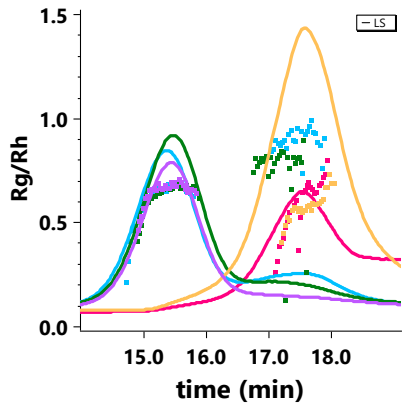

Figure S10. $R_{\mathrm{g}} / R_{\mathrm{h}}$ traces of the $\mathrm{AF}_{4}$-MALS fractograms of nanoparticles made with block copolymer $\mathrm{C}_{\mathrm{mPEG}} \mathrm{F}_{5 \mathrm{~K}}-b$-p $\left(\mathrm{HPMA}_{\mathrm{B}} \mathrm{Bz}\right)_{5.2 \mathrm{~K}}$ with a concentration of $5 \mathrm{mg} / \mathrm{mL}$ and microfluidic flow rates (a) 1600 $\mu \mathrm{L} / \mathrm{min}$, (b) $500 \mu \mathrm{L} / \mathrm{min}$, (c) $350 \mu \mathrm{L} / \mathrm{min}$, (d) $200 \mu \mathrm{L} / \mathrm{min}$, (e) $100 \mu \mathrm{L} / \mathrm{min}$ and (f) all microfluidic flow rates together in one graph.

(a)

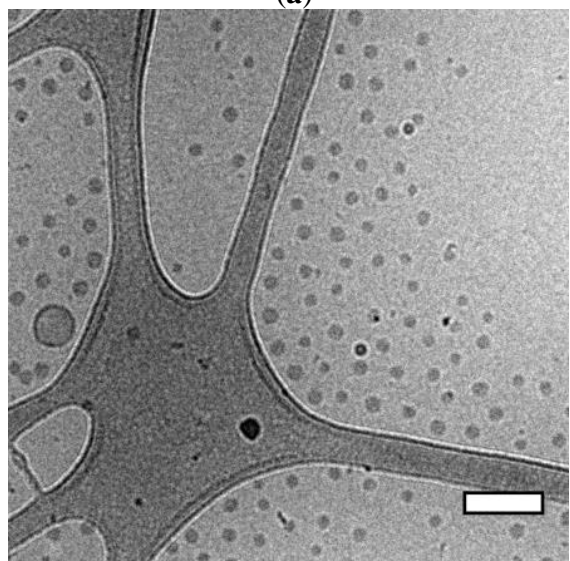

(b)

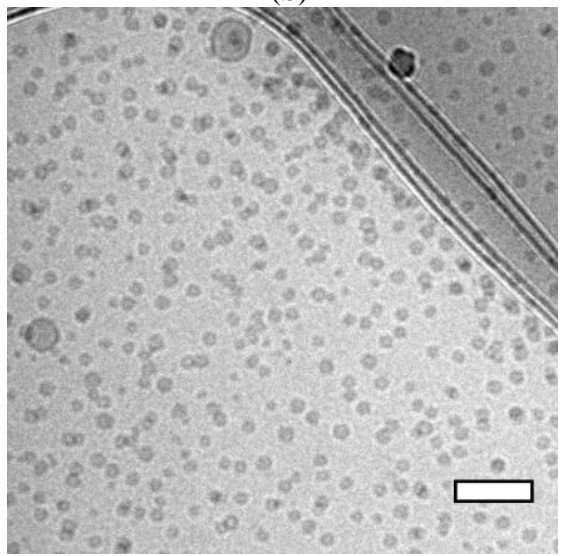

Figure S11. Cryo-TEM pictures of block copolymer C mPEG ${ }_{5 \mathrm{~K}}-b-\mathrm{p}(\mathrm{HPMA}-\mathrm{Bz})_{5.2 \mathrm{~K}}$ nanoparticles prepared at a concentration of $5 \mathrm{mg} / \mathrm{mL}$ and flow rates. Scale bars indicate $100 \mathrm{~nm}$. (a) $100 \mu \mathrm{L} / \mathrm{min}$ and (b) $350 \mu \mathrm{L} / \mathrm{min}$. 
(a)

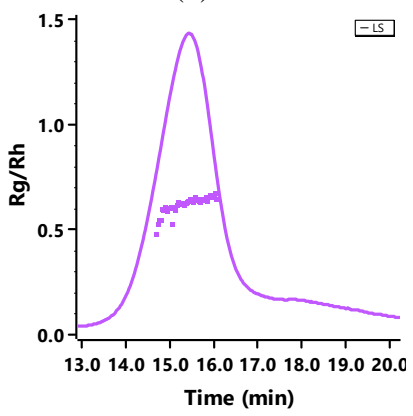

(d)

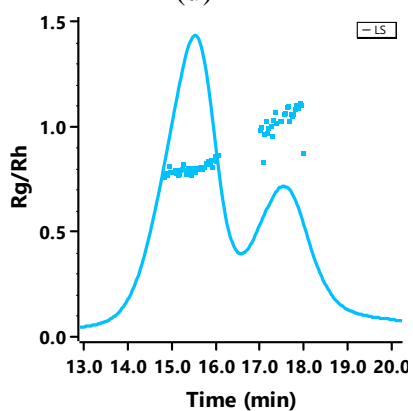

(b)

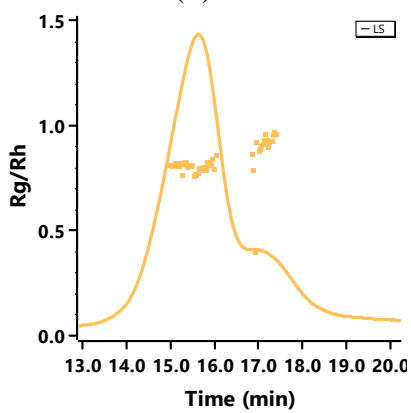

(e)

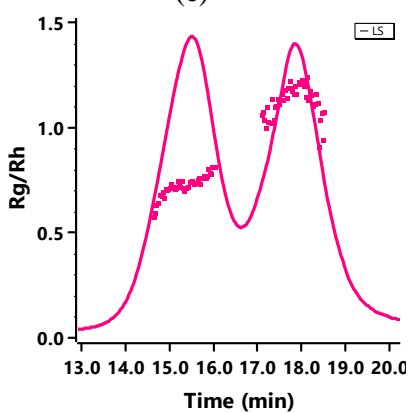

(c)

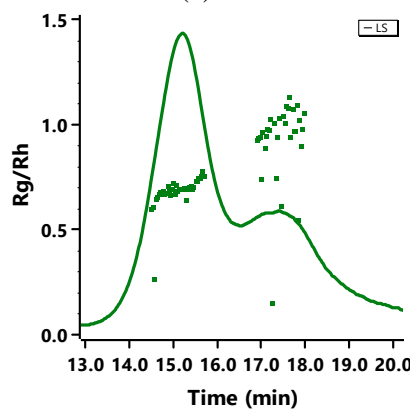

(f)

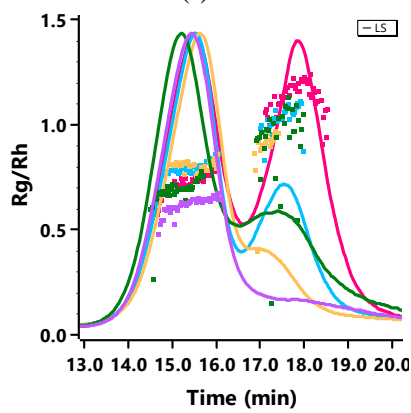

Figure S12. $R_{\mathrm{g}} / R_{\mathrm{h}}$ traces of the $\mathrm{AF}_{4}-\mathrm{MALS}$ fractograms of nanoparticles made with block copolymer D mPEG ${ }_{5 K}-b-\mathrm{p}(\mathrm{HPMA}-\mathrm{Bz})_{2.7 \mathrm{~K}}$ with a concentration of $5 \mathrm{mg} / \mathrm{mL}$ and microfluidic flow rates (a) 1600 $\mu \mathrm{L} / \mathrm{min}$, (b) $500 \mu \mathrm{L} / \mathrm{min}$, (c) $350 \mu \mathrm{L} / \mathrm{min}$, (d) $200 \mu \mathrm{L} / \mathrm{min}$, (e) $100 \mu \mathrm{L} / \mathrm{min}$ and (f) all microfluidic flow rates together in one graph.

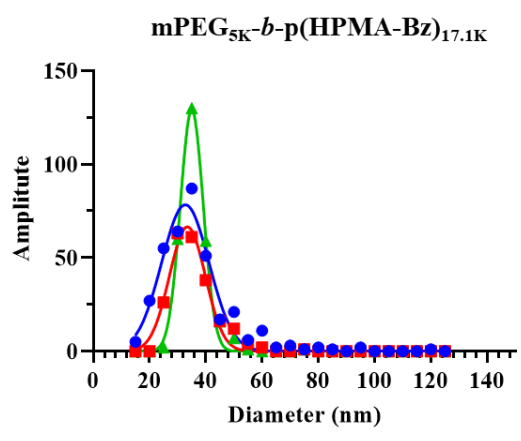

(a)

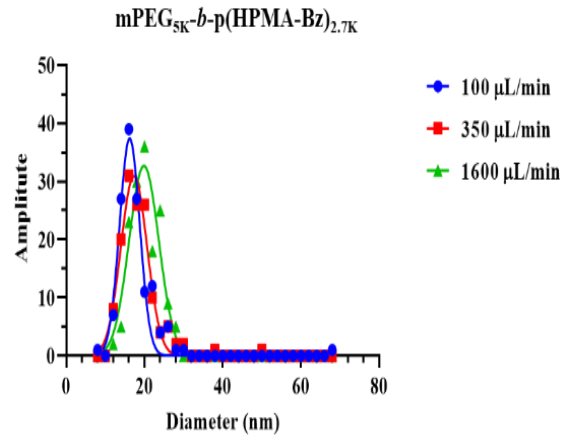

(b)

Figure S13. Histograms of cryo-TEM diameters of (a) block copolymer A (mPEG $5 \mathrm{~K}-b-\mathrm{p}(\mathrm{HPMA}-$

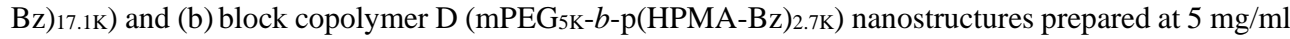
polymer concentration and different flow rates. The data are fitted by Gaussian laws using GraphPad Prism. 


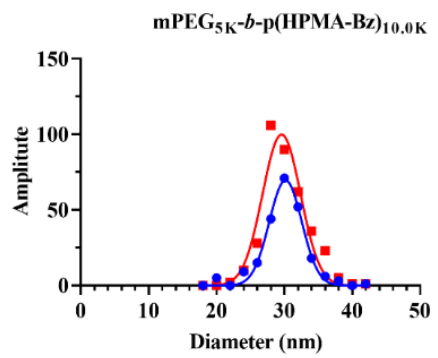

(a)

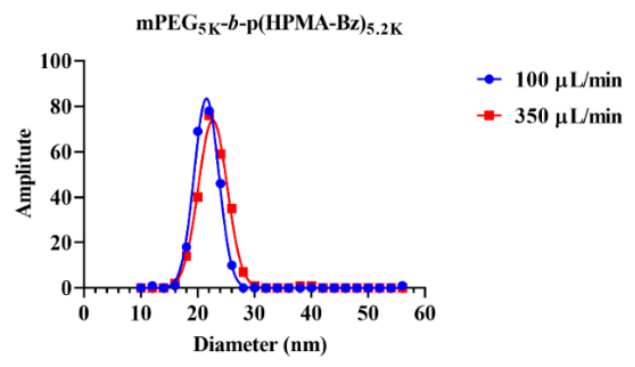

(b)

Figure S14. Histograms of cryo-TEM diameters of (a) block copolymer copolymer B (mPEG5K- $b$ $\left.\mathrm{p}(\mathrm{HPMA}-\mathrm{Bz})_{10.0 \mathrm{~K}}\right)$ and (b) block copolymer $\mathrm{C}\left(\mathrm{mPEG}_{5 \mathrm{~K}}-b-\mathrm{p}(\mathrm{HPMA}-\mathrm{Bz})_{5.2 \mathrm{~K}}\right)$ nanostructures prepared at $5 \mathrm{mg} / \mathrm{ml}$ polymer concentration and different flow rates. The data are fitted by Gaussian laws using GraphPad Prism.

\section{Differential scanning calorimetry}

Differential scanning calorimetry (DSC) was performed using a Discovery DSC (TA Instruments, New Castle, DE, USA) calibrated with indium. Samples (5-10 mg) were heated with a ramp of $2{ }^{\circ} \mathrm{C} / \mathrm{min}$ up to $170{ }^{\circ} \mathrm{C}$ (modulated), kept isothermal for $2 \mathrm{~min}$, cooled down at $1{ }^{\circ} \mathrm{C} / \mathrm{min}$ to $-90{ }^{\circ} \mathrm{C}$ (modulated), isothermal for $10 \mathrm{~min}$, and subsequently heated at $2{ }^{\circ} \mathrm{C}$ $/$ min up to $170{ }^{\circ} \mathrm{C}$ (modulated). The second heating cycle was used to obtain the glass transition temperature $\left(T_{\mathrm{g}}\right) . T_{\mathrm{g}}$ was analyzed by taking the point of inflection of the step change observed in the reversing heat flow curve. For all polymers the $T_{\mathrm{g}}$ is around $98{ }^{\circ} \mathrm{C}$.

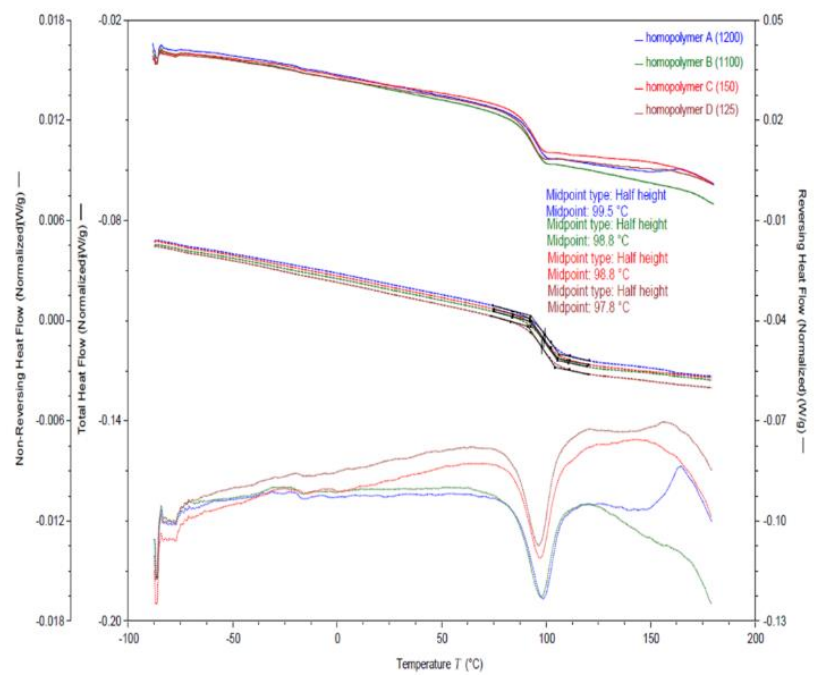

Figure S15. Thermograms of p(HPMA-Bz) homopolymers corresponding to the different molecular weight block copolymers recorded by DSC. 




\section{Chapter 5}

\section{In Vitro and In Vivo Studies on HPMA-Based Polymeric Micelles Loaded with Curcumin}

Mahsa Bagheri ${ }^{1}$, Marcel H. Fens ${ }^{1}$, Tony G. Kleijn ${ }^{1,2}$, Robin B. Capomaccio ${ }^{3}$, Dora Mehn ${ }^{3}$, Przemek M. Krawczyk ${ }^{4}$, Enzo M. Scutigliani ${ }^{4}$, Andrei Gurinov ${ }^{5}$, Marc Baldus ${ }^{5}$, Nicky C.H. van Kronenburg ${ }^{1}$, Robbert J. Kok ${ }^{1}$, Michal Heger ${ }^{1,2}$, Cornelus F. van Nostrum ${ }^{1}$, Wim E.

Hennink $^{1}$

${ }^{1}$ Department of Pharmaceutics, Utrecht Institute for Pharmaceutical Sciences (UIPS), Utrecht University, 3508 TB Utrecht, the Netherlands

${ }^{2}$ Department of Pharmaceutics, Jiaxing Key Laboratory for Photonanomedicine and Experimental Therapeutics, College of Medicine, Jiaxing University, Jiaxing 314001, P. R. China

${ }^{3}$ European Commission, Joint Research Centre (JRC), 21027 Ispra (Va), Italy

${ }^{4}$ Department of Medical Biology, Amsterdam UMC, University of Amsterdam, 1105AZ Amsterdam, the Netherlands

${ }^{5}$ NMR Spectroscopy Group, Bijvoet Center for Biomolecular Research, Utrecht University, Padualaan 8, $3584 \mathrm{CH}$ Utrecht, the Netherlands

Molecular pharmaceutics, 2020, 18 (3), 1247-1263 


\begin{abstract}
Curcumin-loaded polymeric micelles composed of poly(ethylene glycol)- $b$-poly(N-2benzoyloxypropyl methacrylamide) (mPEG- $b$-p(HPMA-Bz)) were prepared to solubilize and improve the pharmacokinetics of curcumin. Accordingly, curcumin-loaded micelles were prepared by a nanoprecipitation method using $\mathrm{mPEG}_{5 \mathrm{kDa}}-b$ - $\mathrm{p}(\mathrm{HPMA}-\mathrm{Bz})$ copolymers with a varying molecular weight of the hydrophobic block $(5.2,10.0$, and $17.1 \mathrm{kDa})$. At equal curcumin loading, micelles composed of $\mathrm{mPEG}_{5 \mathrm{kDa}}-b-\mathrm{p}(\mathrm{HPMA}-\mathrm{Bz})_{17.1 \mathrm{kDa}}$ showed better curcumin retention in both phosphate buffered saline (PBS) and plasma at $37^{\circ} \mathrm{C}$ than micelles based on block copolymers with smaller hydrophobic blocks. No change in micelle size was observed during $24 \mathrm{~h}$ incubation in plasma using asymmetrical flow field-flow fractionation $\left(\mathrm{AF}_{4}\right)$, attesting to particle stability. However, 22 to $49 \%$ of the curcumin loading was released from the micelles during $24 \mathrm{~h}$ from formulations with the highest to the lowest molecular weight $\mathrm{p}(\mathrm{HPMA}-\mathrm{Bz})$, respectively, in plasma. $\mathrm{AF}_{4}$ analysis further showed that the released curcumin was subsequently solubilized by albumin. In vitro analyses revealed that the curcumin-loaded $\mathrm{mPEG}_{5 \mathrm{kDa}}-b-\mathrm{p}(\mathrm{HPMA}-\mathrm{Bz})_{17.1 \mathrm{kDa}}$ micelles were internalized by different types of cancer cells, resulting in curcumin-induced cell death. Intravenously administered curcumin-loaded, Cy7-labeled mPEG $_{5 \mathrm{kDa}}-b$-p (HPMA-Bz) ${ }_{17.1 \mathrm{kDa}}$ micelles in mice at $50 \mathrm{mg}$ curcumin $/ \mathrm{kg}$ showed a long circulation half-life for the micelles $\left(\mathrm{t}_{1 / 2}=42 \mathrm{~h}\right)$, in line with the $\mathrm{AF}_{4}$ results. In contrast, the circulation time of curcumin was considerably shorter than that of the micelles $\left(\mathrm{t}_{1 / 2 \alpha}=0.11, \mathrm{t}_{1 / 2 \beta}=2.5 \mathrm{~h}\right)$ but $\sim 5$ times longer than has been reported for free curcumin $\left(\mathrm{t}_{1 / 2 \alpha}=0.02 \mathrm{~h}\right)$. The faster clearance of curcumin in vivo compared to in vitro studies can be attributed to the interaction of curcumin with blood cells. Despite the excellent solubilizing effect of these micelles, no cytostatic effect was achieved in neuroblastoma-bearing mice, possibly because of low sensitivity of the Neuro2A cells to curcumin.
\end{abstract}

Keywords: nanomedicine; pHPMA; in vitro uptake and localization; human neuroblastoma xenograft model; pharmacokinetics parameters; pharmacodynamics 


\section{Introduction}

Curcumin is a polyphenolic compound that is mainly isolated from the rhizome of Curcuma longa ${ }^{1}$. A plethora of studies has shown that curcumin exerts a wide variety of pharmacological effects against several pathologies, including cancer ${ }^{2}$. Curcumin interacts with numerous vital pathways in cancer cells, resulting in antimutagenic, cytostatic, cytotoxic, and antimetastatic effects ${ }^{3}$. Also, clinical studies have consistently demonstrated that curcumin is safe and well-tolerated at high doses, exhibiting no dose-limiting toxicity ${ }^{4}$. However, curcumin has poor aqueous solubility $(\log P=2.5)^{5}$, degrades under neutral-toalkaline conditions ${ }^{6,7}$, is heavily biotransformed and rapidly eliminated, altogether accounting for poor pharmacokinetics that translate to insufficient curcumin accumulation in tumors to instill significant therapeutic responses ${ }^{8}$. Consequently, much research has been devoted to improve curcumin uptake and prolong circulation time.

Polymer-based nanocarriers have been investigated to improve the solubility and therapeutic efficacy of hydrophobic anticancer drugs ${ }^{9,10}$. In particular, polymeric micelles have attracted substantial attention for solubilization of hydrophobic drugs ${ }^{11,12}$. The nanometer-size range of polymeric micelles facilitates the enhanced permeability and retention (EPR) effect, commonly serving as the pathophysiological basis for intratumoral accumulation of nanoparticulate drug delivery systems ${ }^{13}$. Encapsulation of curcumin in polymeric micelles has also been employed to tackle its poor solubility and stability issues ${ }^{14,15}$. To this end, a variety of amphiphilic polymers such as block copolymers of poly(2-oxazoline)s, poly(ethylene glycol)- $b$-poly( $\varepsilon$-caprolactone), and poly(ethylene glycol)-b-poly(lactic-coglycolic acid) have been used to prepare curcumin-loaded micelles, leading to promising results in vitro and in vivo ${ }^{16-18}$.

Numerous curcumin delivery systems have been designed and evaluated in vivo with respect to pharmacokinetics. The reported 1.3-fold to 5-fold increase in curcumin area under the curve is at best classified as a marginal increase when compared to the improvement rates of other nano-encapsulated drugs versus their respective free form controls (manuscript in preparation). It is therefore warranted to develop more stable nanoformulations to fully exploit curcumin's pharmacodynamic potency. One frequently employed strategy to improve the stability of polymeric micelles is centered on physical interactions between the hydrophobic core and the payload, such as $\pi-\pi$ stacking interactions between aromatic groups ${ }^{19}$. Gong et al. ${ }^{20}$ successfully applied this approach to improve the pharmacokinetics of curcumin using $\mathrm{N}$-(tert-butoxycarbonyl)-L-phenylalanine end-capped mPEG-PCL (mPEG-PCL-Phe(Boc)).

Also, the stability of drug delivery systems needs to be investigated to gain insight into in vitro-in vivo relationships. Drug release studies in relevant media can provide valuable information regarding the performance of a formulation in retaining the encapsulated drug in vitro and in vivo ${ }^{21}$. However, studying the stability of drug delivery systems loaded with hydrophobic drugs in biological fluids such as plasma is challenging because of the difficulties in recovering and separating nanoparticles from other components of the release medium, such as (lipo)proteins. Most often, dialysis or repeated high-speed centrifugation is required for complete separation ${ }^{22,23}$. One possible solution to overcome this challenge is to apply asymmetrical flow field-flow fractionation $\left(\mathrm{AF}_{4}\right)$. This technique separates nanoparticles mainly based on their hydrodynamic size ${ }^{24} . \mathrm{AF}_{4}$ columns do not have a stationary phase, which reduces possible interactions and enables studying highly sensitive 
samples in biological media. Additionally, this technique can separate self-assembled particles such as micelles from other components without disrupting their structure ${ }^{25}$.

In previous studies, mPEG- $b$-p(HPMA-Bz) block copolymers were successfully employed as micellar drug delivery systems and prepared on a relatively large scale ${ }^{26-28}$. A comprehensive study of formulation and processing parameters of polymeric micelles based on these block copolymers allowed proper control of micelle size ${ }^{29}$. Moreover, mPEG- $b$ p(HPMA-Bz) micelles are associated with excellent particle stability and good drug retention due to $\pi-\pi$ stacking interactions $\mathrm{s}^{26}, 27,30,31$. The promising results achieved with paclitaxelloaded mPEG- $b$-p(HPMA-Bz) micelles in terms of pharmaceutical aspects (loading and stability) and in vivo therapeutic efficacy motivated us to assess this platform for its suitability as a delivery system for curcumin.

Accordingly, curcumin-loaded micelles with different (block copolymer) sizes were prepared and evaluated for stability in buffer and plasma. Particle stability due to $\pi-\pi$ stacking interactions in the micellar core was studied by solid-state NMR. Also, the uptake of curcumin-containing mPEG- $b$-p(HPMA-Bz) micelles was analyzed in various human cancer cell lines. Subsequently, the circulation kinetics and biodistribution of mPEG- $b$-p(HPMA$\mathrm{Bz}$ ) micelles and encapsulated curcumin were assessed in mice. Finally, the therapeutic efficacy of curcumin-loaded micelles was evaluated in a mouse model of human neuroblastoma.

\section{Materials and Methods}

\subsection{Materials and Animals}

The $\left(\mathrm{mPEG}_{5 \mathrm{kDa}}\right)_{2}-\mathrm{ABCPA}$ macroinitiator and HPMA-Bz were synthesized and characterized according to previously described protocols ${ }^{28,}{ }^{29}$. N-(2-aminoethyl)methacrylamide hydrochloride (AEMA), triethylamine, HEPES, lithium chloride ( $\mathrm{LiCl})$, sulforhodamine B (SRB) sodium salt, trichloroacetic acid, curcuminoid mixture, human serum albumin (HSA), and Roswell Park Memorial Institute (RPMI) 1640 culture medium were purchased from Sigma-Aldrich (St. Louis, MO, USA). Phosphate buffer saline (PBS) 10× solution was acquired from Fisher bioreagents (Pittsburgh, PA, USA). Tris(hydroxymethyl)aminomethane was obtained from Serva (Heidelberg, Germany). Stellate Cell Growth Supplements (SteCGS) were purchased from ScienCell (Carlsbad, CA, USA). Endothelial Basal Medium (EBM-2) supplemented with growth factors (Growth Medium 2 SupplementMix) were obtained from PromoCell (Heidelberg Germany). Lglutamine, penicillin, and streptomycin were acquired from Lonza (Basel, Switzerland). Accutase was purchased from Global Cell Solutions (Charlottesville, VA, USA). TrypsinEDTA, PBS and gentamicin/amphotericin B were acquired from Gibco (Waltham, MA, USA). PEG standards for gel permeation chromatography (GPC) calibration were obtained from Agilent Technologies (Santa Clara, CA, USA). Cyanine 7 (Cy7) NHS ester and cyanine 5 (Cy5) NHS ester were obtained from Lumiprobe (Hannover, Germany). Radioimmunoprecipitation assay (RIPA) lysis buffer (10×, 0.5 M Tris-HCl, $\mathrm{pH}=7.4,1.5 \mathrm{M}$ $\mathrm{NaCl}, 2.5 \%$ deoxycholic acid, 10\% NP-40,10 mM EDTA) was purchased from Merck KGaA (Darmstadt, Germany). Six and 24-well plates were acquired from Greiner Bio-One (Cellstar; Kremsmünster, Austria). Fetal bovine serum (FBS) was obtained from Bodinco (Alkmaar, the Netherlands). All solvents were purchased from Biosolve (Valkenswaard, the Netherlands) and used as received. Syringe filters of regenerated cellulose were ordered from 
Phenomenex (Torrance, CA, USA). Dialysis bags with 8-kDa cut-off were purchased from Spectrum Chemical (SpectraPor; New Brunswick, NJ, USA). Female BALB/c mice (18-22 g) and female A/J mice (18-22 g) were purchased from Charles River (Den Bosch, the Netherlands) and Envigo (Horst, the Netherlands), respectively.

\subsection{Cell Culture}

Murine neuroblastoma (Neuro2A) cells were purchased from ATCC (Manassas, VA, USA). Human extrahepatic cholangiocarcinoma (TFK-1) was ordered from DMSZ (Brunswick, Germany). Human extrahepatic cholangiocarcinoma (Sk-ChA-1) and gallbladder adenocarcinoma (Mz-ChA-1) cells were licensed to Michal Heger by the University Hospital Zurich, Switzerland. Human biliary adenocarcinoma (EGI-1) cells were provided by the Tytgat Institute for Liver and Intestinal Research to Michal Heger (Amsterdam UMC, location AMC). Primary human umbilical vein endothelial cells (HUVECs) were obtained from Lonza (Verviers, Belgium). Primary human pancreatic stellate cells (hPSC) were obtained from ScienCell Research Laboratories (Carlsbad, CA, USA). TFK-1, Mz-ChA-1, Sk-ChA-1, EGI-1 and Neuro2A cells were cultured in Roswell Park Memorial Institute (RPMI) 1640 culture medium supplemented with $10 \% \mathrm{FBS}, 100 \mathrm{U} / \mathrm{mL}$ penicillin, $100 \mu \mathrm{g} / \mathrm{mL}$ streptomycin, and $2 \mathrm{mM} \mathrm{L-glutamine.} \mathrm{hPSC}$ were cultured in complete stellate cell medium supplemented with $2 \%$ FBS, $1 \%$ penicillin/streptomycin, and $1 \%$ SteCGS. HUVECs were cultured in EBM-2 medium supplemented with growth factors (Growth Medium 2 SupplementMix) and antibiotics/fungicidals (gentamicin/amphotericin B) up to passage number 6. Cells were maintained at $37{ }^{\circ} \mathrm{C}$ in a $5 \% \mathrm{CO}_{2}$ humidified atmosphere (standard culture conditions). EGI-1, Mz-ChA-1, SK-ChA-1, and TFK-1 cells were cultured in $75 \mathrm{~cm}^{2}$ cell culture flasks and passaged once per week at a ratio of 1:10, 1:2, 1:8, and 1:6, respectively.

\subsection{Synthesis of mPEG- $b$-p(HPMA-Bz) Polymers}

mPEG- $b$-p(HPMA-Bz) block copolymers were synthesized via free-radical polymerization and characterized by ${ }^{1} \mathrm{H}-\mathrm{NMR}$ and GPC as described previously ${ }^{28-30}$. Three different $\mathrm{mPEG}-$ $b$-p(HPMA-Bz) block copolymers with fixed mPEG $_{5 \mathrm{kDa}}$ and different molecular weights of the hydrophobic block were synthesized by varying the molar feed ratios of macroinitiator:monomer (1:200, 1:100, 1:50) using acetonitrile (ACN) as solvent. Polymerization was performed under nitrogen at $70{ }^{\circ} \mathrm{C}$ for $24 \mathrm{~h}$. The polymers were collected by precipitation in ice-cold diethyl ether and dried under vacuum. The polymer characteristics were published previously ${ }^{30}$ and reported in the supplementary information (SI) Table S1.

\subsection{Synthesis and Characterization of Fluorescently-Labeled mPEG- $b$-p(HPMA- Bz)}

mPEG- $b$-p(HPMAm-Bz $98 \%$-co-AEMAm $2 \%$ ) with macro-initiator:monomer molar ratio of 1:200 was synthesized as described previously ${ }^{26}$. The primary amine groups in the hydrophobic block were reacted with the Cy7-NHS ester or Cy5-NHS ester. In short, the polymer $(100 \mathrm{mg})$ was transferred into a glass vial and dissolved in $1.4 \mathrm{~mL}$ dimethyl sulfoxide (DMSO). Next, $0.58 \mathrm{~mL}$ dye stock solution $(10 \mathrm{mg} / \mathrm{mL})$ and $3.2 \mu \mathrm{L}$ of dry triethylamine were added. The reaction was conducted in the dark at room temperature overnight. The fluorescently-labeled polymers were dialyzed against a tetrahydrofuran (THF)/water mixture $(1 / 1 \mathrm{v} / \mathrm{v})$ for $72 \mathrm{~h}$. The medium was refreshed 5 times to remove uncoupled dye using an $8-\mathrm{kDa}$ dialysis membrane. The final product was obtained as a dark green and blue powders after lyophilization. GPC was performed to confirm the dye 
conjugation to the polymer as described ${ }^{26}$, using refractive index (RI) and UV detectors (detection wavelength of $700 \mathrm{~nm}$ and 650 for Cy7 and Cy5, respectively). The results are reported in SI Figure S3.

\subsection{Preparation and Characterization of Empty/Curcumin-Loaded Micelles}

Empty and curcumin-loaded mPEG- $b$-p(HPMA-Bz) micelles were prepared by a nanoprecipitation procedure ${ }^{29}$. In short, mPEG- $b$-p(HPMA-Bz) $(30 \mathrm{mg} / \mathrm{mL})$ alone or together with curcumin (varying concentrations depending on the target load) were dissolved in THF and added dropwise at a 1:1 volume ratio to HEPES buffered saline (HBS; containing $20 \mathrm{mM}$ HEPES and $150 \mathrm{mM} \mathrm{NaCl}, \mathrm{pH}=7.4$ ) while stirring. Curcumin micelles were prepared in dim light to avoid photodegradation. Subsequently, THF was removed by evaporation overnight in a fume hood. The micelle dispersions were filtered using $0.2-\mu \mathrm{m}$ regenerated cellulose membranes to remove unencapsulated curcumin and any polymer aggregates. The formulations used for cell culture and animal studies were additionally dialyzed for $24 \mathrm{~h}$ using an $8-\mathrm{kDa}$ dialysis membrane to completely remove the organic solvent. Previously, it was shown that the residual THF content can be reduced to acceptable levels by evaporation and subsequent dialysis to achieve an intravenous formulation with THF content below the threshold concentration $(720 \mathrm{ppm})$ as stipulated by International Council of Harmonization of Technical Requirements for Registration of Pharmaceuticals for Human $\mathrm{Use}^{29}$. The curcumin-loaded polymeric micelles were characterized for size, size distribution, curcumin content, and polymer concentration. Micelle size was determined by dynamic light scattering (DLS) using a Zetasizer (model ZS90, Malvern Instruments, Malvern, UK). Curcumin was quantified as reported earlier ${ }^{6}$. The encapsulation efficacy (EE) and loading capacity (LC) were calculated as follows:

$\mathrm{EE} \%=($ measured amount of curcumin $) /($ amount of curcumin added $) \times 100 \%$

$\mathrm{LC} \%=$ (measured amount of curcumin) $/$ (measured amount of curcumin and polymer $) \times$ $100 \%$

GPC analysis was conducted to measure the polymer concentration using two serial PLgel 5 - $\mu \mathrm{m}$ MIXED-D GPC columns (Polymer Laboratories, Agilent Technologies) at $65{ }^{\circ} \mathrm{C}$. Dimethylformamide (DMF) containing $10 \mathrm{mM} \mathrm{LiCl}$ was used as eluent and results were obtained with a refractive index detector (RI) (Waters 2414, Waters Corporation, Milford, MA, USA) and UV detection (280 nm) (waters 2489, Waters Corporation, Milford, MA, USA). Aqueous micelle dispersions were diluted 10-fold in DMF and the absorbance was measured at $280 \mathrm{~nm}$. Standard curves were obtained with the same polymer in DMF in a 0.1 $10 \mathrm{mg} / \mathrm{mL}$ concentration range.

\subsection{Analysis of $\pi-\pi$ Stacking in Curcumin-Loaded Micelles by Solid-State NMR Spectroscopy}

${ }^{1} \mathrm{H}-\mathrm{NMR}$ spectra of mPEG- $b$-p(HPMA-Bz) copolymer and curcumin dissolved in DMSO- $\mathrm{d}_{6}$ as the solvent were recorded using a Bruker $600 \mathrm{MHz}$ spectrometer (Billerica, MA, USA). The DMSO peak at $2.52 \mathrm{ppm}$ was used for calibration. The following chemical shifts for mPEG- $b$-p(HPMA-Bz) copolymer were obtained (SI, Figure S1): 8.0 (b, 2H, aromatic $\mathrm{CH}$ ), $7.55(\mathrm{~b}, 1 \mathrm{H}$, aromatic $\mathrm{CH}), 7.65(\mathrm{~b}, 2 \mathrm{H}$, aromatic $\mathrm{CH}), 7.35$ (b, CO-NH- $\left.\mathrm{CH}_{2}\right), 5.0$ (b, NH$\left.\mathrm{CH}_{2}-\mathrm{C} \underline{\mathrm{H}}\left(\mathrm{CH}_{3}\right)-\mathrm{O}-(\mathrm{Bz})\right)$, 3.40-3.60 (b, mPEG 5000 methylene protons, $\left.\overline{\mathrm{O}}_{-\mathrm{C}} \underline{\mathrm{H}}_{2}-\underline{\mathrm{C}}_{2}\right), 3.1$ (b, $\mathrm{NH}-\mathrm{CH}_{2}-\mathrm{CH}$ ) and $0.6-2.2$ (b, the rest of the protons are from the methyl and backbone $\mathrm{CH}_{2}$ 
protons). The chemical shifts of curcumin (SI, Figure S2) were determined as follows: 9.66 (s, 2H, aromatic $\mathrm{OH}), 7.58(\mathrm{~d}, 2 \mathrm{H}, \mathrm{CO}-\mathrm{CH}-\mathrm{C} \underline{\mathrm{H}}), 7.35$ (s, 2H, C-C프-C-OCH 3 ), 7.16 (d, 2H, aromatic $\mathrm{CH}), 6.84(\mathrm{~d}, 2 \mathrm{H}$, aromatic $\mathrm{CH}), 6.78$ (d, 2H, CO-Cㅍ-CH), 6.08 (s, 1H, OH-C-Cㅍ$\mathrm{CO})$ and $3.84\left(\mathrm{~s}, 6 \mathrm{H}\right.$, aromatic $\left.\mathrm{OCH}_{3}\right)$.

Empty mPEG- $b$-p(HPMA-Bz) micelles $(30 \mathrm{mg} / \mathrm{mL})$ and $9 \%(\mathrm{w} / \mathrm{w})$ curcumin-loaded micelles $(30 \mathrm{mg} / \mathrm{mL})$ were prepared in $\mathrm{D}_{2} \mathrm{O}$ according to section 2.5 . Solid-state NMR experiments were performed using a Bruker Avance III spectrometer operating at a ${ }^{1} \mathrm{H}$ Larmor frequency of $500 \mathrm{MHz}$ equipped with a $4 \mathrm{~mm}$ double resonance probe head at MAS rates varying from 340 to $1120 \mathrm{~Hz}$ in order to distinguish isotropic signals from spinning sidebands. Spectral referencing was done using adamantane. Complementary static NMR experiments were performed using a Bruker Avance III spectrometer operating at a ${ }^{1} \mathrm{H}$ Larmor frequency of $600 \mathrm{MHz}$ equipped with a $5 \mathrm{~mm}$ triple resonance probe head.

\subsection{Curcumin Retention in mPEG- $b$-p(HPMA-Bz) Micelles Dispersed in Phosphate Buffered Saline}

The stability of the curcumin micelles in PBS (11.9 mM phosphates, $137 \mathrm{mM}$ sodium chloride, and $2.5 \mathrm{mM}$ potassium chloride, $\mathrm{pH}=7.4$ ), measured by the retention of curcumin in the micelles, was determined as described previously ${ }^{32}$. In short, mPEG- $b$-p(HPMA-Bz) micelles containing 2.0, 4.8, and 9\% curcumin $(w / w)$ were diluted 5-fold in PBS and incubated at $37{ }^{\circ} \mathrm{C}$ with constant shaking. The polymer concentration was kept at $\sim 6 \mathrm{mg} / \mathrm{mL}$ for the different formulations while curcumin concentration was around 0.6, 0.3 and 0.12 $\mathrm{mg} / \mathrm{mL}$ from the highest to the lowest curcumin loading, respectively. The samples were protected from light to avoid photodegradation. At pre-defined time points, $100-\mu \mathrm{L}$ aliquots were removed and centrifuged at $5000 \times \mathrm{g}$ for $10 \mathrm{~min}$ to spin down precipitated curcumin. Next, the supernatant was diluted at least $10 \times$ with methanol and vortexed to disrupt the micelles and solubilize the loaded curcumin. The curcumin concentration was determined by high-performance liquid chromatography (HPLC) ${ }^{6}$. HPLC analysis was performed on a Waters instrument equipped with a C18 column (SunFire, $5 \mu \mathrm{m}, 150 \mathrm{~mm} \times 4.6 \mathrm{~mm}$; Waters Corporation, Milford, MA, USA). A gradient system was applied using 5:95 (v/v) ACN:water and $100 \% \mathrm{ACN}$ as eluent $\mathrm{A}$ and $\mathrm{B}$, respectively. The $\mathrm{pH}$ of the eluents was adjusted by addition of $0.25 \%(v / v)$ acetic acid. The gradient ran from $90 \%$ A to $70 \% \mathrm{~B}$ in $15 \mathrm{~min}$ at a flow rate of $1.2 \mathrm{~mL} / \mathrm{min}$. The injection volume was $20 \mu \mathrm{L}$. Curcumin was detected at $425 \mathrm{~nm}$ and $254 \mathrm{~nm}$.

\subsection{Stability of Curcumin-Loaded Micelles in Plasma}

Curcumin-loaded micelles were prepared as described in section 2.5 using $3.0 \mathrm{mg}$ curcumin and $30 \mathrm{mg}$ of the different polymers. The curcumin-loaded micelles were diluted $10 \times$ in human plasma (total volume of $400 \mu \mathrm{L}$ ) and incubated at $37{ }^{\circ} \mathrm{C}$ in a block thermomixer (Eppendorf, Hamburg, Germany) at $450 \mathrm{rpm}$. The samples were protected from light to avoid photodegradation. At pre-defined time points, $20-\mu \mathrm{L}$ aliquots were collected and analyzed using $\mathrm{AF}_{4}$. Separation was performed using an AF2000 separation system (Postnova Analytics, Landsberg, Germany) that consisted of PN1130 isocratic pumps, a degasser, two UV detectors PN3211 (280 nm, $425 \mathrm{~nm}$ ), and an online-coupled DLS (Zetasizer Nano ZS, Malvern Instruments). The separation channel included a spacer with $350 \mu \mathrm{m}$ thickness, 27 $\mathrm{cm}$ channel length, deltoid shaped channel profile, and a 10-kDa cut-off regenerated cellulose membrane (Postnova Analytics). PBS was used as mobile phase. In order to separate the micelles from plasma components, a cross-flow program using a time delay exponential 
decay was set up. After applying an injection flow of $0.20 \mathrm{~mL} / \mathrm{min}$, the sample was focused for $4 \mathrm{~min}$ at a focus flow rate of $2.3 \mathrm{~mL} / \mathrm{min}$ and cross-flow of $2 \mathrm{~mL} / \mathrm{min}$. At the end of the focusing step and a transition time of $1 \mathrm{~min}$, the cross-flow was kept constant at $2 \mathrm{~mL} / \mathrm{min}$ for $7 \mathrm{~min}$. Next, the cross-flow was decreased at an exponential decay of 0.5 to $0.1 \mathrm{~mL} / \mathrm{min}$ over $20 \mathrm{~min}$. Finally, the cross-flow was kept constant at $0.1 \mathrm{~mL} / \mathrm{min}$ for $15 \mathrm{~min}$. During the entire run (both focus and elution step), the detector flow rate was $0.5 \mathrm{~mL} / \mathrm{min}$. The stability is reported as percentage of release and calculated as:

Release $(\%)=($ area under the curve of curcumin in albumin fraction at $425 \mathrm{~nm}) /($ area under the curve of curcumin in the micelle and albumin fractions at $425 \mathrm{~nm}) \times 100 \%$

\subsection{Association and Uptake Analysis of Free Curcumin, Curcumin-Loaded Micelles, and Cy5-Labeled Micelles by Flow Cytometry and Confocal Microscopy}

Ten percent $(w / w)$ Cy5-labeled $\mathrm{mPEG}_{5 \mathrm{kDa}}-b$-p(HPMA-Bz) $)_{17.1 \mathrm{kDa}}$ micelles (3.0 mg Cy5labeled polymer and $27 \mathrm{mg}$ unlabeled polymer) and curcumin-loaded $\mathrm{mPEG}_{5 \mathrm{kDa}}-b$-p(HPMA$\mathrm{Bz})_{17.1 \mathrm{kDa}}$ micelles (3.0 $\mathrm{mg}$ curcumin and $30 \mathrm{mg}$ polymer) were prepared in HBS as described in section 2.5. Cells were seeded at a density of $5 \times 10^{5}$ cells/well in 6 -well plates and incubated for $18 \mathrm{~h}$. The cell culture medium was subsequently replaced by fresh medium containing $0.3 \mathrm{mg} / \mathrm{mL}$ of $10 \%(w / w)$ Cy5-labeled micelles, free curcumin (dissolved in DMSO; final concentration of DMSO was $0.4 \%)$, or curcumin-loaded micelles $(20 \mu \mathrm{M})$ dispersed in HBS. Before flow cytometry, the cells were rinsed twice with PBS, trypsinized using $0.05 \%$ trypsin-EDTA, and collected in culture medium. The Cy5 and curcumin intensity of approximately 5000 single cells per condition was quantified on a BD FACSCanto II (BD Biosciences, Franklin Lakes, NJ, USA) equipped with dedicated filter sets. Different detector voltages were used for the free curcumin and micellar curcumin groups. Data were processed using FlowJo (BD Biosciences) and plotted using GraphPad Prism (GraphPad Software, La Jolla, CA, USA).

Cells were seeded on glass coverslips in 6 -well plates at a density of $5 \times 10^{5}$ cells/well and incubated for $18 \mathrm{~h}$. The culture medium was subsequently supplemented with the indicated formulations at $0.3 \mathrm{mg} / \mathrm{mL}$ (Cy5-labeled micelles) or $20 \mu \mathrm{M}$ (free curcumin or curcuminloaded micelles). Cells were then incubated for $0.5,1$, and $4 \mathrm{~h}$ and imaged after washing in PBS with a $40 \times$ oil immersion objective using a Leica TCS SP8 SMD scanning unit (Leica Microsystems, Wetzlar, Germany) mounted on a Leica DMI6000 inverted microscope enclosed in an incubator equilibrated at $37^{\circ} \mathrm{C}$. Curcumin and $\mathrm{Cy} 5$ fluorescence were excited at $405 \mathrm{~nm}$ and $633 \mathrm{~nm}$, respectively.

\subsection{Curcumin and Micelle In Vitro Cytotoxicity}

The cytotoxicity of free curcumin, empty $\mathrm{mPEG}_{5 \mathrm{kDa}}-b-\mathrm{p}(\mathrm{HPMA}-\mathrm{Bz})_{17.1 \mathrm{kDa}}$ micelles, and curcumin-loaded $\mathrm{mPEG}_{5 \mathrm{kDa}}-b-\mathrm{p}(\mathrm{HPMA}-\mathrm{Bz})_{17.1 \mathrm{kDa}}$ micelles was investigated in vitro in different cancer cell lines (Neuro2A, EGI-1, TFK-1, SK-ChA-1, and Mz-ChA-1) and noncancerous cells. Cell viability was assessed with the SRB total protein assay ${ }^{33}$. EGI-1, TFK1, SK-ChA-1, Neuro2A, and HUVECs were seeded in 24-well plates at a density of $5 \times 10^{4}$ cells/well and Mz-ChA-1 cells were seeded at $25 \times 10^{4}$ cells/well due to their slow growth rate. The cells were cultured for $24 \mathrm{~h}$ under standard culture conditions. Next, empty and curcumin-loaded micelles dispersed in HBS and curcumin dissolved in DMSO were added at various concentrations (curcumin and polymer concentration ranged between 0-200 $\mu \mathrm{M}$ and $0-800 \mu \mathrm{g} / \mathrm{mL}$, respectively). The final concentration of DMSO used to solubilize 
curcumin was $0.4 \%(v / v)$ in cell culture medium. Cells incubated with HBS were used as control. After 24, 48, and $72 \mathrm{~h}$ of incubation, the wells were washed with PBS and total protein content was determined with the SRB assay as described by Vichai et al. ${ }^{33}$. Absorbance at $564 \mathrm{~nm}$ was recorded using a BioTek Synergy HT microplate reader and the relative cell viability was calculated as follows:

Relative cell viability $(\%)=($ absorbance of the sample $) /($ absorbance of the control $) \times 100 \%$

The $50 \%$ inhibitory concentration $\left(\mathrm{IC}_{50}\right)$ was derived from a nonlinear regression model (curve fit) based on a sigmoidal inhibitor vs. normalized response curve (variable slope) and calculated using GraphPad Prism.

\subsection{Acute Toxicity of Empty Micelles in Healthy BALB/c Mice}

All animal studies were conducted in compliance with guidelines provided by national regulations and approved by Utrecht University's institutional review board for animal experiments. Empty polymeric micelles $(50 \mathrm{mg} / \mathrm{mL})$ were prepared using $\mathrm{mPEG}_{5 \mathrm{kDa}}-b-$ p(HPMA-Bz) ${ }_{17.1 \mathrm{kDa}}$ in HBS as described in section 2.5 . The empty micelles were injected intravenously into BALB/c mice via the tail vein at escalating doses of 100,300 , and 500 $\mathrm{mg} / \mathrm{kg}(100-200 \mu \mathrm{L}, \mathrm{n}=3$ per dose). Control mice received $200 \mu \mathrm{L}$ HBS intravenously. Body weight and general health conditions were monitored before, directly after, and $24 \mathrm{~h}$ after administration. Also, blood cell counts were measured after $24 \mathrm{~h}$. At the end of the experiment, animals were sacrificed via deep isoflurane anesthesia followed by cervical dislocation.

\subsection{Circulation Kinetics and Biodistribution of Cy7-Labeled Curcumin Micelles in Mice}

Cy7-labeled micelles loaded with curcumin were prepared by dissolving $300 \mathrm{mg}$ unlabeled $\mathrm{mPEG}_{5 \mathrm{kDa}}-b-\mathrm{p}(\mathrm{HPMA}-\mathrm{Bz})_{17.1 \mathrm{kDa}}, 4.5 \mathrm{mg}$ Cy7-labeled polymer $(1.5 \% \mathrm{w} / \mathrm{w})$, and $30.9 \mathrm{mg}$ curcumin in $4 \mathrm{~mL}$ of THF. One $\mathrm{mL}$ of the resulting solution was subsequently added to one mL HBS. THF was removed by evaporation overnight in a fume hood followed by dialysis against HBS for one day to completely remove the organic solvent. Finally, the micellar dispersions were filtered using $0.2-\mu \mathrm{m}$ regenerated cellulose membranes to remove unencapsulated curcumin and any polymer aggregates. Higher starting polymer and curcumin concentrations were used to compensate for curcumin and polymer loss during dialysis and to achieve the intended injection dose (maximum volume of injection was 200 $\mu \mathrm{L}$ per mouse). The characteristics of the micellar dispersions are reported in (SI, Table S2).

For the pharmacokinetics (PK) and biodistribution studies the formulation was intravenously administered into female BALB/c mice (100-200 $\mu \mathrm{L}$ injection volume), corresponding to a final concentration of $50 \mathrm{mg}$ curcumin $/ \mathrm{kg}$. Blood samples were collected into EDTA containing tubes after $1 \mathrm{~min}$ (set as $100 \%$ injected dose), $10 \mathrm{~min}$, and 1 hour via submandibular puncture $(50-80 \mu \mathrm{L})$, and after $4 \mathrm{~h}$ and $24 \mathrm{~h}$ via cardiac puncture following animal sacrifice as described in section 2.11. Plasma samples were prepared by centrifugation at $1500 \times \mathrm{g}$ for $10 \mathrm{~min}$. The liver, spleen, heart, kidneys, lungs, and brain were dissected after $4 \mathrm{~h}$ and $24 \mathrm{~h}$ and fluorescence of the excised organs was imaged using a Pearl Impulse small animal imaging system (LI-COR Biosciences, Bad Homburg, Germany). The tissues were rinsed with PBS and snap-frozen in liquid nitrogen and stored at $-80^{\circ} \mathrm{C}$ until further analysis. The plasma samples ( 1 volume) were mixed with 4 volumes of ACN and vortexed for 2 min, 
followed by centrifugation $(15,000 \times \mathrm{g}, 10 \mathrm{~min})$. Curcumin content in the supernatant was determined by reversed-phase HPLC (RP-HPLC) on a Waters system equipped with a C18 column (SunFire, $5 \mu \mathrm{m}, 150 \mathrm{~mm} \times 4.6 \mathrm{~mm}$ ). The eluent consisted of $50 \%$ ACN and $50 \%$ water containing $0.25 \%$ acetic acid as $\mathrm{pH}$ modifier. The injection volume and flow rate were $20 \mu \mathrm{L}$ and $1.2 \mathrm{~mL} / \mathrm{min}$, respectively. Curcumin was detected at 425 and $254 \mathrm{~nm}$. The data were analyzed with Empower software (Waters Corporation). Calibration was done using curcumin in $80 \% / 20 \% \mathrm{ACN} /$ water with concentrations ranging from 0.1 to $100 \mu \mathrm{g} / \mathrm{mL}$.

The concentration of Cy7-labeled micelles in the plasma samples was measured at $800 \mathrm{~nm}$ on an Odyssey imaging system (LI-COR Biosciences) using a calibration curve of Cy7labeled micelles in PBS in a concentration range of $0.2-20 \mu \mathrm{g} / \mathrm{mL}$. Blood samples were diluted 10-40 times in PBS buffer and the intensity of Cy7 was extrapolated from the linear fit function and corrected for the dilution factor.

To determine the curcumin and Cy7-polymer content in different organs, the excised tissue samples were treated as follows. RIPA buffer $(200 \mu \mathrm{L})$ was added to $\sim 100 \mathrm{mg}$ of tissue and the mixture was homogenized at a speed of 6000/s for $60 \mathrm{~s}$ using a tissue homogenizer (Bertin Technologies, Montigny-le-Bretonneux, France). The homogenized tissue was treated with $\mathrm{ACN}$ (1:4 volume ratio) and vortexed for $2 \mathrm{~min}$, followed by centrifugation at $15,000 \times \mathrm{g}$ for $10 \mathrm{~min}$. The curcumin concentration in the supernatant was analyzed by HPLC as described in section 2.7. The concentration of Cy7-polymer in different tissues was analyzed as described above by measuring the fluorescence of diluted tissue homogenates in RIPA buffer. Cy7-polymer dissolved in DMSO and subsequently diluted in tissue homogenate suspension obtained from untreated mice was used for calibration $(0.2-40 \mu \mathrm{g} / \mathrm{mL})$.

Pharmacokinetics parameters were calculated using the PkSolver 2.0 add-in template in Microsoft Excel. Calculations were based on non-compartmental and two-compartmental analyses for the micelles and curcumin, respectively ${ }^{34}$.

\subsection{Therapeutic Efficacy of Curcumin Micelles in Tumor-Bearing Mice}

The anticancer properties of the curcumin-loaded $\mathrm{mPEG}_{5 \mathrm{kDa}}-b-\mathrm{p}(\mathrm{HPMA}-\mathrm{Bz})_{17.1 \mathrm{kDa}}$ micelles (9\% $w / w$, prepared in HBS) were studied in a syngeneic murine subcutaneous neuroblastoma (Neuro2A) model. Tumors were grown from cells subcutaneously injected $\left(3 \times 10^{6}\right.$ cells/100 $\mu \mathrm{L}$ PBS, $\mathrm{pH}=7.4$ ) into the right flank of female $\mathrm{A} / \mathrm{J}$ mice. Nine days after inoculation, mice received an intravenous bolus of curcumin-loaded micelles at $50 \mathrm{mg}$ curcumin $/ \mathrm{kg}$, empty micelles (500 mg polymer $/ \mathrm{kg}$ ), or HBS solution (maximum $200 \mu \mathrm{L} /$ injection) for 10 consecutive days ( $\mathrm{n}=5$ per group). The infusion of solutions was performed slowly. Body weight and tumor size were monitored daily for one month. Tumor volume was calculated using $\mathrm{V}=(\mathrm{A} \times 0.52) \times \mathrm{B}^{2}$, where $\mathrm{A}$ and $\mathrm{B}$ are the largest and the smallest superficial diameters, respectively ${ }^{35}$. Mice were sacrificed as described in section 2.11 upon reaching one of the following human end points: weight less of $>25 \%$, tumor volume reaching 2000 $\mathrm{mm}^{3}$, tumor breaking out of the skin, or when the animal was deemed moribund.

\subsection{Statistical Analysis}

Statistical analysis was performed in GraphPad. A Kruskal-Wallis test with Dunn's post-hoc analysis was used to compare the differences in $\mathrm{IC}_{50}$ values between different incubation times. A Mann-Whitney U test was used to compare the size of micelles before and after curcumin loading. Statistical significance is designated as $* \mathrm{p} \leq 0.05, * * \mathrm{P}$ value $\leq 0.01$. 


\section{Results and Discussion}

\subsection{Characterization of Curcumin-Loaded mPEG- $b$-p(HPMA-Bz) Micelles}

The mPEG- $b$-p(HPMA-Bz) polymers contained a hydrophilic mPEG $_{5 \mathrm{kDa}}$ block and different hydrophobic blocks (molecular weights of $5.2 \mathrm{kDa}, 10.0 \mathrm{kDa}$, and $17.1 \mathrm{kDa}$ ). The polymers were synthesized by free radical polymerization ${ }^{30}$ and their characteristics are reported in Table S1.

Curcumin was loaded into the micelles by a nanoprecipitation technique with an encapsulation efficiency of $>90 \%$ (Table 1). Previously, mPEG- $b$-p(HPMA-Bz) micelles based on $21 \mathrm{kDa}$ copolymer had a maximal loading capacity of $19 \%$ without compromising the entrapment efficiency $(87-97 \%)^{27}$. In the present study a lower loading capacity of $9 \%$ was chosen to avoid the risk of compromising the solubilization capacity of the hydrophobic core. Also, the size of curcumin-loaded micelles was between 40 to $60 \mathrm{~nm}$ for the lowest to the highest molecular weight polymers, respectively, and comparable to that of previously studied paclitaxel-loaded micelles ${ }^{30}$. A PDI of $\leq 0.14$ indicates a narrow size distribution of the particles ${ }^{36,37}$.

Table 1. Physicochemical characteristics of empty and curcumin-loaded micelles ( $9 \% \mathrm{w} / \mathrm{w}$ loading) prepared using copolymers with a different molecular weight of the hydrophobic block.

\begin{tabular}{|c|c|c|c|c|c|c|}
\hline \multirow[b]{2}{*}{ Polymer } & \multicolumn{2}{|c|}{ Empty micelles } & \multicolumn{4}{|c|}{ Curcumin-loaded micelles } \\
\hline & $\begin{array}{c}\text { Z-ave } \\
\text { (nm) }\end{array}$ & PDI & $\begin{array}{c}\text { Z-ave } \\
\text { (nm) }\end{array}$ & PDI & EE $(\%)$ & LC $(\%)$ \\
\hline $\mathrm{mPEG}_{5 \mathrm{kDa}}-b-\mathrm{p}(\mathrm{HPMA}-\mathrm{Bz})_{17.1 \mathrm{kDa}}$ & $52 \pm 1$ & $0.09 \pm 0.02$ & $59 \pm 3$ & $0.09 \pm 0.02$ & $91 \pm 5$ & $9.0 \pm 0.6$ \\
\hline $\mathrm{mPEG}_{5 \mathrm{kDa}}-b-\mathrm{p}(\mathrm{HPMA}-\mathrm{Bz})_{10.0 \mathrm{kDa}}$ & $46 \pm 1$ & $0.05 \pm 0.01$ & $48 \pm 2$ & $0.03 \pm 0.01$ & $94 \pm 6$ & $8.8 \pm 0.5$ \\
\hline $\mathrm{mPEG}_{5 \mathrm{kDa}}-b-\mathrm{p}(\mathrm{HPMA}-\mathrm{Bz})_{5.2 \mathrm{kDa}}$ & $40 \pm 1$ & $0.08 \pm 0.05$ & $38 \pm 2$ & $0.14 \pm 0.10$ & $90 \pm 12$ & $9.0 \pm 1.4$ \\
\hline
\end{tabular}

Abbreviations: Z-ave, Z-average hydrodynamic diameter; PDI, polydispersity index

Data are presented as mean $\pm S D(n \geq 3)$

Differences between Z-ave were not significant $(\mathrm{P}>0.05$, Mann-Whitney U-test)

Curcumin retention in the $\mathrm{mPEG}_{5 \mathrm{kDa}}-b$-p(HPMA-Bz) micelles was studied in PBS at $37{ }^{\circ} \mathrm{C}$ by determining micellar curcumin content as a function of incubation time. The results are plotted as both percentage and curcumin concentration in Figure 1 and SI, Figure S5, respectively. Figure $1 \mathrm{~A}$ depicts the stability of micelles containing a $9 \%(w / w)$ curcumin load that were formulated from polymers with different molecular weights of the hydrophobic block. The curcumin micelles composed of mPEG $_{5 \mathrm{kDa}}-b-\mathrm{p}(\mathrm{HPMA}-\mathrm{Bz})_{17.1 \mathrm{kDa}}$ showed the highest curcumin retention, retaining approximately $60 \%$ of curcumin after $168 \mathrm{~h}$. Micelles comprised of $\mathrm{mPEG}_{5 \mathrm{kDa}}-b-\mathrm{p}(\mathrm{HPMA}-\mathrm{Bz})_{10.0 \mathrm{kDa}}$ and $\mathrm{mPEG}_{5 \mathrm{kDa}}-b-\mathrm{p}(\mathrm{HPMA}-\mathrm{Bz})_{5.2 \mathrm{kDa}}$ retained $25-30 \%$ of the loaded curcumin after $168 \mathrm{~h}$ incubation. The rate of curcumin release from the micelles was inversely proportional to the size of the hydrophobic blocks. Most of the curcumin in micelles with the smallest hydrophobic block $(5.2 \mathrm{kDa} ; 70 \%)$ was released 
during the first 2 days. In line with studies using different polymers ${ }^{38,} 39$ and our previous study on paclitaxel-loaded mPEG- $b$-p(HPMA-Bz $)^{30}$, micelles based on block copolymers with a larger hydrophobic block retained the loaded drug more effectively. Particle stability as measured by curcumin retention seems to be correlated with the ratio of aromatic rings present in the larger polymers relative to curcumin (Table 2). It can therefore be hypothesized that strong hydrophobic and $\pi-\pi$ interactions contribute to the stability of the curcuminloaded micelles. The presence of $\pi-\pi$ interactions in the core of micelles was studied by solidstate ${ }^{1} \mathrm{H}$ NMR (Figure 5 and 6, vide infra).
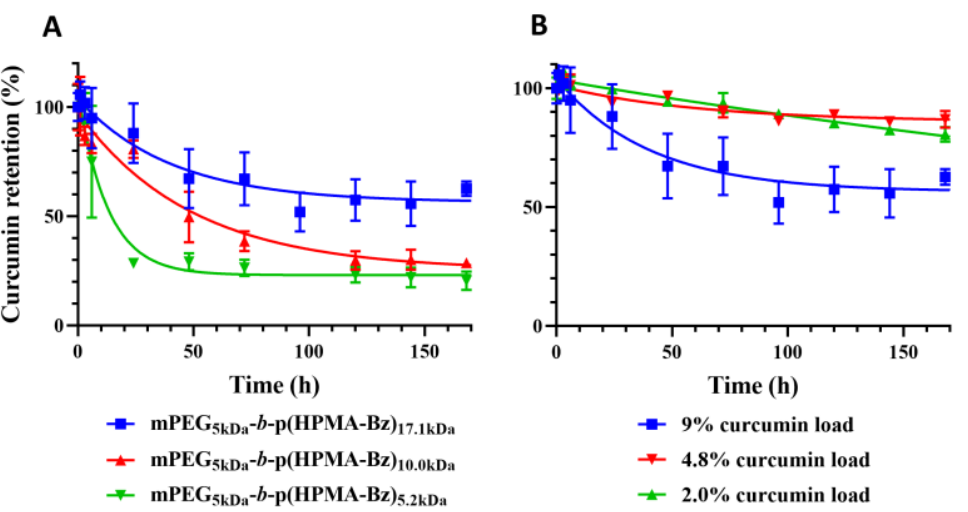

Figure 1. (A) Stability of curcumin-loaded $\mathrm{mPEG}_{5 \mathrm{kDa}}-b-\mathrm{p}(\mathrm{HPMA}-\mathrm{Bz})$ micelles composed of polymers with varying molecular weight of the hydrophobic block $(17.1 \mathrm{kDa}, 10.0 \mathrm{kDa}$, and $5.2 \mathrm{kDa})$. Micelles contained 9\% (w/w) curcumin and were dispersed in PBS, $\mathrm{pH}=7.4$, at $37^{\circ} \mathrm{C}$ during $168 \mathrm{~h}$. (B) Stability of $\mathrm{mPEG}_{5 \mathrm{kDa}}-b-\mathrm{p}(\mathrm{HPMA}-\mathrm{Bz})_{17.1 \mathrm{kDa}}$ micelles as a function of curcumin load $(9 \%, 4.8 \%$, and $2.0 \% \mathrm{w} / \mathrm{w})$ during $168 \mathrm{~h}$ incubation in PBS, $\mathrm{pH}=7.4$, at $37^{\circ} \mathrm{C}$. Data are presented as mean $\pm \mathrm{SD}(\mathrm{n}=3)$.

Table 2. HPMA-Bz monomers:curcumin ratio in different curcumin-loaded micelle formulations at a feed of $3.0 \mathrm{mg} / \mathrm{mL}$ curcumin and $30 \mathrm{mg} / \mathrm{mL}$ polymer.

\begin{tabular}{cccc}
\hline Polymer & Curcumin (mM) & HPMA-Bz (mM) & $\begin{array}{c}\text { Ratio HPMA-Bz/curcumin } \\
(\mathbf{m o l} / \mathbf{m o l})\end{array}$ \\
\hline $\mathrm{mPEG}_{5 \mathrm{kDa}}-b$-p(HPMA-Bz) ${ }_{17.1 \mathrm{kDa}}$ & 7.4 & 94 & 12.7 \\
$\mathrm{mPEG}_{5 \mathrm{kDa}}-b$ - $\mathrm{p}(\mathrm{HPMA}-\mathrm{Bz})_{10.0 \mathrm{kDa}}$ & 7.7 & 81 & 10.6 \\
$\mathrm{mPEG}_{5 \mathrm{k \textrm {Da }}}-b$-p(HPMA-Bz) & 7.3 & 62 & 8.4 \\
\hline
\end{tabular}

An exemplary calculation to obtain the ratio of HPMA-Bz/curcumin is provided for mPEG5kDa- $b$ p(HPMA-Bz) 17.1kDa micelles in the supplementary information.

Based on the retention properties in PBS, studies were continued with the curcumin$\mathrm{mPEG}_{5 \mathrm{kDa}}-b-\mathrm{p}(\mathrm{HPMA}-\mathrm{Bz})_{17.1 \mathrm{kDa}}$ micelles that were deemed most stable. Next, the effect of curcumin load on retention rate was investigated in PBS. The characteristics of the micelles with 2.0 and $4.8 \%$ curcumin load are reported in SI, Table S5. Figure 1B reveals that lower curcumin loads lead to improved micelle stability. Compared to a $9 \%(w / w)$ load, $\mathrm{mPEG}_{5 \mathrm{kDa}}{ }^{-}$ $b$-p(HPMA-Bz) ${ }_{17.1 \mathrm{kDa}}$ micelles containing $2.0 \%$ and $4.8 \%$ curcumin exhibited around $20 \%$ curcumin release during 168 -h incubation at $37{ }^{\circ} \mathrm{C}$, while $40 \%$ of curcumin was released from $\mathrm{mPEG}_{5 \mathrm{kDa}}-b-\mathrm{p}(\mathrm{HPMA}-\mathrm{Bz})_{17.1 \mathrm{kDa}}$ micelles containing $9 \%(w / w)$ curcumin. Similarly, 
Lübtow et al. ${ }^{40}$ evaluated the stability of micelles based on ABA triblock copolymers comprised of hydrophilic poly(2-methyl-2-oxazoline) shell A and poly(2-oxazoline) and poly(2-oxazine)-based hydrophobic blocks B. Although the stability study was different (e.g., higher drug loading and longer monitoring time frame) than the present study, the longterm stability of many of their micellar formulations was higher at lower initial drug feed. Another study reported that the release rate of hydrophobic drugs from hydrotropic polymeric micelles based on N,N-diethylnicotinamide increased with higher drug loading ${ }^{41}$.

As reported previously by Sheybanifard et al. ${ }^{30}$, using DLS and TEM analysis, and as confirmed by $\mathrm{AF}_{4}$ in the present study (Figure 3), mPEG- $b$-p(HPMA-Bz) micelles retained their structural stability upon release of the payload from the micelles. Also, it was demonstrated previously by Naksuriya et al. ${ }^{6}$ that the hydrophobic core of mPEG- $b$ p(HPMA-Bz) micelles significantly reduced the oxidative degradation of curcumin, compared to solubilized curcumin in basic aqueous medium. Protection from degradation is also apparent for curcumin encapsulated in $\mathrm{mPEG}_{5 \mathrm{kDa}}-b-\mathrm{p}(\mathrm{HPMA}-\mathrm{Bz})$ micelles. Only a small additional peak with a retention time of around $5 \mathrm{~min}$ in the HPLC chromatograms at $254 \mathrm{~nm}$ was detected in samples collected at a later time points (SI, Figure S6), which may be ascribed to the formation of dioxygenated bicyclopentadione (the main degradation product of curcumin) as demonstrated by Naksuriya et al. ${ }^{6}$ It is arguable that oxidative modification and alkaline degradation of curcumin mainly occurred after curcumin has dissociated from the micelles, once it became deprotonated.

To mimic quasi-physiological conditions, the stability of curcumin-loaded micelles was studied in human plasma using the $\mathrm{AF}_{4}$ technique. To validate this method, samples consisting of HSA (green line, Figure 2A), plasma spiked with curcumin/DMSO (red lines, Figure 2A), and curcumin-loaded micelles composed of mPEG- $b$-p(HPMA-Bz) with different molecular weights of the hydrophobic block were fractionated and analyzed by measuring absorbance at $280 \mathrm{~nm}$ (Figure 2A; to detect both micelles and protein) and at 425 $\mathrm{nm}$ (Figure 2B; to detect curcumin only). Figure $2 \mathrm{~A}$ confirms that $\mathrm{AF}_{4}$ is a suitable method to separate micelles of different sizes from (lipo)proteins such as albumin, the most abundant plasma protein. The first peak in the plasma sample is albumin, as evidenced by overlaying the fractograms of HSA and plasma (green and red lines Figure 2A). Moreover, the fractogram of curcumin-spiked plasma at $425 \mathrm{~nm}$ (Figure 2B, red line) demonstrates that curcumin binds to and co-elutes with albumin. The observation is in line with previous reports showing that albumin has binding sites for curcumin ${ }^{42,43}$. 
A

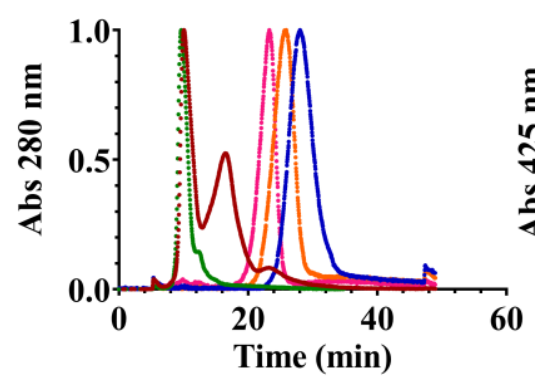

B

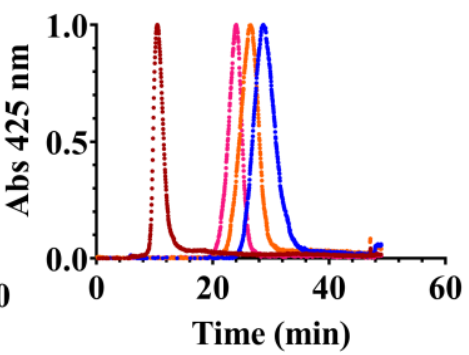

Figure 2. AF4 fractograms of (red) curcumin-spiked plasma, (green) human serum albumin, (blue) curcumin-loaded mPEG5kDa-b-p(HPMA-Bz)17.1kDa micelles, (orange) curcumin-loaded mPEG5kDa-b-p(HPMA-Bz)10.0kDa micelles, and (pink) curcumin-loaded mPEG5kDa-b-p(HPMA$\mathrm{Bz}) 5.2 \mathrm{kDa}$ micelles. Fractograms were recorded at $280 \mathrm{~nm}(\mathrm{~A})$ and $425 \mathrm{~nm}(\mathrm{~B})$. The absorbance (Abs) at each wavelength was normalized to maximum intensity.

Curcumin-loaded micelles were mixed with plasma at a final curcumin concentration of 0.3 $\mathrm{mg} / \mathrm{mL}$, which is in the sink condition range $(0.22-0.44 \mathrm{mg} / \mathrm{mL})$ considering one to two binding sites in albumin (molecular weight $66.5 \mathrm{kDa}$ ) at a physiological concentration of around $40 \mathrm{mg} / \mathrm{mL}$. Figure 3 (right panels) shows that the size and size distribution of different micelles did not change up to $24 \mathrm{~h}$ incubation in plasma. Also, UV absorbance at $280 \mathrm{~nm}$ corresponding to plasma (lipo)proteins and micelles showed a negligible reduction over time (left panels). On the other hand, the absorbance specific for curcumin (425 nm, middle panels) decreased in the micellar fraction, particularly during the first hour. At the same time, curcumin gradually appeared in the albumin fraction, indicating curcumin relocalization from particle to protein.

The rate at which the curcumin area under the curve (AUC) in the albumin fraction increased at the expense of the micellar fraction is reported as percentage of release (Figure 4). At the first measuring point, i.e., after the addition of curcumin-loaded micelles to plasma and before incubation at $37{ }^{\circ} \mathrm{C}$ (taken as time zero), 1.2-10.7\% curcumin release was observed for the largest to the smallest micelles, respectively. This may be ascribed to curcumin release from the micelles within approximately $10 \mathrm{~min}$ after addition to plasma until complete separation by $\mathrm{AF}_{4}$. The release was slower for micelles prepared from $\mathrm{mPEG}_{5 \mathrm{kDa}}-b-\mathrm{p}(\mathrm{HPMA}-$ $\mathrm{Bz})_{17.1 \mathrm{kDa}}$ with the longest hydrophobic block $(22 \%$ in $24 \mathrm{~h})$ versus $\mathrm{mPEG}_{5 \mathrm{kDa}}-b$-p (HPMA$\mathrm{Bz})_{10.0 \mathrm{kDa}}\left(34 \%\right.$ release) and $\mathrm{mPEG}_{5 \mathrm{kDa}}-b-\mathrm{p}(\mathrm{HPMA}-\mathrm{Bz})_{5.2 \mathrm{kDa}}(49 \%$ release). It is remarked that the decrease in AUC of curcumin in the micellar fraction over time is not fully compensated by an increase in curcumins' AUC in the albumin fraction. Partial precipitation and interaction of curcumin with the $\mathrm{AF}_{4}$ membrane may coincide with albumin binding. Disposition of curcumin on the membrane was very evident as the membrane turned yellow over time. In correspondence with the stability data (Figure 1), the $\mathrm{AF}_{4}$ results confirm that curcumin has better retention in micelles prepared from the $\mathrm{mPEG}_{5 \mathrm{kDa}}-b$-p(HPMA-Bz) with a higher molecular weight of the hydrophobic block in the presence of plasma. Correspondingly, Moquin et al. ${ }^{44}$ evaluated the stability of AB3 miktoarm star micelles loaded with curcumin in different media such as Dulbecco's modified Eagle medium (DMEM) enriched with FBS using $\mathrm{AF}_{4}$, and observed that curcumin binds to serum proteins. However, the authors did not clearly explain the role of albumin. 

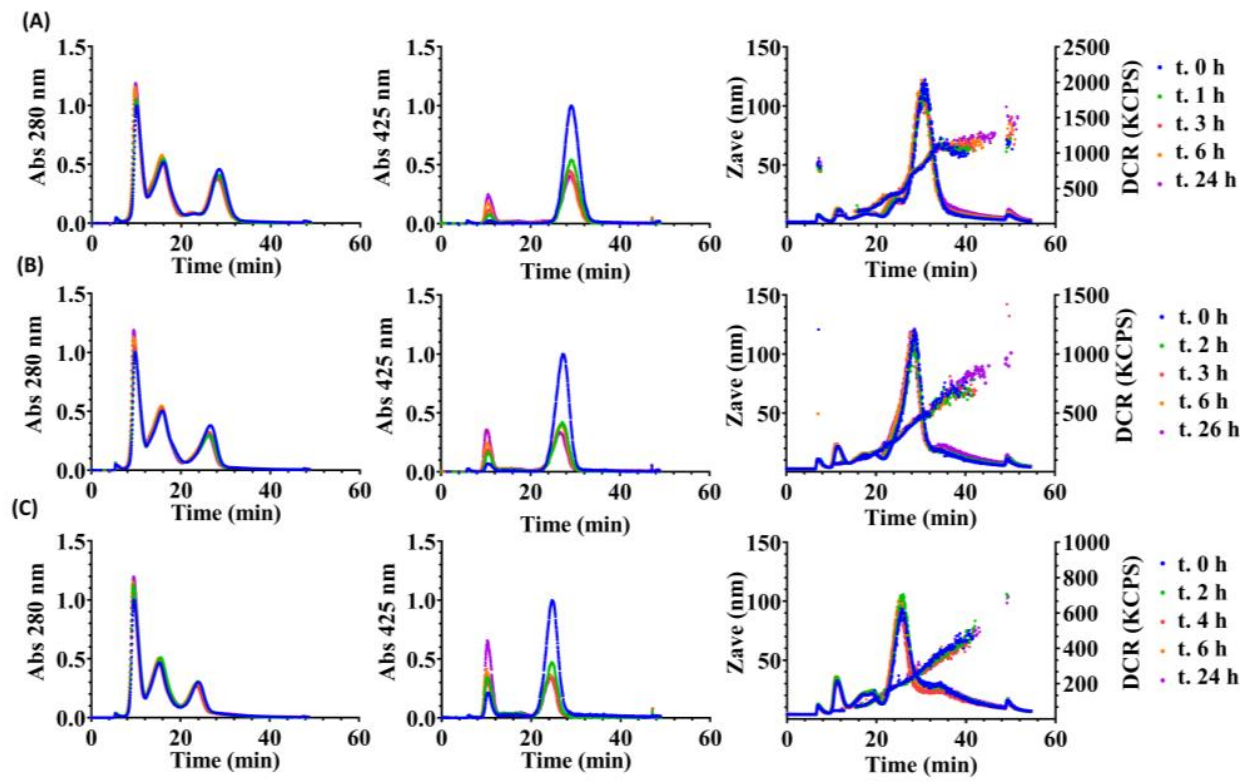

Figure 3. AF4 fractograms of mPEG5kDa- $b$-p (HPMA-Bz) micelles with different molecular weights of the hydrophobic block loaded with $9 \%$ (w/w) curcumin. (A) curcumin-loaded mPEG5kDa- $b$-p (HPMA$\mathrm{Bz})_{17.1 \mathrm{kDa}}$ micelles, (B) curcumin-loaded $\mathrm{mPEG}_{5 \mathrm{kDa}}-b$-p (HPMA-Bz) ${ }_{10.0 \mathrm{kDa}}$ micelles, and (C) curcuminloaded mPEG ${ }_{5 \mathrm{kDa}}-b$-p(HPMA-Bz)5.2kDa micelles incubated in plasma at $37{ }^{\circ} \mathrm{C}$. Fractograms were recorded at $280 \mathrm{~nm}$ (left panel), $425 \mathrm{~nm}$ (middle panel), and by the DLS detector (Z-average size and derived count rate (DCR), right panel). Absorbance was normalized to the signal at time zero (the left and middle panels). Abbreviations: Abs, absorbance; KCPS, kilocounts per second.

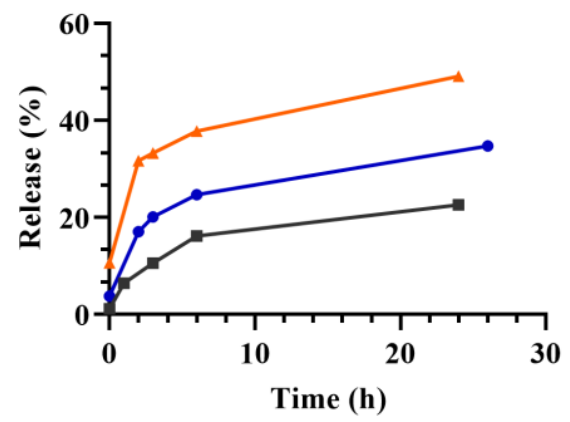

Figure 4. Release of curcumin from mPEG $\mathrm{knDa} b-\mathrm{p}(\mathrm{HPMA}-\mathrm{Bz})$ micelles in plasma at $37{ }^{\circ} \mathrm{C}$. Release (\%) was calculated as (area under the curve of curcumin in albumin fraction at $425 \mathrm{~nm}$ ) / (area under the curve of curcumin in the micelle and albumin fractions at $425 \mathrm{~nm}) \times 100 \%$, based on the areas under the curve presented in Figure 3. Curcumin-loaded $\mathrm{mPEG}_{5 \mathrm{kDa}}-b-\mathrm{p}(\mathrm{HPMA}-\mathrm{Bz})_{17.1 \mathrm{kDa}}, \mathrm{mPEG}_{5 \mathrm{kDa}}-b-$ $\mathrm{p}(\mathrm{HPMA}-\mathrm{Bz}$ ) $10.0 \mathrm{kDa}$, and mPEG5kDa- $b$-p(HPMA-Bz)5.2kDa micelles are depicted as black, blue, and orange, respectively.

\subsection{Detection of $\pi-\pi$ Stacking in Curcumin-loaded Micelles}

Previously, the presence of $\pi-\pi$ stacking interactions among aromatic rings in the core of empty micelles based on a thermosensitive polymer was demonstrated by one-(1D) and two- 
dimensional (2D) solid-state ${ }^{1} \mathrm{H}-\mathrm{NMR}^{32}$. In the current study, the changes in the solid-state ${ }^{1} \mathrm{H}$ NMR spectrum of $\mathrm{mPEG}_{5 \mathrm{kDa}}-b$-p(HPMA-Bz) ${ }_{17.1 \mathrm{kDa}}$ micelles upon loading of curcumin were investigated using a similar approach by applying slow MAS in the order of $1 \mathrm{kHz}$ to prevent micelles destruction (Figure 5).

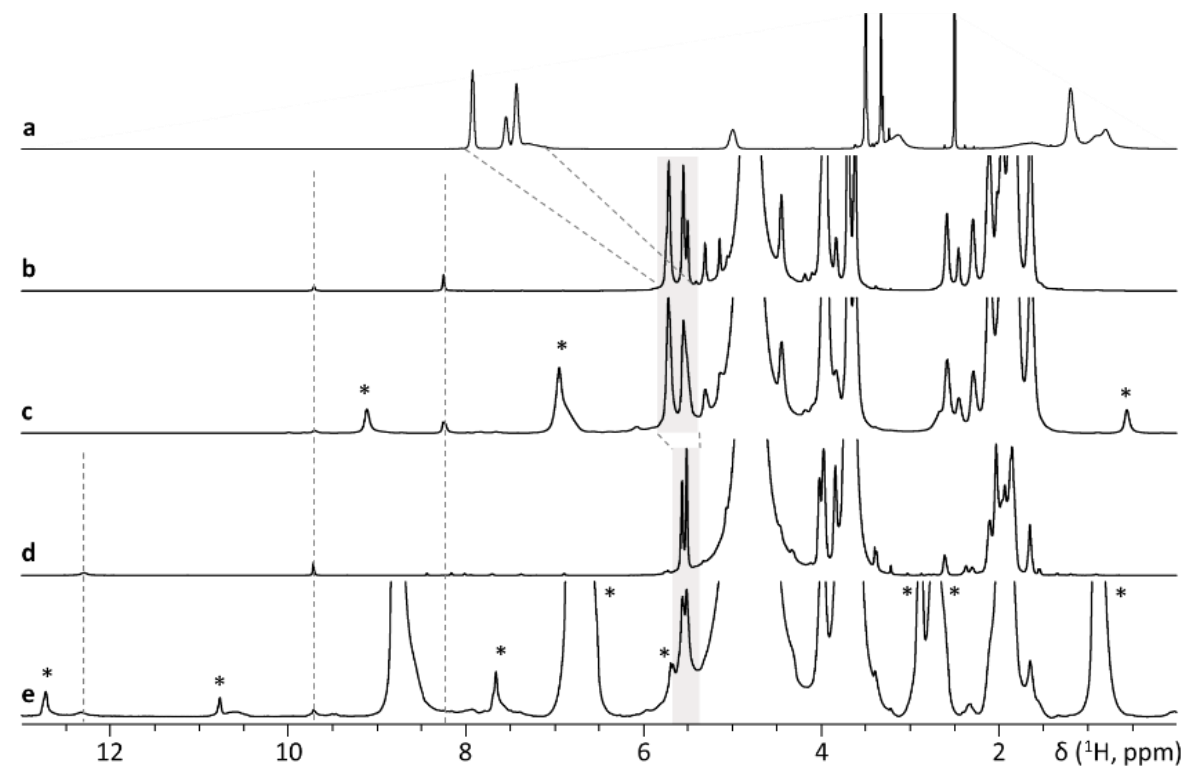

Figure 5. One-dimensional solid-state ${ }^{1} \mathrm{H}$ NMR spectra obtained using different MAS rates compared to solution-state NMR data. (a). ${ }^{1} \mathrm{H}$ NMR spectrum of mPEG $5 \mathrm{kDa}-b$-p (HPMA-Bz) ${ }_{17.1 \mathrm{kDa}}$ copolymer in solution using DMSO as the solvent. (b). Empty micelles dispersed in D2O (solution-state), (c). Empty micelles dispersed in D2O (MAS frequency: $1070 \mathrm{~Hz}$ ), (d). Curcumin-loaded micelles dispersed in $\mathrm{D}_{2} \mathrm{O}$ (solution-state), (e). Curcumin-loaded micelles dispersed in $\mathrm{D}_{2} \mathrm{O}$ (MAS frequency $1110 \mathrm{~Hz}$ ). Asterisks (*) indicate spinning sidebands.

Figure 5a shows the spectrum of copolymer dissolved in DMSO. In line with our previous study, upon formation of micelles in $\mathrm{D}_{2} \mathrm{O}$ using nanoprecipitation as described in section 2.5, our solid-state ${ }^{1} \mathrm{H}$ NMR data showed that aromatic peaks significantly broadened and shifted towards higher field (from $7.4-7.9 \mathrm{ppm}$ to $5.5-5.7 \mathrm{ppm}$ ) as expected for $\pi$ - $\pi$ interactions ${ }^{45}$, ${ }^{46}$. In addition, weak and broad peaks appeared at 8.25 and $9.31 \mathrm{ppm}$ (Figure $5 \mathrm{~b}$ and c). Figure 6 demonstrates that signals resonating at $5.5-5.7 \mathrm{ppm}$ in the spectrum of micelles correlate with signals at $100-120 \mathrm{ppm}$ in ${ }^{13} \mathrm{C}$ dimension. There are no other correlations at higher ppm values except for a thus far unassigned ${ }^{13} \mathrm{C}$ signal at $\sim 170 \mathrm{ppm}$. In the spectra of curcumin-loaded micelles (Figure $5 \mathrm{~d}$ and e), peaks of the aromatic protons shifted further to high field. In addition, the signal at $8.21 \mathrm{ppm}$ either disappeared or further broadened beyond detection and a new very low-field and broad signal appeared at $12.30 \mathrm{ppm}$. The observed spectral changes may indicate the presence of $\pi-\pi$ stacking interactions in the hydrophobic core of the micelles which further strengthen upon addition of curcumin. However, to unambiguously prove these interactions, additional 2D NMR experiments, possibly at a higher magnetic field, will be needed. 


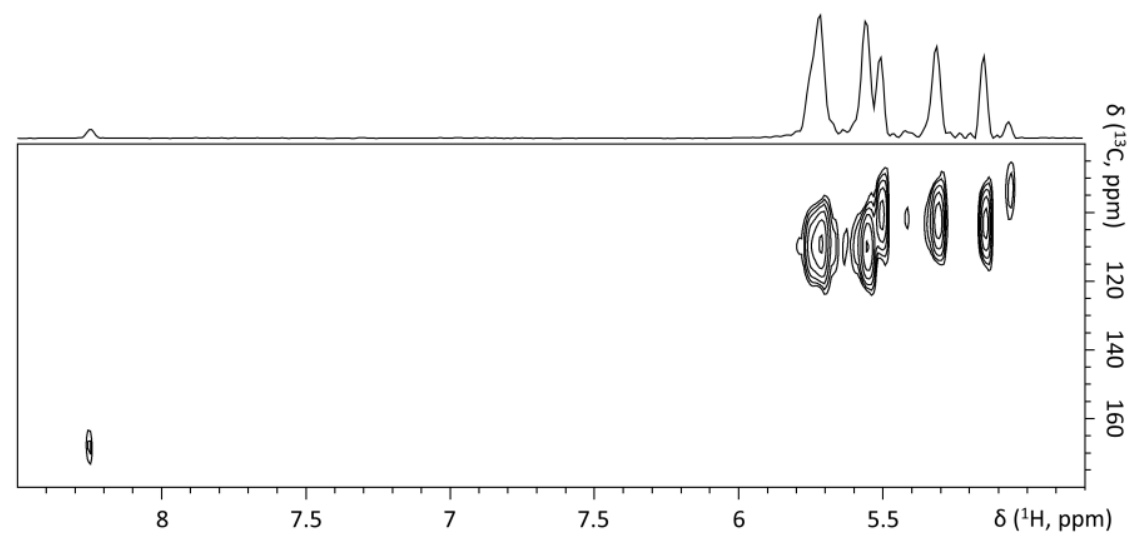

Figure 6. Spectral cutout of a $2 \mathrm{D}{ }^{1} \mathrm{H}-{ }^{13} \mathrm{C}$ heteronuclear single quantum correlation (HSQC) spectrum of empty micelles dispersed in $\mathrm{D}_{2} \mathrm{O}$ (static conditions).

\subsection{Cellular Uptake of mPEG ${ }_{5 \mathrm{kDa}}-\boldsymbol{b}$-p(HPMA-Bz $)_{17.1 \mathrm{kDa}}$ Micelles In Vitro}

In light of the stability data, further studies focused solely on the $\mathrm{mPEG}_{5 \mathrm{kDa}}-b$-p (HPMA$\mathrm{Bz})_{17.1 \mathrm{kDa}}$ formulation. Cell association and internalization were studied by flow cytometry and confocal imaging, respectively. Figure 7 demonstrates that the micelles were internalized by different types of cancer cells. The internalization of the micelles occurred in a timedependent manner (Figure 7A and B). Confocal microscopy revealed that free curcumin, empty micelles, and curcumin-loaded micelles were intracellularly detected in Neuro2A cells during a 4-h time span (Figure $7 \mathrm{C}$ ). At a $\log \mathrm{P}$ of 2.5 , free curcumin can permeate cell membranes passively, which is in line with the chemical behavior of compounds with a $\log \mathrm{P}$ $>1.6^{47}$. Quantification of curcumin by flow cytometry showed different association kinetics for curcumin in its free form and loaded in micelles. Free curcumin was detected intracellularly within $1 \mathrm{~h}$ of incubation. After $4 \mathrm{~h}$ of incubation the intracellular curcumin level remained constant in Neuro2A and EGI-1 cells, suggesting equilibrium between curcumin in the culture medium and the cytosol. In TFK-1 and M-ChA-1 cells curcumin fluorescence intensity had decreased, possibly due to degradation. In line with this study, Sun et al. ${ }^{48}$ reported similar rapid cell uptake of free curcumin followed by a decline in curcumin fluorescence intensity over time due to degradation of unprotected curcumin. Figure 7B shows that the intracellular curcumin fluorescence intensity was substantially lower (by 3 - to 24-fold) for curcumin-loaded micelles incubated at the same curcumin concentration with the cells compared to the free curcumin. This lower fluorescence from intracellular curcumin is possibly attributable to either lower uptake of the micelles or fluorescence quenching of micellar curcumin (SI, Figure S8), which suggests that the kinetics of curcumin uptake are governed by micelle uptake. It should be noted that curcumin is strongly solvatochromic, so differential interaction of curcumin with its chemical environment should not be discounted as a (partial) basis for the differences in fluorescence intensity. Importantly, the intracellular fluorescence intensity increased with time upon incubation of the cells with curcumin-loaded micelles, pointing to a relatively slow internalization of the loaded micelles. Taken together, the results demonstrate that the intracellular presence of curcumin is at least partly related to the uptake of intact curcumin-loaded micelles. However, some release of curcumin from the micelles in the cell culture medium and/or in the cytosol cannot be excluded. 


\section{Chapter 5}

A
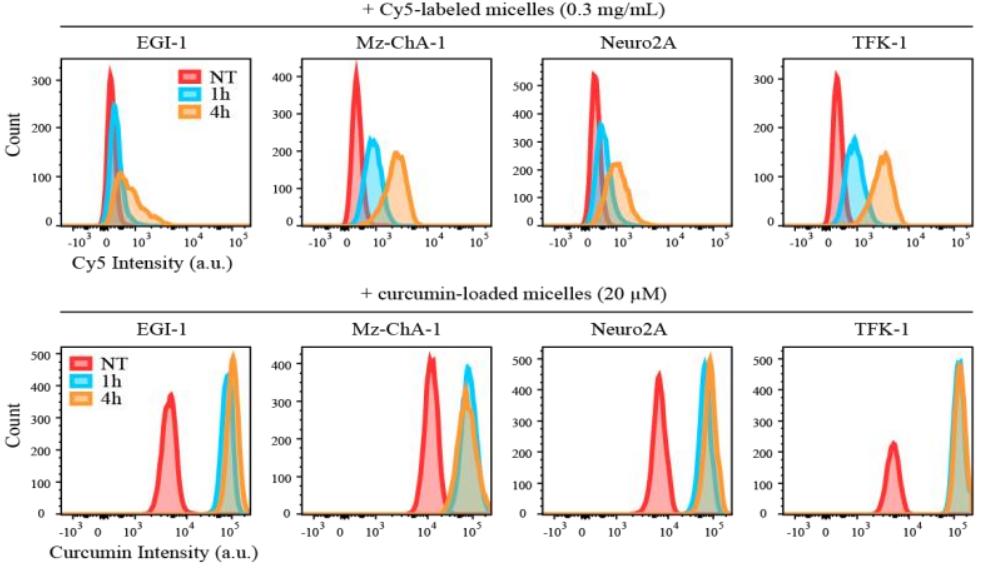

+ free curcumin $(20 \mu \mathrm{M})$
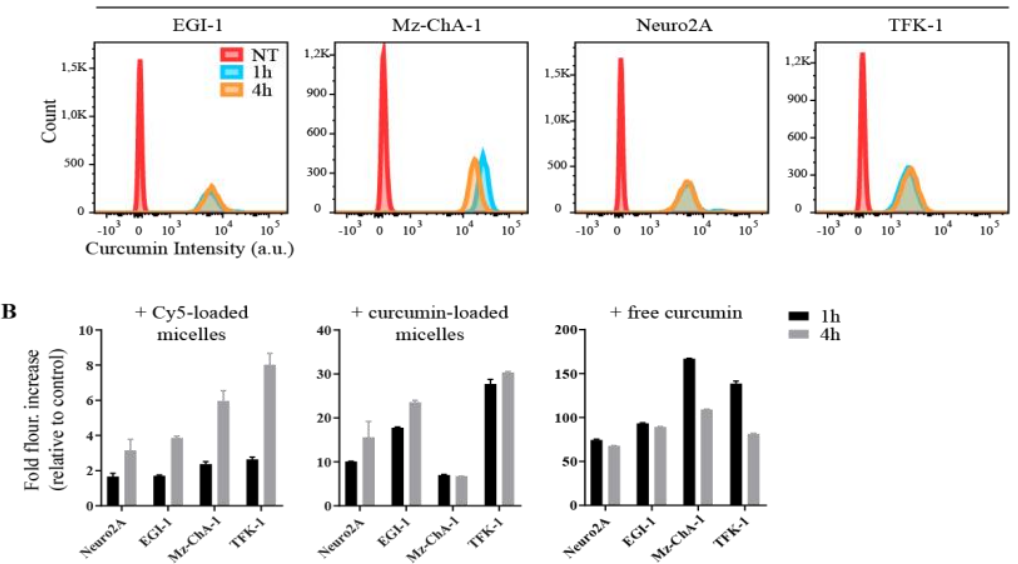

C
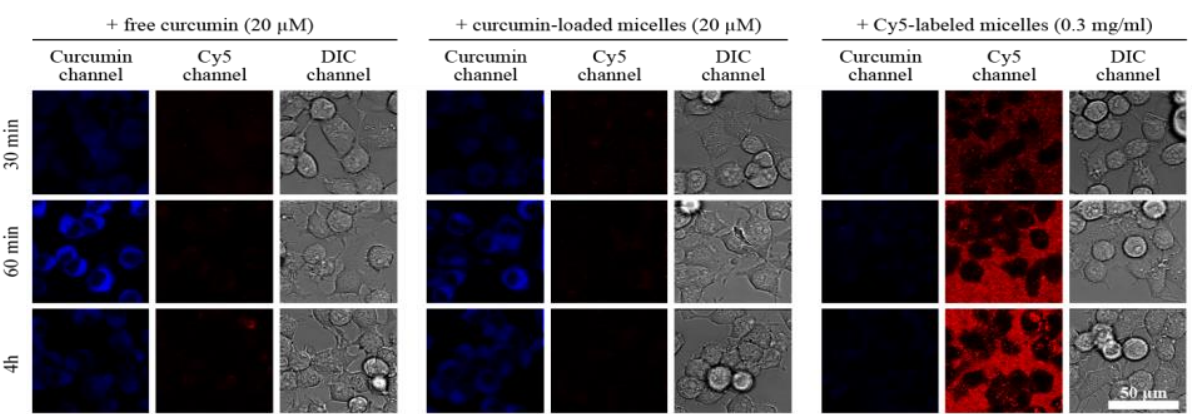

Figure 7. Time-based uptake of free curcumin, empty mPEG5kDa- $b$-p(HPMA-Bz) $17.1 \mathrm{kDa}$ micelles, and curcumin-loaded mPEG $5 \mathrm{kDa}-b$-p(HPMA-Bz) $17.1 \mathrm{kDa}$ micelles. (A) Representative flow cytograms of cancer cells that had been exposed to free curcumin, curcumin-loaded micelles, and Cy5-labeled micelles. Cells were incubated with non-supplemented medium containing curcumin in free form or loaded in micelles at $20 \mu \mathrm{M}$ or Cy5-loaded micelles $(0.3 \mathrm{mg} / \mathrm{mL})$ for 1 or $4 \mathrm{~h}$. (B) Fluorescence quantification of results from (A). The bars represent the mean \pm SD fold-increase in fluorescence intensity relative to non-treated samples ( $\mathrm{n}=3$ per group). (C) Confocal imaging of uptake of free curcumin, 9\% (w/w) curcumin-loaded $\mathrm{mPEG}_{5 \mathrm{kDa}}-b-\mathrm{p}(\mathrm{HPMA}-\mathrm{Bz})_{17.1 \mathrm{kDa}}$ micelles, and Cy5-labeled 
$\mathrm{mPEG}_{5 \mathrm{kDa}}-b$-p(HPMA-Bz) ${ }_{17.1 \mathrm{kDa}}$ micelles. Neuro2A cells seeded on glass coverslips were incubated in medium supplemented with the indicated formulations for $0.5,1$, and $4 \mathrm{~h}$ and imaged by confocal microscopy. Note the binding of the Cy5-labeled micelles to the surface of the coverslips despite 5 washing steps, visible as granular red staining in-between cells. Nevertheless, this non-specific binding does not mask the increasing uptake of micelles at later time-points, discernible as dotted staining of cell membranes. Galleries constitute representative images. Scale bar: $50 \mu \mathrm{m}$. Abbreviations: NT, not treated; DIC, differential interference contrast.

\subsection{In Vitro Cytotoxicity of Curcumin-loaded mPEG $_{5 k D a}-b$-p(HPMA-Bz) ${ }_{17.1 k D a}$ Micelles}

Cytotoxicity of empty and curcumin-loaded mPEG $_{5 \mathrm{kDa}}-b$-p(HPMA-Bz) ${ }_{17.1 \mathrm{kDa}}$ micelles was evaluated in human neuroblastoma (Neuro2A) cells and compared to the cytotoxicity of free curcumin. The data are summarized in Figure 8.

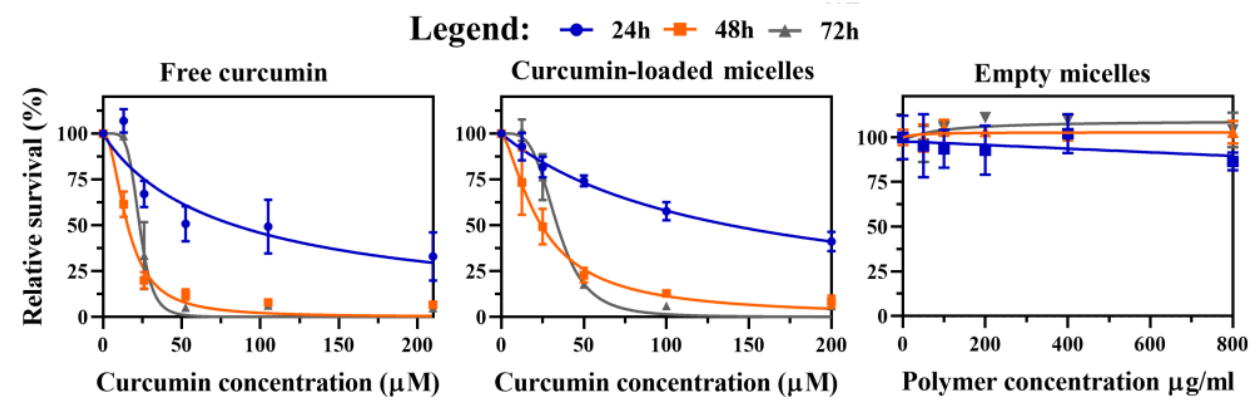

Figure 8. Cytotoxicity of free curcumin, curcumin-loaded $\mathrm{mPEG}_{5 \mathrm{kDa}}-b-\mathrm{p}(\mathrm{HPMA}-\mathrm{Bz})_{17.1 \mathrm{kDa}}$ micelles, and empty mPEG $5 \mathrm{kDa}-b$-p(HPMA-Bz) $17.1 \mathrm{kDa}$ micelles in Neuro2A cells incubated for $24 \mathrm{~h}, 48 \mathrm{~h}$, and 72 h. Cell viability was measured with the SRB total protein assay. Data were normalized to the average value of the control (untreated) cells at the respective incubation time. The highest curcumin concentration in $\mathrm{mPEG}_{5 \mathrm{kDa}}-b$-p $(\mathrm{HPMA}-\mathrm{Bz})_{17.1 \mathrm{kDa}}$ micelles $(200 \mu \mathrm{M})$ corresponds to a polymer concentration of $800 \mu \mathrm{g} / \mathrm{mL}$. Data were fitted using a nonlinear regression model (curve fit) based on a sigmoidal inhibitor vs. normalized response curve (variable slope) and are presented as mean \pm SD $(\mathrm{n}=4$ per incubation time).

Empty micelles did not notably affect Neuro2A cell viability up to a polymer concentration of $800 \mu \mathrm{g} / \mathrm{mL}$, which is in agreement with previous studies ${ }^{26,27}$. The cytotoxicity of free curcumin and curcumin-loaded $\mathrm{mPEG}_{5 \mathrm{kDa}}-b$-p(HPMA-Bz $)_{17.1 \mathrm{kDa}}$ micelles was dose- and time-dependent, whereby the most profound cell death occurred during the first $48 \mathrm{~h}$. Curcumin interferes with several vital pathways and, almost without exception, induces apoptosis in tumor cells ${ }^{3}$ that includes Neuro2A cells. Sidhar et al. ${ }^{49}$ showed that curcumin can induce re-expression of brain expressed X-linked (Bex) genes and thereby activate p53 and cause apoptosis in Neuro2A cells. Also, the induction of apoptosis in Neuro2A cells via mitochondrial pathways was reported by Jana et al. ${ }^{50}$

The $\mathrm{IC}_{50}$ values decreased over time and plateaued around $20 \mu \mathrm{M}$ after 48 and $72 \mathrm{~h}$ (Table 3 ), corresponding to similar $\mathrm{IC}_{50}$ values reported in the literature for other cancer cell lines ${ }^{3}$. The higher $\mathrm{IC}_{50}$ value observed at $72 \mathrm{~h}$ incubation can be explained by several phenomena. First, curcumin was shown to exit the micellar complex (Figures 1 and 4), which also seems to occur following particle internalization (Figure 7). Free curcumin in the intracellular milieu becomes deprotected and may be degraded and/or metabolized to molecular entities 
that are not cytotoxic ${ }^{3}$, a process that culminates in a sublethal intracellular curcumin concentration within $48 \mathrm{~h}$. Second and in parallel, the remaining viable cells recover and/or activate cell survival pathways ${ }^{51}$, which leads to cell proliferation and higher total protein content at $72 \mathrm{~h}$ relative to $48 \mathrm{~h}$, and hence a higher $\mathrm{IC}_{50}$ value.

The cytotoxicity study was repeated in cell lines derived from human cholangiocarcinoma (EGI-1, Sk-ChA-1, and TFK-1) and gallbladder adenocarcinoma (Mz-ChA-1) to validate the Neuro2A results. Mz-ChA-1 did not show cell death up to a polymer concentration of 800 $\mu \mathrm{g} / \mathrm{mL}$, while TFK-1, EGI-1, and Sk-ChA-1 exhibited a moderate decrease in relative survival at the highest polymer concentration (SI, Figure S7). In line with the Neuro2A toxicity data, the $\mathrm{IC}_{50}$ values for free and micellar curcumin in the biliary cancer cell lines decreased and plateaued after 48 (Table 3 ). The $\mathrm{IC}_{50}$ values of free curcumin in Neuro2A, EGI-1, Sk-ChA-1, and TFK-1 after 48 and $72 \mathrm{~h}$ were comparable and gyrated around $20 \mu \mathrm{M}$.

Table 3. IC50 values $(\mu \mathrm{M})$ of free curcumin and curcumin-loaded mPEG5kDa-b-p(HPMABz) 17.1kDa micelles in Neuro2A, EGI-1, Mz-CHA-1, Sk-ChA-1, and TFK-1 cells at different curcumin exposure times.

\begin{tabular}{ccccccc}
\hline & \multicolumn{3}{c}{ Free curcumin } & \multicolumn{3}{c}{ Curcumin-loaded micelles } \\
\hline & $\mathbf{2 4} \mathbf{~ h}$ & $\mathbf{4 8} \mathbf{~ h}$ & $\mathbf{7 2 ~ h}$ & $\mathbf{2 4 ~ h}$ & $\mathbf{4 8 ~ h}$ & $\mathbf{7 2 ~ \mathbf { ~ }}$ \\
\hline Neuro2A & $83.8 \pm 13.5$ & $15.6 \pm 0.8^{*}$ & $23.3 \pm 1.2$ & $138.7 \pm 9.0$ & $24.3 \pm 1.8^{* *}$ & $33.9 \pm 1.2$ \\
EGI-1 & $21.7 \pm 0.5$ & $18.2 \pm 0.3$ & $19.1 \pm 0.3$ & $38.4 \pm 4.4$ & $20.6 \pm 0.5$ & $21.8 \pm 0.5$ \\
Mz-ChA-1 & $47.6 \pm 1.6$ & $38.4 \pm 1.4$ & $37.8 \pm 1.1$ & $132.8 \pm 6.2$ & $80.1 \pm 2.6$ & $69.2 \pm 2.6^{* *}$ \\
Sk-ChA-1 & $33.9 \pm 1.3$ & $21.5 \pm 0.4$ & $21.3 \pm 0.4$ & $46.3 \pm 1.6$ & $36.3 \pm 1.1$ & $33.4 \pm 1.1^{*}$ \\
TFK-1 & $27.7 \pm 0.9$ & $20.1 \pm 0.9$ & $17.5 \pm 0.5$ & $76.7 \pm 4.8$ & $36.9 \pm 1.7$ & $30.8 \pm 0.7^{* *}$ \\
\hline
\end{tabular}

Data are presented as mean \pm SD (free curcumin $(\mathrm{n}=3$ ), free curcumin in Neuro2A $(\mathrm{n}=4)$, and curcumin-loaded micelles $(n=4)$ per time point).

Statistical analysis versus $24 \mathrm{~h}$ incubation; $* \mathrm{P}$ value $\leq 0.05$ and $* * \mathrm{P}$ value $\leq 0.01$ (Kruskal-Wallis test with Dunn's post-hoc correction).

Statistical analysis between 48-h and 72-h incubation time was not significant (Kruskal-Wallis test with Dunn's post-hoc correction).

Neuro2A and Mz-ChA-1 cells showed higher $\mathrm{IC}_{50}$ values ( $84 \mu \mathrm{M}$ and $48 \mu \mathrm{M}$, respectively) compared to the other cell lines, particularly during the first $24 \mathrm{~h}$ of exposure (Table 3 ). This might be due to the slower growth rate and thus slower metabolic rate of Neuro2A and MzChA-1 cells (doubling time of 3-4 days) (2) $^{52}$ compared to the other cell lines (around 2 days) ${ }^{52}$, ${ }^{53}$. Metabolically hyperactive cancer cells are generally more susceptible to pleiotropic agents such as curcumin because these agents can attack multiple biochemical hubs that culminate in metabolic catastrophe and corollary cell death ${ }^{54}$. Generally, the $\mathrm{IC}_{50}$ values of free curcumin for these cell lines are within the range $(1-100 \mu \mathrm{M})$ of those reported for different types of cancer cell lines in the literature, with a mean $\pm \mathrm{SD}$ of $21 \pm 17 \mu \mathrm{M}^{3}$. Among the cell lines, Neuro2A and Mz-ChA-1 with the lowest growth rates showed the highest $\mathrm{IC}_{50}$ values for curcumin-loaded micelles (above $130 \mu \mathrm{M}$ after $24 \mathrm{~h}$ exposure), affirming this inverse metabolic rate-chemical susceptibility relationship. It should be noted that the $\mathrm{IC}_{50}$ substantially decreased at longer exposure times, particularly for Neuro2A cells, reaching around 24-33 $\mu \mathrm{M}$ (Table 3). In support of these findings, relatively high resistance to both free and curcumin-loaded $\gamma$-cyclodextrin liposomal nanoparticles was also reported in a primary cell line established from an untreated patient osteosarcoma biopsy. 
Chemotherapeutic resistance was attributed to the very slow growth rate and low uptake capacity of the cells with inherently low metabolic activity ${ }^{55}$.

The higher $\mathrm{IC}_{50}$ value for curcumin-loaded $\mathrm{mPEG}_{5 \mathrm{kDA}}-b$-p(HPMA-Bz) micelles versus free curcumin is echoed by previous observations by Naksuriya et al. ${ }^{27}$, and is likely due to the slow release of curcumin from the micelles either in the medium or intracellularly after internalization of the micelles (Figure 7 and Figure 8).

\subsection{Pharmacokinetics and Biodistribution of Curcumin-Loaded mPEG $5 \mathrm{kDa}-b$ - p(HPMA-Bz) 17.1kDa Micelles}

To detect any possible acute toxicity, mice received an escalating polymer dose of the empty $\mathrm{mPEG}_{5 \mathrm{kDa}}-b-\mathrm{p}(\mathrm{HPMA}-\mathrm{Bz})_{17.1 \mathrm{kDa}}$ micelles. Mice did not exhibit signs of discomfort, changes in blood cell count, or weight loss $24 \mathrm{~h}$ after intravenous administration (SI, Figure S11 and Figure S12) for any of the tested doses $(100,300,500 \mathrm{mg} / \mathrm{kg})$. Also, the cytocompatibility of the micelles was confirmed in vitro in non-cancer cells such as fibroblasts (up to $800 \mu \mathrm{g} / \mathrm{mL}$ ) and HUVECs even at polymer concentrations up to $3 \mathrm{mg} / \mathrm{mL}$ (Figure $\mathrm{S} 9$ and S10). Therefore, the in vitro and in vivo results suggest that the micelles have good cytocompatibility and do not induce short-term toxicity.

Next, the circulation kinetics and biodistribution of Cy7-labeled micelles loaded with $9 \%$ curcumin were studied in BALB/c mice at a polymer dose of $500 \mathrm{mg} / \mathrm{kg}$, which is equivalent to $50 \mathrm{mg} / \mathrm{kg}$ of curcumin. Figure 9 depicts curcumin and Cy7-labeled micelle concentration in plasma at different time points after intravenous injection. More than $50 \%$ of the injected dose of micelles was still in the circulation after 24 hours, underscoring the in vivo stability of the micelles. This is in agreement with the in vitro stability results obtained by $\mathrm{AF}_{4}$ (Figure 3 ) and previous studies on mPEG- $b$-p(HPMA-Bz) micelles by Varela-Moreira et al. and Shi et al. ${ }^{26,56}$. However, $90 \%$ of the loaded curcumin was rapidly eliminated during the first hour. Accordingly, $\mathrm{AF}_{4}$ experiments demonstrated that curcumin-loaded micelles incubated in plasma at $37{ }^{\circ} \mathrm{C}$ were stable in terms of size and polydispersity for at least $24 \mathrm{~h}$, but that a significant decrease of curcumin content in the micellar fraction occurred within $1 \mathrm{~h}$ (Figure 3 ). The release of curcumin from the micelles during the first hour after injection was substantially faster than the release rate anticipated by the stability studies in plasma. After intravenous administration, curcumin-loaded micelles are in contact with (lipo)proteins and different blood cells. It has been demonstrated by Bolger et al. ${ }^{57}$ that curcumin distributes across blood cells and is even metabolized, particularly in erythrocytes, which can explain the observed discrepancy between the $\mathrm{AF}_{4}$ and $\mathrm{PK}$ data of the curcumin-loaded micelles. 


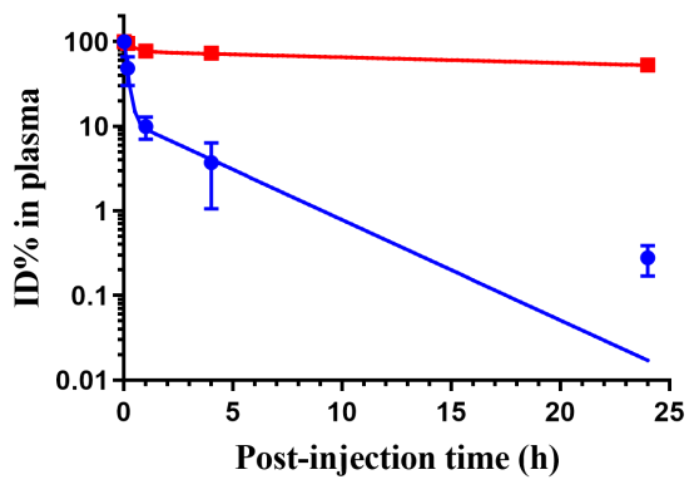

- curcumin

- polymeric micelles

Figure 9. Circulation kinetics of $9 \%(\mathrm{w} / \mathrm{w})$ curcumin-loaded Cy7-labeled mPEG $5 \mathrm{kDa}-b-\mathrm{p}(\mathrm{HPMA}-$ $\mathrm{Bz})_{17.1 \mathrm{kDa}}$ micelles $(500 \mathrm{mg} / \mathrm{kg}$, equivalent to $50 \mathrm{mg}$ curcumin $/ \mathrm{kg}$ ) in BALB/c mice. Plasma samples collected at different time points were used to quantify the percentage of the initial dose (\%ID) present in the systemic circulation. Data are presented as mean $\pm \mathrm{SD}(\mathrm{n}=3$ per time point). Symbols: experimental data of Cy7-labeled polymeric micelles (red squares) and curcumin (blue dots); red and blue lines: fitted curves of compartmental analysis of plasma concentrations.

Non-compartmental and two-compartmental analyses were employed to determine the PK parameters of the micelles and curcumin, respectively (Table 4). The PK profile of curcumin in plasma demonstrated that the initial $\left(\mathrm{t}_{1 / 2 \alpha}\right)$ and terminal elimination phase $\left(\mathrm{t}_{1 / 2 \beta}\right)$ were 6.7 min and $2.5 \mathrm{~h}$, respectively. The alpha and beta phases are primarily attributed to the drug distribution from the central compartment (circulation) and elimination by metabolism and excretion, respectively ${ }^{58}$. Based on the curcumin plasma concentration (ID\%; Figure 9) and PK (Table 4), the crucial part of the circulation kinetics of curcumin encompasses the first 4 $\mathrm{h}$, where most of the curcumin was eliminated from the circulation and during which time the initial half-life is defined. In contrast, $\mathrm{mPEG}_{5 \mathrm{kDa}}-b-\mathrm{p}(\mathrm{HPMA}-\mathrm{Bz})_{17.1 \mathrm{kDa}}$ micelles exhibited a prolonged circulation time with a $\mathrm{t}_{1 / 2}$ of $42 \mathrm{~h}$ (Table 4 ). These results are comparable to the circulation kinetics of intravenously administered PEGylated liposomes ${ }^{59}$.

Table 4. Pharmacokinetic parameters of $\mathrm{mPEG}_{5 \mathrm{kDa}}-b-\mathrm{p}(\mathrm{HPMA}-\mathrm{Bz})_{17.1 \mathrm{kDa}}$ micelles and curcumin after intravenous administration of $9 \%(\mathrm{w} / \mathrm{w})$ curcumin-loaded micelles containing $1.5 \%(\mathrm{w} / \mathrm{w})$ Cy 7 -labeled polymer, analyzed by non-compartmental and two-compartmental models, respectively. The formulation was injected at $50 \mathrm{mg}$ curcumin/ $\mathrm{kg}$ body weight, corresponding to $7.8 \mathrm{mg} / \mathrm{kg}$ Cy7-labeled polymer.

\begin{tabular}{|c|c|c|c|c|c|c|c|c|}
\hline \multirow{2}{*}{ Component } & \multicolumn{2}{|c|}{$\mathbf{t}_{1 / 2}(\mathrm{~h})$} & \multirow{2}{*}{$\begin{array}{l}\mathbf{t}_{1 / 2} \\
\text { (h) }\end{array}$} & \multirow{2}{*}{$\underset{(\boldsymbol{\mu g} \cdot \mathbf{h} / \mathbf{m L})}{\mathbf{A U C}_{\mathbf{0}-\infty}}$} & \multirow{2}{*}{$\begin{array}{c}\mathrm{V}_{1} \\
(\mathrm{~mL} / \mathrm{kg})\end{array}$} & \multirow{2}{*}{$\begin{array}{c}\mathrm{V}_{2} \\
(\mathrm{~mL} / \mathrm{kg})\end{array}$} & \multirow{2}{*}{$\underset{(\mathrm{mL} / \mathrm{kg})}{\mathbf{V}}$} & \multirow{2}{*}{$\begin{array}{c}\mathbf{C L} \\
(\mathrm{mL} / \mathrm{h} / \mathrm{kg})\end{array}$} \\
\hline & $\alpha$-phase & $\beta$-phase & & & & & & \\
\hline curcumin & 0.11 & 2.5 & - & 319 & 86 & 344 & - & 157 \\
\hline micelles & - & - & 42 & 6713 & - & - & 70 & 1.2 \\
\hline
\end{tabular}

Abbreviations: $\mathrm{t}_{1 / 2}$, half-life (initial half-life $\mathrm{t}_{1 / 2 \alpha}$ and terminal half-life $\mathrm{t}_{1 / 2 \beta}$ ); $\mathrm{V}$, volume of distribution; $\mathrm{CL}$, clearance; $\mathrm{AUC}_{0-\infty}$, extrapolated area under the curve (AUC) from time zero to infinity.

The results were compared to the pharmacokinetics of free curcumin (compartmental and non-compartmental analysis) reported in the literature. $\mathrm{A}_{1 / 2 \alpha}$ of $0.023 \pm 0.001 \mathrm{~h}$ (average value \pm standard deviation of three independent studies) has been reported for free curcumin 
in healthy mice ${ }^{18,60,61}$, which is around 5 times shorter than the $t_{1 / 2 \alpha}$ of curcumin after administration of the curcumin-loaded mPEG- $b$-p(HPMA-Bz) micelles (Table 4). In addition, the AUC values reported in the literature were normalized to the injected dose (AUC/mg/kg), which ranged between $0.036-4.08(\mu \mathrm{g} \cdot \mathrm{h} / \mathrm{mL}) /(\mathrm{mg} / \mathrm{kg})$ for free curcumin in mice and had a mean \pm SEM AUC of $1.1 \pm 0.5 \mu \mathrm{g} \cdot \mathrm{h} / \mathrm{mL}$ based on 9 studies, indicating a broad range in reported AUC values ${ }^{16,18,20,60-65}$. Among these studies, Gao et al. reported an AUC value of $60.3 \mu \mathrm{g} \cdot \mathrm{h} / \mathrm{mL}$ for free curcumin at a similar curcumin administration dose (50 $\mathrm{mg} / \mathrm{kg}$ ) in mice using non-compartmental analysis ${ }^{63}$. The normalized AUC of curcumin nanoformulations in two studies were 6.0 and $5.9(\mu \mathrm{g} \cdot \mathrm{h} / \mathrm{mL}) /(\mathrm{mg} / \mathrm{kg})$ compared to normalized AUCs of 1.2 for free curcumin $(\mu \mathrm{g} \cdot \mathrm{h} / \mathrm{mL}) /(\mathrm{mg} / \mathrm{kg})$ in both studies, respectively ${ }^{16}$, 63. These nanoformulations increased the AUC by a factor 5 . In this study, the normalized AUC of curcumin-loaded mPEG- $b$-p(HPMA-Bz) micelles was $6.4(\mu \mathrm{g} \cdot \mathrm{h} / \mathrm{mL}) /(\mathrm{mg} / \mathrm{kg})$. Therefore, it can be concluded that curcumin-loaded mPEG- $b$-p(HPMA-Bz) micelles improved the circulation kinetics of curcumin to the same extent as other nanoformulations.

The PK values of the particles were not reported in relevant publications ${ }^{16,18,20,60-65}$. It is therefore not possible to relate the curcumin retention with the circulation time of the particles. However, since the curcumin concentration declined rapidly in the circulation and the AUC ratio of nanoformulated curcumin to free curcumin is around $5^{16,63}$, these nanoformulations (including mPEG- $b$-p(HPMA-Bz) micelles) thus mainly acted as an efficient solubilizer. Similar to the mPEG- $b$-p(HPMA-Bz) micelles of the present study, Gong et al. ${ }^{20}$ utilized an intermolecular $\pi-\pi$ stacking strategy using mPEG-PCL-Phe(Boc)based micelles to improve the PK of curcumin. These micelles prolonged the circulation kinetics of curcumin compared to both mPEG-PCL micelles and the free form by $\sim 3$-fold. The normalized AUC for the mPEG-PCL-based curcumin formulation was 0.23 $(\mu \mathrm{g} \cdot \mathrm{h} / \mathrm{mL}) /(\mathrm{mg} / \mathrm{kg})$, which is lower than the AUC reported for mPEG- $b-\mathrm{p}$ (HPMA-Bz) micelles $(6.4(\mu \mathrm{g} \cdot \mathrm{h} / \mathrm{mL}) /(\mathrm{mg} / \mathrm{kg}))$. Accordingly, mPEG- $b$-p(HPMA-Bz) micelles can results in higher curcumin exposure per injected dose. The difference might be ascribed to the number of aromatic rings in the polymers since only the end of the mPEG-PCL polymers was modified.

The volume of distribution of mPEG- $b$-p(HPMA-Bz) micelles was $70 \mathrm{~mL} / \mathrm{kg}$ (Table 4), indicating that the micelles were retained in the circulation considering that an average mouse has a blood volume of $85-96 \mathrm{~mL} / \mathrm{kg}^{66}$ or blood volume of around $1.5-2.5 \mathrm{ml}$ (6-8\% of the body weight $)^{67}$. In contrast, the larger volume of distribution $(334 \mathrm{~mL} / \mathrm{kg})$ for curcumin shows that it leaves the vascular space extensively ${ }^{68}$.

Figure $10 \mathrm{~A}$ and Figure 11 show that $20 \%$ and $30 \%$ of the injected dose of Cy7-labeled micelles accumulated in organs that extensively harbor cells of the mononuclear phagocyte system (MPS), namely the liver and spleen, respectively, $24 \mathrm{~h}$ post-administration. Approximately 5\% of Cy7-labeled-polymer was detected in the kidneys, suggesting disintegration of micelles into polymer unimers that have a molecular size below the size threshold for glomerular filtration ${ }^{69}$. The accumulation of Cy7-labeled mPEG- $b$-p(HPMA$\mathrm{Bz}$ ) polymers in the liver, spleen, and kidneys increased over time due to clearance by the MPS and liberation of unimers from the micelles. It is important to note that part of the polymeric micelle disposition in hypervascularized tissue (e.g., liver, lungs, and kidneys) can be ascribed to the micelles that are still in the blood circulation since these micelles had a long circulation time (Table 4). 
Figure 10B shows that curcumin accumulated in the spleen, liver, and lungs (i.e., MPSenriched tissues) after 4-h circulation. The curcumin concentration amounted to $0.2-3 \mu \mathrm{g} / \mathrm{g}$ tissue in the different organs at 4 and 24 h post-administration (SI, Figure S13). In line with other curcumin biodistribution studies, curcumin cleared from the tissues over time (Figure $10 \mathrm{~B})^{16,18,70}$. The difference between curcumin and micelles biodistribution patterns may be attributable to their dissociation and different circulation kinetics. Unlike the stable micelles, curcumin cleared faster from plasma.

A

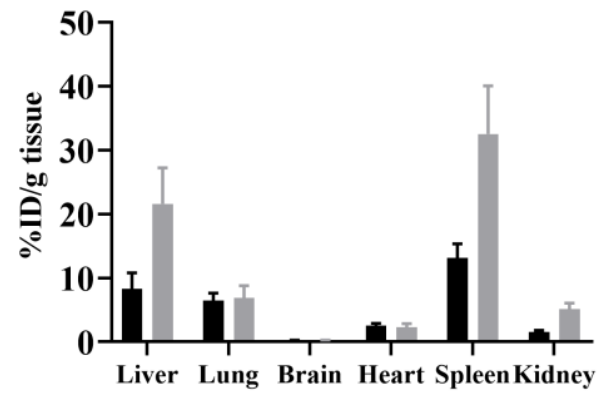

B

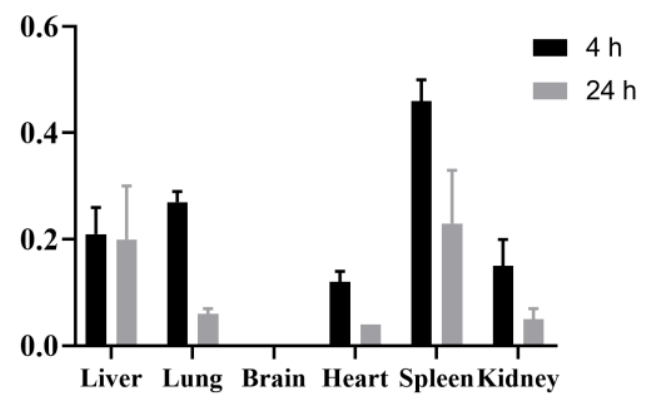

Figure 10. Accumulation of curcumin-loaded Cy7-labeled $\mathrm{mPEG}_{5 \mathrm{kDa}}-b-\mathrm{p}(\mathrm{HPMA}-\mathrm{Bz})_{17.1 \mathrm{kDa}}$ micelles in tissues of $\mathrm{BALB} / \mathrm{c}$ mice at 4 and $24 \mathrm{~h}$ after intravenous injection. (A) Percentage of injected dose (ID\%)/g tissue of Cy7-labeled polymer and (B) ID\%/g tissue of curcumin. Data represent mean $\pm \mathrm{SD}$ $(\mathrm{n}=3$ per time interval).
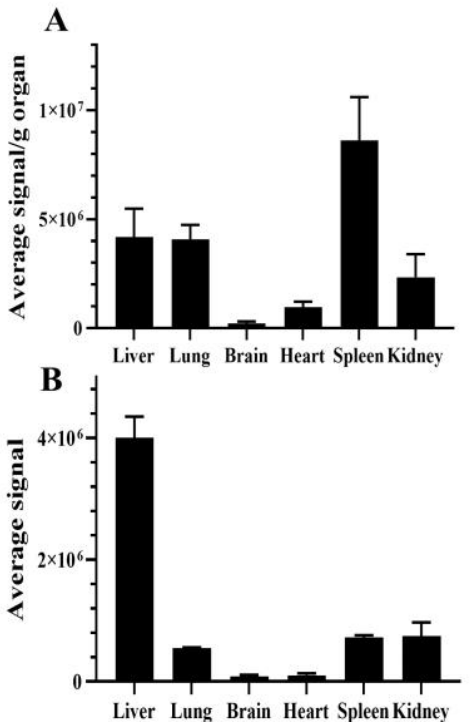

C

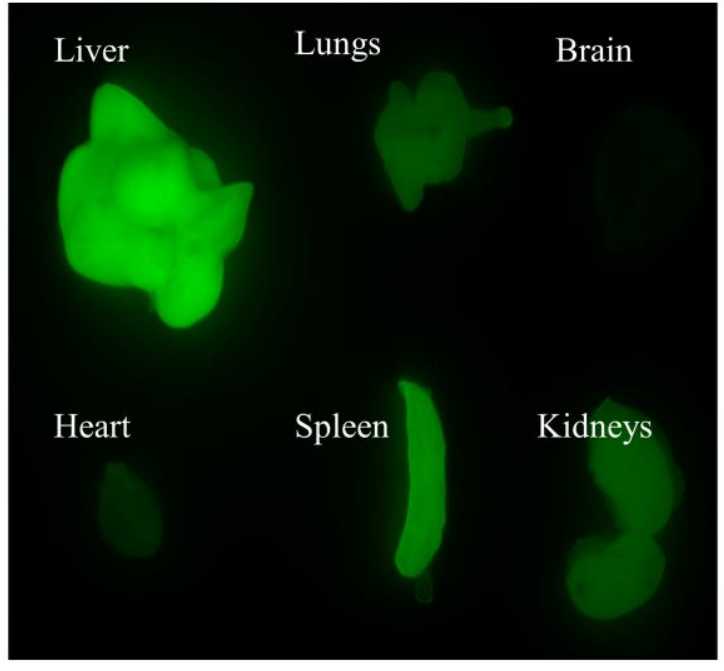

Figure 11. Ex vivo fluorescence reflectance imaging (FRI) analysis of accumulation of the Cy7-labeled $\mathrm{mPEG}_{5 \mathrm{kDa}}-b$-p $(\text { HPMA-Bz })_{17.1 \mathrm{kDa}}$ micelles in organs $24 \mathrm{~h}$ after intravenous administration. Images were obtained at $\lambda \mathrm{ex}=785 \mathrm{~nm}$ with $\lambda \mathrm{em}=820 \mathrm{~nm}(\mathrm{n}=3)$, i.e., at wavelengths at which there is no tissue autofluorescence (data not shown). The extent of Cy7-labeled-micelle accumulation is plotted per gram of tissue (A) and per organ (B). 


\subsection{Therapeutic Efficacy of Curcumin-Loaded mPEG5kDa- $b$-p(HPMA-Bz) 17.1kDa Micelles}

The potential therapeutic benefit of the curcumin-loaded $\mathrm{mPEG}_{5 \mathrm{kDa}}-b-\mathrm{p}(\mathrm{HPMA}-\mathrm{Bz})_{17.1 \mathrm{kDa}}$ micelles was evaluated in Neuro2A tumor-bearing mice. A syngeneic tumor model was selected to avoid xenograft models without a fully functional immune system. VarelaMoreira et al. ${ }^{56}$ demonstrated that compromising the status of the immune system can significantly change the circulation kinetics of mPEG- $b-\mathrm{p}$ (HPMA-Bz) micelles. Also, Neuro2A (neuroblastoma) tumors are reasonably vascularized and can present therapeutic advantages in the form of an EPR effect ${ }^{71,72}$.

Nine days after inoculation, mice received 10 consecutive daily injections of curcuminloaded micelles. Tumor volume and body weight were monitored over time. The group that received curcumin-loaded micelles did not show tumor growth suppression or growth delay compared to the control groups receiving empty micelles and HBS (SI, Figure S14). Since the size of tumors on the day of starting the treatment was relatively large $\left(526 \pm 180 \mathrm{~mm}^{3}\right)$, it was hypothesized that the curcumin dosage was insufficient to exert a therapeutic response, as a result of which mice reached humane endpoints. Therefore, in a follow-up experiment, treatment was started at a time when the tumors were palpable ( 7 days after the inoculation of the tumor cells). Although the overall number of mice that developed tumors was lower (6 out of 27 mice) due to the inoculation with fewer tumor cells, no anti-cancer effect was observed under otherwise similar therapeutic conditions (SI, Figure S15). Contrary to curcumin-loaded nanosized delivery systems that exhibited antitumor effects in different human tumor murine models, the curcumin-loaded mPEG $_{5 \mathrm{kDa}}-b-\mathrm{p}(\mathrm{HPMA}-\mathrm{Bz})$ micelles did not impart tumor inhibition despite similar PK profiles as other tested nanoformulations. The absence of therapeutic efficacy may stem from the possibility that curcumin is extracted too rapidly from the micelles and degraded and/or cleared from the circulation before ample accumulation could have occurred in the tumor to instill notable tumoricidal effects. Another confounding variable may be the relatively low sensitivity of the Neuro2A cells to curcuminloaded micelles, as was shown in vitro (Table 3), which translates to higher curcumin levels needing to be achieved in the Neuro2A tumors than in other types of subcutaneous tumor xenografts for a therapeutic effect.

Taken together, mPEG- $b$-p(HPMA-Bz) micelles are excellent solubilizers for curcumin with a PK profile that is comparable to other curcumin nanoformulations. To date, the majority of the developed curcumin nanoformulations acts as solubilizers since they only improved the AUC by a factor of 1.3-5-fold compared to curcumin in its free form, as pointed out in the introduction. It should be emphasized that this increase was sufficient to confer antitumor activity in non-Neuro2A tumor-bearing mouse models ${ }^{16,20,62,63,70}$, similar to the well-known paclitaxel nanomedicines Genexol and Abraxane. These formulations hardly improve the PK of loaded paclitaxel but do have valuable therapeutic benefits ${ }^{73-76}$. It is therefore possible that curcumin-loaded mPEG- $b$-p(HPMA-Bz) micelles are effective in other human tumor models that were proven susceptible to curcumin and other nanomedicines.

\section{Conclusions}

Curcumin-loaded mPEG $_{5 \mathrm{KDa}}-b$-p(HPMA-Bz) micelles with different block copolymer sizes were prepared, characterized, and subjected to stability analysis. The micelles were stable in plasma, but curcumin was rapidly transferred from the polymeric micelles to albumin. The 
polymeric micelles with the highest molecular weight hydrophobic block were found to be the most stable formulation, exhibiting comparable pharmacokinetics in mice as other curcumin nanoformulations reported in the literature. Nevertheless, the curcumin-loaded micelles did not stall tumor growth or reduce tumor size in a murine human neuroblastoma model, despite exhibiting in vitro cytotoxicity. It might be possible to achieve therapeutic efficacy in this tumor model using combination therapy with other chemotherapeutic agents $^{77,78}$. In the final analysis, curcumin-loaded $\mathrm{mPEG}_{5 \mathrm{KDa}}-b-\mathrm{p}(\mathrm{HPMA}-\mathrm{Bz})_{17.1 \mathrm{kDa}}$ micelles can either be repurposed to target more amenable cancers such as hematological malignancies or cancers of the lymphatic and immune system ${ }^{3}$ or injected directly into the tumor for local uptake and gradual release of the chemotherapeutic. Alternatively, the formulation can be subjected to more research focused on developing more stable curcumin-loaded micelles.

\section{Conflict of Interest Disclosure}

Part of this research has been done in collaboration with the European Commission in Italy. The work was conducted by Dr. Robin B. Capomaccio while working at the European Commission with no connection to AstraZeneca. Dr. Robin B. Capomaccio is currently an employee at AstraZeneca. No further work on the publication has been conducted using AstraZeneca resources. Michal Heger is co-founder of Nurish.Me and has equity in that company (whose business activities are unrelated to the present work).

\section{Acknowledgements}

This work was funded by the European Union's Horizon 2020 research and innovation program Marie Sklodowska-Curie Innovative Training Networks (ITN) under grant No. 676137. Michal Heger is currently supported by grants from the Dutch Cancer Foundation (KWF project \# 10666), National Natural Science Foundation of China (\#81872220), a Zhejiang Provincial Foreign Expert Program Grant, Zhejiang Provincial Key Natural Science Foundation of China (\#Z20H160031), and a grant for the establishment of the Jiaxing Key Laboratory for Photonanomedicine and Experimental Therapeutics. The SK-ChA-1, MzChA-1 cell lines were kindly received under a license agreement to Michal Heger from Alexander Knuth and Claudia Matter (University Hospital Zurich, Switzerland). The $\mathrm{AF}_{4}$ data used in this research were generated through access to the Nanobiotechnology Laboratory under the Framework of access to the Joint Research Centre Physical Research Infrastructures of the European Commission (Stability of polymeric micelles in serum, MicStab, Research Infrastructure Access Agreement RIAA 35050/7). 


\section{References}

1. Patil, S. S.; Bhasarkar, S.; Rathod, V. K. Extraction of curcuminoids from Curcuma longa: comparative study between batch extraction and novel three phase partitioning. Prep. Biochem. Biotechnol. 2019, 49, (4), 407-418.

2. Shanmugam, M. K.; Rane, G.; Kanchi, M. M.; Arfuso, F.; Chinnathambi, A.; Zayed, M. E.; Alharbi, S. A.; Tan, B. K.; Kumar, A. P.; Sethi, G. The multifaceted role of curcumin in cancer prevention and treatment. Molecules 2015, 20, (2), 2728-69.

3. Heger, M.; van Golen, R. F.; Broekgaarden, M.; Michel, M. C. The molecular basis for the pharmacokinetics and pharmacodynamics of curcumin and its metabolites in relation to cancer. Pharmacol. Rev. 2014, 66, (1), 222-307.

4. $\quad$ Vareed, S. K.; Kakarala, M.; Ruffin, M. T.; Crowell, J. A.; Normolle, D. P.; Djuric, Z.; Brenner, D. E. Pharmacokinetics of curcumin conjugate metabolites in healthy human subjects. Cancer Epidemiol., Biomarkers Prev. 2008, 17, (6), 1411-1417.

5. Fujisawa, S.; Atsumi, T.; Ishihara, M.; Kadoma, Y. Cytotoxicity, ROS-generation activity and radical-scavenging activity of curcumin and related compounds. Anticancer Res. 2004, 24, (2B), 563-570.

6. Naksuriya, O.; van Steenbergen, M. J.; Torano, J. S.; Okonogi, S.; Hennink, W. E. A kinetic degradation study of curcumin in its free form and loaded in polymeric micelles. AAPS J. 2016, 18, (3), 777-787.

7. Schneider, C.; Gordon, O. N.; Edwards, R. L.; Luis, P. B. Degradation of curcumin: from mechanism to biological implications. J. Agric. Food Chem. 2015, 63, (35), 7606-7614.

8. Anand, P.; Kunnumakkara, A. B.; Newman, R. A.; Aggarwal, B. B. Bioavailability of curcumin: problems and promises. Mol. Pharmaceutics 2007, 4, (6), 807-818.

9. Wicki, A.; Witzigmann, D.; Balasubramanian, V.; Huwyler, J. Nanomedicine in cancer therapy: challenges, opportunities, and clinical applications. Journal of controlled release : official journal of the Controlled Release Society 2015, 200, 138-157.

10. Bobo, D.; Robinson, K. J.; Islam, J.; Thurecht, K. J.; Corrie, S. R. Nanoparticle-based medicines: a review of FDA-approved materials and clinical trials to date. Pharm. Res. 2016, 33, (10), 2373-2387.

11. Houdaihed, L.; Evans, J. C.; Allen, C. Overcoming the road blocks: advancement of block copolymer micelles for cancer therapy in the clinic. Mol. Pharmaceutics 2017, 14, (8), 2503-2517.

12. Cabral, H.; Miyata, K.; Osada, K.; Kataoka, K. Block copolymer micelles in nanomedicine applications. Chem. Rev. 2018, 118, (14), 6844-6892.

13. Fang, J.; Nakamura, H.; Maeda, H. The EPR effect: Unique features of tumor blood vessels for drug delivery, factors involved, and limitations and augmentation of the effect. Adv. Drug Delivery Rev. 2011, 63, (3), 136-151.

14. Naksuriya, O.; Okonogi, S.; Schiffelers, R. M.; Hennink, W. E. Curcumin nanoformulations: A review of pharmaceutical properties and preclinical studies and clinical data related to cancer treatment. Biomaterials 2014, 35, (10), 3365-3383.

15. Mehanny, M.; Hathout, R. M.; Geneidi, A. S.; Mansour, S. Exploring the use of nanocarrier systems to deliver the magical molecule; Curcumin and its derivatives. Journal of controlled release : official journal of the Controlled Release Society 2016, 225, 1-30.

16. Gong, C.; Deng, S.; Wu, Q.; Xiang, M.; Wei, X.; Li, L.; Gao, X.; Wang, B.; Sun, L.; Chen, Y.; Li, Y.; Liu, L.; Qian, Z.; Wei, Y. Improving antiangiogenesis and anti-tumor activity of curcumin by biodegradable polymeric micelles. Biomaterials 2013, 34, (4), 1413-1432. 


\section{Chapter 5}

17. Lübtow, M. M.; Nelke, L. C.; Seifert, J.; Kühnemundt, J.; Sahay, G.; Dandekar, G.; Nietzer, S. L.; Luxenhofer, R. Drug induced micellization into ultra-high capacity and stable curcumin nanoformulations: Physico-chemical characterization and evaluation in $2 \mathrm{D}$ and $3 \mathrm{D}$ in vitro models. Journal of controlled release : official journal of the Controlled Release Society 2019, 303, 162-180.

18. Song, Z.; Feng, R.; Sun, M.; Guo, C.; Gao, Y.; Li, L.; Zhai, G. Curcumin-loaded PLGAPEG-PLGA triblock copolymeric micelles: Preparation, pharmacokinetics and distribution in vivo. $J$. Colloid Interface Sci. 2011, 354, (1), 116-123.

19. Shi, Y.; Lammers, T.; Storm, G.; Hennink, W. E. Physico-chemical strategies to enhance stability and drug retention of polymeric micelles for tumor-targeted drug delivery. Macromol. Biosci. 2017, 17, (1), 1600160.

20. Gong, F.; Chen, D.; Teng, X.; Ge, J.; Ning, X.; Shen, Y. L.; Li, J.; Wang, S. Curcuminloaded blood-stable polymeric micelles for enhancing therapeutic effect on erythroleukemia. Mol. Pharmaceutics 2017, 14, (8), 2585-2594.

21. D'Souza, S. A review of in vitro drug release test methods for nano-sized dosage forms. Advances in Pharmaceutics 2014, 2014, 304757.

22. Abouelmagd, S. A.; Sun, B.; Chang, A. C.; Ku, Y. J.; Yeo, Y. Release kinetics study of poorly water-soluble drugs from nanoparticles: Are we doing it right? Mol. Pharmaceutics 2015, 12, (3), 997-1003.

23. Gil, D.; Frank-Kamenetskii, A.; Barry, J.; Reukov, V.; Xiang, Y.; Das, A.; Varma, A. K.; Kindy, M. S.; Banik, N. L.; Vertegel, A. Albumin-assisted method allows assessment of release of hydrophobic drugs from nanocarriers. Biotechnol. J. 2018, 13, (1), 1700337.

24. Contado, C. Field flow fractionation techniques to explore the "nano-world". Anal. Bioanal. Chem. 2017, 409, (10), 2501-2518.

25. Wagner, M.; Holzschuh, S.; Traeger, A.; Fahr, A.; Schubert, U. S. Asymmetric flow fieldflow fractionation in the field of nanomedicine. Anal. Chem. 2014, 86, (11), 5201-5210.

26. Shi, Y.; van der Meel, R.; Theek, B.; Oude Blenke, E.; Pieters, E. H. E.; Fens, M. H. A. M.; Ehling, J.; Schiffelers, R. M.; Storm, G.; van Nostrum, C. F.; Lammers, T.; Hennink, W. E. Complete regression of xenograft tumors upon targeted delivery of paclitaxel via $\Pi-\Pi$ stacking stabilized polymeric micelles. ACS Nano 2015, 9, (4), 3740-3752.

27. Naksuriya, O.; Shi, Y.; van Nostrum, C. F.; Anuchapreeda, S.; Hennink, W. E.; Okonogi, S. HPMA-based polymeric micelles for curcumin solubilization and inhibition of cancer cell growth. Eur. J. Pharm. Biopharm. 2015, 94, 501-512.

28. Bresseleers, J.; Bagheri, M.; Storm, G.; Metselaar, J. M.; Hennink, W. E.; Meeuwissen, S. A.; van Hest, J. C. M. Scale-up of the manufacturing process to produce docetaxel-loaded mPEG-bp(HPMA-Bz) block copolymer micelles for pharmaceutical applications. Org. Process Res. Dev. 2019, 23, (12), 2707-2715.

29. Bagheri, M.; Bresseleers, J.; Varela-Moreira, A.; Sandre, O.; Meeuwissen, S. A.; Schiffelers, R. M.; Metselaar, J. M.; van Nostrum, C. F.; van Hest, J. C. M.; Hennink, W. E. Effect of formulation and processing parameters on the size of mPEG-b-p(HPMA-Bz) polymeric micelles. Langmuir 2018, $34,(50), 15495-15506$.

30. Sheybanifard, M.; Beztsinna, N.; Bagheri, M.; Miriam Buhl, E.; Bresseleers, J.; VarelaMoreira, A.; Shi, Y.; van Nostrum, C. F.; van der Pluijm, G.; Storm, G.; Hennink, W. E.; Lammers, T.; Metselaar, J. M. Systematic evaluation of design features enables efficient selection of $\Pi$ electronstabilized polymeric micelles. Int. J. Pharm. 2020, 119409.

31. Zhuang, W.-R.; Wang, Y.; Cui, P.-F.; Xing, L.; Lee, J.; Kim, D.; Jiang, H.-L.; Oh, Y.-K. Applications of $\pi-\pi$ stacking interactions in the design of drug-delivery systems. Journal of controlled release : official journal of the Controlled Release Society 2019, 294, 311-326. 
32. Shi, Y.; van Steenbergen, M. J.; Teunissen, E. A.; Novo, L.; Gradmann, S.; Baldus, M.; van Nostrum, C. F.; Hennink, W. E. Pi-pi stacking increases the stability and loading capacity of thermosensitive polymeric micelles for chemotherapeutic drugs. Biomacromolecules 2013, 14, (6), 1826-1837.

33. Vichai, V.; Kirtikara, K. Sulforhodamine B colorimetric assay for cytotoxicity screening. Nat. Protoc. 2006, 1, (3), 1112-1116.

34. Zhang, Y.; Huo, M.; Zhou, J.; Xie, S. PKSolver: An add-in program for pharmacokinetic and pharmacodynamic data analysis in Microsoft Excel. Comput. Methods Programs Biomed. 2010, 99, (3), 306-314.

35. Fens, M. H.; Hill, K. J.; Issa, J.; Ashton, S. E.; Westwood, F. R.; Blakey, D. C.; Storm, G.; Ryan, A. J.; Schiffelers, R. M. Liposomal encapsulation enhances the antitumour efficacy of the vascular disrupting agent ZD6126 in murine B16.F10 melanoma. Br. J. Cancer 2008, 99, (8), 12561264.

36. Stetefeld, J.; McKenna, S. A.; Patel, T. R. Dynamic light scattering: a practical guide and applications in biomedical sciences. Biophys Rev 2016, 8, (4), 409-427.

37. Bhattacharjee, S. DLS and zeta potential - What they are and what they are not? Journal of controlled release : official journal of the Controlled Release Society 2016, 235, 337-351.

38. Lin, W.-J.; Juang, L.-W.; Lin, C.-C. Stability and release performance of a series of pegylated copolymeric micelles. Pharm. Res. 2003, 20, (4), 668-673.

39. Hussein, Y. H. A.; Youssry, M. Polymeric micelles of biodegradable diblock copolymers: Enhanced encapsulation of hydrophobic drugs. Materials 2018, 11, (5), 688.

40. Lubtow, M. M.; Haider, M. S.; Kirsch, M.; Klisch, S.; Luxenhofer, R. Like dissolves like? A comprehensive evaluation of partial solubility parameters to predict polymer-drug compatibility in ultrahigh drug-loaded polymer micelles. Biomacromolecules 2019, 20, (8), 3041-3056.

41. Huh, K. M.; Lee, S. C.; Cho, Y. W.; Lee, J.; Jeong, J. H.; Park, K. Hydrotropic polymer micelle system for delivery of paclitaxel. J Control Release 2005, 101, (1-3), 59-68.

42. Barik, A.; Mishra, B.; Kunwar, A.; Indira Priyadarsini, K. Interaction of curcumin with human serum albumin: Thermodynamic properties, fluorescence energy transfer and denaturation effects. Chem. Phys. Lett. 2007, 436, (1), 239-243.

43. Basu, A.; Suresh Kumar, G. Elucidating the energetics of the interaction of non-toxic dietary pigment curcumin with human serum albumin: A calorimetric study. J. Chem. Thermodyn. 2014, 70, 176-181.

44. Moquin, A.; Sharma, A.; Cui, Y.; Lau, A.; Maysinger, D.; Kakkar, A. Asymmetric AB3 miktoarm star polymers: Synthesis, self-Assembly, and study of micelle stability using AF4 for efficient drug delivery. Macromol. Biosci. 2015, 15, (12), 1744-1754.

45. Mafra, L.; Santos, S. M.; Siegel, R.; Alves, I.; Almeida Paz, F. A.; Dudenko, D.; Spiess, H. W. Packing interactions in hydrated and anhydrous forms of the antibiotic ciprofloxacin: a solid-state NMR, X-ray diffraction, and computer simulation study. J. Am. Chem. Soc. 2012, 134, (1), 71-74.

46. Carignani, E.; Borsacchi, S.; Bradley, J. P.; Brown, S. P.; Geppi, M. Strong intermolecular ring current influence on $1 \mathrm{H}$ chemical shifts in two crystalline forms of naproxen: a combined solidstate NMR and DFT study. J. Phys. Chem. C 2013, 117, (34), 17731-17740.

47. Bennion, B. J.; Be, N. A.; McNerney, M. W.; Lao, V.; Carlson, E. M.; Valdez, C. A.; Malfatti, M. A.; Enright, H. A.; Nguyen, T. H.; Lightstone, F. C.; Carpenter, T. S. Predicting a drug's membrane permeability: A computational model validated with in vitro permeability assay data. J. Phys. Chem. B 2017, 121, (20), 5228-5237. 


\section{Chapter 5}

48. Sun, J.; Bi, C.; Chan, H. M.; Sun, S.; Zhang, Q.; Zheng, Y. Curcumin-loaded solid lipid nanoparticles have prolonged in vitro antitumour activity, cellular uptake and improved in vivo bioavailability. Colloids Surf., B 2013, 111, 367-375.

49. Sidhar, H.; Giri, R. K. Induction of Bex genes by curcumin is associated with apoptosis and activation of p53 in N2a neuroblastoma cells. Sci. Rep. 2017, 7, 41420.

50. Jana, N. R.; Dikshit, P.; Goswami, A.; Nukina, N. Inhibition of proteasomal function by curcumin induces apoptosis through mitochondrial pathway. J. Biol. Chem. 2004, 279, (12), 1168011685.

51. Broekgaarden, M.; Weijer, R.; van Gulik, T. M.; Hamblin, M. R.; Heger, M. Tumor cell survival pathways activated by photodynamic therapy: a molecular basis for pharmacological inhibition strategies. Cancer Metastasis Rev. 2015, 34, (4), 643-90.

52. Knuth, A.; Gabbert, H.; Dippold, W.; Klein, O.; Sachsse, W.; Bitter-Suermann, D.; Prellwitz, W.; Meyer zum Büschenfelde, K. H. Biliary adenocarcinoma. Characterisation of three new human tumor cell lines. J. Hepatol. 1985, 1, (6), 579-596.

53. Saijyo, S.; Kudo, T.; Suzuki, M.; Katayose, Y.; Shinoda, M.; Muto, T.; Fukuhara, K.; Suzuki, T.; Matsuno, S. Establishment of a new extrahepatic bile duct carcinoma cell line, TFK-1. Tohoku. J. Exp. Med. 1995, 177, (1), 61-71.

54. Lee, K. W.; Bode, A. M.; Dong, Z. Molecular targets of phytochemicals for cancer prevention. Nat. Rev. Cancer 2011, 11, (3), 211-218.

55. Dhule, S. S.; Penfornis, P.; Frazier, T.; Walker, R.; Feldman, J.; Tan, G.; He, J.; Alb, A.; John, V.; Pochampally, R. Curcumin-loaded $\gamma$-cyclodextrin liposomal nanoparticles as delivery vehicles for osteosarcoma. Nanomedicine 2012, 8, (4), 440-451.

56. Varela-Moreira, A.; van Straten, D.; van Leur, H. F.; Ruiter, R. W. J.; Deshantri, A. K.; Hennink, W. E.; Fens, M. H. A. M.; Groen, R. W. J.; Schiffelers, R. M. Polymeric micelles loaded with carfilzomib increase tolerability in a humanized bone marrow-like scaffold mouse model. Int. $J$. Pharm. 2020, 100049.

57. Bolger, G. T.; Licollari, A.; Tan, A.; Greil, R.; Vcelar, B.; Majeed, M.; Helson, L. Distribution and metabolism of Lipocurc ${ }^{\mathrm{TM}}$ (liposomal curcumin) in dog and human blood cells: species selectivity and pharmacokinetic relevance. Anticancer Res 2017, 37, (7), 3483-3492.

58. Brown, D.; Tomlin, M., pharmacokinetic principles. In Pharmacology \& Pharmacokinetics: A Basic Reader, Tomlin, M., Ed. Springer-Verlag London: London, 2010; pp 13-51.

59. Gabizon, A. A.; Pappo, O.; Goren, D.; Chemla, M.; Tzemach, D.; Horowitz, A. T. Preclinical studies with doxorubicin encapsulated in polyethyleneglycol-coated liposomes. J. Liposome Res. 1993, $3,(3), 517-528$.

60. Sun, M.; Gao, Y.; Guo, C.; Cao, F.; Song, Z.; Xi, Y.; Yu, A.; Li, A.; Zhai, G. Enhancement of transport of curcumin to brain in mice by poly(n-butylcyanoacrylate) nanoparticle. J. Nanopart. Res. 2010, 12, (8), 3111-3122.

61. Zhai, S.; Ma, Y.; Chen, Y.; Li, D.; Cao, J.; Liu, Y.; Cai, M.; Xie, X.; Chen, Y.; Luo, X. Synthesis of an amphiphilic block copolymer containing zwitterionic sulfobetaine as a novel $\mathrm{pH}-$ sensitive drug carrier. Polym. Chem. 2014, 5, (4), 1285-1297.

62. Duan, J.; Zhang, Y.; Han, S.; Chen, Y.; Li, B.; Liao, M.; Chen, W.; Deng, X.; Zhao, J.; Huang, B. Synthesis and in vitro/in vivo anti-cancer evaluation of curcumin-loaded chitosan/poly(butyl cyanoacrylate) nanoparticles. Int. J. Pharm. 2010, 400, (1), 211-220.

63. Gao, X.; Zheng, F.; Guo, G.; Liu, X.; Fan, R.; Qian, Z.-y.; Huang, N.; Wei, Y.-q. Improving the anti-colon cancer activity of curcumin with biodegradable nano-micelles. J. Mater. Chem. B 2013, $1,(42), 5778-5790$. 
64. Sun, D.; Zhou, J.-K.; Zhao, L.; Zheng, Z.-Y.; Li, J.; Pu, W.; Liu, S.; Liu, X.-S.; Liu, S.-J.; Zheng, Y.; Zhao, Y.; Peng, Y. Novel curcumin liposome modified with hyaluronan targeting CD44 plays an anti-leukemic role in acute myeloid leukemia in vitro and in vivo. ACS Appl. Mater. Interfaces 2017, 9, (20), 16857-16868.

65. Chen, S.; Li, Q.; Li, H.; Yang, L.; Yi, J.-Z.; Xie, M.; Zhang, L.-M. Long-circulating zeinpolysulfobetaine conjugate-based nanocarriers for enhancing the stability and pharmacokinetics of curcumin. Mater. Sci. Eng., C 2020, 109, 110636.

66. Riches, A. C.; Sharp, J. G.; Thomas, D. B.; Smith, S. V. Blood volume determination in the mouse. J. Physiol. 1973, 228, (2), 279-284.

67. The Johns Hopkins University. Animal care and use committee. http://web.jhu.edu/animalcare/procedures/mouse.html (accessed July 24, 2020),

68. Paul, A., Drug distribution. In Introduction to basics of pharmacology and toxicology, Raj, G. M.; Raveendran, R., Eds. Springer Singapore: 2019; pp 89-98.

69. Seymour, L. W.; Duncan, R.; Strohalm, J.; Kopeček, J. Effect of molecular weight (Mw) of $\mathrm{N}$-(2-hydroxypropyl)methacrylamide copolymers on body distribution and rate of excretion after subcutaneous, intraperitoneal, and intravenous administration to rats. J. Biomed. Mater. Res. 1987, 21, (11), 1341-1358.

70. Hong, J. Y.; Liu, Y. Y.; Xiao, Y.; Yang, X. F.; Su, W. J.; Zhang, M. Z.; Liao, Y. H.; Kuang, H. X.; Wang, X. T. High drug payload curcumin nanosuspensions stabilized by mPEG-DSPE and SPC: in vitro and in vivo evaluation. Drug Deliv. 2017, 24, (1), 109-120.

71. Balza, E.; Carnemolla, B.; Mortara, L.; Castellani, P.; Soncini, D.; Accolla, R. S.; Borsi, L. Therapy-induced antitumor vaccination in neuroblastomas by the combined targeting of IL-2 and TNFalpha. Int. J. Cancer 2010, 127, (1), 101-110.

72. Vader, P.; van der Meel, R.; Symons, M. H.; Fens, M. H.; Pieters, E.; Wilschut, K. J.; Storm, G.; Jarzabek, M.; Gallagher, W. M.; Schiffelers, R. M.; Byrne, A. T. Examining the role of Rac1 in tumor angiogenesis and growth: a clinically relevant RNAi-mediated approach. Angiogenesis 2011, 14, (4), 457-466.

73. Sparreboom, A.; Scripture, C. D.; Trieu, V.; Williams, P. J.; De, T.; Yang, A.; Beals, B.; Figg, W. D.; Hawkins, M.; Desai, N. Comparative preclinical and clinical pharmacokinetics of a cremophorfree, nanoparticle albumin-bound paclitaxel (ABI-007) and paclitaxel formulated in Cremophor (Taxol). Clin. Cancer Res. 2005, 11, (11), 4136-43.

74. Kim, S. C.; Kim, D. W.; Shim, Y. H.; Bang, J. S.; Oh, H. S.; Kim, S. W.; Seo, M. H. In vivo evaluation of polymeric micellar paclitaxel formulation: toxicity and efficacy. Journal of Controlled Release 2001, 72, (1), 191-202.

75. Varela-Moreira, A.; Shi, Y.; Fens, M. H. A. M.; Lammers, T.; Hennink, W. E.; Schiffelers, R. M. Clinical application of polymeric micelles for the treatment of cancer. Mater. Chem. Front. 2017, $1,(8), 1485-1501$.

76. Youn, Y. S.; Bae, Y. H. Perspectives on the past, present, and future of cancer nanomedicine. Adv. Drug Delivery Rev. 2018, 130, 3-11.

77. Tan, B. L.; Norhaizan, M. E. Curcumin combination chemotherapy: The implication and efficacy in cancer. Molecules 2019, 24, (14).

78. Zhai, K.; Brockmüller, A.; Kubatka, P.; Shakibaei, M.; Büsselberg, D. Curcumin's beneficial effects on neuroblastoma: mechanisms, challenges, and potential solutions. Biomolecules 2020, 10, (11). 


\section{Supporting Information}

Table S1. Characteristics of the $\mathrm{mPEG}_{5 \mathrm{kDa}}-b-\mathrm{p}(\mathrm{HPMA}-\mathrm{Bz}) \mathrm{x}$ block copolymers as determined by ${ }^{1} \mathrm{H}-$ NMR and GPC ${ }^{1}$.

\begin{tabular}{|c|c|c|c|c|c|c|}
\hline Polymer & $\begin{array}{l}\text { MI:M } \\
\text { mol/mol }\end{array}$ & $\begin{array}{c}\mathbf{M}_{\mathrm{n}} \\
{ }^{1} \mathrm{H}-\mathrm{NMR} \\
(\mathbf{k D a})\end{array}$ & $\begin{array}{c}\mathbf{M}_{\mathbf{n}} \\
\text { GPC } \\
(\mathbf{k D a})\end{array}$ & $\begin{array}{c}\mathbf{M}_{\mathrm{w}} \\
\mathbf{G P C} \\
(\mathbf{k D a})\end{array}$ & $\begin{array}{l}\text { Polydispersity } \\
\left(\mathbf{M}_{w} / \mathbf{M}_{\mathbf{n}}\right) \\
(\mathbf{G P C})\end{array}$ & $\begin{array}{c}\text { Weight } \\
\text { fraction } \\
\text { p(HPMA-Bz) } \\
(\%)\end{array}$ \\
\hline $\begin{array}{c}\mathrm{mPEG}_{5 \mathrm{kDa}}-b-\mathrm{p}(\mathrm{HPMA}- \\
\mathrm{Bz})_{17.1 \mathrm{kDa}}\end{array}$ & $1: 200$ & 22.1 & 15.8 & 20.7 & 1.31 & 77.4 \\
\hline $\begin{array}{c}\mathrm{mPEG}_{5 \mathrm{kDa} \mathrm{a}}-b-\mathrm{p}(\mathrm{HPMA}- \\
\mathrm{Bz})_{10.0 \mathrm{ka}}\end{array}$ & $1: 100$ & 15.0 & 13.2 & 17.5 & 1.32 & 66.7 \\
\hline $\begin{array}{c}\mathrm{mPEG}_{5 \mathrm{kDa}}-b-\mathrm{p}(\mathrm{HPMA}- \\
\left.\mathrm{Bz}_{5}\right)^{2}\end{array}$ & $1: 50$ & 10.2 & 10.8 & 14 & 1.30 & 51.0 \\
\hline
\end{tabular}

Abbreviations: MI:M, macro-initiator:monomer; $\mathrm{M}_{\mathrm{n}}$, the Number Average Molecular Weight; $\mathrm{M}_{\mathrm{w}}$, the Weight Average Molecular Weight.

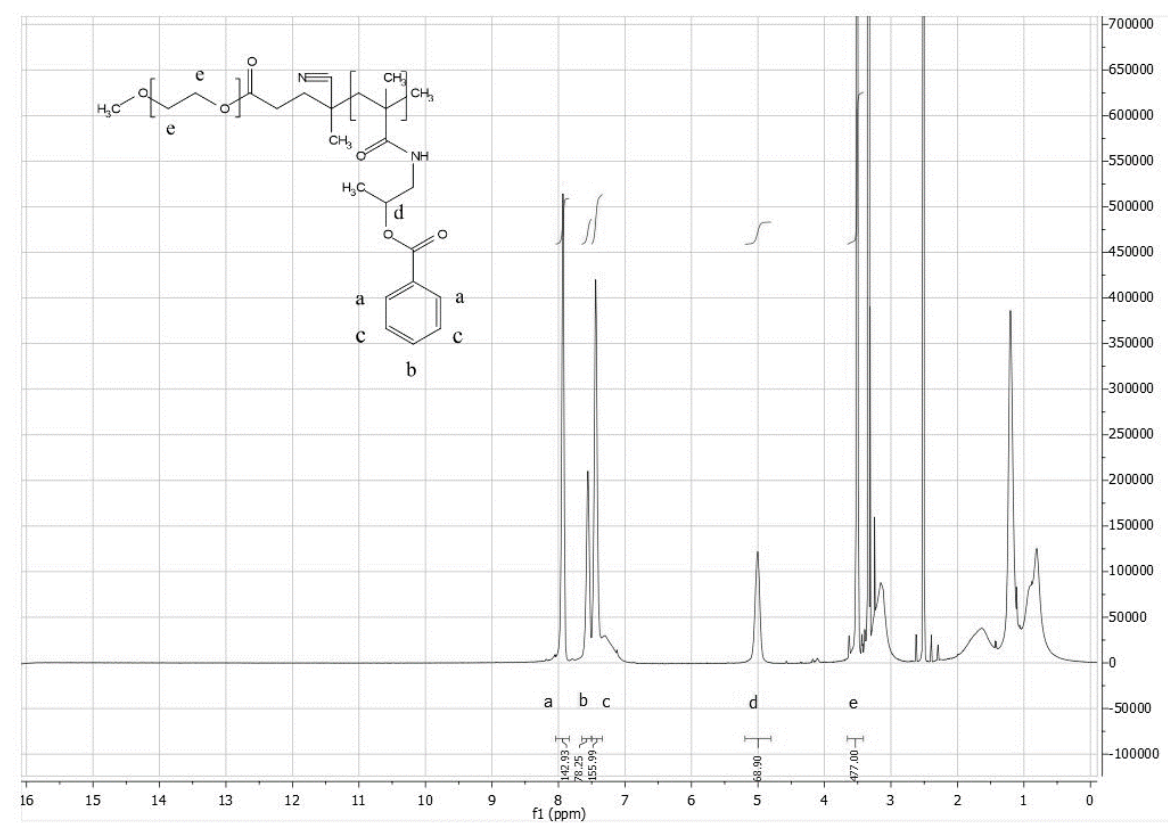

Figure S1. ${ }^{1} \mathrm{H}-\mathrm{NMR}$ spectrum of $\mathrm{mPEG}_{5 \mathrm{kDa}}-b-\mathrm{p}(\mathrm{HPMA}-\mathrm{Bz})_{17.1 \mathrm{kDa}}$ block copolymer in DMSO-d 6 . 


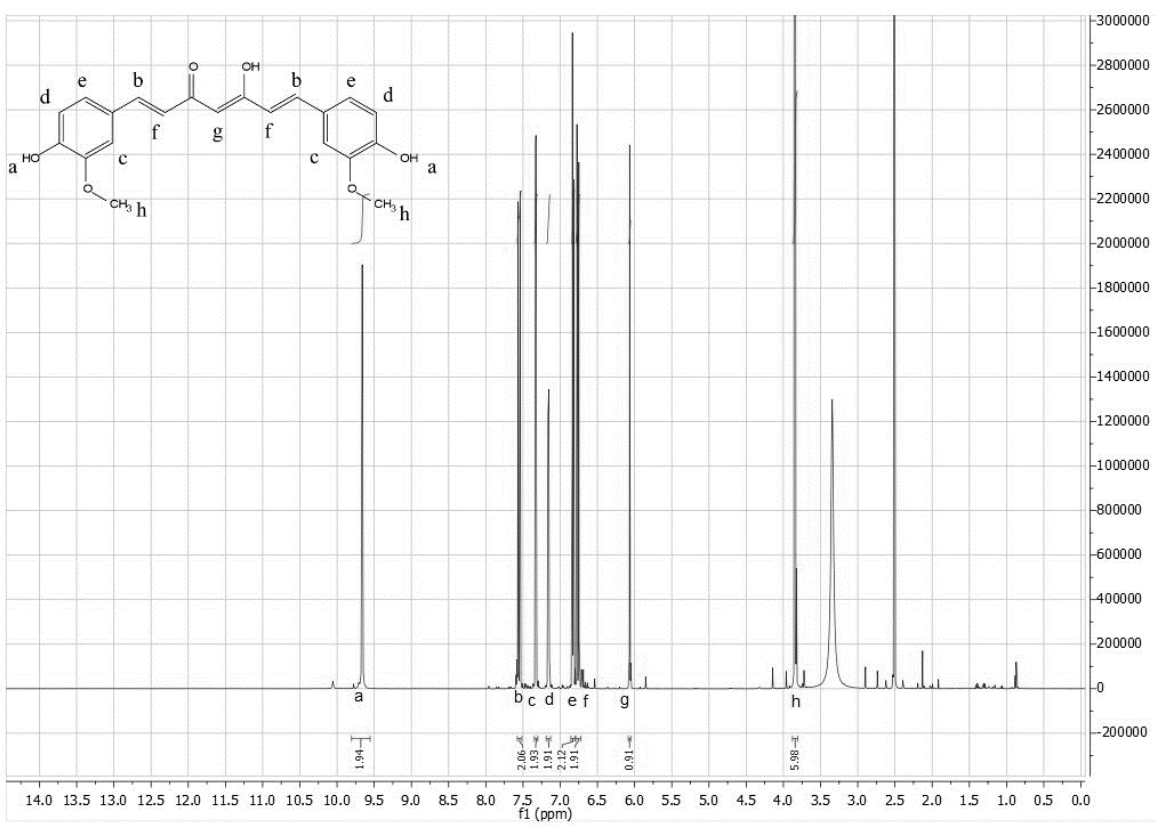

Figure S2. ${ }^{1} \mathrm{H}-\mathrm{NMR}$ spectrum of curcumin in DMSO-d 6 .

The GPC chromatograms of the fluorescently-labeled polymers are shown in Figure S3. The polymer eluted between 10 to 15 min (RI detector), which coincided with the elution time as measured by absorbance at $700 \mathrm{~nm}(\mathrm{Cy} 7)$ and $650 \mathrm{~nm}(\mathrm{Cy} 5)$. The results confirm that the dyes were conjugated to the polymer. On the other hand, free dye eluted at $18 \mathrm{~min}$ (UV detector). The fluorescently-labeled polymers did not show absorbance at this elution time, confirming that free dyes were completely removed by dialysis.

A

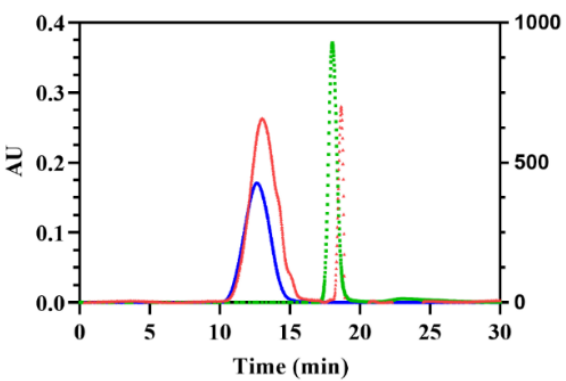

B

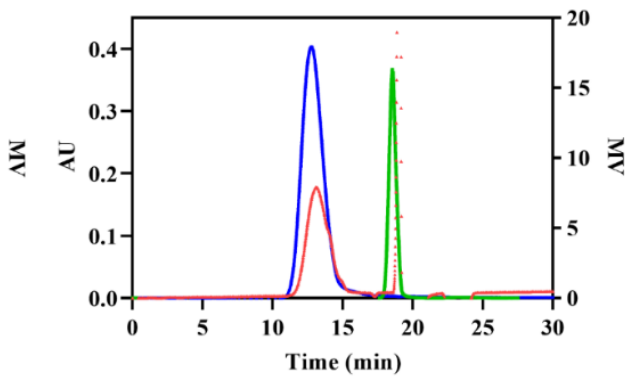

Figure S3. GPC chromatograms of fluorescently labeled mPEG- $b$-p(HPMA-Bz) polymers. (A) Cy7labeled polymer: green and blue lines represent absorbance at $700 \mathrm{~nm}$ for free $\mathrm{Cy} 7$ dye and Cy7-labeled polymer, respectively. Red line is the refractive index (RI) signal of Cy7-labeled polymer under the same chromatography conditions. (B) Cy5-labeled-polymer: green and blue lines are absorbance at 650 $\mathrm{nm}$ for free Cy5 dye and Cy5-labeled polymer, respectively. Red line is the RI signal of Cy5-labeled polymer under the same chromatography conditions. Abbreviations: AU, absorbance unit; MV, voltage offset in millivolts. 
Table S2. Characterization of Cy7-labeled micelles loaded with curcumin.

\begin{tabular}{cccccc}
\hline $\begin{array}{c}\text { Polymer concentration } \\
(\mathbf{m g} / \mathbf{m L})\end{array}$ & $\begin{array}{c}\text { Curcumin concentration } \\
(\mathbf{m g} / \mathbf{m L})\end{array}$ & $\begin{array}{c}\text { Z-ave } \\
(\mathbf{n m})\end{array}$ & PDI & $\begin{array}{c}\text { EE } \\
\mathbf{( \% )}\end{array}$ & $\begin{array}{c}\text { LC } \\
(\boldsymbol{\%})\end{array}$ \\
\hline 5.7 & 56 & 56 & 0.11 & 73.1 & 9.2 \\
\hline
\end{tabular}

Abbreviations: Z-ave, Z-average hydrodynamic diameter; PDI, polydispersity index; EE, encapsulation efficacy and LC, loading capacity.

\section{The effect of dialysis on curcumin-loaded micelles}

Curcumin-loaded micelles (polymer $30.0 \mathrm{mg}$ and curcumin $3.0 \mathrm{mg}$ ) were prepared using the method described in section 2.5. Dialysis was performed in HBS solution for one day at $4{ }^{\circ} \mathrm{C}$ using an 8-kDa dialysis membrane. Samples were taken before and after dialysis and analyzed for polymer and curcumin concentration.

Table S3. Characteristics of curcumin-loaded micelles in terms of curcumin content before and after the dialysis against $\mathrm{HBS}$ at $4{ }^{\circ} \mathrm{C}$.

\begin{tabular}{cccccccc}
\hline \multicolumn{3}{c}{ Before dialysis } & \multicolumn{5}{c}{ After dialysis } \\
\hline $\begin{array}{c}\text { Curcumin } \\
\text { conc. } \\
(\mathbf{m g} / \mathbf{m L})\end{array}$ & $\begin{array}{c}\text { Polymer } \\
\text { conc. } \\
(\mathbf{m g} / \mathbf{m L})\end{array}$ & $\mathbf{E E \%}$ & $\mathbf{L C \%}$ & $\begin{array}{c}\text { Curcumin } \\
\text { conc. } \\
(\mathbf{m g} / \mathbf{m L})\end{array}$ & $\begin{array}{c}\text { Polymer } \\
\text { conc. } \\
(\mathbf{m g} / \mathbf{m L})\end{array}$ & EE\% & LC\% \\
\hline $3.1 \pm 0.1$ & $28.3 \pm 0.4$ & $103 \pm 2.2$ & $9.8 \pm 0.2$ & $2.3 \pm 0.2$ & $22.5 \pm 1.5$ & $77.4 \pm 5.7$ & $9.4 \pm 0.3$ \\
\hline
\end{tabular}

Abbreviations: conc., concentration; EE, encapsulation efficacy; LC, loading capacity.

Data are presented as mean $\pm \mathrm{SD}(\mathrm{n}=5)$.

\section{HPLC method validation}

The method to extract curcumin from plasma was validated by spiking plasma (100 $\mu \mathrm{L})$ with known amounts of curcumin-loaded micelles $(10 \mu \mathrm{L})$. Samples were extracted by addition of 4 volumes of acetonitrile (ACN) to 1 volume of spiked plasma and vortexed for 2 min, followed by centrifugation at $15,000 \times \mathrm{g}$ for $10 \mathrm{~min}$. The concentration of curcumin was determined in the supernatant using an isocratic HPLC method described in the Materials and Methods section 2.12 of the main text. The ratio of the measured curcumin concentration to the known curcumin concentration was used to calculate the recovery percentage (Figure S4 and Table S4). 


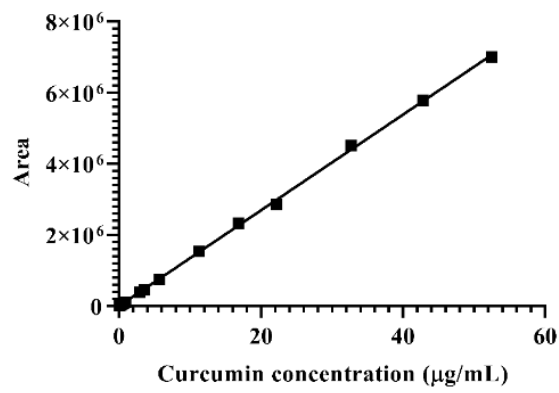

Figure S4. Calibration curve of curcumin in ACN/water 80\%/20\%. Y-axis depicts area under the curve (AUC) of curcumin at $425 \mathrm{~nm}$.

Table S4. Determination of curcumin concentration in plasma samples spiked with curcumin-loaded micelles at different concentrations.

\begin{tabular}{cccc}
\hline Area & $\begin{array}{c}\text { Curcumin } \\
\text { concentration measured } \\
(\boldsymbol{\mu g} / \mathbf{m L})\end{array}$ & $\begin{array}{c}\text { Curcumin } \\
\text { concentration added } \\
(\boldsymbol{\mu g} / \mathbf{m L})\end{array}$ & Recovery $(\%)$ \\
\hline $5004168 \pm 47875$ & $186 \pm 2$ & 203.9 & $91 \pm 1$ \\
$1648332 \pm 8911$ & $61.4 \pm 0.3$ & 68.0 & $90 \pm 1$ \\
$472586 \pm 3349$ & $17.7 \pm 0.1$ & 19.4 & $91 \pm 1$ \\
$104090 \pm 975$ & $4.02 \pm 0.04$ & 4.0 & $100 \pm 1$ \\
$47849 \pm 653$ & $1.93 \pm 0.02$ & 2.0 & $96 \pm 1$ \\
\hline
\end{tabular}

Data are presented as mean \pm SD $(n=3)$.

Table S5. Physicochemical characteristics of $\mathrm{mPEG}_{5 \mathrm{kDa}}-b-\mathrm{p}(\mathrm{HPMA}-\mathrm{Bz})_{17.1 \mathrm{kDa}}$ micelles loaded with 2.0 and $4.8 \% \mathrm{w} / \mathrm{w}$ curcumin.

\begin{tabular}{ccccc}
\hline & \multicolumn{4}{c}{ Curcumin-loaded micelles } \\
\hline Polymer & Z-ave (nm) & PDI & EE (\%) & LC (\%) \\
\hline $2.0 \%$ curcumin-loaded micelles & $53 \pm 1$ & $0.05 \pm 0.01$ & $98 \pm 2$ & $1.9 \pm 0.0$ \\
$4.8 \%$ curcumin-loaded micelles & $55 \pm 1$ & $0.08 \pm 0.02$ & $96 \pm 2$ & $4.5 \pm 0.0$
\end{tabular}

Abbreviations: Z-ave, Z-average hydrodynamic diameter; PDI, polydispersity index; EE, encapsulation efficacy and LC, loading capacity. Data are presented as mean \pm SD $(n=3)$. 


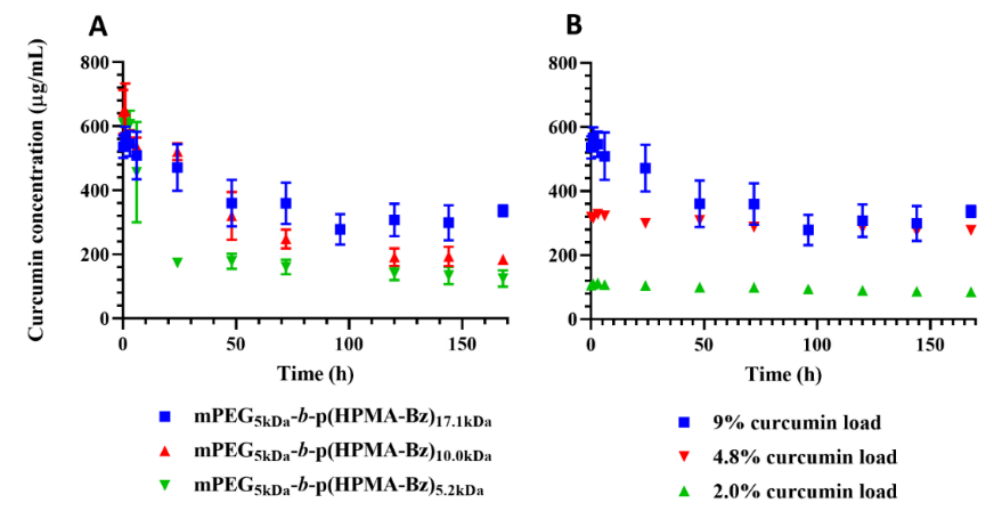

Figure S5. (A) Curcumin retention in $\mathrm{mPEG}_{5 \mathrm{kDa}}-b-\mathrm{p}(\mathrm{HPMA}-\mathrm{Bz})$ micelles composed of polymers with varying molecular weight of the hydrophobic block (17.1, 10.0, and $5.2 \mathrm{kDa}$ ). Micelles contained 9\% (w/w) curcumin and were dispersed in PBS, $\mathrm{pH}=7.4$, at $37^{\circ} \mathrm{C}$ during $168 \mathrm{~h}$. (B) Curcumin retention of mPEG ${ }_{5 \mathrm{kDa}}-b-\mathrm{p}(\mathrm{HPMA}-\mathrm{Bz})_{17.1 \mathrm{kDa}}$ micelles as a function of curcumin loading $(9 \%, 4.8 \%$, and $2.0 \%$ $\mathrm{w} / \mathrm{w})$ during $168 \mathrm{~h}$ incubation in $\mathrm{PBS}, \mathrm{pH}=7.4$, at $37^{\circ} \mathrm{C}$. Data are presented as mean $\pm \mathrm{SD}(\mathrm{n}=3)$.
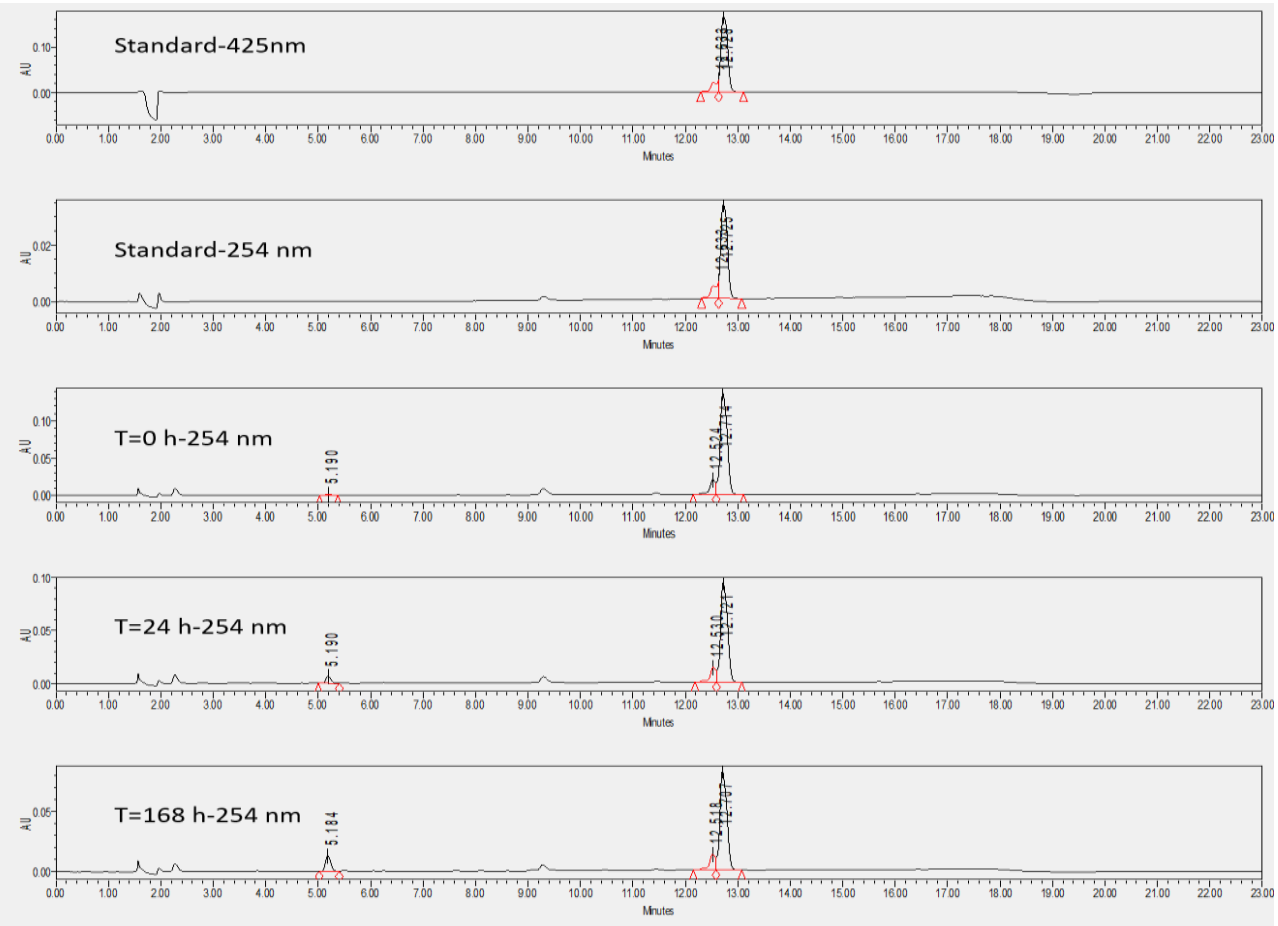

Figure S6. Representative HPLC chromatograms of curcumin samples at different time points collected during the stability study of curcumin-loaded mPEG $5 \mathrm{kDa}-b-\mathrm{p}(\mathrm{HPMA}-\mathrm{Bz})$ micelles in PBS at $37^{\circ} \mathrm{C}$. 


\section{Ratio of HPMA-Bz/curcumin}

The exemplary calculation to obtain the ratio of HPMA-Bz/curcumin $(\mathrm{mol} / \mathrm{mol})$ for $\mathrm{mPEG}_{5 \mathrm{kDa}}-b-\mathrm{p}(\mathrm{HPMA}-\mathrm{Bz})_{17.1 \mathrm{kDa}}$ is provided as an illustration.

Feed ratio: $3.0 \mathrm{mg} / \mathrm{mL}$ curcumin and $30 \mathrm{mg} / \mathrm{mL}$ polymer

Weight fraction\% of p(HPMA-Bz): $77.4 \%$

p(HPMA-Bz): $30 \mathrm{mg} / \mathrm{mL} \times 77.4 \%=23.2 \mathrm{mg} / \mathrm{mL}$

p(HPMA-BZ): $23.2(\mathrm{mg} / \mathrm{mL}) / 247.4$ (HPMA-Bz monomer Mw g/mol) $\times 10^{3}=94 \mathrm{mM}$

Curcumin: $3.0(\mathrm{mg} / \mathrm{mL}) \times 91 \%(\mathrm{EE} \%) / 368.38($ curcumin $\mathrm{Mw} \mathrm{g} / \mathrm{mol}) \times 10^{3}=7.4 \mathrm{mM}$

Ratio HPMA-Bz/curcumin: $94(\mathrm{mM}) / 7.4(\mathrm{mM})=12.7$ 

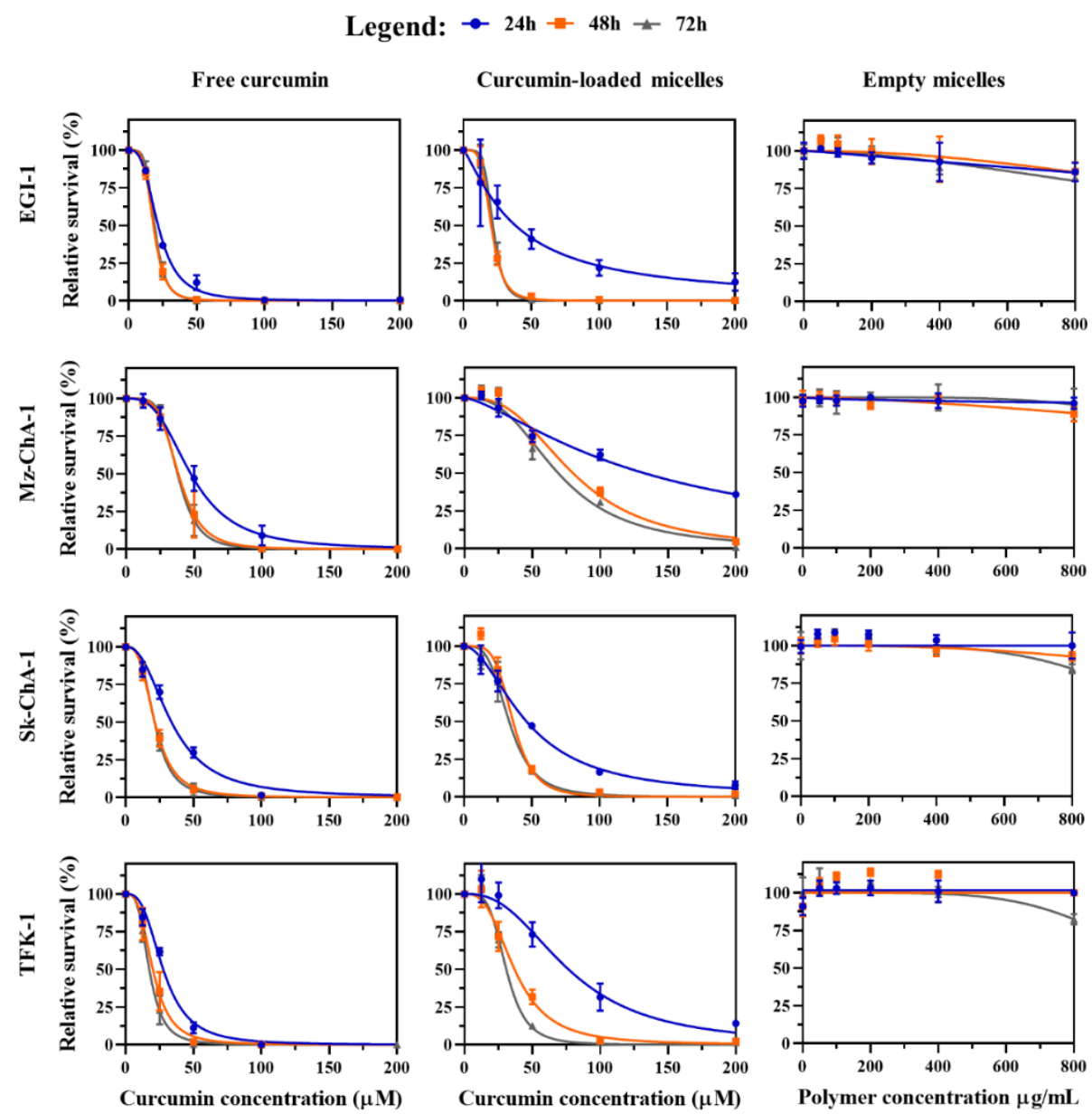

Figure S7. Cytotoxicity of free curcumin, curcumin-loaded mPEG ${ }_{5 \mathrm{kDa}}-b-\mathrm{p}(\mathrm{HPMA}-\mathrm{Bz})_{17.1 \mathrm{kDa}}$ micelles, and empty mPEG 5 kDa- $b$-p(HPMA-Bz) ${ }_{17.1 \mathrm{kDa}}$ micelles in EGI-1, Mz-ChA-1, Sk-ChA-1, and TFK-1 cells incubated for $24 \mathrm{~h}, 48 \mathrm{~h}$, and $72 \mathrm{~h}$. Cell viability was measured with the SRB total protein assay. Data were normalized to the average value of the control (untreated) cells at the respective incubation time. The highest curcumin concentration in $\mathrm{mPEG}_{5 \mathrm{kDa}}-b$-p(HPMA-Bz) ${ }_{17.1 \mathrm{kDa}}$ micelles $(200 \mu \mathrm{M})$ corresponds to a polymer concentration of $800 \mu \mathrm{g} / \mathrm{mL}$. Data were fitted using a nonlinear regression model (curve fit) based on inhibitor vs. normalized response curve (variable slope) and are presented as mean $\pm \mathrm{SD}(\mathrm{n}=4$ per time interval).

\section{Fluorescence spectrum of curcumin-loaded micelles}

Curcumin-loaded micelles were prepared at $30 \mathrm{mg} / \mathrm{mL}$ polymer and varying curcumin concentrations $(0.06-3.0 \mathrm{mg} / \mathrm{mL})$ using the method described in section 2.5 . The samples were diluted to a curcumin concentration of $40 \mu \mathrm{g} / \mathrm{mL}$. The fluorescence spectra were recorded using a Jasco FP8300 spectrofluorometer (Tokyo, Japan). The excitation wavelength was $429 \mathrm{~nm}$. 


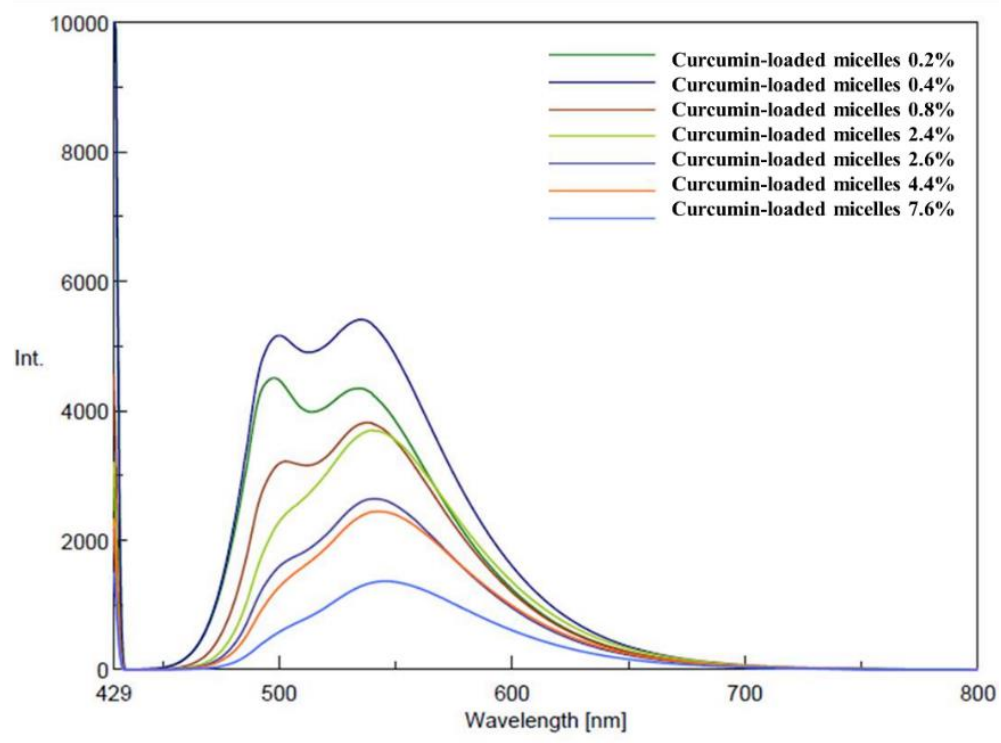

Figure S8. Fluorescence spectra of curcumin-loaded micelles with different loadings.

Figure S8 shows that the fluorescence spectra of curcumin-loaded micelles changed with increasing the curcumin loading. The fluorescence intensity decreased with curcumin loadings $>0.4 \%$. Micelles with a curcumin loading of $9 \%(w / w)$ were used for the in vitro cell studies and Figure 8 demonstrates that the curcumin fluorescence was substantially quenched.

\section{Cytotoxicity of Empty Micelles in Human Pancreatic Stellate Cells (hPSC)}

The cytotoxicity of $\mathrm{mPEG}_{5 \mathrm{kDa}}-b$-p(HPMA-Bz) $17.1 \mathrm{kDa}$ micelles was investigated in hPSC as the main precursor of pancreatic cancer-associated fibroblasts. hPSC cells were seeded in 96well plates at a density of 2500 cells/well. Empty micelles were added to the cells in the range of $0-800 \mu \mathrm{g} / \mathrm{mL}$. Also, HEPES buffer equivalent to the highest concentration of micelles diluted in the medium was used as control. After 24,48 , and $72 \mathrm{~h}$ of incubation, the cells were washed with PBS and AlamarBlue $(\times 1$ in cell culture medium) was added to the cells. After $4 \mathrm{~h}$ of incubation, the medium was removed and collected in a 96 -well plate. The absorbance of the medium was measured with a microplate reader (Tecan Group, Männedorf, Switzerland). 


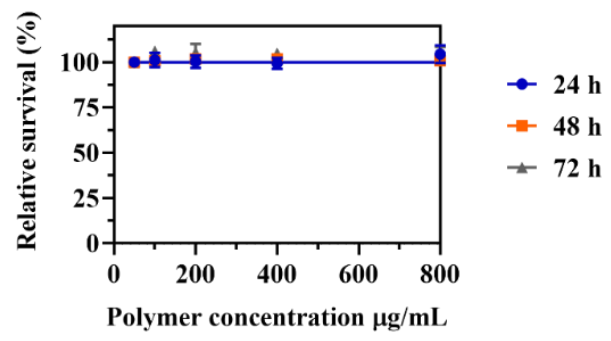

Figure S9. Cytotoxicity of empty micelles in human pancreatic stellate cells (hPSC) using AlmarBlue test. Data were normalized to the average value of the control (untreated) cells at the respective incubation time. Data are presented as mean $\pm S D(n=4$ per time point).

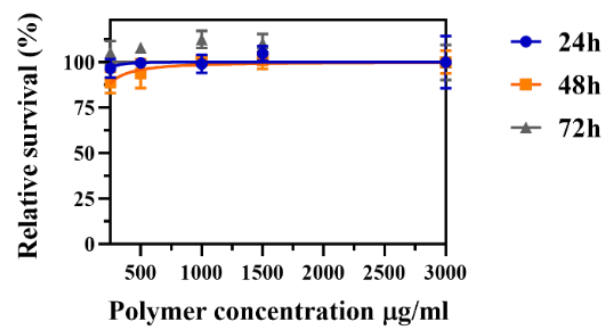

Figure S10. Cytotoxicity of $\mathrm{mPEG}_{5 \mathrm{kDa}}-b-\mathrm{p}(\mathrm{HPMA}-\mathrm{Bz})_{17.1 \mathrm{kDa}}$ micelles in primary human umbilical vein endothelial cells (HUVECs) incubated for $24 \mathrm{~h}, 48 \mathrm{~h}$, and $72 \mathrm{~h}$. Cell viability was measured with the SRB total protein assay. Data were normalized to the average value of the control (untreated) cells at the respective incubation time. Data are presented as mean $\pm \mathrm{SD}(\mathrm{n}=4$ per time point). 


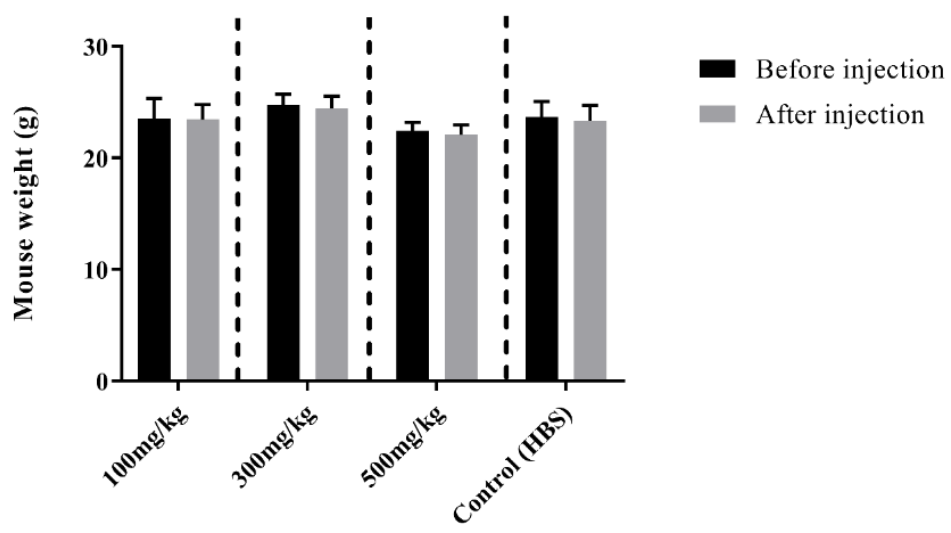

Figure S11. Mouse body weight before and $24 \mathrm{~h}$ after treatment with 100, 300, and $500 \mathrm{mg} / \mathrm{kg}$ empty $\mathrm{mPEG}_{5 \mathrm{KDa}}-b-\mathrm{p}(\mathrm{HPMA}-\mathrm{Bz})_{17.1 \mathrm{kDa}}$ micelles vs control group receiving HBS. Data are presented as mean $\pm \mathrm{SD}(\mathrm{n}=3$ per concentration).

RBC

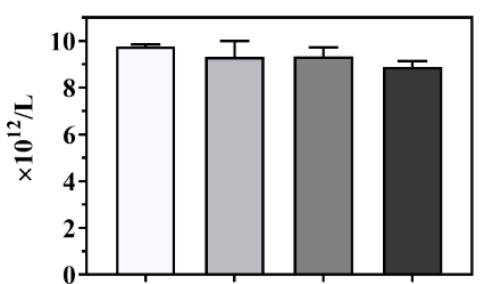

WBC

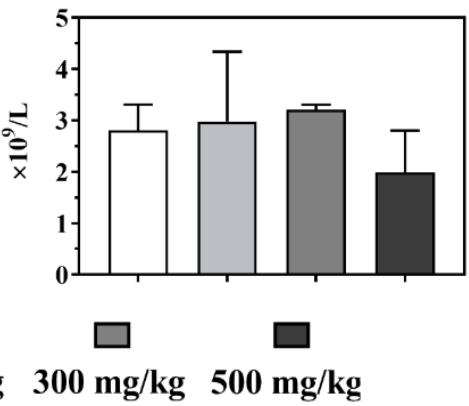

Figure S12. Red blood cell (RBC) and white blood cell (WBC) counts in mice after intravenous administration of 100,300 , and $500 \mathrm{mg} / \mathrm{kg}$ empty $\mathrm{mPEG}_{5 \mathrm{KDa}}-b$-p(HPMA-Bz) ${ }_{17.1 \mathrm{kDa}}$ micelles vs control group receiving HBS. Blood samples were taken after $24 \mathrm{~h}$. Data are presented as mean \pm SD $(n=3$ per concentration).

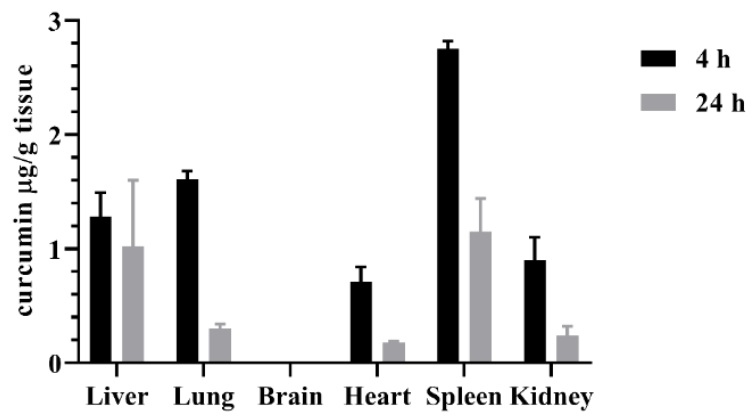

Figure S13. Accumulation of curcumin-loaded Cy7-labeled mPEG5kDa- $b$-p(HPMA-Bz) $17.1 \mathrm{kDa}$ micelles in tissues of BALB/c mice at 4 and $24 \mathrm{~h}$ after intravenous injection. Data are presented as mean $\pm \mathrm{SD}$ ( $\mathrm{n}=3$ per time interval). 
A

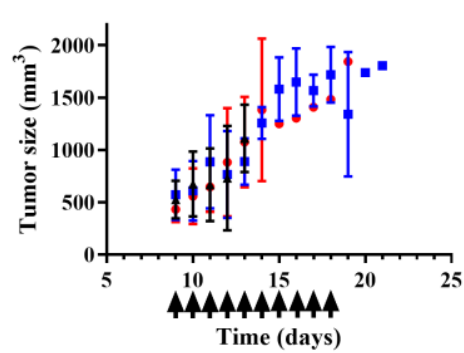

B

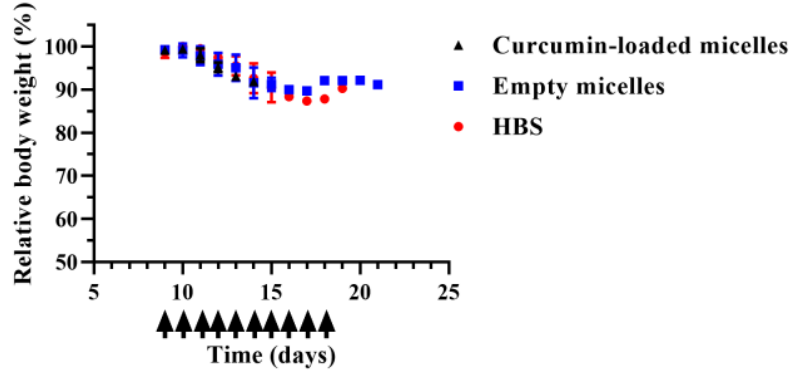

Figure S14. Therapeutic efficacy of curcumin-loaded micelles in Neuro2A tumor-bearing mice. Tumors were grown from cells subcutaneously injected $(3 \times 106$ cells $/ 100 \mu \mathrm{L}$ PBS, $\mathrm{pH}=7.4)$ into the right flank of female $\mathrm{A} / \mathrm{J}$ mice. Treatment consisted of 10 consecutive intravenous injections (starting 9 days after inoculation) of HEPES-buffered saline (HBS), empty mPEG skDa- $b$-p (HPMA-Bz) 17. $_{1 \mathrm{kDa}}$ micelles $(500 \mathrm{mg} / \mathrm{kg}$, polymer), and curcumin-loaded mPEG5kDa- $b$-p(HPMA-Bz) $17.1 \mathrm{kDa}$ micelles (500 $\mathrm{mg} / \mathrm{kg}$ micelles, equivalent to $50 \mathrm{mg} / \mathrm{kg}$ curcumin) dispersed in HBS. The injection volume was between 100-200 $\mu \mathrm{L}$ and infusions were performed slowly. Arrows point to the days on which intravenous injections were administered. (A) Mean \pm SD tumor size $(n=5)$. (B) Mean \pm SD relative body weight of Neuro2A tumor-bearing mice $(n=5)$. The body weights were normalized to the animal's body weight on day 9 (considered as $100 \%$ ).

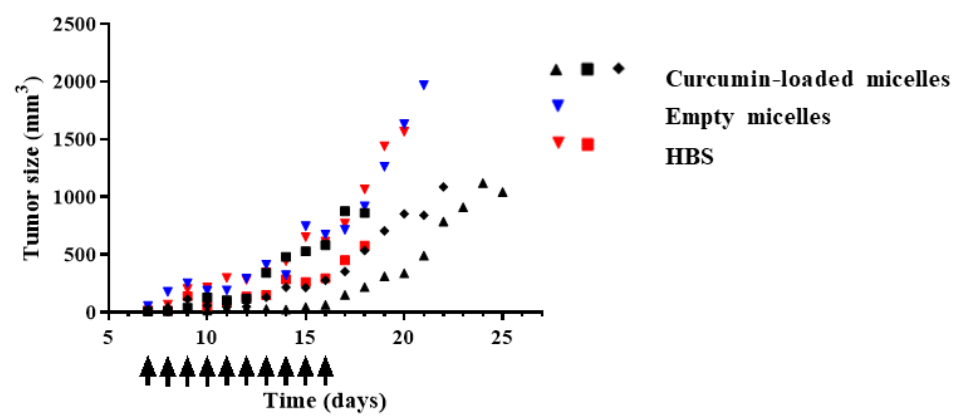

Figure S15. Therapeutic efficacy of curcumin-loaded micelles in Neuro2A tumor-bearing mice. Tumors were grown from cells subcutaneously injected $(1 \times 106$ cells $/ 100 \mu \mathrm{L} \mathrm{PBS,} \mathrm{pH}=7.4)$ into the right flank of female $\mathrm{A} / \mathrm{J}$ mice. Treatment consisted of 10 consecutive intravenous injections (starting 7 days after inoculation) of HEPES-buffered saline (HBS), empty mPEG $_{5 \mathrm{kDa}}-b$-p (HPMA-Bz) 17.1kDa $_{1}$ micelles $\left(500 \mathrm{mg} / \mathrm{kg}\right.$, polymer), and curcumin-loaded $\mathrm{mPEG}_{5 \mathrm{kDa}}-b-\mathrm{p}(\mathrm{HPMA}-\mathrm{Bz}) 17.1 \mathrm{kDa}$ micelles (500 $\mathrm{mg} / \mathrm{kg}$ micelles, equivalent to $50 \mathrm{mg} / \mathrm{kg}$ curcumin) dispersed in HBS. The injection volume was between 100-200 $\mu \mathrm{L}$. Arrows point to the days on which intravenous injections were administered. Data are presented per individual mouse. 


\section{References}

1. Sheybanifard, M.; Beztsinna, N.; Bagheri, M.; Miriam Buhl, E.; Bresseleers, J.; VarelaMoreira, A.; Shi, Y.; van Nostrum, C. F.; van der Pluijm, G.; Storm, G.; Hennink, W. E.; Lammers, T.; Metselaar, J. M. Systematic Evaluation of Design Features Enables Efficient Selection of П ElectronStabilized Polymeric Micelles. Int. J. Pharm. 2020, 119409. 



\section{Chapter 6}

\section{In vitro Evaluation of Curcumin-Loaded $\pi-\pi$ - Stacked Polymeric Micelles in a 2D Mono- Culture and a 3D Stroma-Containing Pancreatic Tumor Model: an Introductory Study}

Mahsa Bagheri ${ }^{1}$, Marcel A. Heinrich ${ }^{2}$, Jai Prakash ${ }^{2}$, Cornelus F. van Nostrum ${ }^{1}$, Michal Heger $^{1,3}$ and Wim E. Hennink ${ }^{1}$

${ }^{1}$ Department of Pharmaceutics, Utrecht Institute for Pharmaceutical Sciences (UIPS), Faculty of Science, Utrecht University, 3508 TB Utrecht, the Netherlands

${ }^{2}$ Targeted Therapeutics, Department of Biomaterials Science and Technology, TechMed Centre, University of Twente, Enschede, the Netherlands

${ }^{3}$ Department of Pharmaceutics, Jiaxing Key Laboratory for Photonanomedicine and Experimental Therapeutics, College of Medicine, Jiaxing University, Jiaxing 314001, P. R. China 


\begin{abstract}
Polymeric micelles composed of poly(ethylene glycol)- $b$-poly(N-2-benzoyloxypropyl methacrylamide) (mPEG- $b$-p(HPMA-Bz)) with two distinct average sizes were prepared via a nanoprecipitation technique and loaded with curcumin as a therapeutic agent to evaluate their performance in 2D and 3D cell culture models. The sizes of the small and large micelles loaded with $5.0 \% \mathrm{w} / \mathrm{w}$ curcumin were 37 and $94 \mathrm{~nm}$, respectively, whereas the empty micelles had a size of 38 and $80 \mathrm{~nm}$ (further referred to small and large micelles). Both types of curcumin-loaded micelles showed around $80 \%$ curcumin retention in phosphate buffer saline at $37{ }^{\circ} \mathrm{C}$ during incubation for 7 days. The empty micellar dispersions showed good cytocompatibility for Panc-1 and primary human pancreatic stellate cells (hPSC) as the main precursor of pancreatic cancer-associated fibroblasts (CAF) in 2D cell culture. In contrast, a cytotoxicity assay showed that the small curcumin-loaded micelles showed higher toxicity with lower $50 \%$ inhibitory concentration $\left(\mathrm{IC}_{50}=52.7 \pm 1.4 \mu \mathrm{M}\right)$ compared to the large micelles $\left(\mathrm{IC}_{50}=73.8 \pm 1.4 \mu \mathrm{M}\right)$ after $72 \mathrm{~h}$ incubation with Panc-1 cells, probably due to the previously demonstrated lower stability of the small curcumin-loaded micelles in a biological fluids and faster release of curcumin. Also, free curcumin demonstrated a slightly higher cytotoxicity $\left(\mathrm{IC}_{50}=43.6 \pm 3.6 \mu \mathrm{M}\right)$ than curcumin-loaded micelles likely because curcumin was slowly released and also partially retained in the micelles, and thus unable to fully exert the pharmacological activity of the curcumin dose present in the culture medium. A qualitative cell uptake study of Cy3-labeled micelles by Panc-1 cells indicated that both formulations were taken up with a higher internalization for the larger micelles. An introductory study showed that the small micelles likely penetrated deeper in 3D stromacontaining spheroids than the large micelles indicating the potential to exert a stronger therapeutic effect for these drug-loaded small micelles. To substantiate this hypothesis, complementary experiments such as cytotoxicity assay in 3D cell cultures are necessary.
\end{abstract}

Keywords: Nanomedicine; drug delivery; 3D cell culture; pHPMA, $\pi-\pi$ stacking; hydrophobic drugs, micelle size 


\section{Introduction}

Nanomedicine is an emerging field in the pharmaceutical sciences with a particular section focusing on the improvement of cancer therapy in terms of efficiency and safety ${ }^{1-6}$. To this end, polymeric based nanoparticles, in particular polymeric micelles, have gained considerable attention ${ }^{7-13}$. Amphiphilic block copolymers form core-shell structures in water, as referred to as 'polymeric micelles'. The hydrophobic core of these micelles is suitable for the solubilization and loading of hydrophobic therapeutics, whereas the hydrophilic shell, usually based on poly(ethylene glycol) (PEG), renders colloidal stability. Moreover, a variety of polymer chemistries can be exploited to design polymeric micelles with different physicochemical characteristics. For clinical translation, polymeric micelles should be sufficiently stable and simultaneously retain the loaded drug while circulating in the bloodstream. Stability can be achieved by using chemical crosslinking ${ }^{14}$ or by exploiting physical interactions ${ }^{15-17}$. Such stable particles with a size $<100 \mathrm{~nm}$ display prolonged circulation times and show penetration through fenestrated blood capillaries in tumors and are deposited in tumors due to lack of proper lymphatic drainage. This phenomenon, the socalled enhanced permeation and retention effect (EPR), was first discovered by Matsumura and $\mathrm{Maeda}^{18}$ and is the underlying mechanism of passive cancer targeting using nanomedicine ${ }^{19-21}$.

Despite many preclinical successes of nanoparticulate drug delivery systems, not many nanomedicine formulations have reached clinical applications. In particular, the limited penetration of drug-loaded nanomedicines into tumor tissues has hindered the therapeutic efficacy of these systems ${ }^{22}$. The effect of size as one of the important physicochemical properties of nanomedicines on tumor penetration has been evaluated in several in vitro and in vivo studies ${ }^{23-28}$. Notably, in recent years, in vitro studies using three-dimensional (3D) cell culture models such as 3D spheroids have been progressed considerably ${ }^{29-32}$. Such 3D spheroids not only better resemble the tumor microenvironment (TME) compared to 2D monolayered cell cultures, but also are potential valuable substitutes for animal studies, considering the current movements toward restricting animal testing in many countries among which the Netherlands ${ }^{23,33}$.

It has been demonstrated that TME significantly influences tumor growth. The composition of TME and its complex interaction with tumor cells regulate many tumor mechanisms from tumorigenesis to metastasis ${ }^{34}$. The TME mainly consists of non-malignant cells surrounded by a dense extracellular matrix (ECM) with fibroblasts as one of the most abundant cells ${ }^{35-}$ ${ }^{36}$. Within tumors, fibroblasts get activated towards cancer associated-fibroblasts (CAFs) that secrete high amounts of ECM, in particular collagen, eventually leading to a fibrotic tissue inside the tumor ${ }^{37}$. This dense fibrotic tumor tissue in turn can cause tumor resistance and treatment failure ${ }^{38-39}$. Therefore, it is essential to use 3D cell models that also reflect the role of TME, including CAFs to model drug and nanomedicine penetration in a biologically relevant fashion. In this regard, Priwitaningrum et al. ${ }^{23}$ developed a 3D co-culture spheroidal array to represent CAFs along with tumor cells. The authors studied the penetration of both organic and inorganic nanoparticles and concluded that the heterospheroids are useful tools for screening of nanomedicines during formulation development and in preclinical efficacy studies. 
Previously, we developed a micellar drug delivery system based on mPEG- $b$-p(HPMA-Bz) polymer with excellent particle stability and good drug retention due to the $\pi-\pi$ stacking interactions ${ }^{40-42}$. Also, the preparation method was optimized to tailor the micellar size ${ }^{43}$. In the present study, curcumin was used as a therapeutic compound due to its potential anticancer activity ${ }^{44-47}$. However, the therapeutic potential of curcumin is significantly hampered due to its low aqueous solubility, low bioavailability after oral administration and chemical instability ${ }^{46,48}$. Importantly, it has been reported that curcumin loaded in the hydrophobic core of mPEG- $b$-p(HPMA-Bz) micelles had substantially higher stability than in its free form ${ }^{41,49}$. In this study, two different micellar formulations based on mPEG- $b$ p(HPMA-Bz) copolymer with sizes of around $38 \mathrm{~nm}$ and $80 \mathrm{~nm}$ were prepared. The in vitro cell uptake of the empty micelles and cytotoxicity profile of the micelles loaded with curcumin was evaluated in $2 \mathrm{D}$ cells. Also, the penetration of the micelles into stroma-rich $3 \mathrm{D}$ heterospheroids consisting of pancreatic tumor cells and $\mathrm{CAFs}^{50}$ was investigated.

\section{Materials and Methods}

\subsection{Materials}

$N$-(2-benzoyloxypropyl) methacrylamide (HPMA-Bz) and mPEG $_{5 \mathrm{kDa}}-\mathrm{ABCPA} \mathrm{mPEG}_{5 \mathrm{kDa}}$ macroinitiator were synthesized according to published methods ${ }^{43},{ }^{51}$. N-(2aminoethyl)methacrylamide hydrochloride (AEMA), 4,4-azobis(4-cyanopentanoic acid) (ABCPA), triethylamine (TEA), lithium chloride ( $\mathrm{LiCl})$, HEPES, sodium chloride, curcuminoid mixture and penicillin/streptomycin were obtained from Sigma-Aldrich (St. Louis, MO, USA). PEG standards were obtained from Agilent (Santa Clara, CA, USA). Cyanine 3 (Cy3) NHS ester was purchased from Lumiprobe (Hannover, Germany). Acetonitrile (ACN), tetrahydrofuran (THF), dimethylformamide (DMF) and diethyl ether were purchased from Biosolve (Valkenswaard, the Netherlands). Syringe filters made of regenerated cellulose (RC) were acquired from Phenomenex (Torrance, CA, USA). Dialysis bags with $8-\mathrm{kDa}$ cut-off were obtained from Spectrum Chemical (SpectraPor; New Brunswick, NJ, USA). Stellate Cell Growth Supplement (SteCGS) was purchased from ScienCell (Carlsbad, CA, USA). Dulbecco's Modified Eagles medium (DMEM) was ordered from GE Healthcare (Vienna, Austria). Fetal bovine serum (FBS) was obtained from Lonza (Basel, Switzerland). DAPI was purchased from Abcam (Cambridge, UK). PAP pen was ordered from DAKO Agilent (Santa Clara, CA, USA). Alamar Blue dye was obtained from Invitrogen (Carlsbad, CA, USA). 96-Well plates were purchased from Greiner Bio-One (Cellstar, Kremsmünster, Austria). Nunc ${ }^{\circledR}$ black 96-well plates were acquired from Thermofisher Scientific (Waltham, MA, USA). Microscopic glass slides were obtained from Menzel-Gläser (Braunschweig, Germany).

\subsection{Cell Culture}

Primary human pancreatic stellate cells (hPSC) (ScienCell; Carlsbad, CA, USA) were cultured in complete stellate cell medium supplemented with $2 \%$ FBS, $1 \%$ penicillin/streptomycin and 1\% SteCGS. Panc-1 cancer cells (ATCC, Rockville, MD, USA) were cultured in DMEM containing high glucose $(4.5 \mathrm{~g} / \mathrm{L})$ supplemented with $2 \mathrm{mM} \mathrm{l-}$ glutamine. The medium was also supplemented with $10 \%$ FBS and $100 \mu \mathrm{g} / \mathrm{mL}$ penicillin/streptomycin. Cells were maintained in a humidified atmosphere at $37^{\circ} \mathrm{C}$ with $5 \%$ $\mathrm{CO}_{2}$ and passaged upon $80 \%$ confluency. 
In vitro Evaluation of Curcumin-Loaded $\pi$ - $\pi$-Stacked Polymeric Micelles in a 2D Mono-Culture and a 3D Stroma-Containing Pancreatic Tumor Model: an Introductory Study

\subsection{Synthesis and Characterization of Copolymers, Homopolymer and Cy3-Labeled Polymer}

$\mathrm{mPEG}_{5 \mathrm{kDa}}-b-\mathrm{p}(\mathrm{HPMA}-\mathrm{Bz})$ block copolymers were synthesized via free-radical polymerization and characterized by ${ }^{1} \mathrm{H}-\mathrm{NMR}$ and gel permeation chromatography (GPC) as described previously ${ }^{43}, 51$. In short, the $\mathrm{mPEG}_{5 \mathrm{kDa}}-\mathrm{ABCPA}-\mathrm{mPEG}_{5 \mathrm{kDa}}$ macro-initiator and HPMA-Bz were dissolved in ACN at two feed ratios of 1:50 and 1:200 (mol:mol). The polymerization was performed at $70{ }^{\circ} \mathrm{C}$ for $24 \mathrm{~h}$ under a nitrogen atmosphere. The polymers were collected by precipitation in ice-cold diethyl ether, followed by filtration. The characteristics of the synthesized polymers were published previously ${ }^{52-53}$ and reported here in the supplementary information Table S1. A p(HMA-Bz) homopolymer was synthesized similarly using ABCPA as initiator and HPMA-Bz at a feed ratio of 1:200 (mol:mol) ${ }^{43}$. mPEG $_{5 \mathrm{kDa}}-b$-p(HPMA-Bz98\%-co-AEMA $2 \%$ ) at macro-initiator to monomer ratio of $1: 200$ (mol:mol) was synthesized as reported earlier ${ }^{40}$. Cy3-NHS was reacted with the primary amines of AEMA in the HPMA-Bz ${ }_{98 \%}$ co-AEMA $_{2 \%}$ part of the copolymer (Scheme S1). Briefly, Cy3-NHS was dissolved in dried DMSO at a concentration of $25 \mathrm{mg} / \mathrm{mL}$. Next, 100 mg mPEG ${ }_{5 \mathrm{kDa}}-b$-p(HPMA-Bz ${ }_{98 \%}$-co-AEMA $2 \%$ ) was transferred into a dried glass vial and subsequently dissolved in $1.76 \mathrm{~mL}$ dried DMSO. Subsequently, $0.23 \mathrm{~mL}$ solution of the Cy3NHS and $3.2 \mu \mathrm{L}$ of TEA were added to the polymer solution. The reaction was conducted in the dark at room temperature overnight. The uncoupled $\mathrm{Cy} 3$ dye was removed by extensive dialysis against THF/water $(1 / 1, v / v)$ for three days using an $8-\mathrm{kDa}$ dialysis membrane. The dialysis medium was refreshed 5 times. Cy3-labeled copolymer was collected as a fluffy powder after freeze-drying. GPC was conducted to confirm the conjugation of $\mathrm{Cy} 3$ to the polymer as described before ${ }^{40}$, using refractive index (RI) and UV detection at $555 \mathrm{~nm}$. The chromatograms are shown in Figure S1.

\subsection{Preparation and Characterization of mPEG- $b$-p(HPMA-Bz) Polymeric Micelles}

$\mathrm{mPEG}_{5 \mathrm{kDa}}-b$-p(HPMA-Bz) micelles were prepared by a nanoprecipitation technique, as described in detail previously ${ }^{43}$. In short, the polymer solution in THF ( $1 \mathrm{~mL}$, different polymer concentrations) was added dropwise to HEPES buffer saline (HBS; 20 mM HEPES and $150 \mathrm{mM} \mathrm{NaCl}, \mathrm{pH} 7.4$ ) in 1:1 ratio under vigorous stirring. Then, THF was removed by evaporation overnight in a fume hood. The samples for the cell studies were dialyzed against HBS overnight to remove residual solvent. The obtained micellar dispersions were subsequently sterilely filtered through $0.2 \mu \mathrm{m}$ regenerated cellulose syringe filters.

Empty micelles with two different sizes were prepared by dissolving the unlabeled polymers in THF. The small micelles were prepared using $\mathrm{mPEG}_{5 \mathrm{kDa}}-b-\mathrm{p}(\mathrm{HPMA}-\mathrm{Bz})_{5.2 \mathrm{kDa}}$ dissolved in THF at a concentration of $30 \mathrm{mg} / \mathrm{mL}$. The large micelles were prepared by addition of homopolymer $(7.5 \mathrm{mg})$ to $\mathrm{mPEG}_{5 \mathrm{kDa}}-b$-p(HPMA-Bz) $17.1 \mathrm{kDa}(30 \mathrm{mg})$ in $1 \mathrm{~mL}$ THF. Micelles were prepared using the nanoprecipitation technique as described above. Similarly, small Cy3-labeled micelles were prepared by dissolving Cy3-labeled polymer $(7.5 \mathrm{mg})$ and unlabeled polymer $(30 \mathrm{mg})$ in $1 \mathrm{~mL}$ THF. The large Cy3-labeled micelles were prepared by the addition of Cy3-labeled polymer $(8 \mathrm{mg})$ to a mixture of homopolymer $(8 \mathrm{mg})$ and $\mathrm{mPEG}_{5 \mathrm{kDa}}-b-\mathrm{p}(\mathrm{HPMA}-\mathrm{Bz})_{17.1 \mathrm{kDa}}(24 \mathrm{mg})$ in $1 \mathrm{~mL}$ THF. Curcumin-loaded micelles were prepared by dissolving curcumin and the polymers in THF. The small micelles were prepared by dissolution of $1.6 \mathrm{mg}$ curcumin and $30 \mathrm{mg} \mathrm{mPEG}_{5 \mathrm{kDa}}-b-\mathrm{p}(\mathrm{HPMA}-\mathrm{Bz})_{5.2 \mathrm{kDa}}$ in $1 \mathrm{~mL}$ THF, while the large micelles were prepared by dissolution of $1.8 \mathrm{mg}$ curcumin, $26 \mathrm{mg} \mathrm{mPEG} \mathrm{kDa}^{-}$ $b$-p(HPMA-Bz) ${ }_{17.1 \mathrm{kDa}}$ and $7 \mathrm{mg}$ homopolymer in $1 \mathrm{~mL}$ THF. Micelles loaded with curcumin 
were prepared as described above for the empty micelles and were protected from light to avoid curcumin's photodegradation.

The size and polydispersity of the micelles were determined using Dynamic Light Scattering (DLS) (Zetasizer nano series ZS90, Malvern Instruments, Malvern, UK) at $1.5 \mathrm{mg} / \mathrm{mL}$ in HEPES solution. The zeta potential of $20 \times$ diluted micelles in HEPES solution $(20 \mathrm{mM}, \mathrm{pH}$ 7.4) was measured by Zetasizer (Nano ZS, Malvern Instruments, Malvern, UK). The polymer concentrations in the different micellar dispersions were measured by GPC using MIXED-D column (Polymer Laboratories, Agilent Technologies) stabilized at $65^{\circ} \mathrm{C}$ and DMF plus 10 $\mathrm{mM} \mathrm{LiCl}$ at $1 \mathrm{~mL} / \mathrm{min}$ as the eluent. RI detection (Waters 2414, Waters Corporation, Milford, MA, USA) and UV detection at $280 \mathrm{~nm}$ (waters 2489, Waters Corporation, Milford, MA, USA) were used to measure the polymer concentrations. Dispersions of polymeric micelles were diluted 10-fold in DMF to solubilize the micelles and the absorbance was measured at $280 \mathrm{~nm}$. Standard curves $(0.1-10 \mathrm{mg} / \mathrm{mL}$ concentration) were prepared using the same polymer in DMF plus $10 \mathrm{mM} \mathrm{LiCl}$. Curcumin concentrations were determined using HighPerformance Liquid Chromatography (HPLC) as described before ${ }^{49}$. In short, C18 column, SunFire $^{\mathrm{TM}}(5 \mu \mathrm{m}, 150 \times 4.6 \mathrm{~mm}$; Waters Corporation, Milford, MA, USA) and a gradient elution consisting of 5:95 (v/v) ACN/water (eluent A) and 100\% ACN (eluent B) were used. Acetic acid $(0.25 \%)$ was added to adjust the $\mathrm{pH}$ of the eluents. The gradient run was performed from $90 \%$ A to $70 \% \mathrm{~B}$ in $15 \mathrm{~min}$ at $1.2 \mathrm{~mL} / \mathrm{min}$ flow rate with a sample injection volume of $20 \mu \mathrm{L}$. The encapsulation efficacy (EE) and loading capacity (LC) were calculated as follows:

$\mathrm{EE} \%=($ measured amount of curcumin $)($ amount of curcumin added $) \times 100 \%$,

LC\% $=($ measured amount of curcumin $)($ measured amount of curcumin and polymer $) \times 100 \%$.

\subsection{Stability of Curcumin-Loaded Micelles in Phosphate Buffered Saline}

The retention of curcumin in the micelles was measured in phosphate buffered saline (PBS; $11.9 \mathrm{mM}$ phosphates, $137 \mathrm{mM}$ sodium chloride, and $2.5 \mathrm{mM}$ potassium chloride, $\mathrm{pH} 7.4$ ), as described previously ${ }^{40}$. The $5.0 \% \mathrm{w} / \mathrm{w}$ curcumin-loaded micelles were diluted 5-fold in PBS and incubated at $37{ }^{\circ} \mathrm{C}$ with constant shaking and protected from light. At different time points, $100 \mu \mathrm{L}$ aliquots were removed and centrifuged at $5000 \times \mathrm{g}$ for $10 \mathrm{~min}$ to spin down released and precipitated curcumin. Then, the collected supernatants were diluted at least $10 \times$ with methanol, followed by vortexing to solubilize the micelles. The curcumin concentrations were measured using HPLC, as described in section 2.4.

\subsection{D Cell Uptake Study}

Cells were seeded into 96-well plate at 5000 cells/well and incubated overnight. Next, the medium was replaced with complete fresh medium containing $2 \%$ FBS and Cy3-labeled polymeric micelles at a concentration of $300 \mu \mathrm{g} / \mathrm{mL}$ with a similar fluorescence signal. The cells were incubated with Cy3-labeled polymeric micelles for $24 \mathrm{~h}$. Cells without treatment were used as a negative control and DAPI was used for nuclei staining. Fluorescent images were obtained by an EVOS fluorescence microscope (Thermofisher; Waltham, MA, USA) at $20 \times$ magnification. 


\subsection{D Heterospheroid Formation}

3D-heterospheroids containing primary human pancreatic stellate cells (hPSCs) and Panc1 cells were prepared using round-bottom low attachment plates. In brief, 96-well round bottom plates were treated with $1 \%$ pluronic F127 overnight to create a non-adherent surface ${ }^{54}$. After washing the wells twice with sterile water and letting the plate air-dried in a flow hood, the cell dispersions (6000 cells/ well in $100 \mu \mathrm{L}$ of medium) were pipetted into the wells. hPSCs and Panc-1 were trypsinized and suspended in their respective culture medium at a cell density of 300,000 cells $/ \mathrm{mL}$. The suspensions of hPSCs and Panc-1 cells were mixed in 5:1 volume ratio. The spheroids were allowed to generate without disturbance for three days before proceeding with the experiments.

\subsection{Penetration of Cy3-Labeled Micelles in Heterospheroids}

Spheroids were incubated with Cy3-labeled micelles at final concentrations of $300 \mu \mathrm{g} / \mathrm{mL}$ in a DMEM/SteCM medium (volume ratio 1/1) containing 2\% FBS allowing the micelles to diffuse passively into the spheroids. After $24 \mathrm{~h}$ incubation, the spheroids were fixed with $4 \%$ formaldehyde, submerged in Cryomatrix ${ }^{\circledR}$ and snap-frozen using isopentane (Acros Organics). Cryosections of around $6 \mu \mathrm{m}$-thickness were prepared using Cryotome ${ }^{\circledR}$ FSE (Thermo Fisher Scientific; Cheshire, UK) protected from light. The cryosections were collected on Superfrost microscopic glass slides. Next, the cryosections were air-dried and either stored at $-20{ }^{\circ} \mathrm{C}$ or immediately fixed with acetone at $25{ }^{\circ} \mathrm{C}$ for 20 min for further process. The slides were marked with a hydrophobic PAP pen before rehydration in PBS. The slides were then mounted with Fluoroshield containing DAPI to stain the nuclei. Fluorescent images of the cryosections were obtained using an EVOS fluorescence microscope (Thermofisher, Waltham, MA, USA). The penetration profile of Cy3-labeled micelles was analyzed using NIH ImageJ software.

\subsection{In Vitro Cytotoxicity of Curcumin-Loaded Micelles in 2D Cell Culture}

The cytotoxicity of free curcumin, empty and curcumin-loaded micelles was investigated in vitro in 2D monolayers of Panc-1 and hPSC. Panc-1 and PSC cells were seeded in 96-well plates at a density of 5,000 and 2,500 cells/well, respectively. Curcumin-loaded micelles dispersed in HBS and free curcumin dissolved in DMSO $(20 \mathrm{mg} / \mathrm{mL})$ were added at various concentrations (curcumin concentrations ranged between 0-100 $\mu \mathrm{M}$, final DMSO concentration was $0.4 \%(\mathrm{v} / \mathrm{v}))$. Empty micelles dispersed in HEPES were used as control and added to the cells in the range of $0-800 \mu \mathrm{g} / \mathrm{mL}$. Also, HEPES buffer equivalent to the highest concentration of micelles diluted in the medium and DMSO $(0.4 \%)$ were additionally taken as controls to correct for effect from the vehicle (buffer or DMSO) only. After 24, 48, and 72 $\mathrm{h}$ of incubation, the cells were washed with PBS and AlamarBlue ( $\times 1$ in cell culture medium) was added to the cells. After $4 \mathrm{~h}$ of incubation, the medium was removed and collected in a Nunc ${ }^{\circledR}$ black 96-well plate. The absorbance of the medium was measured by a Tecan plate reader (Tecan Group Ltd; Männedorf, Switzerland). The 50\% inhibitory concentrations $\left(\mathrm{IC}_{50}\right)$ were obtained from a nonlinear regression model based on an inhibitor versus normalized response with variable slope and calculated using GraphPad Prism (GraphPad Software, La Jolla, CA, USA). 


\section{Results and Discussion}

\subsection{Preparation and Characterization of mPEG- $b$-p(HPMA-Bz) Polymeric Micelles}

Two amphiphilic $\mathrm{mPEG}_{5 \mathrm{kDa}}-b$-p(HPMA-Bz) block copolymers were previously synthesized by a free radical polymerization with varying feed ratios of macro-initiator:monomer (Table $\mathrm{S} 1)$ and used in the present study. The $\mathrm{mPEG}_{5 \mathrm{kDa}}-b-\mathrm{p}(\mathrm{HPMA}-\mathrm{Bz})_{5.2 \mathrm{kDa}}$ block copolymer resulted in polymeric micelles with a hydrodynamic size of $38 \pm 1 \mathrm{~nm}$. $\mathrm{mPEG}_{5 \mathrm{kDa}}-b$-p (HPMA$\mathrm{Bz})_{17.1 \mathrm{kDa}}$ with $20 \% \mathrm{w} / \mathrm{w}$ homopolymer formed micelles with a size of $80 \pm 7 \mathrm{~nm}$. Both micellar dispersions showed narrow size distributions with a polydispersity index (PDI) of $<0.15$ (Figure 1). In line with our previous study, the blockcopolymer with a hydrophobic block of $5.2 \mathrm{kDa}$ self-assembled into smaller $\sim 40 \mathrm{~nm}$ micelles ${ }^{43}$. Micelles based on $\mathrm{mPEG}_{5 \mathrm{kDa}}-b-$ p(HPMA-Bz) $17.1 \mathrm{kDa}$ had a size of $50 \mathrm{~nm}$ which increased to $80 \mathrm{~nm}$ by addition of homopolymer because of its partitioning in the hydrophobic core of the micelles ${ }^{43}$. The size

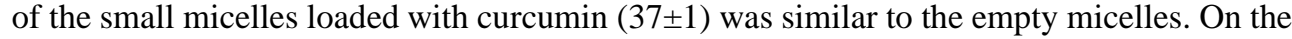
other hand, the size of large micelles loaded with curcumin increased from $80 \pm 7$ to $94 \pm 16$ $\mathrm{nm}$. An increase in the size of $\mathrm{mPEG}_{5 \mathrm{kDa}}-b-\mathrm{p}(\mathrm{HPMA}-\mathrm{Bz})$ micelles upon drug loading was previously observed for other drugs such as paclitaxel (71 to $104 \mathrm{~nm}$ in the range of $0-23 \%$ drug loading) ${ }^{40}$ and DUSP6 Inhibitor - BCI (55 to $75 \mathrm{~nm}$ in the range of $0-25 \%$ drug loading $)^{55}$. Also, these two micellar formulations showed two different appearances with the small micelles being more transparent than the larger micelles (Figure 1B), which might be ascribed to the difference in micelle size and size distribution. Curcumin-loaded micelles showed EE\% and LC\% of more than $85 \%$ and $4.5 \%$, respectively. The zeta potential of the micelles with and without curcumin loading was almost neutral, with values between -0.6 to $-2.1 \mathrm{mV}$.

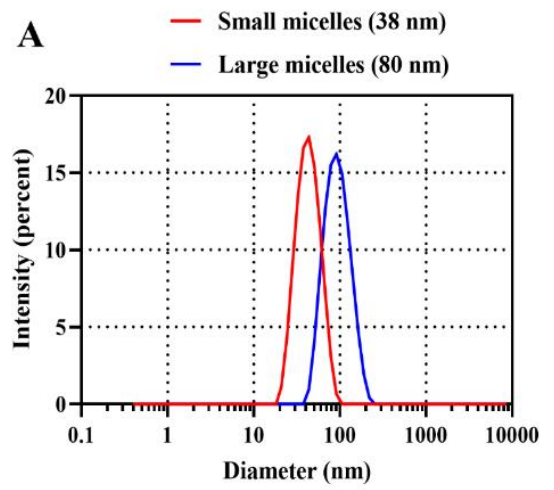

B

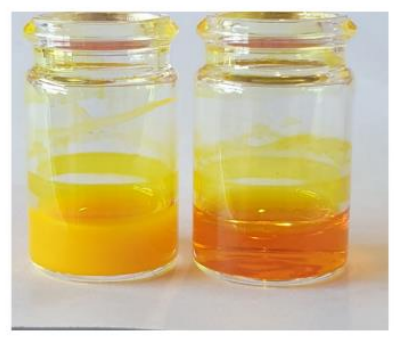

Figure 1. (A) Size distribution of empty mPEGskDa- $b$-p(HPMA-Bz) micelles of $38 \mathrm{~nm}$ (red) and $80 \mathrm{~nm}$ (blue). (B) Photographic image of dispersions of curcumin-loaded micelles with small size $(38 \mathrm{~nm})$ on the right and large size $(80 \mathrm{~nm})$ on the left.

Curcumin retention of the two mPEG5 $\mathrm{kDa}-b-\mathrm{p}(\mathrm{HPMA}-\mathrm{Bz})$ micellar dispersions in PBS was studied at $37{ }^{\circ} \mathrm{C}$. Figure 2 shows that both micellar formulations retained around $80 \%$ of curcumin loading during $168 \mathrm{~h}$ incubation. The small micelles showed a continuous release over $168 \mathrm{~h}$, whereas the large micelles expelled around $20 \%$ of the loading during the first 5 $\mathrm{h}$ of incubation and then hardly any release was observed. 


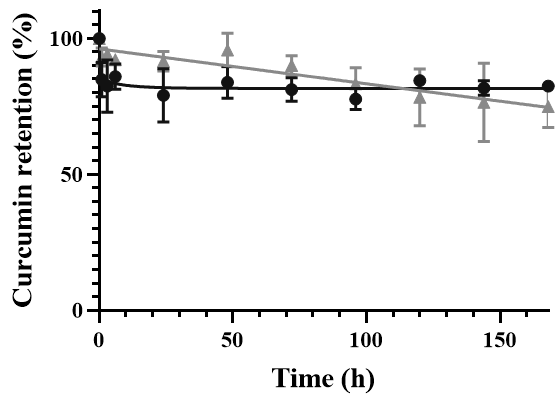

Figure 2. Retention of curcumin in micelles composed of mPEG- $b$-p(HPMA-Bz) with an average size of $37 \mathrm{~nm}$ (gray) and $94 \mathrm{~nm}$ (black). Micelles contained 5.0\% (w/w) curcumin and were dispersed in PBS, pH 7.4, and incubated at $37^{\circ} \mathrm{C}$ for $168 \mathrm{~h}$. Data are presented as mean $\pm \mathrm{SD}(\mathrm{n}=3)$.

\subsection{Cytotoxicity and Uptake of mPEG- $b$-p(HPMA-Bz) Micelles in 2D Pancreatic Tumor Models}

The cytotoxicity of the small and large micelles with and without curcumin loading was investigated in 2D pancreatic tumor models using the AlamarBlue test. In agreement with previous studies using other cell lines ${ }^{40-41,56}$, placebo mPEG- $b$-p(HPMA-Bz) micelles have a good cytocompatibility in both Panc-1 and hPSC cells (Figure S2). On the other hand, free curcumin and curcumin-loaded micelles showed dose and time-dependent cytotoxicity against these cell lines (Figure 3 and Table 1). The observed toxicity is due to the fact that curcumin interferes with many vital pathways and induces apoptosis in tumor cells ${ }^{47}$. Figure 3 shows that curcumin (both in its free form and as micellar formulation) is more toxic for hPSC than for Panc-1 cells as reflected by lower $\mathrm{IC}_{50}$ values (Table 1 ). hPSCs are noncancerous and therefore less resistant to the drugs in contrast to cancer cells ${ }^{57}$.A similar trend was also observed in these cell lines when incubated with cyclopamine-loaded micelles based

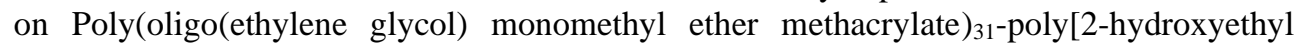
methacrylate-g-((E-caprolactone $)_{5}$-oxycarbonyl aminopropyl triethoxysilane $\left.)\right]_{26}$, showing higher cytotoxic effects against hPSC cells ${ }^{58}$. In general metabolically hyperactive cancer cells are more susceptible to compounds with pleiotropic properties like curcumin and cyclopamine because they can interfere with multiple cellular pathways or pathways with pleiotropic effects that lead to metabolic catastrophe and eventually cause cell death ${ }^{59}$.

Free curcumin $\left(\log P 2.5^{60}\right)$ showed higher toxicity than curcumin-loaded micelles because of the ability to passively permeate over the cell membrane, which is the property of compounds with high permeability $(\log P>1.62)^{61}$. On the other hand, molecules with $\log$ $P>3.5^{62}$ tend to stay in the lipid membrane and do not partition out to the intracellular compartment. Therefore, curcumin is a favorable molecule for passive permeation. Also, the observed $\mathrm{IC}_{50}$ values for free curcumin are in the range of $1-100 \mu \mathrm{M}$, which is in the range reported for different types of cancer cell lines in the literature ${ }^{47}$. Similar to the previous study of Naksuriya et al. ${ }^{41}$, the higher $\mathrm{IC}_{50}$ of curcumin-loaded micelles compared to free curcumin indicates that the cytotoxicity profile is most likely due to the slow release of curcumin from the micelles either in the cell culture medium or intracellularly after internalization of the micelles. It was demonstrated in chapter 5 that the internalization of curcumin-loaded micelles indeed occurs. In both cell lines, the smaller curcumin-loaded micelles demonstrated higher cytotoxicity (thus lower $\mathrm{IC}_{50}$ value) compared to the larger micelles (Table 1). 
Although the stability of the small and large micelles in buffer was comparable (Figure 2), the higher cytotoxicity effect is most likely due to the lower stability of micelles composed of $\mathrm{mPEG}_{5 \mathrm{kDa}}-b$ - $\mathrm{p}(\mathrm{HPMA}-\mathrm{Bz})$ with smaller hydrophobic block in biological media and faster release of the cargo either in the medium and probably also intracellularly (chapter 5). Furthermore, it can be seen that the $\mathrm{IC}_{50}$ values for free curcumin for Panc- 1 cells slightly decreased in time (from 58 to $44 \mu \mathrm{M}$ ). On the other hand, the $\mathrm{IC}_{50}$ values for micellar formulations in Panc-1 cells were strongly time dependent (from $>100$ to $73.8 \mu \mathrm{M}$ for the large micelles and from $>100$ to $52.7 \mu \mathrm{M}$ for the small micelles during $72 \mathrm{~h}$ of incubation). This again points to a slow release of curcumin (intracellularly or extracellularly) from the micelles.

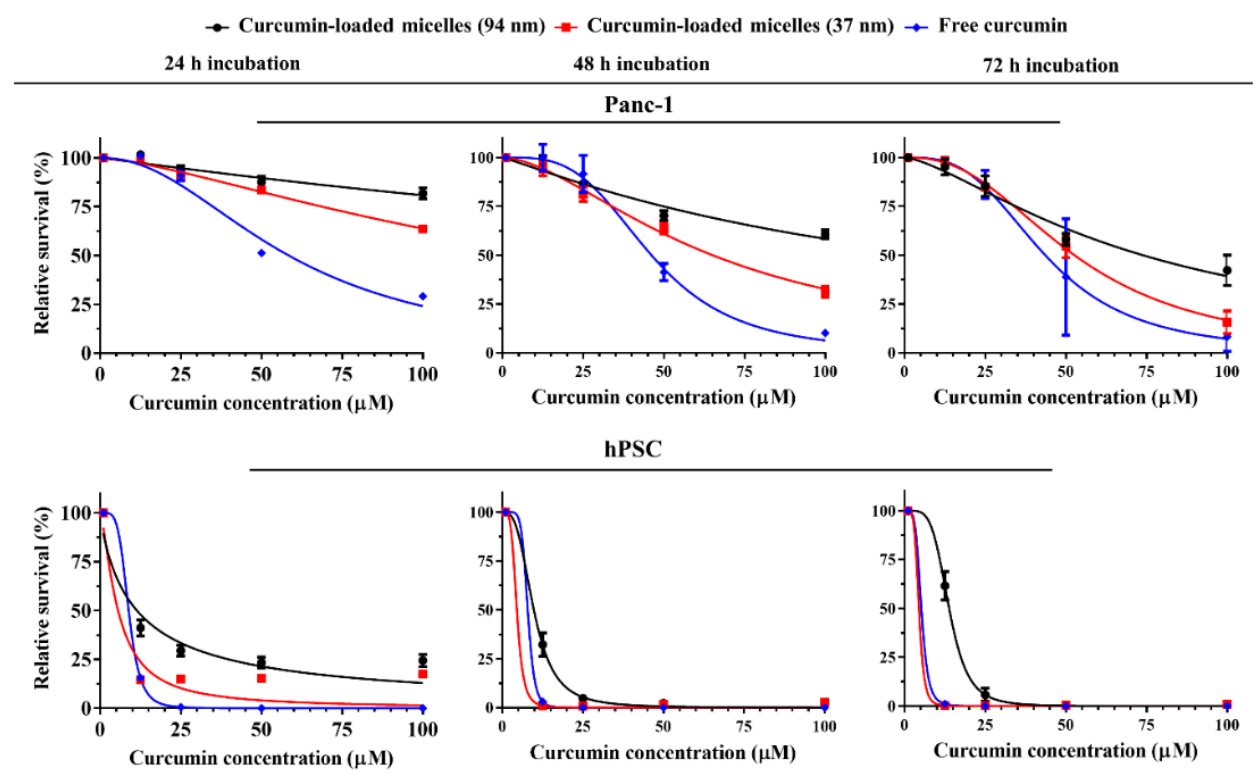

Figure 3. Cytotoxicity of free curcumin as well as small and large curcumin-loaded micelles for 2D Panc-1 and hPSC cells. Cell viability was measured by AlamarBlue test. Data are normalized to the average value of the untreated cells at the respective incubation time. The highest curcumin concentration $(100 \mu \mathrm{M})$ corresponds to a polymer concentration of $800 \mu \mathrm{g} / \mathrm{mL}$. Data were fitted using a nonlinear regression model based on an inhibitor vs. normalized response with variable slope. Data are presented as mean $\pm \operatorname{SD}(n=3$ per time interval).

Table 1. IC 50 values $(\mu \mathrm{M})$ of free curcumin, large curcumin-loaded micelles $(94 \mathrm{~nm})$ and small curcumin-loaded micelles ( $37 \mathrm{~nm}$ ) in Panc-1 and hPSC cells at different curcumin exposure times.

\begin{tabular}{|c|c|c|c|c|c|c|}
\hline \multirow[t]{2}{*}{ Formulations } & \multicolumn{3}{|c|}{ Panc-1 } & \multicolumn{3}{|c|}{ hPSC } \\
\hline & $24 \mathrm{~h}$ & $48 \mathrm{~h}$ & $72 \mathrm{~h}$ & $24 \mathrm{~h}$ & $48 \mathrm{~h}$ & $72 \mathrm{~h}$ \\
\hline $\begin{array}{l}\text { Curcumin-loaded micelle } \\
(94 \mathrm{~nm})\end{array}$ & $>100$ & $>100$ & $73.8 \pm 4.8$ & $11.4 \pm 1.9$ & $9.8 \pm 0.5$ & $13.8 \pm 0.2$ \\
\hline $\begin{array}{l}\text { Curcumin-loaded micelles } \\
\qquad(37 \mathrm{~nm})\end{array}$ & $>100$ & $65.1 \pm 2.0$ & $52.7 \pm 1.4$ & $<10$ & $<10$ & $<10$ \\
\hline Free curcumin & $58.5 \pm 2.6$ & $46.2 \pm 1.5$ & $43.6 \pm 3.6$ & $<10$ & $<10$ & $<10$ \\
\hline
\end{tabular}


In order to get mechanistic insights into the cytotoxicity of the micellar curcumin formulations, the uptake of fluorescently-labeled micelles was examined in Panc-1 2D cell culture. Figure 4 demonstrates that both polymeric micelles were taken up by the cells. However, the Cy3 intensity of cells incubated with the small micelles was much lower than that observed upon incubation with the large micelles. This is contrary to the observed cytotoxicity effects, assuming that the cytotoxicity is dependent only on the uptake of curcumin-loaded micelles. This points to lower stability of smaller curcumin-loaded micelles in the biological medium than the larger micelles resulting in more and faster release of curcumin in the medium and/or intracellularly as discussed above. Moreover, it has been stated in the literature that generally speaking the uptake efficiency of nanoparticles is negatively correlated with the size of nanomedicines most likely because of extensive membrane rearrangement that has to occur for the uptake of larger particles in contrast to smaller particles ${ }^{63}$. Although it is not possible to quantitatively report the extent of uptake as a result of high $\mathrm{Cy} 3$ background in the images obtained from cells incubated with large micelles, the higher Cy3 fluorescent signal in those cells compared to the cells incubated with smaller micelles can probably be ascribed to the presence of more Cy3-labeled polymer chains in the larger micelles than in the smaller micelles. To explain, in the present study, both polymeric micelles were prepared with $20 \% \mathrm{w} / \mathrm{w}$ Cy3-labeled polymer to have an equal fluorescence intensity of the obtained dispersions. As reported earlier, micelles based on $\mathrm{mPEG}_{5 \mathrm{kDa}}-b$-p(HPMA-Bz $)_{18.5 \mathrm{kDa}}$ have a higher aggregation number $\left(\mathrm{N}_{\mathrm{agg}}=904\right)$ than micelles based on $\mathrm{mPEG}_{5 \mathrm{kDa}}-b-\mathrm{p}(\mathrm{HPMA}-\mathrm{Bz})_{4.7 \mathrm{kDa}}\left(\mathrm{N}_{\mathrm{agg}}=416\right)^{43}$. Thus the uptake of larger micelles with higher number of fluorescence probes per particle could result in higher intracellular fluorescence signal than the smaller micelles.
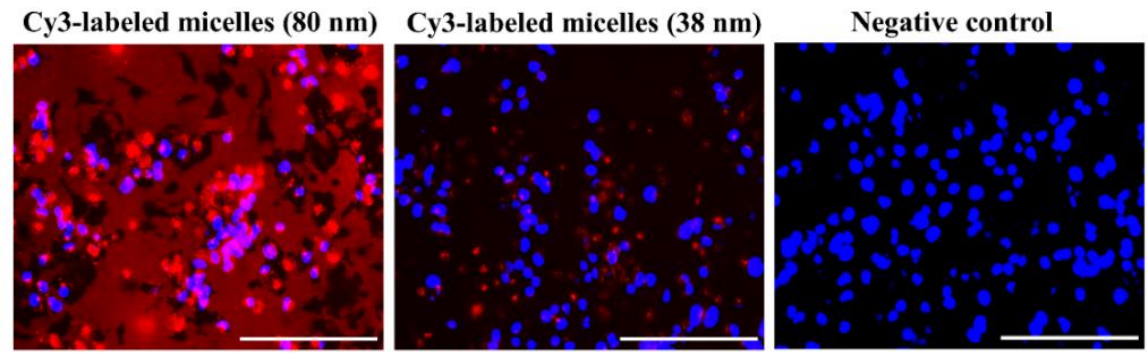

Figure 4. Uptake of Cy3-labeled micelles by Panc-1 cells after $24 \mathrm{~h}$ incubation. Red: Cy3-labeled micelles, blue: stained nuclei with DAPI. (left) $80 \mathrm{~nm}$ micelles, (middle) $38 \mathrm{~nm}$ micelles and (right) negative control. The size of the bar is $200 \mu \mathrm{m}$.

\subsection{Penetration of mPEG- $b$-p(HPMA-Bz) Micelles in 3D Pancreatic Heterospheroids}

The penetration of 38 and $80 \mathrm{~nm}$-sized micelles was investigated in pancreatic heterospheroids consisting of Panc- 1 and hPSC cells, which better mimic the in vivo situation than simple 2D models. The penetration was studied using fluorescence microscopy on cryosections made from the spheroids. The spheroids were incubated at similar micelle concentrations and fluorescence intensity of the dispersions. Figure 5 demonstrates that both small and large micelles penetrated into the heterospheroids after $24 \mathrm{~h}$ of incubation (more than $60 \%$ positive area). However, small micelles showed stronger and deeper penetration into the spheroids compared to the larger micelles. It should be noted that this experiment was done once and repetitions are therefore required to confirm this finding. 

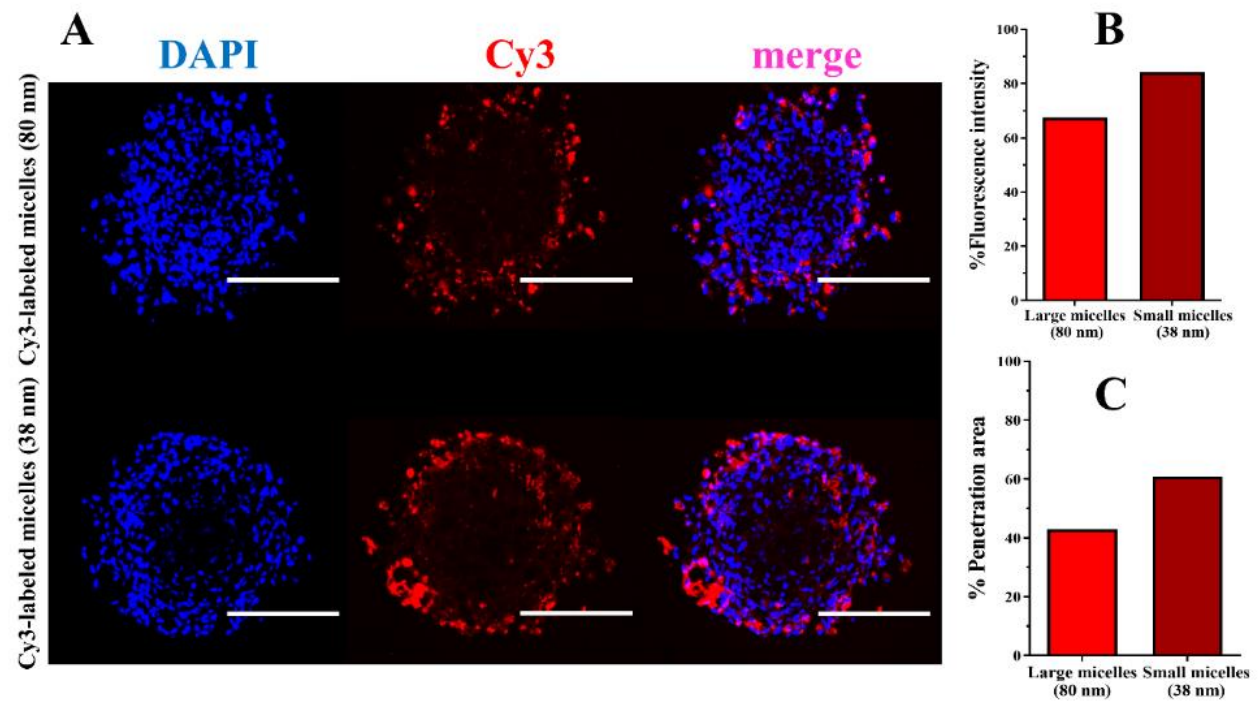

Figure 5. Penetration of Cy3-labeled micelles into Panc-1/hPSC heterospheroids. (A) Fluorescence microscopy images showing the penetration of $38 \mathrm{vs} 80 \mathrm{~nm}$ Cy3-labeled micelles into the spheroids after $24 \mathrm{~h}$ incubation at $37^{\circ} \mathrm{C}$. The size of the bars is $200 \mu \mathrm{m}$. Quantitative analysis of (B) fluorescent intensity of Cy3-labeled micelles and (C) \% penetration area of Cy3-labeled micelles. Images were analyzed using NIH ImageJ software.

\section{Conclusion}

Curcumin-loaded $\mathrm{mPEG}_{5 \mathrm{KDa}}-b$-p(HPMA-Bz) micelles with two different sizes were prepared. The smaller micelles demonstrated higher cytotoxicity in $2 \mathrm{D}$ cell culture than the larger micelles, which is probably attributed to their lower stability in biological environments. In 2D cell culture, both micellar formulations are taken up by pancreatic cancer cells. However, extra experiments (e.g. kinetics of uptake by flow cytometry at shorter incubation times) are required to explain the difference in cell uptake between the two formulations. In 3D heterospheroids, smaller micelles seem to show deeper penetration, which could potentially result in higher cytotoxicity of these drug-loaded micelles. Further experiments are certainly required to support this finding. Also, in future, the cytotoxicity profile of the curcumin-loaded micelles should be tested in 3D heterospheroids, confirming this hypothesis.

\section{Acknowledgement}

The authors are grateful of the funding provided by the European Union's Horizon 2020 research and innovation program Marie Sklodowska-Curie Innovative Training Networks (ITN) under grant No. 676137. 


\section{References}

1. Bobo, D.; Robinson, K. J.; Islam, J.; Thurecht, K. J.; Corrie, S. R., Nanoparticle-based medicines: a review of FDA-approved materials and clinical trials to date. Pharm. Res. 2016, 33 (10), 2373-2387.

2. Duncan, R.; Vicent, M. J., Polymer therapeutics-prospects for 21 st century: the end of the beginning. Adv. Drug Delivery Rev. 2013, 65 (1), 60-70.

3. Hare, J. I.; Lammers, T.; Ashford, M. B.; Puri, S.; Storm, G.; Barry, S. T., Challenges and strategies in anti-cancer nanomedicine development: An industry perspective. Adv. Drug Delivery Rev. 2017, $108,25-38$.

4. Kunjachan, S.; Ehling, J.; Storm, G.; Kiessling, F.; Lammers, T., Noninvasive imaging of nanomedicines and nanotheranostics: Principles, progress, and prospects. Chem. Rev. 2015, 115 (19), 10907-10937.

5. van Elk, M.; Murphy, B. P.; Eufrásio-da-Silva, T.; O’Reilly, D. P.; Vermonden, T.; Hennink, W. E.; Duffy, G. P.; Ruiz-Hernández, E., Nanomedicines for advanced cancer treatments: Transitioning towards responsive systems. Int. J. Pharm. 2016, 515 (1), 132-164.

6. Edgar, J. Y. C.; Wang, H., Introduction for design of nanoparticle based drug delivery systems. Curr. Pharm. Des. 2017, 23 (14), 2108-2112.

7. Houdaihed, L.; Evans, J. C.; Allen, C., Overcoming the road blocks: advancement of block copolymer micelles for cancer therapy in the clinic. Mol. Pharmaceutics 2017, 14 (8), 2503-2517.

8. Varela-Moreira, A.; Shi, Y.; Fens, M. H. A. M.; Lammers, T.; Hennink, W. E.; Schiffelers, R. M., Clinical application of polymeric micelles for the treatment of cancer. Mater. Chem. Front. 2017, 1 (8), 1485-1501.

9. Cabral, H.; Miyata, K.; Osada, K.; Kataoka, K., Block copolymer micelles in nanomedicine applications. Chem. Rev. 2018, 118 (14), 6844-6892.

10. Hwang, D.; Ramsey, J. D.; Kabanov, A. V., Polymeric micelles for the delivery of poorly soluble drugs: From nanoformulation to clinical approval. Adv. Drug Delivery Rev. 2020, In Press.

11. Deng, C.; Jiang, Y.; Cheng, R.; Meng, F.; Zhong, Z., Biodegradable polymeric micelles for targeted and controlled anticancer drug delivery: Promises, progress and prospects. Nano Today 2012, 7 (5), 467-480.

12. Mikhail, A. S.; Allen, C., Block copolymer micelles for delivery of cancer therapy: transport at the whole body, tissue and cellular levels. Journal of controlled release : official journal of the Controlled Release Society 2009, 138 (3), 214-223.

13. Cabral, H.; Kataoka, K., Progress of drug-loaded polymeric micelles into clinical studies. Journal of controlled release : official journal of the Controlled Release Society 2014, 190, 465-476.

14. van Nostrum, C. F., Covalently cross-linked amphiphilic block copolymer micelles. Soft Matter 2011, 7 (7), 3246-3259.

15. Yuan, X.; Jiang, M.; Zhao, H.; Wang, M.; Zhao, Y.; Wu, C., Noncovalently connected polymeric micelles in aqueous medium. Langmuir 2001, 17 (20), 6122-6126.

16. Kang, N.; Perron, M.-È.; Prud'homme, R. E.; Zhang, Y.; Gaucher, G.; Leroux, J.-C., Stereocomplex block copolymer micelles: Core-shell nanostructures with enhanced stability. Nano Lett. 2005, 5 (2), 315-319.

17. Shi, Y.; Lammers, T.; Storm, G.; Hennink, W. E., Physico-chemical strategies to enhance stability and drug retention of polymeric micelles for tumor-targeted drug delivery. Macromol. Biosci. 2017, 17 (1), 1600160 . 


\section{Chapter 6}

18. Matsumura, Y.; Maeda, H., A new concept for macromolecular therapeutics in cancer chemotherapy: mechanism of tumoritropic accumulation of proteins and the antitumor agent smancs. Cancer Res. 1986, 46 (12 Pt 1), 6387-6392.

19. Fang, J.; Nakamura, H.; Maeda, H., The EPR effect: Unique features of tumor blood vessels for drug delivery, factors involved, and limitations and augmentation of the effect. Adv. Drug Delivery Rev. 2011, 63 (3), 136-151.

20. Maeda, H.; Wu, J.; Sawa, T.; Matsumura, Y.; Hori, K., Tumor vascular permeability and the EPR effect in macromolecular therapeutics: a review. Journal of controlled release : official journal of the Controlled Release Society 2000, 65 (1), 271-284.

21. Torchilin, V., Tumor delivery of macromolecular drugs based on the EPR effect. Adv. Drug Delivery Rev. 2011, 63 (3), 131-135.

22. Waite, C. L.; Roth, C. M., Nanoscale drug delivery systems for enhanced drug penetration into solid tumors: current progress and opportunities. Crit. Rev. Bioeng. 2012, 40 (1), 21-41.

23. Priwitaningrum, D. L.; Blondé, J.-B. G.; Sridhar, A.; van Baarlen, J.; Hennink, W. E.; Storm, G.; Le Gac, S.; Prakash, J., Tumor stroma-containing 3D spheroid arrays: A tool to study nanoparticle penetration. Journal of controlled release : official journal of the Controlled Release Society 2016, 244 (Part B), 257-268.

24. Wang, J.; Mao, W.; Lock, L. L.; Tang, J.; Sui, M.; Sun, W.; Cui, H.; Xu, D.; Shen, Y., The role of micelle size in tumor accumulation, penetration, and treatment. ACS Nano 2015, 9 (7), 7195-7206.

25. Cabral, H.; Matsumoto, Y.; Mizuno, K.; Chen, Q.; Murakami, M.; Kimura, M.; Terada, Y.; Kano, M. R.; Miyazono, K.; Uesaka, M.; Nishiyama, N.; Kataoka, K., Accumulation of sub-100 nm polymeric micelles in poorly permeable tumours depends on size. Nat. Nanotechnol. 2011, 6 (12), 815-823.

26. Sun, Q.; Ojha, T.; Kiessling, F.; Lammers, T.; Shi, Y., Enhancing tumor penetration of nanomedicines. Biomacromolecules 2017, 18 (5), 1449-1459.

27 Huang, K.; Ma, H.; Liu, J.; Huo, S.; Kumar, A.; Wei, T.; Zhang, X.; Jin, S.; Gan, Y.; Wang, P. C.; He, S.; Zhang, X.; Liang, X.-J., Size-dependent localization and penetration of ultrasmall gold nanoparticles in cancer cells, multicellular spheroids, and tumors in vivo. ACS Nano 2012, 6 (5), 44834493.

28. Dreher, M. R.; Liu, W.; Michelich, C. R.; Dewhirst, M. W.; Yuan, F.; Chilkoti, A., Tumor vascular permeability, accumulation, and penetration of macromolecular drug carriers. J. Natl. Cancer Inst. 2006, 98 (5), 335-344.

29. Sant, S.; Johnston, P. A., The production of 3D tumor spheroids for cancer drug discovery. Drug Discovery Today: Technol. 2017, 23, 27-36.

30. Nath, S.; Devi, G. R., Three-dimensional culture systems in cancer research: Focus on tumor spheroid model. Pharmacol. Ther. 2016, 163, 94-108.

31. Costa, E. C.; Moreira, A. F.; de Melo-Diogo, D.; Gaspar, V. M.; Carvalho, M. P.; Correia, I. J., 3D tumor spheroids: an overview on the tools and techniques used for their analysis. Biotechnol. Adv. 2016, 34 (8), 1427-1441.

32. Astashkina, A.; Grainger, D. W., Critical analysis of 3-D organoid in vitro cell culture models for high-throughput drug candidate toxicity assessments. Adv. Drug Delivery Rev. 2014, 69-70, 1-18.

33. Lu, H.; Stenzel, M. H., Multicellular tumor spheroids (MCTS) as a 3D in vitro evaluation tool of nanoparticles. Small 2018, 14 (13), 1702858.

34. Nilendu, P.; Sarode, S. C.; Jahagirdar, D.; Tandon, I.; Patil, S.; Sarode, G. S.; Pal, J. K.; Sharma, N. K., Mutual concessions and compromises between stromal cells and cancer cells: driving tumor development and drug resistance. Cell. Oncol. 2018, 41 (4), 353-367. 
35. Zhang, J.; Liu, J., Tumor stroma as targets for cancer therapy. Pharmacol. Ther. 2013, 137 (2), 200215.

36. Xouri, G.; Christian, S., Origin and function of tumor stroma fibroblasts. Semin. Cell Dev. Biol. 2010, 21 (1), 40-46.

37. Bremnes, R. M.; Dønnem, T.; Al-Saad, S.; Al-Shibli, K.; Andersen, S.; Sirera, R.; Camps, C.; Marinez, I.; Busund, L.-T., The role of tumor stroma in cancer progression and prognosis: Emphasis on carcinoma-associated fibroblasts and non-small cell lung cancer. J. Thorac. Oncol. 2011, 6 (1), 209217.

38. Öhlund, D.; Elyada, E.; Tuveson, D., Fibroblast heterogeneity in the cancer wound. J. Exp. Med. 2014, 211 (8), 1503-1523.

39. Prakash, J., Cancer-associated sibroblasts: Perspectives in cancer therapy. Trends Cancer 2016, 2 (6), 277-279.

40. Shi, Y.; van der Meel, R.; Theek, B.; Oude Blenke, E.; Pieters, E. H. E.; Fens, M. H. A. M.; Ehling, J.; Schiffelers, R. M.; Storm, G.; van Nostrum, C. F.; Lammers, T.; Hennink, W. E., Complete regression of xenograft tumors upon targeted delivery of paclitaxel via $\Pi-\Pi$ stacking stabilized polymeric micelles. ACS Nano 2015, 9 (4), 3740-3752.

41. Naksuriya, O.; Shi, Y.; van Nostrum, C. F.; Anuchapreeda, S.; Hennink, W. E.; Okonogi, S., HPMA-based polymeric micelles for curcumin solubilization and inhibition of cancer cell growth. Eur. J. Pharm. Biopharm. 2015, 94, 501-512.

42. Liang, C.; Bai, X.; Qi, C.; Sun, Q.; Han, X.; Lan, T.; Zhang, H.; Zheng, X.; Liang, R.; Jiao, J.; Zheng, Z.; Fang, J.; Lei, P.; Wang, Y.; Möckel, D.; Metselaar, J. M.; Storm, G.; Hennink, W. E.; Kiessling, F.; Wei, H.; Lammers, T.; Shi, Y.; Wei, B., П electron-stabilized polymeric micelles potentiate docetaxel therapy in advanced-stage gastrointestinal cancer. Biomaterials 2021, 266, 120432.

43. Bagheri, M.; Bresseleers, J.; Varela-Moreira, A.; Sandre, O.; Meeuwissen, S. A.; Schiffelers, R. M.; Metselaar, J. M.; van Nostrum, C. F.; van Hest, J. C. M.; Hennink, W. E., Effect of formulation and processing parameters on the size of mPEG-b-p(HPMA-Bz) polymeric micelles. Langmuir 2018, 34 (50), 15495-15506.

44. Allegra, A.; Innao, V.; Russo, S.; Gerace, D.; Alonci, A.; Musolino, C., Anticancer activity of curcumin and its analogues: Preclinical and clinical studies. Cancer Invest. 2017, 35 (1), 1-22.

45. Shehzad, A.; Wahid, F.; Lee, Y. S., Curcumin in Cancer Chemoprevention: Molecular Targets, Pharmacokinetics, Bioavailability, and Clinical Trials. Arch. Pharm. 2010, 343 (9), 489-499.

46. Devassy, J. G.; Nwachukwu, I. D.; Jones, P. J. H., Curcumin and cancer: barriers to obtaining a health claim. Nutr. Rev. 2015, 73 (3), 155-165.

47. Heger, M.; van Golen, R. F.; Broekgaarden, M.; Michel, M. C., The molecular basis for the pharmacokinetics and pharmacodynamics of curcumin and its metabolites in relation to cancer. Pharmacol. Rev. 2014, 66 (1), 222-307.

48. Naksuriya, O.; Okonogi, S.; Schiffelers, R. M.; Hennink, W. E., Curcumin nanoformulations: A review of pharmaceutical properties and preclinical studies and clinical data related to cancer treatment. Biomaterials 2014, 35 (10), 3365-3383.

49. Naksuriya, O.; van Steenbergen, M. J.; Torano, J. S.; Okonogi, S.; Hennink, W. E., A kinetic degradation study of curcumin in its free form and loaded in polymeric micelles. AAPS J. 2016, 18 (3), 777-787.

50. Schnittert, J.; Heinrich, M. A.; Kuninty, P. R.; Storm, G.; Prakash, J., Reprogramming tumor stroma using an endogenous lipid lipoxin A4 to treat pancreatic cancer. Cancer Lett. 2018, 420, 247-258. 
51. Bresseleers, J.; Bagheri, M.; Storm, G.; Metselaar, J. M.; Hennink, W. E.; Meeuwissen, S. A.; van Hest, J. C. M., cale-up of the manufacturing process to produce docetaxel-loaded mPEG-b-p(HPMA$\mathrm{Bz}$ ) block copolymer micelles for pharmaceutical applications. Org. Process Res. Dev. 2019, 23 (12), 2707-2715.

52. Sheybanifard, M.; Beztsinna, N.; Bagheri, M.; Miriam Buhl, E.; Bresseleers, J.; Varela-Moreira, A.; Shi, Y.; van Nostrum, C. F.; van der Pluijm, G.; Storm, G.; Hennink, W. E.; Lammers, T.; Metselaar, J. M., Systematic evaluation of design features enables efficient selection of $\Pi$ electron-stabilized polymeric micelles. Int. J. Pharm. 2020, 119409.

53. Bresseleers, J.; Bagheri, M.; Lebleu, C.; Lecommandoux, S.; Sandre, O.; Pijpers, I. A. B.; Mason, A. F.; Meeuwissen, S.; Nostrum, C. F. V.; Hennink, W. E.; Hest, J., Tuning size and morphology of mPEG-b-p(HPMA-Bz) copolymer self-assemblies using microfluidics. Polymers 2020, 12 (11), 2572.

54. Dadgar, N.; Gonzalez-Suarez, A. M.; Fattahi, P.; Hou, X.; Weroha, J. S.; Gaspar-Maia, A.; Stybayeva, G.; Revzin, A., A microfluidic platform for cultivating ovarian cancer spheroids and testing their responses to chemotherapies. Microsyst. Nanoeng. 2020, 6 (1), 93.

55. Moreira, A. V. $\pi-\pi$ stacked polymeric micelles as carriers of anticancer therapeutics for hematological malignancies. Ph.D. thesis, University Medical Center Utrecht, The Netherlands, 2020.

56. Varela-Moreira, A.; van Straten, D.; van Leur, H. F.; Ruiter, R. W. J.; Deshantri, A. K.; Hennink, W. E.; Fens, M. H. A. M.; Groen, R. W. J.; Schiffelers, R. M., Polymeric micelles loaded with carfilzomib increase tolerability in a humanized bone marrow-like scaffold mouse model. Int. J. Pharm. 2020, 100049.

57. Wong, R. S. Y., Apoptosis in cancer: from pathogenesis to treatment. J. Exp. Clin. Cancer Res. 2011, 30 (1), 87-87.

58. Zhao, J.; Wu, C.; Abbruzzese, J.; Hwang, R. F.; Li, C., Cyclopamine-loaded core-cross-linked polymeric micelles enhance radiation response in pancreatic cancer and pancreatic stellate cells. Mol. Pharmaceutics 2015, 12 (6), 2093-2100.

59. Lee, K. W.; Bode, A. M.; Dong, Z., Molecular targets of phytochemicals for cancer prevention. Nat. Rev. Cancer 2011, 11 (3), 211-218.

60. Fujisawa, S.; Atsumi, T.; Ishihara, M.; Kadoma, Y., Cytotoxicity, ROS-generation activity and radical-scavenging activity of curcumin and related compounds. Anticancer Res. 2004, 24 (2B), 563570.

61. Bennion, B. J.; Be, N. A.; McNerney, M. W.; Lao, V.; Carlson, E. M.; Valdez, C. A.; Malfatti, M. A.; Enright, H. A.; Nguyen, T. H.; Lightstone, F. C.; Carpenter, T. S., Predicting a drug's membrane permeability: A computational model validated with in vitro permeability assay data. J. Phys. Chem. B 2017, 121 (20), 5228-5237.

62. Pandit, N. K.; Soltis, R. P., Introduction to the Pharmaceutical Sciences: An Integrated Approach. Lippincott Williams \& Wilkins: 2011.

63 Francia, V.; Montizaan, D.; Salvati, A., Interactions at the cell membrane and pathways of internalization of nano-sized materials for nanomedicine. Beilstein J Nanotechnol 2020, 11, 338-353. 


\section{Supporting Information}

Table S1. Characteristics of mPEG 5 kDa- $b$-p $(\mathrm{HPMA}-\mathrm{Bz})$ block copolymers as determined by ${ }^{1} \mathrm{H}$ NMR and $\mathrm{GPC}^{1-2}$.

\begin{tabular}{cccccc}
\hline Polymer & $\begin{array}{c}\text { MI:M } \\
\mathbf{M o l} / \mathbf{m o l}\end{array}$ & $\begin{array}{c}\mathbf{M}_{\mathbf{n}} \\
{ }^{1} \mathbf{H}-\mathbf{N M R} \\
(\mathbf{k D a})\end{array}$ & $\begin{array}{c}\mathbf{M}_{\mathbf{n}} \\
\mathbf{G P C} \\
(\mathbf{k D a})\end{array}$ & $\begin{array}{c}\mathbf{M}_{\mathbf{w}} \\
\mathbf{G P D a}\end{array}$ & $\begin{array}{c}\text { Polydispersity index } \\
\left(\mathbf{M} \mathbf{M}_{\mathbf{w}} / \mathbf{M}_{\mathbf{n}}\right) \\
(\mathbf{G P C})\end{array}$ \\
\hline $\mathrm{mPEG}_{5 \mathrm{KDa}}-b-\mathrm{p}(\mathrm{HPMA}-\mathrm{Bz})_{17.1 \mathrm{KDa}}$ & $1: 200$ & 22.1 & 15.8 & 20.7 & 1.31 \\
\hline $\mathrm{mPEG}_{5 \mathrm{KDa}}-b-\mathrm{p}(\mathrm{HPMA}-\mathrm{Bz})_{5.2 \mathrm{KDa}}$ & $1: 50$ & 10.2 & 10.8 & 14 & 1.30 \\
\hline
\end{tabular}

Abbreviations: MI:M, macro-initiator:monomer; $\mathrm{M}_{\mathrm{n}}$, Number Average Molecular Weight; $\mathrm{M}_{\mathrm{w}}$, Weight Average Molecular Weight.

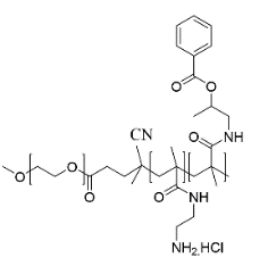

mPEG- $b$-p(HPMA-Bz $z_{98 \%}-$ co-AEMA $2 \%$ )

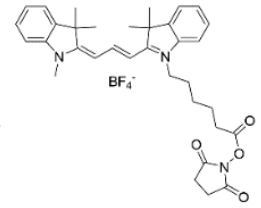

Cy3-NHS

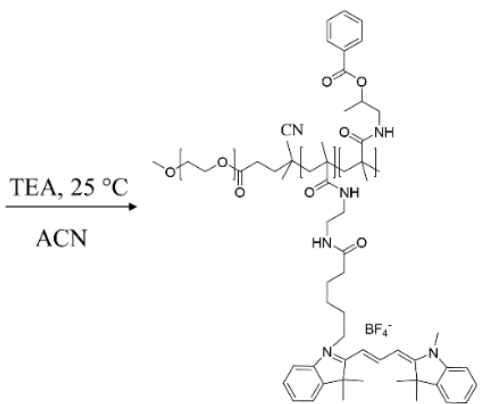

Cy3-labeled mPEG- $b$-p(HPMA-Bz)

Scheme S1. Synthesis of cy3-labeled mPEG- $b$-p(HPMA-Bz).

Figure S1 shows the GPC chromatograms of the Cy3-labeled polymer. The polymer eluted between 10 to $15 \mathrm{~min}$ (RI detection), which coincided with the elution time at $555 \mathrm{~nm}$ (Cy3). On the other hand, free $\mathrm{Cy} 3$ eluted at 18 min (UV detection). The results confirmed Cy3 conjugation to the polymer. The lack of UV signal at $18 \mathrm{~min}$ demonstrated that the concentration of free dye after dialysis was below the detection limit. 


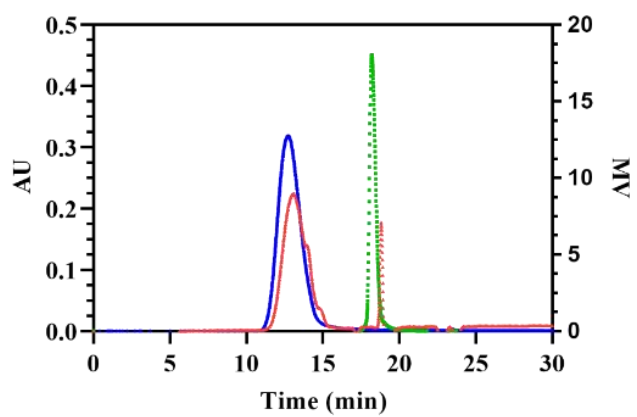

Figure S1. GPC chromatograms of free Cy3 and Cy3-labeled mPEG- $b$-p(HPMA-Bz) polymer after dialysis. Green and blue lines are absorbances at $555 \mathrm{~nm}$ for free $\mathrm{Cy} 3$ and Cy3-labeled polymer, respectively. The red line is the refractive index (RI) signal of Cy3-labeled polymer under the same chromatographic conditions. Abbreviations: AU, absorbance unit; MV, voltage offset in millivolts.
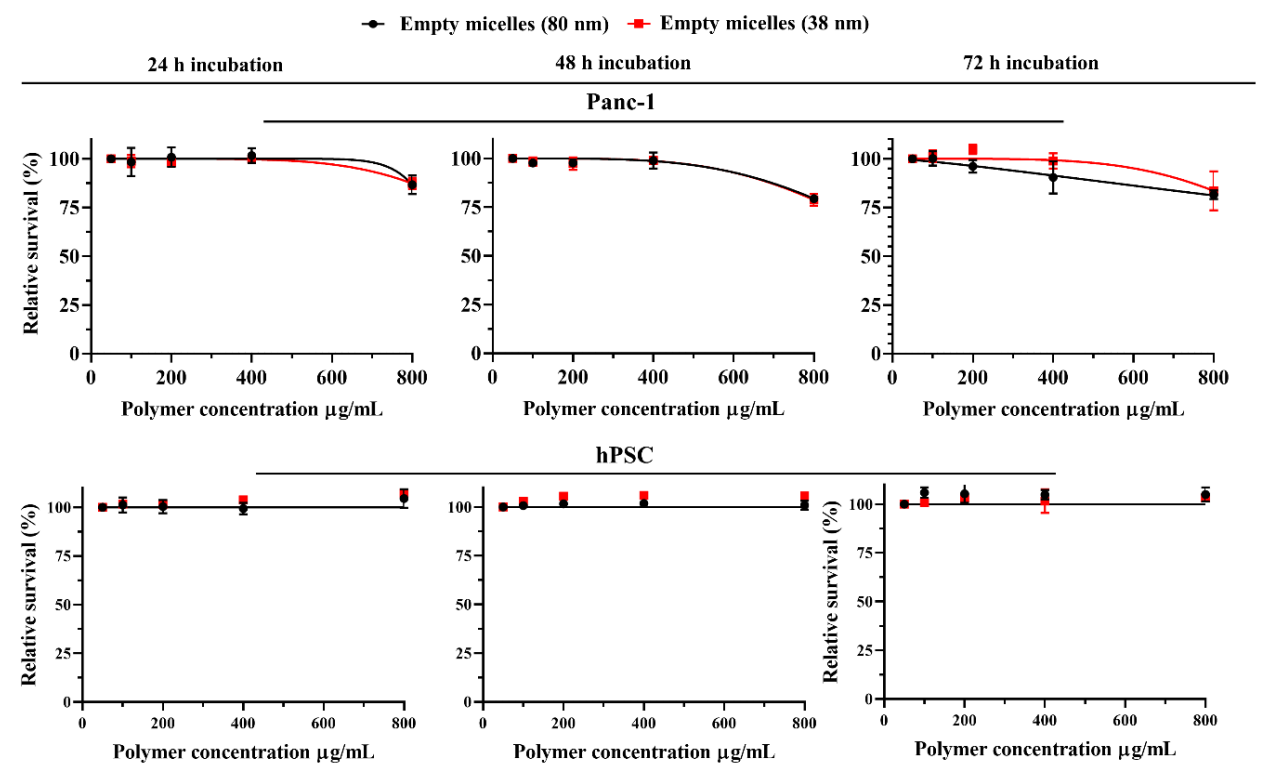

Figure S2. Cytotoxicity of 38 and 80 nm-sized empty micelles on 2D Panc-1 and hPSC cells. Cell viability was measured by AlamarBlue test. Data were normalized to the average value of the untreated cells at the respective incubation time. Data were fitted using a nonlinear regression model based on an inhibitor vs. normalized response with variable slope. Data are presented as mean $\pm \operatorname{SD}(n=3$ per time interval). 


\section{References}

1. Sheybanifard, M.; Beztsinna, N.; Bagheri, M.; Miriam Buhl, E.; Bresseleers, J.; Varela-Moreira, A.; Shi, Y.; van Nostrum, C. F.; van der Pluijm, G.; Storm, G.; Hennink, W. E.; Lammers, T.; Metselaar, J. M., Systematic evaluation of design features enables efficient selection of $\Pi$ electron-stabilized polymeric micelles. Int. J. Pharm. 2020, 119409.

2. Bresseleers, J.; Bagheri, M.; Lebleu, C.; Lecommandoux, S.; Sandre, O.; Pijpers, I. A. B.; Mason, A. F.; Meeuwissen, S.; Nostrum, C. F. V.; Hennink, W. E.; Hest, J., Tuning size and morphology of mPEGb-p(HPMA-Bz) copolymer self-assemblies using microfluidics. Polymers 2020, 12 (11), 2572. 



\section{Chapter 7}

\section{Summary and Discussion}




\section{Summary}

In the past decades, polymeric micelles loaded with chemotherapeutic drugs have been extensively studied for cancer therapy. Polymeric micelles are nano-sized self-assembled core-shell structures with a diameter ranging from $\sim 10-100 \mathrm{~nm}$. Generally, the hydrophobic core of the micelles is suitable for accommodating hydrophobic drugs, while the hydrophilic shell provides colloidal stability and stealth properties ${ }^{1-7}$. These structures should be sufficiently stable and show drug retention in the blood circulation to be clinically interesting for tumor targeting. Various physicochemical strategies have been exploited to develop stable micelles, including the introduction of physical and chemical interactions in the hydrophobic core of the micelles ${ }^{8-12}$. Particularly physical interaction exploiting $\pi-\pi$ stacking, is a promising approach to improve the stability of drug-loaded self-assembled micelles ${ }^{11,13}$. Polymeric micelles with sufficient stability and drug retention in the circulation, can potentially increase the systemic exposure of many anticancer drugs. However, It is also important to note that nanomedicines acting only as solubilizers (e.g. Genexol and Abraxane) have valuable therapeutic benefits in clinics, despite the hardly improved pharmacokinetics ${ }^{6}$, 14-16.

The research described in the current thesis is focused on optimization and comprehensive characterization of polymeric micelles based on poly(ethylene glycol)- $b$-poly(N-2benzoyloxypropyl methacrylamide) (mPEG- $b$-p(HPMA-Bz)). The micelles based on this block copolymer offer excellent particle stability and drug retention in the blood circulation due to the $\pi-\pi$ stacking interactions in the hydrophobic core ${ }^{17-19}$. The project was conducted in the work frame of Nanomed consortium, in close collaboration with Dr. Bresseleers who recently published and successfully defended her thesis about the development of scalable processes for the manufacture of nanocarriers ${ }^{20}$.

Chapter 1 provides a short introduction regarding nanoparticle based drug delivery systems, particularly polymeric micelles for cancer treatment. The nanoprecipitation technique in batch process and microfluidics used to prepare polymeric micelles is briefly explained. The pharmacokinetics and pharmacodynamics of drug delivery systems are shortly discussed. Also, curcumin as a natural compound with anti-cancer activities is briefly introduced. Finally, the importance of 3D tumor spheroids as cell culture platforms that better mimic the tumor microenvironment (TME) than classical 2D cell culture models is discussed.

Curcumin is a natural polyphenolic component with a wide range of pharmacological properties, such as potential therapeutic activities against cancer ${ }^{21}$. However, the extremely low aqueous solubility, low bioavailability after oral administration and high instability of the molecule adversely affect its pharmaceutical properties and consequently hampers its applications in cancer therapy ${ }^{21-25}$. Chapter 2 provides a literature overview regarding the pharmacokinetics (PK) of intravenously administered curcumin-loaded nano-sized delivery systems in two preclinical animal models (mice and rats). Interestingly, the majority of the nanocarriers only marginally improves the area under the curve with a factor 1.3-5 compared to free curcumin. This means that curcumin is not well retained in the particles and the nanomedicines essentially act as solubilizer. Also, studies that report both PK and in vivo antitumor data show higher tumor growth inhibition in groups treated with curcumin nanoformulations than free curcumin, in line with the slightly improved pharmacokinetics. Further analysis indicates only a negative correlation between the relative tumor growth inhibition in treatment groups receiving curcumin nanoformulations and free curcumin vs the 
total injected dose. It is possible that the amount of curcumin in mice after receiving higher dose regimen of free curcumin could exert tumor growth inhibition despite the unfavorable PK. Also, evidently even nanoformulations that act as a solubilizer exert considerable pharmacodynamic effect and reduce the tumor volume compared to the controls. However, the influence of relatively low number of available PK-PD data, the wide variation in the characteristics of nanoformulations such as release profile and some experimental design weaknesses should be considered. Also, the effect of rapid distribution of curcumin into blood cells shortly after injection should be considered in PK studies. Therefore, more wellcontrolled studies are required to reach more conclusive outcomes in the future.

In Chapter 3, parameters affecting the mPEG- $b$-p(HPMA-Bz) polymeric micelles were studied to tune the size of micelles in the range of 25-100 nm. Polymers with varying molecular weights of the hydrophilic ( $\mathrm{mPEG}_{5 \mathrm{kDa}}$ or $\mathrm{mPEG}_{2 \mathrm{kDa}}$ ) and hydrophobic blocks (1.0 to $20.6 \mathrm{kDa}$ ) were synthesized and used in this chapter. A nanoprecipitation batch process was used to prepare micelles with different sizes by varying formulation parameters such as block copolymer molecular weight, hydrophilic-to-hydrophobic ratio, and homopolymer content, as well as processing parameters (e.g. rate of addition and type of solvent). Dynamic light scattering and transmission electron microscopy confirmed the core-shell structure of the micelles. The hydrophobic core of the micelles was in a collapsed state and followed the de Gennes-Alexander theory and scaling law. Also, similar brush height for all the micelles $(10 \mathrm{~nm})$ indicated formation of crew-cut micelles. This size control was dependent on both the molecular weight of the polymers and the processing methods. In short, the micelle size was reduced by tailoring the polymerization step and optimizing the polymer molecular weight using higher hydrophilic-to-hydrophobic ratios. The synthesis of the block copolymer was associated with the formation of homopolymer which partitions into the core of the micelles yielding larger hydrophobic cores. Therefore, it was shown that minimizing the homopolymer content by optimization the synthesis of the macro-initiator resulted in smaller micelles. In terms of processing conditions, using organic solvents with faster mixing rates with the aqueous phase (e.g. acetone, acetonitrile, ethanol, tetrahydrofuran and dioxane), applying faster rates of addition and higher polymer concentration resulted in smaller micelles with a narrow size distribution than solvents with slower mixing rates with water (dimethylformamide and dimethyl sulfoxide). This chapter therefore demonstrates that the size of self-assembled mPEG- $b$-p(HPMA-Bz) micelles can be fine-tuned in a range that is suitable for tumor drug delivery and targeting. Moreover, it shows the effect of critical parameters during the micelle formation, which can be used for the reproducible preparation of micelles in the desired size range.

As discussed in Chapter 3, the size of formed mPEG- $b$-p(HPMA-Bz) micelles is dependent on the mixing rate of the organic solvent and aqueous phase, thereby highlighting the importance of robust mixing features during nanoprecipitation. Therefore the aim of Chapter 4 was to tailor the size and morphology of the mPEG- $b$-p(HPMA-Bz) based micelles using a microfluidic technique. Polymers with a fixed hydrophilic block $\left(\mathrm{mPEG}_{5 \mathrm{kDa}}\right)$ and different molecular weights of the hydrophobic block $(17.5,10.0,5.2$ and $2.7 \mathrm{kDa}$ ) were used in this chapter. Nanostructures were subsequently obtained by nanoprecipitation under well-defined mixing conditions using microfluidics at different concentrations. An increase in particle size of the micelles from 55 to $90 \mathrm{~nm}$ and even formation of vesicles were observed upon using the polymer with the largest hydrophobic block $(17.5 \mathrm{kDa})$, at lower polymer concentration and relatively lower flow rates. In the case of the copolymer with the smallest hydrophobic block $(2.7 \mathrm{kDa})$, regardless of the polymer concentration, the nanoparticle size increased 
from 35 to $70 \mathrm{~nm}$ by decreasing the flow rates. Also polymersomes were formed at slow flow rates using this copolymer. The polymers with hydrophobic blocks of 10 and $5.2 \mathrm{kDa}$ mainly self-assembled into micelles at different applied flow rates and showed negligible size differences in the range of polymer concentrations tested $(5-20 \mathrm{mg} / \mathrm{mL})$. In line with Chapter $\mathbf{3}$ in which a batch process was used, this chapter demonstrates that the self-assembly of mPEG- $b$-p(HPMA-Bz) block copolymers can be easily tailored in terms of size and morphology using microfluidics.

In Chapter 5, curcumin-loaded $\mathrm{mPEG}_{5 \mathrm{kDa}}-b-\mathrm{p}(\mathrm{HPMA}-\mathrm{Bz})$ micelles were prepared to improve the pharmacokinetics of curcumin. Similar mPEG- $b$-p(HPMA-Bz) copolymers as the ones of Chapter 4 with fixed hydrophilic $\left(\mathrm{mPEG}_{5 \mathrm{kDa}}\right)$ and hydrophobic blocks of 5.2, 10.0, and $17.1 \mathrm{kDa}$ were used. At $9 \%$ curcumin loading, micelles with the highest molecular weight of $\mathrm{p}$ (HPMA-Bz) demonstrated better curcumin retention in phosphate-buffered saline (PBS) at $37{ }^{\circ} \mathrm{C}$ than micelles based on block copolymers with smaller hydrophobic blocks. Evaluation of the stability of curcumin-loaded micelles in plasma with asymmetrical flow field-flow fractionation $\left(\mathrm{AF}_{4}\right)$ demonstrated no change in micelle size during $24 \mathrm{~h}$, confirming the particle stability. On the other hand, in line with the stability study in PBS, the release of curcumin in $24 \mathrm{~h}$ was higher from the micelles prepared with the lowest molecular weight $\mathrm{p}(\mathrm{HPMA}-\mathrm{Bz})$. Also, $\mathrm{AF}_{4}$ analysis demonstrated that albumin played an important role in solubilizing the released curcumin. These results indicated that micelles prepared from the larger molecular weight of the hydrophobic block have better curcumin retention in the micelle core. In vitro cell studies revealed that the curcumin-loaded $\mathrm{mPEG}_{5 \mathrm{kDa}}-b$-p(HPMA-Bz $)_{17.1 \mathrm{kDa}}$ micelles were internalized by different cancer cells which in turn resulted in cell cytotoxicity. In vivo, curcumin-loaded $\mathrm{mPEG}_{5 \mathrm{kDa}}-b$-p (HPMA$\mathrm{Bz})_{17.1 \mathrm{kDa}}$ micelles were administered intravenously in mice $(50 \mathrm{mg} / \mathrm{kg}$ of curcumin) and micelles showed prolonged circulation kinetics with more than $50 \%$ of the injected dose in the circulation after $24 \mathrm{~h}$. On the contrary, curcumin half-life was considerably shorter than that of the micelles $\left(\mathrm{t}_{1 / 2 \alpha}=0.11, \mathrm{t}_{1 / 2 \beta}=2.5 \mathrm{~h}\right)$. However, the half-life was substantially improved compared to free curcumin $\left(\mathrm{t}_{1 / 2 \alpha}=0.023 \mathrm{~h}\right)^{26-28}$. Despite the prolonged pharmacokinetics of curcumin by encapsulation in these micelles and comparable pharmacokinetic profile to other curcumin nanoformulations reported in the literature, no therapeutic efficacy was observed in neuroblastoma-bearing mice. The lack of cytotoxic effects in vivo might be attributed to low sensitivity of the Neuro2A cells to curcumin.

In Chapter 6, $\mathrm{mPEG}_{5 \mathrm{kDa}}-b$-p(HPMA-Bz) micelles with two distinct size ranges were prepared and loaded with curcumin to study their performance in $2 \mathrm{D}$ and $3 \mathrm{D}$ cell culture models. These micellar formulations (small and large) demonstrated similar curcumin retention at $5.0 \%$ loading in PBS (80\% curcumin retention in $168 \mathrm{~h})$. $\mathrm{mPEG}_{5 \mathrm{kDa}}-b$-p (HPMA$\mathrm{Bz}$ ) micelles have excellent cytocompatibility as evaluated with both cancer and non-cancer cells (Chapters 5 and 6). Curcumin-loaded micelles showed a higher 50\% inhibitory concentrations $\left(\mathrm{IC}_{50}\right)$ compared to free curcumin, likely because free curcumin passively permeates through the cell membrane while curcumin was partially retained and slowly released from the micelles, preventing complete pharmacological activity of the curcumin available in the medium. Also, the small micelles showed lower $\mathrm{IC}_{50}$ values compared to the large micelles which is possibly due to the faster release of curcumin from the smaller micelles in biological media based on the stability study by $\mathrm{AF}_{4}$ technique (Chapter 5 ). Cell uptake study in Panc-1 cells showed that both small and large micelles were taken up by the cells and based on a qualitative analysis the internalization was higher for the larger micelles. Notably, small micelles demonstrated more extensive and deeper penetration in 3D stroma- 
containing spheroids than the large micelles. This introductory study demonstrates the potential of small micelles in inducing a stronger therapeutic effect which needs to be validated by complementary experiments.

\section{Discussion and Perspectives}

The polymeric micelles studied in the current thesis are based on poly(ethylene glycol)-blockpoly(N-2-benzoyloxypropyl methacrylamide) (mPEG- $b$-p(HPMA-Bz)) block copolymers which were initially synthesized and reported by Shi et al. ${ }^{29}$. In later studies, these micelles were evaluated as carriers of hydrophobic drugs for the treatment of hematological malignancies ${ }^{30}$. Also, the preparation method was further optimized to develop robust and scalable manufacturing processes ${ }^{20}$. These micelles showed excellent particle stability with improved drug retention and circulation kinetics as a result of hydrophobic and $\pi-\pi$ stacking interactions ${ }^{17}$, 19 . In the present study, mPEG- $b$-p(HPMA-Bz) polymers with varying molecular weights of the hydrophilic and hydrophobic blocks were synthesized and used to optimize the polymeric micelle formulations obtained via nanoprecipitation methods. Also, the in vitro and in vivo performance of these micelles loaded with curcumin was studied in 3D spheroid cell culture model (an introductory study) and tumor-bearing mice, respectively.

\subsection{Improving the Preclinical Outcome of Curcumin-Loaded Micelles}

In Chapter 5, curcumin-loaded mPEG- $b$-p(HPMA-Bz) micelles were prepared to solubilize and improve the circulation kinetics of curcumin. The half-life of curcumin loaded in micelles $\left(\mathrm{t}_{1 / 2 \alpha}=0.11, \mathrm{t}_{1 / 2 \beta}=2.5 \mathrm{~h}\right)$ was $\sim 5$ times longer than half-life of free curcumin $\left(\mathrm{t}_{1 / 2 \alpha}=0.023 \mathrm{~h}\right)$ reported in the literature. These results demonstrate that mPEG- $b$-p(HPMA-Bz) micelles only slightly improved the pharmacokinetics of curcumin and thus essentially act as a solubilizer. Further, no therapeutic effect was observed in neuroblastoma-bearing mice, possibly due to low sensitivity of the Neuro2A cells to curcumin. One possible approach to achieve therapeutic efficacy in this tumor model is increasing the dose of curcumin and/or using combination therapy with other chemotherapeutic agents. The dose of curcumin can be increased by increasing the loading content of the micelles in combination with increasing the concentration of the dispersed micelles in the injection fluid. Care should of course be taken that this does not result in instability issue such as substantial leakage and aggregation of the curcumin loaded micelles. Curcumin has already been used in combination with several chemotherapeutic drugs (e.g., paclitaxel, platinum compounds) or in combination with photodynamic therapy and immunotherapy for the treatment of cancer $^{31-34}$. Further research is therefore required to select the optimum drug combination and achieve the best synergistic effects in preclinical tumor models for neuroblastoma and other cancer types. Also, curcumin retention in the micelles could be improved possibly either by lowering the feed ratio of curcumin to polymer or tuning the composition of polymer. The latter could be achieved by combining chemical conjugation and physical entrapment of curcumin in the hydrophobic core of the micelles similar to the approach used by Okano et al. ${ }^{35}$ for doxorubicin or optimizing even the hydrophilic block for better solubilization of hydrophobic molecules beyond the micelle core ${ }^{36-37}$. Currently without any optimization, the micelles developed in this thesis can potentially be used to target cancers of the lymphatic and immune system which are more sensitive and accessible than solid tumors ${ }^{21}$. 


\subsection{Development of a Robust Method to Obtain Tailorable Size Nanoparticles}

It has been shown that the size of drug delivery systems plays an essential factor for improved therapeutic efficacy. To mention, smaller nanoparticles $(<100 \mathrm{~nm})$ have prolonged circulation kinetics as well as better tumor accumulation and penetration via the enhanced permeation and retention effect (EPR) ${ }^{38-40}$. In Chapter 3, the size of the self-assembled micelles was tuned by both the formulation and processing parameters. Our study revealed that the self-assembly of these micelles is based on a nucleation-controlled process, where the size of micelles is dependent on the nucleation rate. The results of Chapter $\mathbf{3}$ evidently showed that the size of mPEG- $b$-p(HPMA-Bz) micelles is dependent on the mixing rate of solvent and non-solvent, thereby emphasizing the need for a technological procedure that controls this feature. Therefore, in Chapter 4 robust mixing rates were achieved using a microfluidics device. We showed in this study that by controlling the processing method and the hydrophilic-to-hydrophobic ratio of mPEG- $b$-p(HPMA-Bz) copolymer, both the size and morphology of the particles can be tuned. In general, at high mPEG- $b$-p(HPMA-Bz) polymer concentration and fast mixing rate, micellar structures were formed. On the other hand, decreasing these two parameters changed the size and morphology of the self-assemblies. These findings gave us the opportunity to tailor the size and morphology of the particles. Adjusting the processing parameters even provided the opportunity to direct the selfassembly of the polymers towards formation of polymersome structures. Polymersoms are spherical vesicles comprised of amphiphilic block copolymers enclosing an aqueous lumen that could encapsulate both hydrophilic (solubilized in the aqueous core) and hydrophobic drugs (solubilized in the hydrophobic bilayer) and offer several advantages such as high colloidal stability and tailorable membrane properties. These structures have also shown prolonged blood circulation times which is prerequisite for drug delivery purposes ${ }^{41-43}$. Formation of polymersomes can be achieved by using lower polymer concentrations or flow rates to tune the nucleation rate and also using mPEG- $b$-p(HPMA-Bz) polymers with a small hydrophilic block ( $\mathrm{mPEG}_{2 \mathrm{kDa}}$ ), as reported in Chapter 3.

\subsection{Considerations for Upscale Production}

It is of great importance to set up an efficient, scalable and reproducible process to obtain both block copolymers and drug-loaded self-assemblies in large batches that are required for clinical studies as well as for commercial manufacturing. The findings of Chapter 3 regarding the critical parameters to control the size of micelles, were used to develop a scalable procedure to obtain well-defined micelles. Also, in Chapter 4, it was shown that controlling the mixing rates of the solvent and non-solvent using microfluidics offers excellent potential in tuning the size and morphology of the self-assemblies and such a continuous process is more favorable compared to the batch mode. In line with this objective, Bresseleers et al. ${ }^{44}$ reported a continuous flow-based method to obtain docetaxel-loaded mPEG- $b$-p(HPMA-Bz) polymeric micelles in a reproducible manner. The procedure described by the authors can be easily translated for large batch production under GMP conditions. In future studies, this method can be applied to formulate other drug-loaded micelles in a scalable and reproducible manner for clinical and commercial purposes.

\subsection{Considerations for Setting Up an Efficient In vitro Stability/Release Study}

A thorough evaluation of the stability and release characteristics of nano-sized drug delivery systems is essential to understand the in vitro-in vivo relationships. Ideally, the drug retention in nano-sized delivery systems should be studied in biologically relevant media ${ }^{45-46}$. However, performing such experiments for nano-drug delivery systems loaded with 
hydrophobic drugs is challenging due to recovering and separating issues of nanoparticles from components of the releasing medium ${ }^{47-48}$. Also it is a challenge to obtain so-called 'sink' conditions for studying the release of (very) hydrophobic drugs and drug candidates from polymeric micelles. In this regard, Sheybanidard et al. ${ }^{18}$ systematically evaluated the stability and release profile of paclitaxel-loaded mPEG- $b-\mathrm{p}(\mathrm{HPMA}-\mathrm{Bz})$ micelles under sink conditions in PBS containing $0.2 \%$ Tween 80 or $4.5 \%$ bovine serum albumin as solubilizers of the released drug using a dialysis technique. The authors concluded that the most promising formulations are based on $\mathrm{mPEG}_{5 \mathrm{kDa}}-b-\mathrm{p}(\mathrm{HPMA}-\mathrm{Bz})_{17.1 \mathrm{kDa}}$ and $\mathrm{mPEG}_{5 \mathrm{kDa}}-b-$ $\mathrm{p}(\text { HPMA-Bz) })_{5.2 \mathrm{kDa}}$ with high drug retention and small size (suitable for deeper tumor penetration), respectively. In Chapter 5, $\mathrm{AF}_{4}$ was used instead of a dialysis method to separate the micelles from the (lipo)proteins present in plasma. $\mathrm{AF}_{4}$ is a robust technique that separates nanoparticles based on their hydrodynamic size ${ }^{49}$. Since it lacks a stationary phase, the possibility of interaction with the stationary phase is low, making it a suitable method for analyzing sensitive samples ${ }^{50}$. Therefore, we were able to study the stability of the curcuminloaded mPEG- $b$-p(HPMA-Bz) micelles in quasi-physiological conditions. The results demonstrated that the size and size distribution of the micelles did not change upon $24 \mathrm{~h}$ incubation in plasma while the released curcumin bound to albumin present in plasma. In this study, $\mathrm{AF}_{4}$ showed to be a promising application in analyzing the stability of drug-loaded polymeric micelles in complex biological fluids. In follow-up studies, the stability of the micelles in whole blood could be measured specially for curcumin-loaded micelles since this molecule can interact with and accumulate in blood cells ${ }^{51}$. Also, in vivo samples can be even analyzed by this technique giving a more comprehensive overview on the stability of the particles and their interactions with blood constituents.

\subsection{Performing In vitro Cell Studies in Relevant and Translatable Cell Culture Models}

Despite numerous preclinical studies, the translation of nanomedicine into clinics is still a great issue in drug delivery development for cancer therapy ${ }^{52-55}$. One of the likely reasons is the insufficient alignment of cell culture models with the patient tumor in clinical stages ${ }^{56}$. Most often, 2D-cell cultures are used that are in monolayers lacking the necessary complex cell-cell or cell-matrix interactions present in in vivo tumor microenvironment ${ }^{57}$. In recent years, 3D cell culture models (e.g. spheroids) are rapidly developing as they represent the in vivo situation and could resemble the $\mathrm{TME}^{58-59}$. In chapter 6 of this thesis, we performed an introductory study on the performance of mPEG- $b-\mathrm{p}$ (HPMA-Bz) micelles in heterospeheroids containing both cancer cells and stroma. In line with earlier research ${ }^{60}$, deeper and more extensive penetration was observed in the heterospheroids using small micelles, which demonstrates their potential in offering a stronger therapeutic effect. In future studies, the cytotoxicity profile of the curcumin-loaded micelles should be evaluated in 3D heterospheroids, confirming this hypothesis.

\section{Conclusions}

This thesis is focused on formulation development, thorough characterization and in vitro and in vivo application of mPEG- $b$-p(HPMA-Bz) self-assemblies. Firstly, the effect of formulation and processing parameters was studied in both a batch process and microfluidic setting to tailor the size and even the morphology of the nanoparticles. The research resulted in a robust and scalable procedure to obtain well-defined micelles potentially suitable as nanomedicine. Particularly the continuous flow process using microfluidics is promising 
since it offers excellent control over the size and morphology as well as the opportunity of large continuous production. The suitability of mPEG- $b$-p(HPMA-Bz) micelles as a drug delivery system was explored by encapsulating curcumin as a hydrophobic therapeutic molecule. It turned out that the micelles essentially acted as a solubilizer and only slightly improved the circulation kinetics of curcumin compared to the free compound. However, no therapeutic efficacy was observed in the selected animal model, which might be due to the relatively low sensitivity of the tumor cells. Further research is therefore required to elucidate the potential of these curcumin-loaded micelles in cancer treatment and possibilities for improvement are provided in this chapter. 


\section{References}

1. Deng, C.; Jiang, Y.; Cheng, R.; Meng, F.; Zhong, Z., Biodegradable polymeric micelles for targeted and controlled anticancer drug delivery: Promises, progress and prospects. Nano Today 2012, 7 (5), 467-480.

2. Cabral, H.; Kataoka, K., Progress of drug-loaded polymeric micelles into clinical studies. Journal of controlled release : official journal of the Controlled Release Society 2014, 190, 465-476.

3. Mikhail, A. S.; Allen, C., Block copolymer micelles for delivery of cancer therapy: transport at the whole body, tissue and cellular levels. Journal of controlled release : official journal of the Controlled Release Society 2009, 138 (3), 214-223.

4. $\quad$ Cagel, M.; Tesan, F. C.; Bernabeu, E.; Salgueiro, M. J.; Zubillaga, M. B.; Moretton, M. A.; Chiappetta, D. A., Polymeric mixed micelles as nanomedicines: Achievements and perspectives. Eur. J. Pharm. Biopharm. 2017, 113, 211-228.

5. Houdaihed, L.; Evans, J. C.; Allen, C., Overcoming the road blocks: advancement of block copolymer micelles for cancer therapy in the clinic. Mol. Pharmaceutics 2017, 14 (8), 2503-2517.

6. Varela-Moreira, A.; Shi, Y.; Fens, M. H. A. M.; Lammers, T.; Hennink, W. E.; Schiffelers, R. M., Clinical application of polymeric micelles for the treatment of cancer. Mater. Chem. Front. 2017, $l(8), 1485-1501$.

7. Cabral, H.; Miyata, K.; Osada, K.; Kataoka, K., Block copolymer micelles in nanomedicine applications. Chem. Rev. 2018, 118 (14), 6844-6892.

8. $\quad$ Ahmad, Z.; Shah, A.; Siddiq, M.; Kraatz, H.-B., Polymeric micelles as drug delivery vehicles. RSC Adv. 2014, 4 (33), 17028-17038.

9. Yuan, X.; Jiang, M.; Zhao, H.; Wang, M.; Zhao, Y.; Wu, C., Noncovalently connected polymeric micelles in aqueous medium. Langmuir 2001, 17 (20), 6122-6126.

10. Kang, N.; Perron, M.-È.; Prud'homme, R. E.; Zhang, Y.; Gaucher, G.; Leroux, J.-C., Stereocomplex block copolymer micelles: Core-shell nanostructures with enhanced stability. Nano Lett. 2005, 5 (2), 315-319.

11. Shi, Y.; Lammers, T.; Storm, G.; Hennink, W. E., Physico-chemical strategies to enhance stability and drug retention of polymeric micelles for tumor-targeted drug delivery. Macromol. Biosci. 2017, 17 (1), 1600160.

12. van Nostrum, C. F., Covalently cross-linked amphiphilic block copolymer micelles. Soft Matter 2011, 7 (7), 3246-3259.

13. Zhuang, W.-R.; Wang, Y.; Cui, P.-F.; Xing, L.; Lee, J.; Kim, D.; Jiang, H.-L.; Oh, Y.-K., Applications of $\pi-\pi$ stacking interactions in the design of drug-delivery systems. Journal of controlled release : official journal of the Controlled Release Society 2019, 294, 311-326.

14. Sparreboom, A.; Scripture, C. D.; Trieu, V.; Williams, P. J.; De, T.; Yang, A.; Beals, B.; Figg, W. D.; Hawkins, M.; Desai, N., Comparative preclinical and clinical pharmacokinetics of a cremophorfree, nanoparticle albumin-bound paclitaxel (ABI-007) and paclitaxel formulated in Cremophor (Taxol). Clin. Cancer Res. 2005, 11 (11), 4136-43.

15. Kim, S. C.; Kim, D. W.; Shim, Y. H.; Bang, J. S.; Oh, H. S.; Kim, S. W.; Seo, M. H., In vivo evaluation of polymeric micellar paclitaxel formulation: toxicity and efficacy. Journal of Controlled Release 2001, 72 (1), 191-202.

16. Youn, Y. S.; Bae, Y. H., Perspectives on the past, present, and future of cancer nanomedicine. Adv. Drug Delivery Rev. 2018, 130, 3-11.

17. Shi, Y.; van der Meel, R.; Theek, B.; Oude Blenke, E.; Pieters, E. H. E.; Fens, M. H. A. M.; Ehling, J.; Schiffelers, R. M.; Storm, G.; van Nostrum, C. F.; Lammers, T.; Hennink, W. E., Complete 


\section{Chapter 7}

regression of xenograft tumors upon targeted delivery of paclitaxel via $\Pi-\Pi$ stacking stabilized polymeric micelles. ACS Nano 2015, 9 (4), 3740-3752.

18. Sheybanifard, M.; Beztsinna, N.; Bagheri, M.; Miriam Buhl, E.; Bresseleers, J.; VarelaMoreira, A.; Shi, Y.; van Nostrum, C. F.; van der Pluijm, G.; Storm, G.; Hennink, W. E.; Lammers, T.; Metselaar, J. M., Systematic evaluation of design features enables efficient selection of $\Pi$ electronstabilized polymeric micelles. Int. J. Pharm. 2020, 119409.

19. Liang, C.; Bai, X.; Qi, C.; Sun, Q.; Han, X.; Lan, T.; Zhang, H.; Zheng, X.; Liang, R.; Jiao, J.; Zheng, Z.; Fang, J.; Lei, P.; Wang, Y.; Möckel, D.; Metselaar, J. M.; Storm, G.; Hennink, W. E.; Kiessling, F.; Wei, H.; Lammers, T.; Shi, Y.; Wei, B., П electron-stabilized polymeric micelles potentiate docetaxel therapy in advanced-stage gastrointestinal cancer. Biomaterials 2021, 266, 120432.

20. Bresseleers, J. Development of scalable processes for the manufacture of nanocarriers. Ph.D. thesis, TU Eindhoven, The Netherlands, 2020, ISBN: 978-90-356-5150-7.

21. Heger, M.; van Golen, R. F.; Broekgaarden, M.; Michel, M. C., The molecular basis for the pharmacokinetics and pharmacodynamics of curcumin and its metabolites in relation to cancer. Pharmacol. Rev. 2014, 66 (1), 222-307.

22. Naksuriya, O.; van Steenbergen, M. J.; Torano, J. S.; Okonogi, S.; Hennink, W. E., A kinetic degradation study of curcumin in its free form and loaded in polymeric micelles. AAPS J. 2016, 18 (3), 777-787.

23. Stohs, S. J.; Ji, J.; Bucci, L. R.; Preuss, H. G., A Comparative Pharmacokinetic Assessment of a Novel Highly Bioavailable Curcumin Formulation with 95\% Curcumin: A Randomized, DoubleBlind, Crossover Study. J. Am. Coll. Nutr. 2018, 37 (1), 51-59.

24. Wang, Y.-J.; Pan, M.-H.; Cheng, A.-L.; Lin, L.-I.; Ho, Y.-S.; Hsieh, C.-Y.; Lin, J.-K., Stability of curcumin in buffer solutions and characterization of its degradation products. J. Pharm. Biomed. Anal. 1997, 15 (12), 1867-1876.

25. Naksuriya, O.; Okonogi, S.; Schiffelers, R. M.; Hennink, W. E., Curcumin nanoformulations: A review of pharmaceutical properties and preclinical studies and clinical data related to cancer treatment. Biomaterials 2014, 35 (10), 3365-3383.

26. Song, Z.; Feng, R.; Sun, M.; Guo, C.; Gao, Y.; Li, L.; Zhai, G., Curcumin-loaded PLGAPEG-PLGA triblock copolymeric micelles: Preparation, pharmacokinetics and distribution in vivo. $J$. Colloid Interface Sci. 2011, 354 (1), 116-123.

27. Sun, M.; Gao, Y.; Guo, C.; Cao, F.; Song, Z.; Xi, Y.; Yu, A.; Li, A.; Zhai, G., Enhancement of transport of curcumin to brain in mice by poly(n-butylcyanoacrylate) nanoparticle. J. Nanopart. Res. 2010, 12 (8), 3111-3122.

28. Zhai, S.; Ma, Y.; Chen, Y.; Li, D.; Cao, J.; Liu, Y.; Cai, M.; Xie, X.; Chen, Y.; Luo, X., Synthesis of an amphiphilic block copolymer containing zwitterionic sulfobetaine as a novel pHsensitive drug carrier. Polym. Chem. 2014, 5 (4), 1285-1297.

29. Shi, Y. Polymeric micelles for drug delivery from synthesis to in vivo studies. Ph.D. thesis, Utrecht University, The Netherlands, 2014, ISBN: 978-90-393-6219-8.

30. Moreira, A. V. $\pi-\pi$ stacked polymeric micelles as carriers of anticancer therapeutics for hematological malignancies. Ph.D. thesis, University Medical Center Utrecht, The Netherlands, 2020.

31. Ruiz de Porras, V.; Layos, L.; Martínez-Balibrea, E., Curcumin: A therapeutic strategy for colorectal cancer? Semin. Cancer Biol. 2020.

32. Postiglione, I.; Chiaviello, A.; Palumbo, G., Enhancing photodynamyc therapy efficacy by combination therapy: dated, current and oncoming strategies. Cancers 2011, 3 (2), 2597-629. 
33. Şueki, F.; Ruhi, M. K.; Gülsoy, M., The effect of curcumin in antitumor photodynamic therapy: In vitro experiments with Caco-2 and PC-3 cancer lines. Photodiagn. Photodyn. Ther. 2019, 27, 95-99.

34. Maletzki, C.; Scheinpflug, P.; Witt, A.; Klar, E.; Linnebacher, M., Targeting Immune-Related Molecules in Cancer Therapy: A Comprehensive In Vitro Analysis on Patient-Derived Tumor Models. BioMed Res. Int. 2019, 2019, 4938285-4938285.

35. Yokoyama, M.; Fukushima, S.; Uehara, R.; Okamoto, K.; Kataoka*, K.; Sakurai, Y.; Okano, $\mathrm{T}$., Characterization of physical entrapment and chemical conjugation of adriamycin in polymeric micelles and their design for in vivo delivery to a solid tumor. Journal of controlled release : official journal of the Controlled Release Society 1998, 50 (1), 79-92.

36. Haider, M. S.; Lübtow, M. M.; Endres, S.; Forster, S.; Flegler, V. J.; Böttcher, B.; Aseyev, V.; Pöppler, A.-C.; Luxenhofer, R., Think Beyond the Core: Impact of the Hydrophilic Corona on Drug Solubilization Using Polymer Micelles. ACS Applied Materials \& Interfaces 2020, 12 (22), 2453124543.

37. Cao, C.; Zhao, J.; Chen, F.; Lu, M.; Khine, Y. Y.; Macmillan, A.; Garvey, C. J.; Stenzel, M. H., Drug-Induced Morphology Transition of Self-Assembled Glycopolymers: Insight into the DrugPolymer Interaction. Chemistry of Materials 2018, 30 (15), 5227-5236.

38. Wang, J.; Mao, W.; Lock, L. L.; Tang, J.; Sui, M.; Sun, W.; Cui, H.; Xu, D.; Shen, Y., The role of micelle size in tumor accumulation, penetration, and treatment. ACS Nano 2015, 9 (7), 71957206.

39. Cabral, H.; Matsumoto, Y.; Mizuno, K.; Chen, Q.; Murakami, M.; Kimura, M.; Terada, Y.; Kano, M. R.; Miyazono, K.; Uesaka, M.; Nishiyama, N.; Kataoka, K., Accumulation of sub-100 nm polymeric micelles in poorly permeable tumours depends on size. Nat. Nanotechnol. 2011, 6 (12), 815823.

40. Sun, Q.; Ojha, T.; Kiessling, F.; Lammers, T.; Shi, Y., Enhancing tumor penetration of nanomedicines. Biomacromolecules 2017, 18 (5), 1449-1459.

41. Lee, J. S.; Feijen, J., Polymersomes for drug delivery: Design, formation and characterization. Journal of Controlled Release 2012, 161 (2), 473-483.

42. Rideau, E.; Dimova, R.; Schwille, P.; Wurm, F. R.; Landfester, K., Liposomes and polymersomes: a comparative review towards cell mimicking. Chemical Society Reviews 2018, 47 (23), $8572-8610$.

43. Williams, D. S.; Pijpers, I. A. B.; Ridolfo, R.; van Hest, J. C. M., Controlling the morphology of copolymeric vectors for next generation nanomedicine. Journal of Controlled Release 2017, 259, 29-39.

44. Bresseleers, J.; Bagheri, M.; Storm, G.; Metselaar, J. M.; Hennink, W. E.; Meeuwissen, S. A.; van Hest, J. C. M., Scale-up of the manufacturing process to produce docetaxel-loaded mPEG-bp(HPMA-Bz) block copolymer micelles for pharmaceutical applications. Org. Process Res. Dev. 2019, 23 (12), 2707-2715.

45. D'Souza, S., A review of in vitro drug release test methods for nano-sized dosage forms. Advances in Pharmaceutics 2014, 2014, 304757.

46. Fu, Y.; Kao, W. J., Drug release kinetics and transport mechanisms of non-degradable and degradable polymeric delivery systems. Expert Opin. Drug Delivery 2010, 7 (4), 429-444.

47. Abouelmagd, S. A.; Sun, B.; Chang, A. C.; Ku, Y. J.; Yeo, Y., Release kinetics study of poorly water-soluble drugs from nanoparticles: Are we doing it right? Mol. Pharmaceutics 2015, 12 (3), 997-1003. 
48. Gil, D.; Frank-Kamenetskii, A.; Barry, J.; Reukov, V.; Xiang, Y.; Das, A.; Varma, A. K.; Kindy, M. S.; Banik, N. L.; Vertegel, A., Albumin-assisted method allows assessment of release of hydrophobic drugs from nanocarriers. Biotechnol. J. 2018, 13 (1), 1700337.

49. Contado, C., Field flow fractionation techniques to explore the "nano-world". Anal. Bioanal. Chem. 2017, 409 (10), 2501-2518.

50. Wagner, M.; Holzschuh, S.; Traeger, A.; Fahr, A.; Schubert, U. S., Asymmetric flow fieldflow fractionation in the field of nanomedicine. Anal. Chem. 2014, 86 (11), 5201-5210.

51. Bolger, G. T.; Licollari, A.; Tan, A.; Greil, R.; Vcelar, B.; Majeed, M.; Helson, L., Distribution and metabolism of Lipocurc ${ }^{\mathrm{TM}}$ (liposomal curcumin) in dog and human blood cells: species selectivity and pharmacokinetic relevance. Anticancer Res 2017, 37 (7), 3483-3492.

52. Venditto, V. J.; Szoka, F. C., Jr., Cancer nanomedicines: so many papers and so few drugs! Adv Drug Deliv Rev 2013, 65 (1), 80-8.

53. Ioannidis, J. P. A.; Kim, B. Y. S.; Trounson, A., How to design preclinical studies in nanomedicine and cell therapy to maximize the prospects of clinical translation. Nat. Biomed. Eng. 2018, 2 (11), 797-809.

54. Lammers, T.; Kiessling, F.; Ashford, M.; Hennink, W.; Crommelin, D.; Storm, G., Cancer nanomedicine: is targeting our target? Nature Reviews Materials 2016, 1 (9), 16069.

55. van der Meel, R.; Lammers, T.; Hennink, W. E., Cancer nanomedicines: oversold or underappreciated? Expert opinion on drug delivery 2017, 14 (1), 1-5.

56. Sant, S.; Johnston, P. A., The production of 3D tumor spheroids for cancer drug discovery. Drug Discovery Today: Technol. 2017, 23, 27-36.

57. Nath, S.; Devi, G. R., Three-dimensional culture systems in cancer research: Focus on tumor spheroid model. Pharmacol. Ther. 2016, 163, 94-108.

58. Lu, H.; Stenzel, M. H., Multicellular tumor spheroids (MCTS) as a 3D in vitro evaluation tool of nanoparticles. Small 2018, 14 (13), 1702858.

59. Astashkina, A.; Grainger, D. W., Critical analysis of 3-D organoid in vitro cell culture models for high-throughput drug candidate toxicity assessments. Adv. Drug Delivery Rev. 2014, 69-70, 1-18.

60. Priwitaningrum, D. L.; Blondé, J.-B. G.; Sridhar, A.; van Baarlen, J.; Hennink, W. E.; Storm, G.; Le Gac, S.; Prakash, J., Tumor stroma-containing 3D spheroid arrays: A tool to study nanoparticle penetration. Journal of controlled release : official journal of the Controlled Release Society 2016, 244 (Part B), 257-268. 




\section{$\underline{\text { Appendices }}$}

Nederlandse Samenvatting

Curriculum Vitae

List of Publications

Acknowledgements 


\section{Nederlandse samenvatting}

In de laatste tientallen jaren zijn polymere micellen die beladen zijn met chemotherapeutica uitgebreid onderzocht voor de therapie van kanker. Polymere micellen zijn zelfgeassembleerde nanostructuren bestaande uit een kern en een schil, met een diameter van $\sim 10-100 \mathrm{~nm}$. In het algemeen is de hydrofobe kern van de micellen geschikt voor het insluiten van hydrofobe geneesmiddelen, terwijl de hydrofiele schil colloïdale stabiliteit en stealth eigenschappen geven ${ }^{1-7}$. Deze structuren zouden voldoende stabiel moeten zijn en het geneesmiddel vast moeten kunnen houden in de bloedcirculatie om de tumor te kunnen bereiken en daarmee klinisch interessant te zijn. Diverse fysisch-chemische strategieën zijn toegepast om stabiele micellen te ontwikkelen, zoals het gebruik van specifieke fysische en chemische interacties binnen de hydrofobe kern van de micellen ${ }^{8-12}$. Vooral zogenaamde $\pi-\pi$ interacties zijn veelbelovend om de stabiliteit van geneesmiddel-beladen zelfgeassembleerde micellen te verbeteren ${ }^{11,13}$. Polymere micellen met voldoende stabiliteit en geneesmiddelretentie in de circulatie kunnen de systemische blootstelling van veel antikanker-geneesmiddelen verhogen. Anderzijds is het ook belangrijk om te vermelden dat nanomedicijnen die slechts als oplosverbeteraars fungeren (zoals Genexol en Abraxane) ook een waardevolle therapeutisch bijdrage kunnen leveren in de kliniek, ondanks de nauwelijks verbeterde farmacokinetiek ${ }^{6,14-16}$.

Het onderzoek dat in dit proefschrift wordt beschreven is gericht op de optimalisatie en diepgaande karakterisering van polymere micellen gebaseerd op poly(ethyleenglycol)- $b$ poly(N-2-benzoyloxypropylmethacrylamide) (mPEG- $b$-p(HPMA-Bz). De micellen gebaseerd op dit blokcopolymeer zijn zeer stabiel in de bloedcirculatie en houden geneesmiddelen vast als gevolg van $\pi-\pi$ interacties in de hydrofobe kern ${ }^{17-19}$. Dit project is uitgevoerd in het kader van het Nanomed consortium, in nauwe samenwerking met Dr. Bresseleers die recent haar proefschrift met betrekking tot opschaalbare processen ten behoeve van de bereiding van nanocarriers heeft gepubliceerd en met succes verdedigd ${ }^{20}$.

Hoofdstuk 1 geeft een korte inleiding met betrekking tot geneesmiddelafgiftesystemen op basis van nanodeeltjes, in het bijzonder polymere micellen voor tumorbehandeling. Er wordt een korte uitleg gegeven over de nanoprecipitatie-techniek in een batchproces en met behulp van microfluidics om polymere micellen te bereiden. De farmacokinetiek en -dynamiek van geneesmiddelafgiftesystemen worden kort bediscussieerd. Ook wordt curcumine geïntroduceerd als een natuurlijke stof met antikanker-activiteit. Tenslotte wordt het belang uiteengezet van 3D tumorsferoïden als celkweekplatform dat de micro-tumoromgeving beter benadert dan klassieke 2D celkweekmodellen.

Curcumine is een natuurlijk polyfenol met veelzijdige farmacologische eigenschappen, onder andere potentiële therapeutische activiteit tegen kanker ${ }^{21}$. De extreem lage oplosbaarheid in water, slechte biologische beschikbaarheid na orale toediening en moleculaire instabiliteit hebben echter een negatieve invloed op de farmaceutische eigenschappen en verhinderen daardoor de toepassing in kankertherapie ${ }^{21-25}$. Hoofdstuk 2 geeft een literatuuroverzicht met betrekking tot de farmacokinetiek (PK) van intraveneus toegediende curcumine-beladen nano-afgiftesystemen in twee preklinische diermodellen (muizen en ratten). Het is interessant dat de meerderheid van de nanocarriers slechts marginaal de area-under-the-curve verbetert, met een factor 1,3-5 ten opzichte van vrij curcumine. Dit betekent dat curcumine niet goed wordt vastgehouden in de deeltjes en de nanomedicijnen in essentie fungeren als solubilisator. Studies die zowel PK als in vivo antitumorgegevens rapporteren, laten een 
sterkere tumorgroeiremming zien in groepen die behandeld werden met curcuminenanoformuleringen dan met vrij curcumine, wat overeenkomt met de licht verbeterde farmacokinetiek. Nadere analyse geeft een negatieve correlatie tussen de relatieve tumorgroeiremming en de totale geïnjecteerde dosis, in groepen die behandeld zijn met de curcumine nanoformuleringen en vrij curcumine. Het is mogelijk dat een hogere dosis vrij curcumine tumorgroeiremming uitoefent in muizen ondanks de ongunstige PK. De conclusie is dat zelfs nanoformuleringen die slechts als solubilisator fungeren een aanzienlijk farmacodynamisch effect sorteren, in de zin van het reduceren van het tumorvolume ten opzichte van de controlegroep. Een en ander moet echter beschouwd worden in het licht van de beperkt beschikbare PK-PD gegevens, de grote variatie in de karakteristieken van de nanoformuleringen zoals het afgifteprofiel, en sommige zwaktes in de experimentele opzet. Ook het effect van de snelle verdeling van curcumine naar bloedcellen kort na injectie zou moeten worden meegenomen in PK studies. Meer en goed opgezette studies zijn derhalve nodig om tot nauwkeurigere conclusies te kunnen komen.

In hoofdstuk 3 zijn de parameters onderzocht die effect hebben op de grootte van mPEG- $b$ p(HPMA-Bz) polymere micellen, met als doel deze in te stellen tussen 25-100 nm. Polymeren met verschillende molecuulmassa's van de hydrofiele ( $\mathrm{mPEG}_{5 \mathrm{kDA}}$ of $\left.\mathrm{mPEG}_{2 \mathrm{KDa}}\right)$ en hydrofobe blokken $(1,0-20,6 \mathrm{kDa})$ zijn gesynthetiseerd en gebruikt in dit hoofdstuk. Er werd batchmatige nanoprecipitatie toegepast om micellen te bereiden van verschillende grootte, middels variatie in de formuleringsparameters zoals molecuulmassa van het blokcopolymeer, hydrofiele-hydrofobe verhouding en homopolymeergehalte, en in de procesparameters (bijv. snelheid van samenvoegen en type oplosmiddel). Dynamische lichtverstrooiing en transmissie elektronenmicroscopie bevestigden de kern-schil structuur van de micellen. De hydrofobe kern van de micellen bevond zich in samengetrokken toestand conform de Gennes-Alexander theorie en schalingswetten. Vergelijkbare schildiktes voor alle micellen $(10 \mathrm{~nm})$ was indicatief voor de vorming van zogenaamde kortharige micellen. De grootte van de micellen was afhankelijk van het molecuulgewicht van de polymeren en de procesmethode. Samengevat kon de micelgrootte worden gereduceerd door de polymerisatie goed te sturen en een hoge hydrofiele-hydrofobe verhouding in te stellen. Immers, tijdens de synthese van het blokcopolymeer wordt er tevens een hoeveelheid homopolymeer gevormd, welke zich verdeelt in de kern van de micellen, leidend tot een grotere hydrofobe kern. De optimalisatie van de synthese van de macro-initiator resulteerde derhalve in het minimaliseren van het gehalte homopolymeer en daarmee in kleinere micellen. In termen van procescondities konden kleinere micellen met een smallere grootteverdeling verkregen worden door gebruik te maken van organische oplosmiddelen met relatief snel mengvermogen met water (bijv. aceton, acetonitril, ethanol, tetrahydrofuraan en dioxaan), samen met snelle menging en hoge polymeerconcentratie, vergeleken met oplosmiddelen met trager mengvermogen (dimethylformamide en dimethylsulfoxide). Dit hoofdstuk laat zien dat de omvang van zelf-geassembleerde mPEG$b$-p(HPMA-Bz) micellen kan worden ingesteld binnen een bereik wat geschikt is voor geneesmiddelafgifte en -gerichtheid naar de tumor. Daarnaast zijn de parameters geïdentificeerd die kritisch zijn voor de vorming van de micellen en voor de reproduceerbare bereiding tot de gewenste omvang.

In hoofdstuk 3 werd bediscussieerd dat de omvang van de gevormde mPEG- $b$-p(HPMA-Bz) micellen afhankelijk is van het mengproces gedurende de nanoprecipitatie, met name de snelheid van mengen van het organisch oplosmiddel met de waterfase. Daarom had hoofdstuk 4 als doel om de grootte en morfologie van de micellen gebaseerd op mPEG- $b$ - 
p(HPMA-Bz) in te stellen met behulp van een microfluidic techniek. In dit hoofdstuk werden polymeren gebruikt met een vastgesteld hydrofiel blok $\left(\mathrm{mPEG}_{5 \mathrm{kDa}}\right)$ maar verschillende molecuulmassa's van het hydrofoob blok (17,5, 10,0, 5,2 en 2,7 kDa). Nanostructuren zijn verkregen onder verschillende omstandigheden door nanoprecipitatie onder goedgedefinieerde mengcondities met behulp van microfluidics. Een toename in de deeltjesgrootte van de micellen van 55 tot $90 \mathrm{~nm}$ werd waargenomen bij lagere polymeerconcentratie en relatief lage stroomsnelheden, en zelfs de vorming van blaasjes in het geval van het polymeer met het grootste hydrofobe blok $(17,5 \mathrm{kDa})$. Bij het polymeer met het kleinste hydrofobe blok $(2,7 \mathrm{kDa})$ nam de omvang van de nanodeeltjes toe van 35 tot 70 $\mathrm{nm}$ met afnemende stroomsnelheden, ongeacht de polymeerconcentratie. Tevens werden bij lage stroomsnelheid polymersomen gevormd met dit copolymeer. De polymeren met een hydrofoob blok van 10 of $5,2 \mathrm{kDa}$ zelf-assembleerden bij verschillend toegepaste stroomsnelheden voornamelijk tot micellen die nauwelijks grootteverschillen vertoonden binnen het bereik van de geteste polymeerconcentraties $(5-20 \mathrm{mg} / \mathrm{mL})$. Overeenkomstig hoofdstuk 3, waarbij een batchproces gebruikt werd, demonstreert dit hoofdstuk dat de zelfassemblage van mPEG- $b$-p(HPMA-Bz) blokcopolymeren met behulp van microfluidics eenvoudig ingesteld kan worden in termen van omvang en morfologie van de micellen.

In hoofdstuk 5 werden curcumine-beladen $\mathrm{mPEG}_{5 \mathrm{kDa}}-b$-p(HPMA-Bz) micellen bereid met het oog op de verbetering van de farmacokinetiek van curcumine. Vergelijkbare mPEG- $b$ p(HPMA-Bz) copolymeren als in hoofdstuk 4 werden gebruikt, met vastgesteld hydrofiel blok $\left(\mathrm{mPEG}_{5 \mathrm{kDa}}\right)$ en hydrofobe blokken van 5,2, 10,0 en 17,1 kDa. Bij een belading van 9\% curcumine lieten micellen met het hoogste molecuulgewicht van p(HPMA-Bz) betere retentie van curcumine zien in fosfaatgebufferde zoutoplossing (PBS) bij $37^{\circ} \mathrm{C}$, ten opzichte van micellen gebaseerd op blokcopolymeren met kleinere hydrofobe blokken. Evaluatie van de stabiliteit van curcumine-beladen micellen in plasma met behulp van asymmetrische stroom-veldstroom fractionering (AF4) toonde aan dat er geen verandering in micelgrootte optrad gedurende 24 uur, daarmee de deeltjesstabiliteit bevestigend. Desalniettemin kwam curcumine in relatief hoge mate vrij uit de micellen die bereid waren met de laagste molecuulmassa van p(HPMA-Bz), overeenkomstig de stabiliteitsstudie in PBS. AF4-analyse toonde aan dat albumine een belangrijke rol speelt bij het solubiliseren van het vrijgekomen curcumine. De resultaten gaven een indicatie dat micellen die bereid waren uit polymeren met de grotere molecuulmassa's van het hydrofobe blok betere curcumineretentie vertoonden binnen de kern van de micellen. In vitro celstudies lieten zien dat de curcumine-beladen $\mathrm{mPEG}_{5 \mathrm{kDa}}-b$-p(HPMA-Bz) $17,1 \mathrm{kDa}$ micellen werden geïnternaliseerd door verschillende kankercellen, wat resulteerde in celtoxiciteit. In vivo werden curcumine-beladen $\mathrm{mPEG}_{5 \mathrm{kDa}}{ }^{-}$ $b$-p(HPMA-Bz) ${ }_{17,1 \mathrm{kDa}}$ micellen intraveneus toegediend bij muizen $(50 \mathrm{mg} / \mathrm{kg}$ curcumine), wat resulteerde in verlengde circulatiekinetiek, met meer dan $50 \%$ van de geïnjecteerde dosis aanwezig in de circulatie na 24 uur. Daarentegen was de halfwaardetijd van curcumine aanzienlijk korter dan van de micellen $\left(\mathrm{t}_{1 / 2 \alpha}=0,11, \mathrm{t}_{1 / 2 \beta}=2,5\right.$ uur), terwijl de halfwaardetijd substantieel verbeterde ten opzichte van vrij curcumine $\left(\mathrm{t}_{1 / 2 \alpha}=0,023 \text { uur }\right)^{26-28}$. Ondanks de verlengde farmacokinetiek van curcumine als gevolg van de insluiting in deze micellen en vergelijkbaar farmacokinetisch profiel als andere nanoformuleringen van curcumine die gerapporteerd zijn in de literatuur, kon er geen therapeutisch effect worden waargenomen in neuroblastoma-dragende muizen. Het gebrek aan in vivo cytotoxische effecten zou kunnen worden toegeschreven aan de lage gevoeligheid van de Neuro2A cellen voor curcumine.

In hoofdstuk 6 werden $\mathrm{mPEG}_{5 \mathrm{kDa}}-b$-p(HPMA-Bz) micellen bereid met twee uiteenlopende deeltjesgroottes, en beladen met curcumine om hun werking in $2 \mathrm{D}$ en $3 \mathrm{D}$ celkweekmodellen 
te bestuderen. Deze (grote en kleine) micelaire formuleringen lieten vergelijkbare curcumineretentie zien bij 5,0\% belading in PBS (80\% curcumineretentie in 168 uur). $\mathrm{mPEG}_{5 \mathrm{kDa}}-b-\mathrm{p}(\mathrm{HPMA}-\mathrm{Bz})$ micellen vertoonden uitstekende cytocompatibiliteit in zowel kanker- als niet-kankercellen (hoofdstukken 5 en 6). Curcumine-beladen micellen lieten een hogere 50\%-remmende concentratie $\left(\mathrm{IC}_{50}\right)$ zien ten opzichte van vrij curcumine, waarschijnlijk omdat vrij curcumine passief diffundeert door het celmembraan, terwijl curcumine deels werd vastgehouden en langzaam afgegeven door de micellen en daarmee volledige farmacologische activiteit werd verminderd van het beschikbare curcumine. Daarnaast lieten de kleine micellen lagere $\mathrm{IC}_{50}$-waarden zien dan de grote micellen, wat mogelijk te wijten is aan de snellere afgifte van curcumine uit de kleinere micellen in het biologisch medium zoals waargenomen uit de stabiliteitsstudie met de AF4-techniek (hoofdstuk 5). Studie van de cel-opname in Panc-1 cellen liet zien dat zowel kleine als grote micellen werden opgenomen door de cellen, waarbij de internalisatie beter bleek te zijn voor de grotere micellen op basis van kwalitatieve analyse. Belangrijk is dat kleine micellen in grotere mate en diepere penetratie lieten zien in 3D stroma-bevattende sferoïden dan de grote micellen. Deze inleidende studie toont het potentieel aan van kleine micellen om een sterker therapeutisch effect te sorteren, hetgeen gevalideerd moet worden in aanvullende experimenten. 



\section{Curriculum Vitae}

Mahsa Bagheri was born on January $6^{\text {th }}, 1988$, in Mashhad, Iran. She obtained her Pharm. D degree from Mashhad University of Medical Sciences in 2013. Then she worked for two years as a manager in a cardiovascular hospital pharmacy. She has always been interested in novel drug delivery systems, and this drove her to apply for a Ph.D. position in the department of pharmaceutical sciences at Utrecht University in 2016. During her Ph.D., she was part of the Nanomed consortium; a multidisciplinary network consisted of several academic and industrial partners. The objective of the network was to develop nanoparticles for biomedical applications. Her research topic was focused on development and optimization of polymeric micelles based on poly(ethylene glycol)- $b$-poly(N-2-benzoyloxypropyl methacrylamide) (mPEG- $b$-p(HPMA-Bz)) as a delivery system for hydrophobic drugs. This system could be suitable for up-scale manufacturing processes and clinical translation. The research was supervised by Prof. dr. ir. Wim Hennink, Dr. Michal Heger and Dr. Rene van Nostrum. She had the opportunity to collaborate with several institutions across Europe, which helped her broaden her network and knowledge. The outcome of her research project is presented in this thesis. Since January 2021, she joined the research group of Prof. dr. Enrico Mastrobattista to explore further in the field of CRISPR delivery. 



\section{List of Publications}

\section{Publications from this thesis}

Bagheri, M.; Fens, M. H. A. M.; Kleijn, T. G.; Capomaccio, R.; Mehn, D., Krawczyk, P. M.; Scutigliani, E. M.; Gurinov, A.; Baldus, M.; van Kronenburg, N. C. H.; Kok, R. J.; Heger, M.; van Nostrum, C. F.; Hennink W. E., In vitro and in vivo Studies on HPMA-based polymeric micelles loaded with curcumin. Mol. Pharmaceutics 2021, 18 (3), 1247-1263.

Bresseleers, J.; Bagheri, M.; Lebleu, C.; Lecommandoux, S.; Sandre, O.; Pijpers, I. A. B.; Mason, A. F.; Meeuwissen, S.; van Nostrum, C. F.; Hennink, W. E.; Hest, J. C. M., Tuning size and morphology of mPEG- $b$-p(HPMA-Bz) copolymer self-assemblies using microfluidics. Polymers 2020, 12 (11), 2572.

Bagheri, M.; Bresseleers, J.; Varela-Moreira, A.; Sandre, O.; Meeuwissen, S. A.; Schiffelers, R. M.; Metselaar, J. M.; van Nostrum, C. F.; van Hest, J. C. M.; Hennink, W. E., Effect of formulation and processing parameters on the size of mPEG-b-p(HPMA-Bz) polymeric micelles. Langmuir 2018, 34 (50), 15495-15506.

\section{Other publications}

Ojha, T.; Hu, Q.; Colombo, C.; Wit, J.; van Geijn, M.; van Steenbergen, M. J.; Bagheri, M.; Königs-Werner, H.; Buhl, E. M.; Bansal, R.; Shi, Y.; Hennink, W. E.; Storm, G.; Rijcken, C. J. F.; Lammers, T., Lyophilization stabilizes clinical-stage core-crosslinked polymeric micelles to overcome cold chain supply challenges. Biotechnol J. 2021, 2000212.

Sheybanifard, M.; Beztsinna, N.; Bagheri, M.; Miriam Buhl, E.; Bresseleers, J.; VarelaMoreira, A.; Shi, Y.; van Nostrum, C. F.; van der Pluijm, G.; Storm, G.; Hennink, W. E.; Lammers, T.; Metselaar, J. M., Systematic evaluation of design features enables efficient selection of $\Pi$ electron-stabilized polymeric micelles. Int. J. Pharm. 2020, 119409.

Bresseleers, J.; Bagheri, M.; Storm, G.; Metselaar, J. M.; Hennink, W. E.; Meeuwissen, S. A.; van Hest, J. C. M., Scale-up of the manufacturing process to produce docetaxel-loaded mPEG- $b$-p(HPMA-Bz) block copolymer micelles for pharmaceutical applications. Org. Process Res. Dev. 2019, 23 (12), 2707-2715.

Amin, M.; Bagheri, M.; Mansourian, M.; Jaafari, M. R.; Ten Hagen, T. L., Regulation of in vivo behavior of TAT-modified liposome by associated protein corona and avidity to tumor cells. Int. J. Nanomed 2018, 13, 7441-7455.

Bagheri, M.; Mohammadi, M.; Steele, T. W.; Ramezani, M., Nanomaterial coatings applied on stent surfaces. Nanomedicine 2016, 11 (10), 1309-26. 



\section{Acknowledgements}

I am filled with utmost joy to express my gratitude to people who have helped me and contributed to this project through these years. Your enormous support was the reason for me to cross the finish line eventually.

Dear Wim, your dedication and scientific knowledge is truly impressive, and I have learned a lot from all the feedback that I received during our progress meetings and writing papers. It would have been impossible to achieve this much without your constant support. I should admit that if I were to chose a supervisor for my Ph.D. project again, it would definitely be you.

Also, I would like to thank Rene, for his kind support during this project. You made it possible for me to go to the Joint Research center (JCR) in Italy and learn firsthand about AF4 as a promising separation technique. Also, your comments during the progress meetings helped me a lot to learn in-depth about polymer chemistry.

Dear Michal, you later joined this project to collaborate with us on developing micellar formulations for curcumin delivery. I appreciate all the commitment and scientific feedback from you and never forget all the emails after 00.00 and meetings with you and Wim. I am delighted that, in the end, we managed to achieve great publications.

I would like to sincerely thank our dedicated technicians, Mies, Roel and Joep, in our department. It goes without saying that the lab can not be operational without your constant watch and maintenance. You are always there to give us a hand for everything. I cannot imagine the synthesis lab without Mies and cell culture and biochemistry labs without Roel. Mies, I have always loved how you laugh out loud from the bottom of your heart and making the lab work fun. I am also grateful that Joep taught me the basics of imaging before leaving the department. Finally, I am happy to continue my work in the biochemistry and bacterial lab with Roel.

Dear Barbara, thanks a lot for arranging all the meetings for me with my supervisors and collaborators. You are always friendly, accessible and helpful with paperwork.

Dear Marcel, thanks for helping me with all the animal work for this project. I enjoyed talking with you when we were playing with mice.

Dear Jai and Marcel, I would like to thank you for your immense help with the spheroid project. I learnt a lot about complex cell cultures through this collaboration.

Dear Robin and Dora, thanks for the fruitful collaboration and for teaching me how to use AF4 system. My stay in Ispra was fun and memorable. Dear Robin, I am happy that we are still in touch and exchange scientific ideas.

Dear Przemek and Enzo, thanks for helping me with cell studies and imaging. We managed to have a great collaboration during the Corona time.

It has been a pleasure to be part of the Nanomed ITN network and work with many outstanding early-stage scientists from all over the world. Doing a Ph.D. project in such an international and multidisciplinary consortium is a rare and valuable opportunity. I enjoyed 
all the scientific and friendly discussions that I had with you guys. Dear Jerry, Molood, Shirin, Jaleesa, Mona, Roxane, Esra, Vangelis, Shoupeng, Roberta, Jelle, Conor, Vijay and Mike, I couldn't have imagined better fellow scientists who are as scientific as caring. I would always follow your progress and cheer for your success. My special thanks go to Jaleesa with whom I have worked closely since the beginning of this project. I am happy to say that we collaborated very efficiently and our joint effort paid off well, and we published several articles together. It wouldn't have possible without your help. You are a very cheerful and cool person. I was fortunate to have you by my side during this journey. I wish the best for you, and hopefully, we can work together again one day. Sweet Jerry, you are the first person that I met on the first day of work. You were a great accompany during Nanomed trips. Your love for science, anime and Japanese culture has always amazed me. Gambatte! To my Iranian friends in Nanomed, Molood and Shirin, it was a pleasant surprise that three out of fifteen students who joined this Network were Iranian. Shirin jan, I really enjoyed our trip and all the shopping in London. I hope we can do this again in future. Molood jan, thanks for being a fantastic host when I was in Gent. I never forget the Persian New Year evening with you and your lovely family. Dear Esra, thanks so much for helping me during my visit to Bordeaux. You kindly helped me with my secondment and showed me around the city. Dear Jan and Silvie, thanks for giving me the opportunity to collaborate with you. I learnt a lot about different drug delivery systems and manufacturing nanomedicines from you. Dear Sebastian and Olivier, thank you for sharing your knowledge about microfluidics with me. Especially, I would like to thank Olivier for our short trip to Saint-Émilion.

To my Iranian friends in our department and DDW building, thank you so much for all the emotional support and fun time that we had together. I would like to especially thank Marzieh, Neda, Negar, Mahnaz and Sanaz for their support and excellent advice during my job hunting. I will never forget your endless support. Dear Maryam, you were an independent and hardworking student. I am happy that your hard work paid off, and you published the data you generated during your internship. Best of luck with your Ph.D. project. Thanks, Amer, Adel and Pouya, for being my alternative lunch buddies.

It is a great pleasure to thank my friends and colleagues at the department of pharmaceutical sciences. Dear Yan, Xiangjie, Bo, Menshang and Yong, it is a privilege to be the only nonChinese speaking person in the Delta sink area. We always had a great time together in the lab. Dear Yan, you are a kind and friendly person, and I am delighted that I could be of help with some parts of your Ph.D. project. Dear Yanna, I will never forget our journey to Spain and Italy. We had memorable experiences exploring different foods and cultures together. I completely trust the Chinese travel tips! Dear Erik, the great borrel master, it is never dull around you, and I have always enjoyed your humour. I hope you can soon throw a great borrel celebrating the end of Corona time. Dear my Italian friends, Cristina, Martina, Blessing and Marko, you have a unique power to light up the department with your energy and humour. I am happy that joyful and energetic Italians like you have taken over the department. Dear Thijs, I miss your company often since you hardly come to the department. I wish you the best of luck with your Ph.D. project. Sweet Barbara, you have a kind heart like no others. I enjoyed the time we had together eating ice cream and strolling alongside the canals during snowfall. Dear CRISPR fellows Danny, Johanna and Mert, I am so lucky to be part of the CRISPR group now. I really appreciate all the help that I get from you. You are smart, skillful and have great ideas and suggestions all the time. Let's make the geneediting great with Casy \& Mady! Dear Lucia and Karina, thanks a lot for the invaluable tips for job searching. Dear Aida, you were my first lab buddy and thanks to you, I learnt how to 
synthesis the mPEG-HPMA-Bz polymer and prepare the micelles when I just started my Ph.D. I also like to thank my colleagues who I enjoyed talking to and sharing lunch with them. Dear Boning, you have always approached me with your funny attitude and thanks for helping me with Photoshop. Dear Locus, Charis, Désirée', Sjaak, Dandan, Yinan, Haili, Carl and Lies, some of you have already left the department, but the memories will always stay with me.

My lovely paranymphs, Blessing and Ada, I really appreciate your help and support with organizing this ceremony although you were so busy with your work. Dear Blessing, I couldn't have been luckier to meet such a passionate and cheerful soul like you. We became close friends, spent so much time together, shared many memories, and helped each other out on gloomy days. You are irreplaceable in my heart. I know that I promised to give you a page of acknowledgement, but I hope you could accept this much for now. Dear Ada, you are always to the point and have an excellent knowledge of many subjects, which makes talking to you very interesting. Also, I enjoy my horseback riding lessons with a talented rider like you. It is nice to share a hobby with someone else in the department.

I am also really grateful to Annemiek, who has always given me emotional support and continuously motivated me to learn Dutch. I still can remember how nervous I was when I had to move from the campus and live with you, but I was the luckiest to find and get to know you.

Dear Yaser and Neda, I could not have started this journey and come to live in the Netherlands if it was not for you. You opened the door of opportunities for me and put me on a path that leads me to stand here now. For that, I am eternally grateful. Having caring relatives like you soothes my heart and makes my homesickness go away.

Last but not least, I would like to thank my family from the bottom of my heart. Your endless support and prayers were the reason to go forward in life successfully. Dear grandma and grandpa, you set great examples as hardworking and committed people. Your love and prayers have always kept me safe. Dear daddy, you have always encouraged me to be strong and brave since I was a little child and fight for things no matter any gender inequality. Words cannot express my gratitude for everything that you have done for me. Dear mom, you trusted me and supported me to pursue my dreams, even if it meant being far apart. Being away from you was hard sometimes, but it helped me become more independent and strong like you. Mina, my lovely sister, I always admire your sense of humour and perception toward life. I enjoyed all our secret chats between sisters. We still need to travel together in Europe, just the two of us, and I bet it would be lots of fun.

If I look back at this trajectory of my life, I see tones of new experiences, ups and downs, as I was advancing in my life. It felt like a river that sets to flow non-stop with all its force to finally get to the tranquil sea. As time passed, I could not believe the magnitude of changes that I went through as if I am not the same person I used to be years earlier. Now, I understand the meaning of this quote that "The Only Thing That Doesn't Change Is The Change Itself."

Mahsa 